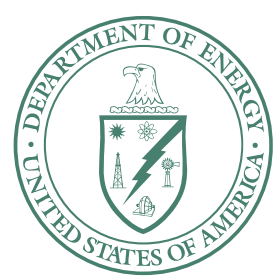

U.S. Department of Energy

Idaho Operations Office

\title{
Remedial Action Report for Operable Units 6-05 and 10-04, Phase III
}

August 2007 
DOE/ID-11315

Revision 0

Project No. 23368

\section{Remedial Action Report for Operable Units 6-05 and 10-04, Phase III}

August 2007

Prepared for the U.S. Department of Energy DOE Idaho Operations Office 



\begin{abstract}
This Phase III remedial action report addresses the remediation of lead-contaminated soils found at the Security Training Facility STF-02 Gun Range at the Idaho National Laboratory Site.

Phase I, consisting of developing and implementing institutional controls at Operable Unit 10-04 sites and developing and implementing Idaho National Laboratory Site-wide plans for both institutional controls and ecological monitoring, was addressed in a previous report. Phase II will remediate sites contaminated with trinitrotoluene and Royal Demolition Explosive. Phase IV will remediate hazards from unexploded ordnance.
\end{abstract}




\section{EXECUTIVE SUMMARY}

The remedial design/remedial action for Operable Unit 6-05 (Waste Area Group 6) and Operable Unit 10-04 (Waste Area Group 10) at the Idaho National Laboratory Site - collectively called Operable Unit 10-04 - has been divided into four phases. Phase I consisted of developing and implementing institutional controls at Operable Unit 10-04 sites and developing and implementing Idaho National Laboratory Site-wide plans for both institutional controls and ecological monitoring. Phase II will remediate sites contaminated with trinitrotoluene and Royal Demolition Explosive. Phase III remediated lead contamination at a gun range, and Phase IV will remediate hazards from unexploded ordnance.

This Phase III remedial action report addresses the remediation of lead-contaminated soils found at the Security Training Facility STF-02 Gun Range. Remediation of the gun range included excavating contaminated soils; physically separating copper and lead for recycling; returning to the site separated soils below the remediation goal; stabilizing contaminated soils, as required, and disposing of the separated soils that exceeded the remediation goal; encapsulating and disposing of creosote-contaminated railroad ties that were characteristic for lead; disposing of creosote-contaminated railroad ties and power poles that were not characteristic for lead; removing and disposing of the wooden building and asphalt pads found at the gun range; sampling and analyzing soil to guide the excavation and determine when the remediation goals had been achieved; backfilling and contouring the excavated area; and reseeding the impacted areas with native species. A recycling facility willing to accept the separated copper and lead could not be located; therefore, this waste stream was shipped off-Site for stabilization and disposal.

The remediation goal of $400 \mathrm{mg} / \mathrm{kg}$ for lead was achieved for the site; therefore, no institutional controls will be required for the area and the site will require no action beyond that which has been completed. 


\section{CONTENTS}

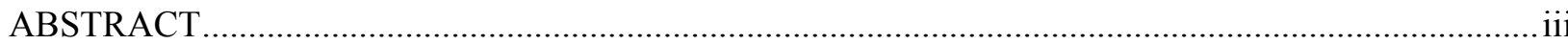

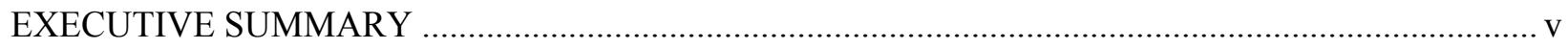

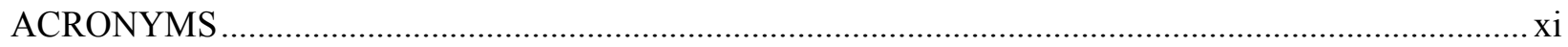

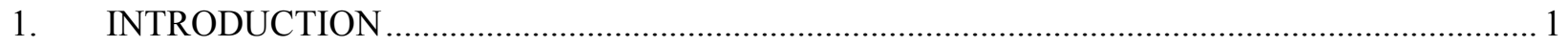

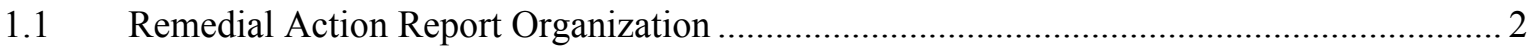

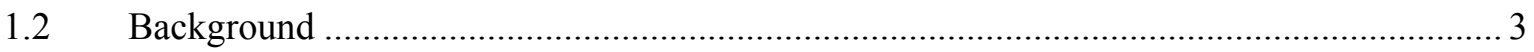

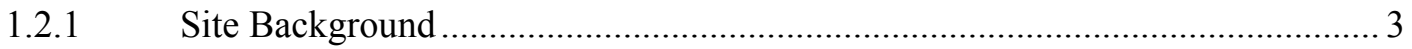

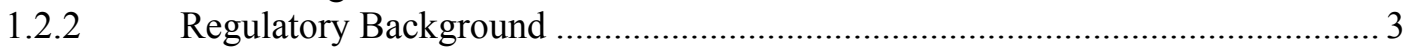

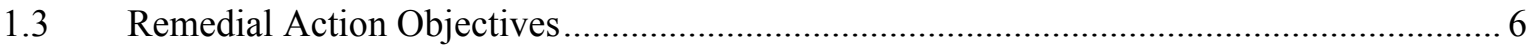

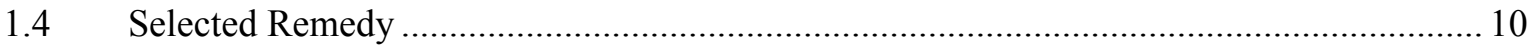

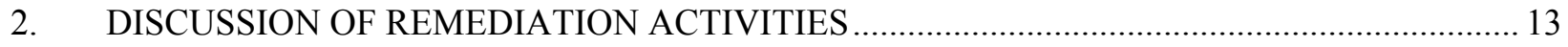

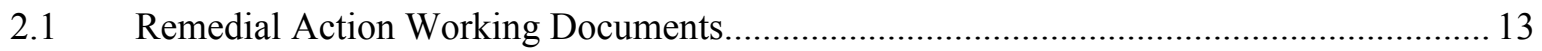

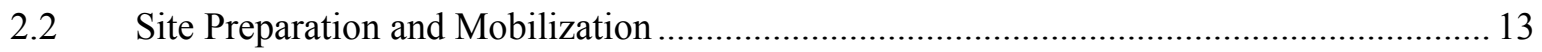

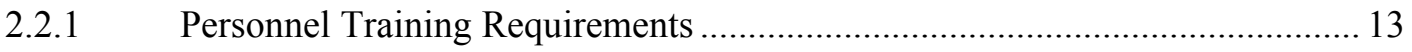

2.2.2 Field Operations and Staging of Equipment and Supplies ................................ 14

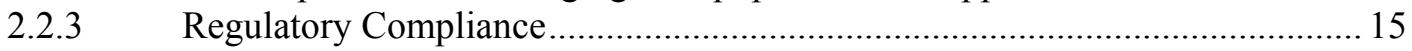

2.2.4 Work Control Requirements at the Idaho National Laboratory Site................. 15

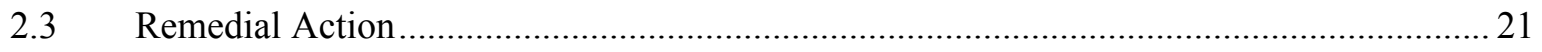

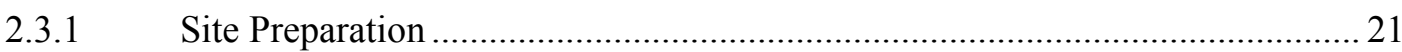

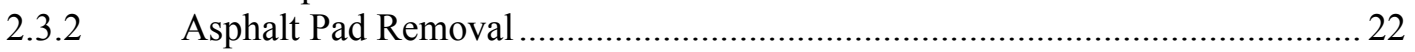

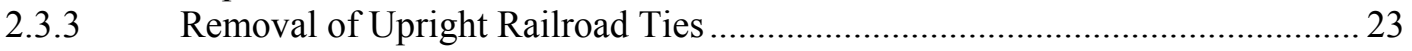

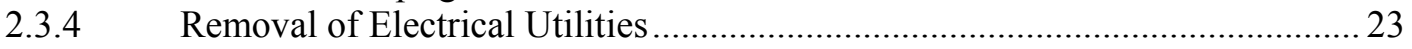

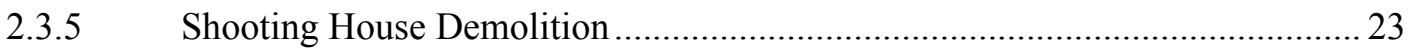

2.3.6 Removal of Test Stand and Burn Barrel ..................................................... 23

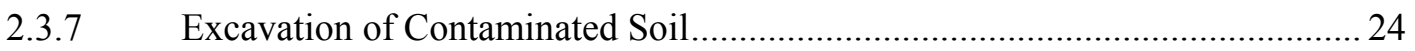

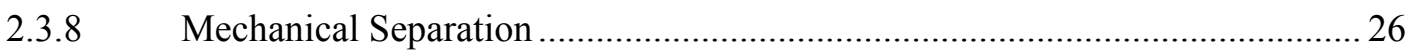

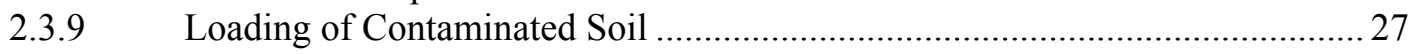

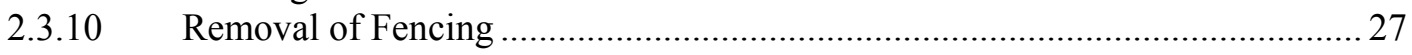

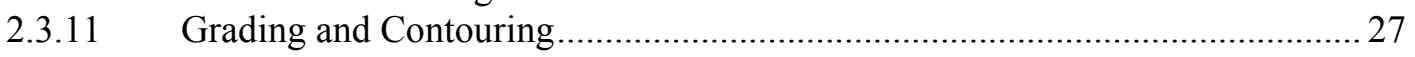

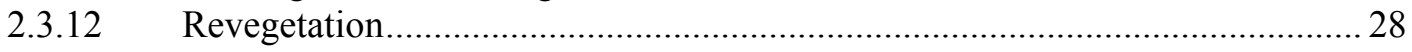

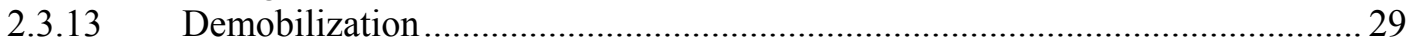




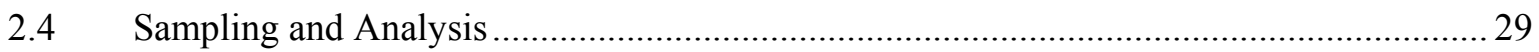

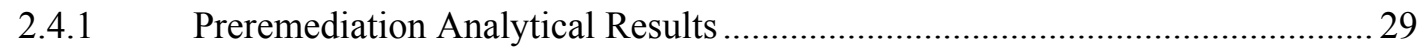

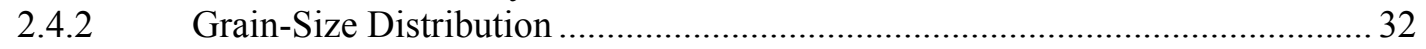

2.4.3 Analytical Results Supporting the Remediation Action.................................... 33

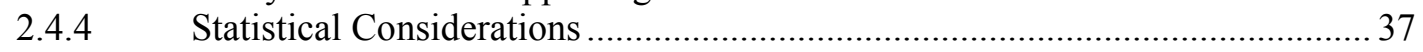

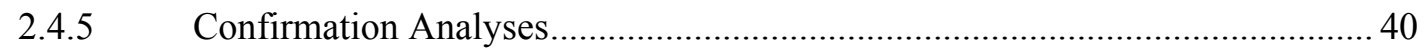

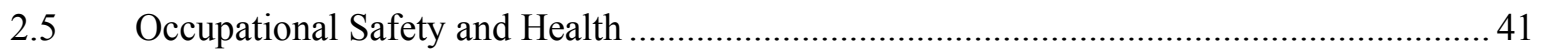

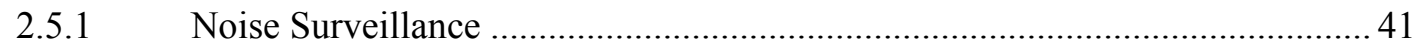

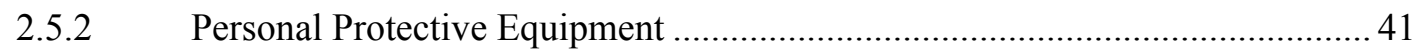

2.5.3 Monitoring for Lead Exposure ........................................................................ 41

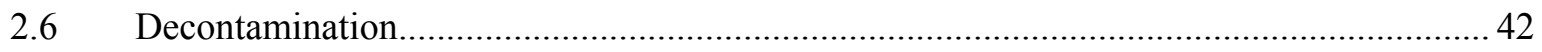

2.7 Lessons Learned and Notable Practices ............................................................... 42

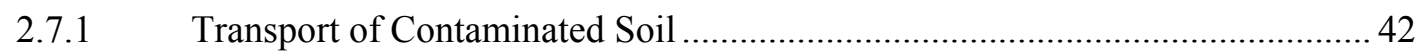

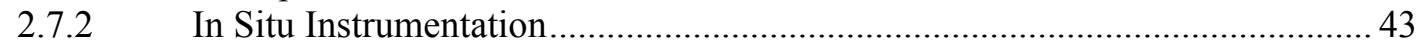

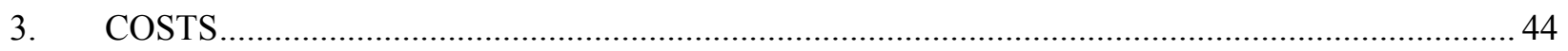

4. MODIFICATIONS TO THE REMEDIAL ACTION WORK PLAN .......................................... 45

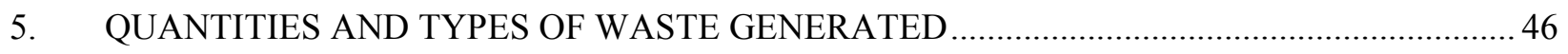

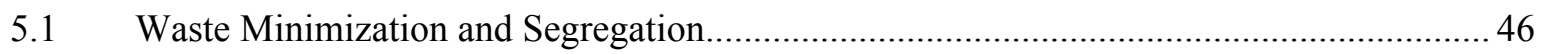

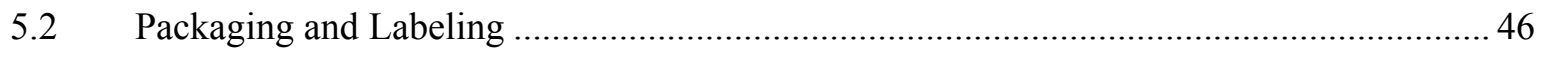

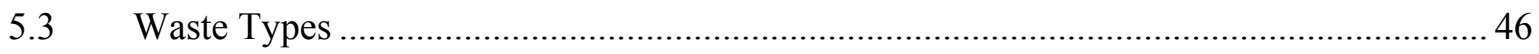

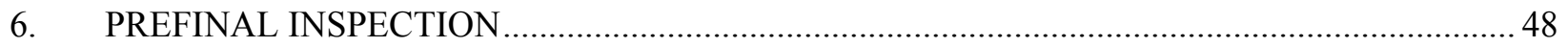

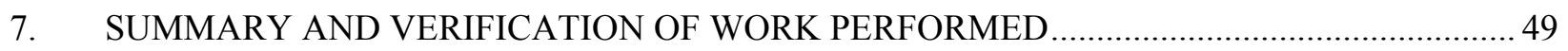

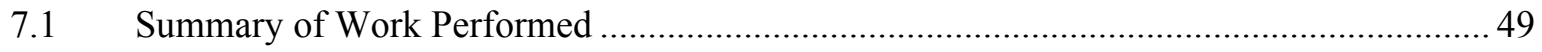

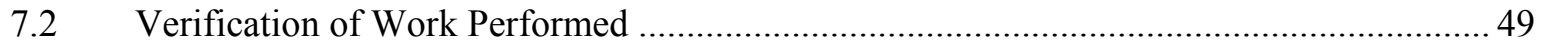

7.3 Performance Standards and Construction Quality Control ........................................... 49

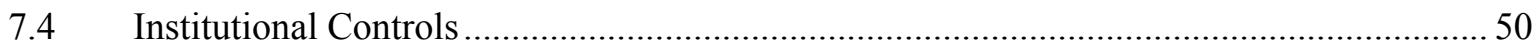

8. CERTIFICATION THAT THE REMEDY IS OPERATIONAL AND FUNCTIONAL ................. 51

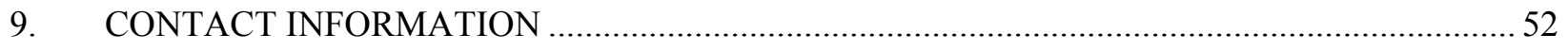

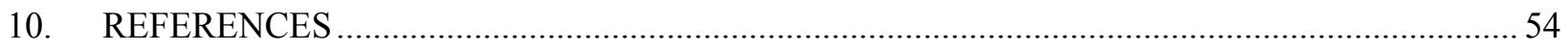


Appendix A-STF-02 Gun Range Photographic Record of Remediation Activities

Appendix B —-Remedial Action Analytical Summaries …........................................................... B-1

Appendix C - Remedial Action Prefinal Inspection Checklist........................................................ C-1

Appendix D—STF-02 Gun Range Site Contour Drawings .......................................................... D-1

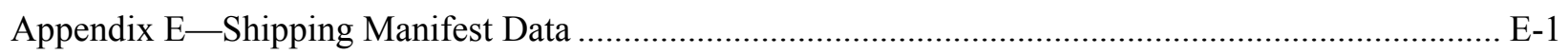

Appendix F—Subcontractor Vendor Data Submittals.......................................................................

\section{FIGURES}

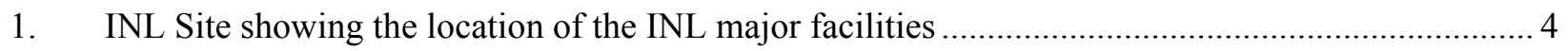

2. Location of the INL Site over the Eastern Snake River Plain Aquifer......................................... 5

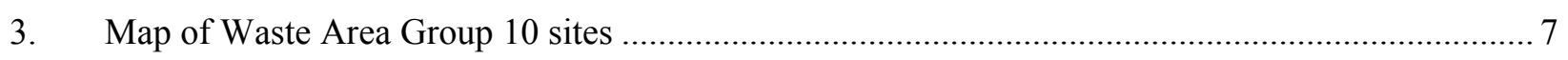

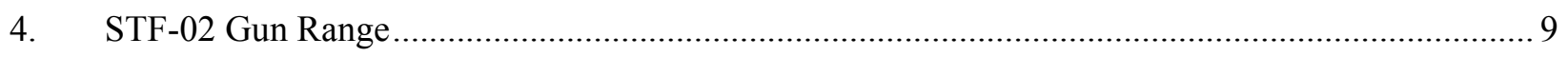

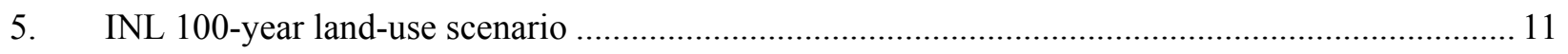

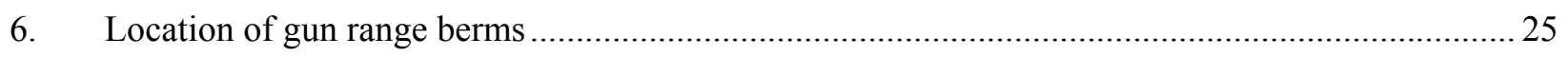

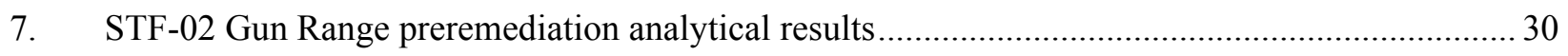

8. Experimental Organic-Cooled Reactor Pond preremediation analytical results ............................ 31

9. Experimental Organic-Cooled Reactor remediation support analytical results............................. 34

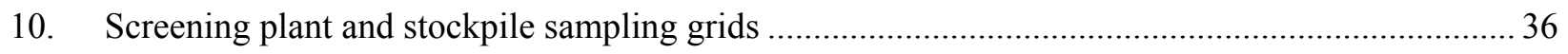

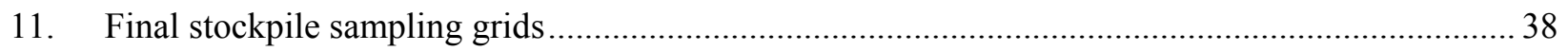

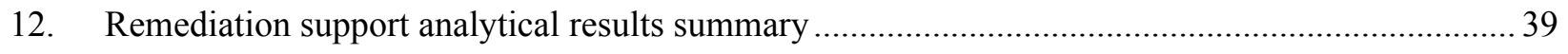

\section{TABLES}

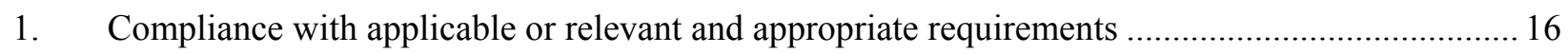

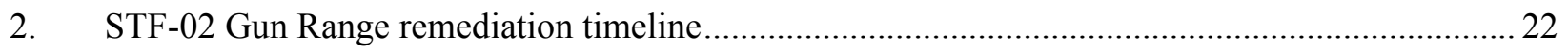

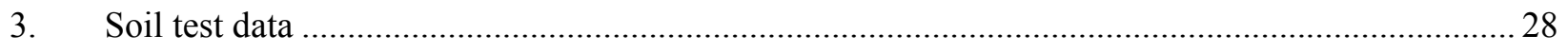

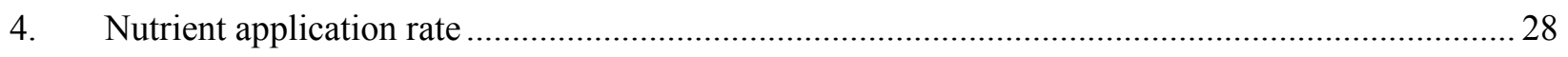




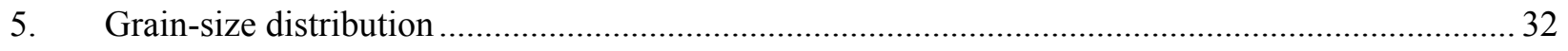

6. Total and toxicity characteristic leaching procedure lead results by fraction................................ 32

7. Experimental Organic-Cooled Reactor Pond lead analytical results........................................... 33

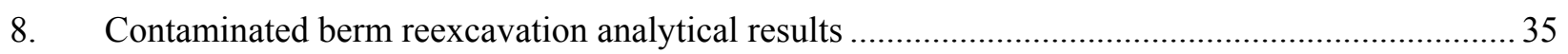

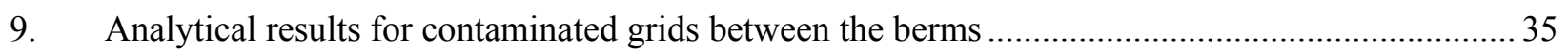

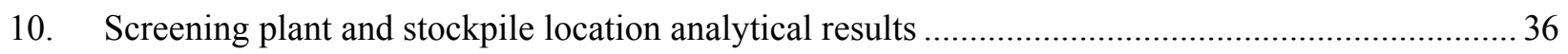

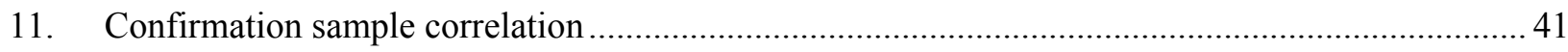

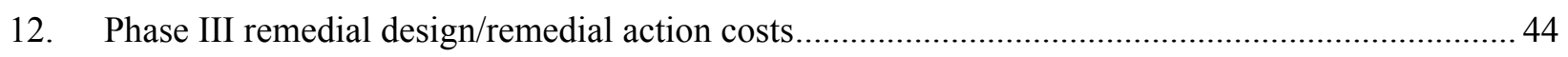

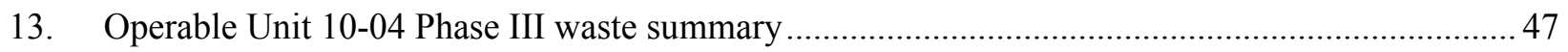




\section{ACRONYMS}

ARAR applicable or relevant and appropriate requirement

BEA Battelle Energy Alliance, LLC

BORAX Boiling Water Reactor Experiment

CERCLA Comprehensive Environmental Response, Compensation, and Liability Act

CFA Central Facilities Area

CFR Code of Federal Regulations

CWI CH2M-WG Idaho, LLC

DEQ Idaho Department of Environmental Quality

DOE U.S. Department of Energy

DOE-ID U.S. Department of Energy Idaho Operations Office

EBR-I $\quad$ Experimental Breeder Reactor I

EOCR Experimental Organic-Cooled Reactor

EPA U.S. Environmental Protection Agency

FFA/CO Federal Facility Agreement and Consent Order for the Idaho National Engineering Laboratory

FR Federal Register

HASP health and safety plan

HAZWOPER hazardous waste operations and emergency response

HEPA high-efficiency particulate air

ICP Idaho Cleanup Project

IDAPA Idaho Administrative Procedures Act

INEEL Idaho National Environmental and Engineering Laboratory

INEL Idaho National Engineering Laboratory

INL Idaho National Laboratory

LDR land disposal restriction

MFC Materials and Fuels Complex 


\begin{tabular}{|c|c|}
\hline NESHAP & National Emission Standards for Hazardous Air Pollutants \\
\hline NIOSH & National Institute of Occupational Safety and Health \\
\hline NRTS & National Reactor Testing Station \\
\hline OSHA & Occupational Safety and Health Administration \\
\hline $\mathrm{OU}$ & operable unit \\
\hline PDD & program description document \\
\hline PLN & plan \\
\hline PPE & personal protective equipment \\
\hline $\mathrm{RAO}$ & remedial action objective \\
\hline RCRA & Resource Conservation and Recovery Act \\
\hline $\mathrm{RD} / \mathrm{RA}$ & remedial design/remedial action \\
\hline $\mathrm{RI} / \mathrm{FS}$ & remedial investigation/feasibility study \\
\hline ROD & record of decision \\
\hline RTC & Reactor Technology Complex \\
\hline STD & standard \\
\hline STF & Security Training Facility \\
\hline TCLP & toxicity characteristic leaching procedure \\
\hline TSDF & treatment, storage, and disposal facility \\
\hline USC & United States Code \\
\hline WAG & waste area group \\
\hline XRF & $\mathrm{x}$-ray fluorescence \\
\hline
\end{tabular}




\section{Remedial Action Report for Operable Units 6-05 and 10-04, Phase III}

\section{INTRODUCTION}

This draft remedial action report for the Security Training Facility (STF) -02 Gun Range was prepared in accordance with the Federal Facility Agreement and Consent Order for the Idaho National Engineering Laboratory (FFA/CO) (DOE-ID 1991) between the U.S. Department of Energy Idaho Operations Office (DOE-ID), the U.S. Environmental Protection Agency (EPA), and the Idaho Department of Environmental Quality (DEQ) - hereafter referred to as the Agencies.

Under the current remediation strategy outlined in the FFA/CO, the location identified for the remedial action is designated as the STF-02 Gun Range within Waste Area Group (WAG) 6, Operable Unit (OU) 6-05, Experimental Breeder Reactor I (EBR-I)/Boiling Water Reactor Experiment (BORAX), and WAG 10, OU 10-04, Miscellaneous Sites, at the Idaho National Laboratory (INL) Site. The WAG 10 comprehensive remedial investigation/feasibility study (RI/FS) was originally defined in the FFA/CO as occurring under OU 10-04 and including the Snake River Plain Aquifer. Because of the intricacies presented by the Snake River Plain Aquifer, a separate remedial investigation was determined to be required to address the aquifer and any potential new sites identified that needed to be addressed. Subsequently, OU 10-08 was added to WAG 10 to address INL-wide groundwater issues and new sites that are passed on to WAG 10 by other groups. The OU 10-08 project will prepare the OU 10-08 comprehensive RI/FS and the record of decision (ROD) to address the groundwater issues and new sites. Therefore, these tasks will not be addressed under OU 10-04.

As required by the Comprehensive Environmental Response, Compensation, and Liability Act (CERCLA) (42 United States Code [USC] § 9601 et seq.), the OU 10-04 remedial action will proceed in accordance with the Record of Decision Experimental Breeder Reactor-I/Boiling Water Reactor Experiment Area and Miscellaneous Sites, Operable Units 6-05 and 10-04 (DOE-ID 2002a). The OU 10-04 ROD (DOE-ID 2002a) presented the selected remedies for 50 surface sites evaluated under the Comprehensive Remedial Investigation/Feasibility Study for Waste Area Groups 6 and 10 Operable Unit 10-04 (DOE-ID 2001).

The remedial action for OU 10-04 is divided into four phases:

- $\quad$ Phase I consisted of developing and implementing institutional controls at OU 10-04 sites and developing and implementing INL Site-wide plans for both institutional controls and ecological monitoring

- $\quad$ Phase II will remediate sites contaminated with trinitrotoluene and Royal Demolition Explosive

- $\quad$ Phase III, the subject of this report, remediated lead contamination at the STF-02 Gun Range

- $\quad$ Phase IV will remediate hazards associated with unexploded ordnance.

A separate remedial action report has or will be prepared for each of the phases with the remedial action report for the final phase, presumably Phase IV, incorporating the information presented in the reports for the previous three phases, providing for one comprehensive remedial action report for OU 10-04. The scope and schedule for implementing these remediation phases were presented in the Operable Units 6-05 and 10-04, Experimental Breeder Reactor-I/Boiling Water Reactor Experiment Area and Miscellaneous Sites, Remedial Design/ Remedial Action Scope of Work (DOE-ID 2003). 
This Phase III remedial action report addresses the remediation of lead-contaminated soil found at the STF-02 Gun Range. The remediation was conducted in accordance with the requirements delineated in the Remedial Design/Remedial Action Work Plan for Operable Units 6-05 and 10-04, Phase III (DOE-ID 2006a). The gun range berms, the surrounding soil, and the adjacent Experimental Organic-Cooled Reactor (EOCR) leach pond, were excavated to remove soil having lead contamination exceeding the $400-\mathrm{mg} / \mathrm{kg}$ remediation goal. Physical separation of the lead and copper fragments (e.g., bullets and casings) from the soil was performed, as feasible, to facilitate possible recycling of the lead and copper. As discussed in Section 2.3.8, a recycler was not found to accept this particular waste stream; therefore, the lead and copper fragments were shipped with the soil that did not meet the Resource Conservation and Recovery Act (RCRA) (42 USC $\S 6901$ et seq.) criteria for direct disposal off-Site for stabilization prior to disposal.

In addition to the soil, creosote-contaminated railroad ties and power poles at the gun range were removed and sent to an approved facility for disposal. A wooden building used as a live-fire training shooting house and asphalt pads were removed and sent for disposal. Soil that met the remediation goal for lead remained at the STF-02 site where it was used to backfill the EOCR pond and contoured to match the surrounding terrain. The area impacted by the remediation activities was subsequently revegetated.

\subsection{Remedial Action Report Organization}

This remedial action report describes the remediation activities associated with Phase III of the OU 10-04 remedial action. The sections and appendixes within this report are briefly described below.

- The remainder of Section 1 describes the background and history of WAG 10 and provides an overview of the selected remedy for the STF-02 Gun Range

- $\quad$ Section 2 summarizes the remedial action activities

- $\quad$ Section 3 outlines the costs incurred during the remedial action

- $\quad$ Section 4 describes the modifications to the Remedial Design/Remedial Action (RD/RA) Work Plan (DOE-ID 2006a)

- Section 5 describes the waste streams generated during the remedial action

- $\quad$ Section 6 addresses the prefinal checklist

- Section 7 includes the summary and verification of the work performed

- Section 8 provides certification that the remedial action functions as designed and meets the remedial action goals and objectives

- $\quad$ Section 9 lists the references cited throughout this report

- $\quad$ Appendix A provides a photographic record of the remediation activities

- Appendix B provides analytical data summaries for the remedial action

- Appendix $\mathrm{C}$ provides the prefinal inspection checklist for the remedial action

- Appendix D provides contour drawings of the site

- $\quad$ Appendix E provides shipping manifest data

- Appendix F provides a compilation of the vendor data submittals from the subcontractor as required by the specification. 


\subsection{Background}

\subsubsection{Site Background}

The INL Site is a government-owned, contractor-operated facility managed by DOE-ID located $51 \mathrm{~km}(32 \mathrm{mi})$ west of Idaho Falls, Idaho. The INL Site and major facilities are shown in Figure 1. The INL Site occupies $2,305 \mathrm{~km}^{2}\left(890 \mathrm{mi}^{2}\right)$ of the northeastern portion of the Eastern Snake River Plain. As shown in Figure 2, the Snake River Plain Aquifer, which is classified as a sole source aquifer (56 Federal Register [FR] 50634), is located within the plain. The INL Site encompasses portions of five Idaho counties: (1) Butte, (2) Jefferson, (3) Bonneville, (4) Clark, and (5) Bingham. The STF-02 Gun Range is located in the south-central quadrant of the INL Site (see Figure 1).

The INL was originally established in 1949 as the National Reactor Testing Station (NRTS). It is managed by the Department of Energy (DOE) for the conduct of nuclear energy research and related activities. The NRTS was redesignated as the Idaho National Engineering Laboratory (INEL) in 1974 to reflect the broad scope of its engineering work being conducted. In 1997, the INEL was redesignated the Idaho National Engineering and Environmental Laboratory (INEEL) to emphasize the focus of the laboratory's mission at that time of environmental research. In 2005, a change in mission that began in 2002 culminated with the division of the INL into two distinct projects: (1) the INL for nuclear energy research to support national energy security and homeland security and (2) the Idaho Cleanup Project (ICP) to perform the large majority of the environmental cleanup at the INL Site and related work including reducing or eliminating risks to the Snake River Plain Aquifer and other resources posed by contamination and wastes left at the INL from past missions, while protecting workers, the public, the environment, national security interests, and the safety of future generations. The INL is managed by Battelle Energy Alliance, LLC (BEA) under a 10-year management and operating contract continuing through September 30, 2014, while the ICP is managed by CH2M-WG Idaho, LLC (CWI) under a 7-year cost-plus-incentives contract continuing through April 30, 2012. The goal of the ICP is to complete as much cleanup work as possible by 2012 to protect the Snake River Plain Aquifer.

The STF-02 Gun Range was used from 1983 to 1990 for security force practice maneuvers, including small arms target practice. Approximately five million rounds were fired, primarily into a main berm behind target posts toward the north end of the gun range. It was estimated that up to 61 tons of lead and 3.4 tons of copper may have been present at the site from bullets fired during target practice. The lead contamination was present as large fragments, as well as finely disseminated fragments in the soil. Based on soil analyses, lead was determined to pose an unacceptable risk to both human health and ecological receptors. To mitigate this risk, a remedial action was implemented to remove contaminated soil with lead concentrations exceeding the remedial action goal of $400 \mathrm{mg} / \mathrm{kg}$ and dispose of the removed soil at an approved treatment, storage, and disposal facility (TSDF) in accordance with all regulatory requirements. In addition, all aboveground structures and components were removed and disposed of appropriately, the remaining berms and soil were contoured to match the surrounding terrain, and the area was reseeded with native species.

\subsubsection{Regulatory Background}

The FFA/CO, and its associated Action Plan, is an agreement reached in 1991 between the Agencies listed in Section 1 to manage environmental cleanup in accordance with CERCLA and other federal and state requirements. Under the FFA/CO, and as shown in Figure 3, WAG 10 comprises miscellaneous surface sites and liquid disposal areas throughout the INL that are not included within other WAGs (WAGs 1 through 9). It also includes regional Snake River Plain Aquifer concerns that cannot be addressed on a WAG-specific basis. 


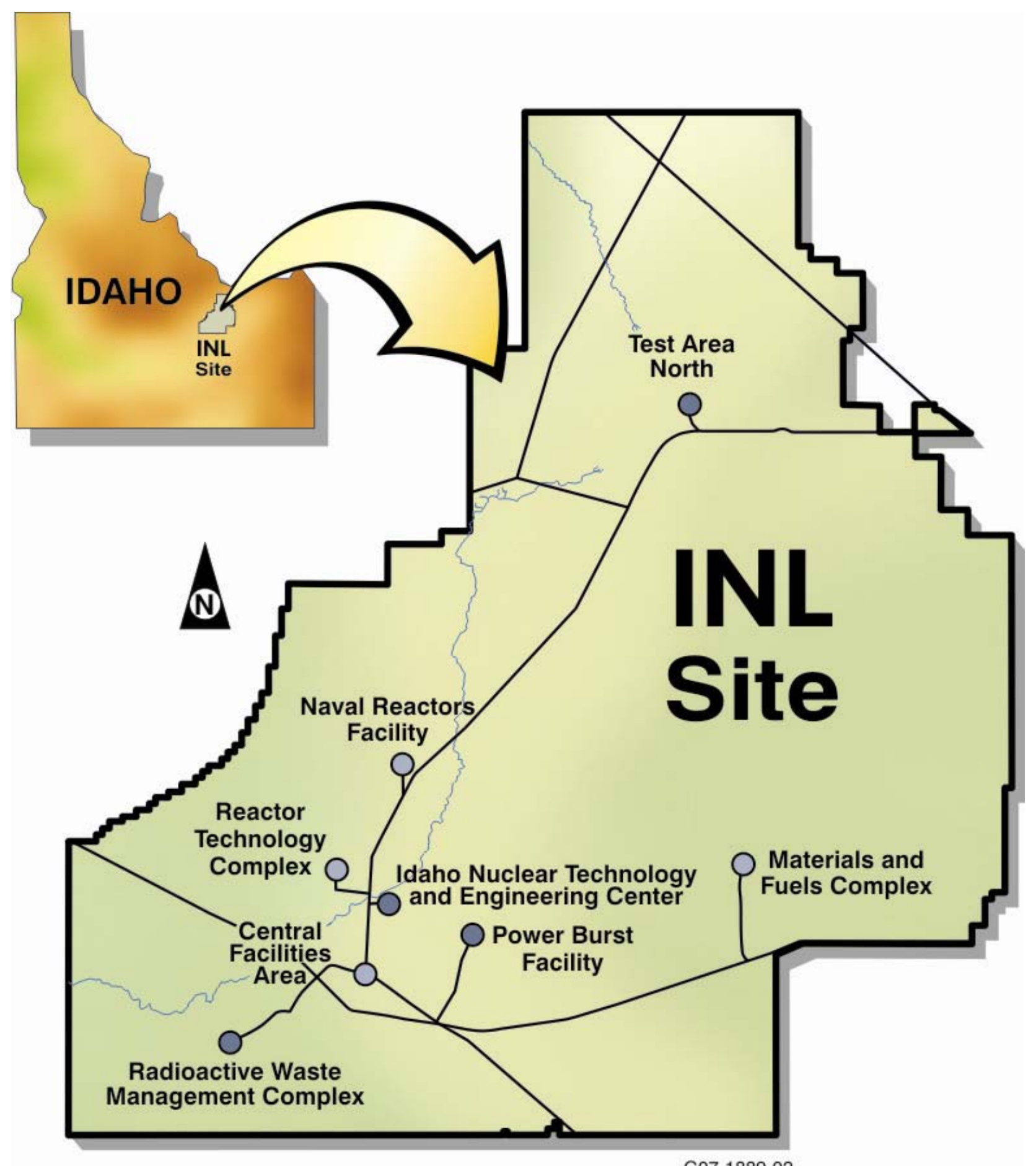

G07-1889-02

Figure 1. INL Site showing the location of the INL major facilities. 


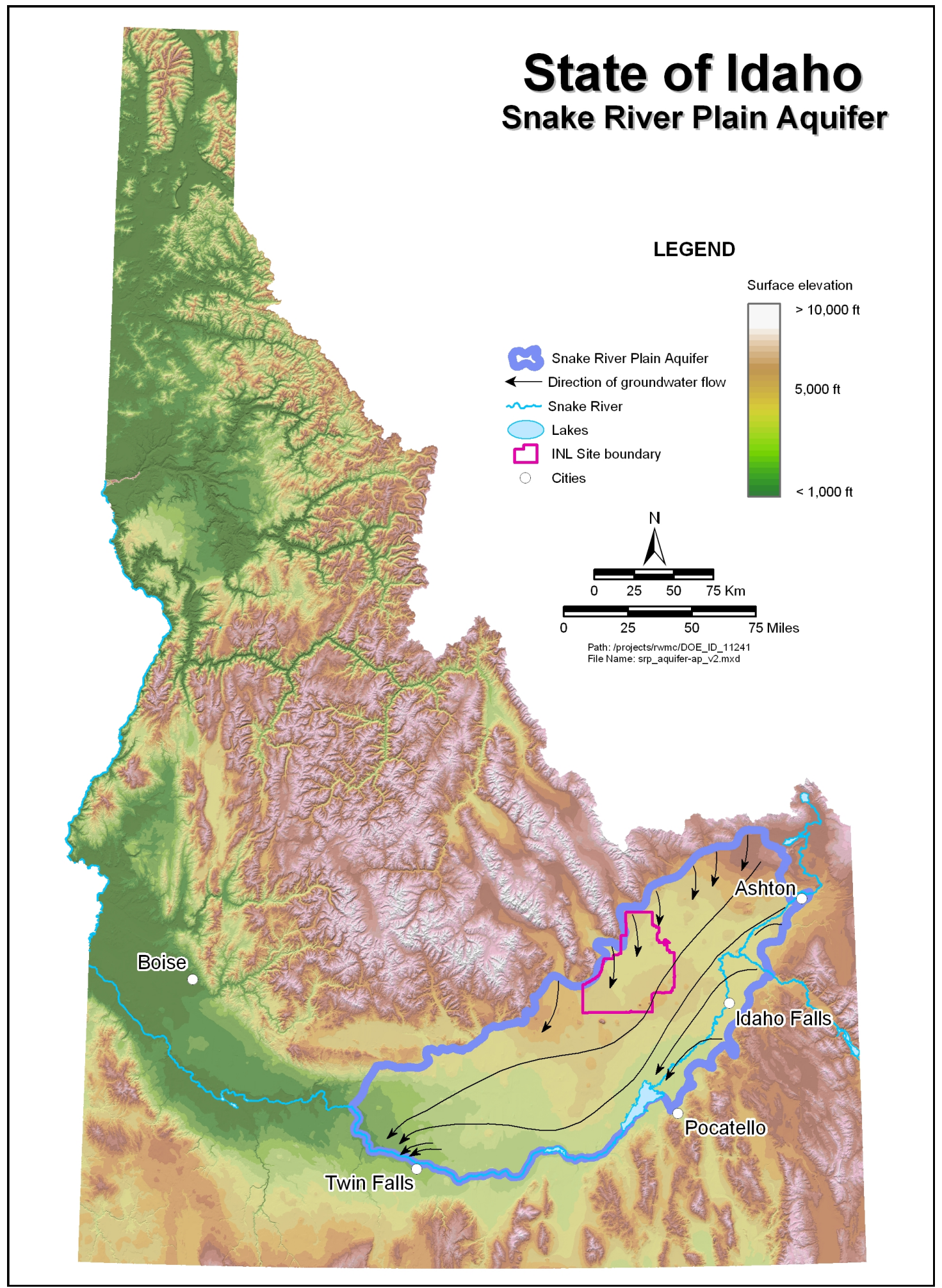

Figure 2. Location of the INL Site over the Eastern Snake River Plain Aquifer. 
The STF area has been used since 1983 for security-force practice maneuvers including small arms target practice in a berm approximately $76 \mathrm{~m}(250 \mathrm{ft})$ northeast of the former STF-601 (see Figure 4). The berm was used from 1983 to 1990. It is estimated that 5 million rounds were fired into the berm including tracer rounds. None of the lead bullets that was fired into or that ricocheted away from the berm into the "kickout" areas has been picked up. Up to 61 tons of lead and 3.4 tons of copper may have been present at the STF-02 site.

During sampling conducted in support of the remedial investigation conducted at the STF-02 Gun Range, two locations within the EOCR leach pond were visually identified as having lead bullets and fragments, presumably from activities conducted at the gun range located immediately adjacent to the pond. These locations were samples and the resulting lead concentrations exceeded the $400-\mathrm{mg} / \mathrm{kg}$ remediation goal. As such, the EOCR leach pond was included with the STF-02 Gun Range for remediation of lead-contaminated soils. The pond was actually never used for its intended purpose; therefore, no other sources of contamination exist.

\subsection{Remedial Action Objectives}

The remedial action objectives (RAOs) for the STF-02 Gun Range were developed in accordance with 40 Code of Federal Regulations (CFR) 300, "National Oil and Hazardous Substances Pollution Contingency Plan," and Guidance for Conducting Remedial Investigations and Feasibility Studies Under CERCLA (EPA 1988) and through the consensus of DOE-ID, EPA, and DEQ participants. The RAOs are based on the results of both human health requirements and the ecological risk assessments and are specific to lead as the only contaminant of concern.

The RAOs specified for protecting human health are expressed both in terms of risk and exposure pathways because protection can be achieved by reducing contaminant levels and restricting or eliminating exposure pathways. The RAOs specified for protecting ecological receptors inhibit adverse effects from contaminated soil on resident populations of flora and fauna. The RAOs developed to protect human health and ecological receptors are as follows:

- $\quad$ Prevent exposure to soils contaminated with lead at concentrations greater than $400 \mathrm{mg} / \mathrm{kg}$

- $\quad$ Prevent groundwater contamination

- Inhibit ecological receptor exposures to soil contaminated with lead, the contaminant of concern, primarily in concentrations in soils that result in a hazard quotient greater than or equal to 10.0 .

The RAOs exclude naturally occurring elements and compounds that are not attributable to historic releases. Remediation goals were established to meet these objectives. The remediation goal for lead-to be protective of both human health and ecological receptors - is $400 \mathrm{mg} / \mathrm{kg}$. A discussion pertaining to the applicability of the $400-\mathrm{mg} / \mathrm{kg}$ remediation goal based on the human health screening level being protective of ecological receptors is provided in Appendix K of the Comprehensive RI/FS for WAGs 6 and 10, OU 10-04 (DOE-ID 2001). The remediation goal can be satisfied by reducing the identified contaminant concentration in the soil to below $400 \mathrm{mg} / \mathrm{kg}$. Removal of the contaminated media and metal debris from the STF-02 Gun Range will reduce further any potential groundwater risk. 


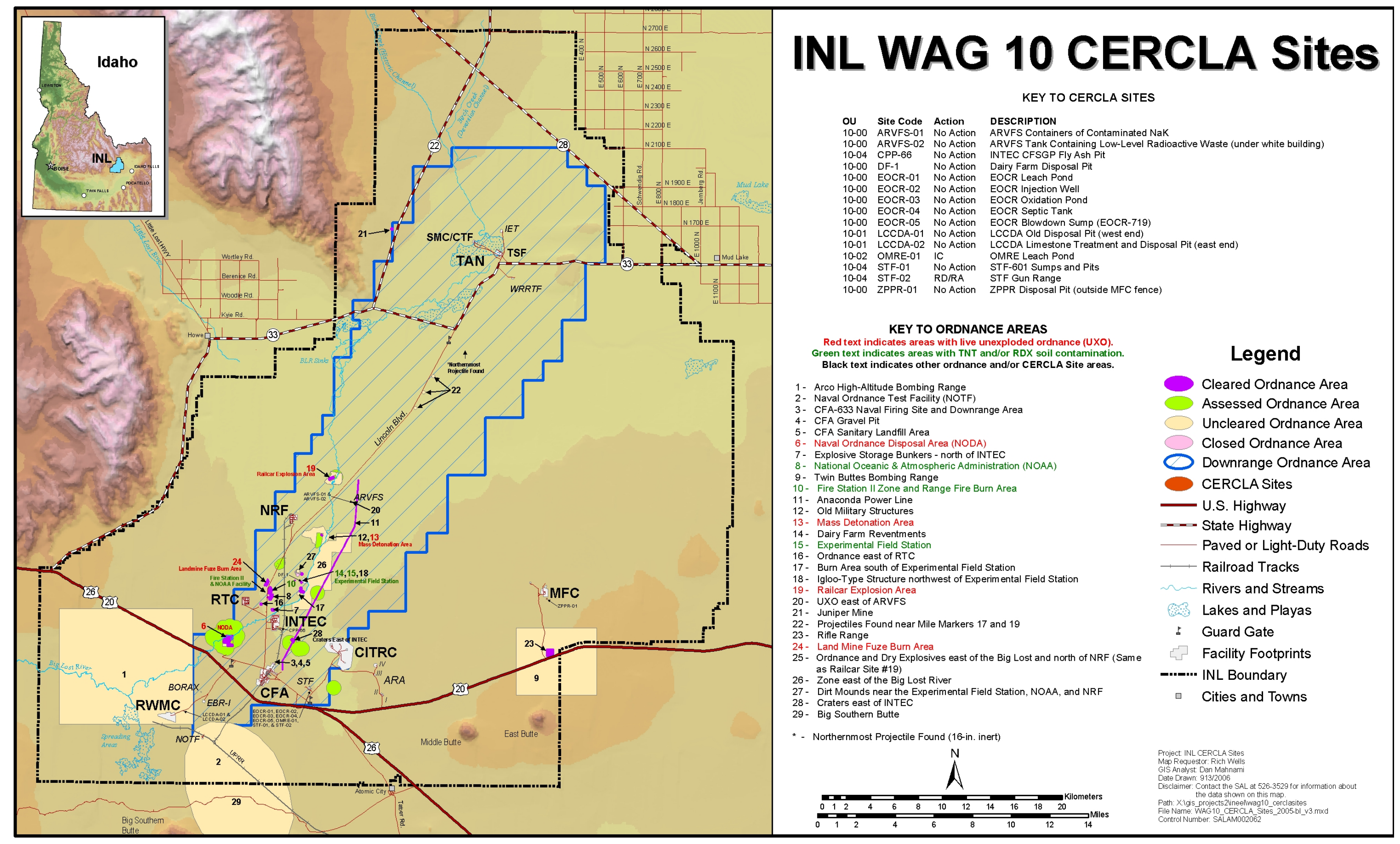

Figure 3. Map of Waste Area Group 10 sites. 



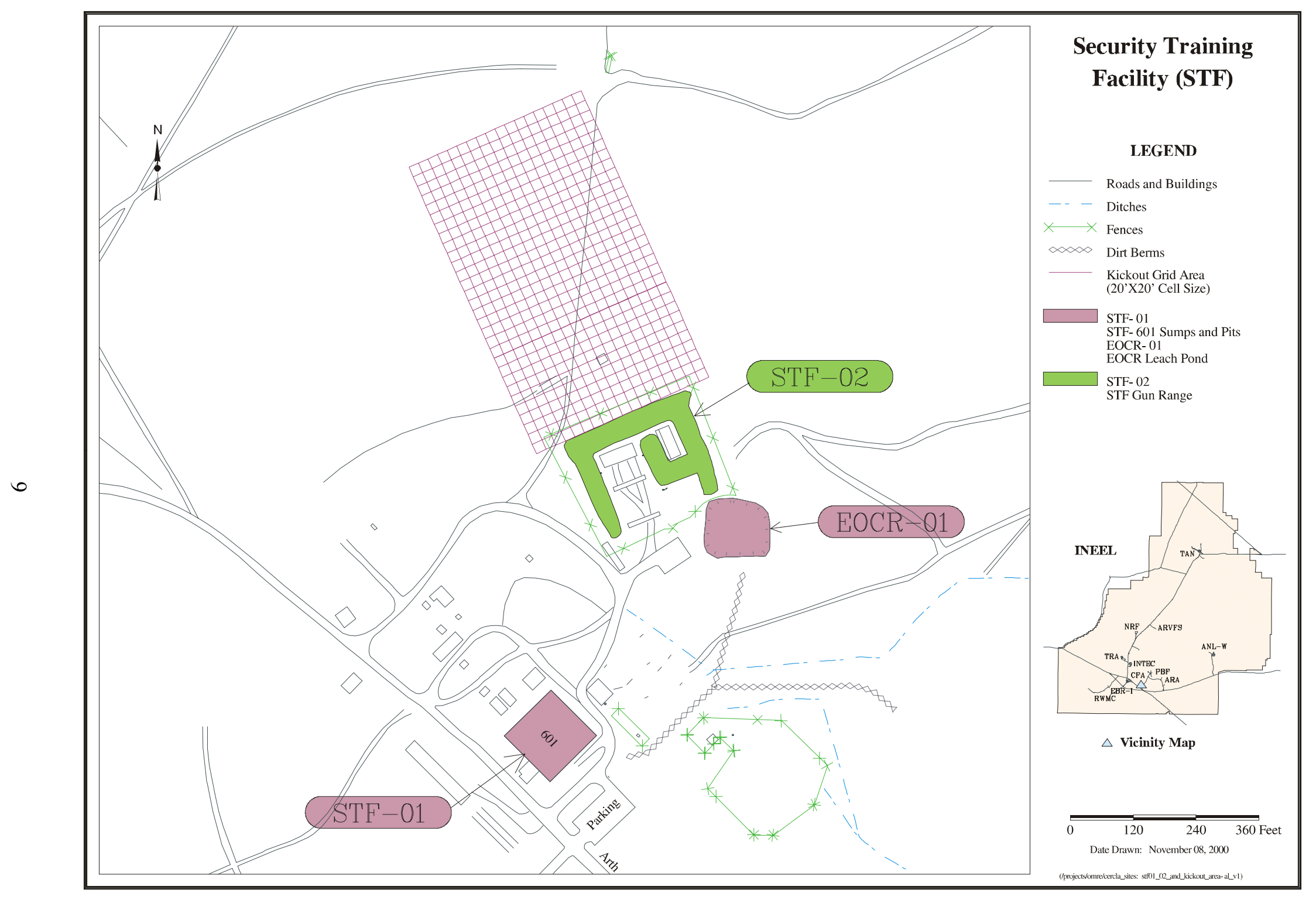

Figure 4. STF-02 Gun Range. 
The Idaho National Laboratory Comprehensive Land Use and Environmental Stewardship Report (INL 2005) presents the INL project land use scenarios from 2005 through 2105 . The INL is in the process of modernizing and optimizing its facility use to create an environment more conducive to research. The INL boundaries and the associated $890 \mathrm{mi}^{2}$ are anticipated to remain under federal government management and control at least until 2105, with portions of the INL (i.e., the Idaho Nuclear Technology and Engineering Center and the Radioactive Waste Management Complex) remaining as such in perpetuity.

Currently the entire area comprising the INL remains under administrative control with access to the INL restricted. Within this area, the current land use consists of industrial areas connected with transportation corridors, vegetated desert and rangelands, wetland and surface water drainage areas, and barren lands. Portions of the vegetated lands are used for grazing, controlled hunting, and ecological preservation. No INL land is used for agricultural or residential purposes.

It is anticipated that over the next 10 years, new on-Site development will occur, major facility decontamination and decommissioning will take place, and specific environmental remediation will be completed. The new development is expected to occur within the existing Core Infrastructure Area of the INL, with specific development taking place at the Materials and Fuels Complex (MFC), the Reactor Technology Complex (RTC), and the Science and Technology Campus. In addition, a new transportation corridor is being considered between the RTC and MFC facilities. The INL facility infrastructure will be reduced over these 10 years with the footprint reductions occurring within existing operations areas. Included in this scenario is the transformation of existing operations areas to decommissioned and institutionally controlled areas.

The 30-year land use scenario is anticipated to be consistent with the 10-year forecast. Specific changes beyond the 10-year horizon include completion of a number of critical environmental remediation efforts. New Development Areas (i.e., RTC and MFC) will continue to serve as the operational staging areas for current and new development within the Core Infrastructure Area. For the 100 -year scenario, the INL land use is consistent with that of the 30 -year scenario. The decommissioned and controlled areas will be the primary focus of the INL long-term stewardship functions. These activities will be coupled with Site-wide administrative controls and the operational areas of RTC and MFC. A determination concerning the proposed transportation corridor will have been made by this time. If the corridor is constructed, it will be maintained consistent with existing infrastructure. Figure 5 shows the proposed INL land use for the 100-year scenario through the year 2105.

Achieving the remedial action objectives specified for the STF-02 Gun Range enables release of the area for use in meeting the long-term objectives for the INL projected land use scenarios. Any residual contamination does not preclude the use of the STF-02 site for any of the proposed scenarios. By achieving the $400-\mathrm{mg} / \mathrm{kg}$ remediation goal for lead, the land is available for free-release under the residential use scenario.

\subsection{Selected Remedy}

Based on consideration of CERCLA requirements, the detailed analysis of alternatives, and public comments, the Agencies selected removal (including physical separation to segregate the metal fragments and bullets), on-site stabilization, and disposal as the remedy for the STF-02 site. Removal of the contaminated soil was to have included physical segregation of lead fragments and bullets from the soil with the segregated lead to be sent for recycling. As a secondary benefit of the physical segregation operation, copper fragments similarly would be removed from the soil and sent for recycling. Performance standards were implemented as design criteria to ensure that the selected remedy remained protective of human health and the environment. 


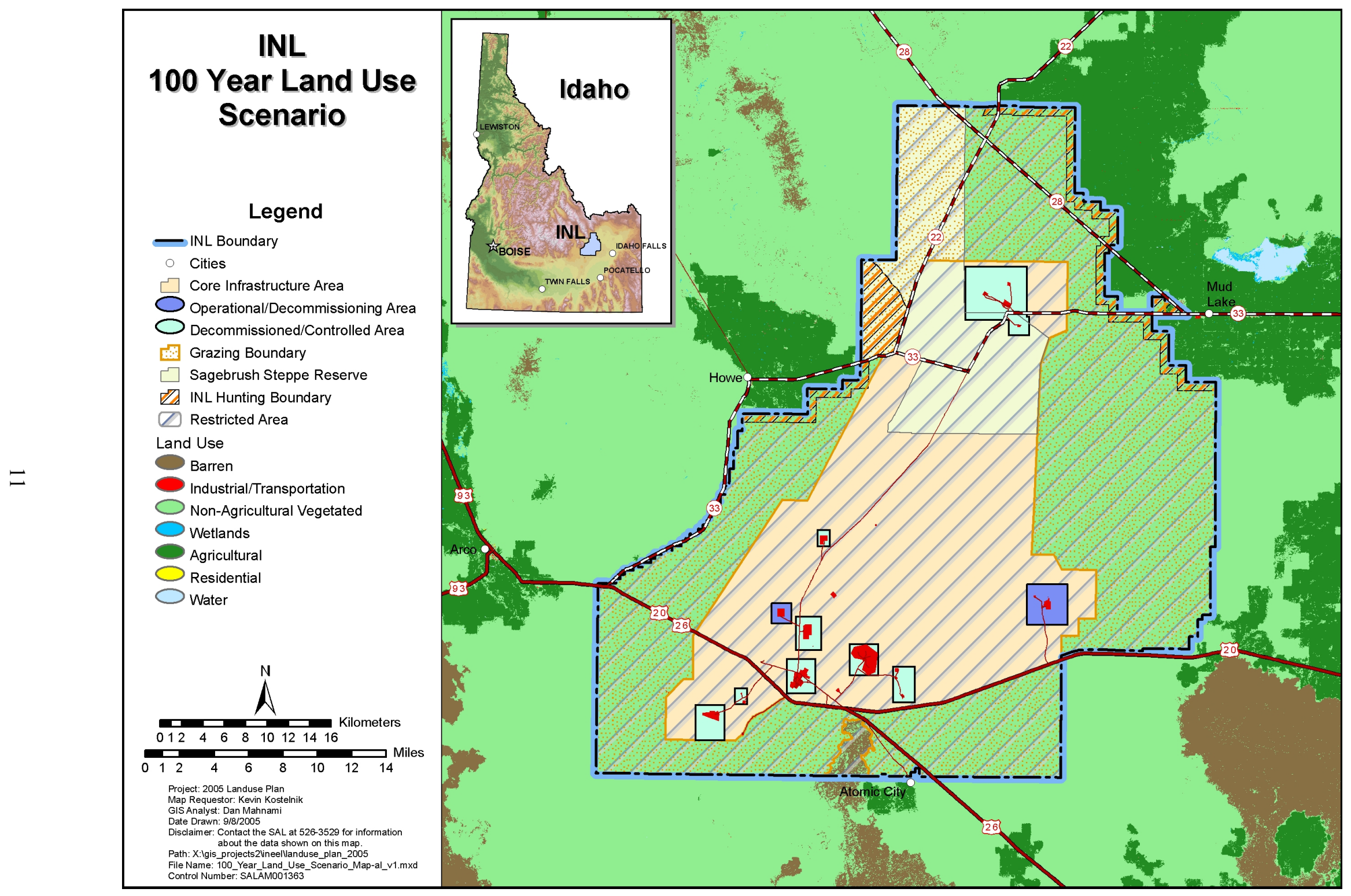

Figure 5. INL 100-year land-use scenario. 
Soil exceeding the remediation goal of $400 \mathrm{mg} / \mathrm{kg}$ for lead was required to meet the "Land Disposal Restrictions" (LDR) (IDAPA 58.01.05.011) and "Alternative LDR Treatment Standards for Contaminated Soil" (40 CFR 268.49). Based on the initial analytical characterization results, soil exceeding the Phase III remediation goal was assumed to also exceed the $5.0-\mathrm{mg} / \mathrm{L}$ toxicity characteristic concentration presented in 40 CFR 261.24, thereby requiring stabilization prior to disposal at an approved facility. Soil with lead concentrations higher than $400 \mathrm{mg} / \mathrm{kg}$ was shipped to the Clean Harbors Grassy Mountain facility west of Salt Lake City for stabilization and disposal. 


\section{DISCUSSION OF REMEDIATION ACTIVITIES}

\subsection{Remedial Action Working Documents}

The Phase III RD/RA Work Plan (DOE-ID 2006a) lists the design criteria for the remediation of the STF-02 Gun Range site, describes the remedial design and how it was implemented for the remedial action, and serves as the guidance document for the OU 10-04 Phase III remedial action. The following documents were included as appendixes to the Phase III RD/RA Work Plan:

- Design drawings detailing the preremediation conditions (e.g., topography and fencing at the STF-02 site) as well as the work to be performed during the remedial action

- Technical specifications providing the general terms and conditions required for completion of the remedial action

- $\quad$ Air emissions modeling results presenting a summary of the required air emission results to satisfy project applicable or relevant and appropriate requirements (ARARs)

- A waste management plan describing the management and disposal of waste generated during Phase III activities

- $\quad$ An operations and maintenance plan describing the operations and maintenance requirements including institutional controls, should the need arise, for the STF-02 Gun Range.

Phase III:

In addition, three separate documents were included with the Phase III RD/RA Work Plan,

- $\quad$ The Field Sampling Plan for the Operable Units 6-05 and 10-04 Remedial Action, Phase III (DOE-ID 2006b) describing the sampling and analyses required during Phase III activities

- $\quad$ The Quality Assurance Project Plan for Waste Area Groups 1, 2, 3, 4, 5, 6, 7, 10, and Deactivation, Decontamination, and Decommissioning (DOE-ID 2006c) describing the necessary steps required to ensure project data quality

- Plan (PLN) -2128, "Miscellaneous Sites Cleanup Project Health and Safety Plan," describing the possible hazards and the required steps to protect the health and safety of project workers.

\subsection{Site Preparation and Mobilization}

Site preparation and mobilization efforts performed prior to commencement of the remedial action are discussed in the sections below.

\subsubsection{Personnel Training Requirements}

Before fieldwork commenced, task-site workers were required to have the following training as specified in Section 6 of the Miscellaneous Sites Health and Safety Plan (HASP) (PLN-2128):

- $\quad$ Site-specific training as required by the HASP

- $\quad$ 40-hour hazardous waste operations and emergency response (HAZWOPER)

- Hazardous waste operations 24-hour "on-the-job" training

- 8-hour HAZWOPER site supervisor, as necessary 
- $\quad$ Radiological Worker I or II (personnel operating the x-ray fluorescence [XRF] spectrometer)

- Hearing conservation

- $\quad$ Respirator qualification and fit test, as necessary

- $\quad$ Personal protective equipment training

- $\quad$ Project-specific HASP training

- $\quad$ Environmental Safety, Health, and Quality Assurance access training

- Hantavirus awareness training

- Heat and cold stress training

- $\quad$ Project-specific job safety analysis training

- $\quad$ Pre-job briefing and post-job review training

- $\quad$ Fire extinguisher training (at least one trained person on the job site)

- $\quad$ Medic first-aid training (at least two trained personnel on the job site)

- Basic industrial ergonomics training

- $\quad$ Pre-job briefings performance evaluation, as necessary

- $\quad$ Point-of-contact for field personnel, as necessary

- Excavation competent person (person conducting daily excavation inspections)

- Unexploded ordnance recognition training.

Specific to the remediation of lead-contaminated soils at the STF-02 Gun Range, all task-site workers were required to be qualified as trained lead workers with those having the potential to come into direct contact with soil during the remedial action being monitored for lead exposure in accordance with RCRA standards. Occasional site workers (e.g., samplers) were required to have undergone lead awareness training. Certifications of training and training updates were maintained in the training database on the INL Intranet.

\subsubsection{Field Operations and Staging of Equipment and Supplies}

A field office trailer was established immediately adjacent to the task site with electrical power obtained from a nearby power pole and an electrical generator maintained onsite if needed. No phone connections were available; therefore, communications contact was maintained through the use of cellular phones. Temporary restroom and wash facilities were established near the trailer for workers in accordance with the Occupational Safety and Health Administration (OSHA) standard "Hazardous Waste Operations and Emergency Response" (29 CFR 1910.120/1926.65). Flammable materials storage was provided in the office trailer with personal protective equipment (PPE) stocked for the field team members including leather gloves, safety glasses with side shields, sunscreen (as needed), hardhats, reflective vests, hearing protection for use in high noise areas, and respiratory protection.

Equipment required to perform the work was staged at the job site as required during the various stages of the remedial action. Equipment included, but was not limited to, the following:

- $\quad$ Appropriate signage

- Excavator 
- Water truck

- $\quad$ Front-end loader

- Road grader

- Crawler dozer

- Backhoe

- $\quad$ Screening plant

- Wheel roller

- Dump trucks

- Tractor

- $\quad$ Tiller

- $\quad$ Seeder

- Chip spreader

- $\quad$ X-ray fluorescence spectrometer

- $\quad$ Sampling supplies

- $\quad$ Absorbent material for containers

- $\quad$ Roll-on/roll-off containers with haul trucks

- Intermodal (i.e., roll-on/roll-off containers with hard lids and gasket seals) containers with haul trucks.

\subsubsection{Regulatory Compliance}

The OU 10-04 Phase III remedial action conformed to the ARARs as outlined in Section 4.2 of the Phase II RD/RA Work Plan. Table 1 summarizes the compliance strategy for each of the ARARs.

\subsubsection{Work Control Requirements at the Idaho National Laboratory Site}

In compliance with CWI procedures and requirements for conducting fieldwork, the following items were required to be completed prior to commencement of the remedial action:

- $\quad$ Standard (STD) -101, "ICP Integrated Work Control Process," planned work order package

- Including the project on the Central Facilities Area (CFA) plan-of-the-week schedule for information

- $\quad$ Project approval for work on the Miscellaneous Sites Cleanup Project plan-of-the-week

- $\quad$ Formal pre-job briefing for the work order package

- $\quad$ Subsurface investigation to identify underground electrical utilities

- Job safety analyses to identify critical procedural safety requirements including personal protective equipment and task-specific training

- $\quad$ Environmental checklist

- $\quad$ Field sampling plan 
Table 1. Compliance with applicable or relevant and appropriate requirements.

\begin{tabular}{|c|c|c|}
\hline Category & Citation & Compliance Strategy \\
\hline \multicolumn{3}{|l|}{ Chemical-Specific ARARs } \\
\hline Idaho Ground Water Quality Rule & $\begin{array}{l}\text { "Ground Water Quality Standards" } \\
\left(\text { IDAPA }^{\mathrm{a}} 58.01 .11 .200\right)\end{array}$ & $\begin{array}{l}\text { The Clean Water Act is not applicable to the remediation of } \\
\text { the STF-02 site based on the following: EPA } \\
\text { representatives met with DOE-ID staff at the INL Site on } \\
\text { August } 27,2003 \text {, to evaluate storm water compliance and } \\
\text { the potential to discharge storm water to waters of the } \\
\text { United States (Ryan 2003). Based on their evaluation of the } \\
\text { Idaho Nuclear Technology and Engineering Center, the } \\
\text { Radioactive Waste Management Complex, and Test Area } \\
\text { North sites, the EPA concluded that these areas do not have } \\
\text { a reasonable potential to discharge storm water to waters of } \\
\text { the United States. The applicability of the Clean Water Act } \\
\text { to the INL also has been evaluated based on the recent } \\
\text { U.S.Supreme Court decision in Rapanos et Ux., et al. } \\
\text { v. United States (2006) issued June 19, 2006. Under the } \\
\text { standard enunciated by Justices Scalia and Kennedy in this } \\
\text { decision, the Clean Water Act has no regulatory jurisdiction } \\
\text { over the Big Lost River and Birch Creek. This is because } \\
\text { they lack any "significant nexus" of surface water flow } \\
\text { connecting them to the Snake River and other traditionally } \\
\text { defined "navigable waters of the United States." In light of } \\
\text { this, the Clean Water Act would no longer be among the } \\
\text { "applicable Federal laws" that would apply to CERCLA } \\
\text { activities at the INL Site. }\end{array}$ \\
\hline
\end{tabular}

\section{Action-Specific ARARs}

Rules for the Control of Air Pollution in Idaho
"Rules for Control of Fugitive Dust" (IDAPA 58.01.01.650) and "General Rules" (IDAPA 58.01.01.651)

“Toxic Substances” (IDAPA 58.01.01.161)

"Toxic Air Pollutants Non-Carcinogenic Increments" (IDAPA 58.01.01.585) and "Toxic Air Pollutants Carcinogenic Increments" (IDAPA 58.01.01.586)
The Clean Water Act is not applicable to the remediation of CF-02 site based on the following. EPA

August 27,2003, to evaluate storm water compliance and the potential to discharge storm water to waters of the Idaho Nuclear Technology and Engineering Center, the Radioactive Waste Management Complex, and Test Area the the United States. The applicability of the Clean Water Act to the INL also has been evaluated based on the recent v. United States (2006) issued June 19, 2006. Under the . over the Big Lost River and Birch Creek. This is because lack any "significant nexus" of surface water flow defined "navigable waters of the United States" In light of this, the Clean Water Act would no longer be among the activities at the INL Site.

Dust suppression methods were used to minimize fugitive dust including use of water sprays, use of tarps, keeping vehicle speeds to a minimum, and implementing dust suppression work controls during periods of high wind.

Air emissions were monitored in accordance with the requirements set forth in the project HASP (PLN-2128), and dust-suppression measures were used to minimize the generation of fugitive dust. 
Table 1. (continued).

\begin{tabular}{|c|c|c|}
\hline Category & Citation & Compliance Strategy \\
\hline & $\begin{array}{l}\text { "Compliance with Rules and Regulations" } \\
\text { (IDAPA 58.01.01.500.02) }\end{array}$ & $\begin{array}{l}\text { Portable equipment used for soil excavation and screening } \\
\text { complied with the appropriate INL Site plans and } \\
\text { procedures and were used in accordance with the project } \\
\text { HASP. }\end{array}$ \\
\hline NESHAP & $\begin{array}{l}\text { "Standards for Process Sources" } \\
(40 \text { CFR 63.543[a]) }\end{array}$ & $\begin{array}{l}\text { Air emissions were monitored in accordance with the } \\
\text { requirements set forth in the project HASP, and } \\
\text { dust-suppression measures were used to minimize the } \\
\text { generation of fugitive dust. The calculated air emissions } \\
\text { will be included in the INL Site annual NESHAP report, } \\
\text { which determines the effective dose equivalent from INL } \\
\text { Site activities to members of the public. }\end{array}$ \\
\hline \multirow[t]{2}{*}{$\begin{array}{l}\text { RCRA-Standards Applicable to } \\
\text { Generators of Hazardous Waste }\end{array}$} & $\begin{array}{l}\text { "Identification and Listing of Hazardous Waste" } \\
\text { (IDAPA 58.01.05.005) and "Requirements for } \\
\text { Recyclable Materials" (40 CFR 261.6[a][b]) }\end{array}$ & No materials were recycled. \\
\hline & $\begin{array}{l}\text { "Standards Applicable to Generators of Hazardous } \\
\text { Waste" (IDAPA 58.01.05.006) and "Hazardous } \\
\text { Waste Determination" (40 CFR 262.11) }\end{array}$ & $\begin{array}{l}\text { Hazardous waste determinations were developed based on } \\
\text { an evaluation of sampling data and process knowledge to } \\
\text { determine disposition of the waste. Waste types included } \\
\text { the following: } \\
\text { - Industrial } \\
\text { - Hazardous contaminated debris } \\
\text { - } \quad \text { Lead-contaminated soils } \\
\text { - } \quad \text { Lead-contaminated light poles and railroad ties } \\
\text { - } \quad \text { Lead recycle (not used) } \\
\text { - } \quad \text { Unexpended ammunition. }\end{array}$ \\
\hline
\end{tabular}




\section{Category}

RCRA—Standards Applicable to

Owners and Operators of

Hazardous Waste Treatment,

Storage, and Disposal Units
Citation

"Standards for Owners and Operators of

Hazardous Waste Treatment, Storage, and Disposal Facilities" (IDAPA 58.01.05.008) and "Purpose, Scope, and Applicability" (40 CFR 264.1[j][1-13])

"Standards for Owners and Operators of Hazardous Waste, Treatment, Storage, and Disposal Facilities" (IDAPA 58.01.05.008) and "Disposal or Decontamination of Equipment, Structures, and Soils" (40 CFR 264.114)

"Standards for Owners and Operators of Hazardous Waste, Treatment, Storage, and Disposal Facilities" (IDAPA 58.01.05.008) and (40 CFR 264.171-177)

"Standards for Owners and Operators of Hazardous Waste, Treatment, Storage, and Disposal Facilities" (IDAPA 58.01.05.008) and "Staging Piles" (40 CFR 264.554)
Compliance Strategy

Analysis of waste was performed in accordance with RCRA requirements. The INL Site controls precluded unauthorized access to the waste. The Site was routinely inspected during management self-assessments. Training was conducted in accordance with the project HASP (PLN-2128).

Equipment decontamination was conducted in accordance with the project HASP, waste management procedures outlined in the RD/RA Work Plan (Appendix B), and PDD-1029, "Pollution Prevention Program."

Hazardous waste generated was stored in compatible containers and the integrity of the storage containers was maintained. Waste Disposal Services conducted weekly inspections. No free liquids were generated during the remedial action requiring secondary containment. The container storage area was graded to provide runoff away from the containers.

Staging piles were established within the area of contamination. Measures taken to preclude the spread of contamination included the use of tarps placed over the waste piles and two dust-suppression techniques: water spray during soil excavation and loading activities. The areas surrounding the staging piles were graded to provide runoff away from the piles. The staging piles have been removed prior to the winter months. The soil underlying the staging piles has been sampled demonstrating that no contamination exceeding the remediation goals for lead remains. No staging piles originating from the STF-02 remediation project remain on-Site. 


\section{Category}

Resource Conservation and

Recovery Act-Land Disposal

Restrictions

Clean Water Act of 1977

(33 USC § 1251 et seq.)
Citation

"Definitions for Purposes of Sections 790 through 799" (IDAPA 58.01.01.11) and "Applicability of Treatment Standards" (40 CFR 268.40[a][b][e])

\section{"Land Disposal Restrictions" (IDAPA}

58.01.05.011) and "Treatment Standards for Hazardous Debris" (40 CFR 268.45[a-d])

"Land Disposal Restrictions"

(IDAPA 58.01.05.011) and "Universal Treatment Standards" (40 CFR 268.48[a])

"Land Disposal Restrictions"

(IDAPA 58.01.05.011) and "Alternative LDR

Treatment Standards for Contaminated Soil" (40 CFR 268.49)

"Storm Water Discharges (Applicable to State NPDES Programs, see $\S 123.25$ ) (40 CFR 122.26)

\section{Location-Specific ARARs}

National Historic Preservation Act
"Historic Properties Owned or Controlled by Federal Agencies" (17 USC § 470h-2)

"Identification of Historic Properties" (36 CFR 800.4)

“Assessment of Adverse Effects" (36 CFR 800.5)
Compliance Strategy

All soil exceeding the remediation goal of $400 \mathrm{mg} / \mathrm{kg}$ for lead was assumed in this report to exceed the $5.0-\mathrm{mg} / \mathrm{L}$ toxicity characteristic concentration, thereby requiring stabilization prior to disposal at an approved facility.

All soil exceeding the remediation goal of $400 \mathrm{mg} / \mathrm{kg}$ for lead was assumed in this report to exceed the $5.0-\mathrm{mg} / \mathrm{L}$ toxicity characteristic concentration, thereby requiring stabilization prior to disposal at an approved facility.

The STF-02 Gun Range was located outside the area of the designated INL Site storm water corridor. Storm water discharges and sediment from remedial activities at STF-02 did not have the potential to impact the Big Lost River or its tributaries. As such, a storm water pollution prevention plan was not required.

Cultural and archaeological resources surveys were performed prior to the remedial action. The surveys showed that no cultural or archaeological resources were within $100 \mathrm{~m}$ of the facility. No cultural or archaeological resources were encountered during the remedial action. 
Table 1. (continued).

\begin{tabular}{|c|c|c|}
\hline Category & Citation & Compliance Strategy \\
\hline $\begin{array}{l}\text { Native American Graves Protection } \\
\text { and Repatriation Act }\end{array}$ & $\begin{array}{l}\text { "Ownership" ( } 25 \S \text { USC } 3002 \text { et seq.) and } \\
\text { "Custody" ( } 43 \text { CFR 10.6) } \\
\text { "Repatriation" ( } 25 \S \text { USC } 3005 \text { et seq.) and } \\
\text { "Repatriation" ( } 43 \text { CFR 10.10) }\end{array}$ & $\begin{array}{l}\text { Cultural and archaeological resources surveys were } \\
\text { performed prior to the remedial action. The surveys showed } \\
\text { that no cultural or archaeological resources were within } \\
100 \mathrm{~m} \text { of the facility. No cultural or archaeological } \\
\text { resources were encountered during the remedial action. }\end{array}$ \\
\hline \multicolumn{3}{|l|}{ Acronyms } \\
\hline $\begin{array}{l}\text { ARAR = applicable or relevant and appropriate } \\
\text { CFR = Code of Federal Regulations } \\
\text { EPA = Environmental Protection Agency } \\
\text { HASP = health and safety plan } \\
\text { IDAPA = Idaho Administrative Procedures Act } \\
\text { INL = Idaho National Laboratory } \\
\text { LDR = land disposal restriction } \\
\text { NESHAP = National Emission Standards for Ha } \\
\text { OU = operable unit } \\
\text { PDD = program description document } \\
\text { RD } / \text { RA = remedial design/remedial action } \\
\text { STF = Security Training Facility } \\
\text { USC = United States Code }\end{array}$ & $\begin{array}{l}\text { equirement } \\
\text { ardous Air Pollutants }\end{array}$ & \\
\hline
\end{tabular}


- Hazard assessment determination

- Health and safety plan

- $\quad$ Spill prevention and control measures

- Cultural resources survey.

Prior to commencement of work, general activities required each day included plan-of-the-day meetings to review the day's work activities, daily equipment inspections, and calibration of instrumentation as required. In addition, as part of the closeout of the work package, a formal post-job review was conducted.

\subsection{Remedial Action}

Appendix A provides a photographic record of the remedial activities conducted at the STF-02 Gun Range. The remedial action commenced on October 12, 2006, with the mobilization of equipment and was completed on November 29, 2006, when revegetation was completed and equipment was demobilized from the site. Table 2 summarizes the progression of the work activities. The Phase III remedial action consisted of the following activities:

- $\quad$ Site preparation including establishing work areas and mobilizing equipment

- $\quad$ Removal of asphalt pads at the STF-02 Gun Range

- Removal of upright railroad ties used to hold targets

- $\quad$ Removal of electrical power poles and electrical equipment

- Demolition of the STF-02 Shooting House

- $\quad$ Removal of a test stand and burn barrel located in the EOCR pond

- Excavation of contaminated soil at the EOCR pond and gun range

- Mechanical separation of lead contaminated soil

- Loading of contaminated soil for off-Site shipment for stabilization and disposal

- Removal of fencing

- $\quad$ Grading and contouring of the site

- Revegetation

- Demobilization.

\subsubsection{Site Preparation}

Site preparation commenced with clearing and grubbing by ICP personnel of the area immediately surrounding the STF-02 Gun Range, as well as the area between the range berms. Upon mobilization to the STF-02 site, a support zone was established including an office trailer, restroom facilities, "lay-down" areas for temporary storage of equipment and waste, vehicle parking area, and an electrical generator if needed. Electrical power was available from an adjacent power pole with the generator never being required. As work in the area progressed, the haul road leading from the personal vehicle parking area into the task site was filled with soil obtained from one of the uncontaminated berms and graded to enable haul trucks ready access to the loading area. 
Table 2. STF-02 Gun Range remediation timeline.

\begin{tabular}{lll}
\hline \multicolumn{1}{c}{ Activity } & \multicolumn{1}{c}{ Start Date } & Completion Date \\
\hline Complete Record of Decision & - & November 5, 2002 \\
Establish Scope of Work & - & February 1, 2003 \\
Complete Remedial Design/Remedial Action Report & - & \\
$\quad$ Revision 0 & - & September 14, 2005 \\
$\quad$ Revision 1 & - September 19, 2006 \\
Complete Explanation of Significant Differences & October 12, 2006 & October 12, 2006 \\
Mobilize & October 12, 2006 & October 12, 2006 \\
Remove electrical utilities & October 16, 2006 & October 24, 2006 \\
Excavate clean berms to pond & October 17, 2006 & October 17, 2006 \\
Remove and dispose of asphalt & October 19, 2006 & October 19, 2006 \\
Excavate pond hot spots & October 19, 2006 & October 23, 2006 \\
Demolish STF-02 Shooting House & October 24, 2006 & October 30, 2006 \\
Excavate and screen contaminated soil & November 1, 2006 & November 29, 2006 \\
Remove clean berm material and contour area & November 2, 2006 & November 29, 2006 \\
Ship contaminated soil off-Site for treatment and disposal & November 10, 2006 & November 29, 2006 \\
Excavate hot spots & November 27, 2006 & November 29, 2006 \\
Reseed and fertilize & November 29, 2006 & November 29, 2006 \\
Demobilize & January 10, 2007 & January 10, 2007 \\
Complete Prefinal Inspection & January 25, 2007 & January 25, 2007 \\
Complete Confirmation Sampling & March 22, 2007 & March 22, 2007 \\
Complete Draft Remedial Action Report & May 2007 & May 2007 \\
Complete Draft Final Remedial Action Report & & Nong \\
\hline
\end{tabular}

The fenced area surrounding the gun range was enlarged to allow equipment to move freely in and around the berms. For the EOCR pond, the fence was removed from the west side of the berm to allow access to the pond from the gun range with the fence on the south side of the pond moved farther south to allow equipment access within the defined work zone. The exclusion zone was established within the work zone surrounding those berm soils and soil within the EOCR pond previously determined to exceed the lead remediation goal of $400 \mathrm{mg} / \mathrm{kg}$. The exclusion zone was modified as appropriate during the various remediation stages to preclude entry by unauthorized personnel into areas of potential lead contamination. Signs were emplaced delineating the construction and exclusion zones with appropriate entry and exit points established.

\subsubsection{Asphalt Pad Removal}

Prior to removal of the pads, the area was visually examined for the presence of spent and unspent ammunition, which was collected and placed in a container for disposal. The three asphalt pads within the gun range plus an asphalt parking pad located immediately to the south of the gun range were removed using conventional earth-moving equipment. The asphalt was loaded into an end dump truck using a front-end loader and transported to the CFA landfill for disposal. 


\subsubsection{Removal of Upright Railroad Ties}

Based on sampling conducted during the RI/FS for WAGs 6 and 10, OU 10-04, upright railroad ties used to hold practice targets at the gun range were determined to be characteristic for lead. As such, the railroad ties had to be shipped off-Site to an approved TSDF for encapsulation and disposal. The railroad ties were removed using conventional heavy equipment. The ties were temporarily stockpiled on a tarp and kept covered to await loading for transport to the TSDF. The railroad ties were loaded into two roll-on/roll-off containers provided by the TSDF and transported to the facility.

\subsubsection{Removal of Electrical Utilities}

Based on results of the subsurface investigation, none of the electrical lines within the gun range was determined to be active. The bang board and power poles were removed using conventional heavy equipment. Electrical lines were cut a minimum of $1 \mathrm{ft}$ below the surface and abandoned in place. Waste materials were loaded and transported to the CFA landfill for disposal.

\subsubsection{Shooting House Demolition}

The STF-02 Shooting House was demolished using an excavator to remove the wood siding, roof, and railroad ties that formed the foundation and were used in the interior walls of the structure to stop bullets. The siding consisted of plywood with roofing materials including fiberglass panels and plywood. The plywood along with the joists and wall studs was sent to the CFA landfill where it could be chipped into wood mulch. Many of the railroad ties used in the foundation were decaying and were removed using a front-end loader, segregated from soil, and loaded for transport along with the fiberglass panels for disposal at the CFA landfill. The railroad ties used in the interior walls of the structure were visually examined for evidence of bullet penetrations. Those identified as having bullets were segregated with the upright railroad ties that had been used to hold targets and held for shipment off-Site to an approved TSDF for encapsulation and disposal. Those railroad ties with no evidence of bullets were sent to the CFA landfill for disposal. The sand that had filled the void between the plywood and the railroad ties in the interior walls was allowed to fall to the dirt floor of the shooting house where it was collected, along with a 3-in. cut of the soil underlying the shooting house, and added to the contaminated soil in the main berm for inclusion with the soils that would undergo physical separation through the screening plant.

\subsubsection{Removal of Test Stand and Burn Barrel}

The test stand and burn barrel located in the EOCR leach pond was identified in July 2000 as new site MISC-33 under OU 10-08. The barrel and test stand consisted of a drum inside a stainless steel cylinder and an apparatus constructed of metal and stainless steel. The annulus between the drum and the stainless steel cylinder contained soil. The actual drum contained ashes, thermocouples, graduated cylinders, beakers, stainless steel blocks, pipettes, crucibles, and other items generated during a series of experiments conducted in the test drum. As a result of the new site identification and subsequent Track 1 Decision Documentation Package - Site 003 (MISC-33) Operable Unit 10-08, Experimental Test Drum in EOCR-01 Leach Pond (DOE-ID 2002b), a Track 2 investigation was performed to assess the potential contaminants of concern at the STF-02 site (DOE-ID 2005a).

As discussed in the Track 2 Summary Report for Operable Unit 10-08 Sites MISC-033, CFA-10A, TRA-60, and TRA-63 (DOE-ID 2005a), sampling of the soil was performed to determine whether the drum contents, soil within the annulus, and soils surrounding the drum and stainless steel cylinder contained lead and nitroaromatics at concentrations that precluded its disposal as industrial (nonhazardous) waste. All contaminants were below the detection limits for the analytical methods employed with the exception of lead from the ash sample collected within the annulus, which had a 
maximum concentration of $32.2 \mathrm{mg} / \mathrm{kg}$. Based on the recommendation provided in the Track 2 Summary Report (DOE-ID 2005a), the test stand and burn barrel were determined to be disposable as industrial waste. Subsequently, the test stand and burn barrel were removed from the pond and shipped to the CFA landfill for disposal.

\subsubsection{Excavation of Contaminated Soil}

Prior to excavation of contaminated soils, the entire gun range was walked down and visually examined for small arms ammunition. This activity was performed at the request of the off-Site TSDF to mitigate the chance that unspent ammunition may be incorporated into soil destined for treatment. Spent cartridges and unspent ammunition were collected by hand to be sent for disposal. In addition, soil undergoing physical separation by screening was visually examined during the process with any unspent ammunition removed.

The primary source of contaminated soil at the STF-02 Gun Range was found in the Berm B with minor quantities also located in Berm $\mathrm{C}$ and the area between the berms. Figure 6 shows the location of each of the berms (A through $\mathrm{E}$ ) at the gun range. Although preremediation sampling did not identify any areas of contamination within the EOCR pond exceeding the remediation goal of $400 \mathrm{mg} / \mathrm{kg}$ for lead, two spots were visually identified as having spent shotgun shells as well as other small arms ammunition. These two spots were initially isolated during the beginning stages of remediation and removed as activities progressed with additional samples collected to confirm that the lead concentrations were below the remediation goal.

Berms $\mathrm{A}, \mathrm{D}, \mathrm{E}$, and much of $\mathrm{C}$ were identified during the preremediation sampling effort as having lead concentrations below the remediation goal of $400 \mathrm{mg} / \mathrm{kg}$. Therefore, soil from Berms A, D, and E, as well as the portion of Berm $\mathrm{C}$ that was below the remediation goal, was used to backfill the EOCR pond. The soil in the berms was too compact for a front-end loader to directly load the soil from the berm and transport it to the pond. Therefore, an excavator was used to cut into the berms, placing the soil in a temporary staging pile immediately adjacent to the berm. The front-end loader was then able to load and move the soil from this temporary staging pile to the pond where it was emplaced in 8-in. lifts. After each 8-in. lift, a roller compacted the fill using three to four passes after which the next lift was emplaced in the pond.

The contaminated soil from Berm B along with that from the portion of Berm $\mathrm{C}$ that exceeded the remediation goal was located in the face and along the top of the berms. The face and top of the berms were removed using the excavator again, staging the soil in a temporary stockpile immediately adjacent to the berm from which the soil was being excavated. The front-end loader was able to scoop soil from this temporary stockpile and place it in the screening plant for segregation by size. The excavated area of each of the berms was sampled and analyzed using both a hand-held XRF spectrometer and conventional analytical methodology to determine whether additional excavation was required to meet the remediation goal of $400 \mathrm{mg} / \mathrm{kg}$ for lead. Any grid determined to exceed the remediation goal was excavated a second time followed by the collection of a second analytical sample to determine whether the goal had been achieved. The second excavation spanned the contaminated grid plus all the adjacent grids known to be below the remediation goal to ensure that contaminated soil exceeding the $400 \mathrm{mg} / \mathrm{kg}$ remediation goal for lead was removed. All excavated soil from the berms was passed through the screening plant. 


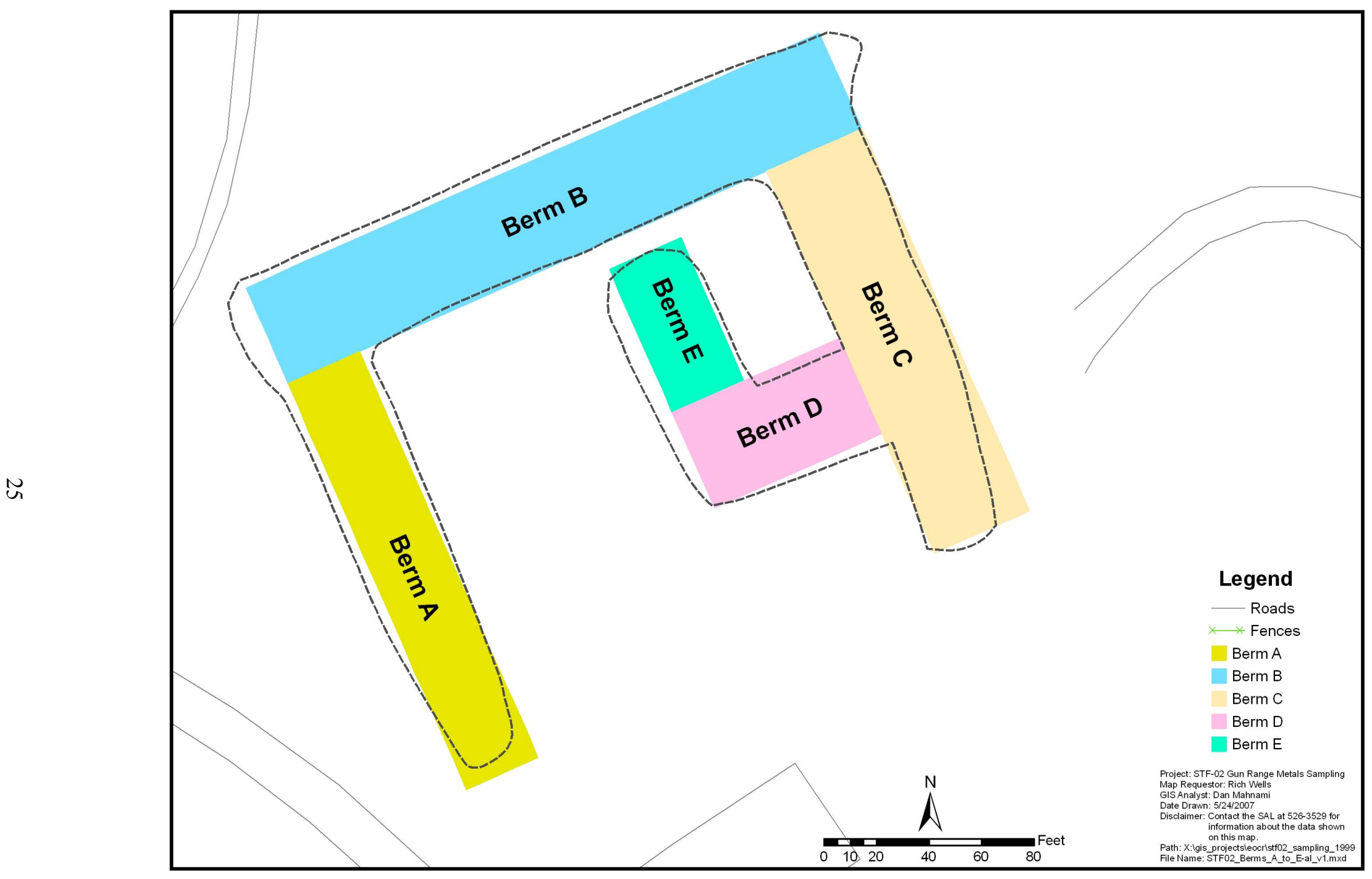

Figure 6. Location of gun range berms. 
Three grid locations between the berms were identified as having lead contamination above the remediation goal of $400 \mathrm{mg} / \mathrm{kg}$. Excavation boundaries for these locations were established using the centers of adjacent grids where the known lead concentrations were below the remediation goal. This resulted in a minimum $20 \times 20$-ft area that was excavated with the soil placed in the contaminated soil stockpile destined for treatment and disposal at the off-Site TSDF. The excavated area was resampled and analyzed for lead to ensure that the remediation goal was achieved.

Following the completion of all screening activities, the footprints of the screening plant and the stockpiles were surveyed using a global positioning system device to aid in establishing excavation areas following the removal of the screening plant and stockpiles. The screening plant was moved off-Site and the stockpiled soil loaded for off-Site shipment for disposal. The impacted areas were subsequently excavated and sampled to ensure that no soil with lead contamination in excess of the $400 \mathrm{mg} / \mathrm{kg}$ remediation goal remained on the STF-02 site. Sample grid locations were established in accordance with the field sampling plan and an iterative process followed whereby, when additional lead contamination was encountered based on analytical results, additional excavation was performed. This process continued until the remediation goal was achieved for the entire area with the final excavated soils loaded directly into an intermodal container for off-Site shipment.

\subsubsection{Mechanical Separation}

Based on the sieve analyses performed during the preremediation sampling effort, using a combination of a 3/4-in. sieve and a 3/8-in. sieve was determined to be sufficient to segregate the majority of the bullets from the larger (greater than $3 / 4$ in.) aggregate and the smaller (less than $3 / 8$ in.) sediment. The segregation of lead from the soil was accomplished using a conventional screening plant outfitted with 3/4- and 3/8-in. screens. The material was segregated into three working stockpiles from which larger stockpiles were formed when a working stockpile became too large for the screening plant setup. Contaminated soil was removed from the berm using an excavator because the soil in the berm was too compacted for a front-end loader to operate efficiently. The excavator would place the soil in a location immediately adjacent to the berm from which the front-end loader was able to obtain soil for loading into the screening plant. Water was used as a dust suppressant during screening operations to mitigate personnel exposure to lead contamination.

The primary reason for screening the contaminated soil was to segregate the bullets from the soil with the end outcome being to ship the lead off-Site for recycling. Unfortunately, the off-Site recycler determined after obtaining a sample of the material proposed for recycling that it was not fiscally viable to recycle the material. Therefore, the fraction less than $3 / 4 \mathrm{in}$. and greater than $3 / 8 \mathrm{in}$. that contained the majority of the lead contamination was combined with other contaminated soil and shipped off-Site for treatment and disposal.

Two secondary reasons existed for performing the segregation. The first was that preliminary analytical results obtained from the preremediation samples indicated that the greater than 3/4-in. fraction consisting primarily of large aggregate may not exceed the $400-\mathrm{mg} / \mathrm{kg}$ remediation goal for lead and could be returned to the area for backfill and contouring rather than shipping off-Site for disposal. Analytical results obtained from the greater than 3/4-in. stockpile did not support this assertion and the material was shipped off-Site for treatment and disposal. The second was that the off-Site disposal facility requested that the soils destined for treatment and disposal be visually examined for any unspent rounds (see Section 2.3.7), which would be removed during the screening operation. 
The soil remaining in Berms 2 and 3, which was below the lead remediation goal of $400 \mathrm{mg} / \mathrm{kg}$, was used to backfill the excavated areas in the gun range with the remaining soil contoured to match the surrounding terrain.

\subsubsection{Loading of Contaminated Soil}

Contaminated soil was loaded into intermodal containers for off-Site shipment to the approved TSDF. For the majority of the operation, two loading locations were established in the support zone immediately adjacent to the exclusion zone. The disposal facility's drivers would back a haul truck with the intermodal container on a trailer into a loading location, off-load the intermodal container, and leave the area prior to the loading operation. The remediation subcontractor would subsequently open the intermodal container, load contaminated soil into the container using a front-end loader, and close the container ensuring the integrity of the lid's seal. Following this operation, the driver was allowed back into the support zone to load the intermodal container back onto the trailer and transport the tractor/trailer to the scale located at CFA for weighing, and ultimately to the off-Site TSDF. If during the weighing operation the truck either exceeded the 80,000 -lb total weight limit or was significantly underweight, the tractor/trailer was returned to the job site where soil was either removed or added as appropriate. The truck was returned to CFA to reweigh prior to continuing to the disposal facility. A total of 96 shipments of contaminated soil were made consisting of a total weight of 3,149,808 lb (1,574.9 tons).

\subsubsection{Removal of Fencing}

Fencing surrounding the STF-02 gun range and EOCR pond was used to establish the work zone, and fencing along the north and west sides of the gun range was moved farther out to allow for equipment movement. The fence between the EOCR pond and the gun range was removed at the beginning of the project to allow equipment to remove the two contaminated spots within the pond and to move backfill soil into the pond where it was subsequently compacted in lifts as the pond was filled. The gate on the south fence of the gun range was removed along with the gate posts and fencing. T-posts were reused to form the support zone fence slightly to the south of the original fence from which yellow and black construction rope was hung and entry and exit points into the support zone were established.

Following the completion of all remediation activities, all fencing materials were removed from the site. Fence posts were located in areas where lead concentrations were at or near background levels and did not exceed the $400 \mathrm{mg} / \mathrm{kg}$ remediation goal. T-posts that were in good condition were sent for reuse on the INL Site while those that were not acceptable were sent for recycle along with the wire that formed the three-strand fence around the facility.

\subsubsection{Grading and Contouring}

Following removal of soils exceeding the remediation goal of $400 \mathrm{mg} / \mathrm{kg}$ for lead, the remaining soil at the site was contoured to match the surrounding terrain. Topsoil was obtained from Rye Grass Flats and spread to a minimum depth of 6 in. across the area impacted by the remediation activities. An analysis of the topsoil was performed in accordance with the Phase III RD/RA Work Plan. Results of the soil test are provided in Table 3. 
Table 3. Soil test data.

\begin{tabular}{lc}
\hline \multicolumn{1}{c}{ Parameter } & Result \\
\hline $\mathrm{pH}$ & 8.1 \\
Salts & $0.7 \mathrm{mmhos} / \mathrm{cm}$ \\
Sodium & $0.3 \mathrm{meq} / 100 \mathrm{~g}$ \\
Cation exchange capacity & $20.8 \mathrm{meq} / 100 \mathrm{~g}$ \\
Excess lime & $1.3 \%$ \\
Organic matter & $1.83 \%$ \\
Organic nitrogen & $70 \mathrm{lb} / \mathrm{acre}$ \\
Nitrate-nitrogen & $13 \mathrm{ppm}$ \\
Phosphorus & $7 \mathrm{ppm}$ \\
Potassium & $590 \mathrm{ppm}$ \\
Calcium & $13.9 \mathrm{meq} / 100 \mathrm{~g}$ \\
Magnesium & $4.6 \mathrm{ppm}$ \\
Sulfate-sulfur & $4 \mathrm{ppm}$ \\
Zinc & $0.6 \mathrm{ppm}$ \\
Iron & $4.8 \mathrm{ppm}$ \\
Manganese & $2.0 \mathrm{ppm}$ \\
Copper & $1.6 \mathrm{ppm}$ \\
Boron & $0.90 \mathrm{ppm}$ \\
\hline
\end{tabular}

\subsubsection{Revegetation}

Site restoration included reseeding those areas affected by the field activities including the gun range, the EOCR pond, adjacent staging areas, and Rye Grass Flats, from which topsoil was obtained. After preparation of a seedbed using a disc to till the top $7.6 \mathrm{~cm}$ (3 in.) of the surface, seed was drilled to a maximum depth of $1.3 \mathrm{~cm}(0.5 \mathrm{in}$.) at a rate of $12.5 \mathrm{lb} /$ acre for the seed mixture. The seeding was performed on November 29 and 30, 2006, meeting the prescribed window of October 1 through November 30, 2006.

Based on the soil analysis results, a fertilizer application rate of $221 \mathrm{lb} /$ acre was determined to be appropriate to amend the soil. Table 4 provides the application rate for the recommended nutrients. To maintain soil moisture levels, wood chip mulch was placed using a modified manure spreader on the reseeded areas in accordance with the specification at a rate of 15 tons/acre.

Table 4. Nutrient application rate.

\begin{tabular}{lc}
\hline \multicolumn{1}{c}{ Nutrient } & $\begin{array}{c}\text { Rate } \\
\text { (lb/acre) }\end{array}$ \\
\hline Nitrogen & 110 \\
$\mathrm{P}_{2} \mathrm{O}_{5}-$ Phosphate & 70 \\
Sulfate-Sulfur & 40 \\
Zinc & 8 \\
Manganese & 3 \\
\hline
\end{tabular}




\subsubsection{Demobilization}

Final demobilization from the STF-02 Gun Range was completed on November 30, 2006. Equipment was decontaminated in accordance with the Miscellaneous Sites Cleanup Project Health and Safety Plan (PLN-2128), and equipment was visually surveyed and decontaminated as necessary prior to release for use on other jobs. The construction trailer was removed, and all waste material had been disposed of at appropriate facilities. Metal T-posts in good condition were sent for reuse, while other metal (e.g., bent T-posts and wire) was sent for recycle.

\subsection{Sampling and Analysis}

The results from the preremediation sampling conducted during August 2006, the grain-size distribution analyses, and the analyses performed to support the remedial action are discussed in the sections below. Sampling and analysis were performed in accordance with the requirements delineated in the Field Sampling Plan for the Operable Units 6-05 and 10-04 Remedial Action, Phase III (DOE-ID 2006b) and complied with the quality assurance/quality control requirements specified in the Quality Assurance Project Plan for Waste Area Groups 1, 2, 3, 4, 5, 6, 7, 10, and Deactivation, Decontamination, and Decommissioning (DOE-ID 2006c). Appendix B provides summaries of the analytical data.

\subsubsection{Preremediation Analytical Results}

Preremediation sampling was performed during August 2006 to refine the contamination boundaries for the project and to provide characterization data to determine whether the lead contamination was associated with a particular grain-size fraction. A $10 \times 10$-ft grid system was established for both the gun range and the EOCR pond.

For Berms A, D, and E, samples were collected from the 0 to 6-in. depth at every fourth grid location, as were samples from the top and back sides of Berms B and C. From the face of Berms B and $\mathrm{C}$, samples were collected from both the 0 to 6 -in. and 6 to 24 -in. depth intervals at every grid location. Based on these samples, Berms A, D, and E were determined to not contain lead at concentrations exceeding the 400-mg/kg remediation goal. Similarly, lead concentrations for the samples collected from the back sides of Berms B and C were below this level. For Berm B, nearly the entire face and the top were determined to exceed the remediation goal. For Berm $\mathrm{C}$, the end of the berm closest to Berm B also exceeded the remediation goal with the portion of the berm farther away from Berm B being below the remediation goal for lead.

Of the samples collected from the 0 to 6-in. depth at the "F" grid locations between Berms A, B, and $\mathrm{E}$, and to the south of Berm D, several immediately adjacent to Berm B were determined to have lead concentrations above the $400 \mathrm{mg} / \mathrm{kg}$ limit (Grids F17, F18, F47, F75, F76, F103, F104, F135, F136, and F159). In addition, six other grid locations were determined to exceed the remediation goal and were delineated for remediation following completion of the berms (Grids F30, F83, F127, F133, F134, and F156). Samples were collected from the 0 to 6-in. depth at the "G" grid locations between Berms B, C, D, and $\mathrm{E}$, demonstrating that the soil in this area was below the remediation goal. Figure 7 summarizes the preremediation analytical results for the STF-02 Gun Range grid locations. Highlighting indicates locations exceeding the lead remediation goal of $400 \mathrm{mg} / \mathrm{kg}$.

Samples were collected from all the grid locations within the EOCR pond with the exception of those that resided on top of the basalt with insufficient soil available to sample. None of the samples collected from locations within the pond exceeded the $400 \mathrm{mg} / \mathrm{kg}$ remediation goal for lead. Figure 8 summarizes the preremediation analytical results for the EOCR pond grid locations. 


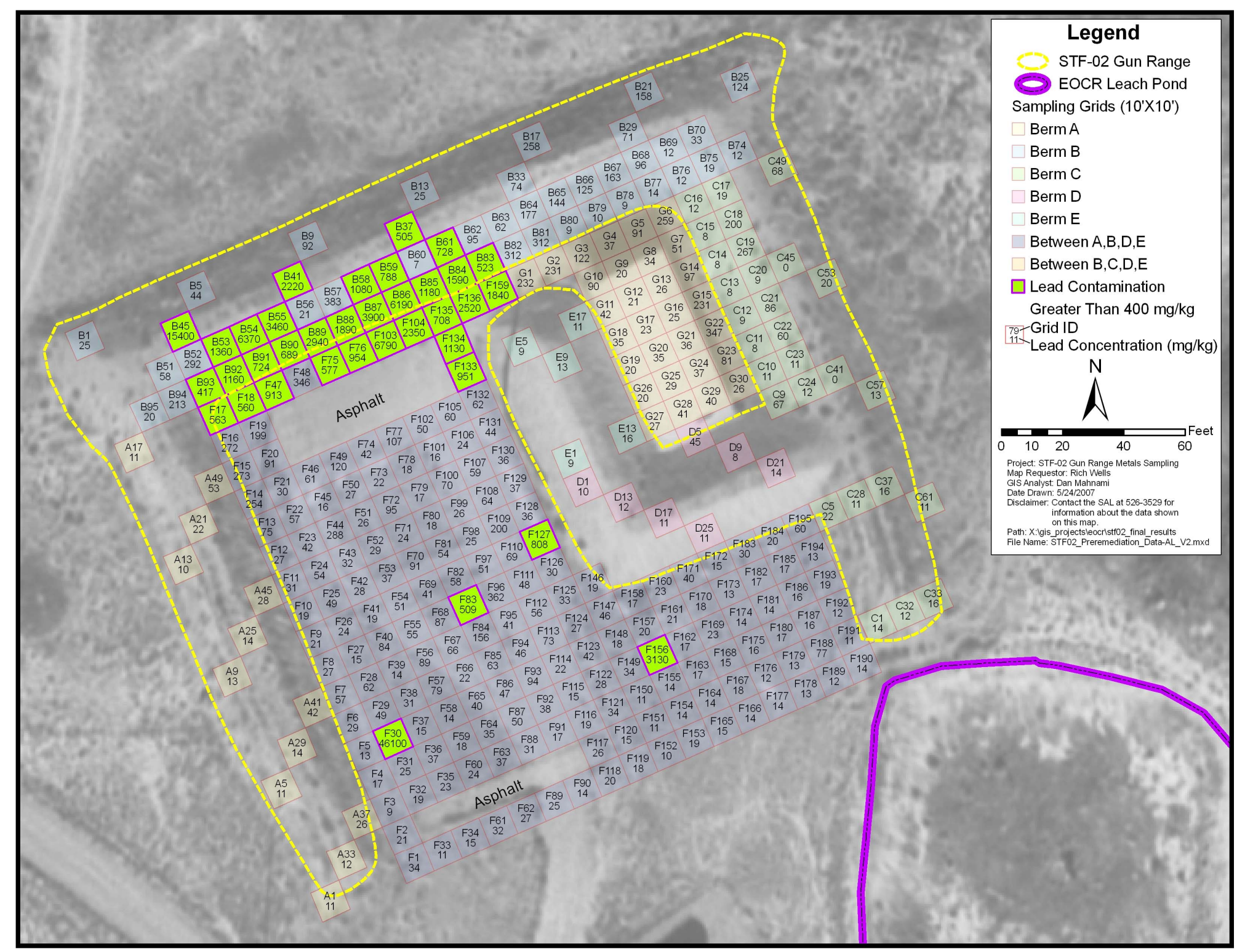

Figure 7. STF-02 Gun Range preremediation analytical results. 


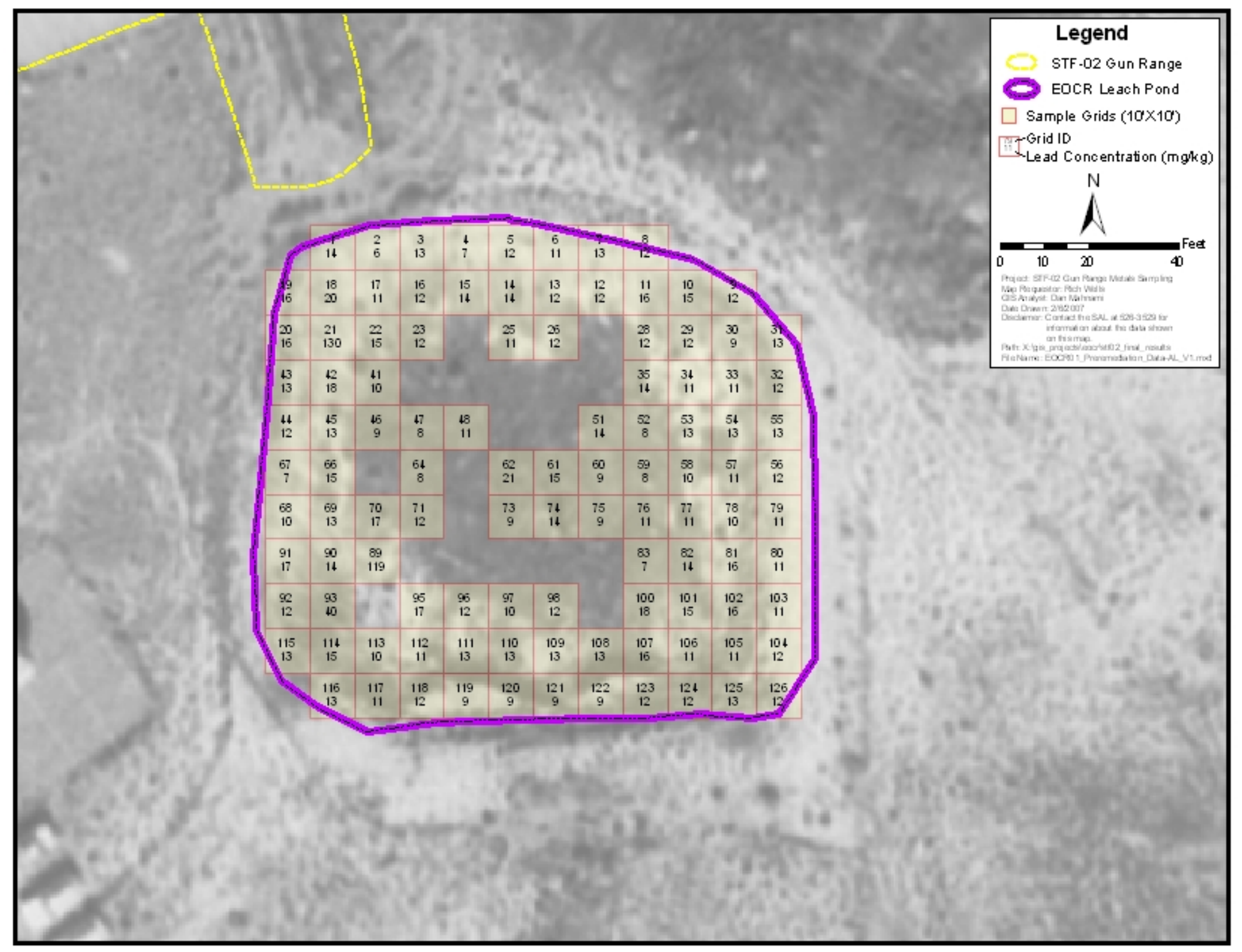

Figure 8. Experimental Organic-Cooled Reactor Pond preremediation analytical results. 


\subsubsection{Grain-Size Distribution}

To determine whether the lead contamination resided within a specific grain-size fraction and to assess the efficacy with which screening would separate lead bullets from the soil, a single sample was collected from Berm B consisting of the sample material collected from across the berm face that was left over after analytical aliquots had been taken. This sample, consisting of approximately 15 gal of material, was submitted for grain-size distribution analysis with each of the subsequent fractions undergoing total and toxicity characteristic leaching procedure (TCLP) lead analyses in accordance with SW-846 Methods 1311, 3010A, and 6010B (EPA 1992a, 1992b, 1996a). Table 5 summarizes the results of the grain-size distribution analysis with Table 6 summarizing the analytical data for the total and TCLP lead analyses of the fractions.

Table 5. Grain-size distribution.

\begin{tabular}{|c|c|c|c|c|c|}
\hline Fraction & $\begin{array}{l}\text { Weight } \\
\text { Retained } \\
(\mathrm{g})\end{array}$ & $\begin{array}{l}\text { Weight } \\
\text { Passing } \\
\text { (g) }\end{array}$ & $\%$ Retained & $\%$ Passing & $\begin{array}{l}\text { Fragments } \\
(\mathrm{g})\end{array}$ \\
\hline$+3 / 4$ in. & $2,263.07$ & $83,968.47$ & 2.6 & 97.4 & None \\
\hline$-3 / 4$ in. $/+3 / 8$ in. & $5,137.84$ & $78,650.63$ & 6.2 & 91.2 & $8,059.75$ \\
\hline $\begin{array}{l}-3 / 8 \mathrm{in} . /+ \text { No. } 4 \\
(4.75 \mathrm{~mm})\end{array}$ & $5,171.18$ & $73,479.45$ & 6.0 & 85.2 & $1,069.69$ \\
\hline $\begin{array}{l}\text { - No. } 4(4.75 \mathrm{~mm}) \\
\text { /+ No. } 10(2.00 \mathrm{~mm})\end{array}$ & $4,278.18$ & $69,201.27$ & 4.9 & 80.3 & 21.67 \\
\hline $\begin{array}{l}\text { - No. } 10(2.00 \mathrm{~mm}) \\
/+ \text { No. } 50(300 \mu \mathrm{m})\end{array}$ & $27,878.13$ & $41,323.14$ & 32.4 & 47.9 & None \\
\hline $\begin{array}{l}\text { - No. } 50(300 \mu \mathrm{m}) \\
/+ \text { No. } 200(75 \mu \mathrm{m})\end{array}$ & $38,952.98$ & $2,370.16$ & 45.2 & 2.7 & None \\
\hline - No. $200(75 \mu \mathrm{m})$ & $2,370.16$ & 0 & 2.7 & 0 & None \\
\hline
\end{tabular}

Table 6 . Total and toxicity characteristic leaching procedure lead results by fraction.

\begin{tabular}{|c|c|c|c|c|}
\hline \multirow[b]{2}{*}{ Fraction } & \multicolumn{2}{|c|}{$\begin{array}{l}\text { Total Lead } \\
(\mathrm{mg} / \mathrm{kg})\end{array}$} & \multicolumn{2}{|c|}{$\begin{array}{l}\text { TCLP Lead } \\
(\mathrm{mg} / \mathrm{L})\end{array}$} \\
\hline & Sample No. 1 & Sample No. 2 & Sample No. 1 & Sample No. 2 \\
\hline$+3 / 4$ in. & 25.1 & 136 & 0.347 & 0.451 \\
\hline$-3 / 4$ in. $/+3 / 8$ in. & 2,030 & 50.5 & 1.07 & 2.56 \\
\hline $\begin{array}{l}-3 / 8 \mathrm{in} . /+ \text { No. } 4 \\
(4.75 \mathrm{~mm})\end{array}$ & 4,310 & 5,440 & 107 & 73.1 \\
\hline $\begin{array}{l}\text { - No. } 4(4.75 \mathrm{~mm}) /+ \\
\text { No. } 10(2.00 \mathrm{~mm})\end{array}$ & 159 & 257,000 & 380 & 558 \\
\hline $\begin{array}{l}\text { - No. } 10(2.00 \mathrm{~mm}) \\
\text { /+ No. } 50(300 \mu \mathrm{m})\end{array}$ & 867 & 3,130 & 378 & 208 \\
\hline $\begin{array}{l}\text { - No. } 50(300 \mu \mathrm{m}) /+ \\
\text { No. } 200(75 \mu \mathrm{m})\end{array}$ & 1,410 & 1,350 & 20.2 & 7.82 \\
\hline - No. $200(75 \mu \mathrm{m})$ & 4,780 & 4,780 & 31.6 & 29.6 \\
\hline
\end{tabular}


As can be seen from the total and TCLP analytical data for the individual grain-size fractions, the only fraction that could possibly be segregated and meet the remediation goal for lead was the greater than $3 / 4$ in. that consisted primarily of large aggregate. The other fractions exceeded the remediation goal with the less than 3/4-in. and greater than 3/8-in. fraction being below the lead toxicity characteristic concentration of $5 \mathrm{mg} / \mathrm{L}$. Based on these analytical data, screening the soil during remediation was determined to have the potential effect of reducing the quantity of soil requiring shipment off-Site for treatment and disposal.

\subsubsection{Analytical Results Supporting the Remediation Action}

During the remediation action, analytical results were obtained for the EOCR pond, the gun range berms, and the area between the berms including the hot spots identified during the preremediation sampling. The sections below summarize the results for these sampling activities.

2.4.3.1 EOCR Pond. The two locations within the EOCR pond visually identified as potentially having elevated concentrations of lead were sampled after being excavated. The analytical results are summarized in Table 7, with locations shown in Figure 9. The results demonstrate that lead concentrations for the soils in the two locations are well below the $400-\mathrm{mg} / \mathrm{kg}$ remediation goal.

Table 7. Experimental Organic-Cooled Reactor Pond lead analytical results.

\begin{tabular}{lccc}
\hline \multicolumn{1}{c}{ Grid Number } & Sample ID No. & $\begin{array}{c}\text { XRF Data } \\
(\mathrm{mg} / \mathrm{kg})\end{array}$ & $\begin{array}{c}\text { Laboratory Data } \\
(\mathrm{mg} / \mathrm{kg})\end{array}$ \\
\hline EOCR \#1 & GR1F0101LD & $<28.9$ & 6.6 \\
EOCR \#2 & GR1F0201LD & $<32.7$ & 15.17 \\
EOCR \#2 (field duplicate) & GR1F0202LD & N/A & 18.43 \\
\hline XRF = X-ray fluorescence & & & \\
\hline
\end{tabular}

2.4.3.2 Contaminated Berms. Following excavation of the areas of Berms B and C identified during the preremediation sampling effort as having lead contamination in excess of the remediation goal of $400 \mathrm{mg} / \mathrm{kg}$, all grid locations of the excavated area were sampled and analyzed by both the XRF spectrometer and the classical SW-846 Laboratory Method 6020 (EPA 1994) to determine whether the remediation goal had been achieved for the soil remaining in the berm.

Based on the first round of sampling, the lead remediation goal was determined to have been achieved for the soils remaining in Berm C with two grid locations (B-87 and B-90) within Berm B as being questionable. Although the initial analysis of the sample collected from the B-90 grid following the first excavation indicated that the remediation goal may have been achieved for the location, the sample was reanalyzed by XRF following a more detailed sample preparation yielding a maximum lead concentration of $432.7 \mathrm{mg} / \mathrm{kg}$. Based on this result for the B-90 grid and the results for B-87, these two locations were excavated a second time with the second round of samples collected from the two grids. The second sample round demonstrated that the remediation goal had been achieved. Table 8 summarizes the analytical results for these two grid locations for both the first and second round of remediation support samples. A summary of all the remedial action support analytical results collected from the contaminated berms is provided in Appendix B. 


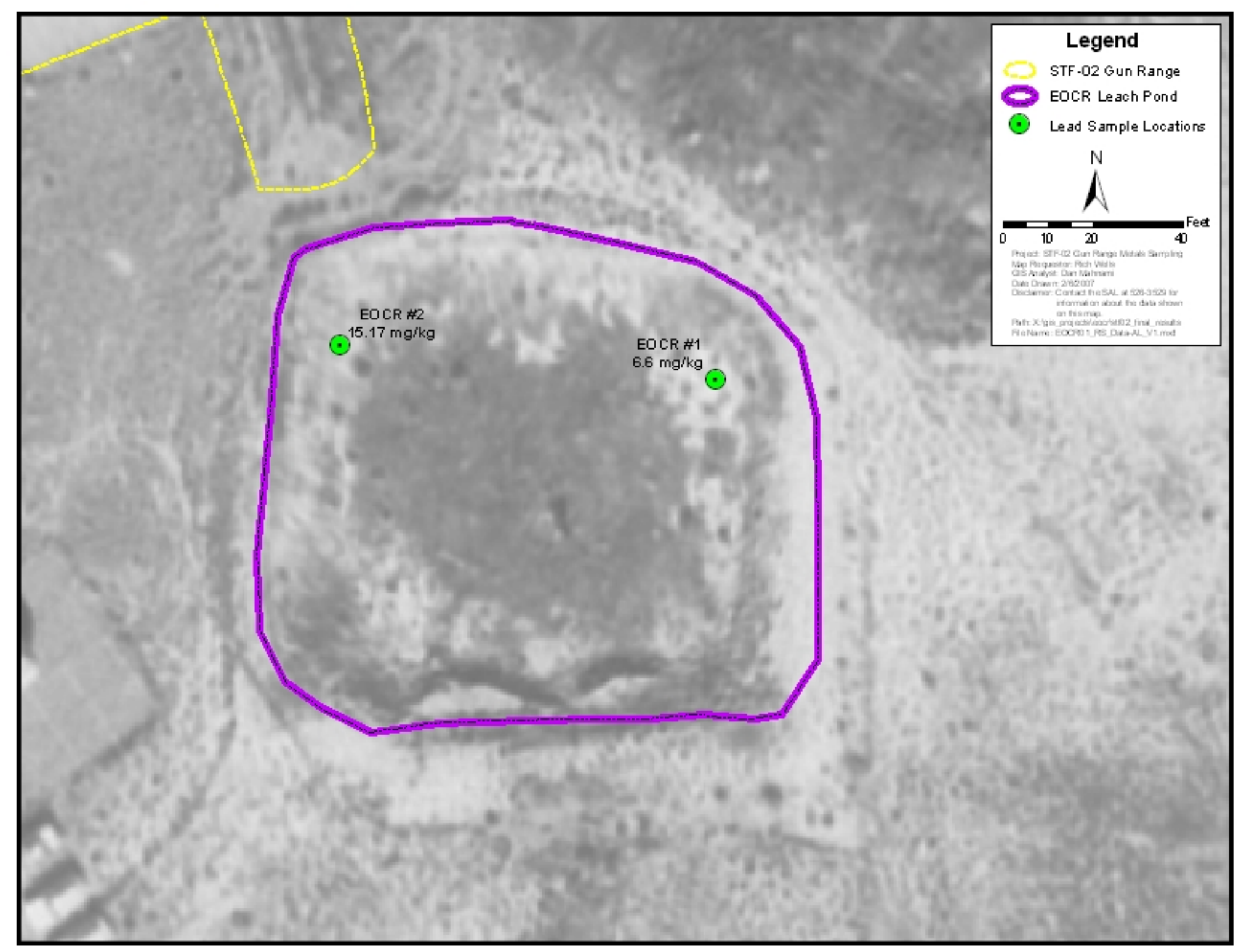

Figure 9. Experimental Organic-Cooled Reactor remediation support analytical results. 
Table 8. Contaminated berm reexcavation analytical results.

\begin{tabular}{|c|c|c|c|c|c|}
\hline \multirow[b]{2}{*}{ Grid No. } & \multicolumn{3}{|c|}{$\begin{array}{l}\text { XRF Results } \\
(\mathrm{mg} / \mathrm{kg})\end{array}$} & \multirow[b]{2}{*}{ Sample No. } & \multirow{2}{*}{$\begin{array}{c}\text { Lab Result } \\
(\mathrm{mg} / \mathrm{kg})\end{array}$} \\
\hline & Run \#1 & Run \#2 & Run \#3 & & \\
\hline B-87 (first excavation) & 276.2 & 237.2 & 781.8 & GR1F7201LD & 621.38 \\
\hline B-87 (first excavation) & N/A & N/A & N/A & $\begin{array}{l}\text { GR1F7202LD } \\
\text { (field duplicate) }\end{array}$ & 779.75 \\
\hline B-87 (second excavation) & $<33.3$ & $<33.9$ & $<26.9$ & GR1F9001LD & 6.22 \\
\hline B-90 (first excavation) & 252.3 & 245.3 & 239.7 & GR1F6701LD & 303.33 \\
\hline B-90 (second excavation) & $<33.1$ & $<35.8$ & $<32.9$ & GR1F8901LD & 7.79 \\
\hline
\end{tabular}

2.4.3.3 Area Between the Berms. As discussed in Section 2.4.1, six grid areas between the berms were identified during the preremediation sampling as having lead concentrations in excess of the $400-\mathrm{mg} / \mathrm{kg}$ remediation goal. These areas were excavated to remove the contaminated soil and sampled to demonstrate whether the remediation goal had been achieved. Table 9 summarizes the preremediation and post-excavation analytical results for these six grid locations between the berms.

Table 9. Analytical results for contaminated grids between the berms.

\begin{tabular}{|c|c|c|c|c|c|}
\hline \multirow[b]{2}{*}{ Grid No. } & \multirow{2}{*}{$\begin{array}{l}\text { Preremediation } \\
\text { Lead Results } \\
(\mathrm{mg} / \mathrm{kg})\end{array}$} & \multicolumn{4}{|c|}{$\begin{array}{l}\text { Post-Excavation Lead Results } \\
(\mathrm{mg} / \mathrm{kg})\end{array}$} \\
\hline & & XRF \#1 & $\mathrm{XRF} \# 2$ & XRF \#3 & $\mathrm{Lab}$ \\
\hline $\mathrm{F}-30$ & 46,100 & $<35.8$ & $<32.8$ & $<36.5$ & 6.73 \\
\hline F-83 & 509 & 40.2 & $<31.4$ & $<36.9$ & 14.75 \\
\hline F-127 & 808 & $<31.3$ & $<32.0$ & $<29.3$ & 14.13 \\
\hline F-133 & 951 & 106.1 & 107.4 & 99.5 & 95.15 \\
\hline F-134 & 1,130 & 70.4 & 48.3 & 60.8 & N/A \\
\hline F-156 & 3,130 & $<34.9$ & $<29.6$ & $<36.3$ & 12.62 \\
\hline
\end{tabular}

2.4.3.4 Sampling Beneath the Screening Plant and Stockpile Locations. Following the completion of all soil screening activities, the subsequent removal of the stockpiles formed from the screened soils and excavation of 6 in. from these locations, the grids potentially impacted by the screening and stockpiling activities were sampled to determine whether any additional excavation was required to achieve the remediation goal of $400 \mathrm{mg} / \mathrm{kg}$ for lead. Figure 10 shows the grid locations with soils that were potentially impacted by the screening plant operations and the stockpiles formed from the soils sent through the plant. Samples were analyzed by both the XRF spectrometer and the classical SW-846 laboratory method to determine whether the remediation goal had been achieved for the remaining soil. Based on the first round of sampling, the lead remediation goal was determined to have been achieved for all the locations. Three of the grid locations (Grids F-84, F-86, and F-87) were potentially suspect based on the XRF data. These grid locations were reexcavated with a new stockpile formed. Table 10 summarizes the analytical results for the three grid locations for the sampling performed after the initial excavation versus the sampling performed after the second excavation. Because Grid F- 85 was potentially impacted by the excavation activity, it was included for reanalysis. For Grid F-87, a third excavation was required to achieve the remediation goal, as shown by the analytical results presented in the table. 


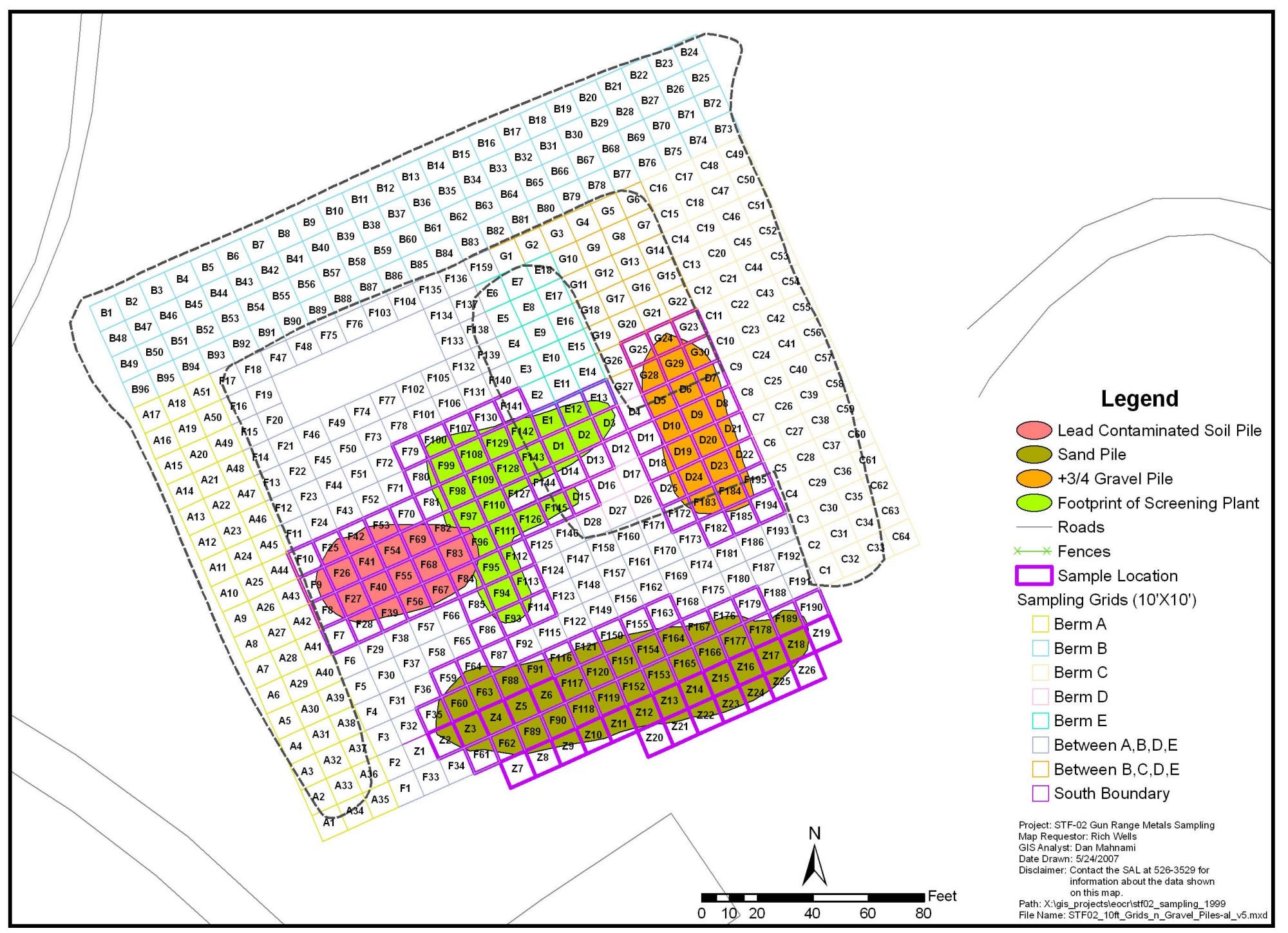

Figure 10. Screening plant and stockpile sampling grids. 
Table 10. Screening plant and stockpile location analytical results.

\begin{tabular}{llcccc}
\hline & Remediation Step & XRF \#1 & XRF \#2 & XRF \#3 & Lab \\
\hline \multirow{2}{*}{ F-84 } & Initial Excavation & 215.3 & 1,040 & 747.3 & 173.23 \\
& Second Excavation & $<40.2$ & 47.3 & $<34.3$ & 16.0 \\
\multirow{2}{*}{ F-85 } & Initial Excavation & $<37.5$ & $<33.7$ & $<40.1$ & 38.96 \\
& Second Excavation & $<37.3$ & $<36.9$ & $<34.5$ & 43.41 \\
\multirow{2}{*}{ F-86 } & Initial Excavation & 81.1 & 160.1 & 58.8 & 226.58 \\
& Second Excavation & $<38.6$ & 56.4 & 67.1 & 14.37 \\
F-87 & Initial Excavation & 187.6 & 170.7 & 222.3 & 294.76 \\
& Second Excavation & 187.6 & 187.5 & 238.4 & N/A \\
& Third Excavation & $<30.9$ & 45.2 & $<33.5$ & 21.96 \\
\hline
\end{tabular}

Figure 11 shows the grid locations with soils that were potentially impacted by this new stockpile. Figure 12 summarizes the analytical results for the remediation support samples collected during the course of the STF-02 remedial action. If a grid had multiple samples collected as a result of additional excavation required, the analytical result from the final sample collected from that grid is presented in the figure because that result is representative of the remaining soil.

\subsubsection{Statistical Considerations}

Lead concentrations were measured on a $10 \times 10$ grid using two methods: XRF and standard laboratory analysis following SW-846 protocol. As well as for estimating the average concentration and planning the required samples for confirmation in attaining remediation goals, the data were used to compare results from the two methods.

A total of 258 locations were measured for lead contamination. For the majority of locations, three XRF measurements were taken and one sample was sent for laboratory analysis. In addition, 14 field duplicates were collected and submitted for laboratory analysis. Five of the samples were submitted for reanalysis by the laboratory because results were inconsistent with the XRF measurements. Two locations were reexcavated based on elevated lead concentrations.

Substantial censoring issues were associated with the XRF data given that $52 \%$ of the locations had three results below the instrument's detection limit. For all analyses, the maximum XRF result was used, with a "U" flag attached if all XRF values were nondetects. This is a conservative approach for estimating the mean concentration, but not for estimating the variance. To assess the impact, the between-location variance was compared to the within-location variance. The median within-location variance for locations with three detectable results was approximately $440 \mathrm{mg} / \mathrm{kg}$, while the between-location variance for locations with three detectable results was approximately $2,500 \mathrm{mg} / \mathrm{kg}$. Although not insignificant, the within-location variance is only $20 \%$ of the between-location variance. The variance among the maximum $\mathrm{XRF}$ results is expected to account for the measurement variance within locations. 


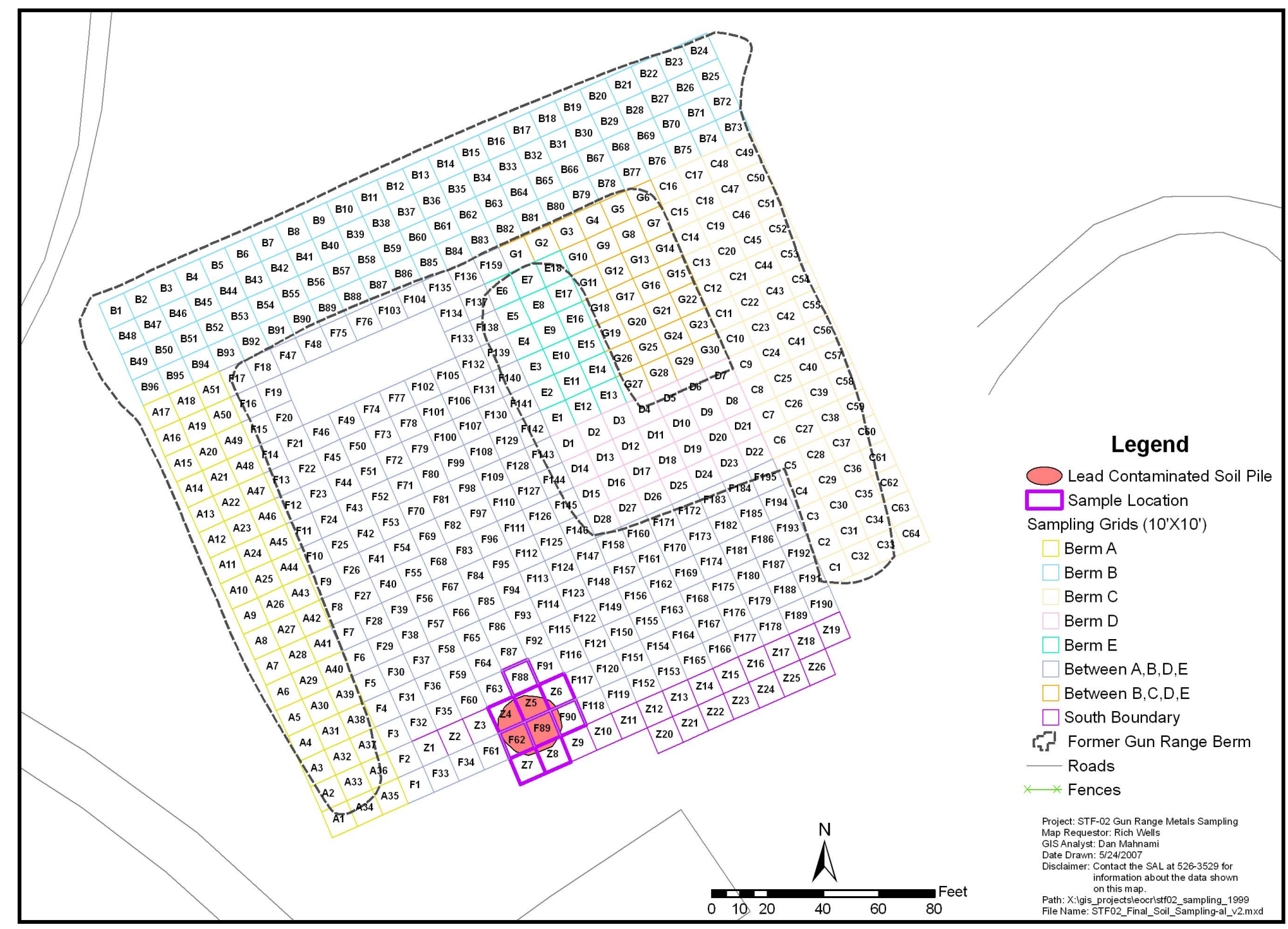

Figure 11. Final stockpile sampling grids. 


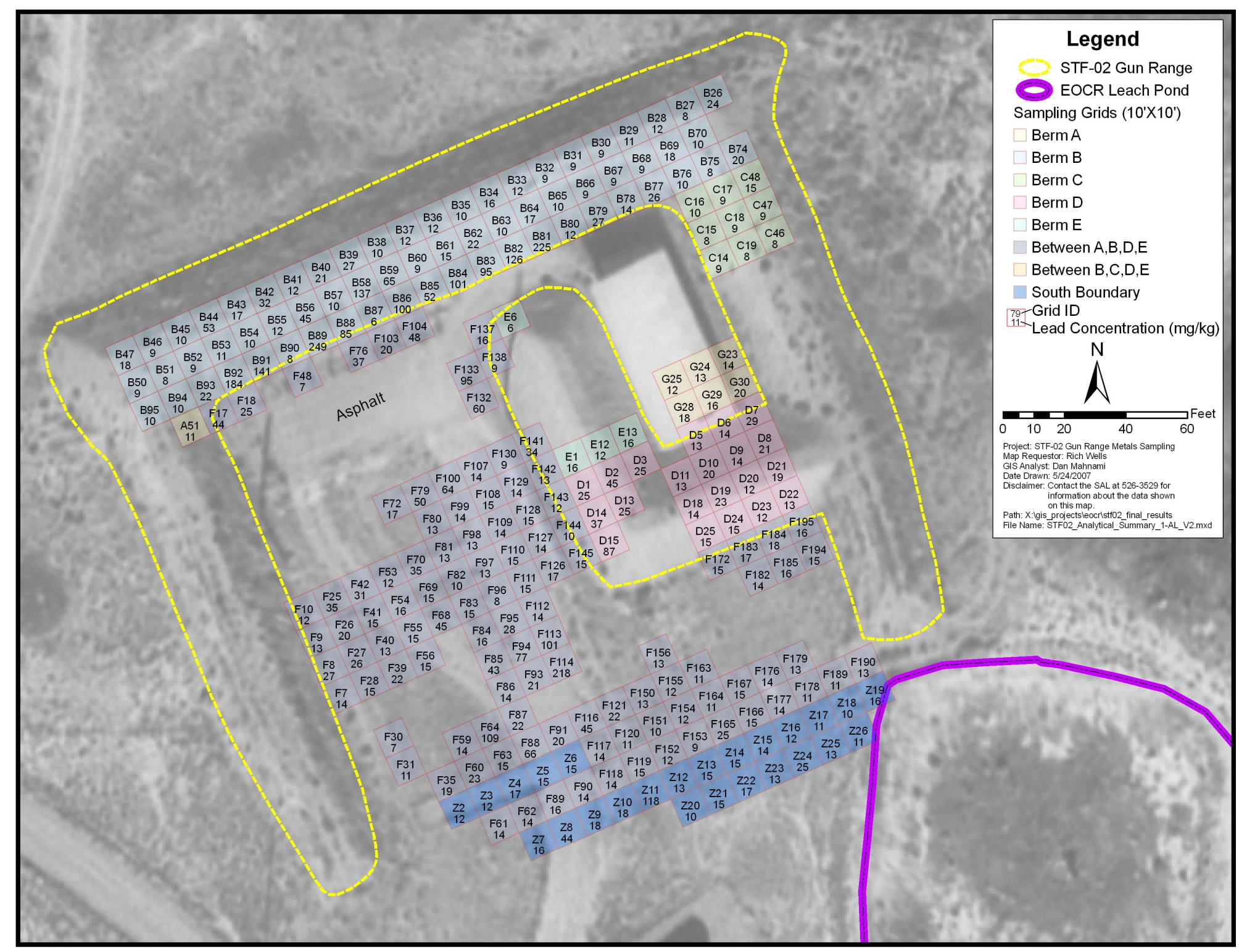

Figure 12. Remediation support analytical results summary. 
Only nine laboratory results were censored. For both data sets, an imputation method based on quantile-quantile regression was used to estimate the censored values to calculate the upper confidence limit as described below.

The three XRF concentrations per location were not very strongly correlated (Spearman rank correlation $\sim 0.4$ ), although the correlation was significantly greater than 0 . The laboratory data were also not strongly (although, again significantly) correlated with any of the XRF concentrations or the maximum XRF concentration (Spearman rank correlation $\sim 0.4$ to 0.5 ). Using only detected values, the correlation was 0.7 , an expected increase when the truncated values are removed.

The XRF results were assumed to be comparable to the laboratory data. At first glance, the XRF concentrations are greater than those generated by the laboratory. A modified sign test (Helsel 2005) is most appropriate for these paired data that have so many nondetects. For each record, a value of $-1,0$, or 1 is assigned based on whether the laboratory result was greater than, tied to, or less than the maximum XRF result. Fully half the records were assumed tied. The laboratory result was less than the detection limit of a nondetected maximum XRF result. The p-value for the modified test was 0.9. Although 12 of the laboratory results were greater than the maximum XRF concentration and 103 were lower, the 121 ties indicate that the methods are comparable.

The distribution of the data was troublesome. For all the data as well as for just the detected results, the distribution was neither normal nor lognormal. In the end, a lognormal distribution was assumed for estimating censored values and upper confidence limits.

The censored values were estimated using an imputation of values based on quantile-quantile regression assuming a lognormal distribution. Quantile-quantile regression models the ordered data values on quantiles of the assumed distribution (in this case, lognormal). The model-fitted values estimate the censored values.

The upper confidence limits are presented on a natural logarithmic scale because back-transformation introduces bias. The 95\% upper confidence limit for the XRF data is 3.5 and for the laboratory data is 3 . The remediation goal on the natural logarithmic scale is 6 . Based on either data set, a sample size of 3 is required to confirm that the remediation goal has been met with $95 \%$ confidence. In accordance with Section 3.7.4 of the Field Sampling Plan for the Operable Units 6-05 and 10-04 Remedial Action, Phase III (DOE-ID 2006b), if the calculated number of confirmation samples is less than 10, then 10 samples will be submitted for confirmation analyses.

\subsubsection{Confirmation Analyses}

The locations for confirmation samples were randomly selected from the 244 discrete grids within the STF-02 Gun Range. The samples were submitted to an independent off-Site laboratory for total lead analyses in accordance with SW-846 Method 3050B/6020 (EPA 1996b, 1994). Table 11 summarizes the correlation of the on-Site laboratory analytical data versus that generated by the off-Site laboratory for the selected grid locations. The calculated correlation coefficient $(\mathrm{R})$ for the two data sets is 0.95 indicating an excellent correlation. For the on-Site laboratory, the 95\% upper confidence limit calculated using ProUCL Version 3.0 (EPA 2004) is 68.6, assuming a gamma distribution of the data. For the off-Site laboratory, the $95 \%$ limit is $91.6 \mathrm{mg} / \mathrm{kg}$, again assuming a gamma distribution of the data. 
Table 11. Confirmation sample correlation.

\begin{tabular}{lcc}
\hline & \multicolumn{2}{c}{$\begin{array}{c}\text { Total Lead Analytical Results } \\
(\mathrm{mg} / \mathrm{kg})\end{array}$} \\
\cline { 2 - 3 } Grid & On-Site Laboratory & Off-Site Laboratory \\
\hline F132 & 60.3 & 85.3 \\
B60 & 8.85 & 7.97 \\
B59 & 65.1 & 109 \\
B37 & 11.6 & 10.8 \\
D18 & 13.6 & 16 \\
F64 & 109.3 & 108 \\
Z24 & 25.1 & 27.3 \\
F88 & 65.8 & 91.1 \\
Z10 & 16.8 & 21.7 \\
F107 & 14.3 & 13.9 \\
\hline
\end{tabular}

\subsection{Occupational Safety and Health}

Monitoring of personnel industrial hygiene was conducted during the STF-02 Gun Range remedial actions and is discussed in the sections below.

\subsubsection{Noise Surveillance}

Personnel who operated heavy equipment and personnel working near the heavy equipment could have been exposed to average noise levels above 84 decibels for a 10-hour time-weighted average. Working in excess of the 84-dB time-weighted average noise level exceeds the OSHA standard, "Occupational Noise Exposure" (29 CFR 1910.95), requiring the project to implement the company's Hearing Conservation Program. Employees at the task site wore acceptable hearing protection when working in and around heavy equipment generating noise levels in excess of the 84-dB requirement.

\subsubsection{Personal Protective Equipment}

In addition to wearing hearing protection, personnel entering the exclusion zone were required to wear safety footwear, hard hats, eye protection (i.e., safety glasses with side shields as a minimum), and leather gloves. When working in dust-generating conditions that had not yet been evaluated for potential lead exposure by an industrial hygienist, personnel in the exclusion zone who were not working in heavy equipment cabs equipped with high-efficiency particulate air (HEPA) filters wore appropriate respiratory protection and Tyvek suits. Full-face respirators were selected, maintained, and inspected daily in accordance with 29 CFR 1910.134. When collecting samples, leather gloves were replaced with either neoprene or latex gloves. Personal protective equipment was worn at all times except in designated areas (e.g., break areas, office area, and designated pathways).

\subsubsection{Monitoring for Lead Exposure}

Lead exposure monitoring for remediation of the STF-02 Gun Range began on October 19, 2006, and ended on November 9, 2006. Testing was conducted for the first 3 days of each distinct phase of work (e.g., demolition and removal, excavation, stockpiling, screening, and vehicle loading) to ensure that adequate measures were taken to mitigate exposure to lead particles. Work phases were monitored in 
accordance with 29 CFR 1926.62(d)(7) with sampling performed in accordance with the established exposure monitoring plan, which followed the OSHA 29 CFR 1926.62 lead standard. All necessary training was documented and maintained in accordance with 29 CFR 1926.62 and 29 CFR 1926.21.

Air sampling and analysis was performed in accordance with National Institute of Occupational Safety and Health (NIOSH) Analytical Method 7082, "Lead by Flame AAS" (NIOSH 1994). Sampling consisted of both personnel and area samples collected to reflect any exposure over the duration of work activities. Representative samples were collected from the work area and the employee determined to be at the highest risk of exposure in accordance with 29 CFR 1926.62(d)(3)(ii). Personnel were notified in person the day of receipt of monitoring results with an explanation of posted results in accordance with 29 CFR 1926.62(d)(8)(i) and in writing within the prescribed 5-day timeframe.

Engineered exposure controls consisted of working upwind of an area when it was outside the exclusion zone and the use of water was unavailable for dust suppression. Only personnel directly involved in operations were allowed in the area. During loading operations, truck drivers would off-load the intermodal containers adjacent to the exclusion zone and then leave the area, with the site subcontractor responsible for opening, loading, and resealing the container. Afterward, the driver was allowed to return to load the container back onto the truck for transport. Respirators were used in accordance with the established respiratory program with employees using respirator protection, complying with the respirator standard requirements outlined in 29 CFR 1910.134.

All area and personal sampling results were below both the OSHA lead permissible exposure limit of $50 \mu \mathrm{g} / \mathrm{m}^{3}$ (29 CFR 1910.1025) and the action level of $30 \mu \mathrm{g} / \mathrm{m}^{3}$ with all sample results reported as nondetectable for lead. The main contributing factors for low lead exposure levels included good work practices such as positioning personnel upwind from visible dust and limiting the number of personnel in high-risk areas for lead exposure in addition to wet weather conditions and low wind that provided favorable working conditions.

\subsection{Decontamination}

Equipment was subject to decontamination prior to removal from the exclusion zone. Contaminated equipment was identified by visual examination for the presence of dirt. Decontamination was performed in accordance with requirements set forth in Section 10.4 of PLN-2128, "Miscellaneous Sites Cleanup Project Health and Safety Plan." To limit the generation of secondary waste, dry decontamination methods were used to remove contamination amenable to those methods. Equipment was decontaminated in the exclusion zone. Decontamination was performed as required and all equipment was released from the STF-02 site without incident.

\subsection{Lessons Learned and Notable Practices}

During the course of the STF-02 Gun Range remediation, a proactive lessons learned approach was taken to further increase the efficiency of operations. In addition, and as a result of some of the lessons learned, notable practices were implemented during the OU 10-04 Phase III remedial action from which other similar remedial actions could benefit.

\subsubsection{Transport of Contaminated Soil}

Intermodal containers were used for remediation of the STF-02 Gun Range because of their potential capability to mitigate release of contaminated soil with their hard lids equipped with gasket seals. However, lessons learned from the STF-02 project suggest instead using end dump trucks for a more cost-effective approach for two reasons. The end dump trucks can be customized to mitigate release 
of contaminants by outfitting the trucks with liners and appropriately sealed end gates. The intermodal containers had to be off-loaded from the trailer prior to loading because they are too high to be reached by a front-end loader when on the trailer. The off-loading and subsequent loading operations require additional time and manpower to accomplish. Also, opening the container's lid if performed at the height while the container is on the trailer is inherently dangerous.

The second reason for using end dump trucks for future remediation activities over intermodal containers is the weight of material that can be hauled. A truck/trailer with an intermodal weighs an average of 45,000 lb versus an end dump truck weighing in at 33,000 lb. With an over-the-road weight limit of $80,000 \mathrm{lb}$, an intermodal can be loaded with only up to $35,000 \mathrm{lb}$ of contaminated soil versus $57,000 \mathrm{lb}$ for an end dump truck. Using intermodals resulted in an increase of shipments required by approximately $60 \%$ and additional costs incurred not only for the shipments but for the overtime required for loading the additional trucks to meet the project's schedule.

\subsubsection{In Situ Instrumentation}

The use of in situ instrumentation such as the hand-held XRF spectrometer used on this project greatly enhanced the rate with which the field crew was able to excavate contaminated soil. The field instrumentation provided timely characterization data to field and project management personnel enabling the team to make decisions about where additional excavation was required. The iterative process of excavation, analysis, and subsequent excavation minimized the quantity of contaminated soil requiring shipment for off-Site treatment and disposal, thereby leading to reduced cost for the overall project. The near real-time data provided by the field instrumentation minimized any downtime for field personnel, which would have been experienced waiting for analytical results obtained by laboratory-based methods. This approach ultimately led to the completion of all field work in 7 weeks. 


\section{COSTS}

Total project costs for the OU 10-04 Phase III remediation activities incurred for the STF-02 Gun Range are provided in Table 12. These costs include the ICP contractor's project management, materials, and labor costs associated with the remediation, as well as the subcontracted field work and disposal fees. No long-term monitoring or maintenance of institutional controls is projected to be required to meet the ARARs associated with the STF-02 Gun Range. Future reporting pertaining to STF-02 consisting of 5 -year reviews will be performed under the purview of the ICP Long-Term Stewardship organization.

Table 12. Phase III remedial design/remedial action costs.

\begin{tabular}{|c|c|}
\hline Activity & Cost \\
\hline Phase III Remedial Design & $\$ 93,872$ \\
\hline Remediation Technical Support Activities & $\$ 40,845$ \\
\hline Phase III Sampling and Analysis & $\$ 155,609$ \\
\hline Phase III Fieldwork & $\$ 281,158$ \\
\hline Phase III Disposal Costs ${ }^{\mathrm{a}}$ & $\$ 427,855$ \\
\hline Phase III Prefinal Inspection & $\$ 783$ \\
\hline Phase III Final Report ${ }^{\mathrm{b}}$ & $\$ 35,000$ \\
\hline Phase III Remedial Design/Remedial Action Total Cost & $\$ 1,035,122$ \\
\hline \multicolumn{2}{|c|}{$\begin{array}{l}\text { a. Costs associated with disposal of the Phase III waste were incurred by Waste Disposal Services and } \\
\text { were not directly accrued by the Waste Area Group } 10 \text {, Operable Unit } 10-04 \text {, Project. } \\
\text { b. Preparation of the final report is in progress. Final costs are not yet available, but estimated to be an } \\
\text { additional } \$ 5 \mathrm{~K} \text { above the } \$ 30 \mathrm{~K} \text { already expended. }\end{array}$} \\
\hline
\end{tabular}

Costs associated with the implementation and performance of the OU 10-04 Phase III remedial action were significantly below the costs outlined in the Record of Decision Experimental Breeder Reactor-I/Boiling Water Reactor Experiment Area and Miscellaneous Sites, Operable Units 6-05 and 10-04 (DOE-ID 2002a). The total cost projected in the record of decision was $\$ 2,600,000$ as compared to the actual cost of $\$ 1,035,122$. The actual cost was $39.8 \%$ of the estimate. Significant cost savings were realized for the remedial design with an estimate of $\$ 438,000$ provided in the ROD versus $\$ 93,872$ actual costs. This is attributed to $\$ 344,128$ in cost savings realized for preparation of the RD/RA Work Plan and associated documents.

Total costs incurred for the remedial action including sampling and analysis, fieldwork, and disposal costs were $\$ 864,622$ as compared to the estimate in the ROD of $\$ 1,929,000$. The remedial action cost savings is largely attributed to treatment and disposal costs being less than projected $(\$ 427,855$ versus $\$ 870,770$ from the ROD) and fieldwork consisting of excavation, loading for transport, soil screening, backfill, reseeding, and sampling and analysis activities being less ( $\$ 461,548$ versus $\$ 737,211)$. These two activities account for $\$ 718,578$ of the overall cost savings realized for the project. 


\section{MODIFICATIONS TO THE REMEDIAL ACTION WORK PLAN}

Modifications to the STF-02 Gun Range remedial action work plan dealt with the disposal of waste. The Waste Management Plan provided in Appendix B to the Remedial Design/Remedial Action Work Plan for Operable Units 6-05 and 10-04, Phase III (DOE-ID 2006a) specified that the lead and copper debris segregated from the soil through the screening plant would be sent off-Site for recycling. A sample of the material designated for recycling was provided to the off-Site recycling subcontractor. After analysis of the material, recycling the material was determined to not be cost effective. The recycling facility determined that the lead and copper fragments would require further segregation from the soil than the screening plant would provide. To pursue recycling of the lead and copper fragments would have required a much more intensive separation method (e.g., soil washing) resulting in added costs to the project and the generation of additional waste streams requiring disposal. Therefore, the material was shipped off-Site for encapsulation and disposal.

The waste management plan also designated creosote-treated railroad ties as being shipped off-Site for encapsulation and disposal. The reason behind requiring encapsulation of the railroad ties was that many of the ties contained lead bullets from target practice. Those railroad ties with visual evidence of lead (e.g., bullet holes) were shipped for encapsulation and disposal. The railroad ties identified as not having any lead (e.g., those used for the foundation of the shooting house) were acceptable for disposal at the CFA landfill. 


\section{QUANTITIES AND TYPES OF WASTE GENERATED}

Waste generated during the OU 10-04 Phase III remedial activities was managed in accordance with the requirements delineated in the Waste Management Plan provided in Appendix B to the Phase III RD/RA Work Plan. The ICP Waste Disposal Services was responsible for managing all waste generated during the Phase III remediation activities. Waste management was performed in accordance with resident procedures.

\subsection{Waste Minimization and Segregation}

Waste minimization during the STF-02 Gun Range remediation activities was achieved primarily through design and planning to maintain efficient operations. To achieve this goal, waste streams were segregated primarily by the field activity being conducted at the time. Those items that could be recycled or reused were segregated from those requiring disposal. Waste types generated included conditional industrial waste, recyclable/reusable materials, and RCRA hazardous waste. Appropriate waste containers were provided for the waste streams and maintained inside the work area until removed for either storage or disposal.

\subsection{Packaging and Labeling}

Containers for storing hazardous waste met the requirements of 40 CFR 264, Subpart I, "Use and Management of Containers." Waste was packaged in accordance with the criteria set forth in Idaho National Engineering and Environmental Laboratory Waste Acceptance Criteria (DOE-ID 2005b). The types of containers used included the following:

- $\quad$ 208-L (55-gal) open-top drums

- $\quad$ 76-L (20-gal) open-top drums

- Intermodal containers.

Bulk waste destined for disposal at the CFA landfill was shipped in an end-dump truck. All containers were labeled in accordance with resident procedures and all state, federal, and local regulations.

For contaminated soil shipped off-Site for treatment and disposal, the containers of choice were intermodal containers fitted with a liner. Each container had a lightweight rigid lid with a rubber gasket to provide a tight seal between the lid and the container. The lid was hinged for ease of opening by an individual allowing the lid to swing out of the way to one side of the container remaining off the ground.

\subsection{Waste Types}

Table 13 summarizes the waste streams that were generated during the OU 10-04, Phase III, remediation of the STF-02 Gun Range. Appendix E provides a summary of the shipping manifests for the contaminated soil that was sent off-Site for treatment and disposal. A total of 96 shipments were made to Clean Harbors Environmental Services consisting of 3,149,808 lb of soil and lead/copper fragments requiring encapsulation and disposal. The EPA Region 8 has instituted a policy requiring periodic verification of the continued acceptability on facilities that previously have been found acceptable under the CERCLA Off-Site Rule. Verifications of continued acceptability are conducted when a request for Off-Site Rule status is received and the previous verification was conducted more than 60 days previously. The verifications of continued acceptability are valid for a period of 60 days. A verification of 
continued acceptability was completed on the Clean Harbors Grassy Mountain facility on October 30, 2006, and was valid until December 30, 2006. With shipments to this facility completed in November 2006, the shipments were in full compliance with the Off-Site Rule.

Table 13. Operable Unit 10-04 Phase III waste summary.

\begin{tabular}{|c|c|c|}
\hline Waste Stream & Disposal Site & Disposal Status \\
\hline Contaminated soil (characteristic) & Clean Harbors & Disposed of on November 2, 2006 \\
\hline STF-612 Wooden Building & CFA landfill & Disposed of on October 2, 2006 \\
\hline Asphalt Pads & CFA landfill & Disposed of on October 2, 2006 \\
\hline $\begin{array}{l}\text { Creosote-treated railroad ties } \\
\text { (characteristic) }\end{array}$ & Clean Harbors & Disposed of on November 2, 2006 \\
\hline Creosote-treated railroad ties & CFA landfill & Disposed of on October 2, 2006 \\
\hline Lead and copper fragments & Clean Harbors & Disposed of on November 2, 2006 \\
\hline Miscellaneous debris & CFA landfill & Disposed of on November 2, 2006 \\
\hline Metal t-posts in good condition & INL reuse & Sent for reuse on November 2, 2006 \\
\hline Scrap Metal & INL recycle & Sent to recycle on November 2,2006 \\
\hline Unspent rounds and shell casings & Clean Harbors & Disposed of on October 2, 2006 \\
\hline
\end{tabular}




\section{PREFINAL INSPECTION}

The prefinal inspection of the WAG 10, OU 10-04, Phase III, STF-02 Gun Range remediation site was conducted on January 11, 2007, in accordance with the prefinal inspection checklist. The project had most items completed with the exception of the final confirmation sample analyses and validation.

Progress was accepted as satisfactory by the Agency representatives in attendance. In the time since the completion of the prefinal inspection, the final confirmation sample analyses and validation activities have been completed and the results included herein. No further actions are necessary. 


\section{SUMMARY AND VERIFICATION OF WORK PERFORMED 7.1 Summary of Work Performed}

The OU 10-04, Phase III, remediation of the STF-02 Gun Range has been completed in accordance with the Remedial Design/Remedial Action Work Plan for Operable Units 6-05 and 10-04, Phase III (DOE-ID 2006a). The remedial action for the site included the following activities:

- $\quad$ Removal of the test stand and burn barrel located in the EOCR leach pond

- $\quad$ Removal of the three asphalt pads located within the gun range as well as the asphalt pad located just south of the gun range boundary

- Removal of the aboveground electrical utilities and abandonment of belowground inactive electrical lines

- Demolition of the shooting house

- $\quad$ Excavation and screening of contaminated soil

- $\quad$ Packaging and transporting remedial action waste

- Treatment and disposal of soil that exceeds the toxicity characteristic concentration for lead

- $\quad$ Sampling and analysis of soil to guide the remedial action and confirm that the remedial action objectives have been achieved

- $\quad$ Backfilling the EOCR leach pond with berm soils that did not exceed the $400-\mathrm{mg} / \mathrm{kg}$ remedial action goal for lead

- $\quad$ Contouring the remaining soils to match the surrounding terrain

- $\quad$ Backfilling the affected area with topsoil followed by reseeding with native species.

\subsection{Verification of Work Performed}

Verification of the work performed was documented throughout the duration of the project. The subcontract technical representative and the subcontractor's job site supervisor maintained daily logsheets that detailed each day's work activities including pre-job briefings, number and names of personnel on the job site and their functions, and ongoing tasks being undertaken at the job site. Periodic management assessments were conducted during the remedial action to verify that work was being completed in accordance with the Phase III RD/RA Work Plan and the planned work order and that the work was on schedule.

A prefinal inspection of the STF-02 Gun Range was conducted with the Agencies on January 11, 2007, to verify that the work outlined in the Phase III RD/RA Work Plan was accomplished. Results of the prefinal inspection are presented in Appendix B. Drawings detailing the contours prior to, during, and after the remedial action are provided in Appendix D.

\subsection{Performance Standards and Construction Quality Control}

For the STF-02 Gun Range, contaminated soil exceeding the remediation goal of $400 \mathrm{mg} / \mathrm{kg}$ for lead was removed in accordance with the requirements delineated in the Record of Decision Experimental Breeder Reactor-I/Boiling Water Reactor Experiment Area and Miscellaneous Sites, Operable Unit 6-05 and 10-04 (DOE-ID 2002a), as propagated in the Phase III RD/RA Work Plan. Removal and disposal of 
soil contaminated with lead mitigated the human health risk associated with this site as well as reduced the ecological risk associated with lead. The 95\% upper confidence limit for lead from the sampling results was 3.5 based on the XRF data and 3.0 based on the laboratory data presented on a natural logarithmic scale because of bias introduced by back-transformation. This is compared to the remedial action goal of $6(400 \mathrm{mg} / \mathrm{kg})$ on a natural logarithmic scale, indicating that the remedial action was successful. The data set for the STF-02 Gun Range site was analyzed following the EPA guidance provided in Calculating Upper Confidence Limits for Exposure Point Concentrations at Hazardous Waste Sites (EPA 2002). The upper confidence limit was calculated using the ProUCL, Version 3.0, software package. Based on comparison of the postremediation lead concentration to the remediation goal, as prescribed by the Phase III Field Sampling Plan, the remediation of the STF-02 Gun Range is successful.

\subsection{Institutional Controls}

In accordance with the OU 10-04 ROD (DOE-ID 2002a), institutional controls will not be required at STF-02 following remediation if the remedial action goals are achieved. Based on the postremediation lead concentrations being below the remediation goal of $400 \mathrm{mg} / \mathrm{kg}$, institutional controls at the STF-02 site are not required. 


\section{CERTIFICATION THAT THE REMEDY IS OPERATIONAL AND FUNCTIONAL}

As stated in the OU 10-04 ROD (DOE-ID 2002a), the remedial action objectives and the remedial action goals were established to reduce or eliminate the risk to human health and the environment. Given that the remedial action objectives were achieved by evidence that lead concentrations at the STF-02 Gun Range have been reduced below the remedial action goal of $400 \mathrm{mg} / \mathrm{kg}$, institutional controls will not be required at the site. This report certifies that the remedy selected in the Record of Decision-Experimental Breeder Reactor-I/Boiling Water Reactor Experiment Area and Miscellaneous Sites, Operable Unit 6-05 and 10-04 (DOE-ID 2002a) and detailed in the Phase III RD/RA Work Plan has been completed and the remedy is operational and functional. 


\section{CONTACT INFORMATION}

The OU 10-04 Phase III remedial action was completed by CH2M-WG Idaho, LLC, the primary contractor for the Idaho Cleanup Project, operating under DOE-ID Contract DE-AC07-05ID14516. The potentially responsible parties used the following contractor for the Phase III remedial action:

Primary Contact Name and Title: $\quad$ Lance G. Peterson, President

Company Name:

Address:

Phenix of Idaho, Inc.

3655 Professional Way

P.O. Box 1626

Idaho Falls, ID 83403-1626

Phone Number:

(208) 524-6488

The following laboratories provided analytical services for the potentially responsible parties:

Company Name:

Address:

Phone Number:

Company Name:

Address:

Phone Number:
General Engineering Laboratories, LLC

2040 Savage Road

Charleston, SC 29407

(843) 556-8171

CH2M-WG Idaho, LLC

Analytical Laboratories Department

P.O. Box 1625

Scoville, ID 83415

(208) 526-3060

The project manager for the potentially responsible parties was:

Name:

Richard P. Wells

Company Name:

CH2M-WG Idaho, LLC

Address:

P.O. Box 1625, MS 3940

Idaho Falls, ID 83415-3940

Phone Number:

(208) 526-2920

The project manager for DOE-ID was:

Name:

Organization:

Address:

Phone Number:
R. Mark Shaw

U.S. Department of Energy Idaho Operations Office

P.O. Box 1625, MS 1222

Idaho Falls, ID 83415-1222

(208) 526-6442

The project manager for the EPA was:

Name:

Organization:

Address:

Phone Number:
Matt Wilkening

U.S. Environmental Protection Agency, Region 10 1435 North Orchard St.

Boise, ID 83706

(208) 378-5760 
The project manager for the DEQ was:

Name:

Organization:

Address:

Phone Number:
Margaretha English

Idaho Department of Environmental Quality

1410 N. Hilton

Boise, ID 83706

(208) 373-0306 


\section{REFERENCES}

29 CFR 1910.95, Title 29, "Labor," Part 1910, “Occupational Safety and Health Standards," Subpart G, "Occupational Health and Environmental Control," Section 95, "Occupational Noise Exposure," Code of Federal Regulations, Office of the Federal Register.

29 CFR 1910.120, Title 29, "Labor," Part 1910, "Occupational Safety and Health Standards," Subpart H, "Hazardous Materials," Code of Federal Regulations, Office of the Federal Register.

29 CFR 1910.134, Title 29, "Labor," Part 1910, "Occupational Safety and Health Standards," Subpart I, "Personal Protective Equipment," Section 134, "Respiratory Protection," Code of Federal Regulations, Office of the Federal Register.

29 CFR 1910.1025, Title 29, "Labor," Part 1910, "Occupational Safety and Health Standards," Section 1025, "Lead," Code of Federal Regulations, Office of the Federal Register.

29 CFR 1926.21, Title 29, "Labor," Part 1926, "Safety and Health Regulations for Construction," Subpart C, "General Safety and Health Provisions," Section 21, "Safety Training and Education," Code of Federal Regulations, Office of the Federal Register.

29 CFR 1926.62, Title 29, "Labor," Part 1926, "Safety and Health Regulations for Construction," Subpart D, "Occupational Health and Environmental Controls," Section 62, "Lead," Code of Federal Regulations, Office of the Federal Register.

29 CFR 1926.65, Title 29, "Labor," Part 1926, "Safety and Health Regulations for Construction," Subpart D, "Occupational Health and Environmental Controls," Section .65, "Hazardous Waste Operations and Emergency Response," Code of Federal Regulations, Office of the Federal Register.

40 CFR 261.24, Title 40, "Protection of Environment," Part 261, "Identification and Listing of Hazardous Waste," Section 24, "Toxicity Characteristic," Code of Federal Regulations, Office of the Federal Register.

40 CFR 264, Title 40, "Protection of Environment," Part 264, "Standards for Owners and Operators of Hazardous Waste Treatment Storage and Disposal Facilities," Subpart I, "Use and Management of Containers," Code of Federal Regulations, Office of the Federal Register.

40 CFR 268.49, Title 40, "Protection of Environment," Part 268, "Land Disposal Restrictions," Section 49, "Alternative LDR Treatment Standards for Contaminated Soil," Code of Federal Regulations, Office of the Federal Register.

42 USC $\S 6901$ et seq., 1976, "Resource Conservation and Recovery Act (Solid Waste Disposal Act)," United States Code, October 21, 1976.

42 USC $\S 9601$ et seq., 1980, "Comprehensive Environmental Response, Compensation and Liability Act of 1980 (CERCLA/Superfund)," United States Code, December 11, 1980.

56 FR 50634, 1991, "Sole Source Designation of the Eastern Snake River Plain Aquifer, Southern Idaho; Final Determination, U.S. Environmental Protection Agency," Federal Register, U.S. Environmental Protection Agency, October 7, 1991. 
DOE-ID, 2006a, Remedial Design/Remedial Action Work Plan for Operable Units 6-05 and 10-04, Phase III, DOE/NE-ID-1 1202, Rev. 1, U.S. Department of Energy Idaho Operations Office.

DOE-ID, 2006b, Field Sampling Plan for the Operable Units 6-05 and 10-04 Remedial Action, Phase III, DOE/NE-ID-11212, Rev. 1, U.S. Department of Energy Idaho Operations Office.

DOE-ID, 2006c, Quality Assurance Project Plan for Waste Area Groups 1, 2, 3, 4, 5, 6, 7, 10, and Deactivation, Decontamination, and Decommissioning, DOE/ID-10587, Rev. 9, U.S. Department of Energy Idaho Operations Office.

DOE-ID, 2005a, Track 2 Summary Report for Operable Unit 10-08 Sites MISC-033, CFA-10A, TRA-60, and TRA-63, DOE/NE-ID-11232, Rev. 0, U.S. Department of Energy Idaho Operations Office.

DOE-ID, 2005b, Idaho National Engineering and Environmental Laboratory Waste Acceptance Criteria, DOE/ID-10381, Rev. 21, U.S. Department of Energy Idaho Operations Office.

DOE-ID, 2003, Operable Units 6-05 and 10-04, Experimental Breeder Reactor-I/Boiling Water Reactor Experiment Area and Miscellaneous Sites, Remedial Design/Remedial Action Scope of Work, DOE/ID-11035, Rev. 0, U.S. Department of Energy Idaho Operations Office.

DOE-ID, 2002a, Record of Decision Experimental Breeder Reactor-I/Boiling Water Reactor Experiment Area and Miscellaneous Sites, Operable Units 6-05 and 10-04, DOE/ID-10980, Rev. 0, U.S. Department of Energy Idaho Operations Office.

DOE-ID, 2002b, Track 1 Decision Documentation Package - Site 003 (MISC-33) Operable Unit 10-08, Experimental Test Drum in EOCR-01 Leach Pond, 24882, U.S. Department of Energy Idaho Operations Office.

DOE-ID, 2001, Comprehensive Remedial Investigation/Feasibility Study for Waste Area Groups 6 and 10 Operable Unit 10-04, DOE/ID-10807, Rev. 0, U.S. Department of Energy Idaho Operations Office.

DOE-ID, 1991, Federal Facility Agreement and Consent Order for the Idaho National Engineering Laboratory, Administrative Record No. 1088-06-29-120, U.S. Department of Energy Operations Office; U.S. Environmental Protection Agency, Region 10; Idaho Department of Health and Welfare, December 4, 1991.

EPA, 2004, ProUCL, Version 3, Environmental Protection Agency Technical Support Center, Las Vegas, Nevada.

EPA, 2002, Calculating Upper Confidence Limits for Exposure Point Concentrations at Hazardous Waste Sites, OSWER 9285.6-10, U.S. Environmental Protection Agency.

EPA, 1996a, SW-186, Method 6010B, "Inductively Coupled Plasma-Atomic Emission Spectrometry," Rev. 2, On-Line Test Methods for Evaluating Solid Wastes, Physical/Chemical Methods, http://www.epa.gov/epaoswer/hazwaste/test/under.htm, U.S. Environmental Protection Agency, Office of Solid Waste.

EPA, 1996b, SW-186, Method 3050B, “Acid Digestion of Sediments, Sludges, and Soils,” Rev. 2, On-Line Test Methods for Evaluating Solid Wastes, Physical/Chemical Methods, http://www.epa.gov/epaoswer/hazwaste/test/under.htm, U.S. Environmental Protection Agency, Office of Solid Waste. 
EPA, 1994, SW-186, Method 6020, "Inductively Coupled Plasma-Mass Spectrometry," Rev. 0, On-Line Test Methods for Evaluating Solid Wastes, Physical/Chemical Methods, http://www.epa.gov/epaoswer/hazwaste/test/under.htm, U.S. Environmental Protection Agency, Office of Solid Waste.

EPA, 1992a, SW-186, Method 1311, "Toxicity Characteristic Leaching Procedure," Rev. 0, On-Line Test Methods for Evaluating Solid Wastes, Physical/Chemical Methods, http://www.epa.gov/epaoswer/hazwaste/test/under.htm, U.S. Environmental Protection Agency, Office of Solid Waste.

EPA, 1992b, SW-186, Method 3010A, “Acid Digestion of Aqueous Samples and Extracts for Total Metals for Analysis by FLAA or ICP Spectroscopy," Rev. 1, On-Line Test Methods for Evaluating Solid Wastes, Physical/Chemical Methods, http://www.epa.gov/epaoswer/hazwaste/test/under.htm, U.S. Environmental Protection Agency, Office of Solid Waste.

EPA, 1988, Guidance for Conducting Remedial Investigations and Feasibility Studies Under CERCLA, Interim Final, EPA/540/G-89/004, U.S. Environmental Protection Agency.

Helsel, D. R., 2005, Nondetects and Data Analysis: Statistics for Censored Environmental Data, John Wiley and Sons, New York.

IDAPA 58.01.05.011, "Land Disposal Restrictions," Idaho Administrative Procedures Act.

INL, 2005, Idaho National Laboratory Comprehensive Land Use and Environmental Stewardship Report, INL/EXT-05-00726, Rev. 0, Idaho National Laboratory, September 2005.

NIOSH, 1994, "Lead by Flame AAS," Analytical Method 7082, Issue 2, National Institute of Occupational Safety and Health.

PLN-2128, "Miscellaneous Sites Cleanup Project Health and Safety Plan,” Rev. 2, October 31, 2006.

Rapanos et Ux., et al. v. United states, No. 04-1034, June 19, 2006.

Ryan, Mark A., 2003, "Stormwater Compliance at the INEEL," CCN 46063, U.S. Environmental Protection Agency, Region X, Idaho Operations Office, letter to Amy Grose, U.S. Department of Energy Idaho Operations Office, October 27, 2003.

STD-101, “ICP Integrated Work Control Process,” Rev. 19, October 26, 2006. 


\section{Appendix A}

\section{STF-02 Gun Range Photographic Record of Remediation Activities}




$$
\text { A-2 }
$$




\section{Appendix A}

\section{STF-02 Gun Range Photographic Record of Remediation Activities}

The photographic record in this appendix provides a pictorial summary of the progression of the remedial action at the STF-02 Gun Range from the initial preremediation sampling through the revegetation and mulching of the site.

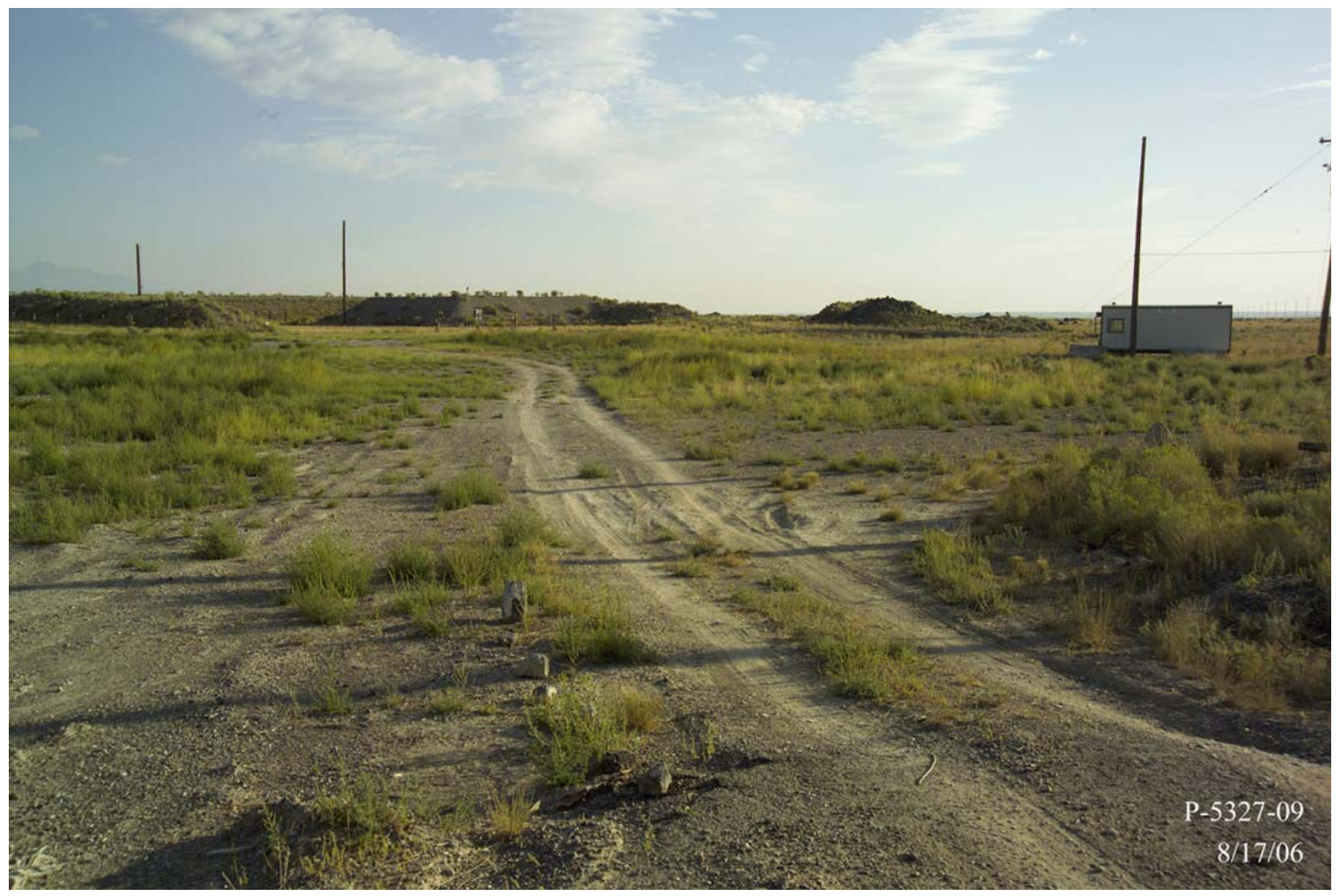

Figure A-1. STF-02 Gun Range prior to remediation. 


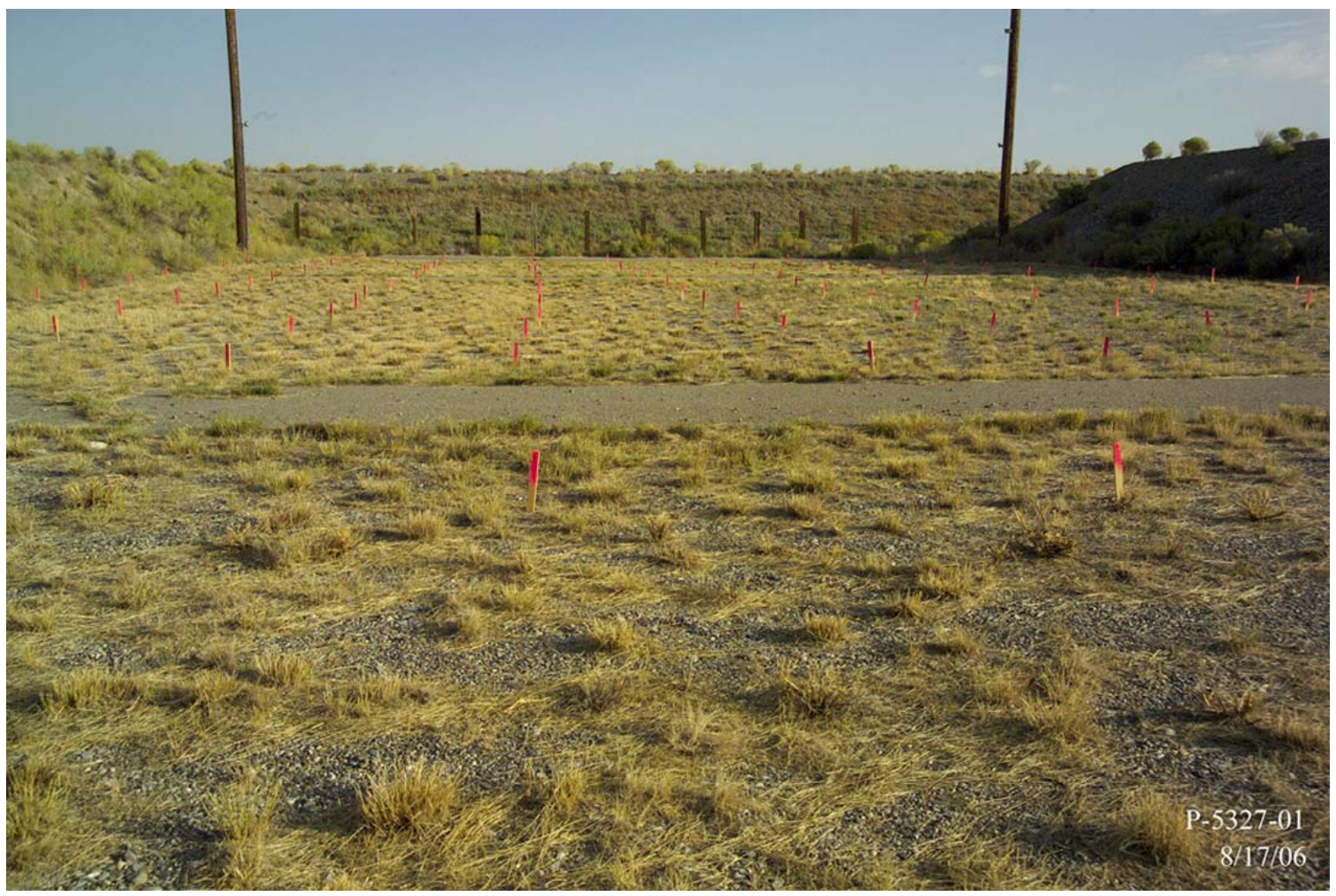

Figure A-2. STF-02 Gun Range preremediation sample locations.

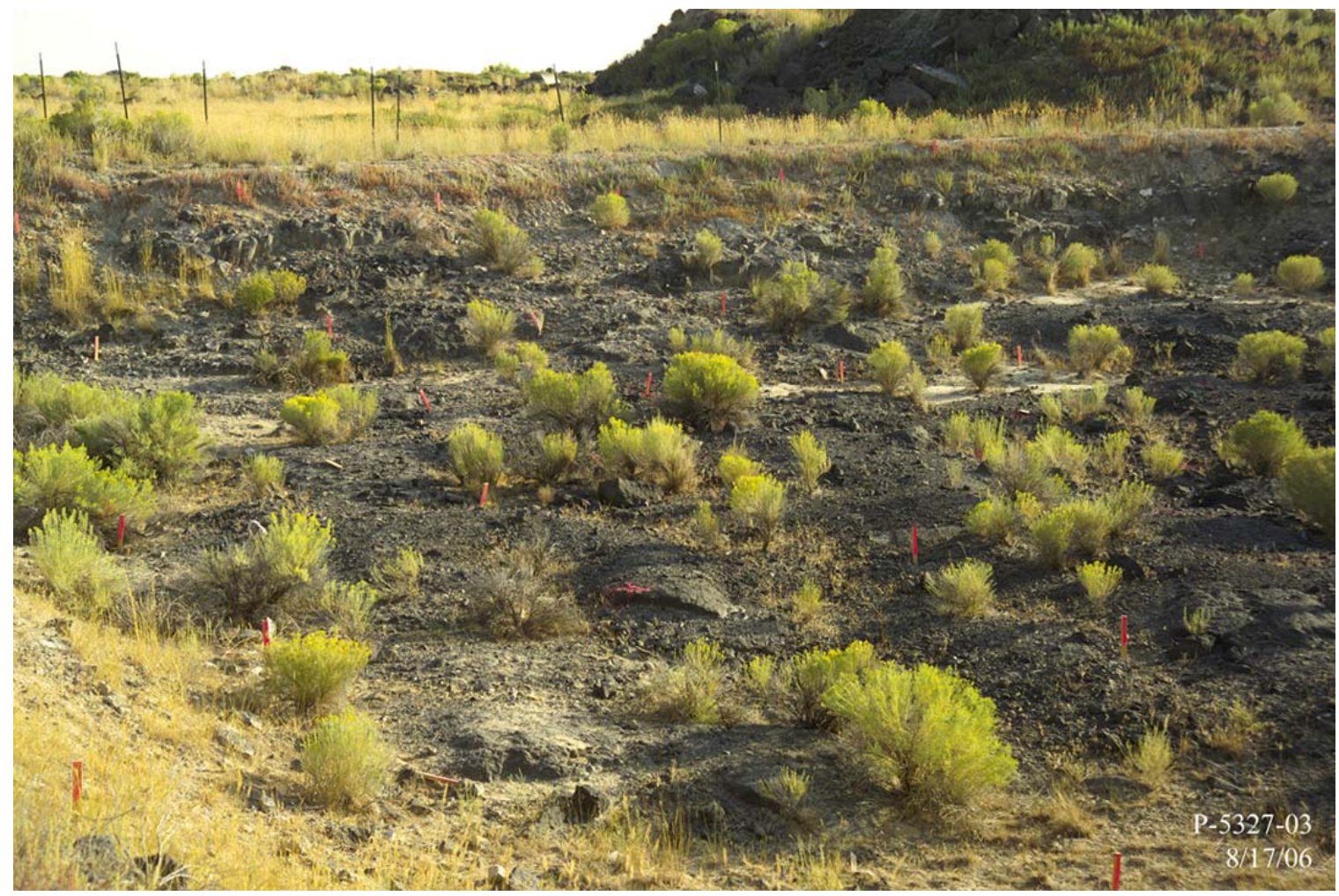

Figure A-3. Experimental Organic-Cooled Reactor Pond preremediation sample locations. 


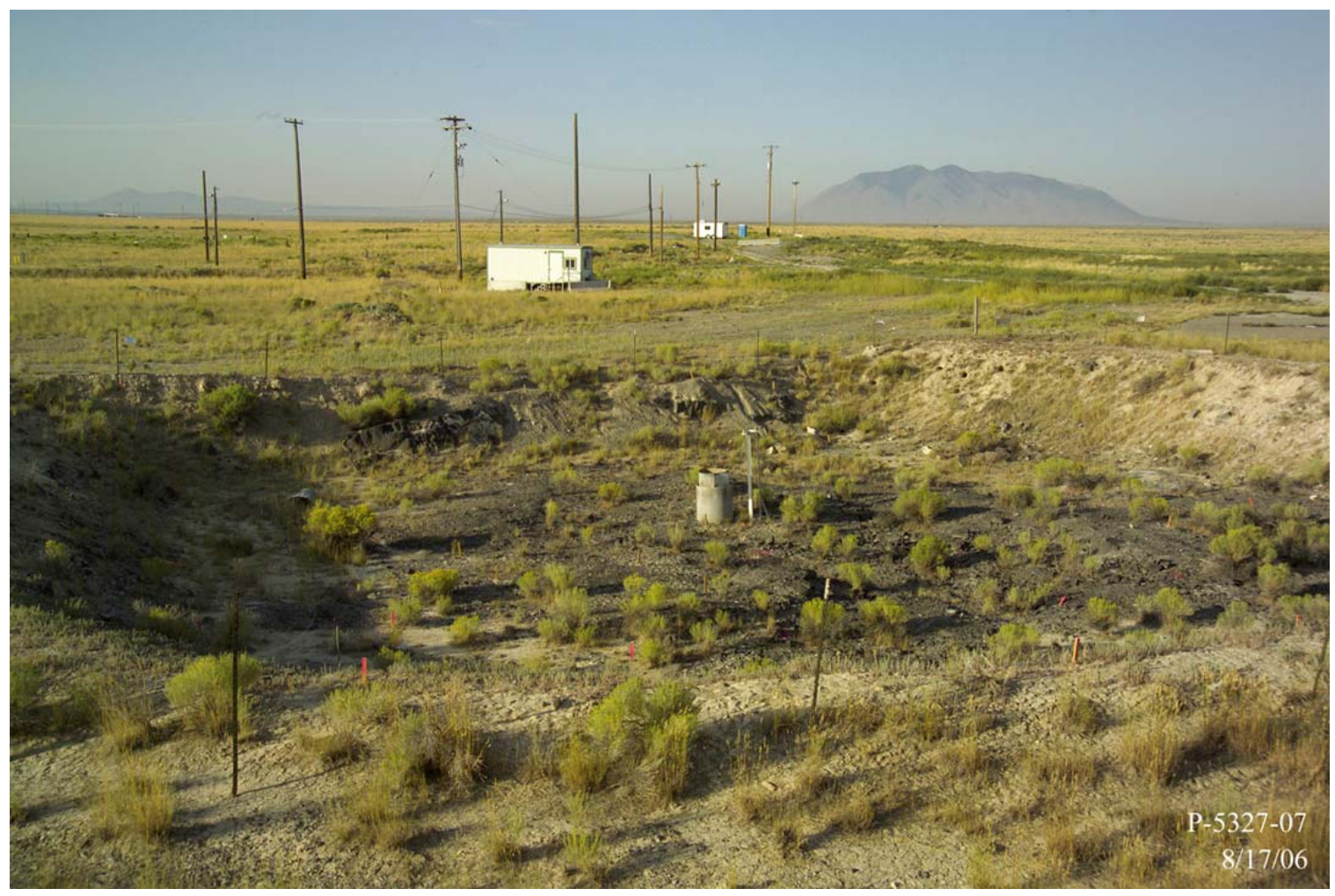

Figure A-4. Test barrel in Experimental Organic-Cooled Reactor Pond.

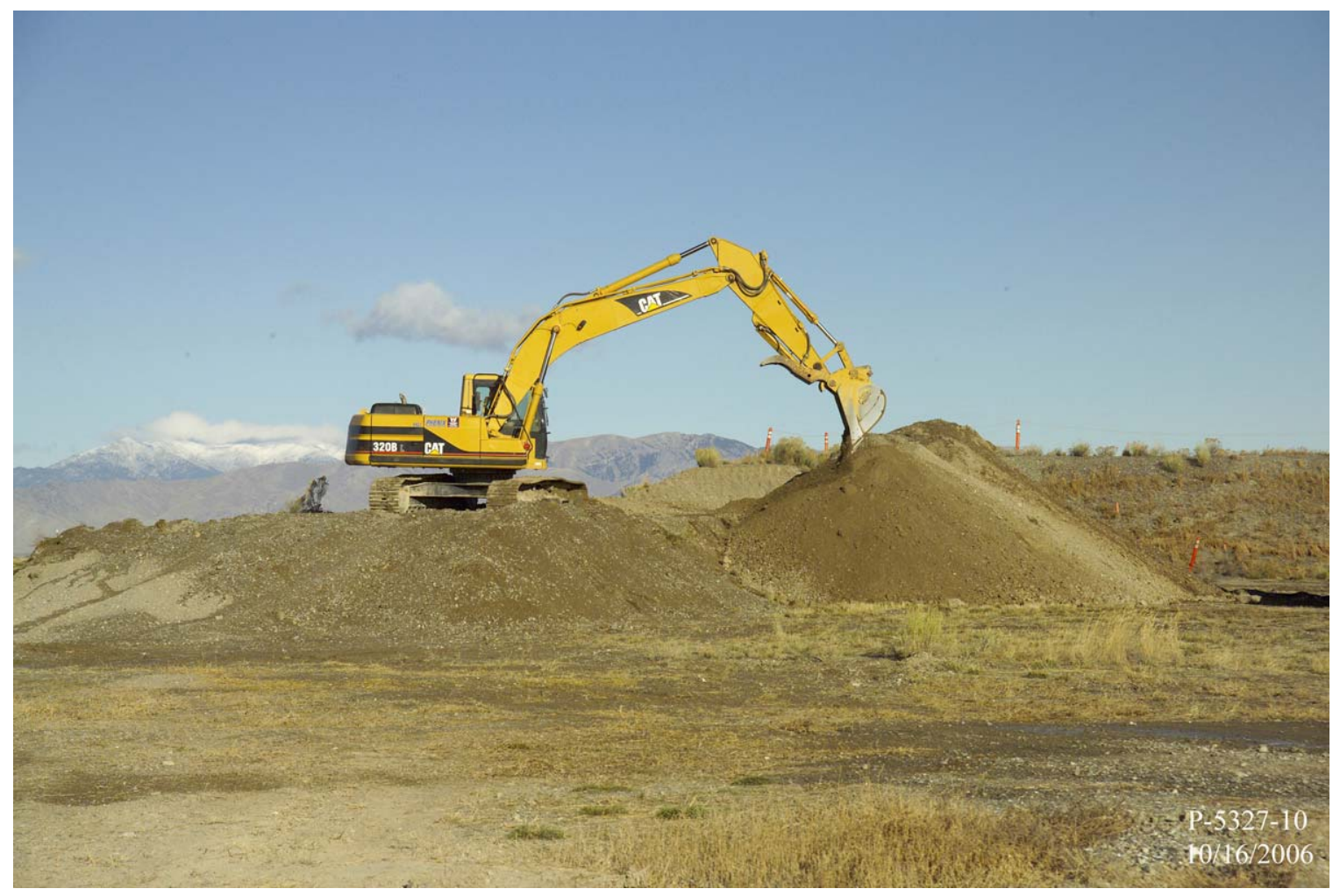

Figure A-5. Excavation of Berm A. 


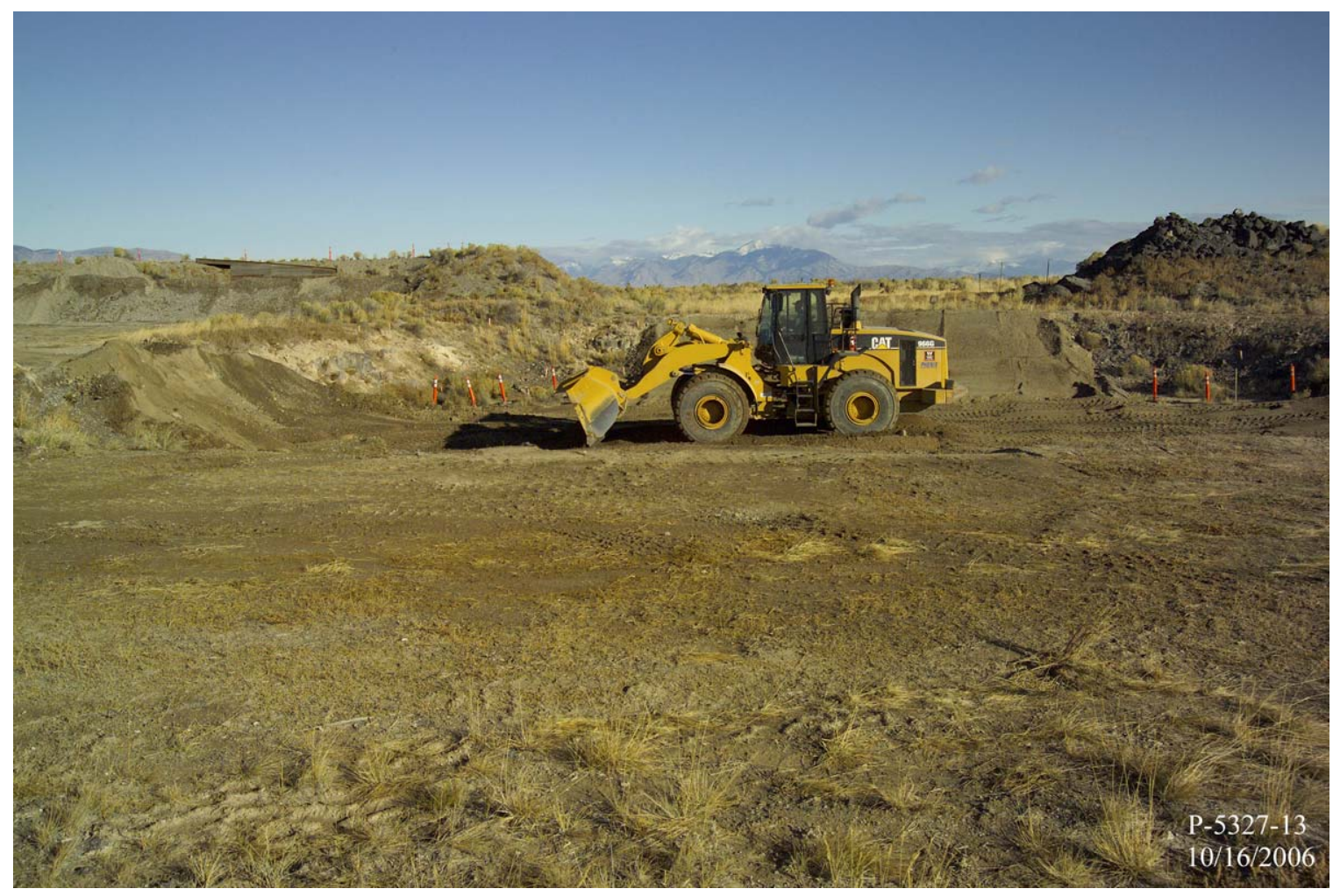

Figure A-6. Backfilling the Experimental Organic-Cooled Reactor Pond.

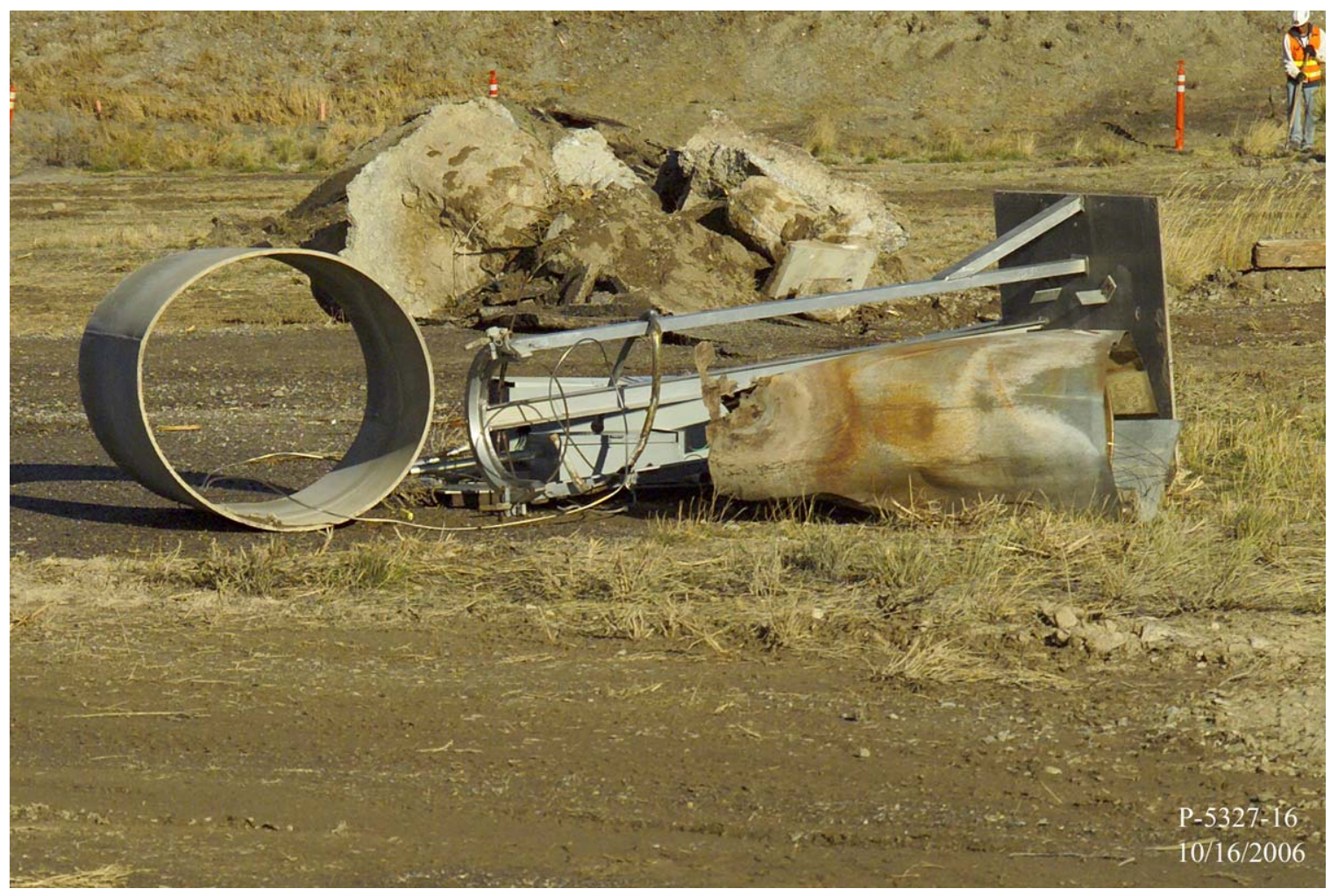

Figure A-7. Test barrel following removal. 


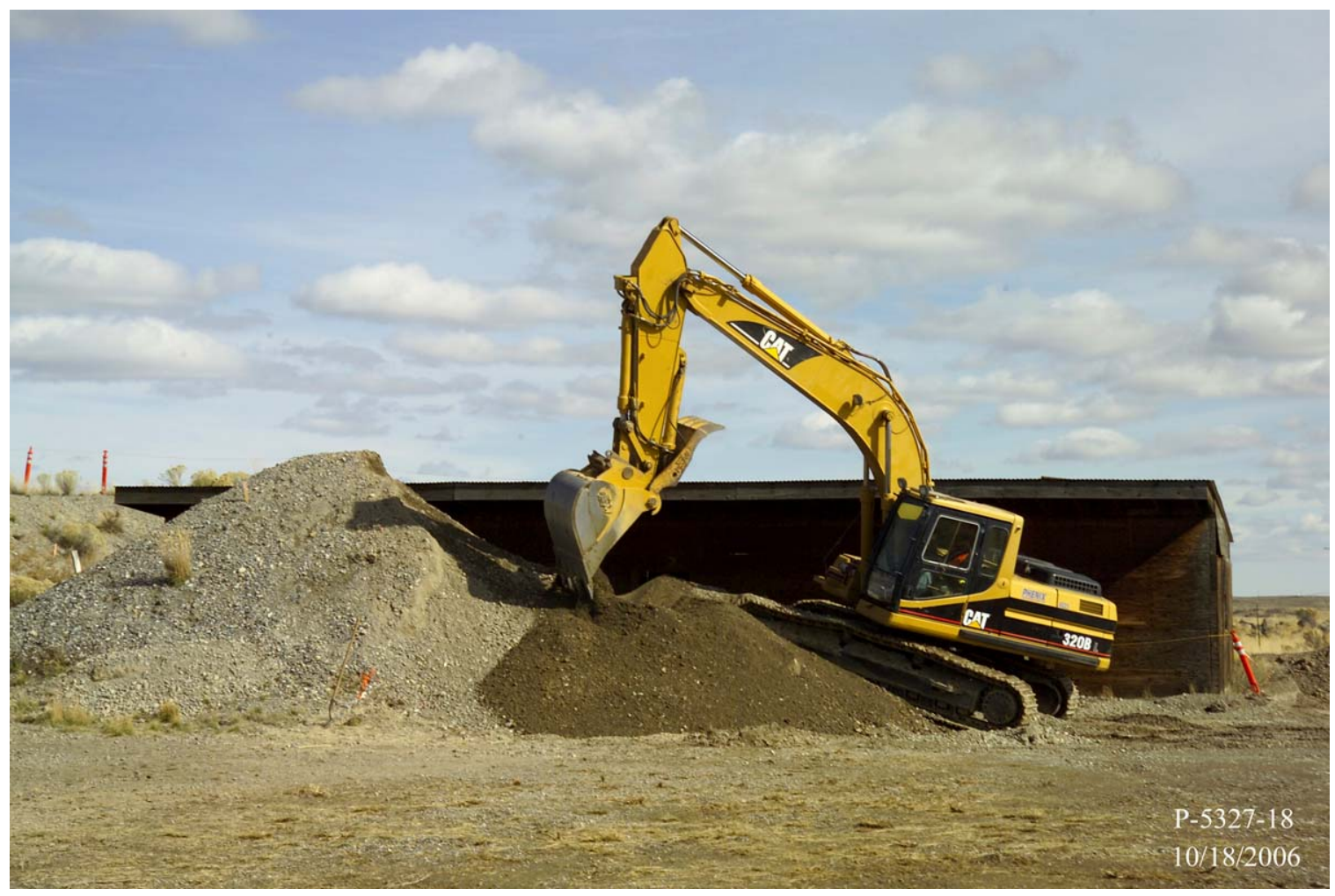

Figure A-8. Excavating Berm E. 


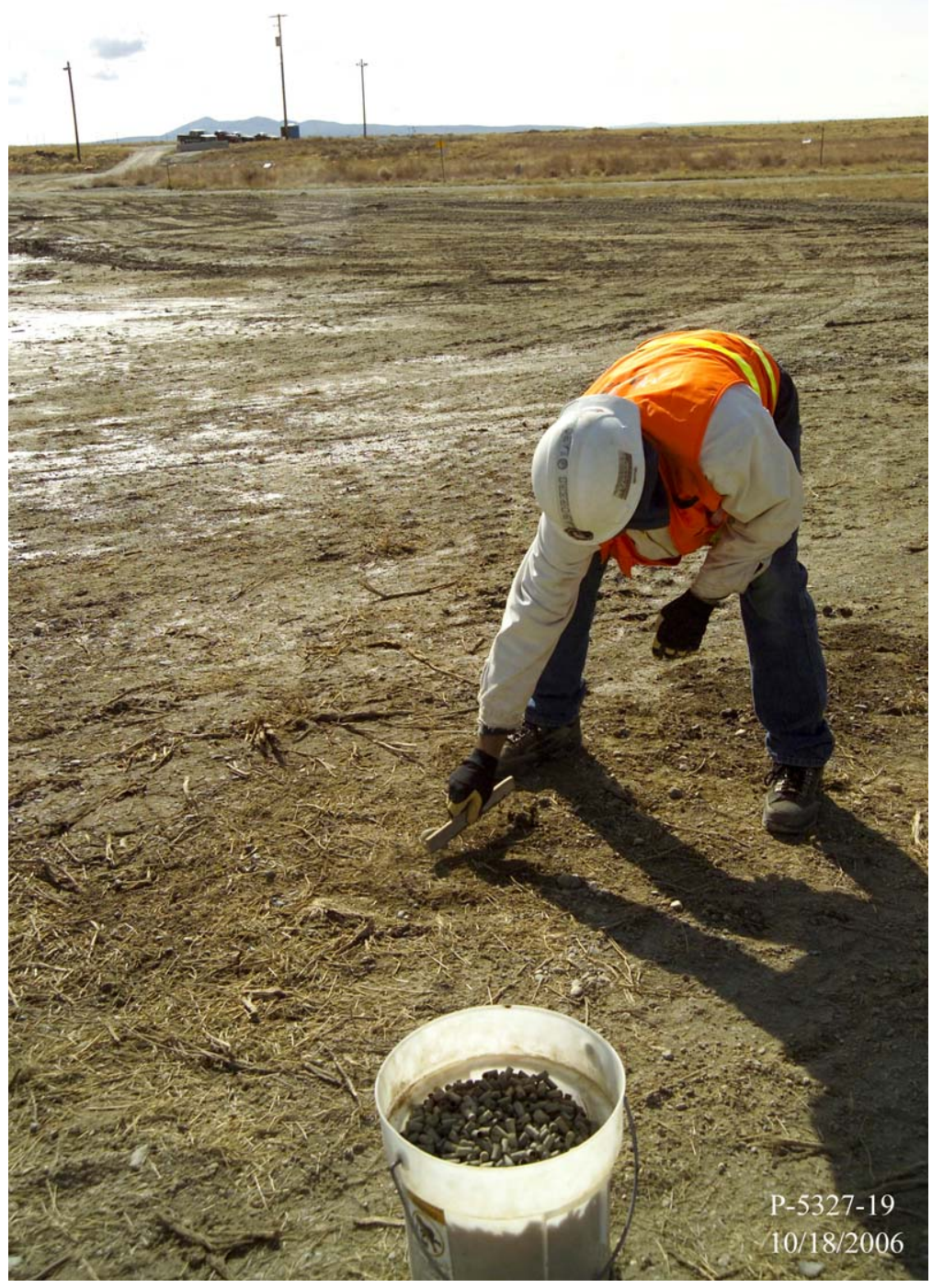

Figure A-9. Removing spent ammunition casings by hand. 


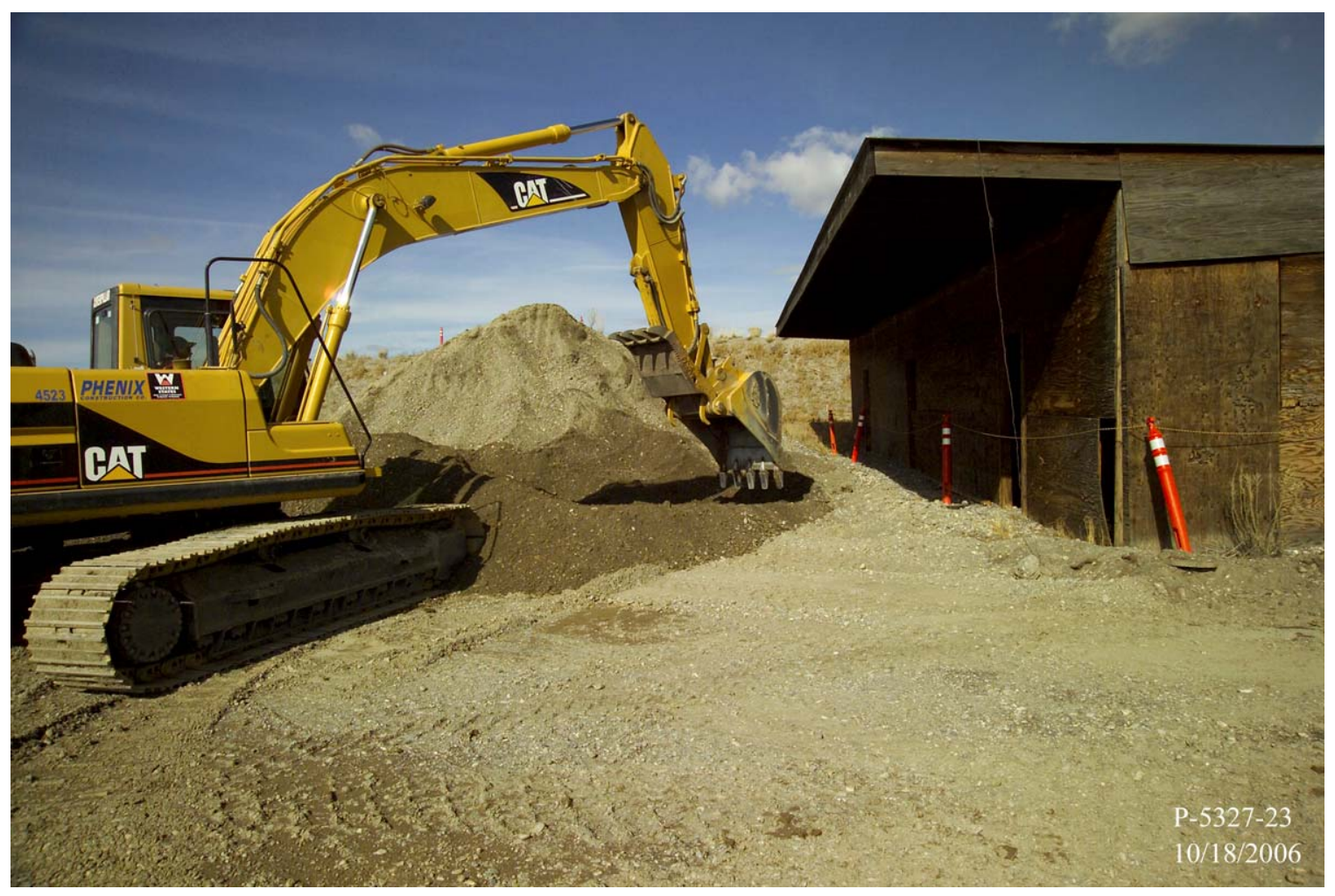

Figure A-10. Berm E excavation prior to shooting house demolition.

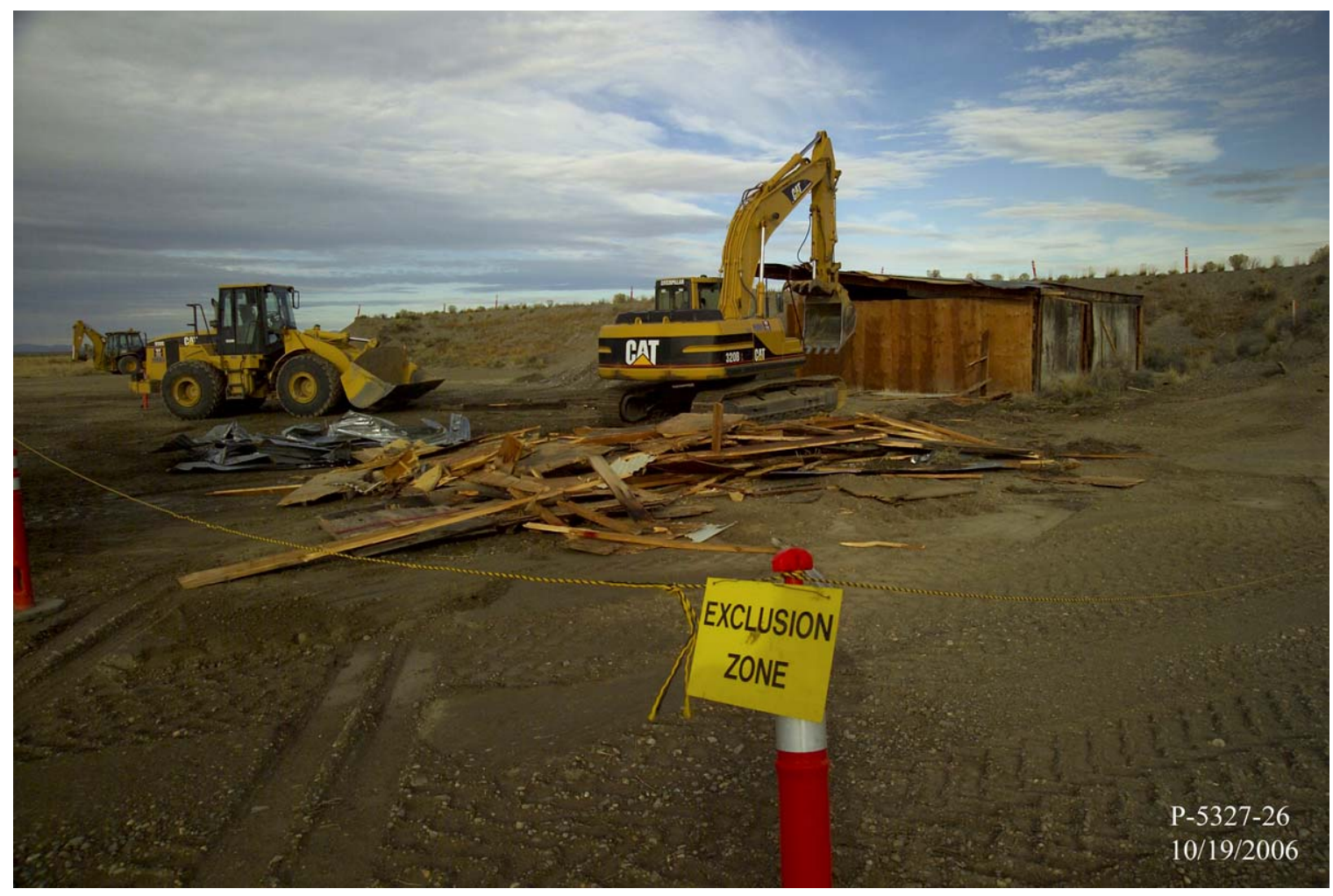

Figure A-11. Shooting house removal. 


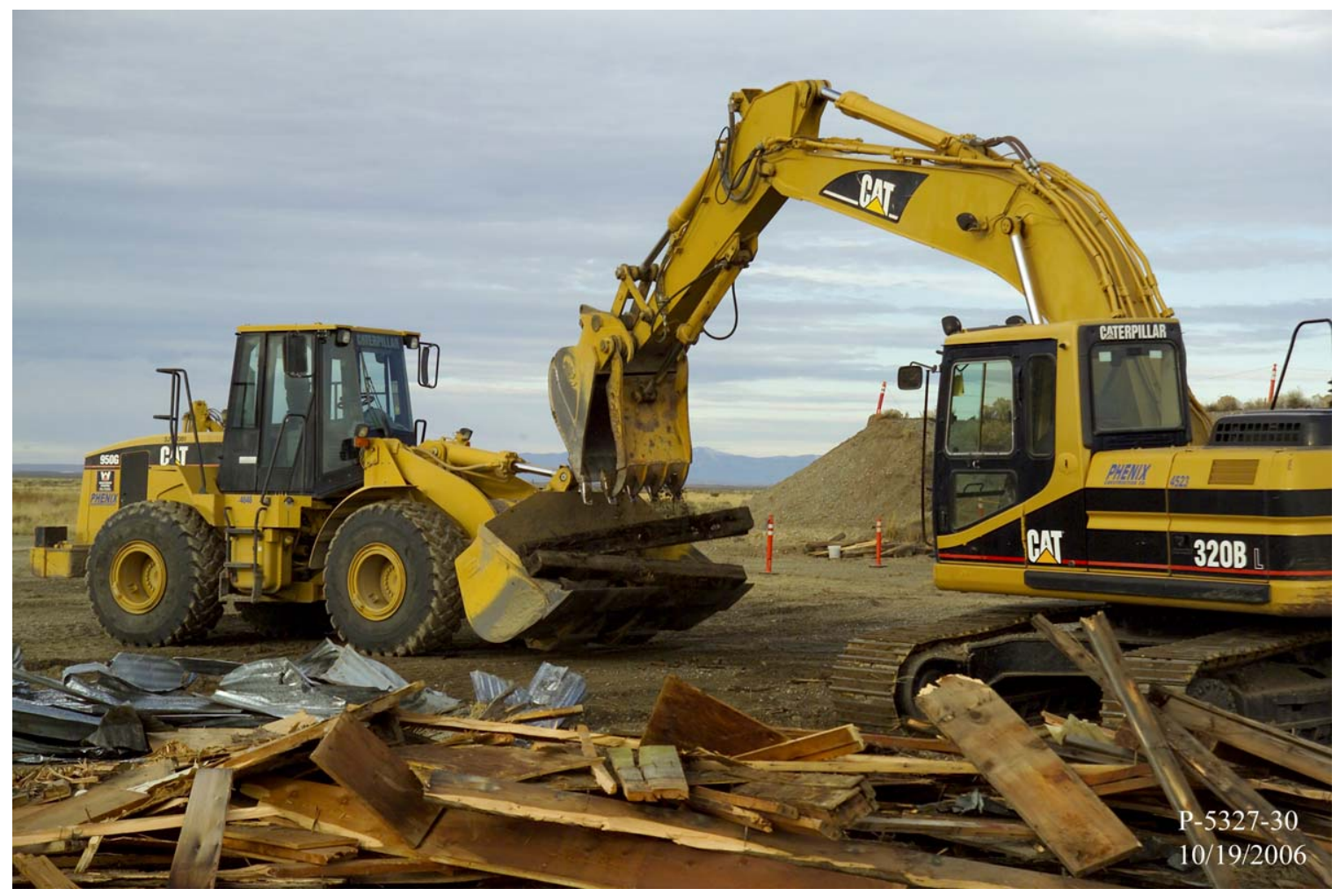

Figure A-12. Segregation of lead-contaminated railroad ties.

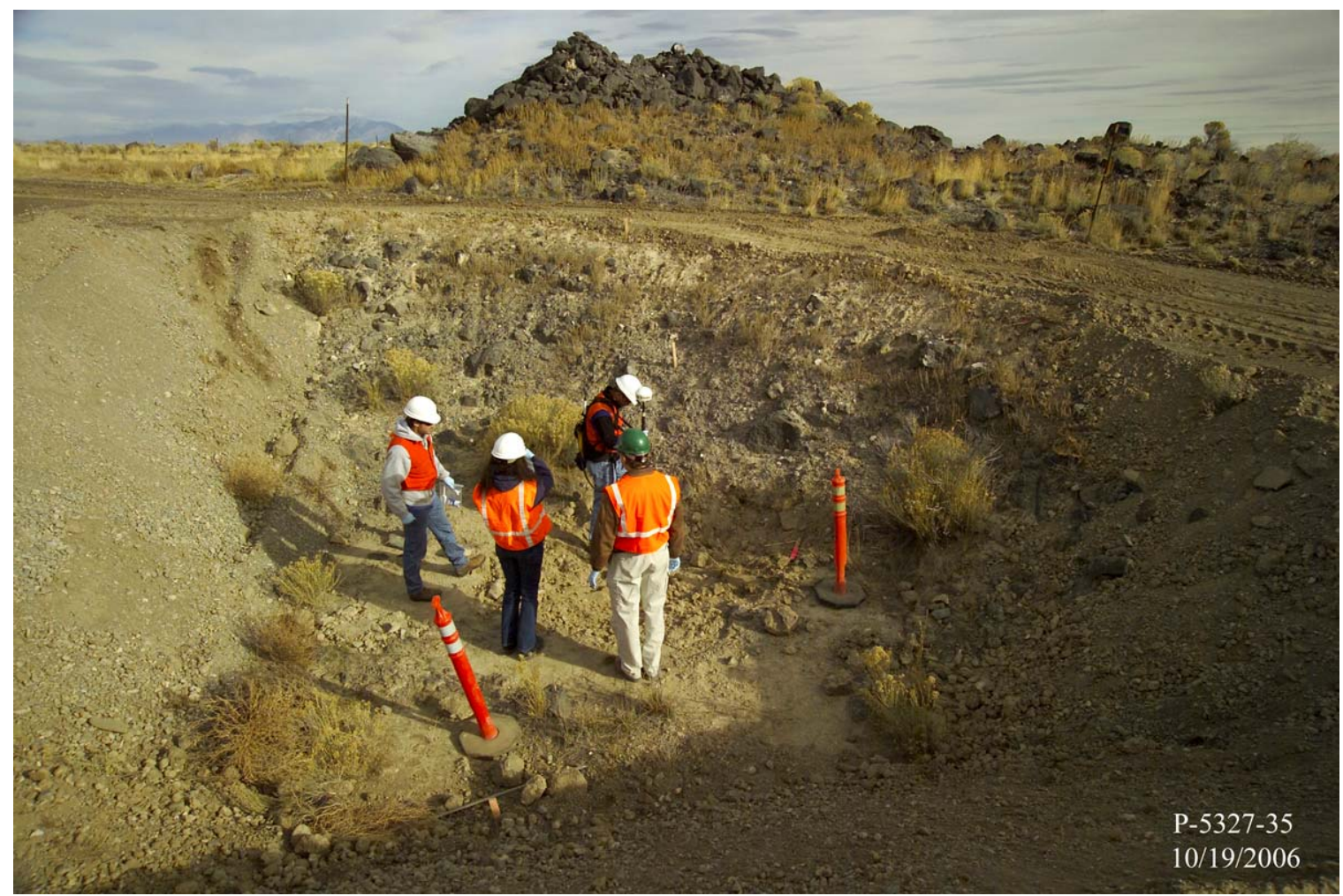

Figure A-13. Sampling an excavation location in the Experimental Organic-Cooled Reactor Pond. 


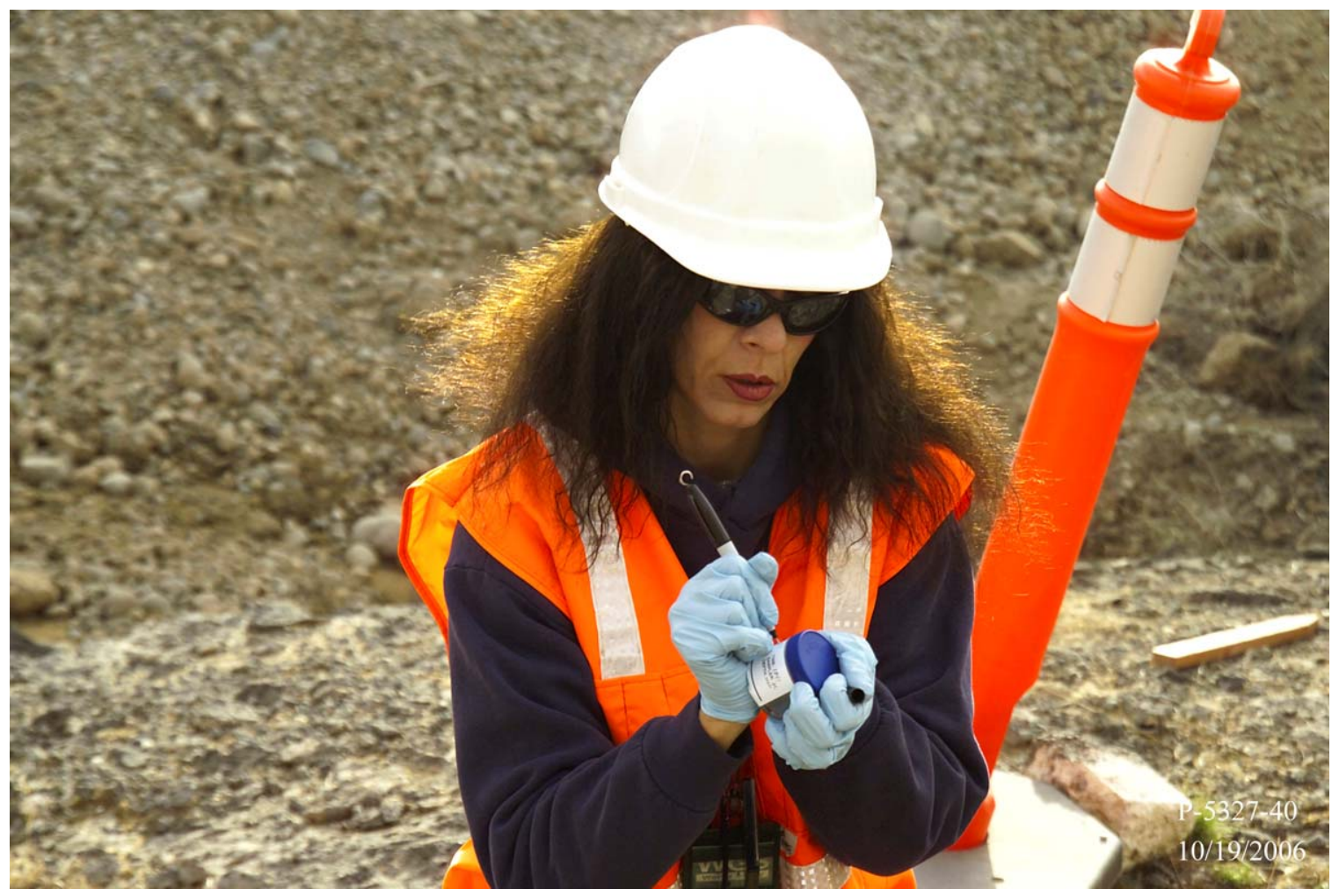

Figure A-14. Labeling a soil sample container.

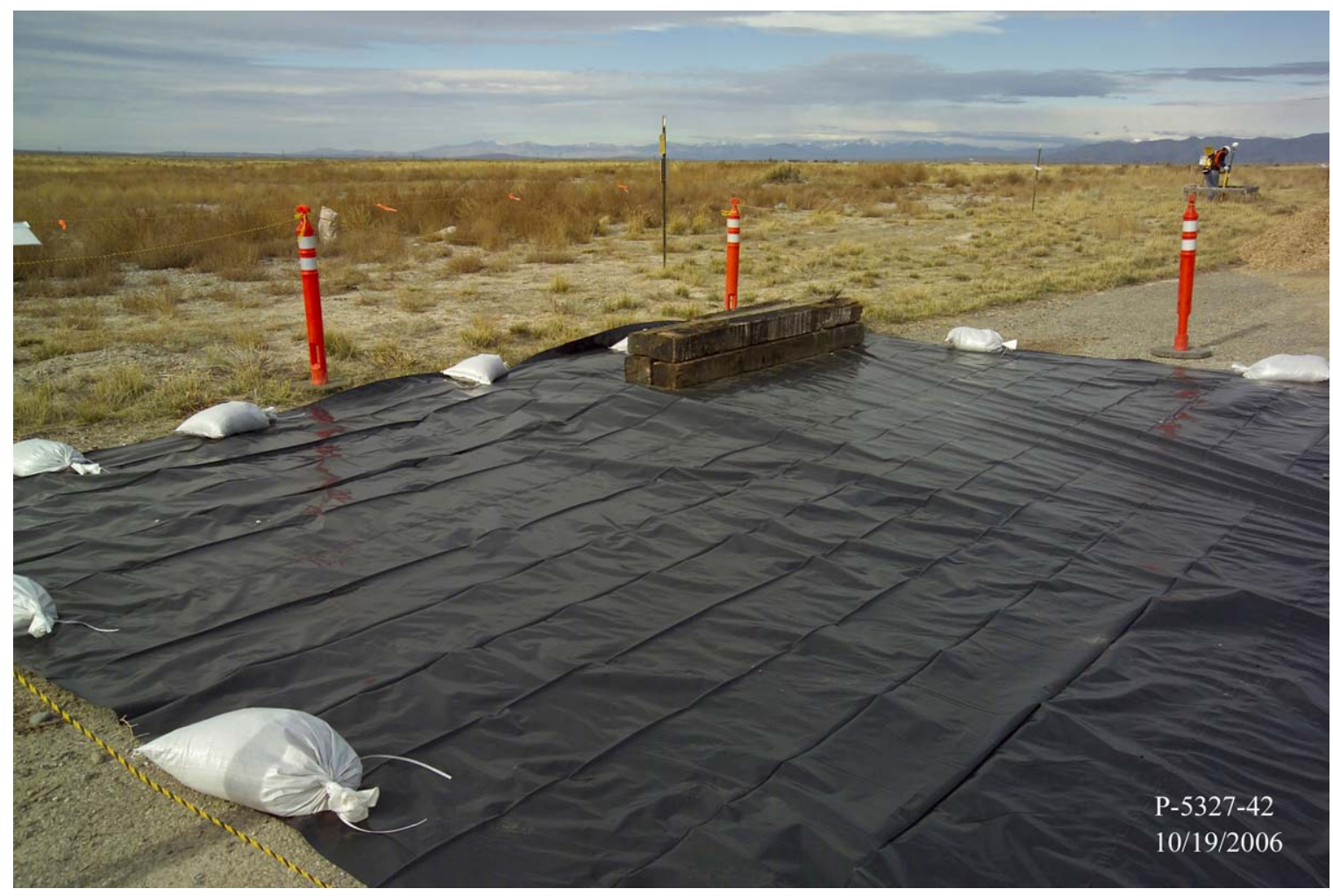

Figure A-15. Temporary staging of railroad ties prior to shipment. 


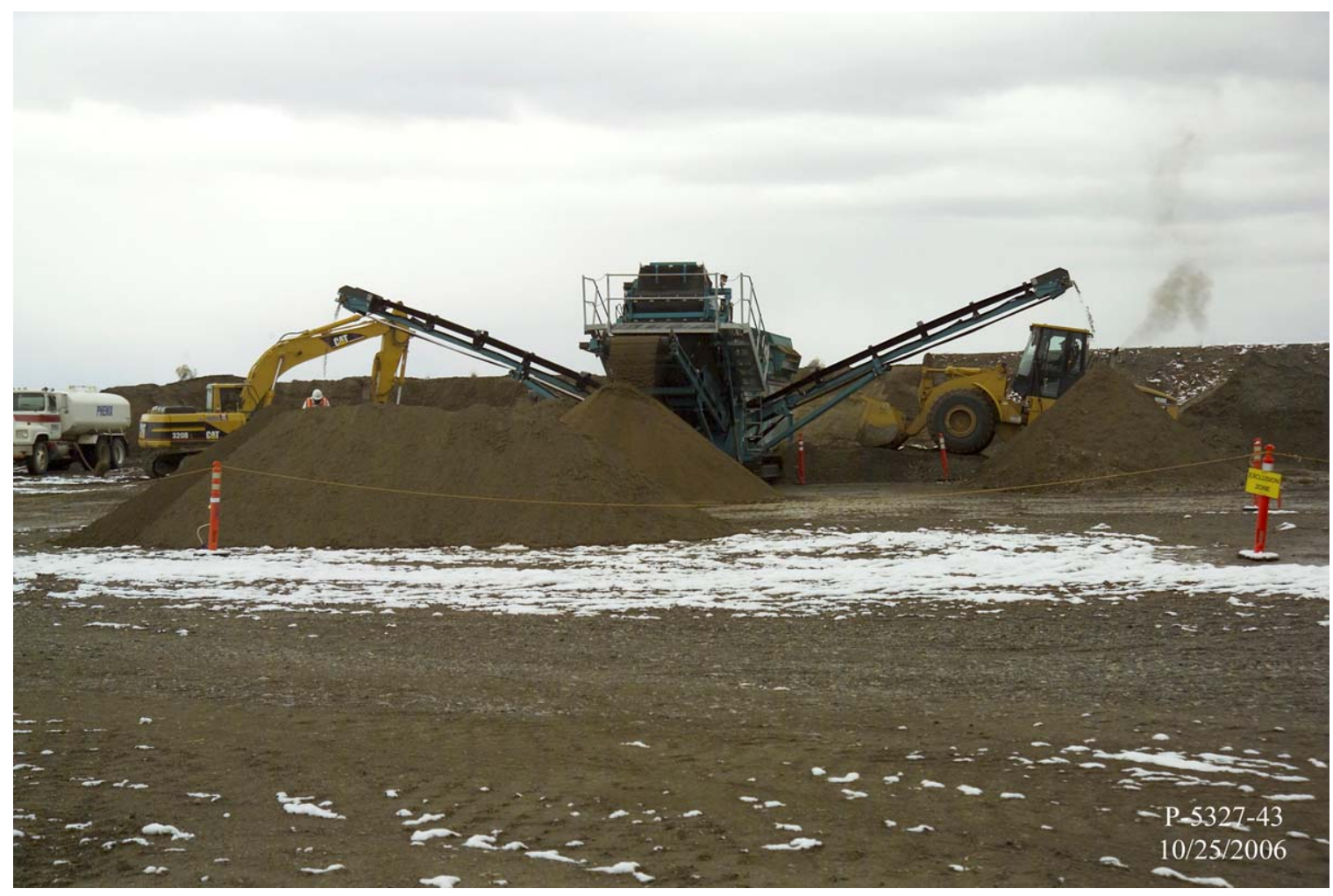

Figure A-16. Screening of lead-contaminated soils.

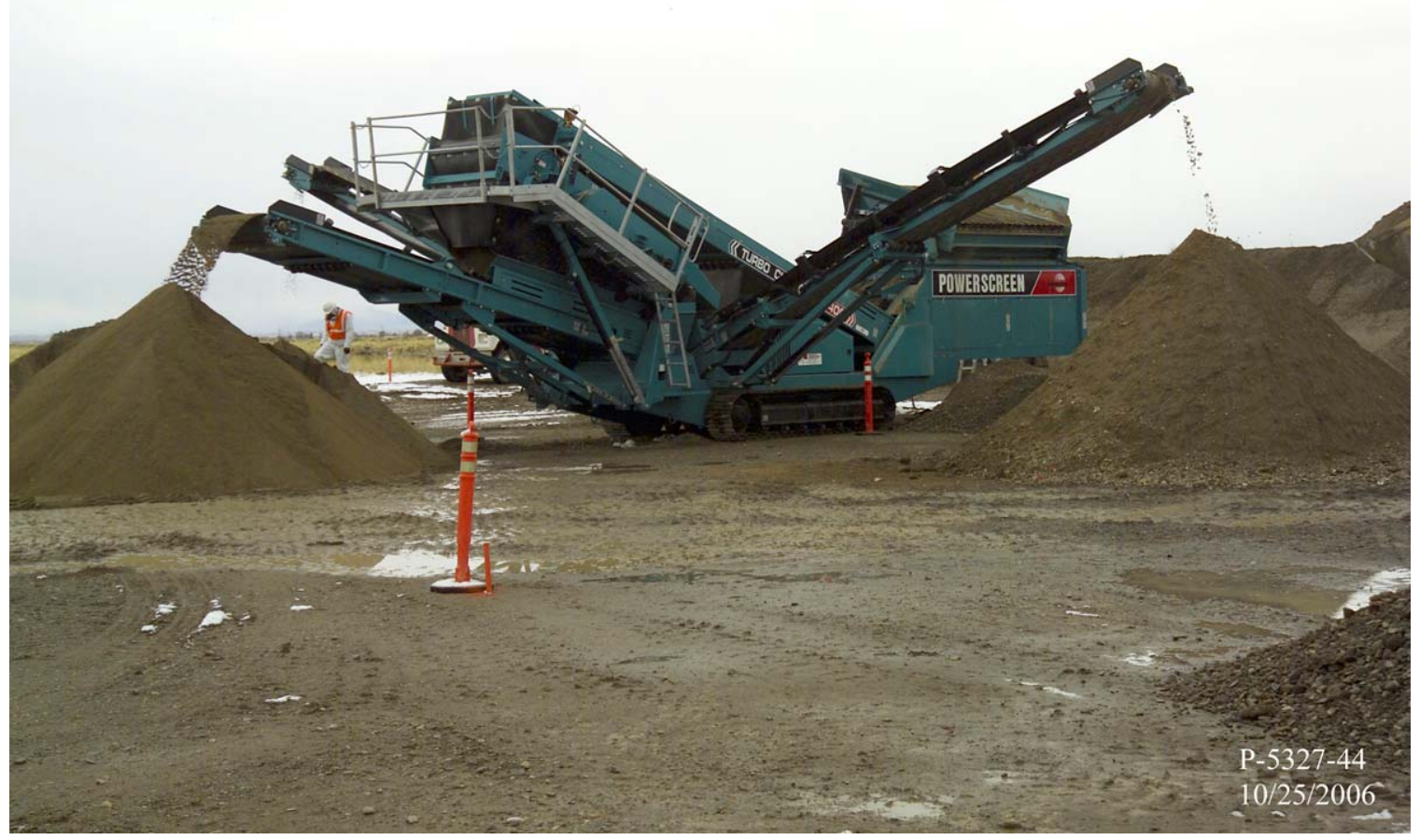

Figure A-17. Temporary soil staging piles. 


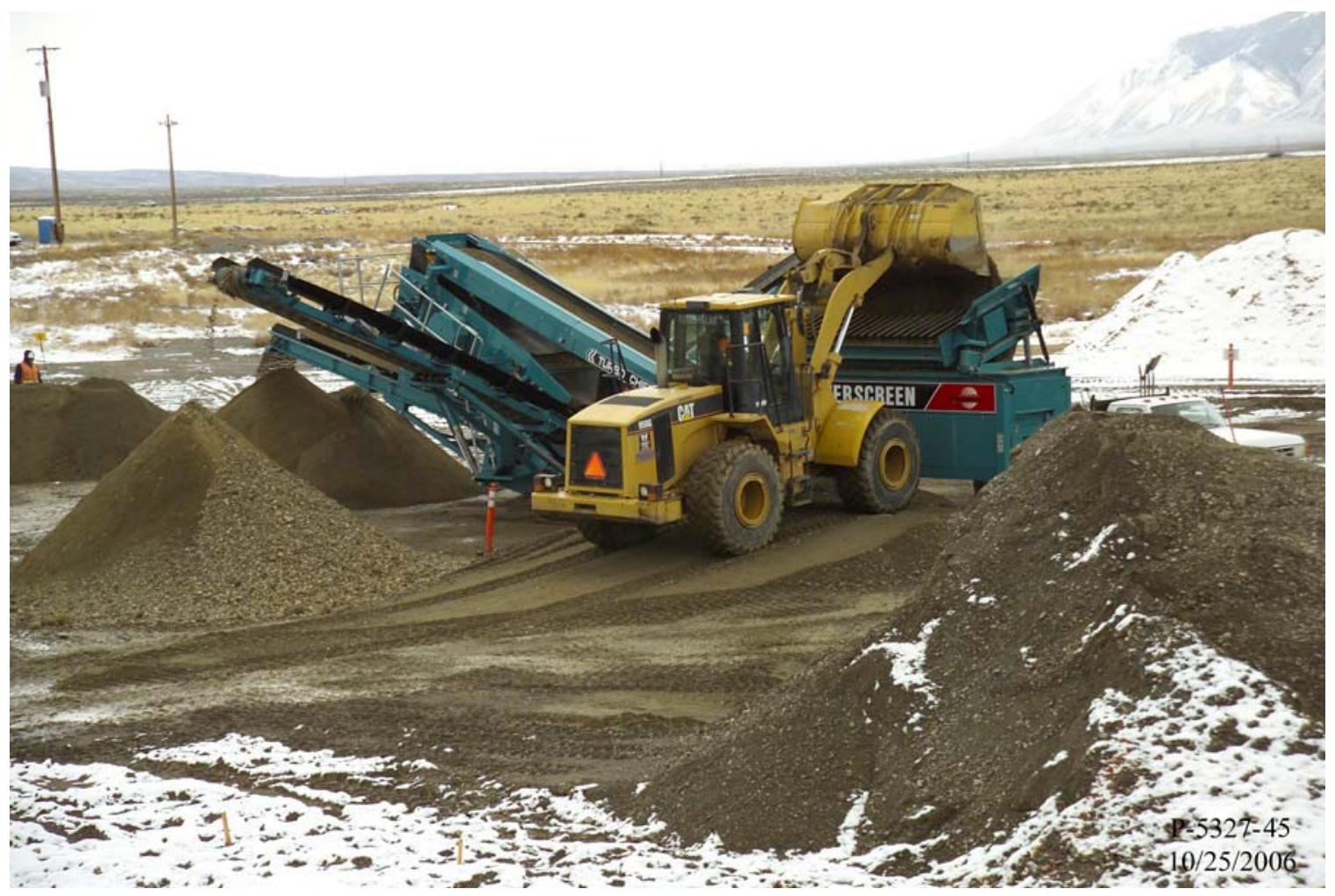

Figure A-18. Loading contaminated soil into the screening plant.

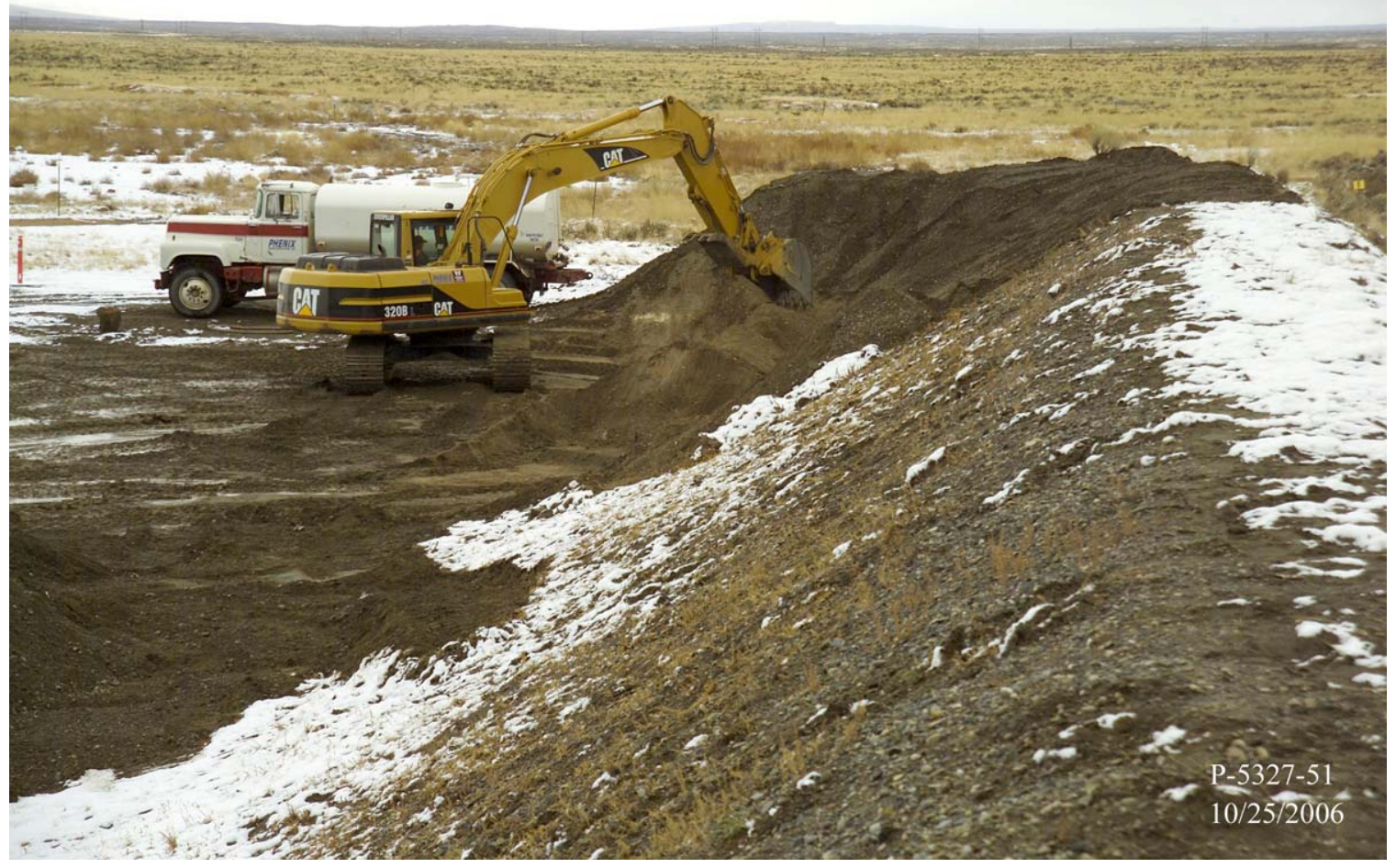

Figure A-19. Excavation of Berm B prior to screening. 


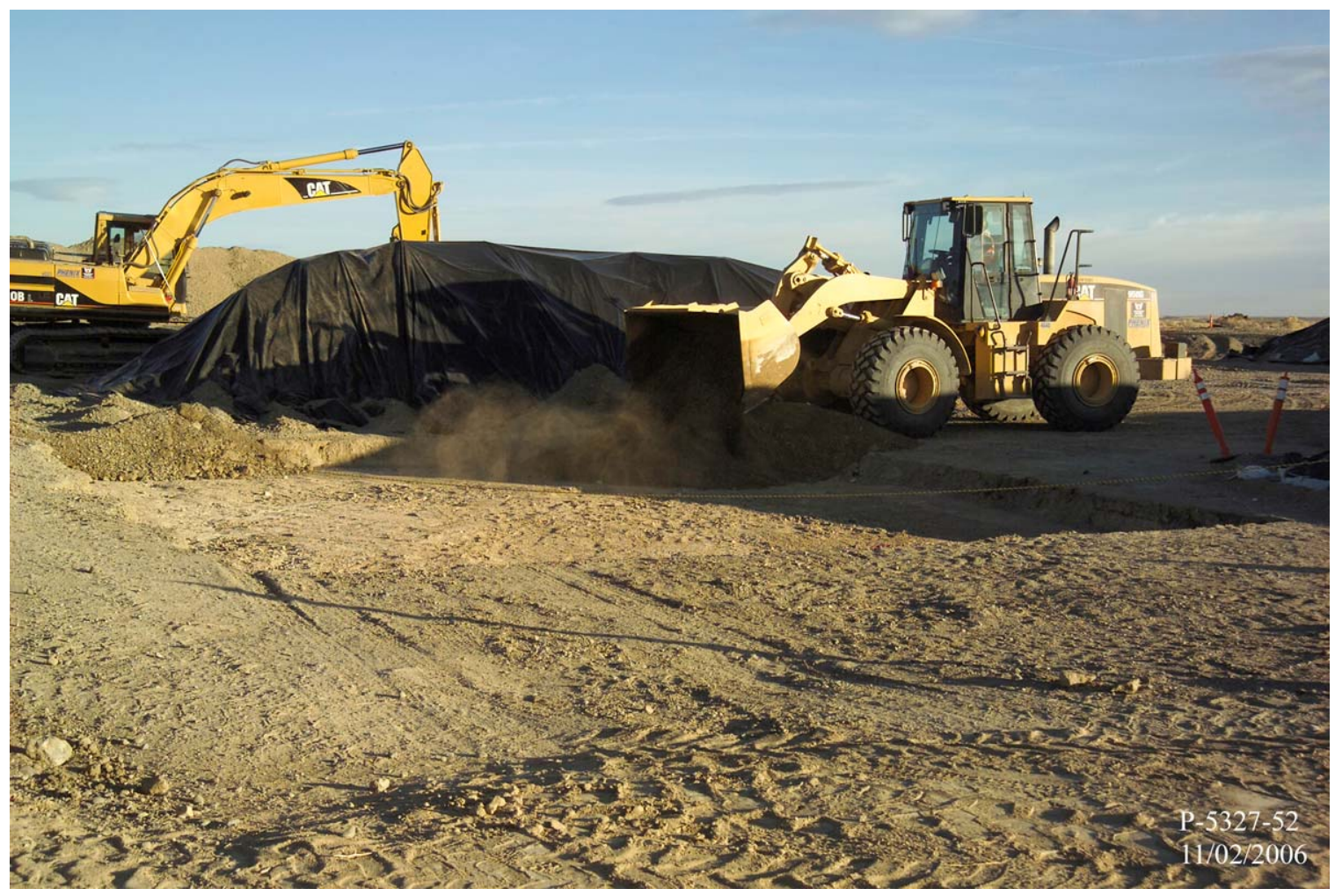

Figure A-20. Excavation of contaminated location between the berms.

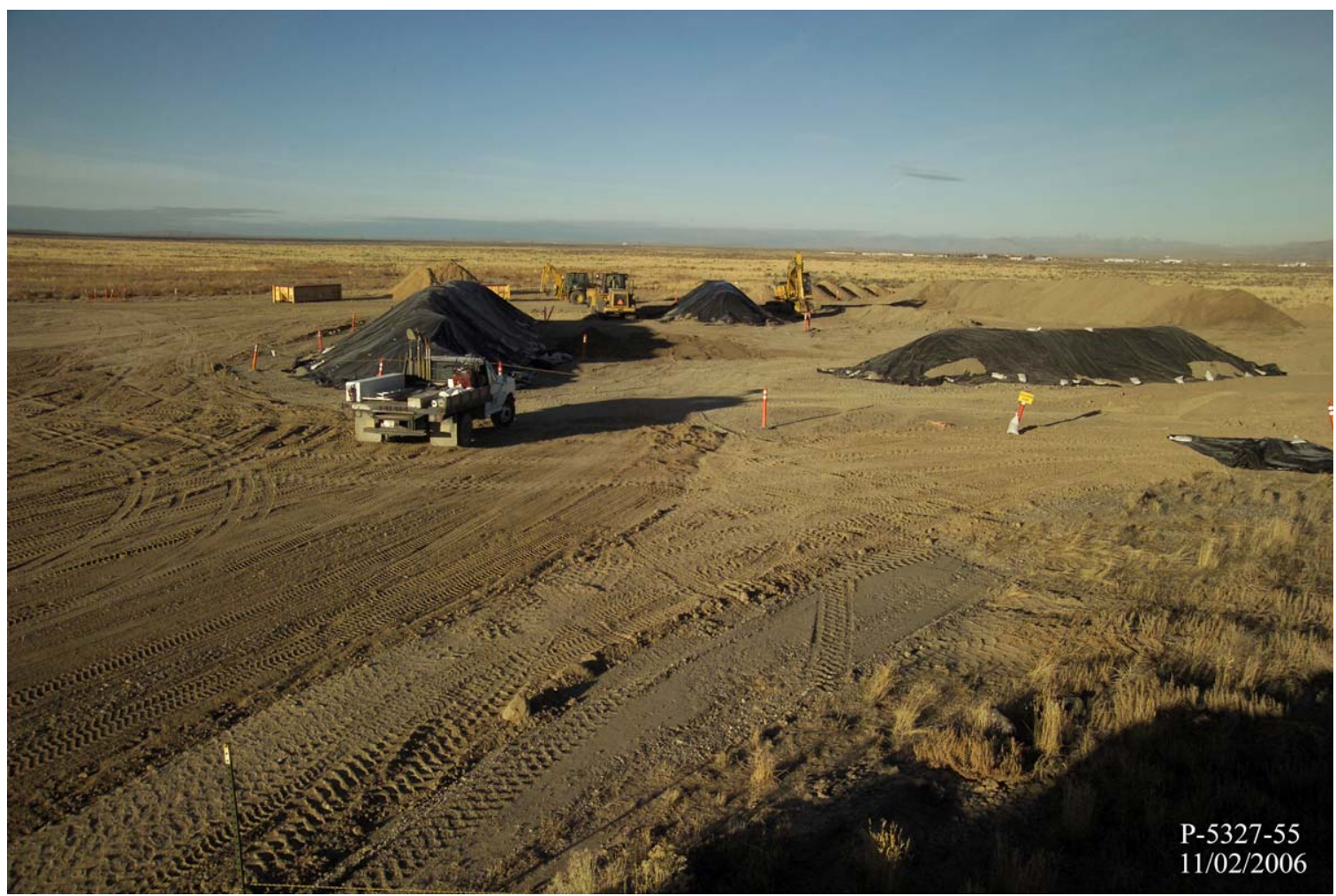

Figure A-21. View of staging piles following completion of screening. 


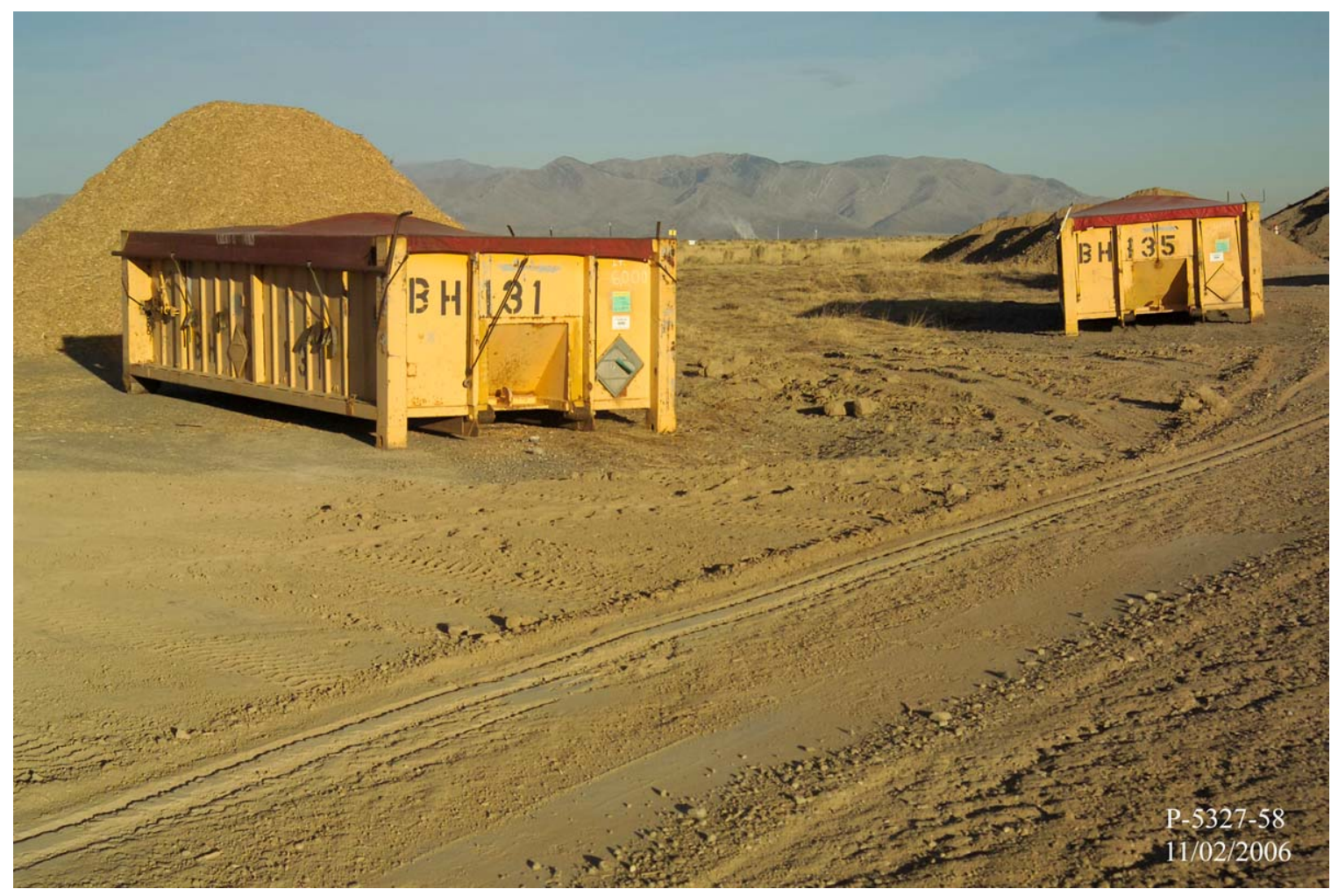

Figure A-22. Railroad tie shipping containers with wood chip mulch in background.

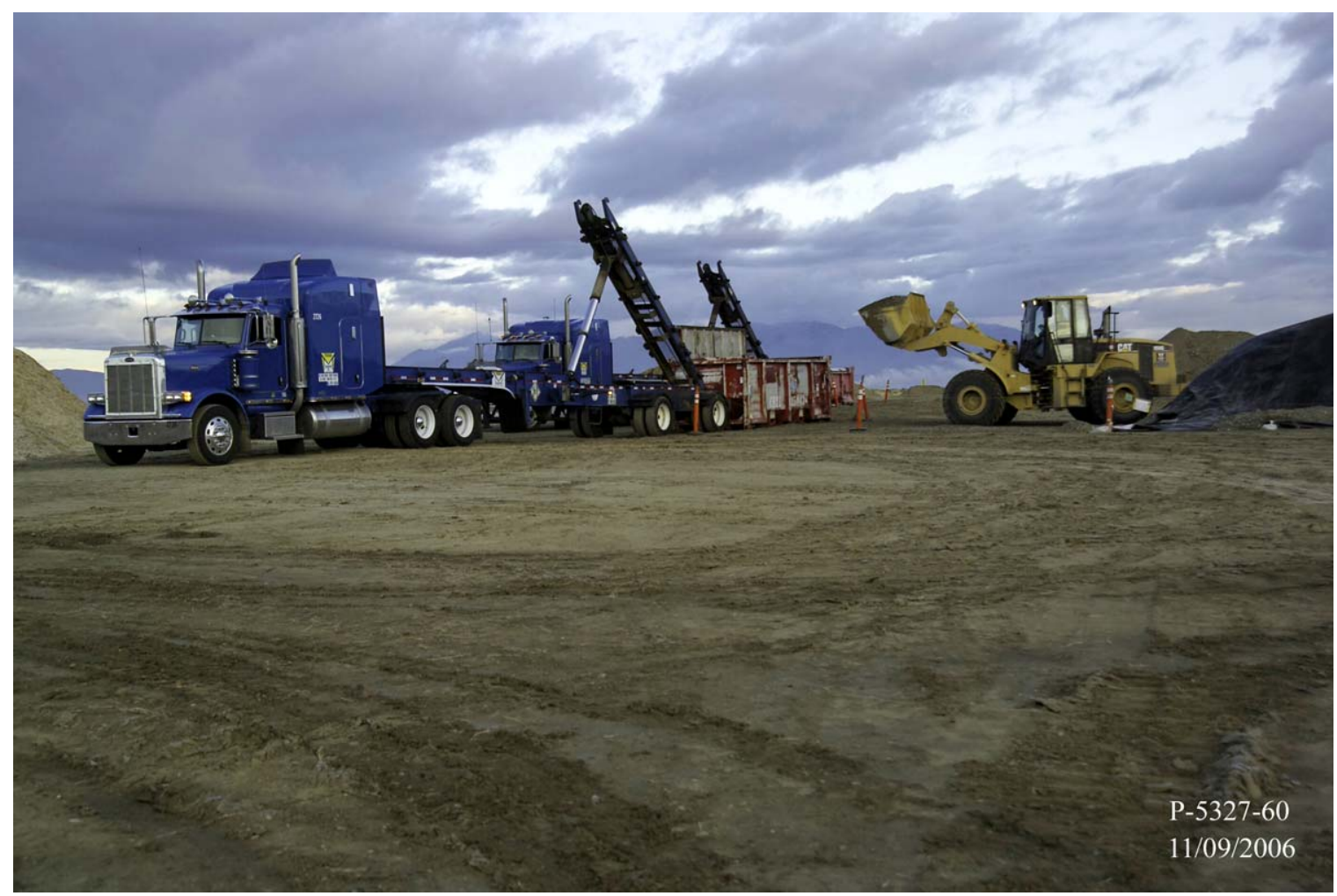

Figure A-23. Loading of soil shipping intermodal containers. 


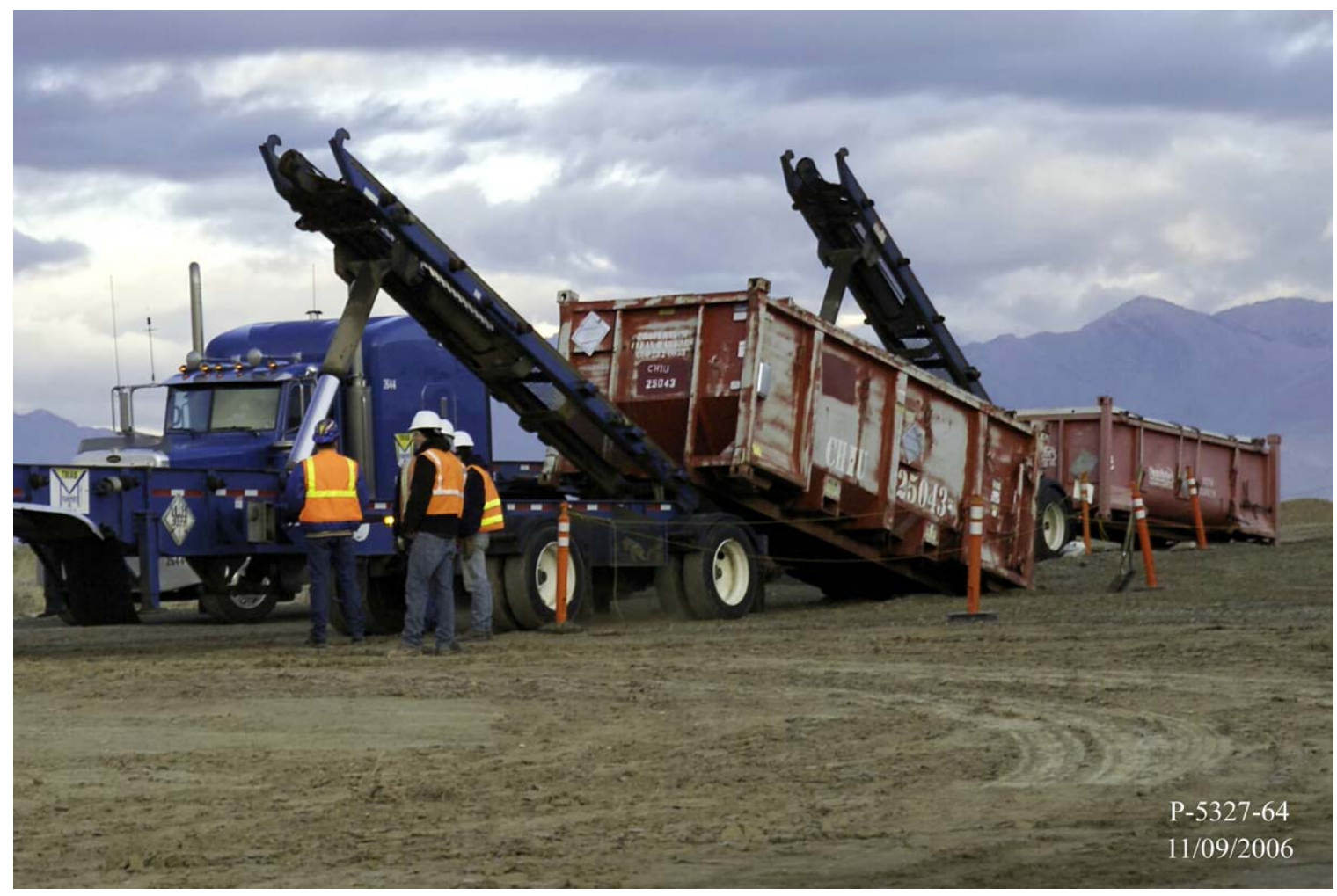

Figure A-24. Placing intermodal containers on shipping trailer.

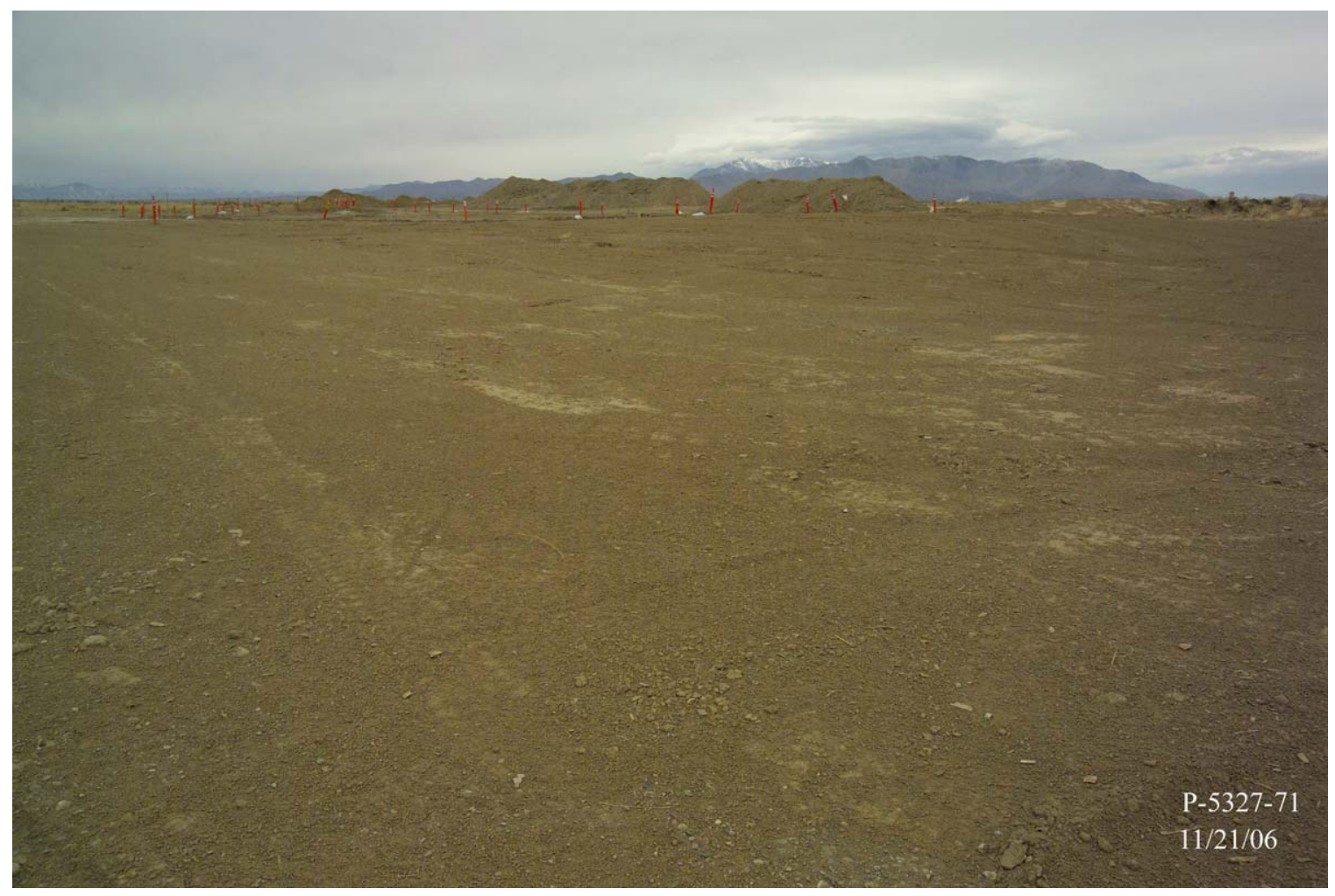

Figure A-25. STF-02 Gun Range prior to final contouring. 


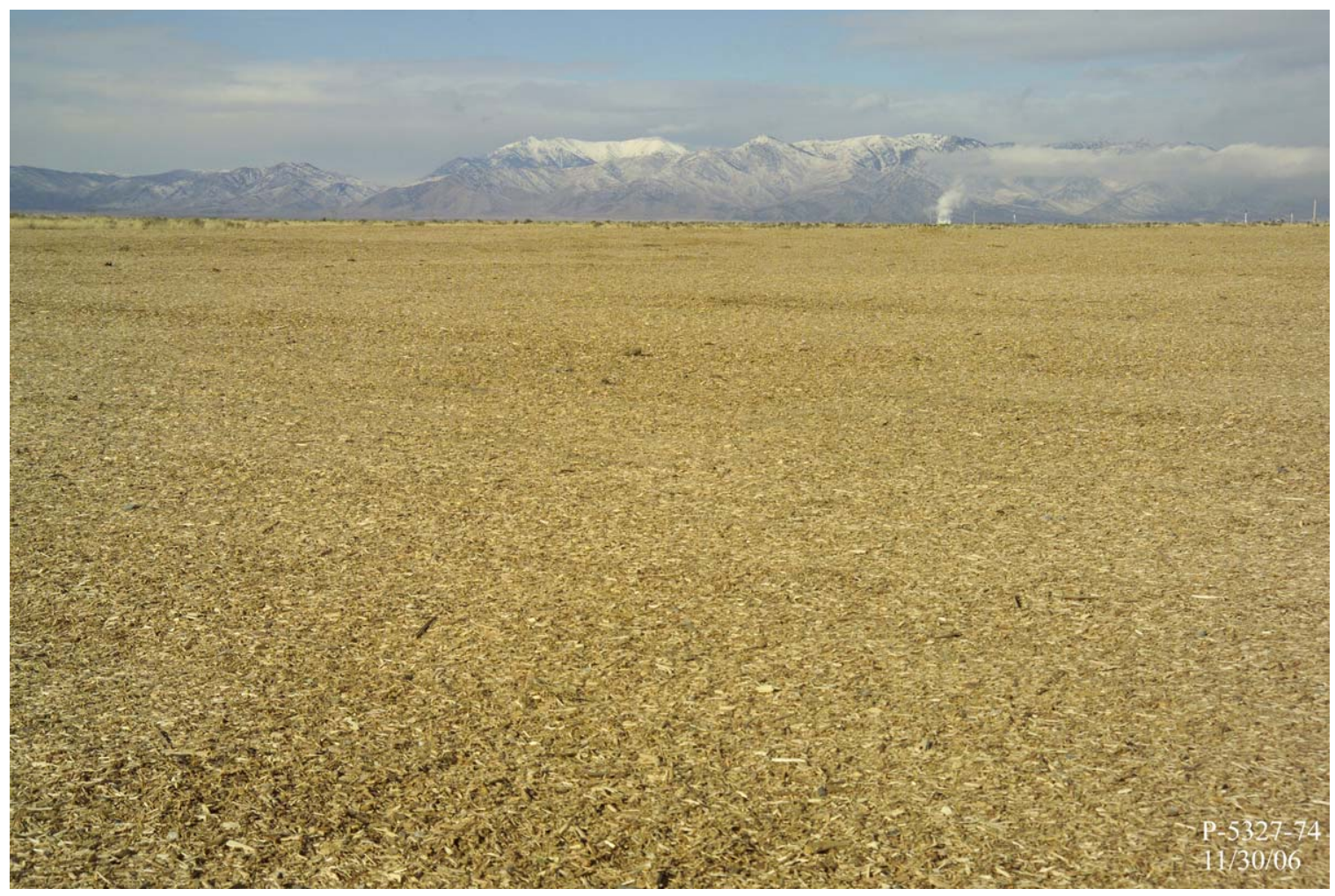

Figure A-26. STF-02 Gun Range following reseeding and mulching.

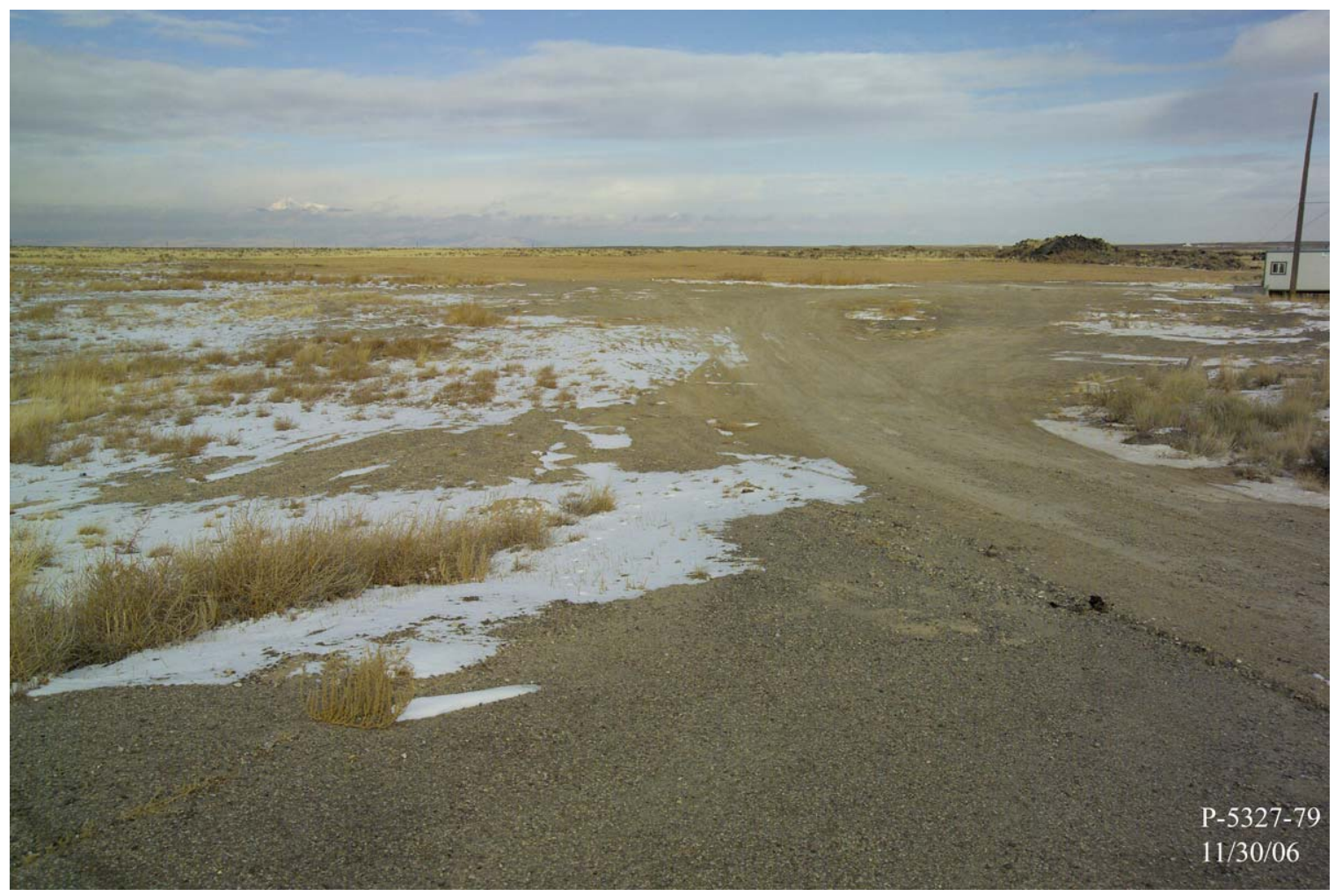

Figure A-27. STF-02 Gun Range with remediation complete. 
A-18 
Appendix B

\section{Remedial Action Analytical Summaries}


B-2 


\section{Appendix B}

\section{Remedial Action Analytical Summaries}

The analytical summaries in this appendix present data from the various sampling conducted during the remedial action at the STF-02 Gun Range, including the data generated by analytical laboratories and by the portable $\mathrm{x}$-ray fluorescence spectrometer.

Table B-1. Preremediation analytical results.

\begin{tabular}{lcccc}
\hline & & & Lead Conc. \\
Sample ID \# & Location & Depth & (mg) & Q \\
\hline GR110101LD & A1 & $0-0.5 \mathrm{ft}$ & 11.3 & $\mathrm{~J}$ \\
GR110201LD & A5 & $0-0.5 \mathrm{ft}$ & 10.7 & $\mathrm{~J}$ \\
GR110202LD & A5 & $0-0.5 \mathrm{ft}$ & 11.7 & $\mathrm{~J}$ \\
GR110301LD & A9 & $0-0.5 \mathrm{ft}$ & 12.7 & $\mathrm{~J}$ \\
GR110401LD & A13 & $0-0.5 \mathrm{ft}$ & 10.2 & $\mathrm{~J}$ \\
GR110501LD & A17 & $0-0.5 \mathrm{ft}$ & 11.3 & $\mathrm{~J}$ \\
GR110601LD & A21 & $0-0.5 \mathrm{ft}$ & 21.8 & $\mathrm{~J}$ \\
GR110701LD & A25 & $0-0.5 \mathrm{ft}$ & 14.4 & $\mathrm{~J}$ \\
GR110801LD & A29 & $0-0.5 \mathrm{ft}$ & 13.6 & $\mathrm{~J}$ \\
GR110901LD & A33 & $0-0.5 \mathrm{ft}$ & 11.6 & $\mathrm{~J}$ \\
GR111001LD & A37 & $0-0.5 \mathrm{ft}$ & 26.3 & $\mathrm{~J}$ \\
GR111101LD & A41 & $0-0.5 \mathrm{ft}$ & 42.2 & $\mathrm{~J}$ \\
GR111201LD & A45 & $0-0.5 \mathrm{ft}$ & 28.1 & $\mathrm{~J}$ \\
GR111301LD & A49 & $0-0.5 \mathrm{ft}$ & 53.3 & $\mathrm{~J}$ \\
GR111401LD & B1 & $0-0.5 \mathrm{ft}$ & 24.9 & $\mathrm{~J}$ \\
GR111501LD & B5 & $0-0.5 \mathrm{ft}$ & 43.9 & $\mathrm{~J}$ \\
GR111601LD & B9 & $0-0.5 \mathrm{ft}$ & 92 & $\mathrm{~J}$ \\
GR111701LD & B13 & $0-0.5 \mathrm{ft}$ & 24.8 & $\mathrm{~J}$ \\
GR111801LD & B17 & $0-0.5 \mathrm{ft}$ & 258 & $\mathrm{~J}$ \\
GR111901LD & B21 & $0-0.5 \mathrm{ft}$ & 158 & $\mathrm{~J}$ \\
GR112001LD & B25 & $0-0.5 \mathrm{ft}$ & 124 & \\
GR112101LD & B29 & $0-0.5 \mathrm{ft}$ & 71.1 & \\
GR112102LD & B29 & $0-0.5 \mathrm{ft}$ & 1,940 & \\
GR112201LD & B33 & $0-0.5 \mathrm{ft}$ & 74.2 & \\
GR112301LD & B37 & $0-0.5 \mathrm{ft}$ & 505 & \\
GR112401LD & B41 & $0-0.5 \mathrm{ft}$ & 2,220 & \\
GR112501LD & B45 & $0-0.5 \mathrm{ft}$ & 15,400 & \\
GR112601LD & B74 & $0-0.5 \mathrm{ft}$ & 12.3 & $\mathrm{~J}$ \\
GR112701LD & B95 & $0-0.5 \mathrm{ft}$ & 19.7 & \\
GR112801LD & C1 & $0-0.5 \mathrm{ft}$ & 13.9 & $\mathrm{~J}$ \\
GR112901LD & $0-0.5 \mathrm{ft}$ & 21.8 & $\mathrm{~J}$ \\
GR113001LD & $0-0.5 \mathrm{ft}$ & 67 & $\mathrm{~J}$
\end{tabular}


Table B-1. (continued).

\begin{tabular}{|c|c|c|c|c|}
\hline Sample ID \# & Location & Depth & $\begin{array}{c}\text { Lead Conc. } \\
(\mathrm{mg} / \mathrm{kg})\end{array}$ & $\mathrm{Q}$ \\
\hline GR113101LD & C24 & $0-0.5 \mathrm{ft}$ & 12.1 & $\mathrm{~J}$ \\
\hline GR113201LD & $\mathrm{C} 28$ & $0-0.5 \mathrm{ft}$ & 10.8 & $\mathrm{~J}$ \\
\hline GR113301LD & $\mathrm{C} 32$ & $0-0.5 \mathrm{ft}$ & 11.7 & $\mathrm{~J}$ \\
\hline GR113401LD & $\mathrm{C} 33$ & $0-0.5 \mathrm{ft}$ & 15.6 & $\mathrm{~J}$ \\
\hline GR113501LD & $\mathrm{C} 37$ & $0-0.5 \mathrm{ft}$ & 15.7 & $\mathrm{~J}$ \\
\hline GR113601LD & $\mathrm{C} 41$ & $0-0.5 \mathrm{ft}$ & 0.12 & $\mathrm{~J}$ \\
\hline GR113701LD & $\mathrm{C} 45$ & $0-0.5 \mathrm{ft}$ & 0.17 & $\mathrm{~J}$ \\
\hline GR113801LD & C49 & $0-0.5 \mathrm{ft}$ & 67.6 & $\mathrm{~J}$ \\
\hline GR113901LD & C53 & $0-0.5 \mathrm{ft}$ & 20.3 & $\mathrm{~J}$ \\
\hline GR114001LD & C57 & $0-0.5 \mathrm{ft}$ & 13.3 & $\mathrm{~J}$ \\
\hline GR114101LD & C61 & $0-0.5 \mathrm{ft}$ & 11.1 & $\mathrm{~J}$ \\
\hline GR114102LD & C61 & $0-0.5 \mathrm{ft}$ & 0.12 & $\mathrm{~J}$ \\
\hline GR114201LD & D1 & $0-0.5 \mathrm{ft}$ & 9.6 & \\
\hline GR114301LD & D5 & $0-0.5 \mathrm{ft}$ & 45.1 & \\
\hline GR114401LD & D9 & $0-0.5 \mathrm{ft}$ & 8.1 & \\
\hline GR114501LD & D13 & $0-0.5 \mathrm{ft}$ & 11.6 & \\
\hline GR114601LD & D17 & $0-0.5 \mathrm{ft}$ & 11.3 & \\
\hline GR114701LD & D21 & $0-0.5 \mathrm{ft}$ & 14.1 & \\
\hline GR114801LD & D25 & $0-0.5 \mathrm{ft}$ & 10.8 & \\
\hline GR114901LD & E1 & $0-0.5 \mathrm{ft}$ & 9.2 & \\
\hline GR115001LD & E5 & $0-0.5 \mathrm{ft}$ & 8.8 & \\
\hline GR115101LD & E9 & $0-0.5 \mathrm{ft}$ & 12.5 & \\
\hline GR115201LD & E13 & $0-0.5 \mathrm{ft}$ & 16.1 & \\
\hline GR115301LD & E17 & $0-0.5 \mathrm{ft}$ & 11.1 & \\
\hline GR115401LD & B51 & $0-0.5 \mathrm{ft}$ & 58 & \\
\hline GR115501LD & B51 & $0.5-1.5 \mathrm{ft}$ & 10.5 & \\
\hline GR115601LD & B52 & $0-0.5 \mathrm{ft}$ & 292 & \\
\hline GR115701LD & B52 & $0.5-1.5 \mathrm{ft}$ & 21.8 & \\
\hline GR115801LD & B53 & $0-0.5 \mathrm{ft}$ & 1,360 & \\
\hline GR115901LD & B53 & $0.5-1.5 \mathrm{ft}$ & 58.2 & \\
\hline GR116001LD & B54 & $0-0.5 \mathrm{ft}$ & 6,370 & \\
\hline GR116101LD & B54 & $0.5-1.5 \mathrm{ft}$ & 174 & \\
\hline GR116102LD & B54 & $0.5-1.5 \mathrm{ft}$ & 257 & \\
\hline GR116201LD & B55 & $0-0.5 \mathrm{ft}$ & 3,460 & \\
\hline GR116301LD & B56 & $0.5-1.5 \mathrm{ft}$ & 20.6 & \\
\hline GR116401LD & B57 & $0-0.5 \mathrm{ft}$ & 383 & \\
\hline GR116501LD & B57 & $0.5-1.5 \mathrm{ft}$ & 74.3 & \\
\hline GR116601LD & B58 & $0-0.5 \mathrm{ft}$ & 1,080 & \\
\hline GR116701LD & B58 & $0.5-1.5 \mathrm{ft}$ & 94.1 & \\
\hline GR116801LD & B59 & $0-0.5 \mathrm{ft}$ & 788 & \\
\hline GR116901LD & B59 & $0.5-1.5 \mathrm{ft}$ & 33.5 & \\
\hline
\end{tabular}


Table B-1. (continued).

\begin{tabular}{|c|c|c|c|c|}
\hline Sample ID \# & Location & Depth & $\begin{array}{l}\text { Lead Conc. } \\
(\mathrm{mg} / \mathrm{kg})\end{array}$ & $\mathrm{Q}$ \\
\hline GR117001LD & B60 & $0-0.5 \mathrm{ft}$ & 7.4 & $\mathrm{~J}$ \\
\hline GR117101LD & B60 & $0.5-1.5 \mathrm{ft}$ & 333 & $\mathrm{~J}$ \\
\hline GR117201LD & B61 & $0-0.5 \mathrm{ft}$ & 728 & $\mathrm{~J}$ \\
\hline GR117301LD & B61 & $0.5-1.5 \mathrm{ft}$ & 882 & $\mathrm{~J}$ \\
\hline GR117401LD & B62 & $0-0.5 \mathrm{ft}$ & 94.6 & $\mathrm{~J}$ \\
\hline GR117501LD & B62 & $0.5-1.5 \mathrm{ft}$ & 68.5 & $\mathrm{~J}$ \\
\hline GR117601LD & B63 & $0-0.5 \mathrm{ft}$ & 62.2 & $\mathrm{~J}$ \\
\hline GR117701LD & B63 & $0.5-1.5 \mathrm{ft}$ & 8.4 & $\mathrm{~J}$ \\
\hline GR117801LD & B64 & $0-0.5 \mathrm{ft}$ & 177 & $\mathrm{~J}$ \\
\hline GR117901LD & B64 & $0.5-1.5 \mathrm{ft}$ & 10.4 & $\mathrm{~J}$ \\
\hline GR118001LD & B65 & $0-0.5 \mathrm{ft}$ & 144 & $\mathrm{~J}$ \\
\hline GR118101LD & B65 & $0.5-1.5 \mathrm{ft}$ & 9.8 & $\mathrm{~J}$ \\
\hline GR118102LD & B65 & $0.5-1.5 \mathrm{ft}$ & 7.3 & $\mathrm{~J}$ \\
\hline GR118201LD & B66 & $0-0.5 \mathrm{ft}$ & 125 & $\mathrm{~J}$ \\
\hline GR118301LD & B66 & $0.5-1.5 \mathrm{ft}$ & 13.4 & $\mathrm{~J}$ \\
\hline GR118401LD & B67 & $0-0.5 \mathrm{ft}$ & 163 & $\mathrm{~J}$ \\
\hline GR118501LD & B67 & $0.5-1.5 \mathrm{ft}$ & 83.7 & $\mathrm{~J}$ \\
\hline GR118601LD & B68 & $0-0.5 \mathrm{ft}$ & 96 & $\mathrm{~J}$ \\
\hline GR118701LD & B68 & $0.5-1.5 \mathrm{ft}$ & 9.9 & $\mathrm{~J}$ \\
\hline GR118801LD & B69 & $0-0.5 \mathrm{ft}$ & 11.9 & $\mathrm{~J}$ \\
\hline GR118901LD & B69 & $0.5-1.5 \mathrm{ft}$ & 8.3 & $\mathrm{~J}$ \\
\hline GR119001LD & B70 & $0-0.5 \mathrm{ft}$ & 32.9 & $\mathrm{~J}$ \\
\hline GR119101LD & B70 & $0.5-1.5 \mathrm{ft}$ & 34.7 & $\mathrm{~J}$ \\
\hline GR119201LD & B75 & $0-0.5 \mathrm{ft}$ & 18.6 & $\mathrm{~J}$ \\
\hline GR119301LD & B75 & $0.5-1.5 \mathrm{ft}$ & 9.7 & $\mathrm{~J}$ \\
\hline GR119401LD & B76 & $0-0.5 \mathrm{ft}$ & 11.6 & $\mathrm{~J}$ \\
\hline GR119501LD & B76 & $0.5-1.5 \mathrm{ft}$ & 28.1 & $\mathrm{~J}$ \\
\hline GR119601LD & B77 & $0-0.5 \mathrm{ft}$ & 13.9 & $\mathrm{~J}$ \\
\hline GR119701LD & B77 & $0.5-1.5 \mathrm{ft}$ & 31.5 & $\mathrm{~J}$ \\
\hline GR119801LD & B78 & $0-0.5 \mathrm{ft}$ & 9.1 & $\mathrm{~J}$ \\
\hline GR119901LD & B78 & $0.5-1.5 \mathrm{ft}$ & 12.8 & $\mathrm{~J}$ \\
\hline GR120001LD & \multicolumn{4}{|c|}{ Grain-size Distribution } \\
\hline GR120101LD & \multicolumn{4}{|c|}{ Grain-size Distribution } \\
\hline GR120201LD & \multicolumn{4}{|c|}{ Grain-size Distribution } \\
\hline GR120301LD & \multicolumn{4}{|c|}{ Grain-size Distribution } \\
\hline GR120401LD & \multicolumn{4}{|c|}{ Grain-size Distribution } \\
\hline GR120501LD & \multicolumn{4}{|c|}{ Grain-size Distribution } \\
\hline GR120601LD & \multicolumn{4}{|c|}{ Grain-size Distribution } \\
\hline GR120701LD & \multicolumn{4}{|c|}{ Grain-size Distribution } \\
\hline GR120801LD & \multicolumn{4}{|c|}{ Grain-size Distribution } \\
\hline GR120901LD & \multicolumn{4}{|c|}{ Grain-size Distribution } \\
\hline
\end{tabular}


Table B-1. (continued).

\begin{tabular}{|c|c|c|c|c|}
\hline Sample ID \# & Location & Depth & $\begin{array}{c}\text { Lead Conc. } \\
(\mathrm{mg} / \mathrm{kg})\end{array}$ & Q \\
\hline GR121001LD & B55 & $0.5-1.5 \mathrm{ft}$ & 412 & \\
\hline GR121101LD & B56 & $0-0.5 \mathrm{ft}$ & 3,360 & \\
\hline GR121102LD & B56 & $0-0.5 \mathrm{ft}$ & 4,880 & \\
\hline GR1A0101LD & B79 & $0-0.5 \mathrm{ft}$ & 9.6 & $\mathrm{~J}$ \\
\hline GR1A0201LD & B79 & $0.5-1.5 \mathrm{ft}$ & 10.2 & $\mathrm{~J}$ \\
\hline GR1A0301LD & B80 & $0-0.5 \mathrm{ft}$ & 8.9 & $\mathrm{~J}$ \\
\hline GR1A0401LD & B80 & $0.5-1.5 \mathrm{ft}$ & 12.6 & $\mathrm{~J}$ \\
\hline GR1A0501LD & B81 & $0-0.5 \mathrm{ft}$ & 312 & $\mathrm{~J}$ \\
\hline GR1A0601LD & B81 & $0.5-1.5 \mathrm{ft}$ & 341 & $\mathrm{~J}$ \\
\hline GR1A0701LD & B82 & $0-0.5 \mathrm{ft}$ & 312 & $\mathrm{~J}$ \\
\hline GR1A0801LD & B82 & $0.5-1.5 \mathrm{ft}$ & 83.6 & $\mathrm{~J}$ \\
\hline GR1A0901LD & B83 & $0-0.5 \mathrm{ft}$ & 523 & $\mathrm{~J}$ \\
\hline GR1A1001LD & B83 & $0.5-1.5 \mathrm{ft}$ & 448 & $\mathrm{~J}$ \\
\hline GR1A1101LD & B84 & $0-0.5 \mathrm{ft}$ & 1,590 & $\mathrm{~J}$ \\
\hline GR1A1201LD & B84 & $0.5-1.5 \mathrm{ft}$ & 12,700 & $\mathrm{~J}$ \\
\hline GR1A1301LD & B85 & $0-0.5 \mathrm{ft}$ & 1,180 & $\mathrm{~J}$ \\
\hline GR1A1401LD & B85 & $0.5-1.5 \mathrm{ft}$ & 841 & $\mathrm{~J}$ \\
\hline GR1A1501LD & B86 & $0-0.5 \mathrm{ft}$ & 6,190 & $\mathrm{~J}$ \\
\hline GR1A1601LD & B86 & $0.5-1.5 \mathrm{ft}$ & 1,950 & $\mathrm{~J}$ \\
\hline GR1A1701LD & B87 & $0-0.5 \mathrm{ft}$ & 3,900 & $\mathrm{~J}$ \\
\hline GR1A1801LD & B87 & $0.5-1.5 \mathrm{ft}$ & 1,760 & $\mathrm{~J}$ \\
\hline GR1A1901LD & B88 & $0-0.5 \mathrm{ft}$ & 1,890 & $\mathrm{~J}$ \\
\hline GR1A2001LD & B88 & $0.5-1.5 \mathrm{ft}$ & 1,730 & $\mathrm{~J}$ \\
\hline GR1A2002LD & B88 & $0.5-1.5 \mathrm{ft}$ & 1,310 & $\mathrm{~J}$ \\
\hline GR1A2101LD & B89 & $0-0.5 \mathrm{ft}$ & 2,940 & $\mathrm{~J}$ \\
\hline GR1A2201LD & B89 & $0.5-1.5 \mathrm{ft}$ & 1,800 & $\mathrm{~J}$ \\
\hline GR1A2301LD & B90 & $0-0.5 \mathrm{ft}$ & 689 & $\mathrm{~J}$ \\
\hline GR1A2401LD & B90 & $0.5-1.5 \mathrm{ft}$ & 761 & \\
\hline GR1A2501LD & B91 & $0-0.5 \mathrm{ft}$ & 724 & \\
\hline GR1A2601LD & B91 & $0.5-1.5 \mathrm{ft}$ & 4,570 & \\
\hline GR1A2701LD & B92 & $0-0.5 \mathrm{ft}$ & 1,160 & \\
\hline GR1A2801LD & B92 & $0.5-1.5 \mathrm{ft}$ & 23.4 & \\
\hline GR1A2901LD & B93 & $0-0.5 \mathrm{ft}$ & 417 & \\
\hline GR1A3001LD & B93 & $0.5-1.5 \mathrm{ft}$ & 37.5 & \\
\hline GR1A3101LD & B94 & $0-0.5 \mathrm{ft}$ & 213 & \\
\hline GR1A3201LD & B94 & $0.5-1.5 \mathrm{ft}$ & 8.6 & \\
\hline GR1A3301LD & $\mathrm{C} 10$ & $0-0.5 \mathrm{ft}$ & 10.9 & $\mathrm{~J}$ \\
\hline GR1A3401LD & $\mathrm{C} 10$ & $0.5-1.5 \mathrm{ft}$ & 11.8 & $\mathrm{~J}$ \\
\hline GR1A3501LD & $\mathrm{C} 11$ & $0-0.5 \mathrm{ft}$ & 8.3 & $\mathrm{~J}$ \\
\hline GR1A3601LD & $\mathrm{C} 11$ & $0.5-1.5 \mathrm{ft}$ & 14.9 & $\mathrm{~J}$ \\
\hline GR1A3701LD & $\mathrm{C} 12$ & $0-0.5 \mathrm{ft}$ & 8.5 & $\mathrm{~J}$ \\
\hline
\end{tabular}


Table B-1. (continued).

\begin{tabular}{|c|c|c|c|c|}
\hline Sample ID \# & Location & Depth & $\begin{array}{c}\text { Lead Conc. } \\
(\mathrm{mg} / \mathrm{kg})\end{array}$ & Q \\
\hline GR1A3801LD & C12 & $0.5-1.5 \mathrm{ft}$ & 27.8 & $\mathrm{~J}$ \\
\hline GR1A3901LD & $\mathrm{C} 13$ & $0-0.5 \mathrm{ft}$ & 7.8 & $\mathrm{~J}$ \\
\hline GR1A4001LD & $\mathrm{C} 13$ & $0.5-1.5 \mathrm{ft}$ & 26.8 & $\mathrm{~J}$ \\
\hline GR1A4002LD & $\mathrm{C} 13$ & $0.5-1.5 \mathrm{ft}$ & 27.4 & $\mathrm{~J}$ \\
\hline GR1A4101LD & $\mathrm{C} 14$ & $0-0.5 \mathrm{ft}$ & 8.2 & $\mathrm{~J}$ \\
\hline GR1A4201LD & C14 & $0.5-1.5 \mathrm{ft}$ & 14.7 & $\mathrm{~J}$ \\
\hline GR1A4301LD & $\mathrm{C} 15$ & $0-0.5 \mathrm{ft}$ & 8.1 & $\mathrm{~J}$ \\
\hline GR1A4401LD & $\mathrm{C} 15$ & $0.5-1.5 \mathrm{ft}$ & 203 & $\mathrm{~J}$ \\
\hline GR1A4501LD & $\mathrm{C} 16$ & $0-0.5 \mathrm{ft}$ & 11.7 & $\mathrm{~J}$ \\
\hline GR1A4601LD & $\mathrm{C} 16$ & $0.5-1.5 \mathrm{ft}$ & 334 & $\mathrm{~J}$ \\
\hline GR1A4701LD & $\mathrm{C} 17$ & $0-0.5 \mathrm{ft}$ & 18.8 & $\mathrm{~J}$ \\
\hline GR1A4801LD & $\mathrm{C} 17$ & $0.5-1.5 \mathrm{ft}$ & 807 & $\mathrm{~J}$ \\
\hline GR1A4901LD & $\mathrm{C} 18$ & $0-0.5 \mathrm{ft}$ & 200 & $\mathrm{~J}$ \\
\hline GR1A5001LD & $\mathrm{C} 18$ & $0.5-1.5 \mathrm{ft}$ & 426 & $\mathrm{~J}$ \\
\hline GR1A5101LD & C19 & $0-0.5 \mathrm{ft}$ & 267 & $\mathrm{~J}$ \\
\hline GR1A5201LD & C19 & $0.5-1.5 \mathrm{ft}$ & 42.8 & $\mathrm{~J}$ \\
\hline GR1A5301LD & $\mathrm{C} 20$ & $0-0.5 \mathrm{ft}$ & 9.2 & $\mathrm{~J}$ \\
\hline GR1A5401LD & $\mathrm{C} 20$ & $0.5-1.5 \mathrm{ft}$ & 238 & $\mathrm{~J}$ \\
\hline GR1A5501LD & $\mathrm{C} 21$ & $0-0.5 \mathrm{ft}$ & 86 & $\mathrm{~J}$ \\
\hline GR1A5601LD & $\mathrm{C} 21$ & $0.5-1.5 \mathrm{ft}$ & 212 & $\mathrm{~J}$ \\
\hline GR1A5701LD & $\mathrm{C} 22$ & $0-0.5 \mathrm{ft}$ & 59.6 & $\mathrm{~J}$ \\
\hline GR1A5801LD & $\mathrm{C} 22$ & $0.5-1.5 \mathrm{ft}$ & 260 & $\mathrm{~J}$ \\
\hline GR1A5901LD & $\mathrm{C} 23$ & $0-0.5 \mathrm{ft}$ & 10.6 & $\mathrm{~J}$ \\
\hline GR1A6001LD & $\mathrm{C} 23$ & $0.5-1.5 \mathrm{ft}$ & 15 & $\mathrm{~J}$ \\
\hline GR1A6002LD & $\mathrm{C} 23$ & $0.5-1.5 \mathrm{ft}$ & 7.9 & \\
\hline GR1A6101LD & $\mathrm{F} 1$ & $0-0.5 \mathrm{ft}$ & 33.9 & $\mathrm{~J}$ \\
\hline GR1A6201LD & $\mathrm{F} 2$ & $0-0.5 \mathrm{ft}$ & 20.5 & $\mathrm{~J}$ \\
\hline GR1A6301LD & F3 & $0-0.5 \mathrm{ft}$ & 8.5 & $\mathrm{~J}$ \\
\hline GR1A6401LD & $\mathrm{F} 4$ & $0-0.5 \mathrm{ft}$ & 17.4 & $\mathrm{~J}$ \\
\hline GR1A6501LD & F5 & $0-0.5 \mathrm{ft}$ & 13 & $\mathrm{~J}$ \\
\hline GR1A6601LD & F6 & $0-0.5 \mathrm{ft}$ & 28.9 & $\mathrm{~J}$ \\
\hline GR1A6701LD & F7 & $0-0.5 \mathrm{ft}$ & 56.8 & $\mathrm{R}$ \\
\hline GR1A6801LD & F8 & $0-0.5 \mathrm{ft}$ & 26.5 & $\mathrm{R}$ \\
\hline GR1A6901LD & F9 & $0-0.5 \mathrm{ft}$ & 21 & $\mathrm{R}$ \\
\hline GR1A7001LD & F10 & $0-0.5 \mathrm{ft}$ & 19.2 & $\mathrm{~J}$ \\
\hline GR1A7101LD & F11 & $0-0.5 \mathrm{ft}$ & 31.3 & $\mathrm{~J}$ \\
\hline GR1A7201LD & F12 & $0-0.5 \mathrm{ft}$ & 27.4 & $\mathrm{~J}$ \\
\hline GR1A7301LD & F13 & $0-0.5 \mathrm{ft}$ & 75.4 & $\mathrm{~J}$ \\
\hline GR1A7401LD & F14 & $0-0.5 \mathrm{ft}$ & 254 & $\mathrm{~J}$ \\
\hline GR1A7501LD & F15 & $0-0.5 \mathrm{ft}$ & 273 & $\mathrm{~J}$ \\
\hline GR1A7601LD & F16 & $0-0.5 \mathrm{ft}$ & 272 & $\mathrm{~J}$ \\
\hline
\end{tabular}


Table B-1. (continued).

\begin{tabular}{|c|c|c|c|c|}
\hline Sample ID \# & Location & Depth & $\begin{array}{c}\text { Lead Conc. } \\
(\mathrm{mg} / \mathrm{kg})\end{array}$ & Q \\
\hline GR1A7701LD & F17 & $0-0.5 \mathrm{ft}$ & 563 & $\mathrm{~J}$ \\
\hline GR1A7801LD & F18 & $0-0.5 \mathrm{ft}$ & 560 & $\mathrm{~J}$ \\
\hline GR1A7901LD & F19 & $0-0.5 \mathrm{ft}$ & 199 & $\mathrm{~J}$ \\
\hline GR1A8001LD & F20 & $0-0.5 \mathrm{ft}$ & 91.3 & $\mathrm{~J}$ \\
\hline GR1A8102LD & $\mathrm{F} 20$ & $0-0.5 \mathrm{ft}$ & 89.2 & $\mathrm{~J}$ \\
\hline GR1A8101LD & F21 & $0-0.5 \mathrm{ft}$ & 29.8 & $\mathrm{~J}$ \\
\hline GR1A8201LD & $\mathrm{F} 22$ & $0-0.5 \mathrm{ft}$ & 56.6 & $\mathrm{~J}$ \\
\hline GR1A8301LD & F23 & $0-0.5 \mathrm{ft}$ & 42.1 & $\mathrm{~J}$ \\
\hline GR1A8401LD & F24 & $0-0.5 \mathrm{ft}$ & 53.6 & $\mathrm{~J}$ \\
\hline GR1A8501LD & F25 & $0-0.5 \mathrm{ft}$ & 48.6 & $\mathrm{~J}$ \\
\hline GR1A8601LD & F26 & $0-0.5 \mathrm{ft}$ & 24.4 & $\mathrm{~J}$ \\
\hline GR1A8701LD & F27 & $0-0.5 \mathrm{ft}$ & 14.5 & $\mathrm{~J}$ \\
\hline GR1A8801LD & F28 & $0-0.5 \mathrm{ft}$ & 62.3 & $\mathrm{~J}$ \\
\hline GR1A8901LD & F29 & $0-0.5 \mathrm{ft}$ & 49.4 & $\mathrm{~J}$ \\
\hline GR1A9001LD & F30 & $0-0.5 \mathrm{ft}$ & 46,100 & $\mathrm{~J}$ \\
\hline GR1A9101LD & F31 & $0-0.5 \mathrm{ft}$ & 25 & $\mathrm{~J}$ \\
\hline GR1A9201LD & F32 & $0-0.5 \mathrm{ft}$ & 19.1 & $\mathrm{~J}$ \\
\hline GR1A9301LD & F33 & $0-0.5 \mathrm{ft}$ & 11.2 & $\mathrm{~J}$ \\
\hline GR1A9401LD & F34 & $0-0.5 \mathrm{ft}$ & 15.1 & $\mathrm{~J}$ \\
\hline GR1A9501LD & F35 & $0-0.5 \mathrm{ft}$ & 22.6 & $\mathrm{~J}$ \\
\hline GR1A9601LD & F36 & $0-0.5 \mathrm{ft}$ & 36.5 & $\mathrm{~J}$ \\
\hline GR1A9701LD & F37 & $0-0.5 \mathrm{ft}$ & 15.3 & $\mathrm{~J}$ \\
\hline GR1A9801LD & F38 & $0-0.5 \mathrm{ft}$ & 31 & $\mathrm{~J}$ \\
\hline GR1A9901LD & F39 & $0-0.5 \mathrm{ft}$ & 14.2 & $\mathrm{~J}$ \\
\hline GR1B0101LD & F40 & $0-0.5 \mathrm{ft}$ & 84.4 & $\mathrm{~J}$ \\
\hline GR1B0102LD & F40 & $0-0.5 \mathrm{ft}$ & 88.6 & $\mathrm{~J}$ \\
\hline GR1B0201LD & F41 & $0-0.5 \mathrm{ft}$ & 18.9 & $\mathrm{~J}$ \\
\hline GR1B0301LD & F42 & $0-0.5 \mathrm{ft}$ & 27.5 & \\
\hline GR1B0401LD & F43 & $0-0.5 \mathrm{ft}$ & 31.7 & \\
\hline GR1B0501LD & F44 & $0-0.5 \mathrm{ft}$ & 288 & \\
\hline GR1B0601LD & F45 & $0-0.5 \mathrm{ft}$ & 16.3 & \\
\hline GR1B0701LD & F46 & $0-0.5 \mathrm{ft}$ & 60.9 & \\
\hline GR1B0801LD & F47 & $0-0.5 \mathrm{ft}$ & 913 & \\
\hline GR1B0901LD & F48 & $0-0.5 \mathrm{ft}$ & 346 & \\
\hline GR1B1001LD & F49 & $0-0.5 \mathrm{ft}$ & 120 & \\
\hline GR1B1101LD & F50 & $0-0.5 \mathrm{ft}$ & 27.2 & \\
\hline GR1B1201LD & F51 & $0-0.5 \mathrm{ft}$ & 25.5 & \\
\hline GR1B1301LD & F52 & $0-0.5 \mathrm{ft}$ & 29.3 & \\
\hline GR1B1401LD & F53 & $0-0.5 \mathrm{ft}$ & 36.8 & \\
\hline GR1B1501LD & F54 & $0-0.5 \mathrm{ft}$ & 50.5 & \\
\hline GR1B1601LD & F55 & $0-0.5 \mathrm{ft}$ & 55.2 & \\
\hline
\end{tabular}


Table B-1. (continued).

\begin{tabular}{|c|c|c|c|c|}
\hline Sample ID \# & Location & Depth & $\begin{array}{l}\text { Lead Conc. } \\
(\mathrm{mg} / \mathrm{kg})\end{array}$ & $\mathrm{Q}$ \\
\hline GR1B1701LD & F56 & $0-0.5 \mathrm{ft}$ & 89 & \\
\hline GR1B1801LD & F57 & $0-0.5 \mathrm{ft}$ & 79.1 & \\
\hline GR1B1901LD & F58 & $0-0.5 \mathrm{ft}$ & 13.6 & \\
\hline GR1B2001LD & F59 & $0-0.5 \mathrm{ft}$ & 17.5 & \\
\hline GR1B2101LD & F60 & $0-0.5 \mathrm{ft}$ & 24.4 & \\
\hline GR1B2102LD & F60 & $0-0.5 \mathrm{ft}$ & 17.6 & \\
\hline GR1B2201LD & F61 & $0-0.5 \mathrm{ft}$ & 32.2 & $\mathrm{R}$ \\
\hline GR1B2301LD & F62 & $0-0.5 \mathrm{ft}$ & 26.6 & $\mathrm{R}$ \\
\hline GR1B2401LD & F63 & $0-0.5 \mathrm{ft}$ & 36.8 & $\mathrm{R}$ \\
\hline GR1B2501LD & F64 & $0-0.5 \mathrm{ft}$ & 35.3 & $\mathrm{R}$ \\
\hline GR1B2601LD & F65 & $0-0.5 \mathrm{ft}$ & 39.8 & \\
\hline GR1B2701LD & F66 & $0-0.5 \mathrm{ft}$ & 21.6 & \\
\hline GR1B2801LD & F67 & $0-0.5 \mathrm{ft}$ & 66.4 & \\
\hline GR1B2901LD & F68 & $0-0.5 \mathrm{ft}$ & 86.5 & \\
\hline GR1B3001LD & F69 & $0-0.5 \mathrm{ft}$ & 40.7 & \\
\hline GR1B3101LD & F70 & $0-0.5 \mathrm{ft}$ & 90.5 & \\
\hline GR1B3201LD & F71 & $0-0.5 \mathrm{ft}$ & 23.6 & \\
\hline GR1B3301LD & F72 & $0-0.5 \mathrm{ft}$ & 94.5 & \\
\hline GR1B3401LD & F73 & $0-0.5 \mathrm{ft}$ & 21.8 & \\
\hline GR1B3501LD & F74 & $0-0.5 \mathrm{ft}$ & 41.7 & \\
\hline GR1B3601LD & F75 & $0-0.5 \mathrm{ft}$ & 577 & \\
\hline GR1B3701LD & F76 & $0-0.5 \mathrm{ft}$ & 954 & \\
\hline GR1B3801LD & F77 & $0-0.5 \mathrm{ft}$ & 107 & \\
\hline GR1B3901LD & F78 & $0-0.5 \mathrm{ft}$ & 17.9 & \\
\hline GR1B4001LD & F79 & $0-0.5 \mathrm{ft}$ & 17.2 & \\
\hline GR1B4101LD & F80 & $0-0.5 \mathrm{ft}$ & 17.7 & \\
\hline GR1B4102LD & F80 & $0-0.5 \mathrm{ft}$ & 18.6 & \\
\hline GR1B4201LD & F81 & $0-0.5 \mathrm{ft}$ & 53.9 & \\
\hline GR1B4301LD & F82 & $0-0.5 \mathrm{ft}$ & 57.8 & \\
\hline GR1B4401LD & F83 & $0-0.5 \mathrm{ft}$ & 509 & \\
\hline GR1B4501LD & F84 & $0-0.5 \mathrm{ft}$ & 156 & $\mathrm{~J}$ \\
\hline GR1B4601LD & F85 & $0-0.5 \mathrm{ft}$ & 62.7 & $\mathrm{~J}$ \\
\hline GR1B4701LD & F86 & $0-0.5 \mathrm{ft}$ & 47.2 & $\mathrm{~J}$ \\
\hline GR1B4801LD & F87 & $0-0.5 \mathrm{ft}$ & 49.8 & $\mathrm{~J}$ \\
\hline GR1B4901LD & F88 & $0-0.5 \mathrm{ft}$ & 30.7 & $\mathrm{~J}$ \\
\hline GR1B5001LD & F89 & $0-0.5 \mathrm{ft}$ & 24.9 & $\mathrm{~J}$ \\
\hline GR1B5101LD & F90 & $0-0.5 \mathrm{ft}$ & 14.4 & $\mathrm{~J}$ \\
\hline GR1B5201LD & F91 & $0-0.5 \mathrm{ft}$ & 16.9 & $\mathrm{~J}$ \\
\hline GR1B5301LD & F92 & $0-0.5 \mathrm{ft}$ & 37.7 & $\mathrm{~J}$ \\
\hline GR1B5401LD & F93 & $0-0.5 \mathrm{ft}$ & 94 & $\mathrm{~J}$ \\
\hline GR1B5501LD & F94 & $0-0.5 \mathrm{ft}$ & 45.9 & $\mathrm{~J}$ \\
\hline
\end{tabular}


Table B-1. (continued).

\begin{tabular}{|c|c|c|c|c|}
\hline Sample ID \# & Location & Depth & $\begin{array}{l}\text { Lead Conc. } \\
(\mathrm{mg} / \mathrm{kg})\end{array}$ & $\mathrm{Q}$ \\
\hline GR1B5601LD & F95 & $0-0.5 \mathrm{ft}$ & 41.3 & $\mathrm{~J}$ \\
\hline GR1B5701LD & F96 & $0-0.5 \mathrm{ft}$ & 362 & $\mathrm{~J}$ \\
\hline GR1B5801LD & F97 & $0-0.5 \mathrm{ft}$ & 51 & $\mathrm{~J}$ \\
\hline GR1B5901LD & F98 & $0-0.5 \mathrm{ft}$ & 24.6 & $\mathrm{~J}$ \\
\hline GR1B6001LD & F99 & $0-0.5 \mathrm{ft}$ & 26.3 & $\mathrm{~J}$ \\
\hline GR1B6101LD & F100 & $0-0.5 \mathrm{ft}$ & 69.6 & $\mathrm{~J}$ \\
\hline GR1B6102LD & F100 & $0-0.5 \mathrm{ft}$ & 28.3 & $\mathrm{~J}$ \\
\hline GR1B6201LD & F101 & $0-0.5 \mathrm{ft}$ & 15.9 & $\mathrm{~J}$ \\
\hline GR1B6301LD & F102 & $0-0.5 \mathrm{ft}$ & 49.8 & $\mathrm{~J}$ \\
\hline GR1B6401LD & F103 & $0-0.5 \mathrm{ft}$ & 6,790 & \\
\hline GR1B6501LD & F104 & $0-0.5 \mathrm{ft}$ & 2,350 & \\
\hline GR1B6601LD & F105 & $0-0.5 \mathrm{ft}$ & 59.8 & \\
\hline GR1B6701LD & F106 & $0-0.5 \mathrm{ft}$ & 23.7 & \\
\hline GR1B6801LD & F107 & $0-0.5 \mathrm{ft}$ & 58.6 & \\
\hline GR1B6901LD & F108 & $0-0.5 \mathrm{ft}$ & 63.5 & \\
\hline GR1B7001LD & F109 & $0-0.5 \mathrm{ft}$ & 200 & \\
\hline GR1B7101LD & F110 & $0-0.5 \mathrm{ft}$ & 68.5 & \\
\hline GR1B7201LD & F111 & $0-0.5 \mathrm{ft}$ & 48.4 & \\
\hline GR1B7301LD & F112 & $0-0.5 \mathrm{ft}$ & 55.6 & \\
\hline GR1B7401LD & F113 & $0-0.5 \mathrm{ft}$ & 72.7 & \\
\hline GR1B7501LD & F114 & $0-0.5 \mathrm{ft}$ & 22.1 & \\
\hline GR1B7601LD & F115 & $0-0.5 \mathrm{ft}$ & 15.3 & \\
\hline GR1B7701LD & F116 & $0-0.5 \mathrm{ft}$ & 19.3 & \\
\hline GR1B7801LD & F117 & $0-0.5 \mathrm{ft}$ & 26.1 & \\
\hline GR1B7901LD & F118 & $0-0.5 \mathrm{ft}$ & 19.9 & \\
\hline GR1B8001LD & F119 & $0-0.5 \mathrm{ft}$ & 17.8 & \\
\hline GR1B8101LD & F120 & $0-0.5 \mathrm{ft}$ & 14.8 & \\
\hline GR1B8102LD & F120 & $0-0.5 \mathrm{ft}$ & 16.2 & \\
\hline GR1B8201LD & F121 & $0-0.5 \mathrm{ft}$ & 34.3 & \\
\hline GR1B8301LD & F122 & $0-0.5 \mathrm{ft}$ & 28.4 & $\mathrm{~J}$ \\
\hline GR1B8401LD & F123 & $0-0.5 \mathrm{ft}$ & 42.3 & $\mathrm{~J}$ \\
\hline GR1B8501LD & F124 & $0-0.5 \mathrm{ft}$ & 27.4 & $\mathrm{~J}$ \\
\hline GR1B8601LD & F125 & $0-0.5 \mathrm{ft}$ & 33.3 & $\mathrm{~J}$ \\
\hline GR1B8701LD & F126 & $0-0.5 \mathrm{ft}$ & 29.9 & $\mathrm{~J}$ \\
\hline GR1B8801LD & F127 & $0-0.5 \mathrm{ft}$ & 808 & $\mathrm{~J}$ \\
\hline GR1B8901LD & F128 & $0-0.5 \mathrm{ft}$ & 35.6 & $\mathrm{~J}$ \\
\hline GR1B9001LD & F129 & $0-0.5 \mathrm{ft}$ & 37.1 & $\mathrm{~J}$ \\
\hline GR1B9101LD & F130 & $0-0.5 \mathrm{ft}$ & 35.5 & $\mathrm{~J}$ \\
\hline GR1B9201LD & F131 & $0-0.5 \mathrm{ft}$ & 43.5 & $\mathrm{~J}$ \\
\hline GR1B9301LD & F132 & $0-0.5 \mathrm{ft}$ & 61.6 & $\mathrm{~J}$ \\
\hline GR1B9401LD & F133 & $0-0.5 \mathrm{ft}$ & 951 & $\mathrm{~J}$ \\
\hline
\end{tabular}


Table B-1. (continued).

\begin{tabular}{|c|c|c|c|c|}
\hline Sample ID \# & Location & Depth & $\begin{array}{c}\text { Lead Conc. } \\
(\mathrm{mg} / \mathrm{kg})\end{array}$ & Q \\
\hline GR1B9501LD & F134 & $0-0.5 \mathrm{ft}$ & 1,130 & $\mathrm{~J}$ \\
\hline GR1B9601LD & F135 & $0-0.5 \mathrm{ft}$ & 708 & $\mathrm{~J}$ \\
\hline GR1B9701LD & F136 & $0-0.5 \mathrm{ft}$ & 2,520 & $\mathrm{~J}$ \\
\hline No Sample - In Berm & F137 & $0-0.5 \mathrm{ft}$ & N/A & \\
\hline No Sample - In Berm & F138 & $0-0.5 \mathrm{ft}$ & N/A & \\
\hline No Sample - In Berm & F139 & $0-0.5 \mathrm{ft}$ & N/A & \\
\hline No Sample - In Berm & F140 & $0-0.5 \mathrm{ft}$ & $\mathrm{N} / \mathrm{A}$ & \\
\hline No Sample - In Berm & F141 & $0-0.5 \mathrm{ft}$ & N/A & \\
\hline No Sample - In Berm & F142 & $0-0.5 \mathrm{ft}$ & N/A & \\
\hline No Sample - In Berm & F143 & $0-0.5 \mathrm{ft}$ & N/A & \\
\hline No Sample - In Berm & F144 & $0-0.5 \mathrm{ft}$ & $\mathrm{N} / \mathrm{A}$ & \\
\hline No Sample - In Berm & F145 & $0-0.5 \mathrm{ft}$ & N/A & \\
\hline GR1C0801LD & F146 & $0-0.5 \mathrm{ft}$ & 18.8 & $\mathrm{~J}$ \\
\hline GR1C0901LD & F147 & $0-0.5 \mathrm{ft}$ & 45.5 & $\mathrm{~J}$ \\
\hline GR1C1001LD & F148 & $0-0.5 \mathrm{ft}$ & 18.2 & $\mathrm{~J}$ \\
\hline GR1C1101LD & F149 & $0-0.5 \mathrm{ft}$ & 33.6 & $\mathrm{~J}$ \\
\hline GR1C1201LD & F150 & $0-0.5 \mathrm{ft}$ & 10.8 & $\mathrm{~J}$ \\
\hline GR1C1301LD & F151 & $0-0.5 \mathrm{ft}$ & 10.7 & $\mathrm{~J}$ \\
\hline GR1C1401LD & F152 & $0-0.5 \mathrm{ft}$ & 9.6 & $\mathrm{~J}$ \\
\hline GR1C1501LD & F153 & $0-0.5 \mathrm{ft}$ & 19.4 & $\mathrm{~J}$ \\
\hline GR1C1601LD & F154 & $0-0.5 \mathrm{ft}$ & 14 & $\mathrm{~J}$ \\
\hline GR1C1701LD & F155 & $0-0.5 \mathrm{ft}$ & 14.3 & $\mathrm{~J}$ \\
\hline GR1C1801LD & F156 & $0-0.5 \mathrm{ft}$ & 3,130 & $\mathrm{~J}$ \\
\hline GR1C1901LD & F157 & $0-0.5 \mathrm{ft}$ & 19.5 & $\mathrm{~J}$ \\
\hline GR1C2001LD & F158 & $0-0.5 \mathrm{ft}$ & 17.4 & $\mathrm{~J}$ \\
\hline GR1C2101LD & F159 & $0-0.5 \mathrm{ft}$ & 1,840 & $\mathrm{~J}$ \\
\hline GR1C2102LD & F159 & $0-0.5 \mathrm{ft}$ & 407 & $\mathrm{~J}$ \\
\hline GR1C2201LD & F160 & $0-0.5 \mathrm{ft}$ & 23.1 & $\mathrm{~J}$ \\
\hline GR1C2301LD & F161 & $0-0.5 \mathrm{ft}$ & 21 & $\mathrm{~J}$ \\
\hline GR1C2401LD & F162 & $0-0.5 \mathrm{ft}$ & 17 & $\mathrm{~J}$ \\
\hline GR1C2501LD & F163 & $0-0.5 \mathrm{ft}$ & 16.6 & $\mathrm{~J}$ \\
\hline GR1C2601LD & F164 & $0-0.5 \mathrm{ft}$ & 13.9 & $\mathrm{~J}$ \\
\hline GR1C2701LD & F165 & $0-0.5 \mathrm{ft}$ & 15.4 & $\mathrm{~J}$ \\
\hline GR1C2801LD & F166 & $0-0.5 \mathrm{ft}$ & 13.6 & $\mathrm{~J}$ \\
\hline GR1C2901LD & F167 & $0-0.5 \mathrm{ft}$ & 17.6 & $\mathrm{~J}$ \\
\hline GR1C3001LD & F168 & $0-0.5 \mathrm{ft}$ & 14.8 & $\mathrm{~J}$ \\
\hline GR1C3101LD & F169 & $0-0.5 \mathrm{ft}$ & 22.9 & $\mathrm{~J}$ \\
\hline GR1C3201LD & F170 & $0-0.5 \mathrm{ft}$ & 17.8 & $\mathrm{~J}$ \\
\hline GR1C3301LD & F171 & $0-0.5 \mathrm{ft}$ & 39.9 & $\mathrm{~J}$ \\
\hline GR1C3401LD & F172 & $0-0.5 \mathrm{ft}$ & 15.3 & $\mathrm{~J}$ \\
\hline GR1C3501LD & F173 & $0-0.5 \mathrm{ft}$ & 12.8 & $\mathrm{~J}$ \\
\hline
\end{tabular}


Table B-1. (continued).

\begin{tabular}{|c|c|c|c|c|}
\hline Sample ID \# & Location & Depth & $\begin{array}{l}\text { Lead Conc. } \\
(\mathrm{mg} / \mathrm{kg})\end{array}$ & Q \\
\hline GR1C3601LD & F174 & $0-0.5 \mathrm{ft}$ & 14.1 & $\mathrm{~J}$ \\
\hline GR1C3701LD & F175 & $0-0.5 \mathrm{ft}$ & 16.3 & $\mathrm{~J}$ \\
\hline GR1C3801LD & F176 & $0-0.5 \mathrm{ft}$ & 12.1 & $\mathrm{~J}$ \\
\hline GR1C3901LD & F177 & $0-0.5 \mathrm{ft}$ & 13.7 & $\mathrm{~J}$ \\
\hline GR1C4001LD & F178 & $0-0.5 \mathrm{ft}$ & 13.1 & $\mathrm{~J}$ \\
\hline GR1C4101LD & F179 & $0-0.5 \mathrm{ft}$ & 13.1 & $\mathrm{~J}$ \\
\hline GR1C4102LD & F179 & $0-0.5 \mathrm{ft}$ & 14.5 & $\mathrm{~J}$ \\
\hline GR1C4201LD & F180 & $0-0.5 \mathrm{ft}$ & 17.3 & $\mathrm{~J}$ \\
\hline GR1C4301LD & F181 & $0-0.5 \mathrm{ft}$ & 13.8 & $\mathrm{~J}$ \\
\hline GR1C4401LD & F182 & $0-0.5 \mathrm{ft}$ & 17 & $\mathrm{~J}$ \\
\hline GR1C4501LD & F183 & $0-0.5 \mathrm{ft}$ & 29.7 & $\mathrm{~J}$ \\
\hline GR1C4601LD & F184 & $0-0.5 \mathrm{ft}$ & 19.8 & $\mathrm{~J}$ \\
\hline GR1C4701LD & F185 & $0-0.5 \mathrm{ft}$ & 16.7 & $\mathrm{~J}$ \\
\hline GR1C4801LD & F186 & $0-0.5 \mathrm{ft}$ & 16.3 & $\mathrm{~J}$ \\
\hline GR1C4901LD & F187 & $0-0.5 \mathrm{ft}$ & 16.4 & $\mathrm{~J}$ \\
\hline GR1C5001LD & F188 & $0-0.5 \mathrm{ft}$ & 77 & $\mathrm{~J}$ \\
\hline GR1C5101LD & F189 & $0-0.5 \mathrm{ft}$ & 11.7 & $\mathrm{~J}$ \\
\hline GR1C5201LD & F190 & $0-0.5 \mathrm{ft}$ & 13.6 & $\mathrm{~J}$ \\
\hline GR1C5301LD & F191 & $0-0.5 \mathrm{ft}$ & 10.7 & $\mathrm{~J}$ \\
\hline GR1C5401LD & F192 & $0-0.5 \mathrm{ft}$ & 11.6 & $\mathrm{~J}$ \\
\hline GR1C5501LD & F193 & $0-0.5 \mathrm{ft}$ & 19.3 & $\mathrm{~J}$ \\
\hline GR1C5601LD & F194 & $0-0.5 \mathrm{ft}$ & 13.3 & $\mathrm{~J}$ \\
\hline GR1C5701LD & F195 & $0-0.5 \mathrm{ft}$ & 60.4 & $\mathrm{~J}$ \\
\hline GR1C5801LD & G1 & $0-0.5 \mathrm{ft}$ & 232 & \\
\hline GR1C5901LD & $\mathrm{G} 2$ & $0-0.5 \mathrm{ft}$ & 231 & \\
\hline GR1C6001LD & G3 & $0-0.5 \mathrm{ft}$ & 122 & \\
\hline GR1C6101LD & G4 & $0-0.5 \mathrm{ft}$ & 36.7 & \\
\hline GR1C6102LD & G4 & $0-0.5 \mathrm{ft}$ & 51.5 & \\
\hline GR1C6201LD & G5 & $0-0.5 \mathrm{ft}$ & 91.4 & \\
\hline GR1C6301LD & G6 & $0-0.5 \mathrm{ft}$ & 259 & \\
\hline GR1C6401LD & G7 & $0-0.5 \mathrm{ft}$ & 50.9 & \\
\hline GR1C6501LD & G8 & $0-0.5 \mathrm{ft}$ & 34.4 & $\mathrm{~J}$ \\
\hline GR1C6601LD & G9 & $0-0.5 \mathrm{ft}$ & 19.8 & $\mathrm{~J}$ \\
\hline GR1C6701LD & G10 & $0-0.5 \mathrm{ft}$ & 89.7 & $\mathrm{~J}$ \\
\hline GR1C6801LD & G11 & $0-0.5 \mathrm{ft}$ & 42.3 & $\mathrm{~J}$ \\
\hline GR1C6901LD & G12 & $0-0.5 \mathrm{ft}$ & 21.4 & $\mathrm{~J}$ \\
\hline GR1C7001LD & G13 & $0-0.5 \mathrm{ft}$ & 26.4 & $\mathrm{~J}$ \\
\hline GR1C7101LD & G14 & $0-0.5 \mathrm{ft}$ & 97 & \\
\hline GR1C7201LD & G15 & $0-0.5 \mathrm{ft}$ & 231 & \\
\hline GR1C7301LD & G16 & $0-0.5 \mathrm{ft}$ & 25.1 & $\mathrm{~J}$ \\
\hline GR1C7401LD & G17 & $0-0.5 \mathrm{ft}$ & 23 & $\mathrm{~J}$ \\
\hline
\end{tabular}


Table B-1. (continued).

\begin{tabular}{|c|c|c|c|c|}
\hline Sample ID \# & Location & Depth & $\begin{array}{c}\text { Lead Conc. } \\
(\mathrm{mg} / \mathrm{kg})\end{array}$ & $\mathrm{Q}$ \\
\hline GR1C7501LD & G18 & $0-0.5 \mathrm{ft}$ & 34.6 & $\mathrm{~J}$ \\
\hline GR1C7601LD & G19 & $0-0.5 \mathrm{ft}$ & 19.7 & $\mathrm{~J}$ \\
\hline GR1C7701LD & $\mathrm{G} 20$ & $0-0.5 \mathrm{ft}$ & 34.6 & $\mathrm{~J}$ \\
\hline GR1C7801LD & $\mathrm{G} 21$ & $0-0.5 \mathrm{ft}$ & 36.3 & $\mathrm{~J}$ \\
\hline GR1C7901LD & $\mathrm{G} 22$ & $0-0.5 \mathrm{ft}$ & 347 & \\
\hline GR1C8001LD & $\mathrm{G} 23$ & $0-0.5 \mathrm{ft}$ & 81.3 & \\
\hline GR1C8101LD & $\mathrm{G} 24$ & $0-0.5 \mathrm{ft}$ & 36.9 & $\mathrm{~J}$ \\
\hline GR1C8102LD & G24 & $0-0.5 \mathrm{ft}$ & 67.4 & $\mathrm{~J}$ \\
\hline GR1C8201LD & $\mathrm{G} 25$ & $0-0.5 \mathrm{ft}$ & 28.6 & $\mathrm{~J}$ \\
\hline GR1C8301LD & G26 & $0-0.5 \mathrm{ft}$ & 19.5 & $\mathrm{~J}$ \\
\hline GR1C8401LD & $\mathrm{G} 27$ & $0-0.5 \mathrm{ft}$ & 26.9 & $\mathrm{~J}$ \\
\hline GR1C8501LD & G28 & $0-0.5 \mathrm{ft}$ & 41 & $\mathrm{~J}$ \\
\hline GR1C8601LD & G29 & $0-0.5 \mathrm{ft}$ & 40.3 & $\mathrm{~J}$ \\
\hline GR1C8701LD & G30 & $0-0.5 \mathrm{ft}$ & 26.1 & \\
\hline GR1C8801LD & 1 & $0-0.5 \mathrm{ft}$ & 14.2 & $\mathrm{~J}$ \\
\hline GR1C8901LD & 2 & $0-0.5 \mathrm{ft}$ & 6 & $\mathrm{~J}$ \\
\hline GR1C9001LD & 3 & $0-0.5 \mathrm{ft}$ & 12.8 & $\mathrm{~J}$ \\
\hline GR1C9101LD & 4 & $0-0.5 \mathrm{ft}$ & 7.2 & $\mathrm{~J}$ \\
\hline GR1C9201LD & 5 & $0-0.5 \mathrm{ft}$ & 11.6 & $\mathrm{~J}$ \\
\hline GR1C9301LD & 6 & $0-0.5 \mathrm{ft}$ & 11.3 & $\mathrm{~J}$ \\
\hline GR1C9401LD & 7 & $0-0.5 \mathrm{ft}$ & 12.5 & $\mathrm{~J}$ \\
\hline GR1C9501LD & 8 & $0-0.5 \mathrm{ft}$ & 12 & $\mathrm{~J}$ \\
\hline GR1C9601LD & 9 & $0-0.5 \mathrm{ft}$ & 11.5 & $\mathrm{~J}$ \\
\hline GR1C9701LD & 10 & $0-0.5 \mathrm{ft}$ & 15.4 & $\mathrm{~J}$ \\
\hline GR1C9801LD & 11 & $0-0.5 \mathrm{ft}$ & 15.9 & $\mathrm{~J}$ \\
\hline GR1C9901LD & 12 & $0-0.5 \mathrm{ft}$ & 12.2 & $\mathrm{~J}$ \\
\hline GR1D0101LD & 13 & $0-0.5 \mathrm{ft}$ & 11.7 & $\mathrm{~J}$ \\
\hline GR1D0102LD & 13 & $0-0.5 \mathrm{ft}$ & 12.2 & $\mathrm{~J}$ \\
\hline GR1D0201LD & 14 & 5 in. & 13.9 & $\mathrm{~J}$ \\
\hline GR1D0301LD & 15 & 4 in. & 13.7 & $\mathrm{~J}$ \\
\hline GR1D0401LD & 16 & $4 \mathrm{in.}$ & 11.7 & $\mathrm{~J}$ \\
\hline GR1D0501LD & 17 & 3 in. & 10.8 & $\mathrm{~J}$ \\
\hline GR1D0601LD & 18 & $0-0.5 \mathrm{ft}$ & 20 & $\mathrm{~J}$ \\
\hline GR1D0701LD & 19 & $0-0.5 \mathrm{ft}$ & 15.9 & $\mathrm{~J}$ \\
\hline GR1D0801LD & 20 & $0-0.5 \mathrm{ft}$ & 15.7 & $\mathrm{~J}$ \\
\hline GR1D0901LD & 21 & $0-0.5 \mathrm{ft}$ & 130 & $\mathrm{~J}$ \\
\hline GR1D1001LD & 22 & $0-0.5 \mathrm{ft}$ & 14.9 & $\mathrm{~J}$ \\
\hline GR1D1101LD & 23 & 3 in. & 12.3 & $\mathrm{~J}$ \\
\hline GR1D1201LD & 24 & \multicolumn{3}{|c|}{ No Sample - On Basalt } \\
\hline GR1D1301LD & 25 & $0-0.5 \mathrm{ft}$ & 11.1 & $\mathrm{~J}$ \\
\hline GR1D1401LD & 26 & $0-0.5 \mathrm{ft}$ & 12.3 & $\mathrm{~J}$ \\
\hline
\end{tabular}


Table B-1. (continued).

\begin{tabular}{|c|c|c|c|c|}
\hline Sample ID \# & Location & Depth & $\begin{array}{c}\text { Lead Conc. } \\
(\mathrm{mg} / \mathrm{kg})\end{array}$ & Q \\
\hline GR1D1501LD & 27 & \multicolumn{3}{|c|}{ No Sample - On Basalt } \\
\hline GR1D1601LD & 28 & $0-0.5 \mathrm{ft}$ & 12.1 & $\mathrm{~J}$ \\
\hline GR1D1701LD & 29 & $0-0.5 \mathrm{ft}$ & 11.7 & $\mathrm{~J}$ \\
\hline GR1D1801LD & 30 & 4 in. & 8.6 & $\mathrm{~J}$ \\
\hline GR1D1901LD & 31 & $0-0.5 \mathrm{ft}$ & 12.5 & $\mathrm{~J}$ \\
\hline GR1D2001LD & 32 & $0-0.5 \mathrm{ft}$ & 11.8 & $\mathrm{~J}$ \\
\hline GR1D2101LD & 33 & $0-0.5 \mathrm{ft}$ & 11.2 & $\mathrm{~J}$ \\
\hline GR1D2102LD & 33 & $0-0.5 \mathrm{ft}$ & 12.5 & $\mathrm{~J}$ \\
\hline GR1D2201LD & 34 & 4 in. & 10.9 & $\mathrm{~J}$ \\
\hline GR1D2301LD & 35 & 5 in. & 14.1 & $\mathrm{~J}$ \\
\hline GR1D2401LD & 36 & \multicolumn{3}{|c|}{ No Sample - On Basalt } \\
\hline GR1D2501LD & 37 & \multicolumn{3}{|c|}{ No Sample - On Basalt } \\
\hline GR1D2601LD & 38 & \multicolumn{3}{|c|}{ No Sample - On Basalt } \\
\hline GR1D2701LD & 39 & \multicolumn{3}{|c|}{ No Sample - On Basalt } \\
\hline GR1D2801LD & 40 & \multicolumn{3}{|c|}{ No Sample - On Basalt } \\
\hline GR1D2901LD & 41 & 1 in. & 10.1 & $\mathrm{~J}$ \\
\hline GR1D3001LD & 42 & 2 in. & 18 & $\mathrm{~J}$ \\
\hline GR1D3101LD & 43 & $0-0.5 \mathrm{ft}$ & 13.2 & $\mathrm{~J}$ \\
\hline GR1D3201LD & 44 & $0-0.5 \mathrm{ft}$ & 11.7 & $\mathrm{~J}$ \\
\hline GR1D3301LD & 45 & 3 in. & 13.4 & $\mathrm{~J}$ \\
\hline GR1D3401LD & 46 & $2 \mathrm{in.}$ & 8.6 & \\
\hline GR1D3501LD & 47 & 4 in. & 7.5 & \\
\hline GR1D3601LD & 48 & $0-0.5 \mathrm{ft}$ & 11.2 & \\
\hline GR1D3701LD & 49 & \multicolumn{3}{|c|}{ No Sample - On Basalt } \\
\hline GR1D3801LD & 50 & \multicolumn{3}{|c|}{ No Sample - On Basalt } \\
\hline GR1D3901LD & 51 & 3 in. & 14.4 & \\
\hline GR1D4001LD & 52 & 4 in. & 7.6 & \\
\hline GR1D4101LD & 53 & $0-0.5 \mathrm{ft}$ & 12.9 & \\
\hline GR1D4102LD & 53 & $0-0.5 \mathrm{ft}$ & 11.3 & \\
\hline GR1D4201LD & 54 & $0-0.5 \mathrm{ft}$ & 12.9 & \\
\hline GR1D4301LD & 55 & $0-0.5 \mathrm{ft}$ & 12.8 & \\
\hline GR1D4401LD & 56 & $0-0.5 \mathrm{ft}$ & 12.2 & \\
\hline GR1D4501LD & 57 & $0-0.5 \mathrm{ft}$ & 10.9 & \\
\hline GR1D4601LD & 58 & $0-0.5 \mathrm{ft}$ & 9.9 & \\
\hline GR1D4701LD & 59 & $0-0.5 \mathrm{ft}$ & 7.5 & \\
\hline GR1D4801LD & 60 & $0-0.5 \mathrm{ft}$ & 9.4 & \\
\hline GR1D4901LD & 61 & $0-0.5 \mathrm{ft}$ & 15.1 & \\
\hline GR1D5001LD & 62 & 5 in. & 21.3 & \\
\hline GR1D5101LD & 63 & \multicolumn{3}{|c|}{ No Sample - On Basalt } \\
\hline GR1D5201LD & 64 & 4 in. & 8.2 & \\
\hline GR1D5301LD & 65 & \multicolumn{3}{|c|}{ No Sample - On Basalt } \\
\hline
\end{tabular}


Table B-1. (continued).

\begin{tabular}{|c|c|c|c|c|}
\hline Sample ID \# & Location & Depth & $\begin{array}{l}\text { Lead Conc. } \\
(\mathrm{mg} / \mathrm{kg})\end{array}$ & Q \\
\hline GR1D5401LD & 66 & $0-0.5 \mathrm{ft}$ & 14.6 & \\
\hline GR1D5501LD & 67 & $0-0.5 \mathrm{ft}$ & 6.6 & \\
\hline GR1D5601LD & 68 & 3 in. & 10 & \\
\hline GR1D5701LD & 69 & 4 in. & 12.5 & $\mathrm{~J}$ \\
\hline GR1D5801LD & 70 & 5 in. & 16.5 & $\mathrm{~J}$ \\
\hline GR1D5901LD & 71 & 3 in. & 11.6 & $\mathrm{~J}$ \\
\hline GR1D6001LD & 72 & \multicolumn{3}{|c|}{ No Sample - On Basalt } \\
\hline GR1D6101LD & 73 & 3 in. & 9.1 & $\mathrm{~J}$ \\
\hline GR1D6102LD & 73 & 3 in. & 9.3 & $\mathrm{~J}$ \\
\hline GR1D6201LD & 74 & $1 \mathrm{in.}$ & 14.4 & $\mathrm{~J}$ \\
\hline GR1D6301LD & 75 & $2 \mathrm{in.}$ & 8.5 & $\mathrm{~J}$ \\
\hline GR1D6401LD & 76 & $5 \mathrm{in.}$ & 11 & $\mathrm{~J}$ \\
\hline GR1D6501LD & 77 & $0-0.5 \mathrm{ft}$ & 10.6 & $\mathrm{~J}$ \\
\hline GR1D6601LD & 78 & $0-0.5 \mathrm{ft}$ & 10.1 & $\mathrm{~J}$ \\
\hline GR1D6701LD & 79 & $0-0.5 \mathrm{ft}$ & 11.4 & $\mathrm{~J}$ \\
\hline GR1D6801LD & 80 & $0-0.5 \mathrm{ft}$ & 11 & $\mathrm{~J}$ \\
\hline GR1D6901LD & 81 & $0-0.5 \mathrm{ft}$ & 16 & $\mathrm{~J}$ \\
\hline GR1D7001LD & 82 & $0-0.5 \mathrm{ft}$ & 14.2 & $\mathrm{~J}$ \\
\hline GR1D7101LD & 83 & 2 in. & 7.1 & $\mathrm{~J}$ \\
\hline GR1D7201LD & 84 & \multicolumn{3}{|c|}{ No Sample - On Basalt } \\
\hline GR1D7301LD & 85 & \multicolumn{3}{|c|}{ No Sample - On Basalt } \\
\hline GR1D7401LD & 86 & \multicolumn{3}{|c|}{ No Sample - On Basalt } \\
\hline GR1D7501LD & 87 & \multicolumn{3}{|c|}{ No Sample - On Basalt } \\
\hline GR1D7601LD & 88 & \multicolumn{3}{|c|}{ No Sample - On Basalt } \\
\hline GR1D7701LD & 89 & $0-0.5 \mathrm{ft}$ & 119 & $\mathrm{~J}$ \\
\hline GR1D7801LD & 90 & $0-0.5 \mathrm{ft}$ & 14 & $\mathrm{~J}$ \\
\hline GR1D7901LD & 91 & $0-0.5 \mathrm{ft}$ & 17.2 & $\mathrm{~J}$ \\
\hline GR1D8001LD & 92 & $0-0.5 \mathrm{ft}$ & 12.1 & $\mathrm{~J}$ \\
\hline GR1D8101LD & 93 & $0-0.5 \mathrm{ft}$ & 40.3 & $\mathrm{~J}$ \\
\hline GR1D8102LD & 93 & $0-0.5 \mathrm{ft}$ & 23.4 & \\
\hline GR1D8201LD & 94 & \multicolumn{3}{|c|}{ No Sample - On Basalt } \\
\hline GR1D8301LD & 95 & 3 in. & 16.5 & \\
\hline GR1D8401LD & 96 & $0-0.5 \mathrm{ft}$ & 11.5 & \\
\hline GR1D8501LD & 97 & 2 in. & 10 & \\
\hline GR1D8601LD & 98 & 3 in. & 11.9 & \\
\hline GR1D8701LD & 99 & \multicolumn{3}{|c|}{ No Sample - On Basalt } \\
\hline GR1D8801LD & 100 & 2 in. & 18.2 & \\
\hline GR1D8901LD & 101 & $4 \mathrm{in.}$ & 14.6 & \\
\hline GR1D9001LD & 102 & $0-0.5 \mathrm{ft}$ & 15.8 & \\
\hline GR1D9101LD & 103 & $0-0.5 \mathrm{ft}$ & 10.7 & \\
\hline GR1D9201LD & 104 & $0-0.5 \mathrm{ft}$ & 11.8 & \\
\hline
\end{tabular}


Table B-1. (continued).

\begin{tabular}{lccc}
\hline Sample ID \# & Location & Depth & $\begin{array}{c}\text { Lead Conc. } \\
(\mathrm{mg} / \mathrm{kg})\end{array}$ \\
\hline GR1D9301LD & 105 & $4 \mathrm{in.}$ & 11.1 \\
GR1D9401LD & 106 & $0-0.5 \mathrm{ft}$ & 11.4 \\
GR1D9501LD & 107 & $0-0.5 \mathrm{ft}$ & 16.2 \\
GR1D9601LD & 108 & $0-0.5 \mathrm{ft}$ & 12.8 \\
GR1D9701LD & 109 & $0-0.5 \mathrm{ft}$ & 13.1 \\
GR1D9801LD & 110 & $0-0.5 \mathrm{ft}$ & 13.2 \\
GR1D9901LD & 111 & $0-0.5 \mathrm{ft}$ & 13 \\
GR1E0101LD & 112 & $0-0.5 \mathrm{ft}$ & 11.1 \\
GR1E0102LD & 112 & $0-0.5 \mathrm{ft}$ & 11.2 \\
GR1E0201LD & 113 & $0-0.5 \mathrm{ft}$ & 9.9 \\
GR1E0301LD & 114 & $0-0.5 \mathrm{ft}$ & 15.1 \\
GR1E0401LD & 115 & $0-0.5 \mathrm{ft}$ & 12.6 \\
GR1E0501LD & 116 & $0-0.5 \mathrm{ft}$ & 13.2 \\
GR1E0601LD & 117 & $0-0.5 \mathrm{ft}$ & 11.4 \\
GR1E0701LD & 118 & $0-0.5 \mathrm{ft}$ & 11.6 \\
GR1E0801LD & 119 & $0-0.5 \mathrm{ft}$ & 9.3 \\
GR1E0901LD & 120 & $0-0.5 \mathrm{ft}$ & 8.8 \\
GR1E1001LD & 121 & $0-0.5 \mathrm{ft}$ & 9.2 \\
GR1E1101LD & 122 & $0-0.5 \mathrm{ft}$ & 9.2 \\
GR1E1201LD & 123 & $0-0.5 \mathrm{ft}$ & 11.8 \\
GR1E1301LD & 124 & $0-0.5 \mathrm{ft}$ & 12.2 \\
GR1E1401LD & 125 & $0-0.5 \mathrm{ft}$ & 12.9 \\
GR1E1501LD & 126 & $0-0.5 \mathrm{ft}$ & 12.4 \\
\hline
\end{tabular}

Table B-2. Sieve fraction data.

\begin{tabular}{|c|c|c|c|c|c|c|c|c|c|}
\hline \multirow[b]{2}{*}{ Fraction } & \multicolumn{2}{|c|}{ Totals $(\mathrm{mg} / \mathrm{kg})$} & \multicolumn{2}{|c|}{ TCLP $(\mu \mathrm{g} / \mathrm{L})$} & \multirow{2}{*}{$\begin{array}{l}\text { Wt. Ret. } \\
\text { (g) }\end{array}$} & \multirow{2}{*}{$\begin{array}{l}\text { Wt. Pass } \\
(\mathrm{g})\end{array}$} & \multirow{2}{*}{$\begin{array}{c}\% \\
\text { Pass }\end{array}$} & \multirow{2}{*}{$\begin{array}{c}\% \\
\text { Ret }\end{array}$} & \multirow{2}{*}{$\begin{array}{l}\text { Fragments } \\
\text { (g) }\end{array}$} \\
\hline & Sample & Duplicate & Sample & Duplicate & & & & & \\
\hline$>3 / 4$ & 25.1 & 136 & 347 & 451 & $2,263.07$ & $83,968.47$ & 97.4 & 2.6 & \\
\hline $3 / 4->3 / 8$ & 2,030 & 50.5 & 1,070 & 2,560 & $5,317.84$ & $78,650.63$ & 91.2 & 6.2 & $8,059.75$ \\
\hline $\begin{array}{l}3 / 8->\text { No. } 4 \\
(4.75 \mathrm{~mm})\end{array}$ & 4,310 & 5,440 & 107,000 & 73,100 & $5,171.18$ & 73479.45 & 85.2 & 6 & $1,069.69$ \\
\hline $\begin{array}{l}\text { No. } 4(4.75 \mathrm{~mm}) \\
->\text { No. } 10 \\
(2.00 \mathrm{~mm})\end{array}$ & 159 & 257,000 & 380,000 & 558,000 & $4,278.18$ & $69,201.27$ & 80.3 & 4.9 & 21.67 \\
\hline $\begin{array}{l}\text { No. } 10(2.00 \mathrm{~mm}) \\
->\text { No. } 50 \\
(300 \mu \mathrm{m})\end{array}$ & 867 & 3,130 & 378,000 & 208,000 & $27,878.13$ & $41,323.14$ & 47.9 & 32.4 & \\
\hline $\begin{array}{l}\text { No. } 50(300 \mu \mathrm{m}) \\
->\text { No. } 200 \\
(75 \mu \mathrm{m})\end{array}$ & 1,410 & 1,350 & 20,200 & 7,820 & $38,952.98$ & $2,370.16$ & 2.7 & 45.2 & \\
\hline $\begin{array}{l}<\text { No. } 200 \\
(75 \mu \mathrm{m})(\mathrm{Pan})\end{array}$ & 4,780 & 4,780 & 31,600 & 29,600 & $2,370.16$ & 0 & 0 & 2.7 & \\
\hline
\end{tabular}


Table B-3. EOCR Pond remediation support analytical results.

\begin{tabular}{cccccc}
\hline Grid \# & Sample ID & $\begin{array}{c}\text { Lab Data } \\
(\mathrm{mg} / \mathrm{kg})\end{array}$ & Q & V & $\begin{array}{c}\text { XRF Data } \\
(\mathrm{mg} / \mathrm{kg})\end{array}$ \\
\hline EOCR \#1 & GR1F0101LD & 6.6 & & $<28.9$ \\
EOCR \#2 & GR1F0201LD & 15.17 & & $<32.7$ \\
EOCR \#2 & GR1F0202LD & 18.43 & & N/A \\
\hline
\end{tabular}

Table B-4. STF-02 Gun Range berm remediation support analytical results.

\begin{tabular}{|c|c|c|c|c|c|c|c|}
\hline \multirow[b]{2}{*}{ Grid \# } & \multirow[b]{2}{*}{ Sample ID } & \multirow[b]{2}{*}{ Lab Data } & \multirow[b]{2}{*}{ Q } & \multirow[b]{2}{*}{$\mathrm{V}$} & \multicolumn{3}{|c|}{ XRF Data } \\
\hline & & & & & Run \#1 & Run \#2 & Run \#3 \\
\hline B47 & GR1F0301LD & 17.84 & & & 52.6 & $<36.9$ & $<36.5$ \\
\hline B50 & GR1F0401LD & 9.37 & & & 47.1 & $<30.3$ & $<40.5$ \\
\hline B95 & GR1F0501LD & 10.27 & & & $<37.5$ & $<34.5$ & $<35.9$ \\
\hline B51 & GR1F0601LD & 8.16 & & & $<30.5$ & $<38.8$ & $<35.4$ \\
\hline B94 & GR1F0701LD & 10.38 & & & $<47.7$ & $<33.3$ & $<36.8$ \\
\hline B46 & GR1F0801LD & 8.86 & & & $<33.1$ & 41.7 & $<32.7$ \\
\hline B45 & GR1F0901LD & 9.95 & & & 37.8 & $<39.0$ & $<36.2$ \\
\hline B44 & GR1F1001LD & 52.56 & & & 176.8 & 188.2 & 178.8 \\
\hline B41 & GR1F1101LD & 11.69 & & & $<37.4$ & $<35.9$ & $<37.6$ \\
\hline B42 & GR1F1201LD & 31.88 & & & 99.8 & 45.4 & 67.8 \\
\hline B43 & GR1F1301LD & 16.64 & & & $<39.4$ & $<39.0$ & $<40.2$ \\
\hline B55 & GR1F1401LD & 11.68 & & & $<32.1$ & $<33.4$ & $<31.9$ \\
\hline B56 & GR1F1501LD & 45.41 & & & 52.4 & 76.8 & 67.7 \\
\hline B54 & GR1F1601LD & 9.79 & & & $<33.6$ & 95.7 & $<31.6$ \\
\hline B53 & GR1F1701LD & 10.5 & & & $<36.0$ & $<38.1$ & $<38.8$ \\
\hline B36 & GR1F1801LD & 11.59 & & & $<34.4$ & $<37.4$ & $<35.0$ \\
\hline B37 & GR1F1901LD & 11.64 & & & $<38.4$ & $<38.6$ & $<33.7$ \\
\hline B40 & GR1F2001LD & 20.82 & & & $<40.8$ & $<36.3$ & $<36.3$ \\
\hline B52 & GR1F2101LD & 8.88 & & & $<36.2$ & $<31.8$ & $<33.4$ \\
\hline B39 & GR1F2201LD & 27.49 & $\mathrm{~N}$ & $\mathrm{R}$ & 41.9 & 40.2 & $<36.3$ \\
\hline B39 & GR1F2202LD & 23.01 & $\mathrm{~N}$ & $\mathrm{R}$ & N/A & N/A & N/A \\
\hline B29 & GR1F2301LD & 10.68 & $\mathrm{~N}$ & $\mathrm{R}$ & $<38.5$ & $<34.0$ & $<35.9$ \\
\hline B38 & GR1F2401LD & 10.39 & $\mathrm{~N}$ & $\mathrm{R}$ & 48.3 & $<38.0$ & $<38.0$ \\
\hline $\mathrm{C} 16$ & GR1F2501LD & 9.55 & $\mathrm{~N}$ & $\mathrm{R}$ & $<33.9$ & $<30.3$ & $<31.2$ \\
\hline $\mathrm{C} 15$ & GR1F2601LD & 8.42 & $\mathrm{~N}$ & $\mathrm{R}$ & 50.9 & $<38.9$ & $<32.2$ \\
\hline B69 & GR1F2701LD & 17.67 & $\mathrm{~N}$ & $\mathrm{R}$ & $<36.6$ & $<37.2$ & $<33.3$ \\
\hline B68 & GR1F2801LD & 8.98 & $\mathrm{~N}$ & $\mathrm{R}$ & $<31.4$ & $<45.0$ & $<27.0$ \\
\hline B75 & GR1F2901LD & 8.14 & $\mathrm{~N}$ & $\mathrm{R}$ & $<33.8$ & $<39.9$ & 38.4 \\
\hline B64 & GR1F3001LD & 16.53 & $\mathrm{~N}$ & $\mathrm{R}$ & $<35.7$ & $<34.2$ & $<32.8$ \\
\hline B70 & GR1F3101LD & 10.11 & $\mathrm{~N}$ & $\mathrm{R}$ & 48.6 & $<36.3$ & $<31.9$ \\
\hline B26 & GR1F3201LD & 24.48 & $\mathrm{~N}$ & $\mathrm{R}$ & $<33.5$ & $<28.9$ & 36.6 \\
\hline $\mathrm{C} 18$ & GR1F3301LD & 8.71 & $\mathrm{~N}$ & $\mathrm{R}$ & 44.4 & $<35.9$ & $<34.3$ \\
\hline $\mathrm{C} 46$ & GR1F3401LD & 7.72 & $\mathrm{~N}$ & $\mathrm{R}$ & $<37.6$ & $<32.7$ & $<37.6$ \\
\hline C14 & GR1F3501LD & 8.68 & $\mathrm{~N}$ & $\mathrm{R}$ & $<51.4$ & 142.3 & $<33.2$ \\
\hline $\mathrm{C} 17$ & GR1F3601LD & 8.54 & $\mathrm{~N}$ & $\mathrm{R}$ & $<30.1$ & $<34.4$ & $<42.1$ \\
\hline
\end{tabular}


Table B-4. (continued).

\begin{tabular}{|c|c|c|c|c|c|c|c|}
\hline \multirow[b]{2}{*}{ Grid \# } & \multirow[b]{2}{*}{ Sample ID } & \multirow[b]{2}{*}{ Lab Data } & \multirow[b]{2}{*}{ Q } & \multirow[b]{2}{*}{$\mathrm{V}$} & \multicolumn{3}{|c|}{ XRF Data } \\
\hline & & & & & Run \#1 & Run \#2 & Run \#3 \\
\hline B74 & GR1F3701LD & 20.06 & $\mathrm{~N}$ & $\mathrm{R}$ & $<40.2$ & 42.1 & 50.9 \\
\hline $\mathrm{C} 47$ & GR1F3801LD & 8.84 & $\mathrm{~N}$ & $\mathrm{R}$ & $<37.2$ & $<36.1$ & $<32.4$ \\
\hline B35 & GR1F3901LD & 10.08 & $\mathrm{~N}$ & $\mathrm{R}$ & $<34.5$ & $<31.6$ & $<33.6$ \\
\hline B28 & GR1F4001LD & 11.76 & & & $<35.2$ & $<38.0$ & $<35.3$ \\
\hline B65 & GR1F4101LD & 10.24 & & & $<28.3$ & $<13.8$ & $<38.5$ \\
\hline B31 & GR1F4201LD & 8.88 & & & $<40.9$ & $<38.1$ & $<34.1$ \\
\hline B31 & GR1F4202LD & 9.80 & & & N/A & N/A & N/A \\
\hline B60 & GR1F4301LD & 8.85 & & & $<38.7$ & $<30.8$ & $<44.2$ \\
\hline $\mathrm{C} 48$ & GR1F4401LD & 14.55 & & & $<36.4$ & $<34.6$ & $<36.9$ \\
\hline C19 & GR1F4501LD & 7.70 & & & $<31.6$ & $<35.9$ & $<35.1$ \\
\hline B76 & GR1F4601LD & 9.52 & & & $<33.4$ & $<36.1$ & $<37.8$ \\
\hline B62 & GR1F4701LD & 21.56 & & & $<33.4$ & $<34.0$ & $<43.0$ \\
\hline B67 & GR1F4801LD & 9.49 & & & $<37.7$ & 47.3 & $<39.7$ \\
\hline B27 & GR1F4901LD & 8.01 & & & $<37.0$ & $<35.2$ & 91.0 \\
\hline B66 & GR1F5001LD & 8.87 & & & $<30.4$ & $<38.2$ & $<35.7$ \\
\hline B61 & GR1F5101LD & 14.93 & & & $<35.9$ & $<31.3$ & $<35.8$ \\
\hline B63 & GR1F5201LD & 9.75 & & & $<27.0$ & $<28.8$ & 36.3 \\
\hline B63 & GR1F5202LD & 11.58 & & & N/A & N/A & N/A \\
\hline B59 & GR1F5301LD & 65.09 & & & 49.3 & 89.0 & 79.7 \\
\hline B57 & GR1F5401LD & 10.45 & & & $<35.6$ & $<35.0$ & $<31.4$ \\
\hline B32 & GR1F5501LD & 9.25 & & & $<36.5$ & $<32.0$ & $<39.6$ \\
\hline B30 & GR1F5601LD & 8.68 & & & $<35.1$ & $<29.5$ & $<32.6$ \\
\hline B58 & GR1F5701LD & 137 & & & $<32.9$ & $<37.4$ & $<40.0$ \\
\hline B34 & GR1F5801LD & 15.73 & & & $<39.2$ & $<36.2$ & $<37.9$ \\
\hline B33 & GR1F5901LD & 11.59 & & & $<43.2$ & $<33.8$ & $<36.2$ \\
\hline B77 & GR1F6001LD & 26.10 & & & $<35.9$ & $<43.1$ & 48.9 \\
\hline B78 & GR1F6101LD & 13.92 & & & 38.6 & $<39.3$ & 45.9 \\
\hline B85 & GR1F6201LD & 52.27 & & & 52.3 & 47.9 & 89.0 \\
\hline F133 & GR1F6301LD & 95.15 & & & 106.1 & 107.4 & 99.5 \\
\hline B82 & GR1F6401LD & 125.84 & & & 97.8 & 112.5 & 134.9 \\
\hline B84 & GR1F6501LD & 100.99 & & & 108.6 & 135.0 & 77.6 \\
\hline B83 & GR1F6601LD & 94.93 & & & 77.2 & 50.9 & 149.2 \\
\hline B90 & GR1F6701LD & 303.33 & & & 252.3 & 245.3 & 239.7 \\
\hline F132 & GR1F6801LD & 60.33 & & & 98.1 & 58.3 & 71.0 \\
\hline F103 & GR1F6901LD & 20.19 & & $\mathrm{U}$ & 39.5 & $<37.1$ & $<34.4$ \\
\hline F76 & GR1F7001LD & 36.68 & & $\mathrm{U}$ & 40.5 & 211.5 & $<39.0$ \\
\hline B86 & GR1F7101LD & 99.64 & & & 55.1 & 63.4 & $<35.9$ \\
\hline B87 & GR1F7201LD & 621.38 & & & 276.2 & 237.2 & 781.8 \\
\hline B87 & GR1F7202LD & 779.75 & & & N/A & N/A & N/A \\
\hline B79 & GR1F7301LD & 27.11 & & $\mathrm{U}$ & 59.8 & 44.3 & 52.6 \\
\hline B80 & GR1F7401LD & 11.62 & & $\mathrm{U}$ & $<38.4$ & 42.4 & $<33.7$ \\
\hline B81 & GR1F7501LD & 225.38 & & & 93.0 & 112.2 & 47.6 \\
\hline F17 & GR1F7601LD & 43.94 & & $\mathrm{U}$ & 42.5 & 55.9 & 59.2 \\
\hline F104 & GR1F7701LD & 47.86 & & $\mathrm{U}$ & 237.4 & 51.9 & 58.1 \\
\hline F138 & GR1F7801LD & 9.02 & & $\mathrm{U}$ & $<39.9$ & $<36.3$ & $<37.7$ \\
\hline
\end{tabular}


Table B-4. (continued).

\begin{tabular}{lcrrrrrr}
\hline & & & & \multicolumn{3}{c}{ XRF Data } \\
\cline { 5 - 7 } Grid \# & Sample ID & Lab Data & Q & V & Run \#1 & Run \#2 & Run \#3 \\
F18 & GR1F7901LD & 24.94 & & $\mathrm{U}$ & 39.6 & $<38.0$ & 48.3 \\
E6 & GR1F8001LD & 6.48 & & $\mathrm{U}$ & $<35.7$ & $<31.5$ & $<26.5$ \\
B89 & GR1F8101LD & 248.53 & & 135.8 & 122.0 & 117.7 \\
B91 & GR1F8201LD & 141.26 & & 126.5 & 112.2 & 158.2 \\
B93 & GR1F8301LD & 21.72 & & $\mathrm{U}$ & $<35.1$ & 42.3 & $<37.6$ \\
B92 & GR1F8401LD & 183.61 & & 61.7 & 61.2 & 54.7 \\
A51 & GR1F8501LD & 11.05 & & $\mathrm{U}$ & $<35.6$ & $<38.5$ & 41.0 \\
B88 & GR1F8601LD & 84.95 & & $<35.6$ & 89.3 & $<39.7$ \\
F137 & GR1F8701LD & 15.61 & & $<36.2$ & $<29.5$ & $<33.2$ \\
F48 & GR1F8801LD & 6.85 & & $<30.7$ & $<30.9$ & $<37.1$ \\
B90-2 & GR1F8901LD & 7.79 & & $<33.1$ & $<35.8$ & $<32.9$ \\
B87 & GR1F9001LD & 6.22 & & $<33.3$ & $<33.9$ & $<26.9$ \\
\hline
\end{tabular}

Table B-5. Remediation support analytical results for the area between the berms.

\begin{tabular}{|c|c|c|c|c|c|c|}
\hline \multirow[b]{2}{*}{ Grid \# } & \multirow[b]{2}{*}{ Sample ID } & \multirow[b]{2}{*}{ Lab Data } & \multirow[b]{2}{*}{ Q } & \multicolumn{3}{|c|}{ XRF Data } \\
\hline & & & & Run \#1 & Run \#2 & Run \#3 \\
\hline F30 & GR1F9101LD & 6.73 & & $<35.8$ & $<32.8$ & $<36.5$ \\
\hline F156 & GR1F9201LD & 12.62 & & $<34.9$ & $<29.6$ & $<36.3$ \\
\hline F156 & GR1F9202LD & 12.76 & & N/A & N/A & N/A \\
\hline F182 & GR1F9301LD & 13.78 & & 41.0 & $<40.2$ & $<37.0$ \\
\hline F185 & GR1F9401LD & 16.34 & & 73.0 & 50.1 & 34.0 \\
\hline F194 & GR1F9501LD & 15.17 & & $<32.0$ & $<34.2$ & $<36.0$ \\
\hline F195 & GR1F9601LD & 16.36 & & $<32.4$ & $<38.5$ & $<38.7$ \\
\hline F184 & GR1F9701LD & 17.96 & & $<35.0$ & $<34.3$ & 40.6 \\
\hline F183 & GR1F9801LD & 17.40 & & $<30.9$ & 43.4 & $<30.0$ \\
\hline F172 & GR1F9901LD & 15.11 & & 44.4 & $<34.8$ & $<37.2$ \\
\hline D25 & GR1G0101LD & 14.60 & & $<34.3$ & $<31.6$ & $<34.4$ \\
\hline D24 & GR1G0201LD & 14.75 & & $<28.7$ & $<36.3$ & $<32.9$ \\
\hline D24 & GR1G0202LD & 19.92 & & N/A & N/A & N/A \\
\hline D23 & GR1G0301LD & 12.25 & & $<34.1$ & $<30.3$ & $<32.6$ \\
\hline D20 & GR1G0401LD & 12.44 & & $<30.6$ & $<34.3$ & $<26.5$ \\
\hline D19 & GR1G0501LD & 23.18 & & 54.0 & $<27.5$ & $<32.3$ \\
\hline D18 & GR1G0601LD & 13.61 & & $<33.1$ & $<32.2$ & $<35.5$ \\
\hline D11 & GR1G0701LD & 12.84 & & $<37.0$ & $<38.4$ & $<31.6$ \\
\hline D10 & GR1G0801LD & 20.28 & & 43.6 & $<35.8$ & 63.3 \\
\hline D9 & GR1G0901LD & 14.13 & & $<40.3$ & $<32.2$ & $<30.3$ \\
\hline D8 & GR1G1001LD & 21.03 & & $<31.3$ & 43.9 & 39.3 \\
\hline D21 & GR1G1101LD & 19.10 & & $<34.2$ & 41.4 & 54.0 \\
\hline D22 & GR1G1201LD & 12.73 & & $<31.6$ & $<33.5$ & $<34.7$ \\
\hline D7 & GR1G1301LD & 28.84 & & 65.1 & 46.7 & 45.4 \\
\hline D6 & GR1G1401LD & 14.48 & & $<31.3$ & $<28.9$ & 47.9 \\
\hline
\end{tabular}


Table B-5. (continued).

\begin{tabular}{|c|c|c|c|c|c|c|}
\hline \multirow[b]{2}{*}{ Grid \# } & \multirow[b]{2}{*}{ Sample ID } & \multirow[b]{2}{*}{ Lab Data } & \multirow[b]{2}{*}{$\mathrm{Q}$} & \multicolumn{3}{|c|}{ XRF Data } \\
\hline & & & & Run \#1 & Run \#2 & Run \#3 \\
\hline D5 & GR1G1501LD & 12.89 & & $<35.4$ & 34.2 & $<29.3$ \\
\hline $\mathrm{G} 28$ & GR1G1601LD & 17.87 & & $<29.5$ & $<38.4$ & $<34.4$ \\
\hline $\mathrm{G} 29$ & GR1G1701LD & 15.98 & & $<33.2$ & $<31.4$ & $<36.6$ \\
\hline G30 & GR1G1801LD & 19.63 & & $<34.5$ & $<38.1$ & $<31.4$ \\
\hline $\mathrm{G} 23$ & GR1G1901LD & 13.55 & & $<27.6$ & $<33.8$ & $<36.4$ \\
\hline G24 & GR1G2001LD & 13.20 & & $<34.3$ & $<31.2$ & $<33.8$ \\
\hline $\mathrm{G} 25$ & GR1G2101LD & 11.65 & & $<30.3$ & $<36.5$ & $<33.6$ \\
\hline E13 & GR1G2201LD & 16.45 & & $<35.7$ & $<35.9$ & $<33.7$ \\
\hline E13 & GR1G2202LD & 16.40 & & N/A & N/A & N/A \\
\hline D3 & GR1G2301LD & 25.46 & & 51.6 & $<30.7$ & $<33.5$ \\
\hline D13 & GR1G2401LD & 25.06 & & $<35.1$ & $<33.4$ & $<30.4$ \\
\hline D2 & GR1G2501LD & 44.50 & & $<35.8$ & 36.3 & $<37.8$ \\
\hline E12 & GR1G2601LD & 11.95 & & 51.6 & $<37.7$ & $<37.1$ \\
\hline E1 & GR1G2701LD & 16.22 & & 35.9 & 45.1 & $<33.3$ \\
\hline D1 & GR1G2801LD & 24.58 & & 40.6 & 61.0 & 47.7 \\
\hline D14 & GR1G2901LD & 36.86 & & $<37.1$ & $<31.8$ & 62.4 \\
\hline D15 & GR1G3001LD & 86.63 & & 57.1 & 61.6 & 150.4 \\
\hline F145 & GR1G3101LD & 15.49 & & $<32.9$ & $<31.6$ & $<34.2$ \\
\hline F144 & GR1G3201LD & 10.40 & & $<34.5$ & $<34.5$ & 39.9 \\
\hline F143 & GR1G3301LD & 11.76 & & $<34.7$ & $<34.9$ & $<36.4$ \\
\hline F142 & GR1G3401LD & 13.21 & & $<34.3$ & $<32.8$ & $<38.7$ \\
\hline F141 & GR1G3501LD & 33.78 & & 38.3 & $<36.3$ & $<28.9$ \\
\hline F130 & GR1G3601LD & 9.15 & & $<37.1$ & $<28.7$ & $<34.2$ \\
\hline F129 & GR1G3701LD & 13.92 & & $<30.0$ & $<32.6$ & $<34.0$ \\
\hline F128 & GR1G3801LD & 14.62 & & $<33.6$ & $<32.2$ & $<33.2$ \\
\hline F127 & GR1G3901LD & 14.13 & & $<31.3$ & $<32.0$ & $<29.3$ \\
\hline F126 & GR1G4001LD & 17.11 & & 57.7 & $<34.8$ & $<38.7$ \\
\hline F111 & GR1G4101LD & 15.07 & & $<31.7$ & $<37.7$ & $<30.7$ \\
\hline F110 & GR1G4201LD & 15.23 & & 63.0 & $<27.2$ & $<33.8$ \\
\hline F110 & GR1G4202LD & 13.82 & & N/A & N/A & N/A \\
\hline F109 & GR1G4301LD & 14.45 & & $<34.2$ & $<32.0$ & $<36.7$ \\
\hline F108 & GR1G4401LD & 15.04 & & $<33.8$ & $<29.8$ & $<36.3$ \\
\hline F107 & GR1G4501LD & 14.31 & & $<39.3$ & $<33.5$ & $<32.0$ \\
\hline F100 & GR1G4601LD & 63.61 & & 78.5 & 62.2 & 92.0 \\
\hline F99 & GR1G4701LD & 14.10 & & $<37.8$ & $<33.7$ & $<35.3$ \\
\hline F98 & GR1G4801LD & 12.84 & & $<34.6$ & $<29.4$ & $<36.0$ \\
\hline F97 & GR1G4901LD & 12.62 & & $<35.6$ & 36.9 & $<30.8$ \\
\hline F96 & GR1G5001LD & 7.63 & & $<30.4$ & 38.2 & $<33.8$ \\
\hline F83 & GR1G5101LD & 14.75 & & 40.2 & $<31.4$ & $<36.9$ \\
\hline F82 & GR1G5201LD & 10.11 & & $<34.2$ & $<32.2$ & $<30.1$ \\
\hline F81 & GR1G5301LD & 12.60 & & $<33.9$ & $<35.1$ & $<30.6$ \\
\hline F80 & GR1G5401LD & 13.16 & & $<30.1$ & $<30.7$ & $<39.0$ \\
\hline
\end{tabular}


Table B-5. (continued).

\begin{tabular}{|c|c|c|c|c|c|c|}
\hline \multirow[b]{2}{*}{ Grid \# } & \multirow[b]{2}{*}{ Sample ID } & \multirow[b]{2}{*}{ Lab Data } & \multirow[b]{2}{*}{$\mathrm{Q}$} & \multicolumn{3}{|c|}{ XRF Data } \\
\hline & & & & Run \#1 & Run \#2 & Run \#3 \\
\hline F79 & GR1G5501LD & 50.40 & & 51.8 & 54.6 & 48.0 \\
\hline F7 & GR1G5601LD & 13.61 & & $<34.9$ & $<33.8$ & $<36.6$ \\
\hline F8 & GR1G5701LD & 27.12 & & $<40.9$ & $<36.8$ & $<38.3$ \\
\hline F9 & GR1G5801LD & 12.93 & & $<40.2$ & $<36.2$ & $<35.1$ \\
\hline F10 & GR1G5901LD & 11.81 & & $<33.4$ & $<36.2$ & $<31.7$ \\
\hline F25 & GR1G6001LD & 34.54 & & 49.3 & $<36.9$ & $<35.2$ \\
\hline F26 & GR1G6101LD & 19.52 & & $<37.7$ & $<30.5$ & $<31.4$ \\
\hline F27 & GR1G6201LD & 25.89 & & 46.8 & 51.8 & $<35.4$ \\
\hline F27 & GR1G6202LD & 28.92 & & $\mathrm{~N} / \mathrm{A}$ & N/A & N/A \\
\hline F28 & GR1G6301LD & 14.94 & & $<30.4$ & 35.8 & 54.5 \\
\hline F40 & GR1G6401LD & 13.40 & & $<29.9$ & 37.3 & $<38.1$ \\
\hline F41 & GR1G6501LD & 15.33 & & 35.8 & $<32.2$ & $<35.0$ \\
\hline F42 & GR1G6601LD & 31.22 & & $<30.7$ & $<34.3$ & $<37.3$ \\
\hline F39 & GR1G6701LD & 21.64 & & $<29.5$ & 33.8 & $<39.2$ \\
\hline F53 & GR1G6801LD & 12.46 & & 40.2 & $<37.6$ & $<41.5$ \\
\hline F54 & GR1G6901LD & 15.83 & & $<35.2$ & $<28.7$ & $<34.4$ \\
\hline F55 & GR1G7001LD & 14.72 & & $<32.9$ & 41.1 & $<33.9$ \\
\hline F56 & GR1G7101LD & 15.19 & & $<32.9$ & $<31.5$ & 42.0 \\
\hline F72 & GR1G7201LD & 17.38 & & $<37.7$ & 43.8 & $<32.5$ \\
\hline F68 & GR1G7301LD & 45.07 & & $<38.7$ & 49.9 & 40.7 \\
\hline F69 & GR1G7401LD & 14.93 & & $<38.7$ & $<33.0$ & $<36.6$ \\
\hline F70 & GR1G7501LD & 35.33 & & 78.0 & 83.5 & $<34.7$ \\
\hline F83 & GR1G7601LD & 15.33 & & $<38.2$ & $<39.9$ & $<36.3$ \\
\hline F114 & GR1G7701LD & 217.66 & & 138.8 & 83.7 & 78.9 \\
\hline F163 & GR1G7801LD & 10.97 & & $<34.7$ & $<32.4$ & $<33.4$ \\
\hline F155 & GR1G7901LD & 12.32 & & $<37.5$ & $<36.4$ & 45.2 \\
\hline F150 & GR1G8001LD & 12.70 & & $<35.1$ & $<29.2$ & $<36.8$ \\
\hline F116 & GR1G8101LD & 45.22 & & 172.2 & 61.3 & 83.1 \\
\hline F121 & GR1G8201LD & 22.04 & & $<32.4$ & $<34.6$ & 47.9 \\
\hline F121 & GR1G8202LD & 35.46 & & N/A & N/A & N/A \\
\hline F91 & GR1G8301LD & 20.12 & & 92.7 & $<30.4$ & $<35.6$ \\
\hline F93 & GR1G8401LD & 21.25 & & $<38.7$ & $<34.1$ & $<40.5$ \\
\hline F87 & GR1G8501LD & 294.76 & & 187.6 & 170.7 & 222.3 \\
\hline F112 & GR1G8601LD & 13.75 & & $<43.7$ & $<35.9$ & $<32.5$ \\
\hline F86 & GR1G8701LD & 226.58 & & 81.1 & 160.1 & 58.8 \\
\hline F113 & GR1G8801LD & 100.95 & & 178.9 & 102.5 & 64.1 \\
\hline F85 & GR1G8901LD & 38.96 & & $<37.5$ & $<33.7$ & $<40.1$ \\
\hline F95 & GR1G9001LD & 28.15 & & 47.5 & $<39.2$ & $<33.0$ \\
\hline F94 & GR1G9101LD & 76.64 & & 49.9 & 103.4 & 53.3 \\
\hline F84 & GR1G9201LD & 173.23 & & 215.3 & 1,040 & 747.3 \\
\hline F60 & GR1G9301LD & 23.35 & & $<36.2$ & $<37.0$ & $<37.6$ \\
\hline F59 & GR1G9401LD & 13.82 & & $<36.3$ & $<30.1$ & $<30.5$ \\
\hline
\end{tabular}


Table B-5. (continued).

\begin{tabular}{|c|c|c|c|c|c|c|}
\hline \multirow[b]{2}{*}{ Grid \# } & \multirow[b]{2}{*}{ Sample ID } & \multirow[b]{2}{*}{ Lab Data } & \multirow[b]{2}{*}{$\mathrm{Q}$} & \multicolumn{3}{|c|}{ XRF Data } \\
\hline & & & & Run \#1 & Run \#2 & Run \#3 \\
\hline F35 & GR1G9501LD & 19.02 & & $<34.5$ & $<39.1$ & $<39.0$ \\
\hline F31 & GR1G9601LD & 11.17 & & $<26.7$ & $<32.0$ & $<37.0$ \\
\hline F64 & GR1G9701LD & 109.27 & & $<40.2$ & $<36.6$ & $<34.7$ \\
\hline F63 & GR1G9801LD & 15.22 & & $<39.9$ & $<32.9$ & $<34.2$ \\
\hline Z26 & GR1G9901LD & 11.12 & & $<32.1$ & $<35.4$ & $<34.1$ \\
\hline Z25 & GR1H0001LD & 13.10 & & $<36.8$ & $<27.9$ & $<29.9$ \\
\hline Z24 & GR1H0101LD & 25.14 & & $<33.9$ & $<32.9$ & 64.9 \\
\hline Z23 & GR1H0201LD & 12.85 & & $<29.3$ & 40.4 & $<34.3$ \\
\hline Z23 & GR1H0202LD & 13.82 & & N/A & $\mathrm{N} / \mathrm{A}$ & N/A \\
\hline Z22 & GR1H0301LD & 16.85 & & $<31.6$ & $<35.3$ & $<31.5$ \\
\hline $\mathrm{Z} 21$ & GR1H0401LD & 14.57 & & $<31.6$ & $<33.8$ & $<36.4$ \\
\hline $\mathrm{Z} 20$ & GR1H0501LD & 10.42 & & $<34.3$ & $<35.6$ & $<31.5$ \\
\hline Z19 & GR1H0601LD & 16.06 & & $<34.9$ & 42.6 & $<34.9$ \\
\hline Z18 & GR1H0701LD & 9.76 & & $<36.3$ & $<31.8$ & $<25.9$ \\
\hline Z17 & GR1H0801LD & 11.08 & & $<35.4$ & $<34.6$ & $<34.7$ \\
\hline Z16 & GR1H0901LD & 11.56 & & $<32.3$ & $<29.0$ & $<36.2$ \\
\hline Z15 & GR1H1001LD & 13.89 & & 40.1 & $<32.3$ & $<36.6$ \\
\hline Z14 & GR1H1101LD & 14.91 & & $<33.4$ & $<33.5$ & 44.1 \\
\hline Z13 & GR1H1201LD & 14.73 & & $<33.4$ & $<32.8$ & $<32.3$ \\
\hline $\mathrm{Z} 12$ & GR1H1301LD & 13.08 & & $<32.2$ & $<31.4$ & $<35.9$ \\
\hline Z11 & GR1H1401LD & 18.92 & & 39.0 & 43.0 & $<35.0$ \\
\hline Z10 & GR1H1501LD & 17.80 & & 38.1 & $<32.6$ & $<31.4$ \\
\hline Z9 & GR1H1601LD & 18.04 & & $<33.6$ & $<36.2$ & $<36.3$ \\
\hline Z8 & GR1H1701LD & 44.14 & & 52.8 & 51.0 & $<31.8$ \\
\hline $\mathrm{Z7}$ & GR1H1801LD & 15.54 & & $<35.2$ & $<33.1$ & 47.0 \\
\hline Z6 & GR1H1901LD & 15.27 & & $<36.8$ & $<29.3$ & $<38.7$ \\
\hline $\mathrm{Z5}$ & GR1H2001LD & 14.51 & & $<38.1$ & $<32.7$ & $<34.7$ \\
\hline $\mathrm{Z} 4$ & GR1H2101LD & 17.27 & & 51.2 & $<39.7$ & $<32.3$ \\
\hline $\mathrm{Z3}$ & GR1H2201LD & 12.32 & & 54.1 & $<37.3$ & 44.2 \\
\hline $\mathrm{Z3}$ & GR1H2202LD & 12.56 & & N/A & N/A & N/A \\
\hline $\mathrm{Z} 2$ & GR1H2301LD & 12.46 & & $<33.5$ & $<33.0$ & $<37.0$ \\
\hline F190 & GR1H2401LD & 12.74 & & $<33.4$ & $<37.0$ & $<36.3$ \\
\hline F189 & GR1H2501LD & 11.15 & & $<30.5$ & $<33.1$ & $<33.7$ \\
\hline F179 & GR1H2601LD & 13.39 & & $<34.4$ & $<32.9$ & $<38.8$ \\
\hline F178 & GR1H2701LD & 10.87 & & 41.1 & $<34.4$ & $<34.7$ \\
\hline F177 & GR1H2801LD & 14.28 & & $<33.6$ & $<33.7$ & $<35.4$ \\
\hline F176 & GR1H2901LD & 14.00 & & $<31.0$ & $<39.0$ & $<37.9$ \\
\hline F167 & GR1H3001LD & 15.14 & & $<30.4$ & $<37.7$ & $<32.4$ \\
\hline F166 & GR1H3101LD & 15.46 & & $<38.1$ & $<33.5$ & $<31.0$ \\
\hline F165 & GR1H3201LD & 25.41 & & $<38.9$ & $<32.3$ & $<32.4$ \\
\hline F164 & GR1H3301LD & 10.58 & & $<34.9$ & $<33.8$ & $<38.1$ \\
\hline F154 & GR1H3401LD & 11.92 & & $<48.0$ & $<34.3$ & $<37.5$ \\
\hline
\end{tabular}


Table B-5. (continued).

\begin{tabular}{|c|c|c|c|c|c|c|}
\hline \multirow[b]{2}{*}{ Grid \# } & \multirow[b]{2}{*}{ Sample ID } & \multirow[b]{2}{*}{ Lab Data } & \multirow[b]{2}{*}{ Q } & \multicolumn{3}{|c|}{ XRF Data } \\
\hline & & & & Run \#1 & Run \#2 & Run \#3 \\
\hline F153 & GR1H3501LD & 8.72 & & 41.2 & $<33.4$ & $<34.0$ \\
\hline F152 & GR1H3601LD & 11.77 & & 38.8 & 41.8 & $<31.0$ \\
\hline F151 & GR1H3701LD & 9.69 & & $<29.5$ & $<31.0$ & $<34.1$ \\
\hline F120 & GR1H3801LD & 11.20 & & $<33.5$ & $<32.6$ & $<35.0$ \\
\hline F119 & GR1H3901LD & 15.08 & & $<37.0$ & $<34.0$ & $<32.5$ \\
\hline F118 & GR1H4001LD & 13.69 & & 51.5 & $<35.5$ & $<38.8$ \\
\hline F117 & GR1H4101LD & 13.93 & & 46.6 & $<36.2$ & $<37.5$ \\
\hline F90 & GR1H4201LD & 13.87 & & $<32.6$ & 51.0 & $<31.7$ \\
\hline F90 & GR1H4202LD & 13.85 & & N/A & N/A & N/A \\
\hline F89 & GR1H4301LD & 15.73 & & $<29.8$ & $<32.1$ & $<33.5$ \\
\hline F62 & GR1H4401LD & 11.84 & & $<32.9$ & $<37.1$ & $<36.0$ \\
\hline F61 & GR1H4501LD & 14.20 & & 40.1 & $<31.0$ & $<32.3$ \\
\hline F84-2 & GR1H4601LD & 16.00 & & $<40.2$ & 47.3 & $<34.3$ \\
\hline F85-2 & GR1H4701LD & 43.41 & & $<37.3$ & $<36.9$ & $<34.5$ \\
\hline F86-2 & GR1H4801LD & 14.37 & & $<38.6$ & 56.4 & 67.1 \\
\hline F87-2 & $\mathrm{N} / \mathrm{A}$ & N/A & & 187.6 & 187.5 & 238.4 \\
\hline F87-3 & GR1H4901LD & 21.96 & & $<30.9$ & 45.2 & $<33.5$ \\
\hline F62 & GR1H5001LD & 14.44 & & $<34.0$ & $<33.3$ & $<34.5$ \\
\hline F90 & GR1H5101LD & 12.66 & & $<39.9$ & $<37.8$ & $<33.0$ \\
\hline F89 & GR1H5201LD & 14.21 & & $<36.6$ & $<29.0$ & $<33.4$ \\
\hline F88 & GR1H5301LD & 65.76 & & 60.0 & 94.4 & 92.8 \\
\hline Z08 & GR1H5401LD & 28.17 & & 49.9 & 38.4 & $<28.3$ \\
\hline Z07 & GR1H5501LD & 16.50 & & $<39.3$ & $<35.8$ & $<38.0$ \\
\hline Z06 & GR1H5601LD & 13.89 & & 33.9 & $<33.3$ & $<31.2$ \\
\hline Z05 & GR1H5701LD & 14.55 & & 37.8 & $<33.6$ & $<35.1$ \\
\hline Z04 & GR1H5801LD & 12.98 & & $<28.8$ & $<35.8$ & $<32.6$ \\
\hline
\end{tabular}


Appendix C

Remedial Action Prefinal Inspection Checklist 


$$
\text { C-2 }
$$




\section{Appendix A}

\section{Remedial Action Prefinal Inspection Checklist}

The prefinal inspection checklist in this appendix summarizes the results of the inspection of the STF-02 Gun Range conducted by the Agencies on January 10, 2007.

Table C-1. STF-02 Gun Range prefinal inspection checklist.

\begin{tabular}{|c|c|c|c|c|c|}
\hline \multirow{2}{*}{\multicolumn{2}{|c|}{ Inspection Item }} & \multirow[b]{2}{*}{$\begin{array}{l}\text { Verification } \\
\text { Information }\end{array}$} & \multicolumn{2}{|c|}{ Satisfactory } & \multirow[b]{2}{*}{ Comments } \\
\hline & & & Yes & No & \\
\hline 1. & $\begin{array}{l}\text { Verify that the three asphalt pads have been } \\
\text { removed from the STF-02 Gun Range. }\end{array}$ & $\begin{array}{l}\text { Visual observation } \\
\text { Photographic records }\end{array}$ & $\mathrm{X}$ & & $\begin{array}{l}\text { An inspection was completed on January } 10 \text {, } \\
\text { 2007. The photographic record is provided in } \\
\text { Appendix A of the remedial action report. }\end{array}$ \\
\hline 2. & $\begin{array}{l}\text { Verify that the perimeter fencing has been } \\
\text { removed from the STF-02 Gun Range. }\end{array}$ & $\begin{array}{l}\text { Visual observation } \\
\text { Photographic records }\end{array}$ & $\mathrm{X}$ & & $\begin{array}{l}\text { An inspection was completed on January } 10 \text {, } \\
\text { 2007. The photographic record is provided in } \\
\text { Appendix A of the remedial action report. }\end{array}$ \\
\hline 3. & $\begin{array}{l}\text { Verify that the shooting house has been removed } \\
\text { from the STF- } 02 \text { Gun Range. }\end{array}$ & $\begin{array}{l}\text { Visual observation } \\
\text { Photographic records }\end{array}$ & $X$ & & $\begin{array}{l}\text { An inspection was completed on January } 10 \text {, } \\
\text { 2007. The photographic record is provided in } \\
\text { Appendix A of the remedial action report. }\end{array}$ \\
\hline 4. & $\begin{array}{l}\text { Verify that the berms have been removed from the } \\
\text { STF-02 Gun Range. }\end{array}$ & $\begin{array}{l}\text { Visual observation } \\
\text { Photographic records }\end{array}$ & $\mathrm{X}$ & & $\begin{array}{l}\text { An inspection was completed on January } 10 \text {, } \\
\text { 2007. The photographic record is provided in } \\
\text { Appendix A of the remedial action report. }\end{array}$ \\
\hline 5. & $\begin{array}{l}\text { Verify that the Experimental Organic-Cooled } \\
\text { Reactor leach pond has been backfilled and } \\
\text { contoured to match the surrounding terrain. }\end{array}$ & $\begin{array}{l}\text { Visual observation } \\
\text { Photographic records }\end{array}$ & $\mathrm{X}$ & & $\begin{array}{l}\text { An inspection was completed on January } 10 \text {, } \\
\text { 2007. The photographic record is provided in } \\
\text { Appendix A of the remedial action report. }\end{array}$ \\
\hline 6. & $\begin{array}{l}\text { Verify that the STF- } 02 \text { Gun Range has been } \\
\text { regraded and contoured to match the surrounding } \\
\text { terrain. }\end{array}$ & $\begin{array}{l}\text { Visual observation } \\
\text { Photographic records }\end{array}$ & $\mathrm{X}$ & & $\begin{array}{l}\text { An inspection was completed on January } 10 \text {, } \\
\text { 2007. The photographic record is provided in } \\
\text { Appendix A of the remedial action report. }\end{array}$ \\
\hline & $\begin{array}{l}\text { Verify that the soil stockpiles have been removed } \\
\text { from the STF-02 Gun Range. }\end{array}$ & $\begin{array}{l}\text { Visual observation } \\
\text { Photographic records }\end{array}$ & $\mathrm{X}$ & & $\begin{array}{l}\text { An inspection was completed on January } 10 \text {, } \\
2007 \text {. The photographic record is provided in } \\
\text { Appendix A of the remedial action report. }\end{array}$ \\
\hline
\end{tabular}


Table C-1. (continued).

\begin{tabular}{|c|c|c|c|c|c|}
\hline \multirow{2}{*}{\multicolumn{2}{|c|}{ Inspection Item }} & \multirow[b]{2}{*}{$\begin{array}{l}\text { Verification } \\
\text { Information }\end{array}$} & \multicolumn{2}{|c|}{ Satisfactory } & \multirow[b]{2}{*}{ Comments } \\
\hline & & & Yes & No & \\
\hline 8. & $\begin{array}{l}\text { Verify that the electrical power poles, conduits and } \\
\text { abovegrade lines, and ancillary electrical } \\
\text { equipment (e.g., electrical panel) have been } \\
\text { removed from the STF-02 Gun Range. }\end{array}$ & $\begin{array}{l}\text { Visual observation } \\
\text { Photographic records }\end{array}$ & $\mathrm{X}$ & & $\begin{array}{l}\text { An inspection was completed on January } 10 \text {, } \\
\text { 2007. The photographic record is provided in } \\
\text { Appendix A of the remedial action report. }\end{array}$ \\
\hline 9. & $\begin{array}{l}\text { Verify that the contaminated soil has been } \\
\text { transported and disposed of at an approved facility. }\end{array}$ & CleanHarbors Invoice & $\mathrm{X}$ & & $\begin{array}{l}\text { An inspection was completed on January } 10 \text {, } \\
2007 \text {. The photographic record is provided in } \\
\text { Appendix A of the remedial action report. }\end{array}$ \\
\hline 10. & $\begin{array}{l}\text { Verify that the seed mix for revegetation has been } \\
\text { tested to verify compliance with specification } \\
\text { requirements. }\end{array}$ & Vendor data submittal & $X$ & & $\begin{array}{l}\text { An inspection was completed on January } 10 \text {, } \\
\text { 2007. The photographic record is provided in } \\
\text { Appendix A of the remedial action report. }\end{array}$ \\
\hline & $\begin{array}{l}\text { Verify that appropriate sampling data appear to } \\
\text { have been collected to enable the Agencies to } \\
\text { determine in the remedial action report whether the } \\
\text { remedial action objectives were met. }\end{array}$ & Data summary & $X$ & & $\begin{array}{l}\text { An inspection was completed on January } 10 \text {, } \\
\text { 2007. The photographic record is provided in } \\
\text { Appendix A of the remedial action report. }\end{array}$ \\
\hline
\end{tabular}


Appendix D

\section{STF-02 Gun Range Site Contour Drawings}


D-2 


\section{Appendix D}

\section{STF-02 Gun Range Site Contour Drawings}

The contour drawings in this appendix present the results of the topographical surveys conducted by a subcontractor at the STF-02 Gun Range prior to remediation, after soil removal, and after final grading and contouring of the site to match the surrounding terrain. The topographical surveys were performed by a professional land surveyor registered in the State of Idaho. 


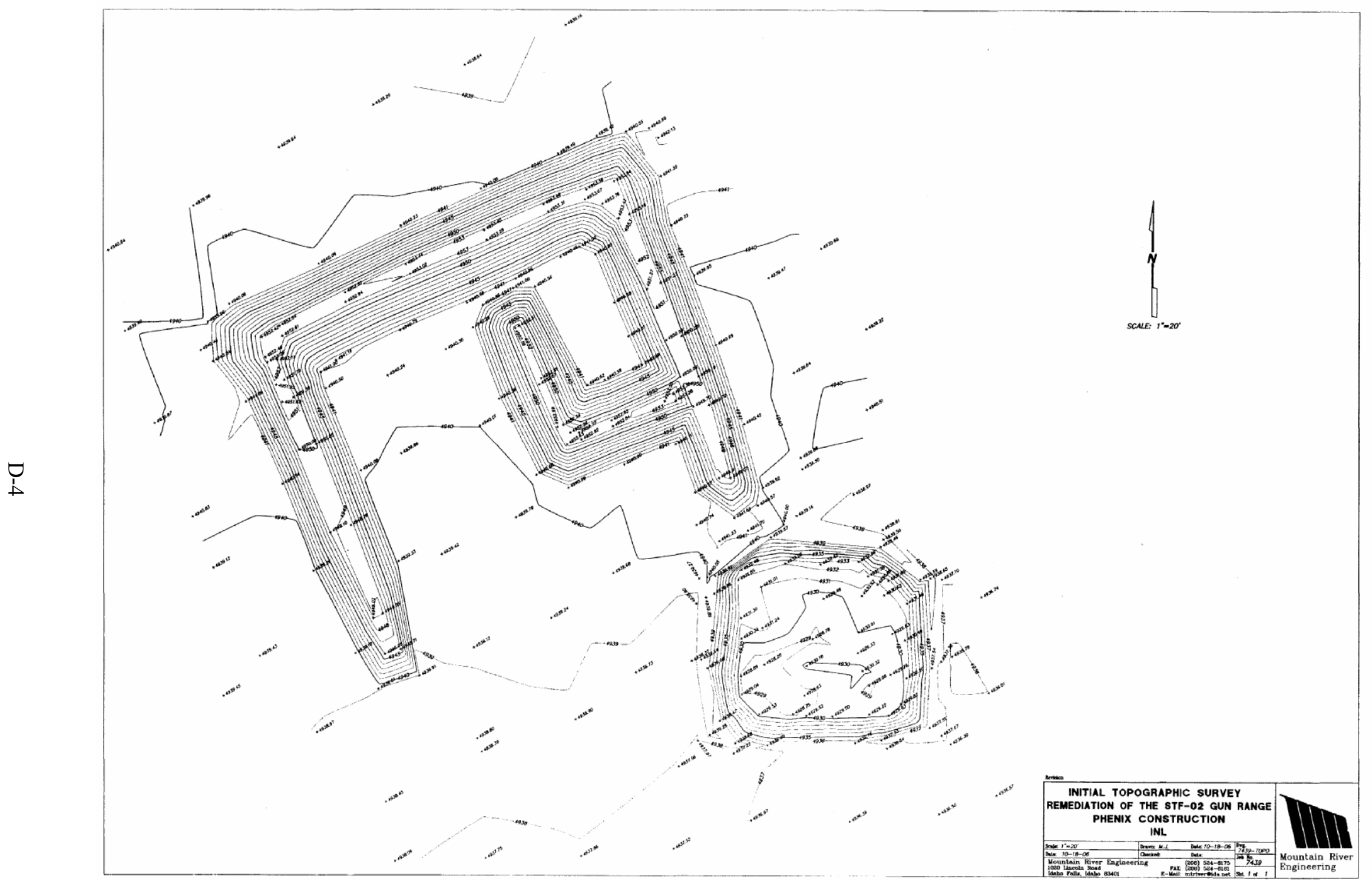

Figure D-1. Initial topographic survey of the STF-02 Gun Range. 


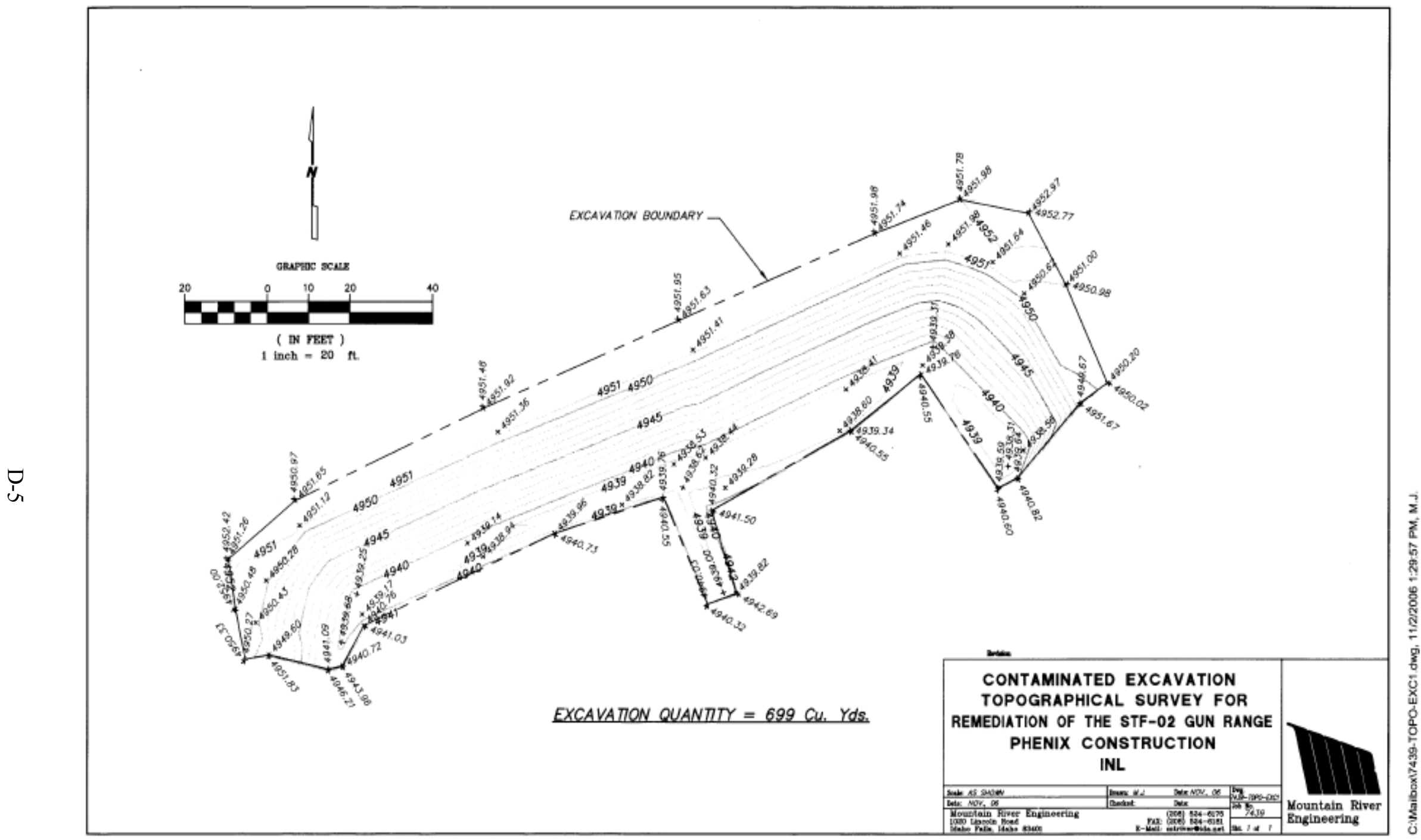

Figure D-2. Topographic survey of berms following excavation of contaminated soil. 


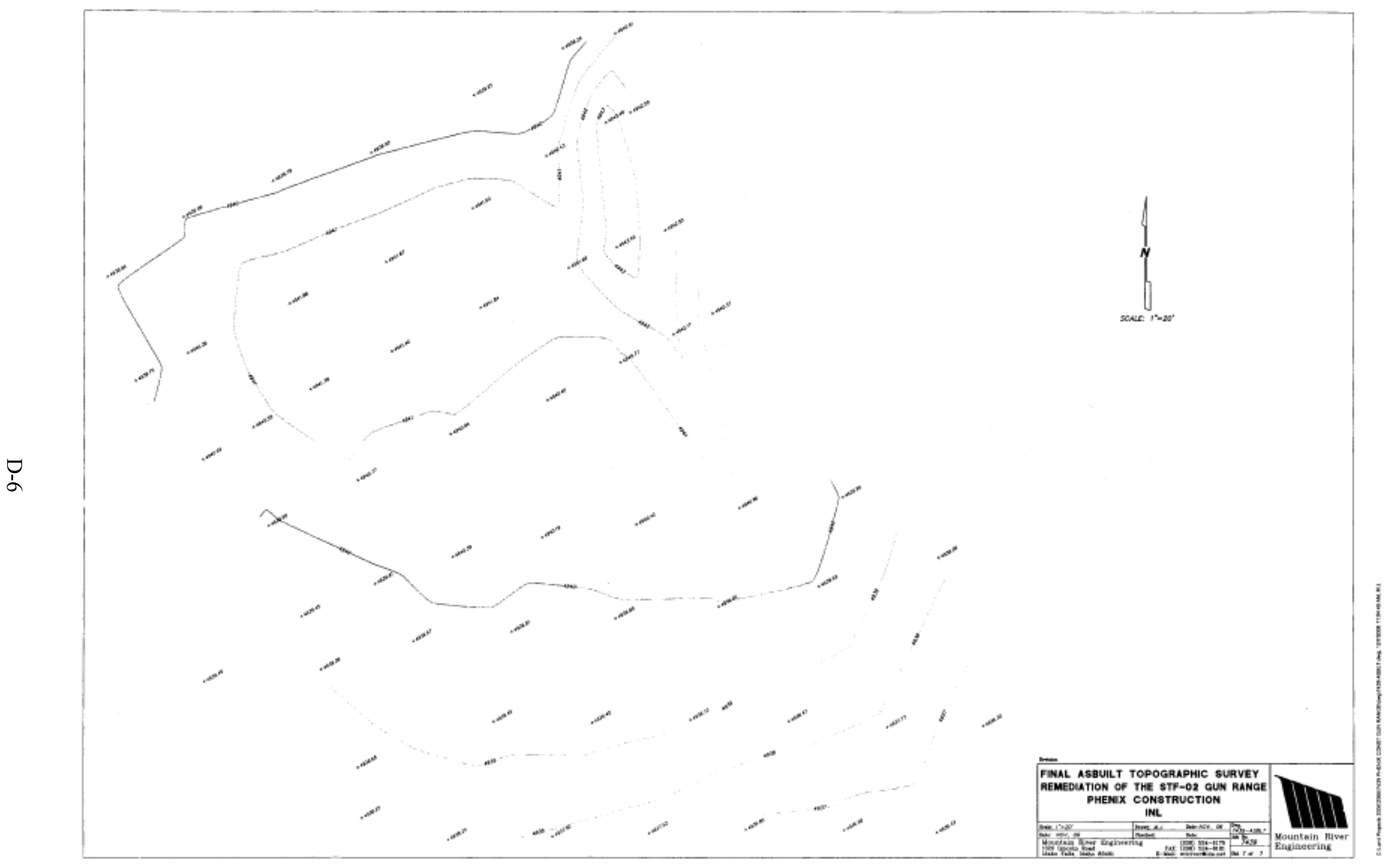

Figure D-3. Final topographic survey of the STF-02 Gun Range. 
Appendix E

Shipping Manifest Data 
E-2 


\section{Appendix E}

\section{Shipping Manifest Data}

The shipping manifest in this appendix is a summary of the contaminated soil shipments made from the STF-02 Gun Range off-Site to the approved treatment, storage, and disposal facility at which the soil was stabilized prior to disposal.

Table E-1. Shipping manifests.

\begin{tabular}{|c|c|c|c|c|}
\hline $\begin{array}{l}\text { Weight } \\
\text { (lb) }\end{array}$ & Manifest Number & Bar Code & Date & Transporter \\
\hline 34,400 & 001499045JJK-9/1 & CFA060128 & Nov. 6, 2006 & Clean Harbors Environmental Services \\
\hline 30,300 & 001499046JJK-9/1 & CFA060129 & Nov. 6, 2006 & Clean Harbors Environmental Services \\
\hline 29,300 & 001499047JJK-9/1 & CFA060130 & Nov. 6, 2006 & Clean Harbors Environmental Services \\
\hline 30,480 & 001499048JJK-9/1 & CFA060131 & Nov. 6, 2006 & Clean Harbors Environmental Services \\
\hline 29,380 & 001499050JJK-9/1 & CFA060132 & Nov. 6, 2006 & Clean Harbors Environmental Services \\
\hline 30,040 & 001499051JJK-9/1 & CFA060133 & Nov. 6, 2006 & Clean Harbors Environmental Services \\
\hline 32,160 & 001499052JJK-9/1 & CFA060134 & Nov. 6, 2006 & Triad Transport \\
\hline 32,540 & 001499053JJK-9/1 & CFA060135 & Nov. 6, 2006 & Triad Transport \\
\hline 29,180 & 001499054JJK-9/1 & CFA060136 & Nov. 6, 2006 & Triad Transport \\
\hline 29,560 & 001499055JJK-9/1 & CFA060137 & Nov. 6, 2006 & Triad Transport \\
\hline 31,000 & 001499056JJK-9/1 & CFA060138 & Nov. 6, 2006 & MP Environmental Services, Inc. \\
\hline 31,240 & 001499069JJK-9/1 & CFA060139 & Nov. 7, 2006 & Clean Harbors Environmental Services \\
\hline 32,220 & 001499070JJK-9/1 & CFA060140 & Nov. 7, 2006 & Clean Harbors Environmental Services \\
\hline 35,240 & 001499071JJK-9/1 & CFA060141 & Nov. 7, 2006 & Clean Harbors Environmental Services \\
\hline 33,640 & 001499072JJK-9/1 & CFA060142 & Nov. 7, 2006 & Clean Harbors Environmental Services \\
\hline 25,060 & 001499073JJK-9/1 & CFA060143 & Nov. 8, 2006 & Clean Harbors Environmental Services \\
\hline 35,920 & 001499074JJK-9/1 & CFA060144 & Nov. 8, 2006 & Clean Harbors Environmental Services \\
\hline 31,720 & 001499075JJK-9/1 & CFA060145 & Nov. 7, 2006 & Triad Transport \\
\hline 26,980 & 001499076JJK-9/1 & CFA060146 & Nov. 8, 2006 & Triad Transport \\
\hline 29,420 & 001499077JJK-9/1 & CFA060147 & Nov. 8, 2006 & Triad Transport \\
\hline 30,920 & 001499079JJK-9/1 & CFA060148 & Nov. 8, 2006 & Triad Transport \\
\hline 35,060 & 001499081JJK-9/1 & CFA060149 & Nov. 7, 2006 & MP Environmental Services \\
\hline 34,800 & 001499085JJK-9/1 & CFA060150 & Nov. 9, 2006 & MP Environmental Services \\
\hline 33,400 & 001499086JJK-9/1 & CFA060151 & Nov. 9, 2006 & Triad Transport \\
\hline 36,920 & 001499087JJK-9/1 & CFA060152 & Nov. 9, 2006 & Clean Harbors Environmental Services \\
\hline 30,100 & 001499088JJK-9/1 & CFA060153 & Nov. 9, 2006 & Clean Harbors Environmental Services \\
\hline 31,920 & 001499089JJK-9/1 & CFA060154 & Nov. 9, 2006 & Clean Harbors Environmental Services \\
\hline
\end{tabular}


Table E-1. (continued).

\begin{tabular}{|c|c|c|c|c|}
\hline $\begin{array}{l}\text { Weight } \\
\text { (lb) }\end{array}$ & Manifest Number & Bar Code & Date & Transporter \\
\hline 33,740 & 001499095JJK-9/1 & CFA060155 & Nov. 9, 2006 & Triad Transport \\
\hline 29,380 & 001499096JJK-9/1 & CFA060156 & Nov. 9, 2006 & Triad Transport \\
\hline 29,740 & 001499097JJK-9/1 & CFA060157 & Nov. 9, 2006 & Triad Transport \\
\hline 34,820 & 001499098JJK-9/1 & CFA060158 & Nov. 9, 2006 & Clean Harbors Environmental Services \\
\hline 33,900 & 001499100JJK-9/1 & CFA060159 & Nov. 10, 2006 & Clean Harbors Environmental Services \\
\hline 35,140 & 001499101JJK-9/1 & CFA060160 & Nov. 10, 2006 & Clean Harbors Environmental Services \\
\hline 30,940 & 001499107JJK-9/1 & CFA060161 & Nov. 10, 2006 & Clean Harbors Environmental Services \\
\hline 34,800 & 001499108JJK-9/1 & CFA060162 & Nov. 11, 2006 & Clean Harbors Environmental Services \\
\hline 33,300 & 001499109JJK-9/1 & CFA060163 & Nov. 10, 2006 & Triad Transport \\
\hline 32,140 & 001499110JJK-9/1 & CFA060164 & Nov. 10, 2006 & Triad Transport \\
\hline 34,440 & 001499111JJK-9/1 & CFA060165 & Nov. 10, 2006 & Triad Transport \\
\hline 34,300 & 001499112JJK-9/1 & CFA060166 & Nov. 10, 2006 & Triad Transport \\
\hline 33,560 & 001499113JJK-9/1 & CFA060167 & Nov. 10, 2006 & MP Environmental Services \\
\hline 33,860 & 001499114JJK-9/1 & CFA060168 & Nov. 13, 2006 & Clean Harbors Environmental Services \\
\hline 35,260 & 001499115JJK-9/1 & CFA060169 & Nov. 13, 2006 & Clean Harbors Environmental Services \\
\hline 34,700 & 001499116JJK-9/1 & CFA060170 & Nov. 14, 2006 & Clean Harbors Environmental Services \\
\hline 34,180 & 001499117JJK-9/1 & CFA060171 & Nov. 13, 2006 & Triad Transport \\
\hline 34,420 & 001499119JJK-9/1 & CFA060172 & Nov. 13, 2006 & Triad Transport \\
\hline 34,480 & 001499120JJK-9/1 & CFA060173 & Nov. 13, 2006 & Triad Transport \\
\hline 36,280 & 001499121JJK-9/1 & CFA060174 & Nov. 13, 2006 & Tri-State Motor Transport \\
\hline 36,340 & 001499122JJK-9/1 & CFA060175 & Nov. 13, 2006 & Tri-State Motor Transport \\
\hline 34,020 & 001499123JJK-9/1 & CFA060176 & Nov. 13, 2006 & MP Environmental Services \\
\hline 32,780 & 001499125JJK-9/1 & CFA060177 & Nov. 13, 2006 & Triad Transport \\
\hline 33,440 & 001499126JJK-9/1 & CFA060178 & Nov. 13, 2006 & MP Environmental Services \\
\hline 34,680 & 001499127JJK-9/1 & CFA060179 & Nov. 14, 2006 & Clean Harbors Environmental Services \\
\hline 33,120 & 001499128JJK-9/1 & CFA060180 & Nov. 14, 2006 & Clean Harbors Environmental Services \\
\hline 32,520 & 001499129JJK-9/1 & CFA060181 & Nov. 14, 2006 & Triad Transport \\
\hline 33,840 & 001499130JJK-9/1 & CFA060182 & Nov. 14, 2006 & Triad Transport \\
\hline 32,440 & 001499131JJK-9/1 & CFA060183 & Nov. 14, 2006 & Triad Transport \\
\hline 31,160 & 001499132JJK-9/1 & CFA060184 & Nov. 14, 2006 & Triad Transport \\
\hline 37,820 & 001499133JJK-9/1 & CFA060185 & Nov. 14, 2006 & MP Environmental Services \\
\hline 35,800 & 001499134JJK-9/1 & CFA060186 & Nov. 16, 2006 & MP Environmental Services \\
\hline 37,200 & 001499135JJK-9/1 & CFA060187 & Nov. 14, 2006 & Tri-State Motor Transport \\
\hline 37,580 & 001499136JJK-9/1 & CFA060188 & Nov. 14, 2006 & Tri-State Motor Transport \\
\hline 34,280 & 001499146JJK-9/1 & CFA060189 & Nov. 15, 2006 & Clean Harbors Environmental Services \\
\hline
\end{tabular}


Table E-1. (continued).

\begin{tabular}{|c|c|c|c|c|}
\hline $\begin{array}{l}\text { Weight } \\
\text { (lb) }\end{array}$ & Manifest Number & Bar Code & Date & Transporter \\
\hline 36,508 & 001499147JJK-9/1 & CFA060190 & Nov. 15, 2006 & Clean Harbors Environmental Services \\
\hline 34,980 & 001499140JJK-9/1 & CFA060191 & Nov. 15, 2006 & Clean Harbors Environmental Services \\
\hline 33,600 & 001499141JJK-9/1 & CFA060192 & Nov. 15, 2006 & Triad Transport \\
\hline 33,280 & 001499142JJK-9/1 & CFA060193 & Nov. 15, 2006 & Triad Transport \\
\hline 32,780 & 001499143JJK-9/1 & CFA060194 & Nov. 15, 2006 & Triad Transport \\
\hline 30,580 & 001499144JJK-9/1 & CFA060195 & Nov. 16, 2006 & Triad Transport \\
\hline 32,580 & 001499148JJK-9/1 & CFA060196 & Nov. 16, 2006 & Clean Harbors Environmental Services \\
\hline 34,060 & 01499160JJK-9/1 & CFA060197 & Nov. 16, 2006 & Clean Harbors Environmental Services \\
\hline 36,340 & 001499150JJK-9/1 & CFA060198 & Nov. 16, 2006 & Clean Harbors Environmental Services \\
\hline 32,860 & 001499151JJK-9/1 & CFA060199 & Nov. 17, 2006 & Clean Harbors Environmental Services \\
\hline 33,680 & 001499152JJK-9/1 & CFA060200 & Nov. 16, 2006 & Triad Transport \\
\hline 34,660 & 001499153JJK-9/1 & CFA060201 & Nov. 16, 2006 & Triad Transport \\
\hline 32,960 & 001499158JJK-9/1 & CFA060202 & Nov. 16, 2006 & Triad Transport \\
\hline 35,000 & 001499163JJK-9/1 & CFA060203 & Nov. 17, 2006 & Clean Harbors Environmental Services \\
\hline 26,780 & 001499164JJK-9/1 & CFA060204 & Nov. 17, 2006 & Clean Harbors Environmental Services \\
\hline 33,900 & 00149165JJK-9/1 & CFA060205 & Nov. 17, 2006 & Clean Harbors Environmental Services \\
\hline 32,740 & 001499166JJK-9/1 & CFA060206 & Nov. 17, 2006 & Triad Transport \\
\hline 27,220 & 001499167JJK-9/1 & CFA060207 & Nov. 17, 2006 & Triad Transport \\
\hline 30,200 & 001499168JJK-9/1 & CFA060250 & Nov. 17, 2006 & Triad Transport \\
\hline 30,860 & 001499169JJK-9/1 & CFA060251 & Nov. 17, 2006 & Triad Transport \\
\hline 33,300 & 001499170JJK-9/1 & CFA060252 & Nov. 17, 2006 & Triad Transport \\
\hline 33,260 & 001499171JJK-9/1 & CFA060253 & Nov. 17, 2006 & MP Environmental Services \\
\hline 35,120 & 001499179JJK-9/1 & CFA060254 & Nov. 17, 2006 & Clean Harbors Environmental Services \\
\hline 34,620 & 001499180JJK-9/1 & CFA060255 & Nov. 20, 2006 & Clean Harbors Environmental Services \\
\hline 32,460 & 001499181JJK-9/1 & CFA060256 & Nov. 20, 2006 & Clean Harbors Environmental Services \\
\hline 34,340 & 001499182JJK-9/1 & CFA060257 & Nov. 20, 2006 & Clean Harbors Environmental Services \\
\hline 32,600 & 001499183JJK-9/1 & CFA060258 & Nov. 20, 2006 & Clean Harbors Environmental Services \\
\hline 33,960 & 001499184JJK-9/1 & CFA060259 & Nov. 20, 2006 & Triad Transport \\
\hline 28,880 & 001499185JJK-9/1 & CFA060260 & Nov. 20, 2006 & Triad Transport \\
\hline 30,640 & 001499186JJK-9/1 & CFA060261 & Nov. 20, 2006 & Triad Transport \\
\hline 33,760 & 001499187JJK-9/1 & CFA060262 & Nov. 20, 2006 & Triad Transport \\
\hline 29,240 & 001499188JJK-9/1 & CFA060263 & Nov. 20, 2006 & MP Environmental Services \\
\hline 32,840 & 001499198JJK-9/1 & CFA060264 & Nov. 28, 2006 & Clean Harbors Environmental Services \\
\hline 27,100 & 001499199JJK-9/1 & CFA060265 & Nov. 29, 2006 & Clean Harbors Environmental Services \\
\hline
\end{tabular}




\section{Appendix F}

\section{Subcontractor Vendor Data Submittals}


F-2 


\section{Appendix F}

\section{Subcontractor Vendor Data Submittals}

Table F-1 summarizes all subcontractor vendor data submittals received to meet the requirements set forth in the vendor data schedule in accordance with Specification (SPC) -646, "Construction

Specification - Remediation of the STF-02 Gun Range." The compilation of full vendor data submittals is provided subsequent to the table. 
Table F-1. Vendor data submittals.

\begin{tabular}{|c|c|c|c|c|c|c|}
\hline $\begin{array}{l}\text { Drawing } \\
\text { Section }\end{array}$ & Description & $\begin{array}{c}\text { Schedule } \\
\text { Item } \\
\text { Number }\end{array}$ & $\begin{array}{l}\text { Transaction } \\
\text { Number }\end{array}$ & $\begin{array}{l}\text { Transaction } \\
\text { Date }\end{array}$ & $\begin{array}{l}\text { Revision } \\
\text { Number }\end{array}$ & VDR Number \\
\hline 01005 & Chemical Inventory List - Form 432.21 Quarterly Report & 1 & S-507296-011 & $11 / 27 / 2006$ & 0 & VDR-157349 \\
\hline 01005 & Chemical Inventory List - Form 432.21 - Final Report & 2 & S-507296-012 & $12 / 5 / 2006$ & 0 & VDR-158077 \\
\hline 01005 & $\begin{array}{l}\text { Chemical Inventory List - Form } 432.21 \\
\text { Initial and Resubmittal with Supporting MSDS }\end{array}$ & 3 & S-507296-01 & $9 / 22 / 2006$ & 0 & VDR-153307 \\
\hline 01005 & $\begin{array}{l}\text { Chemical Inventory List - Form } 432.21 \\
\text { Initial and Resubmittal with Supporting MSDS }\end{array}$ & 3 & S-507296-07 & $10 / 9 / 2006$ & 0 & VDR-154111 \\
\hline 01051 & Land Surveyor Registered Professional Certification & 4 & S-507296-02 & $9 / 22 / 2006$ & 0 & VDR-153312 \\
\hline 01051 & Topographical Survey - Original Topography & 5 & S-507296-09 & $10 / 30 / 2006$ & 0 & VDR-155376 \\
\hline 01051 & Topographical Survey - After Soil Removal & 6 & S-507296-010 & $11 / 8 / 2006$ & 0 & VDR-155906 \\
\hline 01051 & Topographical Survey - Final Surface & 7 & S-507296-013 & $12 / 13 / 2006$ & 0 & VDR-158614 \\
\hline 02200 & Emissions and Dust Control Plan & 8 & S-507296-04 & $10 / 2 / 2006$ & 0 & VDR-153746 \\
\hline 02200 & Emissions and Dust Control Plan & 8 & S-507296-04R.1 & $10 / 9 / 2006$ & 1 & VDR-153746 \\
\hline 02486 & Seed Mix Certification & 9 & S-507296-08 & $10 / 26 / 2006$ & 0 & VDR-155291 \\
\hline 02486 & Soil Analysis & 10 & S-507296-03 & $9 / 22 / 2006$ & 0 & VDR-153317 \\
\hline SC-17 & Personnel Lead Exposure Assessment Plan & 11 & S-507296-05 & $10 / 2 / 2006$ & 0 & VDR-153751 \\
\hline SC-17 & Personnel Lead Exposure Assessment Plan & 11 & S-507296-05R.1 & $10 / 16 / 2006$ & 1 & VDR-153751 \\
\hline SC-17 & Personnel Lead Exposure Assessment Plan & 11 & S-507296-05R.2 & $10 / 18 / 2006$ & 2 & VDR-153751 \\
\hline $\mathrm{SC}-3$ & Job Safety Analysis & 12 & S-507296-06 & $10 / 2 / 2006$ & 0 & VDR-153746 \\
\hline $\mathrm{SC}-3$ & Job Safety Analysis & 12 & S-507296-06R.1 & $10 / 11 / 2006$ & 1 & VDR-153756 \\
\hline
\end{tabular}




\section{VDS \#1_157349}


F-6 
To be completed by Supplier/Subeontractor

Purchase Order or Subcontract Number: $00507296-Q / / B . Q$ Project Title/Number: Remediation of The STF - 02 Gun Range

Submittal Number: 6131-11_ Supplier/Subcontractor Name: Phenix Construction

Submittal Date: $\quad$ 11-25-06 Address: P.O. Box 1626 ldaho Falls Idaho 83403

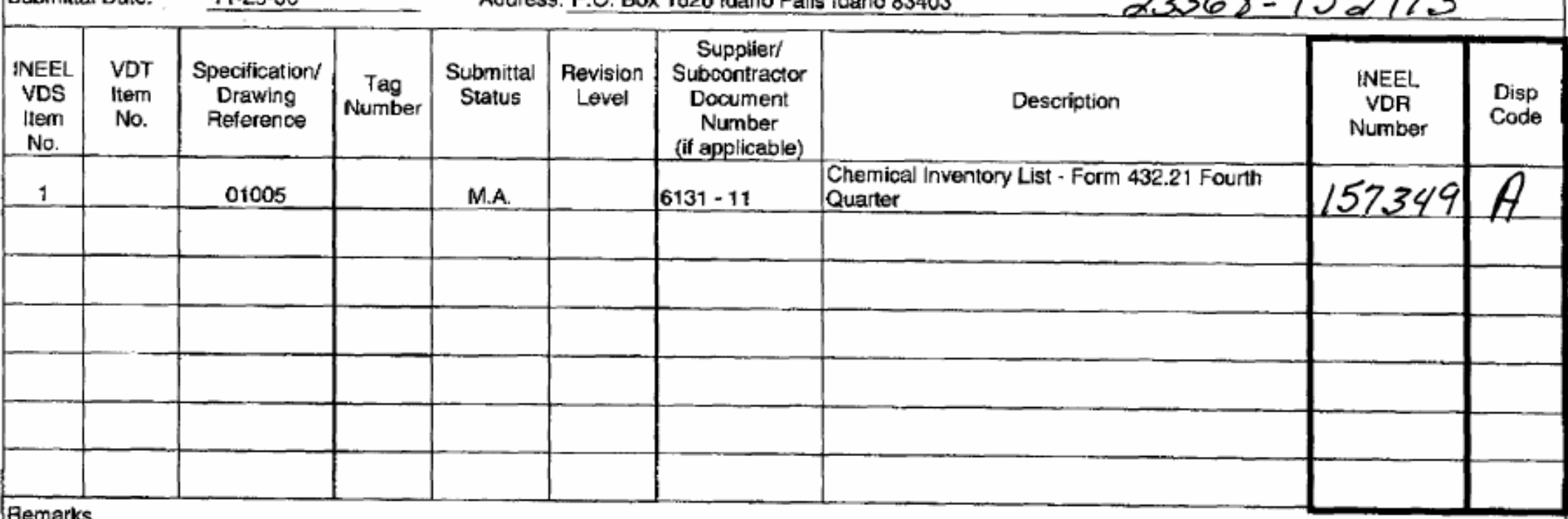

Remarks

21 St $11.25-06$

To Be Completed by Contractor/AE 
432.21

02/17/2000

Rev. 05
SUBCONTRACTOR REPORTING FORM CHEMICAL INVENTORY LIST

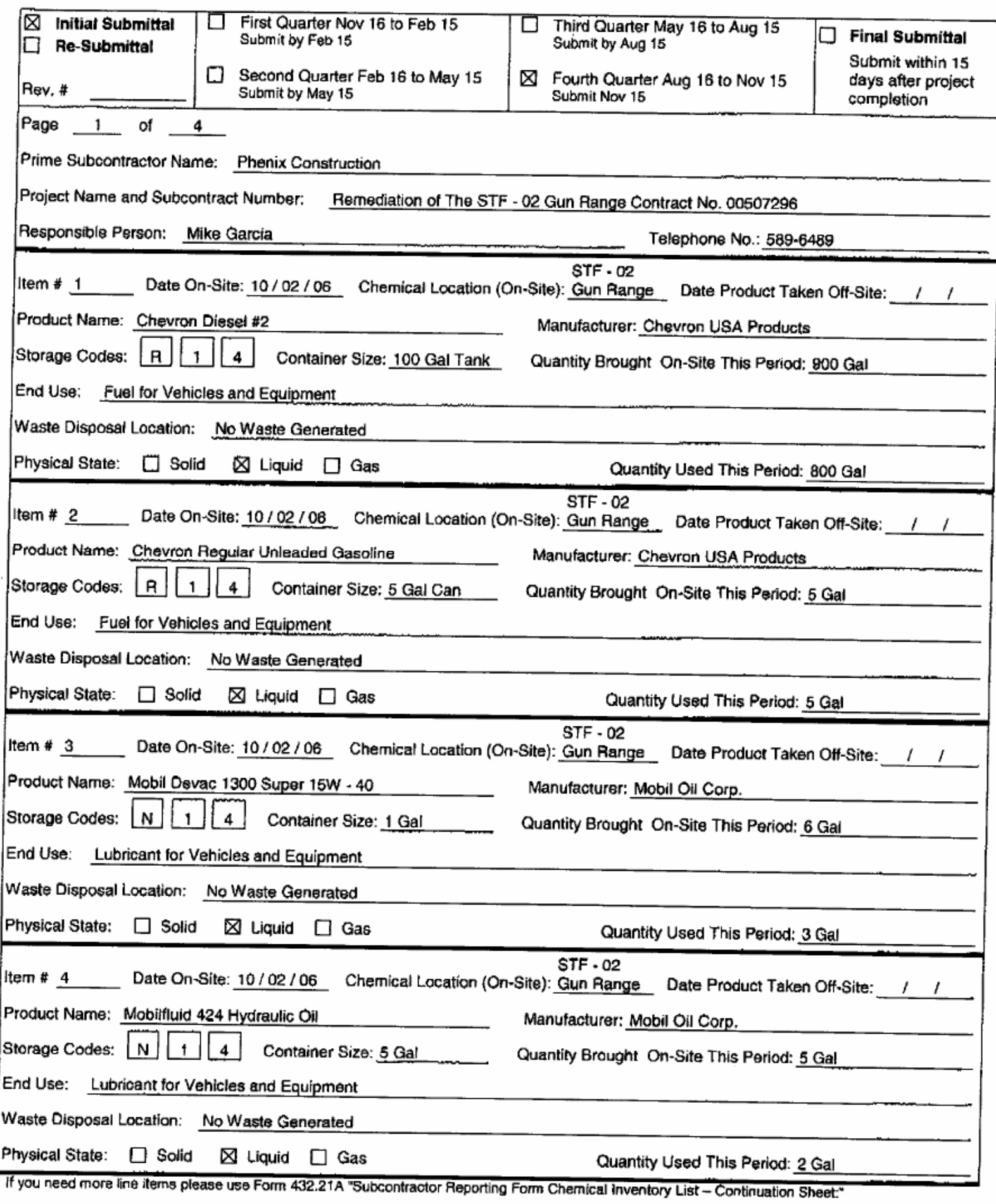


432.21A

$04 / 13 / 99$

Rev. 01

\section{SUBCONTRACTOR REPORTING FORM CHEMICAL INVENTORY LIST \\ (CONTINUATION SHEET)}

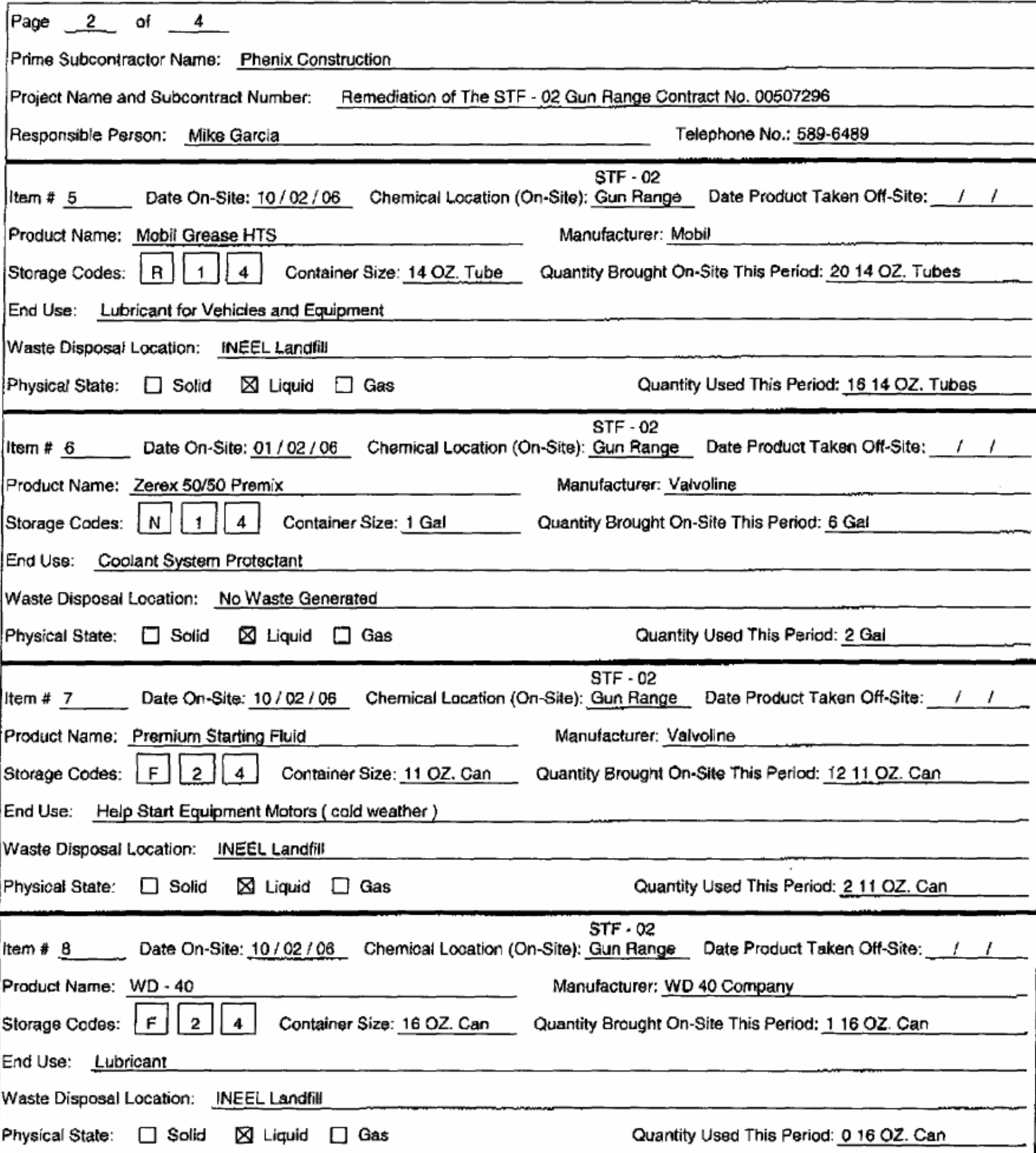


432.21A

$04 / 13 / 99$

Rev. 01

\section{SUBCONTRACTOR REPORTING FORM CHEMICAL INVENTORY LIST (CONTINUATION SHEET)}

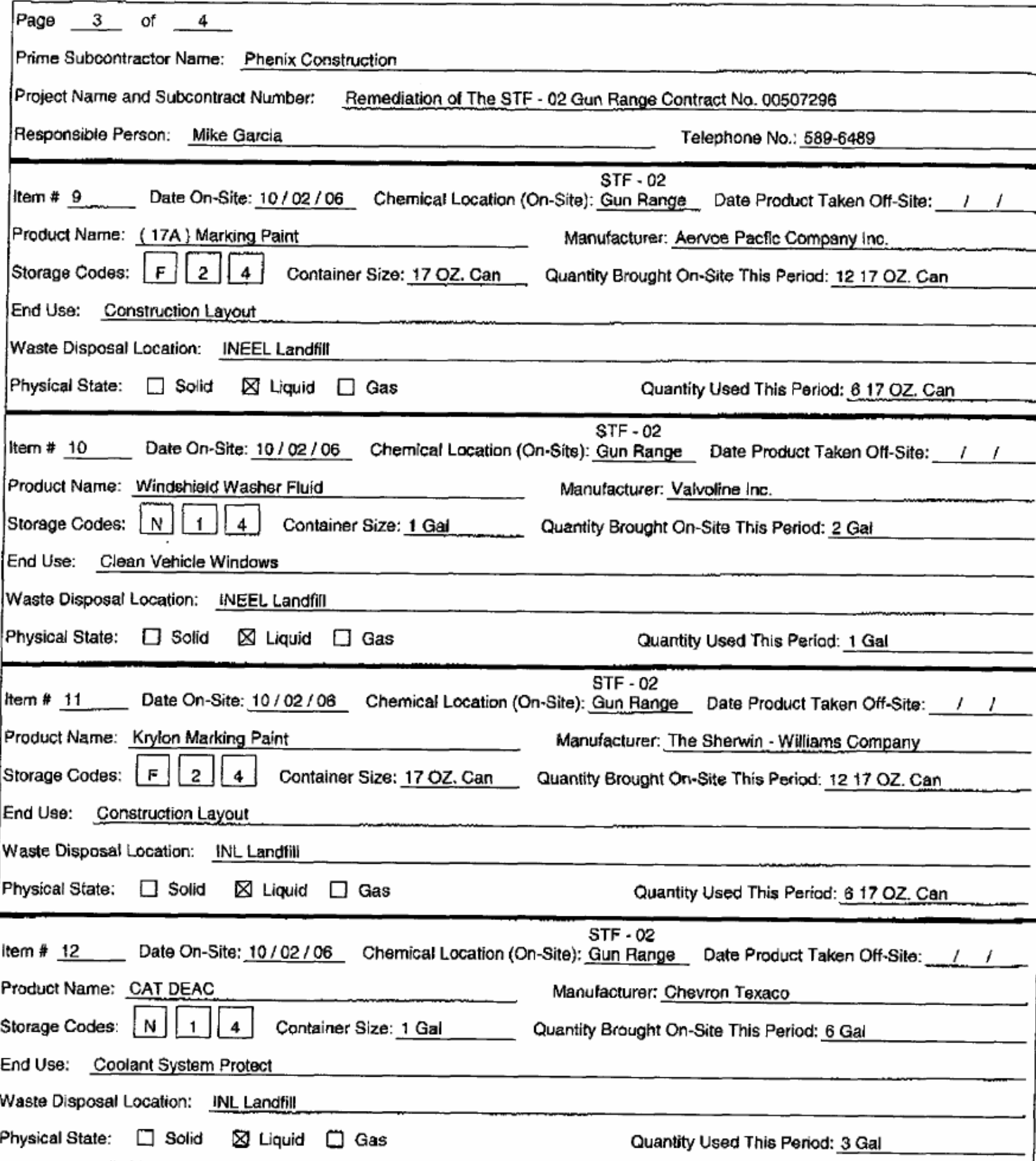


432.21A

$04 / 13 / 99$

Rev. 01

\section{SUBCONTRACTOR REPORTING FORM \\ CHEMICAL INVENTORY LIST \\ (CONTINUATION SHEET)}

Page 4 of 4

Prime Subcontractor Name: Phenix Construction

Project Name and Subcontract Number: Remediation of The STF - 02 Gun Range Contract №. 00507296

Responsible Person: Mike Garcia_____ Telephone No.: $\underline{\text { 589-6489 }}$

STF . 02

Item \# 13 Date On-Site: 10/02/06 Chemical Location (On-Site): Gun Range Date Product Taken Off-Site:

Product Name: RONEX EXTRA DUTY MOLY 2 Manufacturer: EXXONMOBIL Oil Corporation

Storage Codes: $R\left[\begin{array}{l}\mathrm{A} \\ \mathrm{A}\end{array}\right.$

End Use: Lubricant for Vehicles and Equipment

Waste Disposal Location: INL Landfill

Physical State: $\square$ Solid $\otimes$ Liquid $\square$ Gas Quantity Used This Period: $1614 \mathrm{OZ}$. Tube

Item \# 14 Date On-Site: 10/16/06 Chemical Location (On-Site): Gun Range Date Product Taken Off-Site: $11 / 15 / 06$

Product Name: Stockopam - Terra Bond

Manufacturer: Stockhausen Inc.

Storage Codes: $\left[\mathrm{J}[1]\left[\begin{array}{l}4 \\ 4\end{array}\right]\right.$ Container Size: $30 \mathrm{PD}$ Tote Quantity Brought On-Site This Period: 0 PD

End Use: Stabilize soil to minimize dust

Waste Disposal Location: INL Landfill

Physical State: $\triangle$ Solid $\square$ Liquid $\square$ Gas Quantity Used This Period: $\underline{0 P D}$

Item \# Date On-Site: 11 Chemical Location (On-Site):

Date Product Taken Off-Site:

Manufacturer:

Product Name:

Storage Codes:

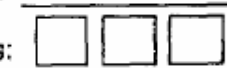

Container Size

Quantity Brought On-Site This Period:

End Use:

Waste Disposal Location:

Physicat State: $\square$ Solid $\square$ Liquid $\square$ Gas Quantity Used This Poriod:

Item \# D Date On-Site: 11 Chemical Location (On-Site):

Manufacturer:

Product Name:

Storage Codes: $\square \square \square$ Container Size:

Quantity Brought On-Site This Period:

End Use:

Waste Disposal Location:

Physical State: $\square$ Solid $\square$ Liquid $\square$ Gas Quantity Used This Period 


\section{Vendor Data Review System Final Disposition Screen}

This vendor data item has been given the following disposition codes

$\begin{array}{lllll}\text { Reviewer } & \text { Revision Level } & \text { Date } & \text { Disposition Code } & \text { Comments } \\ \text { DRIEVER MIKEL K } & 0 & 27-N O V-06 & \text { A } & \\ \text { FRITZ KURT D } & 0 & 14-D E C-06 & \text { D } & \\ \text { TUOTT LEE C } & 0 & 27-N O V-06 & \text { A } & \text { no comment } \\ \text { LANDIS JOSEPH A } & 0 & 27-N O V-06 & \text { A } & \\ \text { MCMANAMON LAWRENCE E } & 0 & 27-N O V-06 & \text { A } \\ \text { VANDEL DOUG S } & 0 & 27-N O V-06 & \text { A }\end{array}$

The following reviewers have NOT yet reviewed this vendor data item

JOLLEY WENDELL L

VDR Number:

Revision Level:

Project Number:

Transmittal Number:

Transmittal Status:

Line Item:

Disposition Code:

A
VDR-157349

0

$23368-152173$

S-507296-011

Mandatory Approval

1

Final Comments:: 


\section{VDS \#2_158077}

F-13 
F-14 
431.13

$07 / 23 / 2002$

Rev, 04
VENDOR DATA

TRANSAAITTAL \& DISPOSITION FORM
ORIGINAL

Page 2 of 3

To be completed by Supplier/Subcontractor

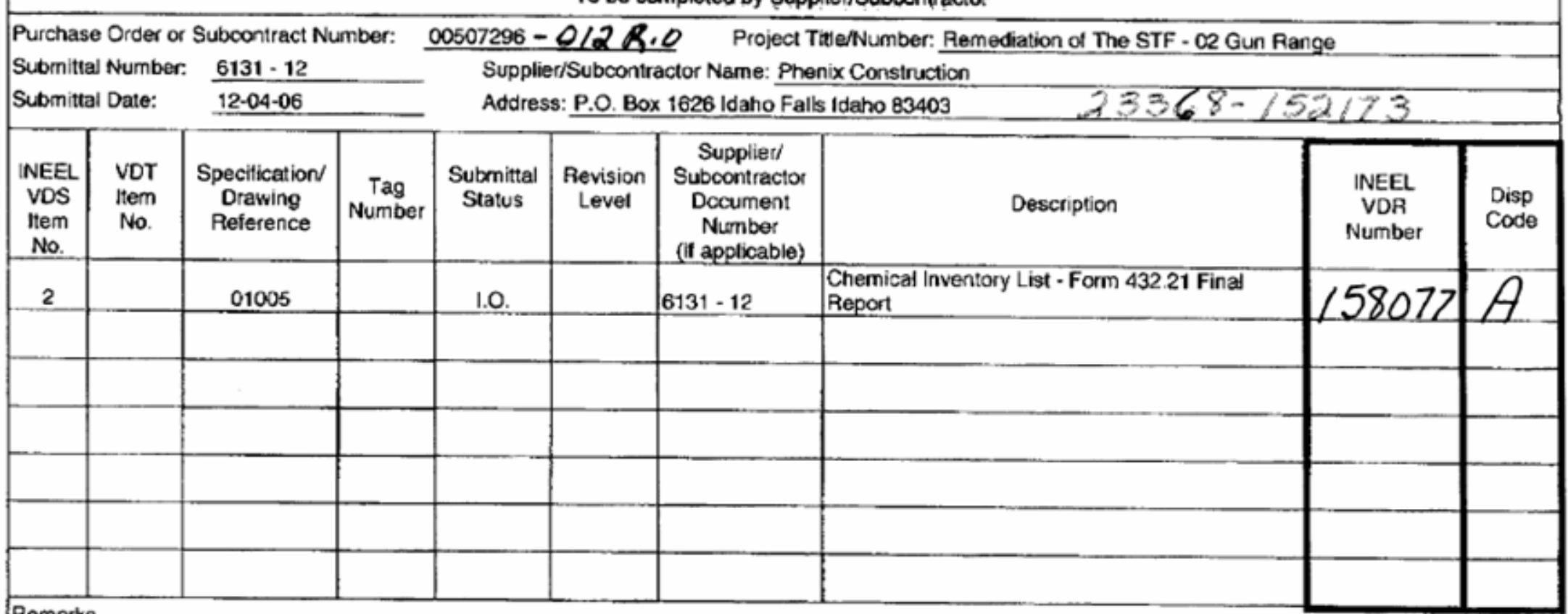

Remarks

$12-04-06$

Supplier/Subcontractor Authorized Signature/Date

To Be Completed by Contractor/AE 


\section{SUBCONTRACTOR REPORTING FORM CHEMICAL INVENTORY UST}

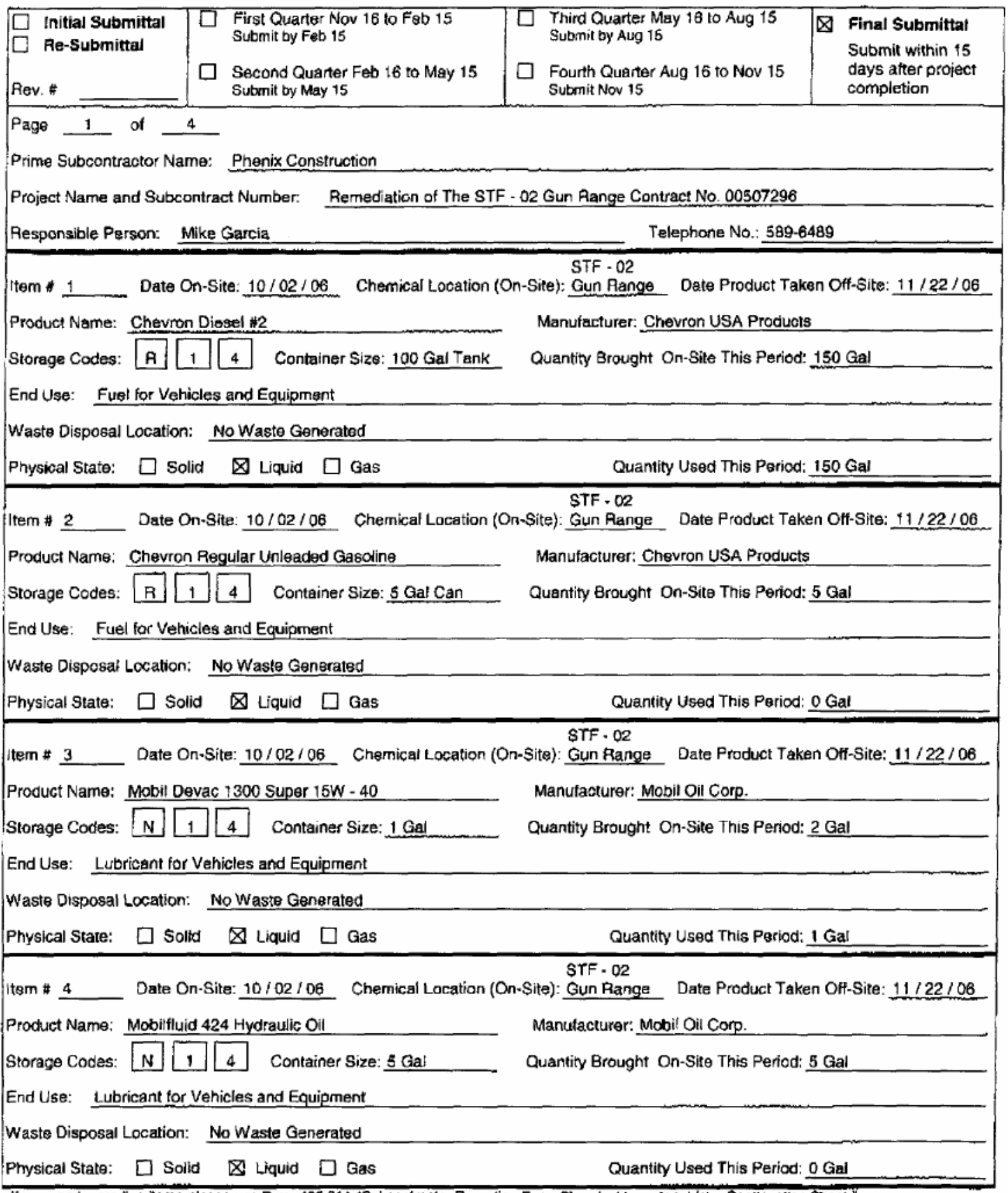

If you need mare line items please use Form 432.21A "Subcontraotor Reporting Form Chemical Inventory List - Continuation Shes:" 
432.21A

$04 / 13 / 99$

Pev. 01

\section{SUBCONTRACTOR REPORTING FORM \\ CHEMICAL INVENTORY LIST \\ (CONTINUATION SHEET)}

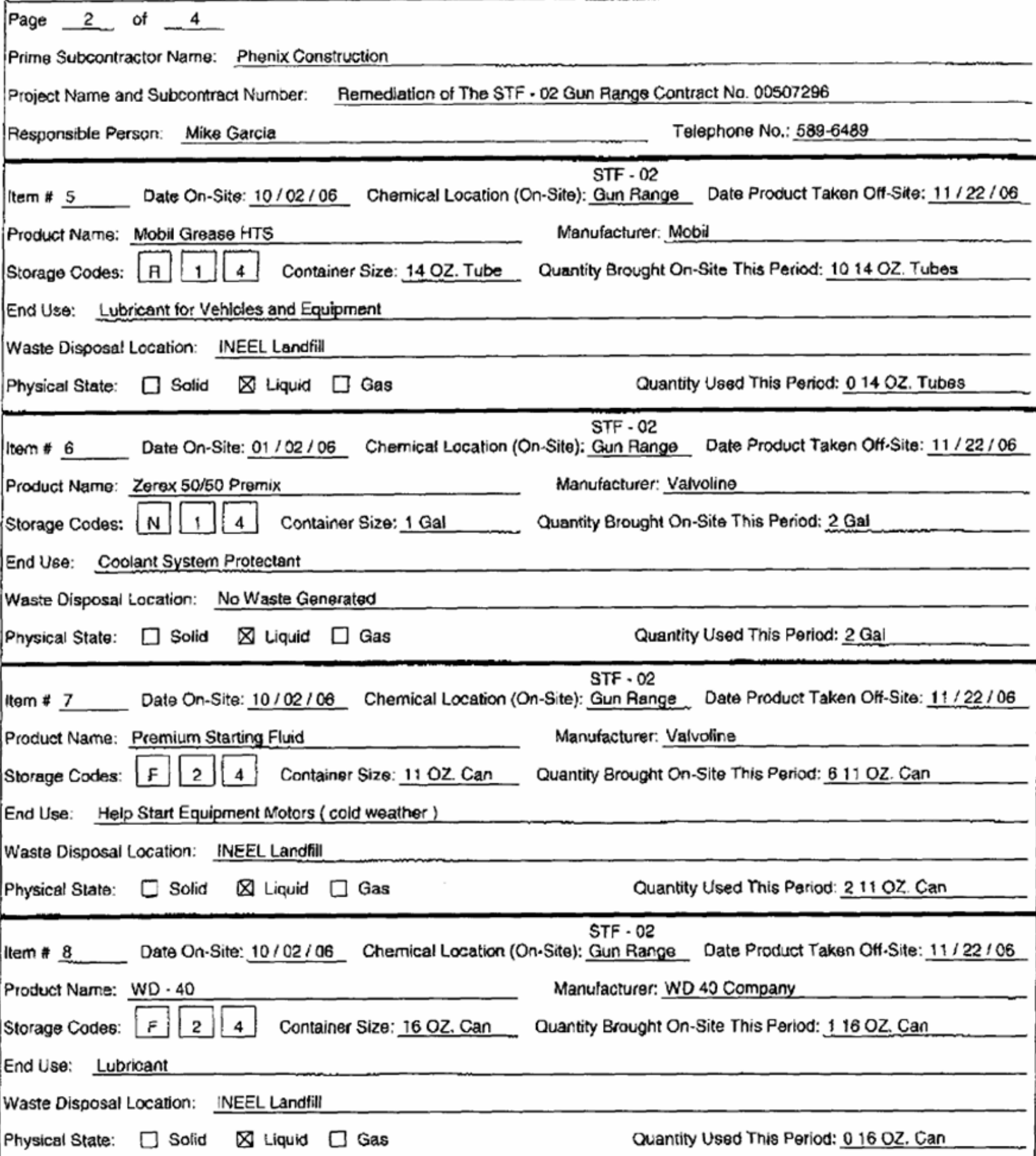


$432.21 \mathrm{~A}$

$04 / 13 / 99$

Rev. 01

\section{SUBCONTRACTOR REPORTING FORM \\ CHEMICAL INVENTORY LIST \\ (CONTINUATION SHEET)}

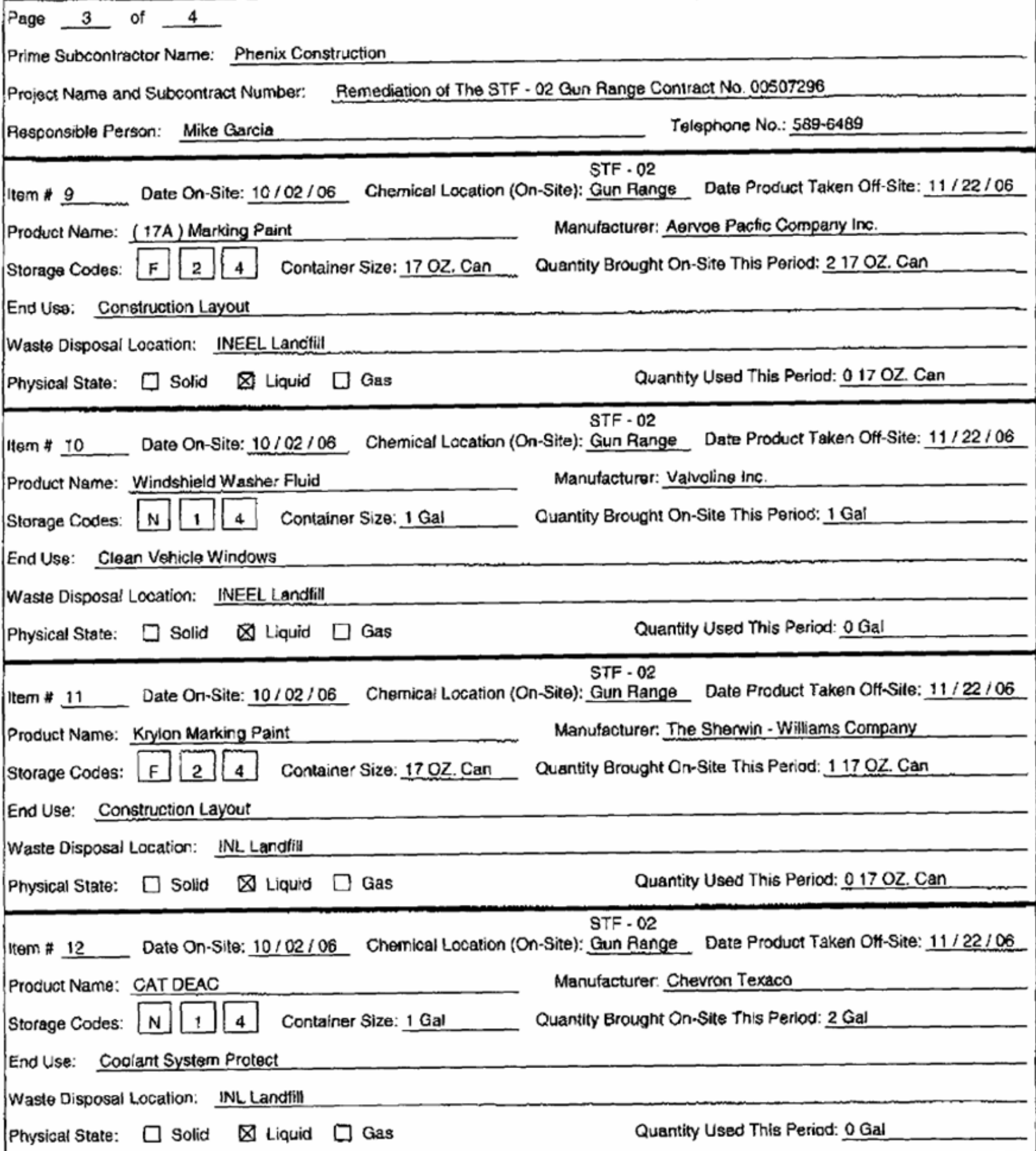


432.21A

$04 / 13 / 99$

Rev. 01

\section{SUBCONTRACTOR REPORTING FORM \\ CHEMICAL INVENTORY LIST \\ (CONTINUATION SHEET)}

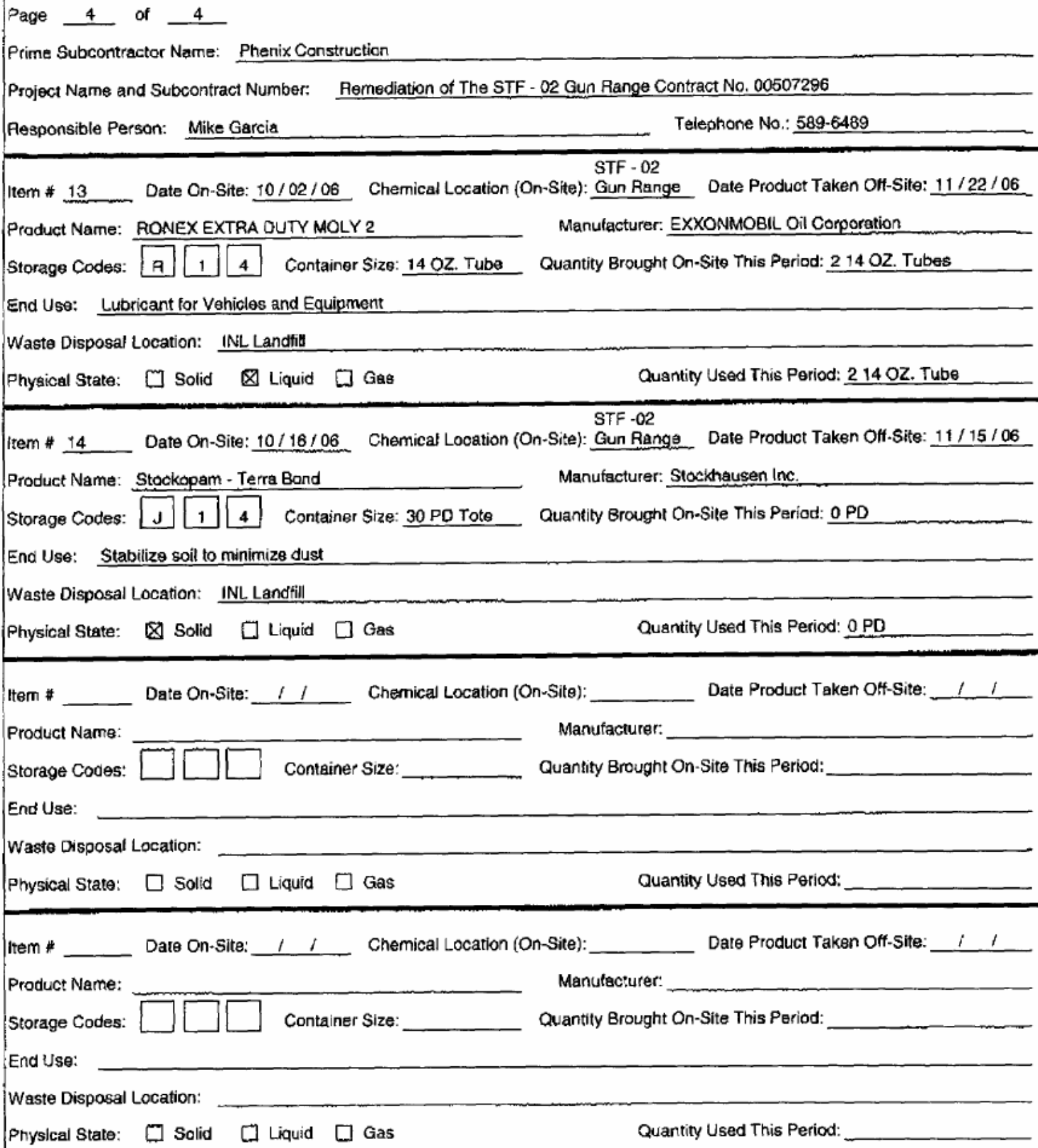




\title{
Vendor Data Review System Final Disposition Screen
}

\author{
This vendor data item has been given the following disposition codes
}

\begin{tabular}{|c|c|c|c|c|}
\hline Reviewer & Revision Level & Date & Disposition Code & Comments \\
\hline FRITZ KURT D & 0 & 14-DEC-06 & D & \\
\hline VANDEL DOUG S & 0 & 05-DEC-06 & A & \\
\hline TUOTT LEE C & 0 & 05-DEC-06 & $\mathrm{D}$ & \\
\hline LANDIS JOSEPH A & 0 & 05-DEC-06 & A & no comment \\
\hline MCMANAMON LAWRENCE E & 0 & 05-DEC-06 & $\mathrm{D}$ & \\
\hline DRIEVER MIKEL K & 0 & 05-DEC-06 & $A$ & \\
\hline
\end{tabular}

The following reviewers have NOT yet reviewed this vendor data item

JOLLEY WENDELL L

VDR Number:

Revision Level:

Project Number:

Transmittal Number:

Transmittal Status:

Line Item:

Disposition Code::

A

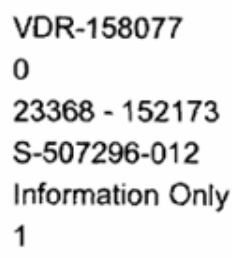

Final Comments:: 


\section{VDS \#3_153307}


F-22 
VENDOR DATA TRANSMITTAL \& DISPOSITION FORM

\section{ORIGINAL}

To be completed by Supplier/Subcontractor

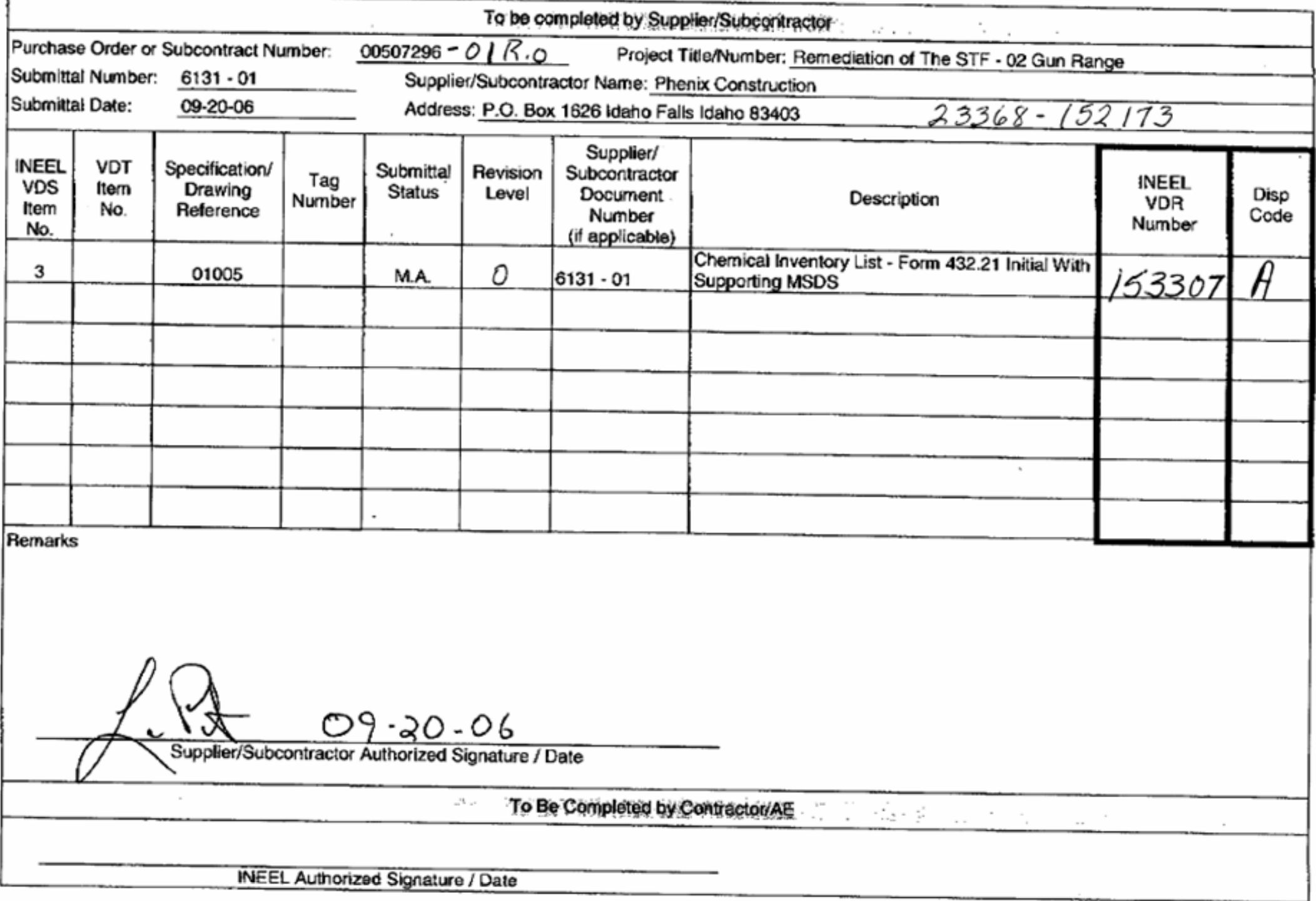


432.21

02/17/2000

Rev. 05
SUBCONTRACTOR REPORTING FORM

CHEMICAL INVENTORY LIST

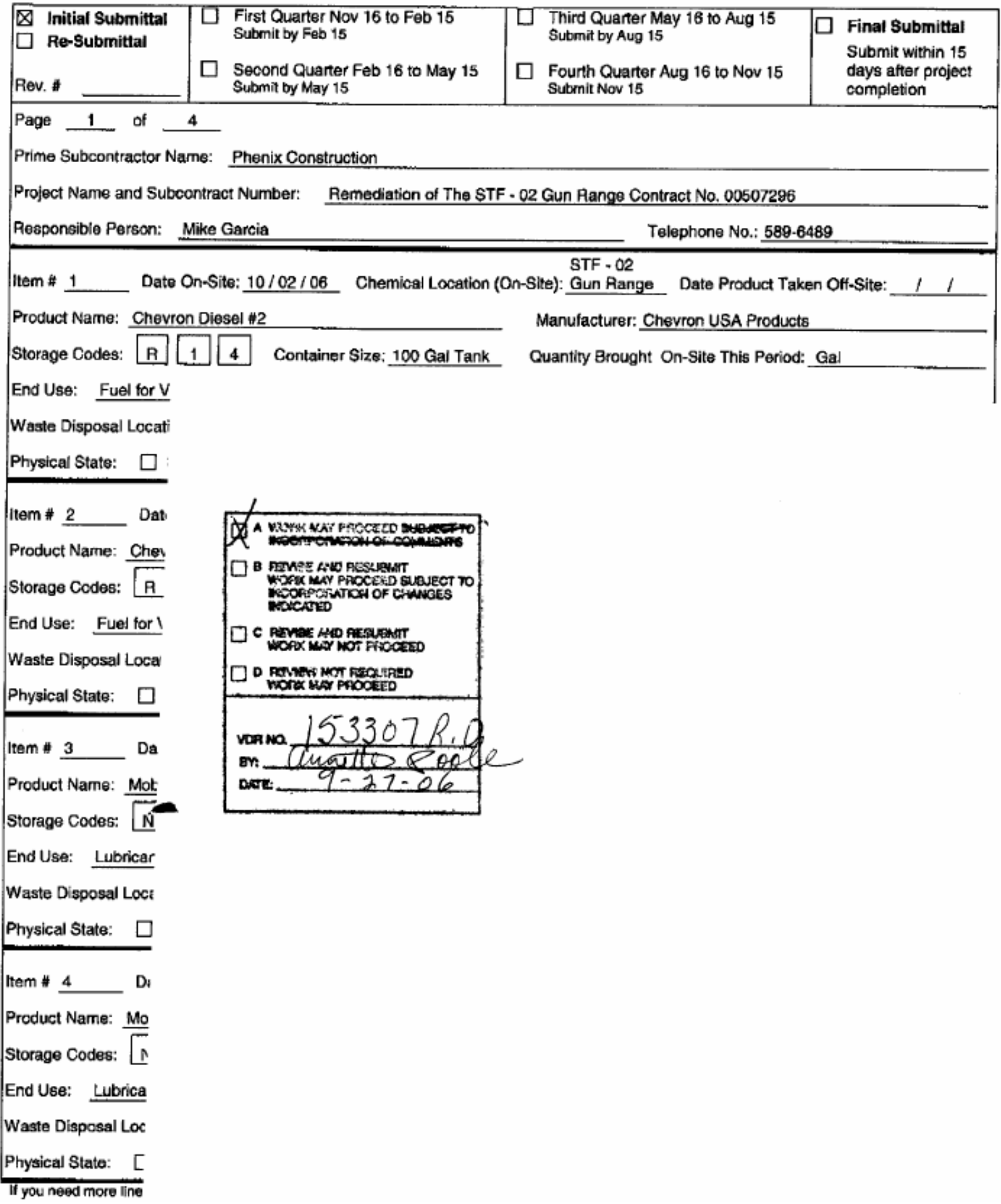


432.21

02/17/2000

Rev. 05

\section{SUBCONTRACTOR REPORTING FORM} CHEMICAL INVENTORY LIST

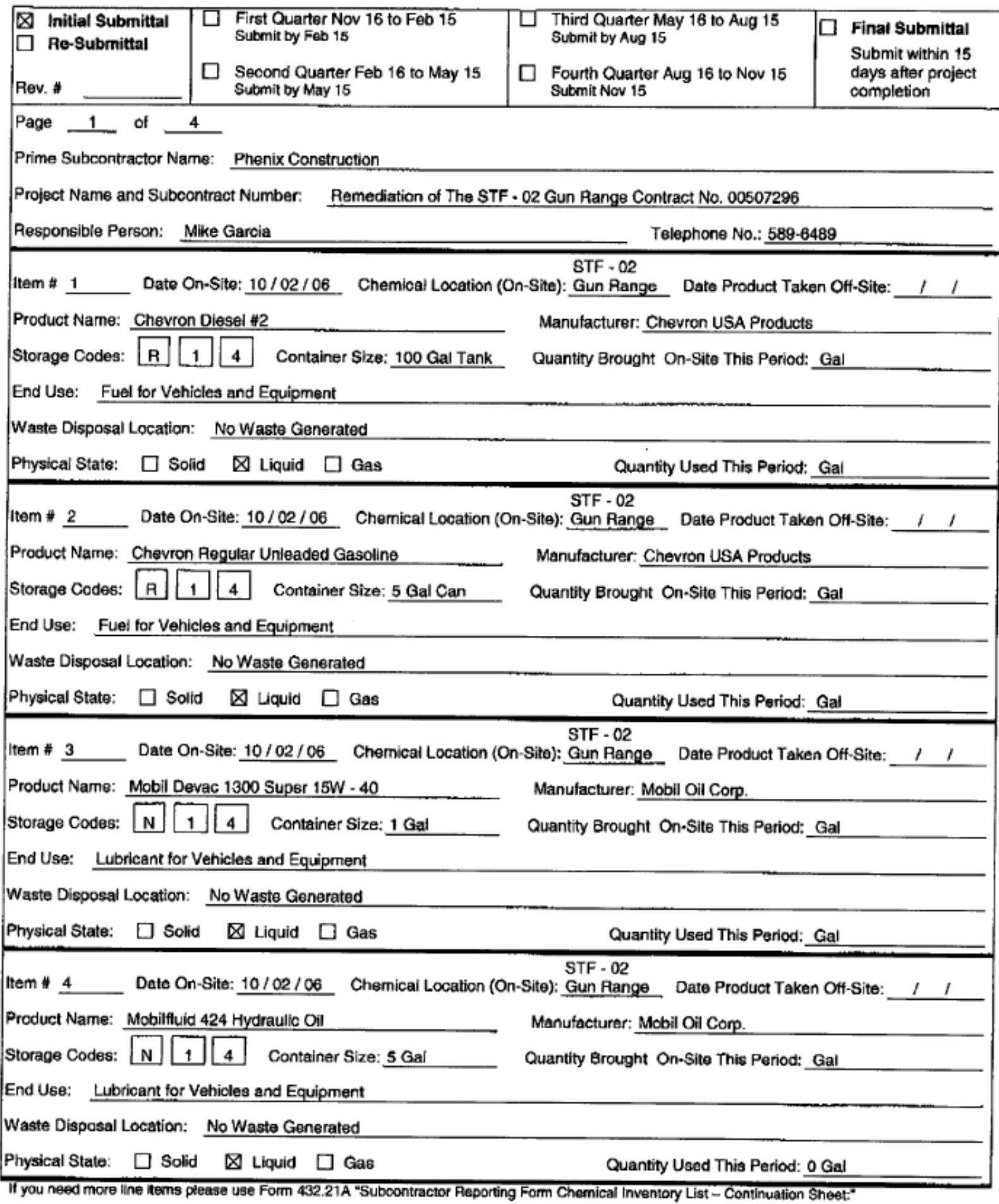


432.21A

$04 / 13 / 99$

Rev. 01

\section{SUBCONTRACTOR REPORTING FORM \\ CHEMICAL INVENTORY LIST \\ (CONTINUATION SHEET)}

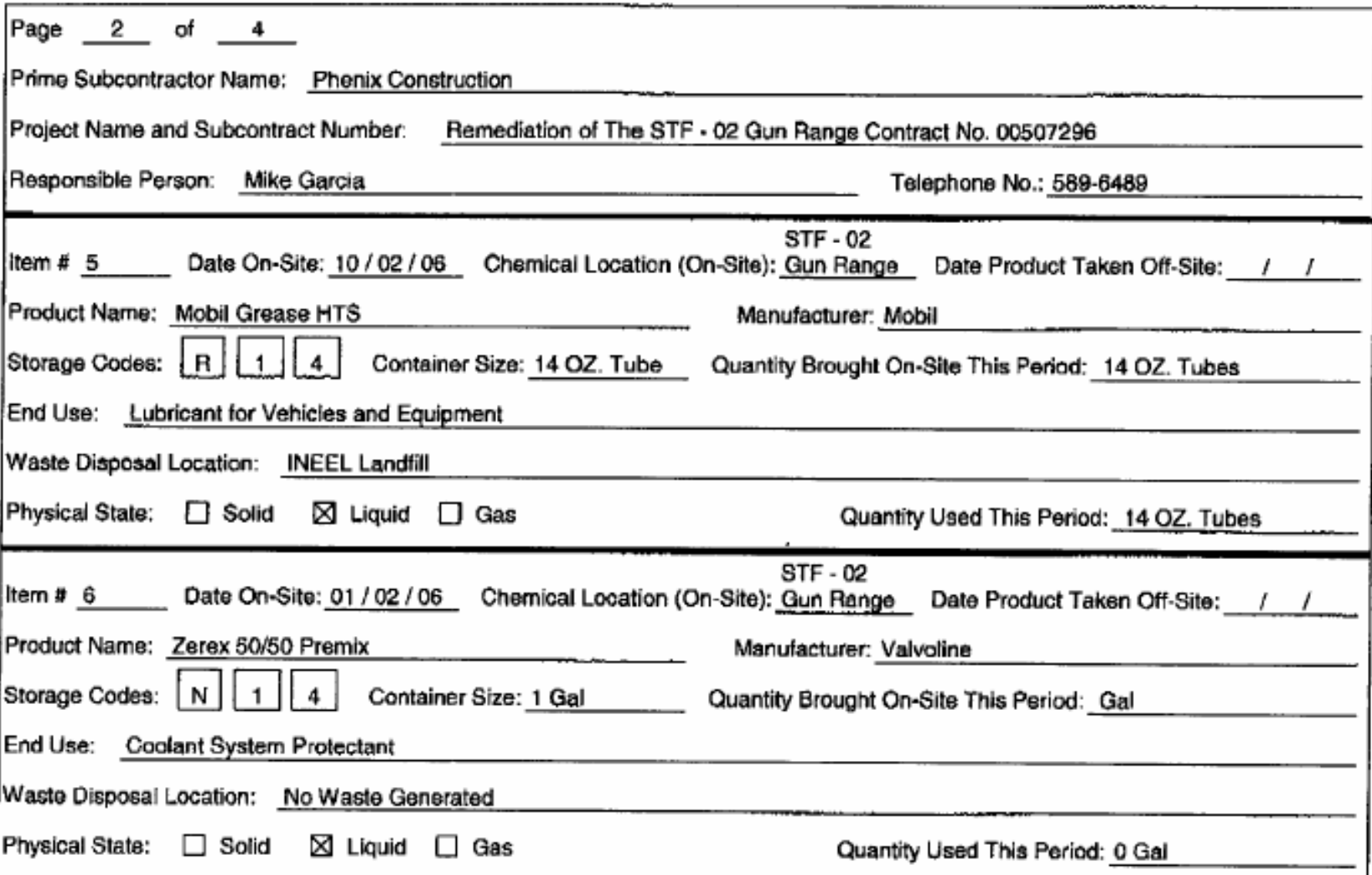

STF - 02

Item \# 7 Date On-Site: $10 / 02 / 06$ Chemical Location (On-Site): Gun Range Date Product Taken Off-Site: $/$,

Product Name: Premium Starting Fluid

Storage Codes: $\mathrm{F}\left[\begin{array}{l}2 \\ 4\end{array}\right.$ Container Size: 11 OZ. Can Quantity Brought On-Sito This Period: 11 OZ. Can

End Use: Help Start Equipment Motors (cold weather)

Waste Disposal Location: INEEL Landfill

Physical State: $\square$ Solid $\otimes$ Liquid $\square$ Gas Quantity Used This Period: $011 \mathrm{OZ}$. Can

STF - 02

Itern \# 8 Date On-Site: 10/02/06 Chemical Location (On-Site): Gun Range Date Product Taken Off-Site:

Product Name: WD-40 Manufacturer: WD 40 Company

Storage Codes: $\mathrm{F} 2 \mathrm{~F}$ Container Size: $16 \mathrm{OZ}$. Can Quantity Brought On-Site This Period: 16 OZ. Can

End Use: Lubricant

Waste Disposal Location: INEEL Landfill

Physical State: $\square$ Solid $\bigotimes$ Liquid $\square$ Gas Quantity Used This Period: $016 \mathrm{OZ}$. Can 
432.21A

04/13/99

Rev. 01

\section{SUBCONTRACTOR REPORTING FORM \\ CHEMICAL INVENTORY LIST \\ (CONTINUATION SHEET)}

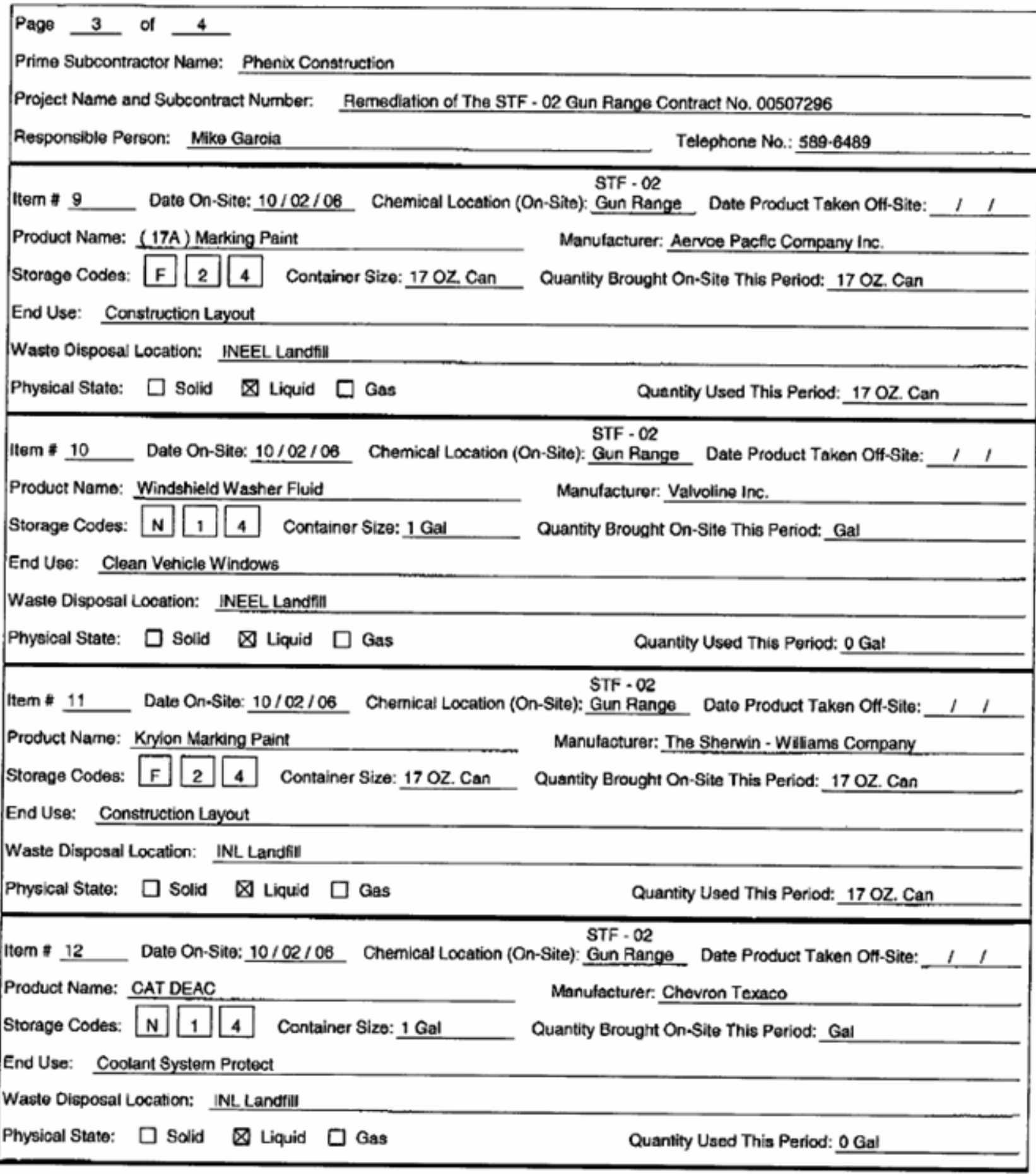


432.21A

04/13/99

Rev. 01

\section{SUBCONTRACTOR REPORTING FORM \\ CHEMICAL INVENTORY LIST}

(CONTINUATION SHEET)

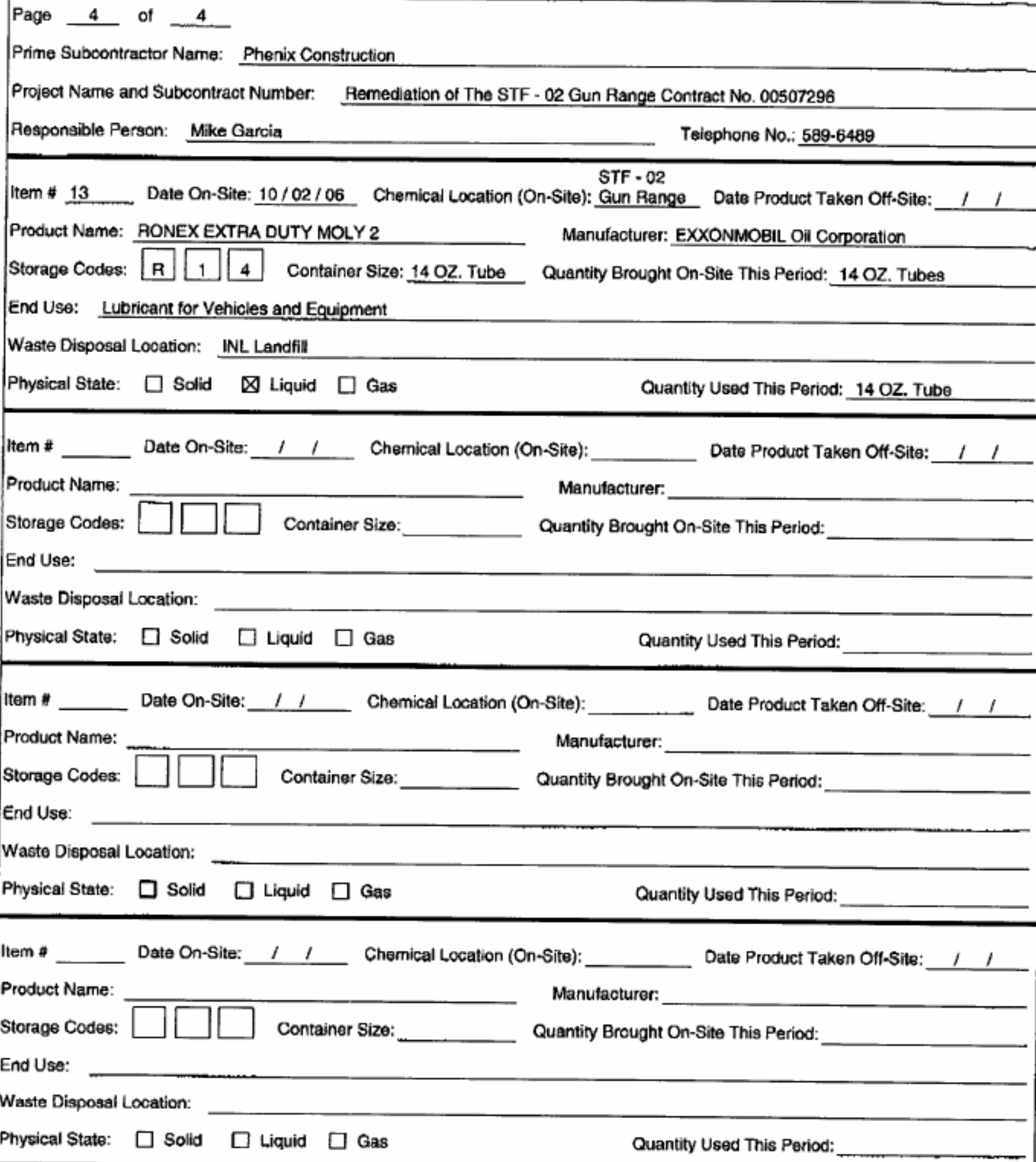




\section{Material Safety Data Sheet}

\section{SECTION 1 PRODUCT AND COMPANY IDENTIFICATION}

\section{DIESEL FUEL No. 2}

\section{Product Use: Fuel}

Product Number(s): CPS220122 (See Section 16 for Additional Product Numbers)

Synonyms: $15 \mathrm{~S}$ Diesel Fuel 2, Alternative Low Aromatic Diesel (ALAD), Calco LS Diesel 2, Calco ULS DF2.

Calco ULS Diesel 2, Chevron LS Diesel 2, Chevron ULS Diesel 2, Diesel Fuel Oil, Diesel Grade No. 2, Diesei No. 2-D S15, Diesel No. 2-D S500, Diesel No. 2-D S5000, Gas Oil, HS Diesel 2, HS Heating Fuel 2, Light Diesel Oil Grade No. 2-D, LS Diesel 2, LS Heating Fuel 2, Marine Diesel, RR Diesel Fuel, Texaco Diesel, Texaco Diesel No. 2. Uitra Low Sulfur Diesel 2

Company Identification

Chevron Products Company

Marketing. MSDS Coordinator

6001 Bollinger Canyon Road

San Ramon, CA 94583

United States of America

Transportation Emergency Response

CHEMTREC: (800) $424-9300$ or (703) $527-3887$

Health Emergency

Chevron Texaco Emergency information Center: Located in the USA. International collect calls accepted. (800)

231-0623 or (510) 231-0623

Proctuct information

MSOS Requests: (800) 689-3998

Technical Information: (510) 242-5357

SPECIAL NOTES: This MSDS covers all Chevron and Calco non-CARB Diesel No. 2 Fuels. The sulfur content is less than $0.5 \%$ (mass). Red dye is added to non-taxable fuel. (MSOS 6894)

\begin{tabular}{|l|l|l|}
\hline \multicolumn{2}{|l|}{ SECTION 2 COMPOSITION/ INFORMATION ON INGREDIENTS } \\
\hline COMPONENTS & CAS NUMBER & AMOUNT \\
\hline Diesel Fuel No. 2 & $68476-34-6$ & $100 \%$ weight \\
\hline Distillates, hydrodesulfurized, middle & $64742-80-9$ & $0-100 \%$ weight \\
\hline Distillates, straight run middle (gas oil, light) & $64741-44-2$ & $0-100 \%$ weight \\
\hline Kerosine & $8008-20-6$ & $0-25 \%$ weight \\
\hline Kerosine, hydrodesulfurized & $64742-81-0$ & $0-25 \%$ weight \\
\hline Distillates (petroleum), light catalytic cracked & $64741-59-9$ & $0-50 \%$ weight \\
\hline Naphthalene & $91-20-3$ & $0.02-0.2 \%$ weight \\
\hline Total sulfur & None & $0-0.5 \%$ weight \\
\hline
\end{tabular}

SECTION 3 HAZARDS IDENTIFICATION 


\section{EMERGENCY OVERVIEW}

- COMBUSTIBLE LIQUID AND VAPOR

- HARMFUL OR FATAL IF SWALLOWED - MAY CAUSE LUNG DAMAGE IF SWALLOWED

- CAUSES SKIN IRRITATION

- POSSIBLE CANCER HAZARD - MAY CAUSE CANCER BASED ON ANIMAL DATA

- TOXIC TO AQUATIC ORGANISMS

\section{IMMEDIATE HEALTH EFFECTS}

Eye: Not expected to cause prolonged or significant eye irritation.

Skin: Contact with the skin causes irritation. Skin contact may cause drying or defatting of the skin. Contact with the skin is not expected to cause an allergic skin response. Symptoms may include pain, itching, discoloration. swelling, and blistering. Not expected to be harmitul to internal organs if absorbed through the skin.

Ingestion: Because of its low viscosity, this material can directly enter the lungs, if swallowed, or if subsequently vomited. Once in the lungs it is very difficult to remove and can cause severe injury or death. May be irritating to mouth, throat, and stomach. Symptoms may include pain, nausea, vomiting, and diarrhea.

Inhalation: Mists of this material may cause respiratory irritation. Symptoms of respiratory irritation may include coughing and difficulty breathing. Breathing this material at concentrations above the recommended exposure limits may cause central nervous system effects. Central nervous system effects may include headache, dizziness, nausea, vomiting, weakness, loss of coordination, blurred vision, drowsiness, confusion, or disorientation. At extreme exposures, central nervous system effects may include respiratory depression, tremors or convulsions, loss of consciousness, coma or death.

\section{DELAYED OR OTHER HEALTH EFFECTS:}

Cancer: Prolonged or repeated exposure to this material may cause cancer. Whole diesel angine exhaust has been classified as a Group 2A carcinogen (probably carcinogenic to humans) by the International Agency for Research on Cancer (IARC). Dlesel exhaust particulate has been classiffed as reasonably anticipated to be a human carcinogen in the National Toxicology Program's Ninth Report on Carcinogens. The National Institute of Occupational Safety and Health (NIOSH) has recommended that whole diesel exhaust be regarded as potentially causing cancer. Olesel engine exhaust is known to the State of California to cause cancer. Contains naphthalene, which has been classified as a Group 2B carcinogen (possibly carcinogenic to humans) by the international Agency for Research on Cancer (IARC).

See Section 11 for additional intormation. Risk depends on duration and level of exposure.

\section{SECTION 4 FIRST AID MEASURES}

Eye: No specific first aid measures are required. As a precaution, remove contact lenses, if worn, and flush eyes with water.

Skin: Wash skin with water immediately and remove contaminated clothing and shoes. Get medical attention if any symptoms develop. To remove the material from skin, use soap and water. Discard contaminated clothing and shces or thoroughly clean before reuse.

Ingestion: If swallowed, get immediate medical attention. Do not induce vomiting. Never give anything by mouth to an unconscious person. If swallowed, get medical attention. Do not induce vomiting. Never give anything by mouth to an unconscious person.

Inhalation: Move the exposed person to fresh air. If not breathing. give artificial respiration. If breathing is difficult, give oxygen. Get medical attention if breathing difficulties continue.

Note to Physicians: ingestion of this product or subsequent vomiting may result in aspiration of light hydrocarbon liquid, which may cause pneumonitis.

\section{SECTION 6 FIRE FIGHTING MEASURES}

See Section 7 for proper handling and storage.

FIRE CLASSIFICATION:

OSHA Classification (29 CFR 1910.1200): Combustible liquid.

NFPA RATINGS: Health: 0 Flammability: 2 Reactivity: 0 
FLAMMABLE PROPERTIES:

Flashpoint: (Pensky-Martens Closed Cup) $52{ }^{\circ} \mathrm{C}\left(125^{\circ} \mathrm{F}\right)$ (Min)

Autoignition: $257^{\circ} \mathrm{C}\left(494^{\circ} \mathrm{F}\right)$

Flammabillty (Explosive) Limits (\% by volume in air): Lower: 0.6 Upper: 4.7

EXTINGUISHING MEDIA: Use water fog, foam, dry chemical or carbon dioxide ( $\mathrm{CO} 2)$ to extinguish flames. PROTECTION OF FIRE FIGHTERS:

Fire Fighting Instructions: For fires involving this materiai, do not enter any enclosed or confined fire space without proper protective equipment, including self-contained breathing apparatus.

Combustion Products: Highly dependent on combustion conditions. A complex mixture of airborne solids, liquids, and gases including carbon monoxide, carbon dioxide, and unidentified organic compounds will be gvolved when this material undergoes combustion.

\section{SECTION 6 ACCIDENTAL RELEASE MEASURES}

Protective Measures: Eliminate all sources of ignition in the vicinity of the spill or released vapor. If this materiat is released into the work area, evacuate the area immediately. Monitor area with combustible gas indicator. Spill Management: Stop the source of the reiease if you can do it without risk. Contain release to prevent further contamination of soil, surface water or groundwater. Clean up spill as soon as possible, observing precautions in Exposure Controls/Personal Protection. Use appropriate techniques such as applying non-combustible absorbent materials or pumping. All equipment used when handing the product must be grounded. A vapor suppressing foam may be used to reduce vapors. Use clean non-sparking tools to collect absorbed material. Where feasible and appropriate, remove contaminated soil. Place contaminated materials in disposable containers and dispose of in a manner consistent with applicable regulations.

Reporting: Report spills to local authorities and/or the U.S. Coast Guard's National Response Center at (800) 424-8802 as appropriate or required.

\section{SECTION 7 HANDLING AND STORAGE}

Precautionary Measures: Liquid evaporates and forms vapor (fumes) which can catch fire and burn with explosive force. Invisible vapor spreads easily and can be set on fire by many sources such as pilot lights, welding equipment, and electrical motors and switches. Fire hazard is greater as liquid temperature rises above
$29 \mathrm{C}(85 \mathrm{~F})$.

Do not get in eyes, on skin, or on clothing. Do not taste or swallow. Do not breathe vapor or fumes. Do not breathe mist. Wash thoroughly after handling. Keep out of the reach of children.

Unusual Handling Hazards: WARNING! Do not use as portable heater or appliance fuet. Toxic fumes may accumulate and cause death.

General Handling Information: Avoid contaminating soil or reieasing this material into sewage and drainage systems and bodies of water.

Static Mazard: Electrostatic charge may accumulate and create a hazardous condition when handling this material. To minimize this hazard, bonding and grounding may be necessary but may not, by themselves, be sufficient. Review all operations which have the potential of generating and accumulating an electrostatic charge and/or a flammable atmosphere (including tank and container filling, splash filling, tank cleaning, sampling, gauging, switch loading, fittering, mixing, agitation, and vacuum truck operations) and use appropriate mitigating procedures. For more intormation, refer to OSHA Standard 2,9 CFR 1910.106, "Flammable and Combustible Liquids', National Fire Protection Association (NFPA 77, 'Recommended Practice on Static Electricity', and/or the American Petroleum Institute (API) Recommended Practice 2003, 'Protection Against Ignitions Arising Out of
Static, Lightning, and Stray Currents'.

General Storage Information: DO NOT USE OR STORE near heat, sparks, flames, or hot surfaces . USE AND STORE ONLY IN WELL VENTILATED AREA. Keep container closed when not in use,

Container Warnings: Container is not designed to contain pressure. Do not use pressure to empty container or it may rupture with explosive force. Empty containers retain product residue (solid, liquid, and/or vapor) and can be dangerous. Do not pressurize, cut, weid, braze, solder, drill, grind, or expose such containers to heat, flame, sparks. static electricity, or other sources of ignition. They may explode and cause injury or death. Empty containers should be completely drained, properly closed. and promptly returned to a drum reconditioner or
disposed of properly.

SECTION 8 EXPOSURE CONTROLS/PERSONAL PROTECTION

GENERAL CONSIDERATIONS:

Consider the potential hazards of this material (see Section 3), applicable exposure limits, job activities, and other substances in the work place when designing engineering controls and selecting personal protective equipment. If 
engineering controls or work practices are not adequate to prevent exposure to harmful levels of this material, the personal protective equipment listed below is recommended. The user should read and understand all instructions and limitations supplied with the equipment since protection is usually provided for a limited time or under certain circumstances.

\section{ENGINEERING CONTROLS:}

Use process enclosures, local exhaust ventilation, or other engineering controls to control airborne levels below the recommended exposure limits.

PERSONAL PROTECTIVE EQUIPMENT

Eye/Face Protection: No special өye protection is normally required. Where splashing is possible, wear safety glasses with side shields as a good safety practice.

Skin Protection: Wear protective clothing to prevent skin contact. Selection of protective clothing may include gloves, apron, boots, and complete facial protection depending on operations conducted. Suggested materiais for protective gloves include: Chlorinated Polyethylene (or Chlorosulfonated Polyethylene). Nitrile Rubber, Polyurethane, Viton.

Respiratory Protection: Determine if airborne concentrations are below the recommended occupational exposure limits for jurisdiction of use. If airborne concentrations are above the acceptable limits, wear an approved respirator that provides adequate protection from this material, such as: Air-Purifying Respirator for Organic Vapors.

When used as a fuel, this material can produce carbon monoxide in the exhaust. Determine if airborne concentrations are below the occupational exposure limit for carbon monoxide. If not, wear an approved positivepressure air-supplying respirator.

Use a positive pressure air-supplying respirator in circumstances where air-purifying respirators may not provide adequate protection.

Occupational Exposure Limits:

\begin{tabular}{|c|c|c|c|c|c|}
\hline Component & Agency & IIWA & STEL & Ceillng & Notation \\
\hline Diesel Fuel No. 2 & ACGIH & $100 \mathrm{mg} / \mathrm{m} 3$ & - & - & Skin A3 \\
\hline Diesel Fuel No. 2 & $\mathrm{CVX}$ & - & $1000 \mathrm{mg} / \mathrm{m} 3$ & - & - \\
\hline Kerosine & ACGIH & $200 \mathrm{mg} / \mathrm{m} 3$ & - & $E$ & Skin A3 \\
\hline Kerosine & $\operatorname{crx}$ & - & $1000 \mathrm{mg} / \mathrm{m} 3$ & E & $E$ \\
\hline Kerosine, hydrodesulfurized & ACGIH & $200 \mathrm{mg} / \mathrm{m} 3$ & - & E & Skin $\mathrm{A} 3$ \\
\hline Kerosine, hydrodesulfurized & CVX & $E=$ & $1000 \mathrm{mg} / \mathrm{ms}$ & 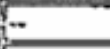 & - \\
\hline Naphthalene & ACGIH & $10 \mathrm{ppm}$ & $15 \mathrm{ppm}$ & - & A4 Skin \\
\hline Naphthalene & OSHAZ-1 & $50 \mathrm{mg} / \mathrm{m} 3$ & - & E & - \\
\hline
\end{tabular}

\section{SECTION 9 PHYSICAL AND CHEMICAL PROPERTIES}

Attention: the data below are typical values and do not constitute a specification.

Color: Varies depending on specification

Physical State: Liquid

Odor: Petroleum odor

$\mathrm{pH}$ : Not Applicable

Vapor Pressure: $0.04 \mathrm{kPa}$ (Approximate) @ $40^{\circ} \mathrm{C}\left(104^{\circ} \mathrm{F}\right.$ )

Vapor Density $($ Air $=1):>1$

Boiling Point: $175.6^{\circ} \mathrm{C}\left(348^{\circ} \mathrm{F}\right)=370^{\circ} \mathrm{C}\left(698^{\circ} \mathrm{F}\right)$

Solubility: Soluble in hydrocarbons; insoluble in water

Freezing Point: Not Applicable

Melting Point: Not Applicable

Specific Gravity: $0.8-0.88$ @ $15.6^{\circ} \mathrm{C}\left(60.1^{\circ} \mathrm{F}\right)$ (Typical)

Viscosity: $1.9 \mathrm{cSt}-4.1 \mathrm{cSt} @ 40^{\circ} \mathrm{C}\left(104^{\circ} \mathrm{F}\right)$

SECTION 10 STABILITY AND REACTIVITY

Chemical Stability: This materiat is considered stable under normal ambient and anticipated storage and handling conditions of temperature and pressure. 
Incompatibility With Other Materials: May react with strong acids or strong oxidizing agents, such as chlorates. nitrates, peroxides, etc.

Hazardous Decomposition Products: None known (None expected)

Hazardous Polymerization: Hazardous polymerization will not occur.

SECTION 11 TOXICOLOGICAL, INFORMATION

IMMEDIATE HEALTH EFFECTS

Eye Irritation: The eye irritation hazard is based on evaluation of data for similar materials or product components.

Skin Irritation: The skin irritation hazard is based on evaluation of data for similar materials or product components.

Skin Sensitization: This material did not cause skin sensitization reactions in a Buehier guinea pig test. Acute Dermai Toxicify: LDSO: $>5 \mathrm{ml} / \mathrm{kg}$ (rabbit).

Acute Oral Toxicity: LD50: $>5 \mathrm{ml} / \mathrm{kg}$ (rat)

Acute Inhalation Toxicity: 4 hour(s) LC50: > 5mg/l (rat).

\section{ADOITIONAL TOXICOLOGY INFORMATION:}

This product contains gas oils.

CONCAWE (product dossier 95/107) has summenzed current health, safety and environmental data available for a number of gas oils, typically hydrodesulfurized middle distillates, CAS 64742-80-9, straight-run middie distillates, CAS 64741-44-2, and/or light cat-cracked distillate CAS 64741-59-9. CARCINOGENICITY: All materials tested have caused the development of skin tumors in mice, but all featured severe skin irritation and sometimes a long latency period before tumors developed. Straight-run and cracked gas oil samples were studied to determine the influence of dermal irritation on the carcinogenic activity of middle distillates. At non-irritant doses the straight-run gas oil was not carcinogenic, but at irritant doses, weak activity was dernonstrated. Cracked gas oils. when diluted with mineral oil, demonstrated carcinogenic activity irrespective of the occurrence of skin irritation. Gas oils were tested on male mice to study tumor initiating/promoting activity. The results demonstrated that while a straight-run gas oil sample was neither an initiator or promotor, a biend of straight-run and FCC stock was both a tumor initiator and a promoter.

GENOTOXICITY. Hydrotreated \& hydrodesulfurized gas oils range in activity from inactive to weakly positive in in-vitro bacterial mutagenicity assays. Mouse lymphoma assays on straight-run gas oils without subsequent hydrodesulphurization gave positive results in the presence of S9 metabolic activation. In-vivo bone marrow cytogenetics and sister chromatic exchange assay exhibited no activity for straight-run components with or without hydrodesulphurization. Thermally or catalytically cracked gas oils tested with in-vitro bacterial mutagenicity assays in the presence of $\mathbf{S 9}$ metabolic activation were shown to be mutagenic. In-vitro sister chromatic exchange assays on cracked gas oil gave equivocal results both with and without $\$ 9$ metabolic activation. In-vivo bone marrow cytogenetics assay was inactive for two cracked gas oil samples. Three hydrocracked gas oils were tested with in-vitro bacterial mutagenicity assays with S9, and one of the three gave positive results. Twelve distillate fuel samples were tested with in-vitro bacterial mutagenicity assays \& with S9 metabolic activation and showed negative to weakly positive results. In one series, activity was shown to be related to the PCA content of samples tested. Two in-vivo studies were also conducted. A mouse dominant lethal assay was negative for a sample of diesel fuel. In the other study, 9 samples of No 2 heating oil containing $50 \%$ cracked stocks caused a slight increase in the number of chromosomal aberrations in bone marrow cytogenetics assays. DEVELOPMENTAL TOXICITY: Diesel furef vapor did not cause fetotoxic or teratogenic effects when pregnant rats were exposed on days 6-15 of pregnancy. Gas oils were applied to the skin of pregnant rats daily on days $0-19$ of gestation. All but one (coker light gas oil) caused fetotoxicity (increased resorptions, reduced litter weight, reduced litter size) at dose levels that were also maternally toxic.

This product contains naphthalene. GENERAL TOXICITY: Exposure to naphthalene has been reported to cause methemoglobinemia and/or hemolytic anemia, especially in humans deficient in the enzyme glucose-6-phosphate dehydrogenase. Laboratory animals given repeated oral doses of naphthaiene have developed cataracts. REPRODUCTIVE TOXICITY AND BIRTH DEFECTS: Naphthalene did not cause birth defects when administered orally to rabbits, rats, and mice during pregnancy, but slightly reduced litter size in mice at dose levels that were lethal to the pregnant females. Naphthalene has been reported to cross the human placenta. GENETIC TOXICITY: Naphthalene caused chromosome aberrations and sister chromatid exchanges in Chinese hamster ovary cells, but was not a mutagen in several other in-vitro tests. CARCINOGENICITY: In a study conducted by the National Toxicology Program (NTP), mice exposed to 10 or $30 \mathrm{ppm}$ of naphthalene by inhalation daily for two years had chronic inflammation of the nose and lungs and increased incidences of metaplasia in those tissues. The incidence of benign lung tumors (aiveolar/bronchiolar adenomas) was significantly increased in the high-cose 
female group but not in the male groups. In another two-year inhalation study conducted by NTP, exposure of rats to 10. 30, and $60 \mathrm{ppm}$ naphthalene caused increases in the incidences of a variety of nonneoplastic lesions in the nose. Increases in nasal tumors were seen in both sexes, including olfactory neuroblastomas in females at 60 ppm and adenomas of the respiratory epithelium in males at all exposure levels. The relevance of these effects to humans has not been established. No carcinogenic effect was reported in a 2 -year feeding study in rats receiving naphthalene at $41 \mathrm{mg} / \mathrm{kg} / \mathrm{day}$. This product may contain significant amounts of Polynuclear Aromatic Hydrocarbons (PAH's) which have been shown to cause skin cancer after prolonged and frequent contact with the skin of test animals. Brief or intermittent skin contact with this product is not expected to have serious effects if it is washed from the skin. While skin cancer is unlikely to occur in human beings following use of this product, skin contact and breathing. of mists, vapors or dusts should be reduced to a minimum.

\section{SECTION 12 ECOLOGICAL INFORMATION}

\section{ECOTOXICITY}

96 hour(s) LC50: 21-210 mg/ (Saimo gairdneri)

48 hour(s) EC50: $20-210 \mathrm{mg} / /$ (Daphnia magna)

72 hour(s) EC50: $2.6-25 \mathrm{mg} / \mathrm{l}$ (Raphidocellus subcapitata)

This material is expected to be toxic to aquatic organisms.

\section{ENVIRONMENTAL FATE}

On release to the environment the lighter components of diesel fuel will generally evaporate but depending on local environmental conditions (temperature. wind, mixing or wave action, soil type, etc.) the remainder may become dispersed in the water column or absorbed to soil or sediment. Diesel fuel would not be expected to be readily biodegradabie. In a modifled Strum test (OECD method 301B) approximately $40 \%$ biodegradation was recorded over 28 days. However, it has been shown that most hydracarbon components of diesel fuel are degraded in soil in the presence of oxygen. Under anaerobic conditions, such as in anoxic sediments, rates of blodegradation are negligible.

\section{SECTION 13 DISPOSAL CONSIDERATIONS}

Use material for its intended purpose or recycle if possible. This material, if it must be discarded, may meet the criteria of a hazardous waste as defined by US EPA under RCRA ( 40 CFR 261) or other State and local regulations. Measurement of certain physical properties and analysis for regulated components may be necessary to make a correct determination. If this material is classifled as a hazardous waste, federal law requires disposal at a licensed hazardous waste disposal facility.

SECTION 14 TRANSPORT INFORMATION

The description shown may not apply to all shipping situations. Consult 49CFR, or appropriate Dangerous Goods Regulations, for additional description requirements (e.g., technical name) and mode-specific or quantity-specific shipping requirements.

DOT Shipping Description: GAS OIL, Combustible Liquid, UN1202,HI

IMOAMDG Shipping Description: GAS OIL.3,UN1202,III, FLASH POINT SEE SECTION 5

ICAO/IATA Shipping Description: GAS OIL,3,UN1202,III, SECTION 15 REGULATORY INFORMATION

EPCRA 311/312 CATEGORIES: 1. Immediate (Acute) Health Effects: YES

2. Delayed (Chronic) Health Effects: YES

3. Fire Hazard: YES

4. Sudden Release of Pressure Hazard: NO

5. Reactivity Hazard: NO

REGULATORY LISTS SEA.RCHED: 


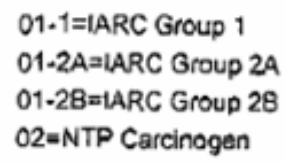

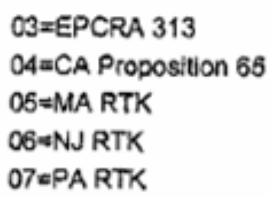

The following components of this material are found on the regulatory lists indicated.

Diesel Fuel No. $2 \quad 07$

Oistillates, straight run middle (gas oil, light) $\quad 06$

Kerosine $05,06,07$

Naphthalene 01-2B, 03, 04, 05, 06, 07

CERCLA REPORTABLE QUANTITIES(RQ)/EPCRA 302 THRESHOLD PLANNING QUANTITIES(TPQ):

\begin{tabular}{|l|l|l|l|}
\hline Component & Component RQ & Component TPQ & Product RQ \\
\hline Naphthalene & $100 \mathrm{lbs}$ & None & $55556 \mathrm{bs}$ \\
\hline
\end{tabular}

CHEMICAL INVENTORIES:

All components comply with the following chemical inventory requirements: AICS (Austrailia), DSL (Canada), EINECS (European Union), IECSC (China), KECI (Korea), PICCS (Philippines), TSCA (United States).

NEW JERSEY RTK CLASSIFICATION:

Refer to components listed in Section 2. Under the New Jersey Right-to-Know Act L. 1983 Chapter 315 N.J.S.A. 34:5A-1 et. seq., the product is to be identified as follows: DIESEL. FUEL

WHMIS CLASSIFICATION:

Class B, Division 3: Combustible Liquids

Class D, Division 2, Subdivision A: Very Toxic Material -

Carcinogenicity

Class D, Division 2. Subdivision B: Toxic Material -

Skin or Eye Irritation

\section{SECTION 16 OTHER INFORMATION}

NFPA RATINGS: Health: 0 Flammability: 2 Reactivity: 0

(O-Least, 1-Slight, 2-Moderate, 3-High. 4-Extreme, PPE:- Personal Protection Equipment Index recommendation. - Chronic Effect indicator). These values are obtained using the guidelines or published evaluations prepared by the National Fire Protection Association (NFPA) or the National Paint and Coating Association (for HMIS ratings).

Additional Product Number(s): CPS225114, CPS225115. CPS225160, CPS266176, CPS270005, CPS270094, CPS270095, CPS270096, CPS271006, CPS272093, CPS272102, CPS272126, CPS272152, CPS272185. CPS272190, CPS272195, CPS272593, CPS272601, CPS272693, CPS272793, CPS273003, CPS273030, CPS273053, CPS275000

REVISION STATEMENT: This revision updates the following sections of this Material Safety Data Sheet: 1. Revision Date: 12/13/2004

\begin{tabular}{|c|c|}
\hline TLV - Throshold Limit Value & TWA - Time Weighted Average \\
\hline STEL - Short-term Exposure Limit & PEL - Permissible Exposure Limit \\
\hline & CAS - Chernical Abstract Servic Number \\
\hline $\begin{array}{l}\text { ACGIH A American Conference of Government } \\
\text { Industrial Hygieniats }\end{array}$ & IMO/MDG - International Maritime Dangerous Goods Code \\
\hline API-American Petroleum Institute & MSDS - Material Safety Data Shøet \\
\hline CVX - ChevronTexaco & NFPA - National Fire PIotection Association (USA) \\
\hline DOT - Department of Transportation (USA) & NTP - National Toxicology Program (USA) \\
\hline
\end{tabular}


HIARC - International Agency for Research on Cancer OSHA - Occupational Safety and Health Administration Prepared according to the OSHA Hazard Communication Standard (29 CFR 1910.1200) and the ANSI MSDS Standard (Z400.1) by the ChevronTexaco Energy Research \& Technology Company, 100 Chevron Way, Rjchmond, California 94802.

The above information is based on the data of which we are aware and is believed to be correct as of the date hereof. Since this information may be applied under conditions beyond our control and with which we may be unfamiliar and sincs data made available subsequent to the date heroof may suggest modifications of the information, we do not assume any responsibility for the results of its use. This information is furnished upon condition that the person receiving it shall make his own determination of tha suitability of the material for his particutar purpose. 


\section{Material Safety Data Sheet}

\section{SECTION 1 PRODUCT AND COMPANY IDENTIFICATION}

\section{CHEVRON and TEXACO REGULAR UNLEADED GASOLINES}

Product Number(s): CPS201000 [See Section 16 for Additional Product Numbers]

Synonyms: Calco Regular Unleaded Gasoline, Chevron Regular Unleaded Gasoline, Texaco Unleaded Gasoline

Company Identification

Chevron Products Company

Marketing, MSDS Coordinator

6001 Boilinger Canyon Road

San Ramon, CA 94583

United States of America

Transportation Emergency Response

CHEMTREC: (800) 424-9300 or (703) $527 \cdot 3887$

Health Emergency

ChevronTexaco Emergency information Center: Located in the USA. International collect calls accepted. (800)

231.0623 or $(510) 231.0623$

Product Information

Technical Information: (510) 242-5357

SPECIAL NOTES: This MSOS applies to: all motor gasoline.

\begin{tabular}{|c|c|c|}
\hline \multicolumn{3}{|c|}{ SECTION 2 COMPOSITION/INFORMATION ON INGREDIENTS } \\
\hline COMPONENTS & CAS NUMBER & AMOUNT \\
\hline Gasoline & $86290-81-5$ & $100 \%$ volume \\
\hline Benzene & $71-43-2$ & $0.1-4.9 \%$ volume \\
\hline Toluene (methylbenzene) & $108-88-3$ & 1 - $17 \%$ volume \\
\hline Ethyl benzene & $100-41-4$ & $0.1 \cdot 3 \%$ volume \\
\hline $\begin{array}{l}\text { Xylene (contains o-, m-, \& p-xylene isomers in varying } \\
\text { amounts) }\end{array}$ & $1330-20-7$ & 1 - $15 \%$ volume \\
\hline Butane & $106-97-8$ & 1 - $12 \%$ volume \\
\hline Heptane & $142-82-5$ & $1.4 \%$ volume \\
\hline Hexane & $110-64-3$ & 1 - 5 \%volume \\
\hline Cyclohexane & $110-82-7$ & 1 - $3 \%$ volume \\
\hline Methylcyclonexane & $108-87-2$ & $1-2 \%$ volume \\
\hline Pentane, 2.2.4-trimethyl- (Isooctane) & $540-84-1$ & 1 - $13 \%$ volume \\
\hline Naphthalene & $91-20-3$ & $0.1-2 \%$ volume \\
\hline Ethanol & $64-17-5$ & $0.10 \%$ volume \\
\hline Methyl tert-butyl ether (NTBE) & $1634-04-4$ & $0-15 \%$ volume \\
\hline
\end{tabular}




\begin{tabular}{|l|l|l|}
\hline Tertiary amyl methyl ether (TAME) & $994-05-8$ & $0-17 \%$ volume \\
\hline Ethyl tert-butyl ether (ETBE) & $637-92-3$ & $0-18 \%$ volume \\
\hline
\end{tabular}

Motor gasoline is considered a mixture by EPA under the Toxic Substances Control Act (TSCA). The refinery streams used to blend motor gasoline are at on the TSCA Chemical Substances Inventory. The appropriate CAS number for refinery blended motor gasoline is $86290-81-5$. The product specifications of motor gasoline sold in your area will depend on applicable Federal and State regulations.

\section{SECTION 3 HAZARDS IDENTIFICATION}

\section{EMERGENCY OVERVIEW}

- EXTREMELY FLAMMABLE LIQUID AND VAPOR. VAPOR MAY CAUSE FLASH FIRE

- HARMFUL OR FATAL IF SWALLOWED - MAY CAUSE LUNG DAMAGE IF SWALLOWED

- VAPOR HARMFUL

- CAUSES EYE AND SKIN IRRITATION

- LONG-TERM EXPOSURE TO VAPOR HAS CAUSED CANCER IN LABORATORY ANIMALS

- KEEP OUT OF REACH OF CHILDREN

- TOXIC TO AQUATIC ORGANISMS

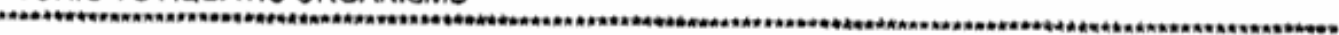

\section{IMMEDIATE HEALTH EFFECTS}

Eye: Contact with the eyes causes irritation. Symptoms may include pain, tearing, reddening, swelling and impaired vision.

Skin: Contact with the skin causes irritation. Skin contact may cause drying or defatting of the skin. Contact with the skin is not expected to cause an allergic skin response. Symptoms may include pain, itching, discoloration, swelling, and blistering. Not expected to be harmful to internal organs if absoroed through the skin.

Ingestion: Because of its low viscosity, this material can diroctly enter the lungs, if swailowed, or if subsequently vomited. Once in the lungs it is very difficult to remove and can cause severe injury or death.

Inhalation: The vapor or fumes from this material may cause respiratory irritation. Symptorns of respiratory irritation may inciude coughing and difficulty breathing. Breathing this materiai at concentrations above the recommended exposure limits may cause central nervous system effects. Central nervous system effects may include headache, dizziness, nausea, vomiting, weakness, loss of coordination, blurred vislon, drowsiness, confusion, or disorientation. At extreme exposures, central nervous system effects may include respiratory depression, tromors or convulsions, loss of consciousness, coma or death.

\section{DELAYED OR OTHER HEALTH EFFECTS:}

Reproduction and Birth Defects: This material is not expected to cause birth defects or other harm to the developing fetus based on animal data.

Cancer: Prolonged or repeated exposure to this material may cause cancer. Gasoline has been classified as a Group 2B carcinogen (possibly carcinogenic to humans) by the Internationai Agency for Research on Cancer (IARC). Whole gasoline exhaust has been classified as a Group 28 carcinogen (possibly carcinogenic to humans) by the
Intemational Agency for Research on Cancor (IARC).

Contains benzene, which has been classifled as a carcinogen by the National Toxicology Program (NTP) and a Group 1 carcinogen (carcinogenic to humans) by the International Agency for Research on Cancer (IARC). Contains ethylbenzene which has been classifted as a Group 28 carcinogen (possibly carcinogenic to humans) by the Intemational Agency for Research on Cancer (IARC).

Contains naphthalene, which has been classified as a Group 28 carcinogen (possibly carcinogenic to humans) by the International Agency for Research on Cancer (IARC).

See Section 11 for additional information. Risk depends on duration and level of exposure.

SECTION $;$ FIRST AID MEASURES 
Eye: Flush eyes with water immediately while holding the eyelids open. Rernove contact lenses, if worn, after initial flushing, and continue flushing for at least 15 minutes. Get medical attention if irritation persists.

Skin: Wash skin with water immediately and remove contaminated clothing and shoes. Get medical attention if any symptoms develop. To remove the material from skin, use soap and water. Discard contaminated clothing and shoes or thoroughly clean before reuse.

Ingestion: If swallowed. get immediate medical attention. Do not induce vomiting. Never give anything by mouth to an unconscious person. If swallowed, get medical attention. Do not induce vomiting. Never give anything by mouth to an unconscious person.

Inhalation: Move the exposed person to fresh air. If not breathing, give artificiai respiration. If breathing is difficuit, give oxygen. Get medical attention if broathing difficulties continue.

Note to Physicians: Ingestion of this product or subsequent vomiting may result in aspiration of light hydrocarbon liquid, which may cause pneumonitis.

SECTION 5 FIRE FIGHTING MEASURES

See Section 7 for proper handling and storage.

FIRE CLASSIFICATION:

OSHA Classification (29 CFR 1910.1200): Flammable liquid.

NFPA RATINGS: Health: 1 Flammability: 3 Reactivity: 0

FLAMMABLE PROPERTIES:

Flashpoint: (Tagliabue Closed Cup ASTM D56) $<-45^{\circ} \mathrm{C}\left(<-49^{\circ} \mathrm{F}\right)$

Autoignition: $>280^{\circ} \mathrm{C}\left(>536^{\circ} \mathrm{F}\right)$

Flammability (Explosive) Limits (\% by volume in air): Lower: 1.4 Upper: 7.6 (Typical)

EXTINGUISHING MEDIA: Dry Chemical, CO2, AFFF Foam or alcohoi resistant foam if $>15 \%$ volume polar solvents (oxygenates).

PROTECTION OF FIRE FIGHTERS:

Fire Fighting Instructions: Use water spray to cool fire-exposed containers and to protect personnel. For fires involving this material, do not enter any enclosed or confined fire space without proper protective equipment, including self-contained breatining apparatus.

Combustion Products: Highly dependent on combustion conditions. A complex mixture of airborne solids. liquids, and gases including carbon monoxide, carbon dioxide, and unidentified organic compounds will be evolved when this material undergoes combustion.

\section{SECTION 6 ACCIDENTAL RELEASE MEASURES}

Protective Measures: Eliminate all sources of ignition in the vicinity of the spill or released vapor. If this material is released into the work area, evacuate the area immediately. Monitor area with combustible gas indicator. Spill Management: Stop the source of the release if you can do it without risk. Contain release to prevent further contamination of soll, surface water or groundwater. Clean up spill as soon as possible, observing precautions in Exposure Controis/Personal Protection. Use appropriate techniques such as applying non-combustible absorbent materials or pumping. All equipment used when handling the product must be grounded. A vapor suppressing foam may be used to reduce vapors. Use clean non-sparking tools to collect absorbed material. Where feasible and appropriate, remove contaminated soil. Place contaminated materials in disposable containers and dispose of in a manner consistent with applicable regulations.

Reporting: Report spills to local authorities and/or the U.S. Coast Guard's National Response Center at $(800)$ 424-8802 as appropriate or required. This material is covered by EPA's Comprehensive Environmental Response, Compensation and Liability Act (CERCLA) Petroleum Exclusion. Therefore, releases to the environment may not be reportable under CERCLA.

\section{SECTION 7 HANDLING AND STORAGE}

Precautionary Measures: READ AND OBSERVE ALL PRECAUTIONS ON PRODUCT LABEL. This product prosents an extreme fire hazard. Liquid very quickly evaporates. oven at low temperatures, and forms vapor (fumes) which can catch fire and burn with explosive violence. Invisible vapor spreads easily and can be set on fire by many scurces 5 . ch as pliot lights, welding equipment, and electrical motors and switches. Do not store in open or unlabeled contaisters. Use only as a motor fuel. Do not use for cleaning, pressure appliance fuet, or any 
other such use. Never siphon gasoline by mouth.

Do not get in eyes, on skin, or on clothing. Do not taste or swallow. Do not breathe vapor or fumes. Wash thoroughly after handling. Keep out of the reach of children.

Unusual Handling Hazards: WARNINGI Do not use as portable heater or appliance fuel. Toxic fumes may accumulate and cause death.

General Handling Information: Avoid contaminating soil or teleasing this material into sewage and drainage systems and bodies of water.

Static Hazard: Electrostatic charge may accumulate and create a hazardous condition when handling this material. To minimize this hazard, bonding and grounding may be necessary but may not, by themselves, be sufficient. Review all operations which have the potential of generating and accumulating an electrostatic charge and/or a flammable atmosphere (including tank and container filling, splash filling, tank cleaning, sampling, gauging, switch loading, filtering, mixing, agitation, and vacuum truck operations) and use appropriate mitigating procedures. For more information, refer to OSHA Standard 29 CFR 1910.106, 'Flammable and Combustible Liquids', National Fire Protection Association (NFPA 77, 'Recommended Practice on Static Electricity', and/or the American Petroleum institute (API) Recommended Practice 2003, "Protection Against ignitions Arising Out of Static, Lightning, and Stray Currents'. Improper filling of portable gasoline containers creates danger of fire. Only dispense gasoline into approved and properly labeled gasoline containers. Always place portabie containers on the ground. Be sure pump nozzle is in contact with the container while filling. Do not use a nozzle's lock-open device. Do not fill portable containers that are inside a vehicle or truck/trailer bed.

General Storage Information: DO NOT USE OR STORE near heat, sparks, flames, or hot surfaces. USE AND STORE ONLY IN WELL VENTILATED AREA, Keep container closed when not in use.

Container Warnings: Container is not designed to contain pressure. Do not use pressure to empty container or it may rupture with explosive force. Empty containers retain product residue (solid, liquid, and/or vapor) and can be dangerous. Do not pressurize, cut, weld, braze, solder, crill, grind, or expose such containers to heat, flame, sparks, static electricity, or other sources of ignition. They may explode and cause injury or death. Empty containers should be completely drained, properly closed, and promptly returned to a drum reconditioner or disposed of properly.

\section{SECTION 8 EXPOSURE CONTROLS/PERSONAL PROTECTION}

\section{GENERAL CONSIDERATIONS:}

Consider the potential hazards of this material (see Section 3), applicable exposure limits, job activities, and other substances in the work place when designing engineering controls and selecting personal protective equipment. If engineering controis or work practices are not adequate to prevent exposure to harmful levels of this material, the personal protective equipment listed below is recommended. The user should read and understand all instructions and limitations supplied with the equipment since protection is usually provided for a limited time or under certain circumstances.

ENGINEERING CONTROLS:

Use process enclosures, local exhaust ventilation, or other engineering controls to control airborne levels below the recommended exposure timits.

PERSONAL PROTECTIVE EQUIPMENT

Eye/Face Protection: No special eye protection is normally required. Where splashing is possible, wear safety glasses with side shieids as a good safety practice.

Skin Protection: No special protective clothing is normally required. Where splashing is possible, select protective clothing depending on operations conducted, physical requirements and other substances in the workplace. Suggested materials for protective gloves include: Chlorinated Polyethylene (or Chlorosulfonated Polyethylene). Nitrile Rubber, Polyurethane, Viton.

Respiratory Protection: Determine if airborne concentrations are beiow the recommended occupational exposure limits for jurisdiction of use. If airborne concentrations are above the acceptable limits, wear an approved respirator that provides adequate protection from this material, such as: Air-Purifying Respirator for Organic Vapors

When used as a fuel, this material can produce carbon monoxide in the exhaust. Determine if airborne concentrations are below the occupational exposure limit for carbon monoxide. If not, wear an approved positivepressure air-supplying respirator.

Use a positive pressure air-supplying respirator in circumstançes where air-purifying respirators may not provide adequate protection.

Occupational Exposure Limits:

\begin{tabular}{|c|c|c|c|c|c|}
\hline Component & Acency & TWA & STEL & Ceiling & Notation \\
\hline Benzene & ACGIH & $.5 \mathrm{ppm}$ (weighi) & $2.5 \mathrm{ppm}$ & - & Skin A1 \\
\hline
\end{tabular}




\begin{tabular}{|c|c|c|c|c|c|}
\hline & & & (weighn) & & \\
\hline Benzene & OSHA SRS & $1 \mathrm{ppm}$ (weight) & $5 \mathrm{ppm}$ (weight) & - & - \\
\hline Benzene & OSHAZ-2 & $10 \mathrm{ppm}$ (weight) & - & $\begin{array}{l}25 \mathrm{ppm} \\
\text { (weight) }\end{array}$ & - \\
\hline Eutane & ACGIH & $\begin{array}{l}800 \mathrm{ppm} \\
\text { (weight) }\end{array}$ & - & - & - \\
\hline Cyciohexane & ACGIH & $\begin{array}{l}100 \mathrm{ppm} \\
\text { (weight) }\end{array}$ & - & - & - \\
\hline Cyclohexane & OSHA Z-1 & $1050 \mathrm{mg} / \mathrm{m3}$ & - & - & - \\
\hline Ethanol & ACGIH & $\begin{array}{l}1000 \text { ppm } \\
\text { (weight) }\end{array}$ & - & 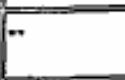 & $\sqrt{A 4}$ \\
\hline Elhanol & OSHAZ-1 & $1900 \mathrm{mg} / \mathrm{m} 3$ & - & - & $=$ \\
\hline Ethyt benzene & ACGIH & $\begin{array}{l}100 \mathrm{ppm} \\
\text { (weight) }\end{array}$ & $\begin{array}{l}125 \mathrm{gpm} \\
\text { (weight }\end{array}$ & - & $A 3$ \\
\hline Ethyl benzene & OSHAZ Z - & $436 \mathrm{mg} / \mathrm{m} 3$ & 5 & 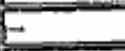 & - \\
\hline Ethyi tert-butyl ether (ETBE) & ACGIH & $5 \mathrm{ppm}$ (weight) & - & $E$ & - \\
\hline Heptane & $\sqrt{\mathrm{ACGIH}}$ & $\begin{array}{l}400 \text { ppm } \\
\text { (weight) } \\
\end{array}$ & $\begin{array}{l}500 \mathrm{ppm} \\
\text { (weight) }\end{array}$ & - & - \\
\hline Heptane & OSHA Z-1 & $2000 \mathrm{mg} / \mathrm{m} 3$ & - & $E$ & - \\
\hline Hexane & ACGIH & $50 \mathrm{ppm}$ (weight) & $E$ & $E$ & Skin \\
\hline Hexane & OSHA Z-1 & $1800 \mathrm{mg} / \mathrm{m} 3$ & $E$ & $E$ & - \\
\hline Methyl tert-butyl ether (MTBE) & ACGIH & $50 \mathrm{pom}$ (weight) & 5 & - & $A 3$ \\
\hline Methyl ter-butyl ether (MTBE) & $\mathrm{c} v \mathrm{x}$ & - & $50 \mathrm{ppm}$ & 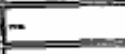 & 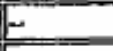 \\
\hline Methylcyctohexsne & ACGIH & $\begin{array}{l}400 \mathrm{ppm} \\
\text { (weight) }\end{array}$ & - & - & $F$ \\
\hline Methylcyclohexane & OSHA Z-1 & $2000 \mathrm{mg} / \mathrm{m}^{3}$ & - & - & 2 \\
\hline Naphthalene & $\mathrm{ACGIH}$ & $10 \mathrm{ppm}$ & $15 \mathrm{ppm}$ & $E$ & A4 Skin \\
\hline Naphthalene & OSHA Z-1 & $50 \mathrm{mg} / \mathrm{m3}$ & - & - & $=$ \\
\hline Pentane, 2,2,4-trimethyl- (Isooctane) & ACGIH & $\begin{array}{l}300 \text { ppm } \\
\text { (weight) }\end{array}$ & - & - & - \\
\hline Pentane, 2,2,4-trimethyl- (Isooctane) & OSHA Z-1 & $2350 \mathrm{mg} / \mathrm{m}^{3}$ & $E$ & - & 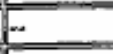 \\
\hline Tertiary amyl methyl ether (TAME) & ACGIH & $20 \mathrm{ppm}$ (weight) & - & - & $E$ \\
\hline Tertiary amyl methyl ether (TAME) & $\mathrm{CVX}$ & - & $50 \mathrm{ppm}$ & E & - \\
\hline Toluene (methyibenzene) & $\mathrm{ACGiH}$ & $50 \mathrm{ppm}$ (weight) & $E$ & - & Skin A4 \\
\hline Toluene (methylbenzene) & OSHA Z.2 & $\begin{array}{l}200 \mathrm{ppm} \\
\text { (weight) }\end{array}$ & - & $\begin{array}{l}300 \mathrm{ppm} \\
\text { (weight) }\end{array}$ & - \\
\hline $\begin{array}{l}\text { Xylene (contains o-. m-, \& p-xylene } \\
\text { (somers in varying amounts) }\end{array}$ & ACGIH & $100 \mathrm{ppm}$ & $\begin{array}{l}150 \mathrm{ppm} \\
\text { (wejght) }\end{array}$ & - & A4 \\
\hline $\begin{array}{l}\text { Xylene (contains o-, m-, \& p-xyiene } \\
\text { isomers in varying amounts) }\end{array}$ & OSHA Z-1 & $435 \mathrm{mg} / \mathrm{m}^{3}$ & - & - & - \\
\hline
\end{tabular}

Refer to the OSHA Benzene Standard (29 CFR 1910.1028) and Table Z-2 for detailed training, exposure monitoring, respiratory protection and medical survellance requirements before using this product.

\section{SECTION 9 PHYSICAL AND CHEMICAL PROPERTIES}

Attention: the data below are typical values and do not constitute a specification.

Color: Colorless to yellow

Physical State: Liquid

Odor: Petroleum odor

$\mathrm{pH}$ : Not Applicable

Vapor Pressure: 5 psi- 15 psi (Typical)@ $37.8^{\circ} \mathrm{C}\left(100^{\circ} \mathrm{F}\right)$

Vapor Density (Air = 1): $3-4$ (Typicai)

Boiling Point: $37.8^{\circ} \mathrm{C}\left(100^{\circ} \mathrm{F}\right)-204.4^{\circ} \mathrm{C}\left(400^{\circ} \mathrm{F}\right.$; (Typicai)

Solubility: Insoluble in water; miscible with most organic solvents. 
Freozing Point: Not Applicable

Melting Point: Not Apolicable

Specific Gravity: $0.7 \mathrm{~g} / \mathrm{ml}-0.8 \mathrm{~g} / \mathrm{ml} @ 15.6^{\circ} \mathrm{C}\left(60.1^{\circ} \mathrm{F}\right)$ (Typical)

Vlscosity: $<1$ SUS Q $37.8^{\circ} \mathrm{C}\left(100^{\circ} \mathrm{F}\right)$

SECTION 10 STABIL.ITY AND REACTIVITY

Chemical Stability: This material is considered stable under normal ambient and anticipated storage and handling conditions of temperature and pressure.

Incompatibility With Other Materials: May react with strong acids or strong oxidizing agents, such as chlorates, nitrates, peroxides, etc.

Hazardous Decomposition Products: None known (None expected)

Hazardous Polymerization: Hazardous polymerization will not cccur.

SECTION 11 TOXICOLOGICAL INFORMATION

IMMEDIATE HEALTH EFFECTS

Eye Irritation: The Draize eye irntation mean score in rabbits for a 24-hour exposure was: 0/110.

Skin Irritation: For a 4-hour exposure, the Primary Irritation Index (PII) in rabbits is: 4.8/8.0.

Skin Sensitization: This material did not cause skin sensitization reactions in a Buehler guinea pig test.

Acute Dermal Toxicity: LDSO: $>3.75 \mathrm{~g} / \mathrm{kg}$ (rabbit)

Acute Oral Toxicity: LD50: $>5 \mathrm{ml} / \mathrm{kg}$ (rat)

Acute Inhalation Toxicity: 4 hour(s) LD50: $>20000 \mathrm{mg} / \mathrm{m} 3$ vapor (rat).

Subchronic Effects: Exposure of rats for 13 weeks ( 6 hriday for 5 days/week) to the light ends of gasoline (up to $20.000 \mathrm{mg} / \mathrm{m} 3$ ) resulted in minimal responses of toxicity. There were no indications of neurotoxicity based morphological, functional and biochemical indices. There was also no evidence of immunotoxicity in the rats. However, when rats were exposed to gasoline vapor containing ethanol up to $20,000 \mathrm{mg} / \mathrm{m} 3$ there was evidence of both humoral immune suppression and mild astrogliosis. Reproduction and Birth Dofects: Exposure of rats to the light ends of gasoline at up to $20,000 \mathrm{mg} / \mathrm{m3}$ had generally no impact upon reproductive abilities and did not cause birth defects.

Genetic Toxicity: Gasoline was not mutagenic, with or without activation, in the Ames assay (Salmonella (yphimurium), Saccharamyces cerevisesae, or mouse lymphoma assays. In addition, point mutations were not induced in human lymphocytes. Gasoline was not mutagenic when tested in the mouse dominant lethal assay. Administration of gascline to rats did not cause chromosomal aberrations in their bon: marrow cells. Inhalation exposure of rats to the light ends of gasoline caused increased sister chromatid exchange in their peripheral white blood cells but did not cause an increase in micronucleated red blood cells in their bone marrow.

ADDITIONAL TOXICOLOGY INFORMATION:

Gasolines are highty volatile and can produce significant concentrations of vapor at ambient temperatures. Gasoline vapor is heavier than air and at high concentrations may accumulate in confined spaces to present both safety and heath hazards. When vapor exposures are low, or short duration and infrequent, such as during refueling and tanker loading/unloading, neither total hydrocarbon nor components such as benzene are likely to result in any adverse health effects. in situations such as accidents or spills where exposure to gasoline vapor is potentially high, attention should be paid to potential toxic effects of specific components. Information about specific components in gasoline can be found in Sections 2, 8 and 15 of this MSDS. More detailed information on the health hazard of specific gasoline components can be obtained calling the ChevronTexaco Emergency Information Center (see Section 1 for phone numbers)

Pathological misuse of solvents and gasoline, involving repeated and prolonged exposure to high concentrations of vapor is a significant exposure on which there are many reports in the medical literature. As with other solvents. persistent abuse involving repeated and prolonged exposures to high concentrations of vapor has been reported to result in central nervous systern damage and eventually, death. In a study in which ten human volunteers were exposed for 30 minutes to approximately 200,500 or 1000 ppm concentrations of gasoline vapor, irritation of the eyes was the only significant effect observed, based on both subjective and objective assessments.

Lifetime inhalation of wholly vaporized unleaded gasoline at $2056 \mathrm{ppm}$ has caused increased liver tumors in female mice and kidney cancer in male rats. In their 1988 review of carcinogenic risk from gasoline, The Intemational Agency for Research on Cancer (IARC) noted that, because published epidemiology studies did not include any exposure data, only occupations where gasoline exposure may have occurred were reviewed. These included gasoline service station attendants and automobile mechanics. IARC also noted that there was no opportunity to separate effects of combustion products from those of gasoline itself. Although IARC allocated gasoline a final overali classification of Group 28 , i.e. possibly carcinoçsnic to humans, this was basod on limited evidencs in experimental animals plus supporting evidence including the presence in gasoline of benzene. The 
actual evidence for carcinogenicity in humans was considered inadequate.

To explore the heaith effects of workers potentially exposed to gasoline vapors in the marketing and distribution sectors of the petroleum industry. the American Petroleum Institute sponsored a cohort mortality study (Publication 4555), a nested case-control study (Publication 4551), and an exposure assessment study (Publication 4552). Histories of exposure to gasoline were reconstructed for cohort of more than 18,000 employees from four companies for the time period between 1946 and 1985 . The results of the cohort mortality study incicated that there was no increased mortality from either kidney cancer or leukemia among marketing and marine distribution employees who were exposed to gasoline in the petroleum industry, when compared to the general population. More importantly, based on internal comparisons, there was no association between mortality from kidney cancer or leukemla and various indices of gasoline exposure. In particular, neither duration of employment, duration of exposure, age at first exposure, year of first exposure. job category. cumulative exposure, frequency of peak exposure. nor average intensity of exposure had any effect on kidney cancer or leukemia mortality. The cesults of the nested case-control study confirmed the findings of the original conort study. That is, exposure to gasoline at the levels experienced by this cohort of distribution workers is not a significant risk factor for ieukemia (all cell types), acute myeioid loukemia, kidney cancer or multiple myeloma.

\section{SECTION 12 ECOLOGICAL INFORMATION}

\section{ECOTOXICITY}

96 hour(s) LCSO: $8.3 \mathrm{mg} /$ (Cyprinodon variegatus)

96 hour(s) LC50: $1.8 \mathrm{mg} /$ (Mysidopsis bahia)

48 hour(s) LC50: $3.0 \mathrm{mg} /$ (Daphnia magna)

96 hour(s) LC50: $2.7 \mathrm{mg} / 1$ (Oncorhynchus mykiss)

This material is expected to be toxic to aquatic organisms. The bulk of the available literature on gasoline relates to the environmental impact of monoaromatic (BTEX) and diaromatic (naphthalene, methylnaphthalenes) constituents. In general, non-oxygenated gasoline exhibits some short-term toxicity to freshwater and marine organisms, especially under closed vessel or flow-through exposure conditions in the laboratory. The components which are the most prominent in the water soluble fraction and cause aquatic toxicity, are also highly volatile and can be reacilly blodegradod by microorganisms.

Gasoline studies have been conducted in the laboratory under a variety of test conditions with a range of fish and invertebrate species. An even more extensive database is available on the aquatic toxicity of individual aromatic constituents. The majority of published studies do not identify the type of gasoline evaluated, or even provide distinguishing characteristics such as aromatic content or presence of lead alkyls. As a result, comparison of results among studies using open and closed vesseís, different ages and species of test animals and different gasoline types, is difficult.

\section{ENVIRONMENTAL FATE}

This material is expected to be readily biodegradable. Following spillage, the more volatile components of gasoline will be rapidly lost, with concurrent dissciution of these and other constituents into the water. Factors such as local environmental conditions (temperature, wind, mixing or wave action. soil type, etc), photo-oxidation, biodegradation and adsorption onto suspended sediments, can contribute to the weathering of spilled gasoline.

The aqueous solubility of non-oxygenated unleaded gasoline, based on analysis of benzene, toluene. ethylbenzene+xylenes and naphthalene, is reported to be $112 \mathrm{mg} /$. Solubility data on individual gasoline constituents also available.

\section{SECTION 13 DISPOSAL CONSIDERATIONS}

Use material for its intended purpose or recycle if possible. This material, if it must be discarded, may meet the criteria of a hazardous waste as defined by US EPA under RCRA ( 40 CFR 261) or other State and local regulations. Measurement of certain physical properties and analysis for regulated components may be necessary to make a correct determination. If this material is classified as a hazardous waste, federal law requires disposal at a licensed hazardous waste disposal facility.

SECTION 14 TRANSPORT INFORMATION


The description shown may not apply to all shipping situations. Consult 49CFR, or appropriate Dangerous Goods Regulations, for additional description requirements ( $\Theta . g$. technical name) and mode-specific or quantity-specific shipping requirements.

DOT Shipping Description: GASOLINE,3,UN1203,il

IMO/IMDG Shipping Description: GASOLINE,3,UN1203,II,FLASH POINT SEE SECTION 5

ICAOIIATA Shipping Description: GASOLINE. 3. UN1203, ॥

\section{SECTION 16 REGULATORY INFORMATION}

EPCRA 311/312 CATEGORIES: 1. Immediate (Acute) Health Effects: YES

2. Delayed (Chronic) Health Effects: YES

3. Fire Hazard: YES

4. Sudden Release of Pressure Hazard: NO

5. Reactivity Hazard: NO

REGULATORY LISTS SEARCHED:

01-1=IARC Group 1

01-2A=|ARC Group 2A

Q3=EPCRA 313

01-2B=[ARC Group 2B

Q4=CA Proposition 85

$02=$ NTP Carcinogen

$05=M A$ RTK

$06=$ NJ RTK

$07=$ PA RTK

The following components of this material are found on the regulatory lists indicated.

\begin{tabular}{|c|c|}
\hline Benzene & $01-1,02,03,04,05,06,07$ \\
\hline Butane & $05,06,07$ \\
\hline Cyclohexane & $03,06,06,07$ \\
\hline Ethanol & $06,06,07$ \\
\hline Ethyl benzene & $01-2 B, 03,05,06,07$ \\
\hline Gasoline & $01-2 B, 07$ \\
\hline Heptane & $05,06,07$ \\
\hline Hexane & $03,05,06,07$ \\
\hline Methyl tert-butyl ether (MTBE) & $03,05,06,07$ \\
\hline Methylcyclohexane & $05,06,07$ \\
\hline Naphthalene & $01-2 B, 03,04,05,06,07$ \\
\hline Pentane, 2,2,4-trimethyl- (Isooctane) & $05,06,07$ \\
\hline Toluene (methylbenzene) & $03,04,05,06,07$ \\
\hline
\end{tabular}

CERCLA REPORTABLE QUANTITIES(RQ)/EPCRA 302 THRESHOLD PLANNING QUANTITIES(TPQ):

\begin{tabular}{|l|l|l|l|}
\hline Component & Component RQ & Component TPQ & Product RQ \\
\hline Benzene & $10 \mathrm{lbs}$ & None & $186 \mathrm{lbs}$ \\
\hline Butane & $100 \mathrm{lbs}$ & None & $725 \mathrm{lbs}$ \\
\hline Cyclohexane & $1000 \mathrm{lbs}$ & None & $34188 \mathrm{lbs}$ \\
\hline Ethanoi & $100 \mathrm{lbs}$ & None & $1934 \mathrm{lbs}$ \\
\hline Ethyl benzene & $1000 \mathrm{lbs}$ & None & $34964 \mathrm{lbs}$ \\
\hline Gasoline & $100 \mathrm{lbs}$ & None & $107 \mathrm{lbs}$ \\
\hline Heptane & $100 \mathrm{lbs}$ & None & $3644 \mathrm{lbs}$ \\
\hline Hexane & $5000 \mathrm{lbs}$ & None & $129149 \mathrm{lbs}$ \\
\hline Hethyl tert-butyl ether (MTSE) & $1000 \mathrm{lbs}$ & None & $7513 \mathrm{lbs}$ \\
\hline
\end{tabular}




\begin{tabular}{|l|l|l|l|}
\hline Methyicyclohexane & $100 \mathrm{lbs}$ & None & $4278 \mathrm{lbs}$ \\
\hline Naphthalene & $100 \mathrm{lbs}$ & None & $4000 \mathrm{lbs}$ \\
\hline Pentane. 2,2,4-trimethyl- (Isooctane) & $1000 \mathrm{lbs}$ & None & $6270 \mathrm{lbs}$ \\
\hline Toluene (methylbenzene) & $1000 \mathrm{lbs}$ & None & $5480 \mathrm{lbs}$ \\
\hline $\begin{array}{l}\text { Xylene (Contains o-, m-. \& p-xylene } \\
\text { isomers in varying amounts) }\end{array}$ & $100 \mathrm{lbs}$ & None & $649 \mathrm{lbs}$ \\
\hline
\end{tabular}

\section{CHEMICAL INVENTORIES:}

All components comply with the following chemical inventory requirements: DSL (Canada), EINECS (European Union), $\mathrm{KECl}$ (Korea), TSCA (United States).

\section{WHMAIS CLASSIFICATION:}

Class B, Division 2: Flammable Liquids

Class D. Division 2. Subdivision A: Very Toxic Material -

Carcinogenicity

Class D, Division 2, Subeivision B: Toxic Material -

Skin or Eye Irritation

\section{SECTION 16 OTHER INFORMATION}

NFPA RATINGS: Health: 1 Flammability: 3 Reactivity: 0

(0-Least, 1-Slight, 2-Moderate, 3-High, 4-Extreme, PPE:- Personal Protection Equipment Index recornmendation. "- Chronic Effect Indicator). These values are obtained using the guidelines or published evaluations prepared by the National Fire Protection Association (NFPA) or the National Paint and Coating Association (for HMiS ratings). Additional Product Number(s): CPS201023, CPS201054, CPS201056, CP\$201075. CPS201090, CP\$201105, CPS201106, CPS201120, CPS201121, CPS201122, CPS201126, CPS201128, CPS201131, CPS201136, CPS201141, CPS201142, CPS201148, CPS201153, CPS201158, CPS201161, CPS201162, CP\$5201168, CPS201181, CPS201185, CPS201186, CPS201188, CPS201216, CPS201217, CPS201218, CP\$201236, CPS201237, CPS201238. CPS201266, CPS201267. CPS201268, CPS201277, CPS201278, CPS201279, CPS201286, CPS201287, CPS201289, CPS201296, CPS201297, CPS201298, CPS201849, CPS201850, CPS201855, CPS201856, CPS201857. CPS204000, CPS204001, CPS204002, CPS204003, CPS204010. CP\$204011, CPS204022, CPS204023, CPS204046, CPS204047, CP\$204070, CPS204071, CPS204088, CPS204089, CPS204104, CPS204105, CP\$204116, CPS204117, CPS204140, CP\$204141. CPS204164, CPS204165, CP\$204188, CPS204189, CPS204200, CP\$204201, CPS204212, CP\$204213, CPS204224. CPS204225, CPS204248, CPS204249, CPS204272, CP\$204273, CPS204290, CPS204291, CPS204322. CPS204323. CPS204324, CPS204350, CPS204352. CPS204354, CP\$204356, CPS204358, CPS204359. CPS204364, CPS204365, CPS204370, CP\$204371, CPS204376, CPS204377, CP\$204382, CPS204383, CPS204388, CPS204389, CPS204394, CPS204395, CPS204400, CPS204401, CPS204406, CPS204407. CPS204412, CPS204413, CPS204418. CPS204419, CPS204424, CPS204425, CPS204430, CP\$204431. CPS204436, CPS204437, CPS204442, CPS204446, CPS204450, CP\$204454, CPS204458, CPS204462, CPS204466, CPS204467, CPS204484, CPS204485, CPS204502, CPS204503, CPS204520, CPS204521. CPS204538, CPS204539, CPS204556, CPS204557, CP\$204574, CPS204575, CPS204592, CPS204593, CPS204610, CPS204611, CPS204628, CPS204629, CPS204646, CP\$204647, CPS204664, CP\$204665. CPS204682, CPS204690. CPS204691, CPS204696, CPS204697, CPS204702, CPS204703, CPS204708, CPS204709, CPS204721, CPS204722, CPS204727, CPS204728, CPS241765

REVISION STATEMENT: This revision updates the following sections of this Material Safety Data Sheet: 1, 2, 8, $11,14,15$.

Revision Date: 06/25/2004

ABBREVIATIONS THAT MAY HAVE BEEN USED IN THIS DOCUMENT:

\begin{tabular}{|c|c|}
\hline TLV - Threshold Limit Value & TWA - Time Weighted Avarage \\
\hline STEL - Short-lerm Exposure Limit & PEL - Permissible Exposure Limit \\
\hline & CAS - Cherrical Abstract Service Number \\
\hline $\begin{array}{l}\text { ACGIH - imerican Conference of Government } \\
\text { industrial Hygienists }\end{array}$ & IN!OilMLG - International Maritime Dangerous Goods Code \\
\hline
\end{tabular}




\begin{tabular}{|l|l|}
\hline API - American Petroleum Institute & MSDS - Material Safely Data Sheot \\
\hline CVX - ChevronTexaco & NFPA - National Fire Protection Association (USA) \\
\hline DOT - Department of Transportation (USA) & NTP - National Toxicology Program (USA) \\
\hline IARC - international Agency for Research on Cancer & OSHA - Occupational Safety and Health Administration \\
\hline Prepared according to the OSHA Hazard Communication Standard (29 CFR 1910.1200) and the ANSI \\
MSDS Standard (Z400.1) by the ChevronTexaco Energy Research \& Technology Company. 100 \\
Chevron Way, Richmond, California 94802. \\
\hline
\end{tabular}

The above information is based on the data of which we are aware and is believed to be correct as of the date hereof. Since this information may be applied under conditions beyond our control and with which we may be unfamiliar and since data made available subsequent to the date hereof may suggest modifications of the information, we do not assume any responsibility for the results of its use. This information is furnished upon condition that the person receiving it shall make his own determination of the suitability of the material for his particular purpose. 


\section{ExxonMobil}

440693-00 MOBII DELVAC 1300 SUPER 25W-40

MATERIAL SAFETY DATA BULLETYN

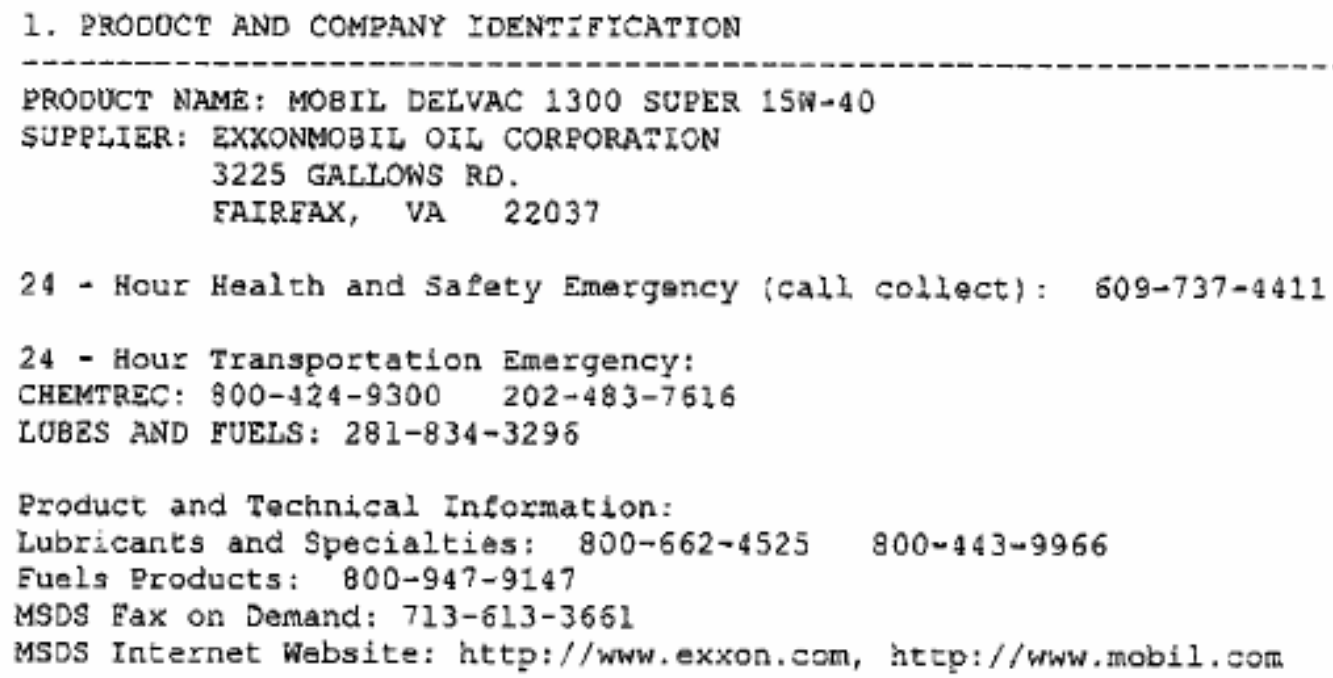


EMERGENCY OVERVIEW: BrOW: Liquid. DOT ERG NO. : NA

POTENTIAL HEALTH EFEECTS: Under normal conditions of interded use, this product does not pose a risk to health. Sxcessive exposure may rasult in eye, skin or respiratory iretcation.

For further health effects/toxicological data, see Section 11.

\section{FIRST AID MEASURES}

EYE CONTACT: Elush thoroughly with water. If irritacion occirs, cali a physician.

SKIN CONTACT: wash contact areas with soap and water. Remove and clean oil soaked cloching daily and wasi affected area. (See Section 16 - Injection Injury)

INHALATION: Not expeceed to be a problam. However, if respiratory irfitation, dizziness, nausea, or unconsciousness occurs due to excessive vapor or mist exposure, saek imnediace medical assistance. If breathing has stopped, assist ventilation with a mechanical device or mouth-to-mouth resuscitation.

INGESTION: Noc expected to be a problem. Seek medical attention if discomfort occurs. Do not induce vomiting.

\section{FIRE-EIGATING MEASURES}

EXrINGUISHING MEDIA: Carbon dioxide, foam, dry chemicai and water fog. SPECIAL FIRE EIGHTING RROCEDURES: Water or foam may cause frothing. Use water to keep fire exposed containers cool. Water spray may be used to flush spills away from exposure. Drevent runoff from fire concrol or dilution from entering streams, sewers, or drinking water supply.

SPECIAL PROTECTIVE EQOTPMENT: For fires in enclosed sareas, fine fighters must use self-contained breathing apparatus.

UNUSUAZ EIRE AND EXPLOSION HAZARDS: None.

COMBUSTYON PRODUCTS: Flumes, smoke, carbon monoxide, sulfuz oxides, aldehydes and othe: decomposition products, in the case of incomplete cotnbustion.

Elash Poine C(E): $230(446)$ (ASTM D-92).

Flammable Limits (approx.s vol.in air) - LEL: 0.98, USL: 7.08 NEPR HAZARD ID: Healtin: 0, Glamability: 1, Reactivity: 0

\section{ACCIDENTAL RELEASE MEASURES}

NOTIEICATION PROCEDURES: Report spills/releases as required to approprtate authorities. U.S. Coast Guard and EPA regulations require imnediate reporting of spiils/releases that could reach any waterway incjuding intermittent dry creeks. Report spill/release to Coast Guard National Response Center coll free number $(800) 424-8802$. In cass of accident or road spi11 notify CAEMTREC (800) 424-9300.

PROCEDURES IF MATERIAL IS RELEASED OR SPILLED:

LAND SPILL: Shut off sourte caking normal safacy precalitions. Take measures to minimize the effects on ground water. Racovar by pumping of concain spilled matarial with sand or other suicable 
absorbent and remove mechanicaliy into containers. If necessary, dispose of adsorbed residues as directed in Section 13. WArER SPIrL: Confine the spill immediacely with boons. Narn other
ships in the vicinity. Notify port and other relevant authorities. Remove from the surface by skimming or with suitable absorbents. If pernitted by regulatory aurhorities the use of suitable dispersants should be considered where recommended in locai oil spill procedures.

ENVIRCNMENTAL PRECAUTIONS: Prevent material from encering sewers, watar sources or low lying areas; advise the relovant authorities if it has, or if it contaminates soil/vegetation.

PERSONAL PRECAOTIONS: See Jection 8

7. RANDLIRG AND STORAGE

TANOLIMG: No special precautions are necessary beyond normal gocd hygiene practices. Seo Section 8 for additional persona! provection advice when handling this product.

STORHGE: Koop containers closed when not in use. Do not store in ogen or unlabelled containers. Store away from strong oxidizing agents and combustible materials. Do not store near heat, spazks, flame or strong oxidants.

SPECIAL PRECAUTIONS: Zzevent small spilis and leakages to avold slip hazard.

EMPTY CONTATNER WARNING: Empty containers retain residue (1iquid and/or vapor) and can be dangerous. DO NOT PRESSURIZE, CUT, WELD, BRAZE, SOLDER, DRILL, GRIND OR EXPOSE SUCH CONTAINERS TO HEAT, FLAME, SPARKS, STATIC ELECTRICITY, OR OTHER SOURCES OE IGNITION; THEY MAY EXPLODE AND CAUSE INJURY OR DEATH. DO not attempt to refill or clean container since residue is difficult ro remove. Empty drums should be complecely drained, properly bunged and promptly returned to a drum reconditioner. All containses should be disposed of in an ervitronmentally safe manner and in accordance wich governmental regulazions.

3. EXPOSURE CONTROLS/PERSONAZ PRCTECTION

OCCUPAT YONAL EXPOSURE LIMITS:

When mists/aerosols can occur, the following are recommended: $3 \mathrm{mg} / \mathrm{m} 3$ (as oil mist) - ACGIH Threshold Limit Value (TLV), $10 \mathrm{mg} / \mathrm{m} 3$ (as oil $\mathrm{mist}$ ) - AcGir Short Term Exposure Limie (STEL), $5 \mathrm{mg} / \mathrm{m3}$ (as oil mise) - OStA Permissible Exposure Limit (2EL)

VENTZLATION: It mists are generaced, use adequate ventilation, local exhaust or anclosures to control below exposure limits.

RESPIRATORY PRCTECIION: If mists are generatəd, and/or when ventilation is not adequace, wear approved respirator.

EYE PROTSCTION: if eye concact is likely, safety giasses with side sinields or chemical type goggles should be worn.

SEIN PROTECZZON: Wot normally required. When splashing or 11quid contact can occur frequencly, woaz oil resistant gloves and/or other protactive clothing. should always be Eollowed. 


\section{PHYSICAL AND CHEMTCAL PROPERTIES}

Typical physical properties are given below. Consulz eroduct Daca Sheer for specific details.

APPEARANCE : Liquid

COLOR: BLOWN

ODOR: MIII

ODOR THRESHOLD-gPa: NE

$\mathrm{pH}: \mathrm{NA}$

BOILING POINT $\mathrm{C}(E):>316(600)$

MELTING POTNT C(E): NA.

ELASA POINT C(E): $230(446)$ (ASTX D-92)

FLAMMABILITY (solids): NE

AUTO FLAMMABILITY $C(\bar{E}):$ NA

EXPLOSIVE PROPERTIES: NA

OXIOIZING PROPERTIES: NA

VAPOR PRESSORE-mmitg $20 \mathrm{C:} \mathrm{NE}$

VAPOR DENSITY: NE

EVAPORATION RATE: NE

RELATIVE DENSITY, $15 / 4$ C: 0.877

SOLUBILITY IN WATER: Negligible

PARTITION COEFEICIENT: > 3.5

VISCOSITY AT $40 \mathrm{C}$, cSt: 217.0

VISCOSITY AT $100 \mathrm{C}$, cSt: 15.5

POUR POINT $C(E):-33(-27)$

FREEZING POINT C $(E)$ : NE

VOLATYLE ORGANIC COAPOUND: NE

DMSO EXTRACT, I?-346 (NT. 3$):<3$, for mineral oil only

NAwNOT AP?LICABLE NE-NO? ESTABLISAED D-DECOMPOSES

ZOR TURTHER TECHNYCAL INEORMATZON, CONTACT YOUR MARKETING REPRESENTATIVE

10. STABILITY AND REACTIVITY

STABILITY (THERMAL, LIGHT, ETC.): STable.

CONDITIONS TO AVOID: Extreme heat and high enezgy sources of ignition.

INCOMPATIBILITY (MATERIALS TO AVOID): Strong oxidizers.

HAZARDOUS DECOMPOSITION PRODUCTS: Pzoduct does not decompose at ambient temperatures.

UAZARDOUS POLYMERIZATION: WIIl not occur.

11. TOXICOLOGICAL DATA

- ACUT2 TOXICOLOGY

CRAL TOXYCYTY (RATS): Practicaliy non-toxic (LD50: greater than 2000 $\mathrm{mg} / \mathrm{kg})$. - - Based on Easting of similar products and/or the components.

DERMAL TOXICITY (RABSITS): Practically non-toxic (LDSO: greacez than $2000 \mathrm{mg} / \mathrm{kg}$ ). --Based on testing of similar products and/or the components.

TNHALATION TOXICYTY (RATS): Practicaliy non-toxic (LCSO: greater than $5 \mathrm{mg} / 1)$. m--Based on testing of similar products and/oz the 
components.

EYE IRRITRTICN (RAaBITS): Practically non-irritating. (Draize score: greater than 6 but 15 or less). ---3ased on testing of similar products and/or the components.

SXIN IRRITATION (RABBZTS): Practicaliy non-irritating. (Primary Irritation Index: greater than 0.5 but less than 3 ). --masod on testing of similaz products and/or the components.

OTHER ACUTE TOXICITY DATA: Although an acute inhalation study was not performed with chis product, a vaziety of mineral and synchetic olls, such as those in this product, have been cestad. These samplas had virtualiy no effect other than a nonspecific inflammatory response in the lung to the aerosolized mineral oil. The presence of additives in ocher tested formulations (in approximately the ame amounts as in the present formulation) did not alter the observed effects.

$$
\text { ---SUBCHRONIC TCXICOLOGY (SUMMARY)--- }
$$

No significant adverse effacts were found in studies using repeated dermal applications of similar formulations to the skin of laboratory animals for 13 weeks at doses significantly higher than those expected during normal industrial exposure. The animals were evaluated extensively for effects of exposure (hematology, serum chemistzy, urinalysis, organ weights, microscopic examination of tissues etc.).

W- -REPRODOCTYVE TOXICOLOGY (SUMMARY) - -

No teratogenic effects would be axpected from dermal exposure, based on laboratory developmental toxicity scudies of major components in this formulation and/or materials of similar composition.

$$
\text { ---CHRONZC TOXICOLCGY (SUMMARY)--- }
$$

Repeated and/or prolonged exposure may cause irritation to the $5 k i n$, eyes or respiratory tract. Ovezexposure to oll mist may result in old droplet deposicion and/oz granulona formation. Eor mineral base olis: Base oils in this product are severely solvent refined and/or severely hydrotreated. Chronic mouse skin painting studies of severely treated ofls showed no evidence of carcinogenic effects. These rasults are confimed on a continuing basis using various screening mechods such as Modified Ames Test, IP-346, and/or other analytical methods. For synthetic base oils: The base oils in this product have been tested in the Ames assay and other tests of mutagenicity with negative results. These base oils are not expected to be carcinogenic with chronic dermal axposures.

$$
\text { ---SENSITIZATION (SUMMARY)--- }
$$

Not expected to be sensitizing based on tests of this product, components, or similaz products.

Used gasoline engine oils have shown evidence of skin carcinogenio activity in laboratory tests when no effort was made to wash the oil off between applications. Used oil trom diesel engines did not groduce this effect.

12. SCOLCGYCAL INEORMATION 


\section{ENVIRONMENTAL EATE AND EEEECTS:}

In the absence of spectific enviromontal dara for this product, this assessment is based on information for representative products.

EcoroxicITY: Available ectoxicity data (LLSO $>1000 \mathrm{mg} / \mathrm{L}$ ) indicates that adverse effects to aquatic organisms are not expected Erom this product.

MCBILITY: When released into the envizonment, adsorption to sadiment and soil will be the predominant behavior.

PERSISTSNCE AND DEGRADABILITY: This product is expected to be inherently biodegradable.

BIOACCUMULATIVE POTENTIAL: BiOaCcimulation is unlikely due to the very low water solubilicy of this product, thecefore bioavailability to aquatic organisms is minimal.

13. DISPOSAL CONSEDERATIONS

WASTE DISPOSAL: Product is suitable for burning in an enclosed, controlled burner for fuel value. Such burning may be linited pursuant to the Resource Conservation and Recovery Act. In addition, the product is suitable for processing by an approved recycling facility or can be disposed of at an appropriate government waste disposal facility. Ose of these methods is subject to user compliance with applicable laws and regulations and consideration of product characteristics at time of disposal.

RCRA INFORMATION: The unused product, in our opinion, is not specifically listed by the S2A as a hazardous waste (40 CER, Part 261D), nor is it formulated to contain macerials which are 1 isted hazardous wastes. It does not exhibit tho hazardous characteriscles of ignicability, corrosivity, or reactivity. The unused product is not formulaced with substances covered by the Toxicity Characteristic Leaching Procedure (TCLP). Rowever, used product may be regulated.

14. TRANSPORT RNEORMATION

USA DOT: NOT REGULATED BY USA DOT.

RID/ADR: NOT REGULATED BY RID/ADR.

IMO: NOT REGULATED BY IMO.

IATA: NOP REGULATED BY IATA.

STATZC ACCFMCLATOR (50 picostemens or iess): YE5 
US OSHA YARARD COMMUNICATION STANDARD: When Used For tes incended gurposes, this product is not classified as hazardcus in accordance with OSAA 29 C $\$ R: 910.2200$.

EU Labeling: Product is not dangerous as defined by the European Union Dangerous Substances/Rzeparations Directives. EU labeling not required.

Governmental Inventory scatus: All zomponents comply with TSCA, EYNECS/EIINCS, AICS, METI, OSL, KOREA, and PHILIPPINES.

U.S. Superfund Amendments and Reauthorization Act (SARA) Title III: This product concains no "EXTREMELY HAZARDOUS SUBSTANCES".

SARA $(311 / 312)$ RS.PORTABLE HAZARD CATEGORIES: NonE.

This product contains the following SARA (313) Toxic Release Chemicals :

\begin{tabular}{lcc} 
CHEMICAE NAME & CAS NUMBER & CONC. \\
\hline ZINC DITHEOPHOSPGATE & $-68649-42-3$ & -2. \\
\end{tabular}

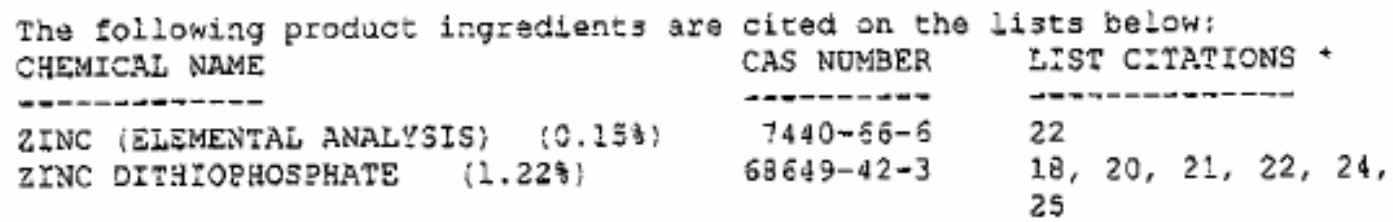

\begin{tabular}{|c|c|c|c|c|c|c|c|c|c|}
\hline \multicolumn{10}{|c|}{--- REGULATORY LISTS SEARCHED --- } \\
\hline $1=A C G I H A L L$ & $6=I A R C$ & 1 & $11=\operatorname{TSCA}$ & 4 & $16=\mathrm{CA}$ & F65 & CARC & $21=1 \mathrm{~A}$ & RTK \\
\hline $2=A C G I H A 1$ & $7=I A R C$ & 2A & $12=T S C A$ & $5 a 2$ & $17 \approx \mathrm{CA}$ & P 65 & RERRO & $22=\mathrm{MI}$ & 293 \\
\hline $3=$ ACGIB A2 & $8=$ YARC & $2 \mathrm{~B}$ & $13=\mathrm{TSCA}$ & 5 se & $18=\mathrm{CA}$ & Rak & & $23=\mathrm{MN}$ & \\
\hline $4=N T P \quad C A R C$ & $\exists=\mathrm{OSHA}$ & CARC & $24=\mathrm{TSCA}$ & 6 & $29 \times E L$ & RTK & & $24=\mathrm{NJ}$ & \\
\hline $5=N T P$ SCS & $10=0 S H A$ & 2 & $15=T S C A$ & $22 b$ & $20=I I$ & RTK & & $\begin{array}{l}25=2 \mathrm{~A} \\
26=R I\end{array}$ & \\
\hline
\end{tabular}

* EPA rocently added new chemical suiostances to izs TSCa Section 4 test rules. ziease contact the supplier to confirm whether the ingredients in this product currently appear on a TSCA 4 or TSCA 12b list. Code key:CARC=Carcinogen; SUS-Suspected Carcinogen; RERRo-Reproductive 
USE: COMMERCIAL ENGINE OIL

NOTE: RROQUCTS OF EXXON MOBIL CORPCRATION AND ITS AEFILIATED COLPANIES ARE NOT EORMULATED TO CONTAIN RCBS.

Health studies have shown that many hydrocasbons pose potontial human healch risks which may vary $\bar{f} x \mathrm{~cm}$ person to pezson. Information provided on this MSOS reflects intended lise. This product should not be used for other applications. In any case, the following advice should be considered:

INJECTION INUURY WARNING: if product is injocted into or under the skin, or into any part of the body, regardiess of the appearance of the wound or its size, the individual should be evaluated immediately by a physician as a surgical emergency. Even though initial symptoms from high pressure infection may be minimal or absent, early surgical treatment within the first few hours may significantly reduce the ultbimate extenc of injury.

INDUSTRIAL LABEL

Under normal conditions of intended use, this product does not pose a risk to health. Excessive exposure thay result in eye, skin or respiratory irritation. Always observe good hygiene measures. First Aid: Wash skin with soap and water. Elush eyes with water. If overcome by fumes or vapor, remove to fresh ait, If ingested do not induce vomiting. If symptoms persist seek medical assistance. Read and understand the MSDS before using this product.

For Internal Use Only: MHC: 1* $1^{*} 1^{*} 1^{*} I^{*}$, MPPEC: R., TRN: $440693-00$, CMCS97: 970529, REQ: US - MARKETING, SĂTE USE: I sis Approval Date: $17 \mathrm{JtN} 2003$ 
Information given herein is offered in good faith as accurate, but without guarantee. Conditions of lise and suitability of che product for parricular uses are beyond cur control; all risks of use of the product are therefore assumed by the usat and WE EXPRESSLY DISCLAIM ALL

WARRANTIES OF EVERY KIND AND NATJRE, INCLUDING WARRANTIES OE MERCHANTABILITY AND EITNESS GOR A RART¿CULAR PURPOSE IN RESPEC $T$ TO THE DSE OR SUITABILITY OE THE PRODCCT. Nothing is intended as a recomnendation for uses which infringe valid patents or as extending license under valid patents. Appropriace warnings and safo randling procedures should be provided to handlers and users. Alteracion of this document is strictly prohibited. Except to the extent required by law, republication or retransmission of this document, in whole or in part, is not permitted. Exxon Mobil Corporation and its affiliated companies assume no responsibility for accuracy of information unless the document is the most current available from an official ExxonMoili distribution system. Exxon Mobil Corporation and its affiliated companies neither represent nor warrant that the format, content or product formulas concained in this document comply with the laws of any other courtry except the United States of America.

Prepared by: ExxonMobil Oil Corporation Environmental fealth and Safecy Department, Clinton, uSA 
Product Name: MOBILFLUID 424

Revision Date: 19May2005

Page 1 of 3

ExonMobil

\section{MATERIAL SAFETY DATA SHEET}

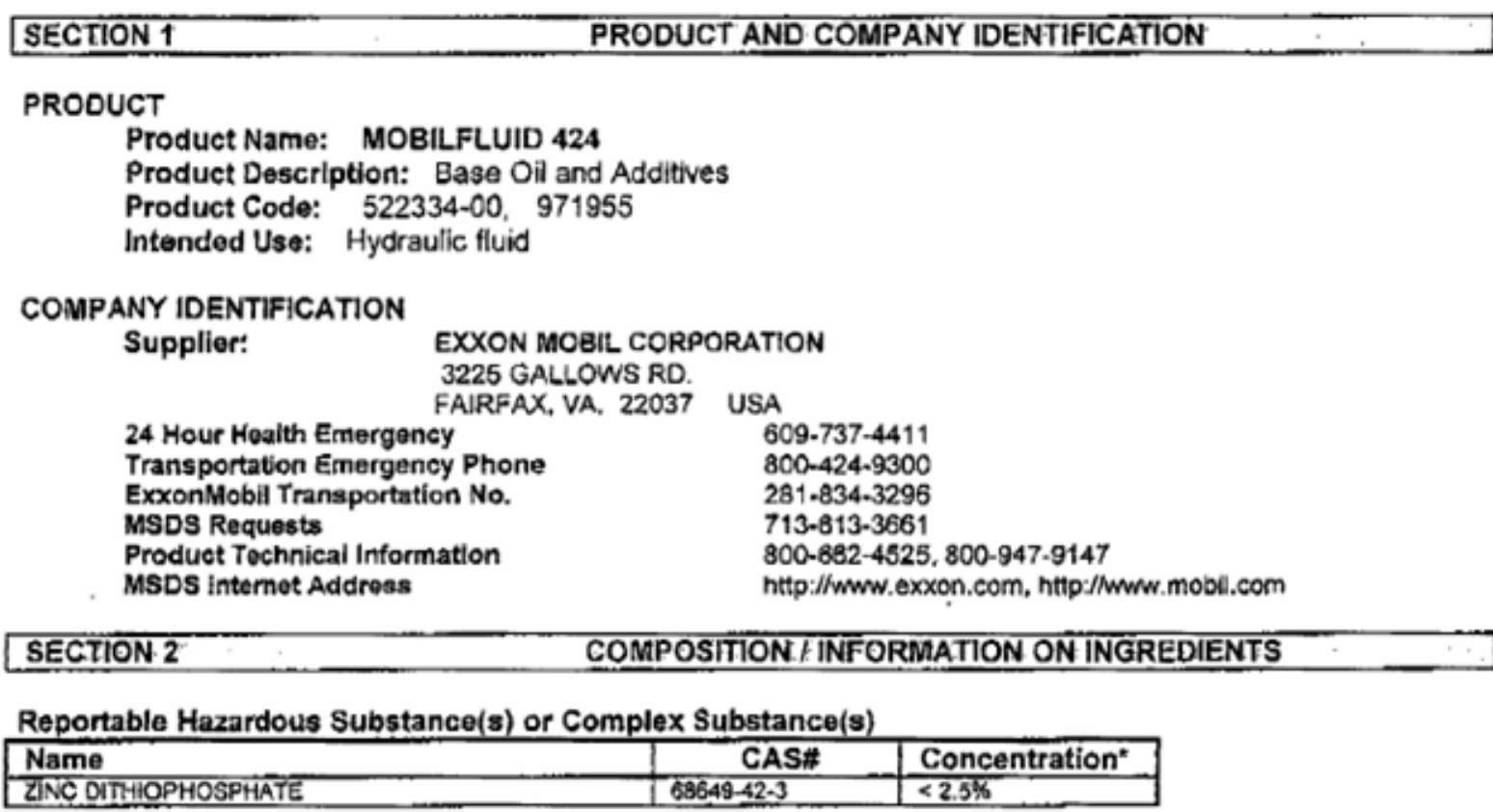

- All concentrations are percent by weight unless material is a gas. Gas concentrations are in percent by volume. SECTION 3. HAZARDS IDENTIFICATION

This material is not considered to be hazardous according to regulatory guidelines (see (M)SDS Section 15).

\section{POTENTIAL HEALTH EFFECTS}

Low order of toxicity. Excessive exposure may result in eye, skin, or respiratory irritation. High-pressure injection under skin may cause serious damage.

$\begin{array}{llll}\text { NFPA Hazard ID: } & \text { Health: } 0 & \text { Flammability: } 1 & \text { Reactivity: } 0 \\ \text { HMIS Hazard ID: } & \text { Health: } 0 & \text { Flammability: } 1 & \text { Reactivity: } 0\end{array}$

NOTE: This material should not be used for any other purpose than the intended use in Section 1 without expert advice. Health studies have shown that chemical exposure may cause potential human health isks which may vary from person to person. 
Product Name: MOBILFLUID 424

Revision Date: 19May2005

Page 2 of 8

ExxonMobil

\begin{abstract}
INHALATION
Remove from further exposure. For those providing assistance, avoid exposure to yourself or others. Use adequate respiratory protection. If respiratory irritation, dizziness, nausea, or unconsclousness occurs, seek immediate medical assistance. If breathing has stopped, assist ventilation with a mechanical device or use mouth-to-mouth resuscitation.

\section{SKIN CONTACT}

Wash contact areas with soap and water. If product is injected into or under the skin, or into any part of the body, regardless of the appearance of the wound or its size, the individual should be evaluated immediateiy by a physician as a surgical ernergency. Even though initial symptoms from high pressure injoction may be minimal or absent, early surgical treatment within the first few hours may significantly reduce the ultimate extent of injury.
\end{abstract}

\title{
EYE CONTACT
}

Flush thoroughly with water. If irritation occurs, get medical assistance.

\section{INGESTION}

First aid is normally not required. Seek medical attention if discomfort occurs.

\section{EXTINGUISHING MEDIA}

Appropriate Extinguishing Medla: Use water fog, foam, dry chemical or carbon dioxide (CO2) to extinguish flames.

Inappropriate Extinguishing Media: Straight Streams of Water

\section{FIRE FIGHTING}

Fire Fighting Instructions: Evacuate area. Prevent runoff from fire control or dilution from entering streams. sewers, or drinking water supply. Firefighters should use standard protective equipment and in enclosed spaces, self-contained breathing apparatus (SCBA). Use water spray to cool fire exposed surfaces and to protect personnel.

Unusual Fire Hazards: Pressurized mists may form a flammable mixture.

Hazardous Combustion Products: Aldehydes, Incomplete combustion products, Smoke, Fume, Oxides of carbon, Sulfur oxides

FLAMMABILITY PROPERTIES

Flash Point [Method]: $>198^{\circ} \mathrm{C}\left(389^{\circ} \mathrm{F}\right)$ [ ASTM D-92]

Flammable Limits (Approximate volume \% in air): LEL: 0.9 UEL: 7.0

Autoignition Tomperature: N/D

SECTION 6 ACCIDENTAL RELEASE MEASURES

NOTIFICATION PROCEDURES

In the event of a spill or accidental release, notify relevant authorities in accordance with all applicable 
Product Name: MOBILFLUID 424

Revision Date: 19May2005

Page 3 of 8

\section{ExxonMobil}

reguiations. U.S. regulations require reporting releases of this material to the environment which exceed the reportabie quantity or oil spills which could reach any waterway including intermittent dry creeks. The National Response Center can be reached at $(800) 424-8802$.

\section{SPILL MANAGEMENT}

Land Spill: Stop leak if you can do it without risk, Recover by pumping or with suitable absorbent.

Water Spill: Confine the spill immediately with booms. \$top leak if you can do it without risk. Warn other shipping. Remove from the surface by skimming or with sultable absorbents. Seek the advice of a specialist before using dispersants.

Water spill and land spill recommendations are based on the most likely spill scenario for this material; however, geographic conditions, wind, temperature, (and in the case of a water spill) wave and current direction and speed may greatly influence the appropriate action to be taken. For this reason, local experts should be consulted. Note: Local regulations may prescribe or limit action to be taken.

\section{ENVIRONMENTAL PRECAUTIONS}

Large Spills: Dike far ahead of liquid spill for later recovery and disposal. Prevent entry into waterways, sewers, basements or confined areas.

SECTION7 HANDEING AND STORAGE

HANDLING

Prevent small spills and leakage to avoid slip hazard.

Static Accumulator: This material is a static accumulator.

STORAGE

Do not store in open or unlabelled containers.

SECTION 8 EXPOSURE CONTROLS / PERSONAL PROTECTION

Exposure limits/standards for materials that can be formed when handling this product: When mists / aerosols can occur, the following are recommended: $5 \mathrm{mg} / \mathrm{m}^{3}$ - ACGIH TLV, $10 \mathrm{mg} / \mathrm{m}^{3}$. ACGIH STEL, $5 \mathrm{mg} / \mathrm{m}^{3}$ - OSHA PEL.

NOTE: Limits/standards shown for guidance only. Follow applicable regulations.

\section{ENGINEERING CONTROLS}

The level of protection and types of controls necessary will vary depending upon potential exposure conditions. Control measures to consider:

No special requirements under ordinary conditions of use and with adequate ventilation.

PERSONAL PROTECTION 
Product Name: MOBILFLUID 424

Rovision Date: 19May2005

Page 4 of 8

\section{ExxonMobil}

Personal protective equipment selections vary based on potential exposure conditions such as applications. handling practices, concentration and ventilation. Information on the selection of protective equipment for use with this material, as provided below, is based upon intended, normal usage.

Respiratory Protection: If engineering controls do not maintain airborne contaminant concentrations at a level which is adequate to protect worker health, an approved respirator may be appropriate. Respirator selection, use, and maintenance must be in accordance with regulatory requirements, if applicable. Types of respirators io be considered for this material include:

No special requirements under ordinary conditions of use and with adequate ventilation.

For high airborne concentrations, use an approved supplied-air respirator, operated in positive pressure mode. Supplied air respirators with an escape bottle may be appropriate when oxygen leveis are inadequate. gas/vapor warning properties are poor, or if air puritying filter capacity/rating may be exceeded.

Hand Protection: Any specific glove information provided is based on published literature and giove manufacturer data. Work conditions can greatly effect glove durability; inspect and replace worn or darnaged gloves. The types of gloves to be considered for this material include:

No protection is ordinarily required under normal conditions of use.

Eye Protection: If contact is likely, safety glasses with side shields are recommended.

Skin and Body Protection: Any specific clothing information provided is based on published literature or manufacturer data. The types of clothing to be considered for this material include:

No skin protection is ordinarily required under normal conditions of use. In accordance with good industrial hygiene practices, precautions should be taken to avoid skin contact.

Specific Hygiene Measures: Always observe good personal hygiene measures, such as washing after handling the material and before eating, drinking, and/or smoking. Routinely wash work clothing and protective equipment to remove contaminants. Discard contaminated clothing and footwear that cannot be cleaned. Practice good housekeeping.

\section{ENVIRONMENTAL CONTROLS}

See Sections 6, 7, 12, 13.

Typical physical and chemical properties are given below. Consult the Supplier in Section 1 for additional data.

GENERAL INFORMATION Physical State: Liquid

Color: Amber

Odor: Characteristic

Odor Threshold: $\mathrm{N} / \mathrm{O}$

IMPORTANT HEALTH, SAFETY, AND ENVIRONMENTAL INFORMATION

Relative Density (at $15^{\circ} \mathrm{C}$ ): 0.884

Flash Point [Method]: $>198^{\circ} \mathrm{C}\left(389^{\circ} \mathrm{F}\right)$ [ ASTM D-92]

Flammable Limits (Approximate volume \% in air): LEL: 0.9 UEL: 7.0

Autoignition Temperature: N/D

Boiling Point / Range: $>316^{\circ} \mathrm{C}\left(600^{\circ} \mathrm{F}\right)$ 
Product Name: MOBILFLUID 424

Revision Date: 19May2005

Page 5 of 3

\section{ExxonMobil}

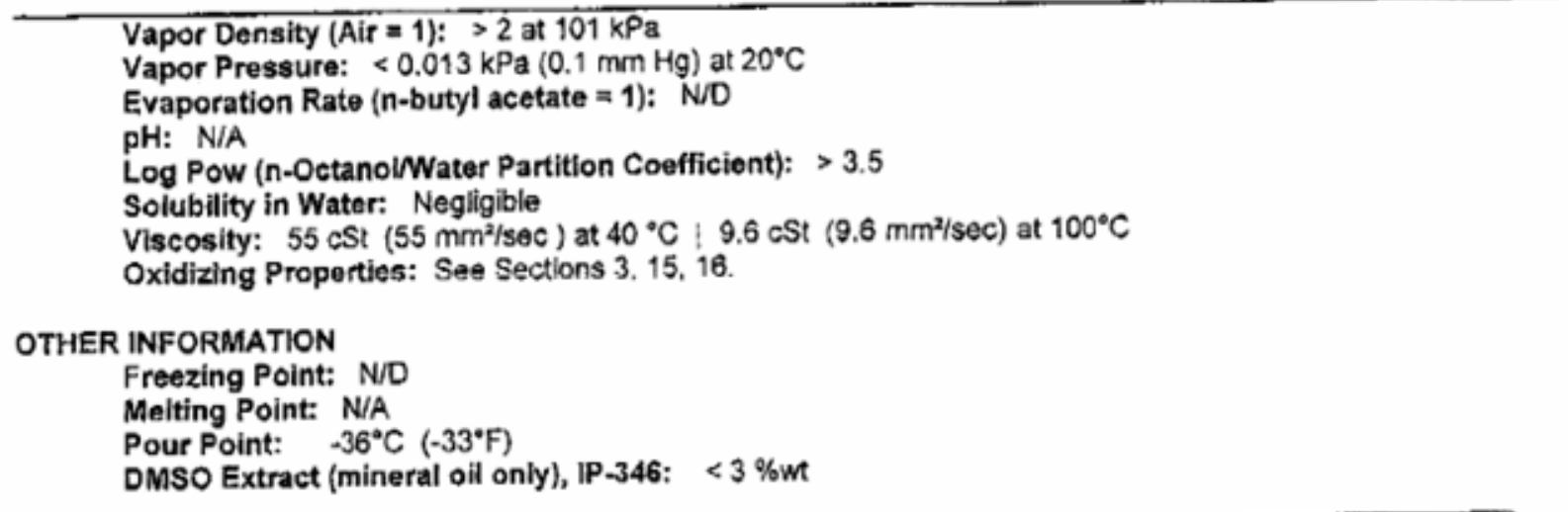

STABILITY: Material is stable under normal conditions.

CONDITIONS TO AVOID: Excessive heat. High energy sources of ignition.

MATERIALS TO AVOID: Strong oxidizers

HAZARDOUS DECOMPOSITION PRODUCTS: Material does not decompose at ambient temperatures.

HAZARDOUS POLYMERIZATION: WIII nOt OCCUR.

SECTION 11 TOXICOLOGICAL INFORMATION

\begin{tabular}{|c|c|}
\hline Route of Exposure & Conclusion / Remarks \\
\hline \multicolumn{2}{|l|}{ Inhalation } \\
\hline Toxicity (Rat): LC50 > $5000 \mathrm{mg} / \mathrm{m}^{3}$ & Minimally Toxic. Based on assessment of the components. \\
\hline Irritation: No end point cata. & $\begin{array}{l}\text { Negligible hazard at ambient/normal handling temperatures. } \\
\text { Based on assessment of the components. }\end{array}$ \\
\hline \multicolumn{2}{|r|}{ (1) } \\
\hline Toxícity (Rat): LD50 > $2000 \mathrm{mg} / \mathrm{kg}$ & $\begin{array}{l}\text { Minimally Toxic. Based on test data for structurally similar } \\
\text { materials. }\end{array}$ \\
\hline \multicolumn{2}{|r|}{ (1) = } \\
\hline Toxicity (Rabbit): L, $\overline{D 50}>2000 \mathrm{mg} / \mathrm{kg}$ & $\begin{array}{l}\text { Minimally Toxic. Basad on test data for structurally similar } \\
\text { materials. }\end{array}$ \\
\hline Irritation (Rabbii): Data available. & $\begin{array}{l}\text { Negligible irntation to sikin at ambient temperatures. Based on } \\
\text { assessment of the components. }\end{array}$ \\
\hline \multicolumn{2}{|r|}{ 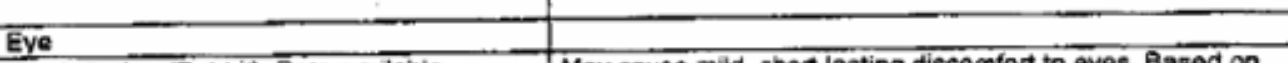 } \\
\hline Eve irritation (Rabbit): Data availabie. & $\begin{array}{l}\text { May cause mild, short-lasting discomfort to eyes. Based on } \\
\text { assessment of the components. }\end{array}$ \\
\hline
\end{tabular}

\section{CHRONICIOTHER EFFECTS}

Contains:

Base oil severely refined: Not carcinogenic in anımal studies. Representative material passes IP-346, Modified 
Product Name: MOBILFLUID 424

Revision Date: 19May2005

Page 6 of 8

\section{ExonMobil}

Ames test, and/or other screening tests. Dermal and inhalation studies showed minimal effects; lung nonspecific infiltration of immune cells, oil deposition and minimal granuloma formation. Not sensitizing in test animals.

Additional information is available by request.

The following ingredients are cited on the lists below: None.

$\begin{array}{lcl} & \text {-REGULATORY LISTS SEARCHED-- } & \\ 1=\text { NTP CARC } & 3=\text { IARC } 1 & 5=\text { IARC 2B } \\ 2=\text { NTP SUS } & 4=\text { IARC 2A } & 6=\text { OSHA CARC }\end{array}$

SECEION 12 ECOLOGICAL INFORMATION

The information given is based on data available for the material, the components of the material, and similar materials.

ECOTOXICITY

Material - Not expected to be harmful to aquatic organisms.

MOBILITY

Base oil component - Low solubility and floats and is axpected to migrate from water to the land. Expected to partition to sediment and wastewater solids.

PERSISTENCE AND DEGRADABILITY

Biodegradation:

Base oil component - Expected to be inherenty biodegradable

BIOACCUMULATION POTENTIAL

Base oil component - Has the potential to bioaccumulate, however metabolism or physical properties may reduce the bioconcentration or limit bioavailabitity.

Disposal recommendations based on material as supplied. Disposal must be in accordance with current applicable laws and regulations, and material characteristics at time of disposal.

\section{DISPOSAL RECOMMENDATIONS}

Product is suitable for burning in an enclosed controlled burner for fuel value or disposal by supervised incineration at very high temperatures to prevent formation of undesirable combustion products.

\section{REGULATORY DISPOSAL, INFORMATION}

RCRA Information: The unused product, in our opinion, is not specifically listed by the EPA as a hazardous waste (40 CFR, Part 261D), nor is it formulated to contain materials which are listed as hazardous wastes. It does not exhibit the hazardous characteristics of ignitability, corrositivity or reactivity and is not formulated with contaminants as determined by the Toxicity Characteristic Leaching Procedure (TCLP). However, used product may be regulated. 
Product Name: MOBILFLUID 424

ExonMobil Revision Date: 19May2005

Page 7 of 8

Empty Container Warning PRECAUTIONARY LABEL TEXT: Empty containers may retain residue and can be dangerous. DO NOT PRESSURIZE, CUT, WELO, BRAZE. SOLDER, ORILL, GRIND OR EXPOSE SUCH CONTAINERS TO HEAT, FLAME, SPARKS, STATIC ELECTRICITY, OR OTHER SOURCES OF IGNITION: THEY MAY EXPLODE AND CAUSE INJURY OR DEATH. Do not attempt to refill or clean container since residue is difficult to remove. Empty drums should be completely drained, properly bunged and promptly returned to a drum reconditioner. All containers should be disposed of in an environmentally safe manner and in accordance with governmental regulations.

SECTION 14 TRANSPORT INFORMATION

LAND (DOT) : Not Regulated for Land Transport

LAND (TDG) : Not Regulated for Land Transport

SEA (IMDG) : Not Regulated for Sea Transport according to IMDG-Code

AIR (IATA) : Not Regulated for Air Transport

SECTION 15 REGULATORY INFORMATION

OSHA HAZARD COMMUNICATION STANDARD: When used for its intended purposes, this material is not classified as hazardous in accordance with OSHA 29 CFR 1910.1200.

NATIONAL CHEMICAL. INVENTORY LISTING: AICS, DSL, EINECS, ENCS. KECI, PICCS, TSCA

EPCRA: This material contains no extremely hazardous substances.

SARA (311/312) REPORTABLE HAZARD CATEGORIES: None.

SARA (313) TOXIC RELEASE INVENTORY:

Chemical Name

ZIINC DITHIOPHÖSPHATE

CAS Number

$68649-42-3$

Typical Value

$<2.5 \%$

The Following ingredients are Cited on the Lists Below:

\begin{tabular}{|l|l|l|}
\hline Chemical Name & CAS Number & List Citations \\
\hline PHOSPHORUS & $7723-14-0$ & 1,4 \\
\hline TOLUENE & $108-88-3$ & 15 \\
\hline ZINC DITHIOPHOSPHATE & $68649-42-3$ & $13,15,17$ \\
\hline
\end{tabular}

$1=$ ACGIH ALL

$2=A C G I H A 1$

$3=\mathrm{ACGIH}$ A2

$4=$ OSHA Z

\begin{tabular}{|c|c|}
\hline- -REG & STS SE \\
\hline $\begin{array}{l}6=\text { TSCA } 5 a 2 \\
7=\text { TSCA } 5 e \\
8=\text { TSCA } 6 \\
9=\text { TSCA } 12 b\end{array}$ & $\begin{array}{l}11=\text { CA P65 REPRO } \\
12=\text { CA RTK } \\
13=\text { IL RTK } \\
14=\text { LA RTK }\end{array}$ \\
\hline
\end{tabular}

11 = CA P65 REPRO

$13=$ IL RTK

$4=$ LA RTK
$16=$ MN RTK

$17=$ NJ RTK

$18=$ PA RTK

19 = RI RTK 
Product Name: MOBILFLUID 424

ExxonMobil

Revision Date: 19May2005

Page 8 of 8

$5=T S \overline{T S C A} 4 \quad 10=$ CA P65 CARC $15=$ MI 293

Code key. $\mathrm{CARC}=\mathrm{Carcinogen;}$ REPRO=Reproductive

- EPA recently added new chemical substances to its TSCA Section 4 test rules. Please contact the supplier to confirm whether the ingredients in this product currently appear on a TSCA 4 or TSCA 12b list.

SECTION 16 OTHER INFORMATION

$\mathrm{N} / \mathrm{D}=$ Not determined, $\mathrm{N} / \mathrm{A}=$ Not applicable

THIS SAFETY DATA SHEET CONTAINS THE FOLLOWING REVISIONS:

No revision information is available.

The information and recommendations contained herein are, to the best of ExxonMobil's knowledge and belief, accurate and reliabie as of the date issued. You can contact ExxonMobil to insure that this document is the most current available from ExxonMobil. The information and recommendations are offered for the user's consideration and examination. It is the user's responsibility to satisfy itself that the product is suitable for the intended use. If buyer repackages this product, it is the user's responsibility to insure proper health, safety and other necessary information is included with and/or on the container. Appropriate warnings and safe-handling procedures should be provided to handiers and users. Alteration of this document is strictly prohibited. Except to the extent required by law, republication or retransmission of this document, in whole or in part, is not permitted. The term, "ExoronMobil" is used for convenience, and may include any one or more of ExxonMobil Chernical Company, Exoxon Mobil Corporation, or any affiliates in which they directly or indirectly hold any interest.

Internal Use Only
MHC: $0,0,0,0,0,0$
PPEC: A

DGN: 2005922XUS (538859)

Copyright 2002 Exxon Mobil Corporation, All rights reserved 


\section{ExxonMobil}

6\$2314-00 MOBILGREASE HTS

MATERIAL SAEETY DATA BULLETTN

1. PROCUCT AND CCMPANY IDENTIEICATION

PRODUCT NAME: MOBILGREASE STS

SUPPLIER: EXXONMOBIL OIL CORPORATION

3225 GALLOWS RD.

FAIREAX, VA 22037

24 - Hour Heslth and Safety Binargency (call collect): 609-737-44I1

24 - Hour Transportation Emezgency:

CHEMTREC: $8 \mathrm{CO}-424-9300$ 202-493-7616

LUBES AND FCELS; 28:-834-3296

Prodict and Technital Information:

Lubricants and Specialties: 800-662-4525 800-443-9966

Euels Products: B00-947-9147

MSDS Fax on Demand: 713-613-3661

MSOS Internet Websice: http://ww' exxon,com, http://ww. nobil.com

2. COMPOSITION/INEORMATION ON INGREDIENTS

CHEMICAL NAMES AND SYNCNYMS: PET. HYDROCARBONS AND ADOITIVES

GLOBALLY REPORTABLE MSDS INGREOIENTS:

Substance Name Approx. Wt
PROSPHORODITHOIC ACID,
O, O-DI C1-14-ALKYL ESTERS,
ZINC SALTS (2:1) (ZDDP)
(68649-42-3)

Sea Section 8 for exposure limits if apolicabla).

3. HAZARDS TDENTISICATYON

Under normal conditions of use, this product is not consiaered hazardous according to regulatory guidelinez (See section i5). 
EMERGSNCY OVERVIEW: BLack Grease. DOT ERG No. : NA

POTENTIAZ HEAETH ERESCTS: Vnder nomal conditions of irtended use, this product does not pose a risk zo health. Excessive exposure thay result in oye, skin or respiratory irzicacion.

For Eurther health effects/toxicological data, see Section il.

4. EIRST AIO MEASURES

EYE CONTACT: Elush thoroughly with water. If irrication occurs, cali a physician.

SKIN CONTACT: Wash contact areas with soap and water. Remove and clean oil soaked cloching datly and wash affected area. INJECTION INJURY WARNING: If product is injected lnto or under the skin, or into any pare of the body, regardless of the appeazance of the wound or its size, the individual should be ovaluated immediately by a physician as a surgical emergency. Even chough initial symptoms from high pressure injection may be minimal or absent, oasly surgical treacment within the first fow hours may significantiy reduce che ultimate extent of infury.

INHALATION: Remove $f=0 m$ further axposure. If zespiratory irritation, dizziness, nausea, or unconsciousness occurs, seek irmediate medical assistance. If breaching has stopped, assist ventilation with mechanical device or use mouth-to-nouch resuscicacion.

INGESTION: Not expected to be a problem. Seek nedical attention if discomfort occurs. Do not induce vomiting.

\section{EIRE-ETGHTING MEASORES}

EXTINGUISHING MEOIA: Carbon dioxide, foam, dry chemical and water fog.

S2ECIAL FIRE EIGHING PROCEDURES: Wacor or foam may cause froting.

Use water to keep fi=a exposed concalners cool. Water spray may be used to flush spills away from exposure. Prevent runoff exom fire control or cilution fron entering streams, sowers, or driaking water supply.

SPECIAL PROTECTIVE EQUTPMENT: For fires in enclosed areas, fire fighters must use sels-contained breazhing apparatus.

UNUSUAL FIRE ANO EXPLOSION GAZARDS: None.

COMBUSTION PRODUCTS: Sumes, smoke, carbon monoxide, sulfur oxides, aldehyces and other decomposition products, in the case of incomplete combustion.

Flash Point $C(E)$ : > 204(40C) (ESTIMATSD EOR OIL, ASTM D-92 (COC)). Flanmable Limits (approx.s vol.in a ${ }^{2}$ ) - LEL: NE, UEL: NE NEPA HAZARD IO: Health: 0, Elammability: 1, Reactivity: 0

\section{ACCIOENTAL REYEASE MEASURES}

NOTIZICATION PROCZDURES: Report splils/releases as required co appropriate authorities. J.S. Coast Guard and EPA regulations requirs impdiate reporzing of spills/roleases that could reach any waterway including incormittent dry creeks. Report spi:2/release to Coast Guarc National Response Center toll exee number (800)424-8802. In civo of accident or road spill notify 
CHEMTREC (800) 424-9300.

PROCEDURES IE MATERIAL IS RELEASED OR SPILEEO:

LAND SPILL: Shut off source taking normal safety precautions. Take measures to aninimize the effects on ground wacer. Recover by pumping or contain spilled material with sand or other suitable absorbent and remove mechanically inco containers. If necessary, dsspose of adsorbed residues as directed in section 13.

WATER SPILL: Confine the spill immediately with booms, Narn other ships in the vicinity. Notify port and other relavant auchorities. Remove from the surface by skimming or with suicable absorbents. If permicted by ragulatory authorities the use of suicable dispersancs should be considezed wheze zecomeaded in local oil spill procedures.

ENVIRONMENTAL PRECAUTIONS: Prevent naterial zrom entering sewers, water sources or low lying areas; advise the relavanc authorities if it has, or if it contaminates soil/vegetation.

PERSONAL PRECACTIONS: See Section 8

7. GANDLING AND STORAGE

HANDLING: HAgh pressura injection under the skin may occur due to the rupture of pressurized lines. Always seek medical attention. No special precautions are necessary beyond normal good hygiene practices. See Section 8 for additional personal protection advice when handling this product.

STORAGE: Kaep containers ciosed when not in use. Do not store in open or unlabelled concainers. Store away from strong oxidizing agonts and combustible materials. Do not store near heat, sparks, \&lame or strong oxidants.

SPECIAL PRECAOTIONS: Prevent small spills and leakages to avoid slip hazard.

EMP'TY CONTAINER WARNING: Empey containers retain residue (14quid and/or vapor) and can be dangerous. DO NOT PRESSORIZS, CUT, NELD, BRAZE, SOLDER, ORILL, GRIND OR EXPOSZ SUCH CONTAINERS TO YEAT, FLAME, STARKS, STATIC ELECTRICITY, OR OTHER SOURCES OE IGNITION; THEY MAY EXYLODE AND CAUSE INJURY OR DEATH. DO not attempt to refill oz clean container since rssidue is difficult to remove. Empty drums should be completely drained, properly bunged and promptly returned to a drum reconditioner. All containers should be disposed of in an environmentally safe manner and in accordance with governnontal regulations.

8. IXPOSURE CONTRCLS/PERSONAL PROTECTION

OCCUPAZTONAL EXPOSURE LEMTS:

This product does not contain any components which have recognized oxposure limies.

VENTTLATION: Use adequate vencization.

RESPIRATORY PROTECTION: No special requirements under ordinary condicions of use and with adequate ventilacion.

EYE PROTECTION: Generaliy sye concace is unlikely with this type material. If eye concact is likely, safety glasses with side shieids or chemical type goggles shouii be worn. 
SKIN PROTSCTION: IE prolonged or repeated $s$ in contact is likely, oil impervious gloves should be worn. Good personal hygiene practices should always be tollowed.

\section{PHYSICAL ANO CHEMICAL PRORERTIES}

Typical physical properties are given below. Consul= eroduct Data Shoet for specialc details.

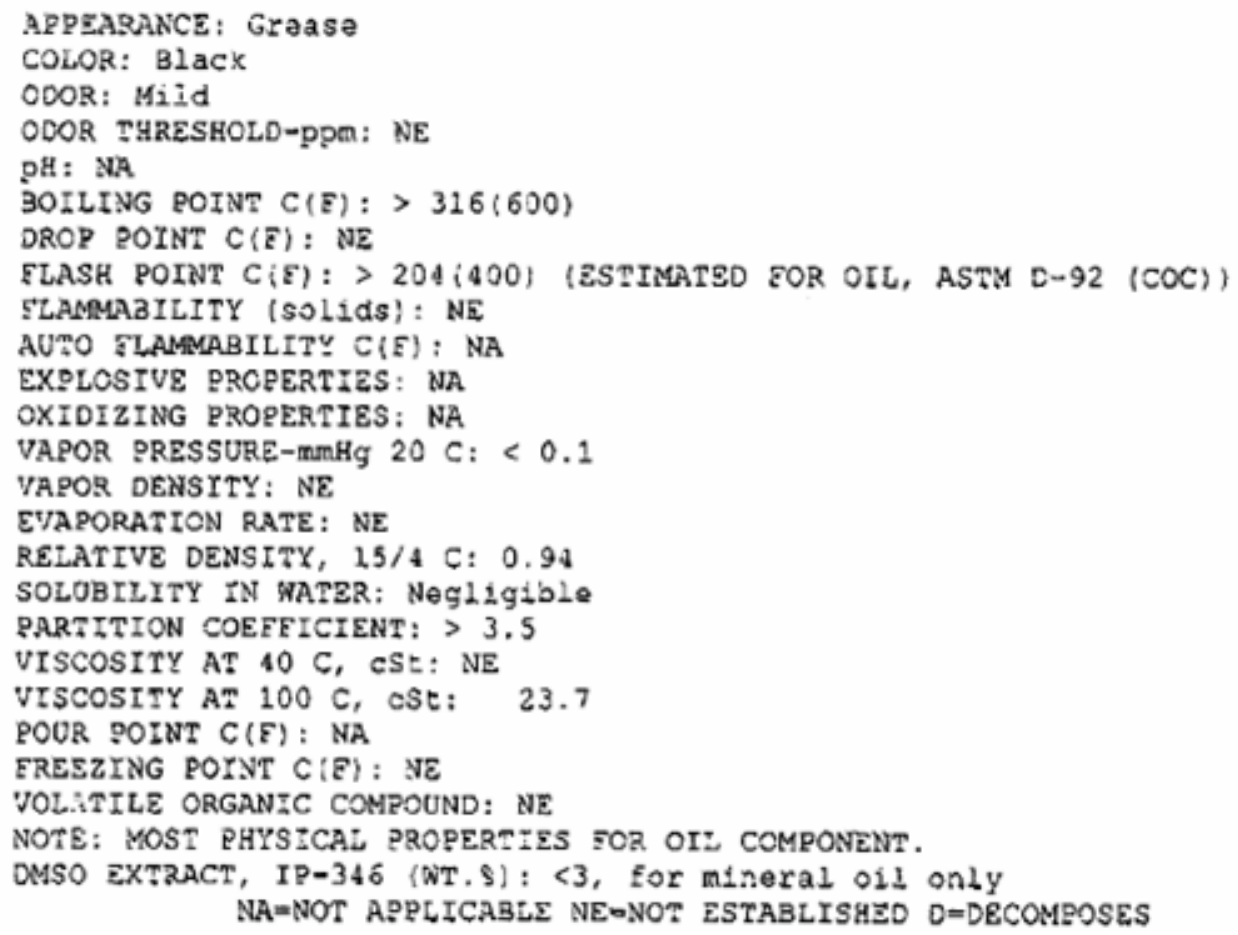


$2000 \mathrm{mg} / \mathrm{kg})$. ---Based on testing of similar products and/or the components.

INAALATION TOXTCTTY (RATS): Practica: Ly non-toxic (LCSO: greace: chan $5 \mathrm{mg} / \mathrm{i}$ ). ---3ased on testing of similar products and/or the components.

EY: IRRITATICN (RABBITS): 2ractically non-izritating. (Draize score: greatar than 5 but 15 or lass). ---Based on testing of similar products and/or the components.

SKIN IRRITATION (RABBITS): Practically non-irricating. (Primary Irritazion Index: greater than 0.5 but less tian 3\}. - - Based on cesting of similar products and/or the components.

OTHER ACUTE TOXICITY OATA: Although an acuto inhalation study was nor performed with this product, a variety of mineral oils and synthecic base oils, such as chose in this product have been tostec. These samplas had virtually no effect other than a nonspecific inflamatory response in the lung to the aerosolized mineral oil. The presence of additives in other tested formulations in approxinately the same amounts as in the present romulation) did not alter the coserved effects.

---SUBCRRCNIC TOXICOLOGY (SUMMARY)---

No stgnificant adverse effects were found in studies using repeated dermal applications of similar formulations to the skin of laboratory animals for 13 weeks at doses significantly higher than those expected during normal industrial exposure. The animais were evaluated extsnsiveiy for effeccs of exposure (hematology, serum chemistry, urinalysis, organ woights, microscopic examination of tissues etc.).

---REPRODUCTYVE TOXICOLOGY (SUMMARY)---

No reratogenic effects would be expected from dermal exposure, based on laboratory developmental toxicity studies of major components in this formulation and/or marezials of similaz composicton.

--CHRONIC TOXICOLOGY (SUMMARY) ---

Repeated and/or prolonged exposure may cause irrication to the skin, syes or rssplracory tract. Eor mineral base oils: Base olls in this product are severely solvent zefined and/or severely hydrotreated. Chronic mouse skin painting studies of severedy traaced oils showed no evidence of carcinogenic effects. These results are contilned on a continuing basis using various screening methods such as Modified Ames Test, IP-346, and/or other analytical methods. For syntheric base oils: The base cils in this product have been restad in the Ames assay and other tests of mutagenicity with negative results. These base oils are not expected to be carcinogenic with chronic dermal exposures.

---SENSITIZATYON (SUMMARY)---

Not expectec to be sensitizing based on tests of this product, componencs, or simizar products.

12. ECOLOGICAL RNEORMATION

\section{EColoGrcal ckeoparion}

ENVIRONMENTAL EATE AND SEEECTS: 
This entironmental assessment was ccnducted lsing informacion ca the individual compcrents as no test data was arailable tor this specizic formulation.

ECoroxicrTy: The major components in the formulation show no aquatic coxicity as $\$ 000 \mathrm{mg} / \mathrm{L}$ Loading, therefore long-cerm adverse effects in the aquatic engironment are roc expected.

MOBIIITY: Not established.

PERSISTENCE AND DEGRADABILITY: This groduct is expected to be inherencly biodegradable, as the principal components have been shown to degrade at siow to moderate rates.

BIOACCDMULATIVE DOTENRIAi: Noc established.

13. DISPOSAL CONSIDERATIONS

WASTE DISPOSAL: Product is suitabie for buring in an enciosed, controlled burner for fuel value. Such buraing may be limited pursuant to the Resource Conservation and Recovery Act. In addition, the product is suitable for processing by an approved recycling facility or can be disposed of at an appropriace governmenc waste disposal factility. Use of these methods is subject to user compliance with applicable laws and regulations and consideration of product characteristics at time of disposal.

RCRA INEORMATION: The unused product, in our opinion, is not spectecally listed by the EPA as a hazardous waste (40 CFR, Part 2610), nor is it formulated to contain materials which are listed hazardous wastes. It does not exhibit the hazardou: characteristics of ignitability, corrosivity, or reactivity. The unused product is not formulated with substances covered by the Toxicity Characteristic Laaching Procedure (TCL?), However, used product may be regulated.

14. TRANSPORT INFORMATION

USA DOT: NOT REGULATED BY USA DOT.

RID/ADR: NOT REGULATED BY RID/ADR.

IMO: NOT REGULATED BY IMO.

IATA: NOT REGULATED BY IATA.

\section{REGULATORY INEORMATZON}

OS OSHA FAZARD COMMUNICATION STANDARD: When used for its intended purposes, this product is hot classified as hazardous in accordance wien OSH. 29 CER 1910.1200. 
EU Labeling: Eroduct is not dangerous as defined by the European Union Dangerous Substances/Preparations Directives. g' labeing not raquired.

Goverumencal Inventory Status; All components compiy with TSCA, EINECS/ELINCS and AICS.

U.S. Superfund Amendments and Reauthorization Act (SARA) Title III: This product contains no "EXTREMEYY uAzARDOUS SUBSTANCES",

SARA (311/312) REPORTABLE HAZARD CATEGORIES: NONE.

This product contains the following SARA (313) Toxic Release Chemicals:

CHEMICAL NAME

2HOSPHCRODITHOIC ACID, 0, O-DI

C1-14-ALKYL ESTERS, ZINC SALTS

(2:1) (ZDOP)

\begin{tabular}{cc} 
CAS NUMBER & CGNC. \\
\hline $68649-42-3$ & -3.38
\end{tabular}

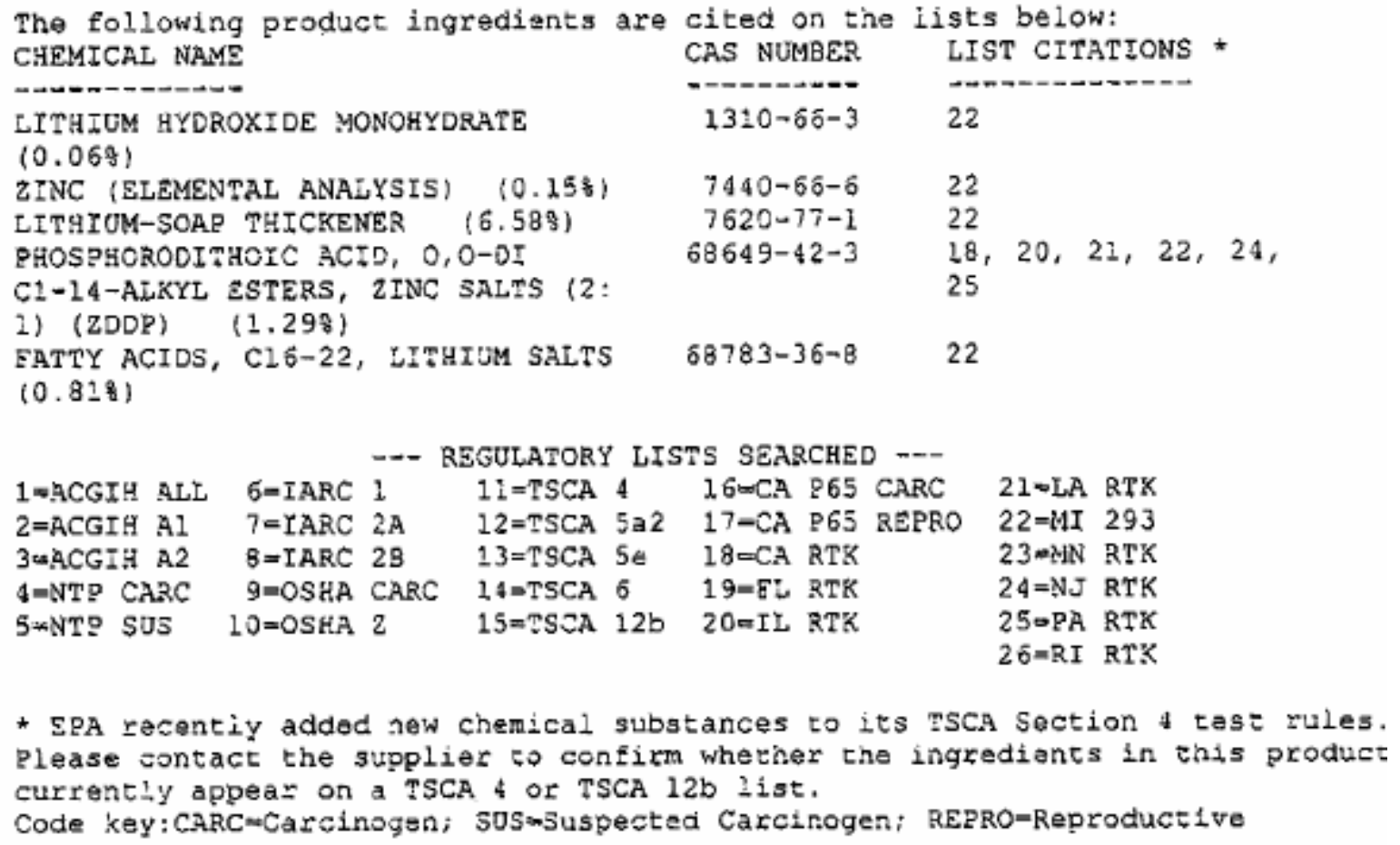

16. OTHER INEORMATION 


\section{USE: EXTREME PRESSURE GREASE}

NOTE: PROCUCTS OE EXXON MOBIL CORPORATION AND ITS AEETLIATED COMPANTES ARE NOT CORMULATED TO CCNTAIN PCBS.

Heaith studies have shown that nany hydrocarbons poso potentiai human health risks which may vary from person to person. Information provided on this MSDS reflects intended use. This product should not be used tor other applications. In any case, the following advice should be considered:

\section{INDUSTRIAL LABEL}

Onder normai conditions of intended use, this product does not pose a risk to health. Excessive exposure may result in eye, skin or zespiratory irritasion. Always observe good hygiene measures. First Aid: Wash skin with soap and watar. Flush eyes with water. If overcome by fumes or vapor, remove to frosh air. If ingested do not induce vomiting. If symptoms persist seek medical assistanco. Read and understand the MSDS before using this product.

For Incernal Use Only: MHC: $1 * 1 * 1 * 1 * 1 *$, MPPEC: A, TRN: $642314-00$, ELIS: 400584, CMCS97: 971554, REQ: OS - MARKETING, SAIE USE: L EHS Approval Date: 20AUG2001

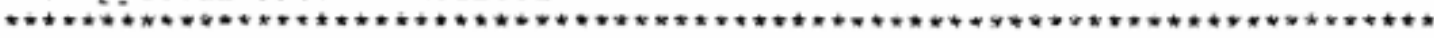

Information given herain is offered in good falth as accurate, but without guarantee. Conditions of use and suicability of the product for particular uses are beyond our control; all risks of use of the product are there Lore assuned by the user and WE EXPRESSLY DISCLAIM ALL WARRANTIES OS EVERY KIND AND NATURE, INCLUDING WARRANTIES OF MERCHANTABILITY AND FITNESS FOR A PARTICCLAR PURPOSE IN RESPECT TO THE USE OR SUITABILITY OE THE PRODOCT. Nothing is intended as a zecommendation for uses which infringe valid patents or as extending license under valid patents. Appropriate warnings and safe handling procedures should be provided to handlers and users. Alteration of this docunent is stzictly prohibited. Except to the extent roquired by law, republication or retransmission of this document, in whole or in part, is not permitted. Exxon Mobil Corpozarion and its affiliaced companies assume no responsibilicy for accuracy of information unless the document is the most current available from an official ExxonMobil distribution system. Exxon Mobil Corporation and its affiliated companies neither represent nor warrant that the format, content or product formulas concained in this document comply with the laws of any ocher country except the United States of America.

Prapazed by: ExxonMobtl Oll Corporation Ervironmencal tealch and Safery Department, Clinton, USA 
MATERIAL SARBTY DATA SHRET

T

Valvolino Company

EEREX $50 / 50$ PREMTX

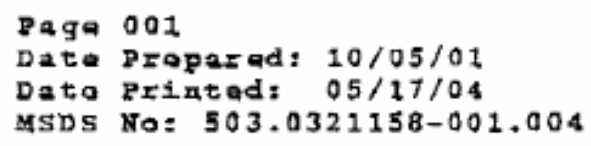

1. CHEMTCAT PRODUC'S AND COMPAWF TDEKTIYICATION

Matorial Idertity

Produat Name: zEREX 50/50. PREMIX

Goneral or Generic ID: GuYCOL

Company

Tha Valroline Compung

P.O. Bax 14000

Iaxingtan, KY 40512
Tolophone sumber̈s

Rinaxgoney:

$1-800-274-5263$

Information: $1-859-357-7206$

2. COMPOSITION/IXzormation ON IMGREDIRNm

Ingradiant (s)

Tम्YIENE GLYCOT

- ETHYIZNR GUYCOL

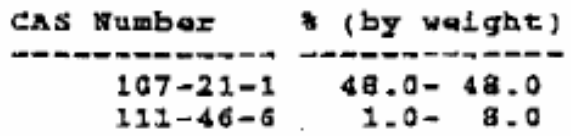

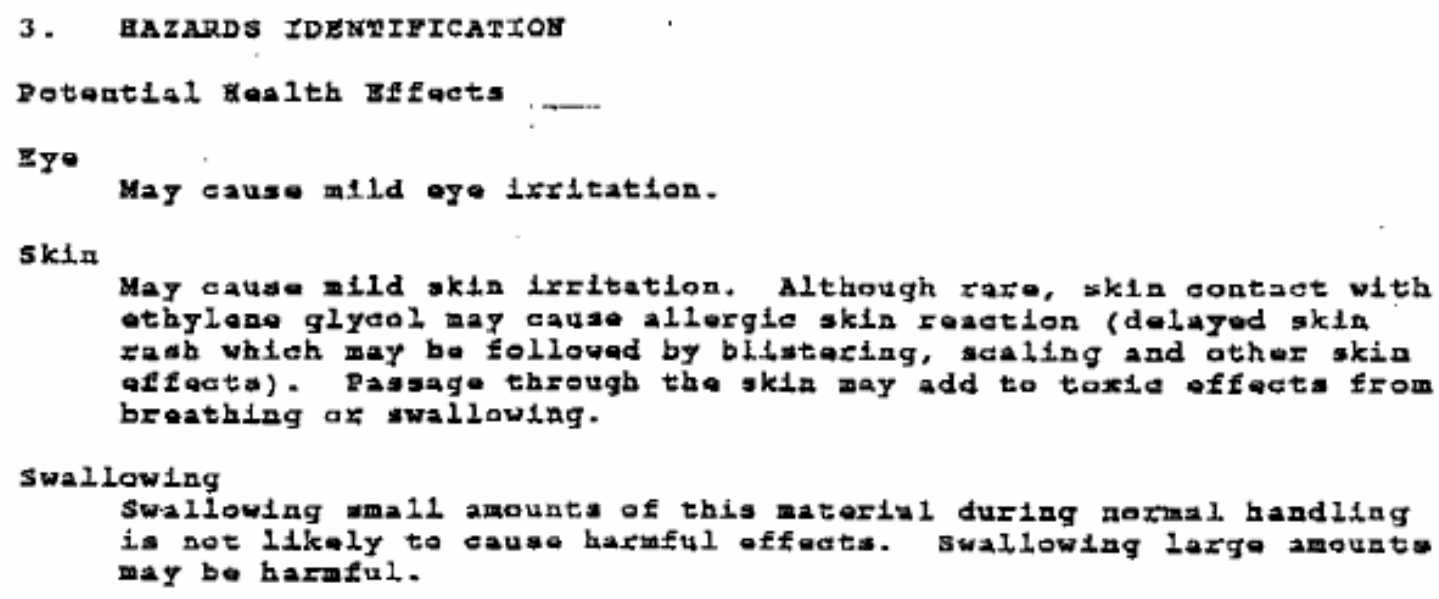

Continuad on naxt paga 
MATBRIAL SAEZTY DATA SHZZT

r. Valvolino Conpany

TEREX 50/50 PREMIX
Page 002

Date Prepared: $10 / 05 / 01$

Data printad: $05 / 17 / 04$

MSDs Ho: 503.0321158-001.004

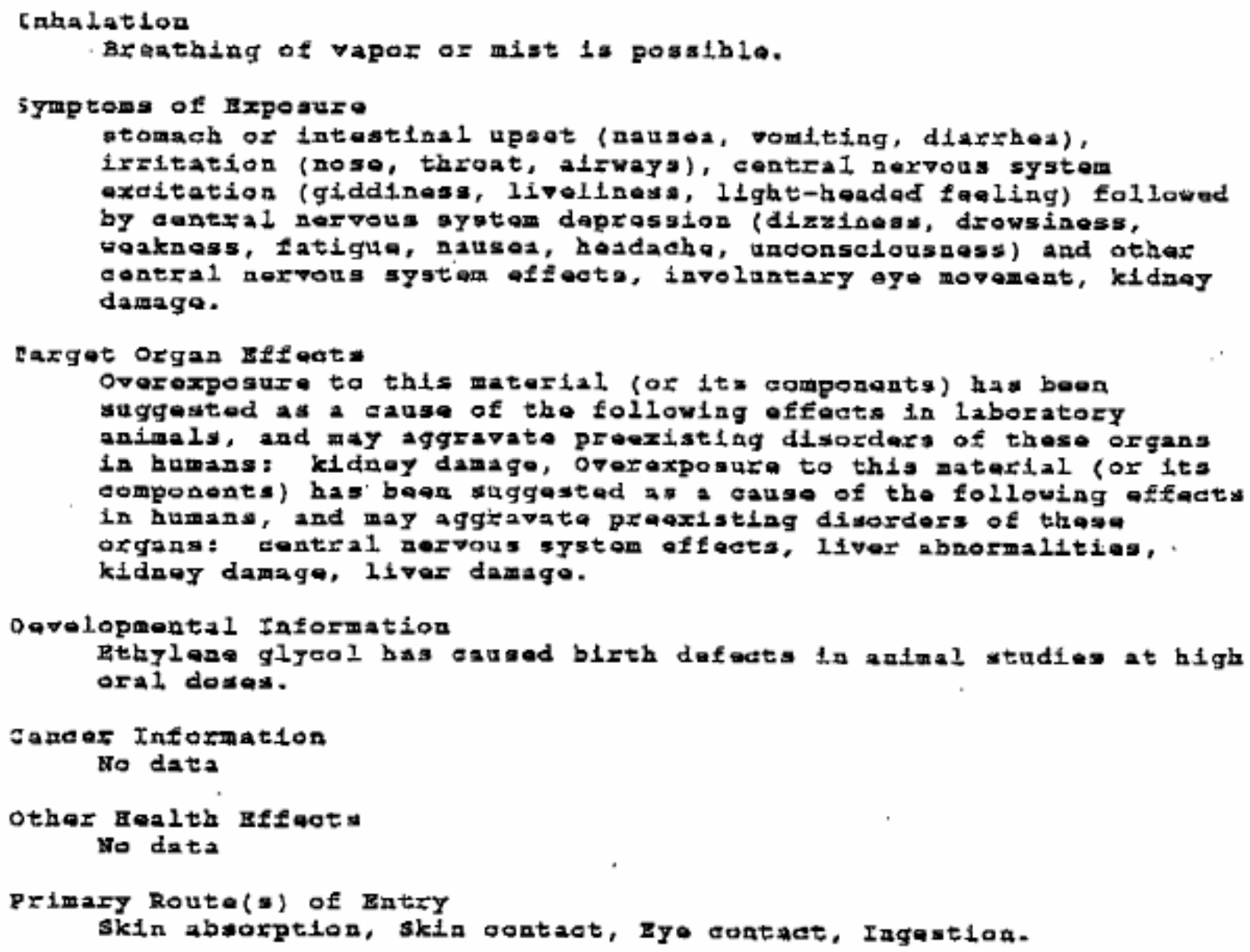

4. FIRST AID MEASURE

Eyas

If ipmptons devaiop, Imadiatoly movo lndividual ayay from exposure and lnto frash air. Plush ayas gantly vith vatar for at loast 15 minution whilo bolding egelld; ppart; seek lmmedinto modidal attontion. 
MATERIAZ SARETY DATA SHEST

T. Valvolina Company

ZBRBX 50/50 PREMIX
Paga 003

Dato Prepareda 10/05/01

Data Pxistad: $05 / 17 / 04$

MSDS No: 503.0321158-001.004

skin

Remove contaminated clothing- Wash axposed area with acap and watar. If syiptoms persist, sook medidal attontion. Launder olothing before rousa.

Stallowing

saak modleal attantion. If lndividual is drowiy or unconscious, do not giva ayything by mouth; place individual on the loft sida aith the head down. Contact a physieian, medidal facility, or poisot control denter for advice about whathar to indugo voniting. If possible, do not loavo individual unattondod.

Inhalation

If symptoms davolop, lmmadatolg mova individual away from

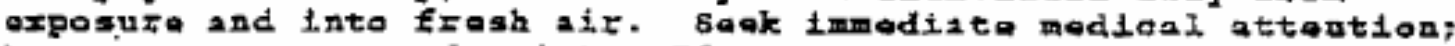
kaip person warm and qu1at. If porson is not braathing, bogin artiflelal roapization. If bzeathing is difficult, adminintor oxgqan.

Nota ta Physialans

This product oontalns athyleno glycal. Zthanol decreases the motabolism of athyleno glyaol to toxia motabolitas, Bthanol.

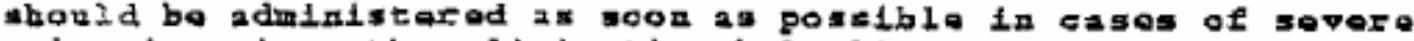
poisoning singa tha elimination half-lifo of athylane glyaol ia 3 hour*. If medidal care vill bo dolaged sovaral hourw, give tha patiant threa to four 1-auneo oral shats of 86-proof or highor whisk (4-methylpyxazola) 1 an affactive thtagonist of aloohol dehydrogana\$a, and as such, may bo usod as an antidoto in tho

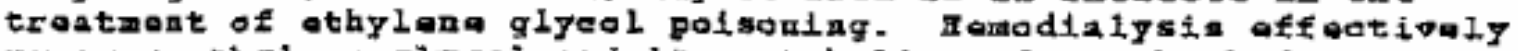
renoves athylene glyaol atad lta metabolitas from tho body. gffects of asute othylone glycol potsoning appear in thraa falliy distindt stagas. The indtinl ataga oeduxs shortly after exposuro, lasta 6-12 houxy, and is chazacterizdd by oontral norvoua systom offects (transiant axhilaration, anusaa, vomiting, and in severe casos, cork, convulatons, and posathla dazth. The socond waga

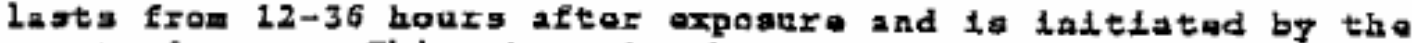
onset of coma. Th1a phase lis oharactorizad by tachypria, tadhycardia, mild hypotension, oyanosis, and in sovoro casos, pulmonary odoma, bronchopneguonta, eardiac onlargomont, and

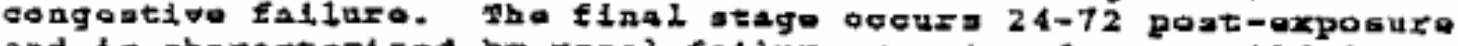
and 1 charactarizod by zanal fallura zanging from a inid increase 1n bload aran aitrogon and croutinina followed by focovary to complato anuria with aduto tubular nooroala that an laad to doath. Oxiluxin in found in mogt ansas. The toost signifigant laboratory finding in othylana glyad intoxidation 13 savaro continued on noxt paga 


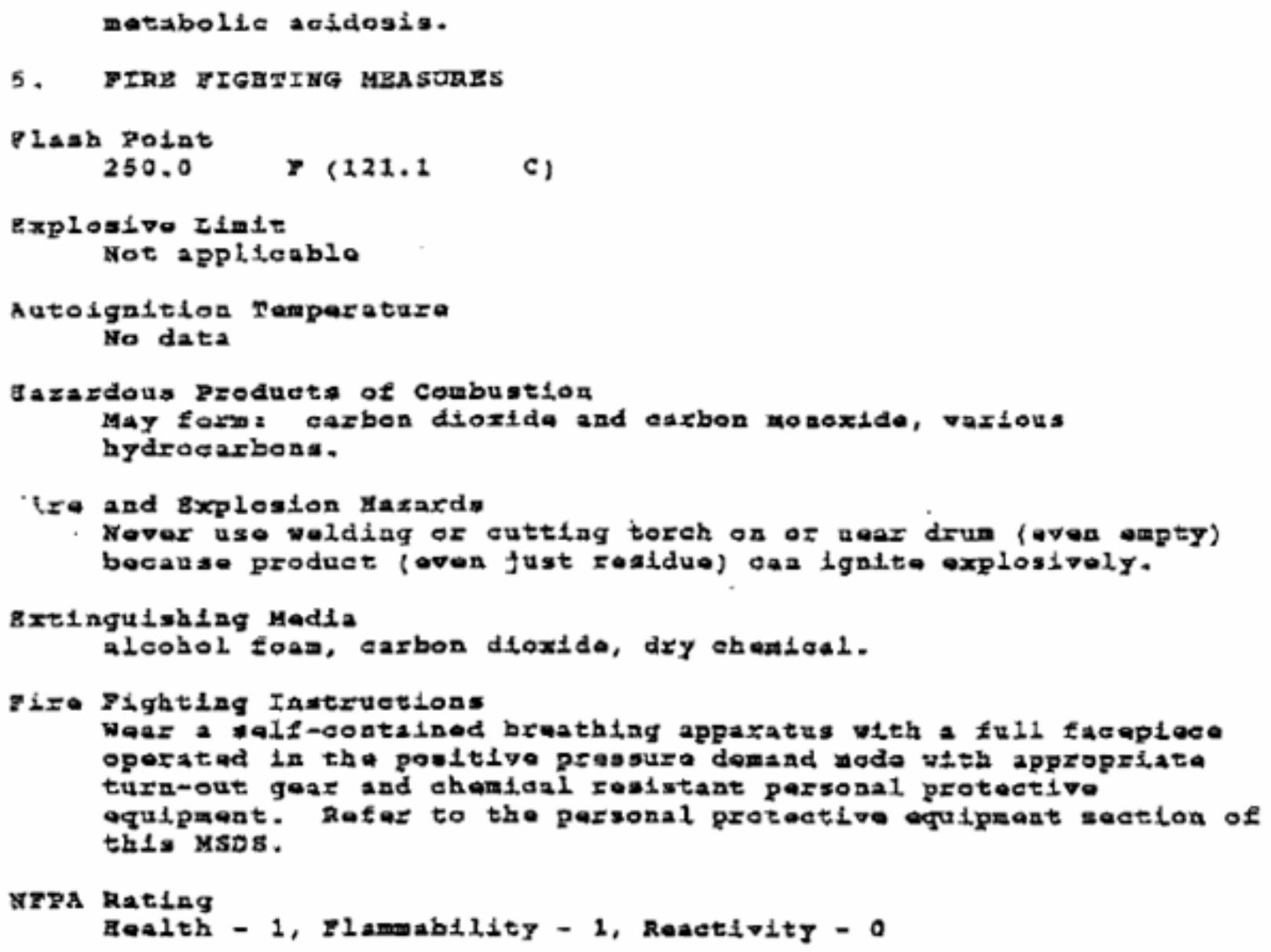

6. ACCIDEWTAY RELBASE MEASURES

$\operatorname{sina11} \operatorname{spi11}$

Abporb liquid on varalculita, floor absorbent ox otbor absorbant matorial. 
r. Valvoling coupany

ZEREX $50 / 50$ PRRMIX
Page 005.

Date Prepared: $10 / 05 / 01$

Data Printed: 05/17/04

MSDS Not $503.0321158-001.004$

Laxge spill

persona not weariag protective equipmant should bo axoluded from araa of apill until clean-up has been completad. Stop spill at sourda, diko arad of epill to provent sproading, purip liguid to salvage tank. Ramainlng 11quid may bo taken up on sand, clay, axth, floor absarbeat, or othar absarbent antarial and shovaled into contalners.

\section{MANDIIXG AND STORAGZ}

Hand $11 \mathrm{ng}$

Contalnars af this matorial mag bo hazardous whur omptied. sinca amptiod sontalnors retain product zasiduos ( solid), all haxaxd precautions givan in tha data abeat must be obaarved.

;ago

Hot appltanbla

\section{EXPOSWRB CONWROLE/PERSONAT PROTRCTIOU}

Bya protaction

chamlant splagh gogglos in aompliane with os advisad; howarar; osan ragulationa also parmit othor typa anoty glassas. Cansult your safaty ragresantativa.

skin protaction Woar Fenlstant gloves wuah as: nqoprena, nftrila rubbor. polyvingl ahleriea, To pravont ropoatad or prolangad skin contact, waar imporvious elothing and boots.

Roaplinatory protactions

If workplace axponaxa limit(a) of praduct ar any componant ia

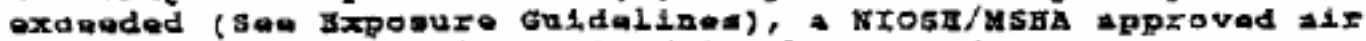
supplied raspirator 1s advigad in absanco of proper onvirontertial dontrol. Osta ragulatians also parmit athor NIOsH/MSAA ragpirators (negetivo prasaure typo) undor spacified aonditions (conault gour lnduxtrial hggtenist). Enginaring or adminfstrativo cantrols mhould bo inplomentad to roduct axposure. 


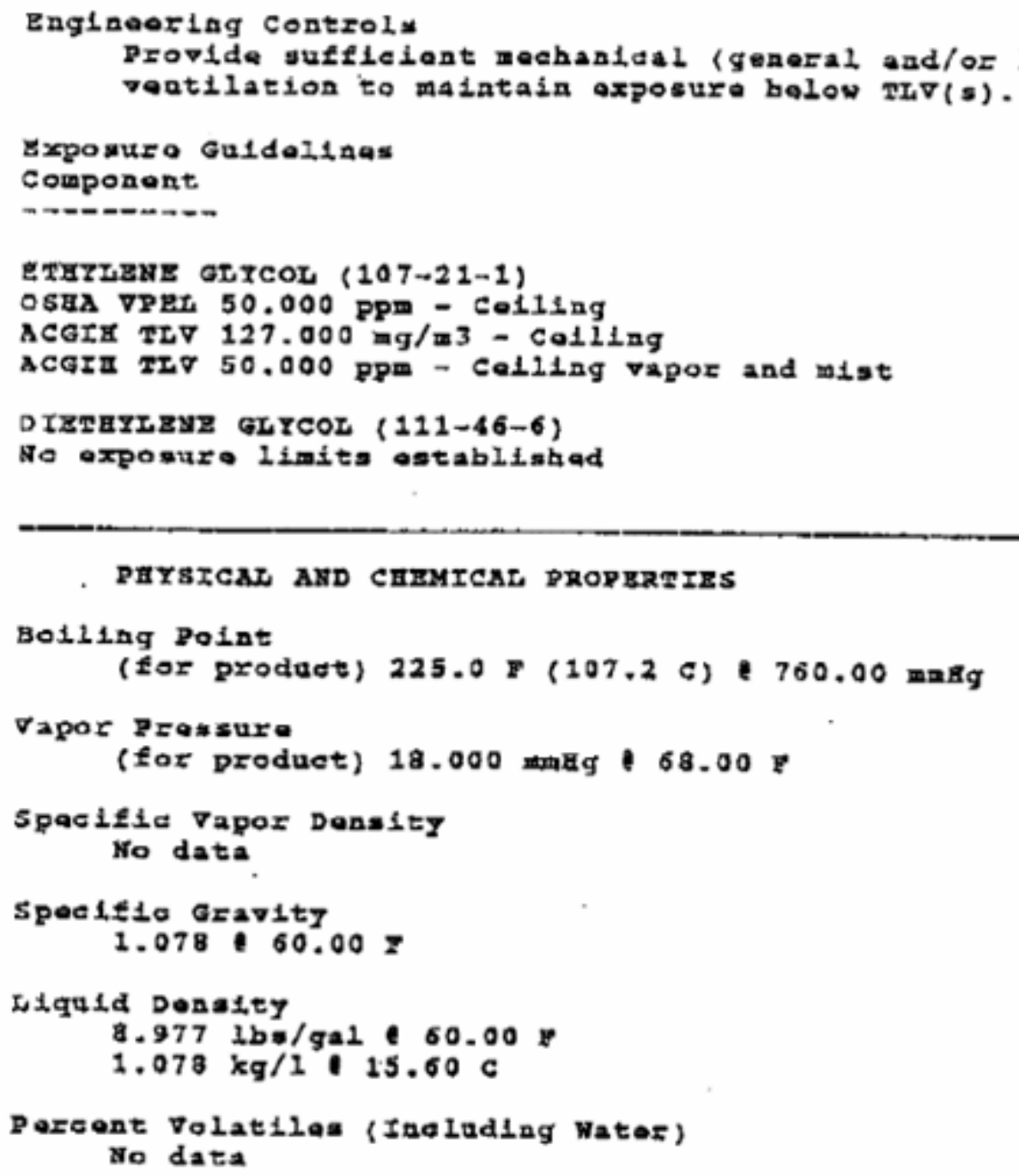


2 Valvoline Compeny

zR28X 50/50 pRzMIX
Page 007

Data Preparad: $10 / 05 / 01$

Date Printod: 05/17/04

MSDS SO: 503.0321158-001.004

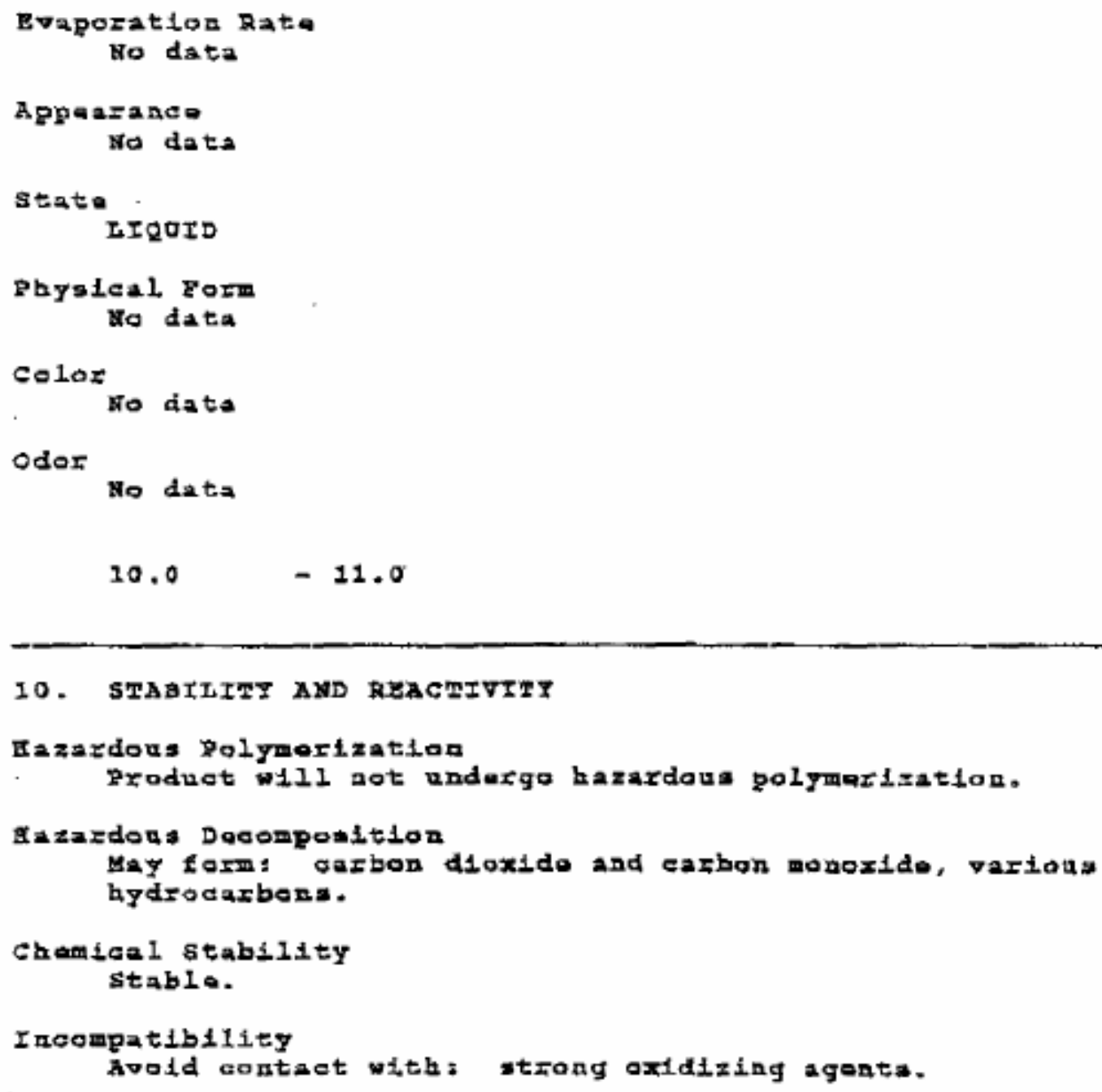


11. TOXICOIOGICAI IXYORMATIOH

No dara

12. ZCOLOGICAL TRZORMATION

No data

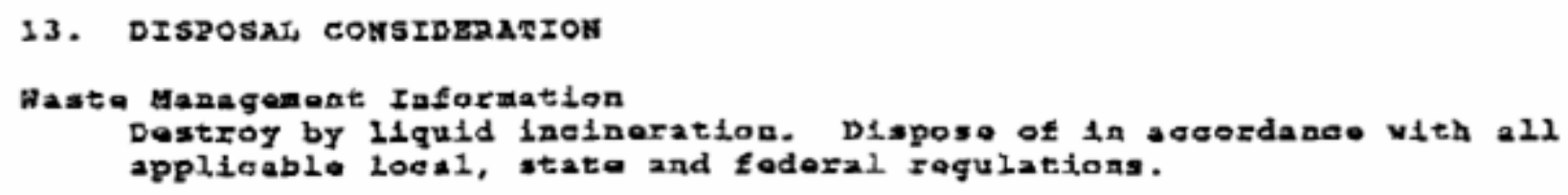

14. TRAMSPORT INPORMATIOK

DOT raformation - $49 \mathrm{CPR} 172.101$

DOT Davaription:

Not Rogulatod

Containar/Mode: CASES/ SORYACZ - SO EXCEPTIOXS

sos Component: Nona

RQ (Raportabla Quant1ty) - $49 \mathrm{crR} 172.101$ produat Quantity (Lba) Caaponont

- 10456 BTHYIEXE GTrCor

15. RZGULATORT IMPORMAIIOX

os Padoral Regulation:

TSCA (Toxto Substancas Gontrol Aat) status TSCA (ONITED STATEs) The Lntantional Lageodionts of this product aro liated.

contiauad on noxt page 
MATERTAL SAFETY DATA SERBT

T

valvolino Company

Page 009

Data Preparod: 10/05/0I

Date Priatad: 05/17/04

ZRRZX 50/50 PREMIX

MSDS NO: 503.032:158-001.004

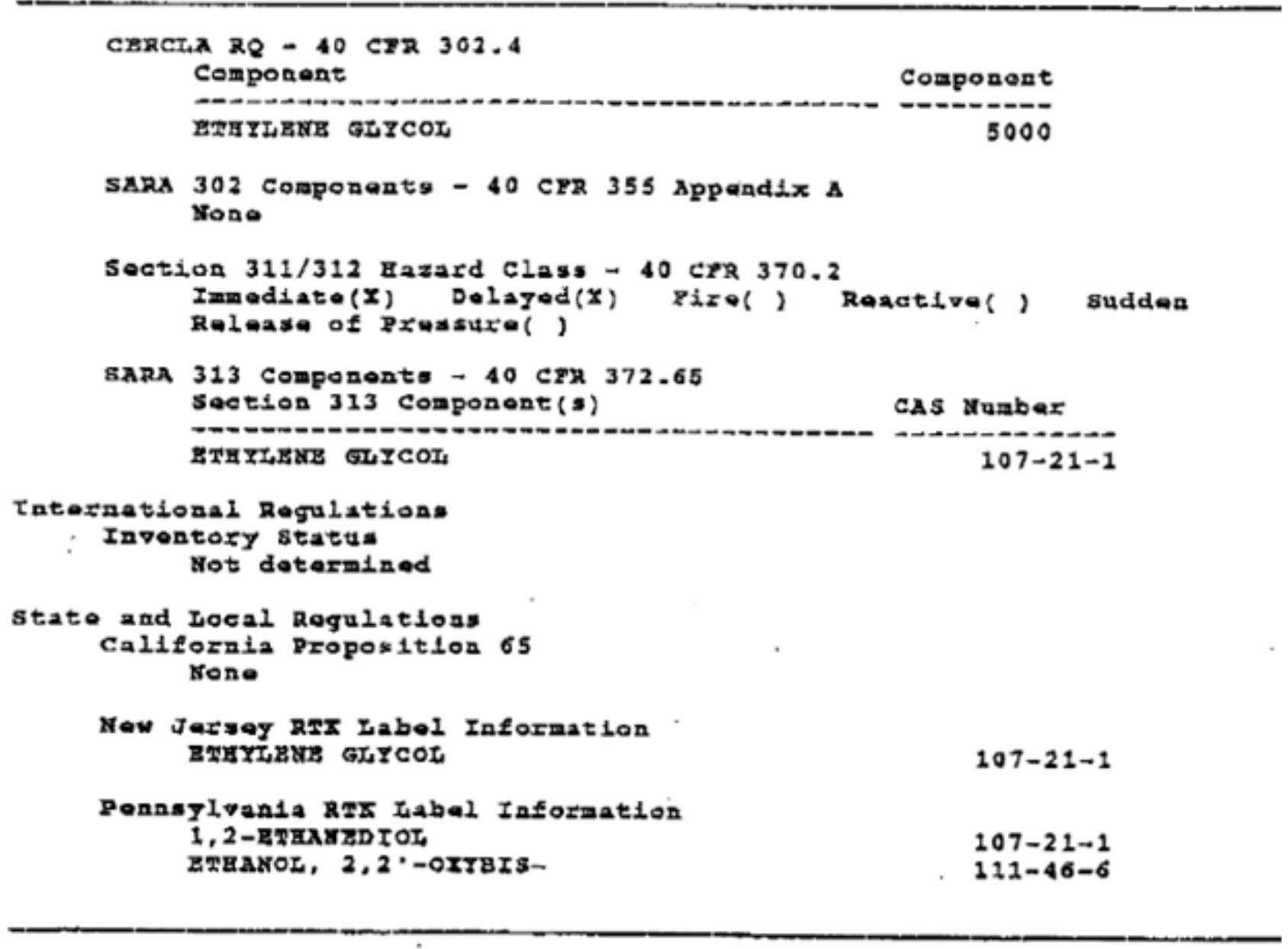

16. OTgEn IXFORMATIO\&

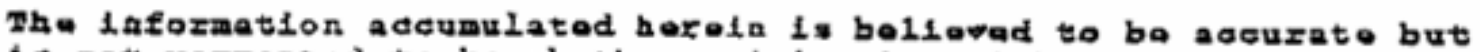
1 a not warranted to be whothor origlaatiag with tho eoppan or not. Recipients aro adplaed to oofira in advaneo of naed that tho inforaatioa is eurront, applicablo, and sultablo to thoir c1roumstanoau.

Last paga 
1. CHEMICAL PRODGCT AND COMPANY IDENTIEICATION

Matorial Identity

Product Name: BLẼND \#0091A PREMIUM STARTING FLUID

General or Generic ID: SOLVENT SLEND

Company

The Valvaline Company

P.0. Box 14000

Lexington, $\mathrm{KY} 40512$

Telaphone Numbers

Emergency: 2-900-274-5263

Information: 1-359-357-7206

\section{COMROSITION/ IXYORMATION ON INGREDIENTS}

$\begin{array}{lrr}\text { Ingzedient }(s) & \text { CAS Number } & \text { (by weight) } \\ \text { HERTANE } & 142-82-5 & 60.0-70.0 \\ \text { ETHYL ETHER ACS REAGENT GRADE } & 60-29-7 & 23.0-33.0 \\ \text { CARBON DIOXIDE } & 124-38-9 & 1.0-11.0\end{array}$

\section{HAZARDS IDENTIEICATION}

Potential Health Effects

aye

May cause mild sye irritazion.

Skin

Can cause skin irritation. Prolonged or repeated contact may dry and crack the skin. Passage of this material into the body through the skin is possible, but it is unitikely that this would result in harmeul effaces during sate handing and use.

\section{Swallowing}

Swallowing smail amounts of chis material during normal handing is not likely to cause hamiul effects. Swallowing large amounts may be harmful. This material can get lato tho lungs during swallowing or voriting. This eesules in lung inflammation and other lung injury.

Iahatarion

3reathing aerosol and/or mist is possible when material is sprayed. Aerosol and nist nay present a greater rigk oe injury because more material may be present in the air than from vapor alone. Breaching small amounts of this matarial during normal banding is not likely to cause hazmful zffects. Breathing large amounts may be harmful. Symptons usually occur at alr concentrations higher than the recommended exposure limits (Seo section B).

Symptoms of Exposure

Signs and symptoms of exposure to this macerial through braathing, swallowing, and/or passage of the materia: through the skin may 
include: stomach or intestina! upset (nausea, vomiting, diazkhea! irrtiation (nose, thrcat, airways), centräl nexvcus system deprossion (dizziness, drowsiness, weakness, fatigue, nausea, headacine, unconsciousnass), Loss of appetits, loss or̆ coordination irregulas heartbeat, narcosis (dazed or sluggish faeling),

Targat Organ Eflects

testis damage, luag damage, visual impairment, centrą trervous system effects.

Developmental Intormation

There are no data availacle for assegsing risk to the fotus from maternal exposuze to this material.

Cancer Information

This material is not listed as a carcinogen by the International Agency for Research on Cancer, the warional roxicology program, or the cccupational Safety and Fealch Adminiszzazion.

Other Health Effects No data

Primary Route(s) of Entry Inhalation, Skin absorption, Skin contact, Eye contact.

4. TIRST AID MEASURES

Eyes

If symptoms develop, move individual away from exposure and iato fresh air. Flush eyes gently with water while holding eyelids apatt. If symptoms persist or there is any visual difficuity, seek nedical attention.

Sikin

Remove contaminated ciothing. Flush exposed area with large amouncs of water. If skin is damaged, seek immediace medical attencion. If skin is not damaged and symptoms persist, seek medical attention. taunder clothing before reuse.

Swallowing

Seek medical attencion. If Individual is drowsy or unconscious, do not give anything by mouth; place sndividual on the left side with the head down. Contact a physician, medical facility, or poison control center for advice about whether to induce vomiting. If possible, do nor leave individual unatended.

Inhalation

If symptoms develop, move individual away from exposure and into fresh air. It symptoms persist, seek medical atcention. If breathing is difficult, administer oxygen. Keep person warm and quiet: saek immediate medical attention.

Note to Physicians

Inhalation of higin concentrations of this material, as could occur in enclosed spaces or curing deliberare abuse, may be associared with cardiac arthythmias. Sympathominetic drugs may iniciare cardiac arrhythmias in persons exposed to this material. This material is an aspiration hazard. Potencial danger from aspiration musc be weighed against possible oral toxicity (See Section 3 Swallowing) when deciding whether to induce vomiting. Preexisting disorders of the folloring organs ( of organ systems) may be 
aggravated by expcsure tc this material: skin, lung (for example, astina-iika conditions), Individuals wich prevexisting heart disorders may be moze susceptible to arrhythmias (irregular heartbeacs) it exposed to high concentrations of this material.

S. TIRE RIGRTING MEASURES

Elash Point

Not applicabla

Explosive timit

(Lor component) Lowez 1.0

8

Autoignition Temperaturs

No data

Hazardous Products of Combustion

May form:

Firg and Explosion Hazards

Material is highly volatile and readily gives off vapors which may travel along the ground or be moved by ventilation and ignized by pilor lights, other flames, sparks, heaters, smoking, electric motors, static discharge, or other ignition sources at locations distant from material handing point. Never use welding or cutting torch on or near drum (ovon empty) because product (even just rosidue) can ignite explosively.

Sxefnguishing Modia

Wo data

Fire Fighting Instructions

Wear a self-contained breathing apparatus with a full Eacopiece operated in the posicivo pressuze demand mode with appropriate tura-out gas and chenical resistant personal protactive equipwent. ReEer to the personal protective aquipment saction of this MSOS.

NFEA Rating

Bealth - 1, Flammability - 4, Reactivity - 0

\section{ACCIDENTAL RELEASE MEASURES}

Small Spizl

Eliminate all sources of ignition such as slares, flames (including piloz lights), and electrical sparks. Absorb isquid on vermiculite, t:oor absorbent or other absorbent material. persons not wearing proper personal procective equipment should be excluded from area of spill.

Large Spi11

Prevent run-ot: to sewers, screams or other bodies of water. If run-off occurs, notify proper auchorities as required, that $a$ gyil has occured. Zersons not wearing protective equipment should be excluded from area of spill uat 1 clean-up has been completed. Elininate all ignition sources (Elares, tlanes, including pilot lights, electrical sparks). 
Hendliag

Containers of this material may be hazardous whan amptied. Since amptied concainers ratain product residues (vapor, iiçuid, andior solid), all hazard grecautions given in the daca sheet must be observed. All five galion pails and larger metal containers including tank cars and tank trucks should be grounded and/or bonded when material is trans lerred. Pracautions during use: avoid prolonged or frequently repeated skin contact with this material. Skin contact can be mininizad by wearlag impervious protective gloves. As with all products of this zature, good personal hyglene $f s$ essential. Hands and otiner exposed areas should be washed thoroughly with soap and water efter contact, especially before eating and/or smoking. Regular laundering of contaminated clothing is essential to raduce ladirect skin contact with this material. Hydrocarbon solvents are casicaily non-conductors of olectricity and can become electrostatically charged during mixing, filtering or pumping at high flow rates. If this charge reaches a surficiently high level, sparks can form that may ignite the vapors of flanmable liquids.

\section{Storage}

Do not srors near extreme heat, open flame, or sources of ignition.

\section{EXPOSURE CONTROLS/PERSONAL PROTECTION}

Bye Protection

Chemical splash goggles in compliance with OSHA regulations ax? advised; however, csta regulations also permit other type safety glasses. Conșult your safecy representarive.

Skin Protection

Wear resistant gloves (corsult you: safety squipment suppliaz). To praven repeated or prolonged skin contact, weaz impervious clothing and boots.

Respiratory Protections

If workplace exposure limit(s) of product of any component is exceeded (See Exposure Guidelines), a NIOSH/MSHA approved ais supplied respirator is advised in absence of propor anvironmental control. OSHA regulations also permit other NIOSA/MSHA respirators (negative pressure type) under specified conditions (consult your industrial hygienist). Engineering or administrative controls should be implemented to reduce exposure.

Zngineering Controls Provide sufficienc mechanical (general and/or local exhaust) ventilation to maintain exposure below TLV $\{3\}$.

Exposuze Guidelines

\section{Component}

- - -

HEFTANE $(142 \sim 32-5)$

OSHA VPEL $1600.000 \mathrm{mg} / \mathrm{m} 3$ - PWA

OSHA VPEZ 400.000 gom - TWA

OSHA VPEL S00.000 PON - STEL

OSHA VDEL $2000.000 \mathrm{mg} / \mathrm{m} 3$ - STEL 


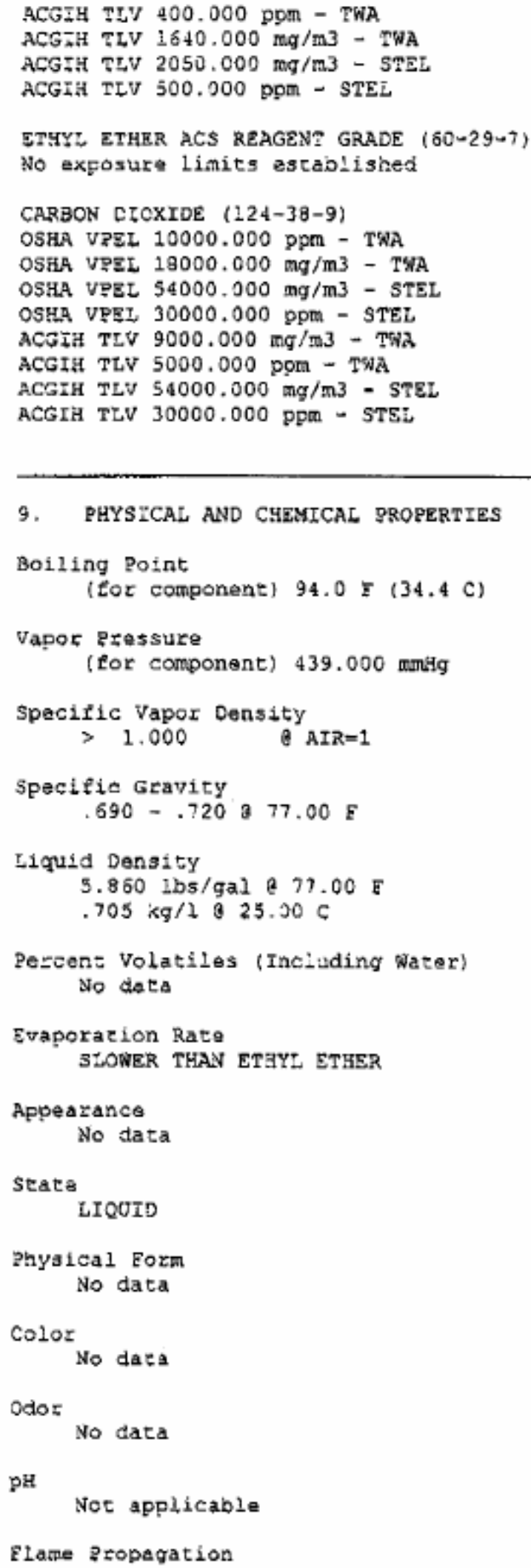

9. PHYSTCAL AND CMEMICAL gROPERTIES

Boiling Point (for component) 94.0 I (34.4 C)

Vapor Pressure 
$>\quad 18,000$ in

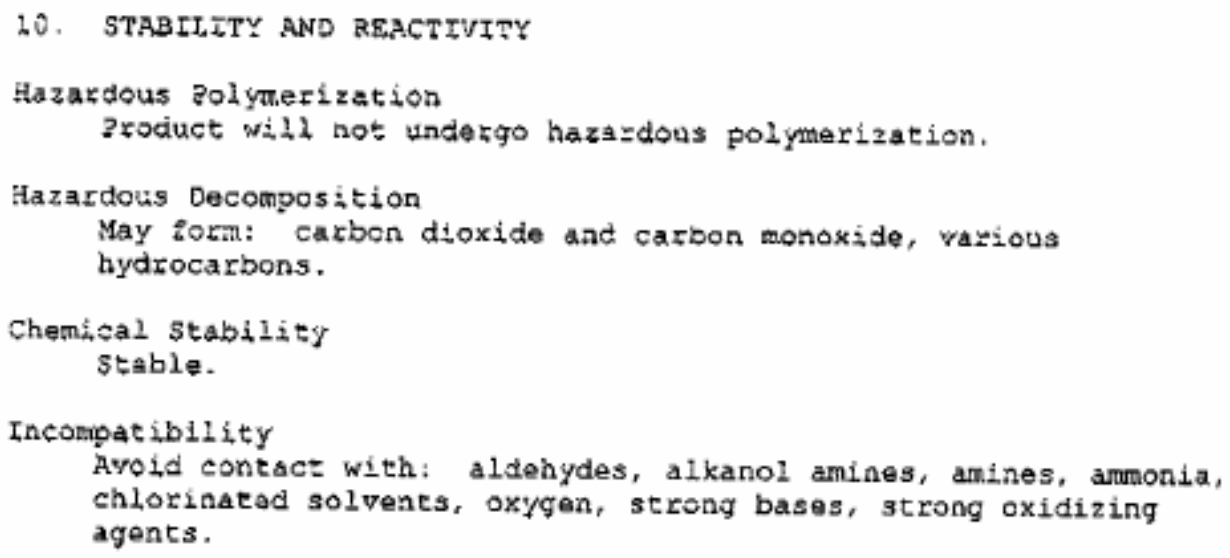

12. TOXICOLOGICAL INEORMATION

No data

12. ECOLOGICAL INFORLATION

No data

13. DYSPOSAL CONSIDERATION

Wasts Management Inf́ornation

Dispose of in accordance with all appliceble local, state and

Eederal regulations.

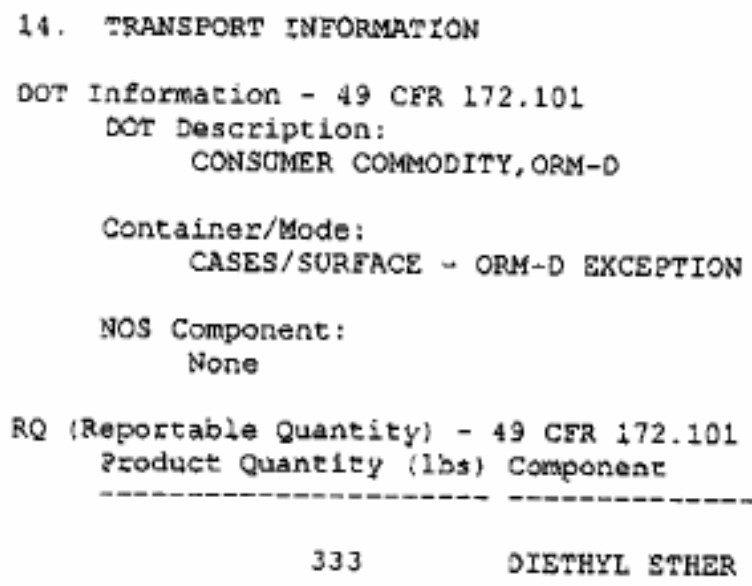




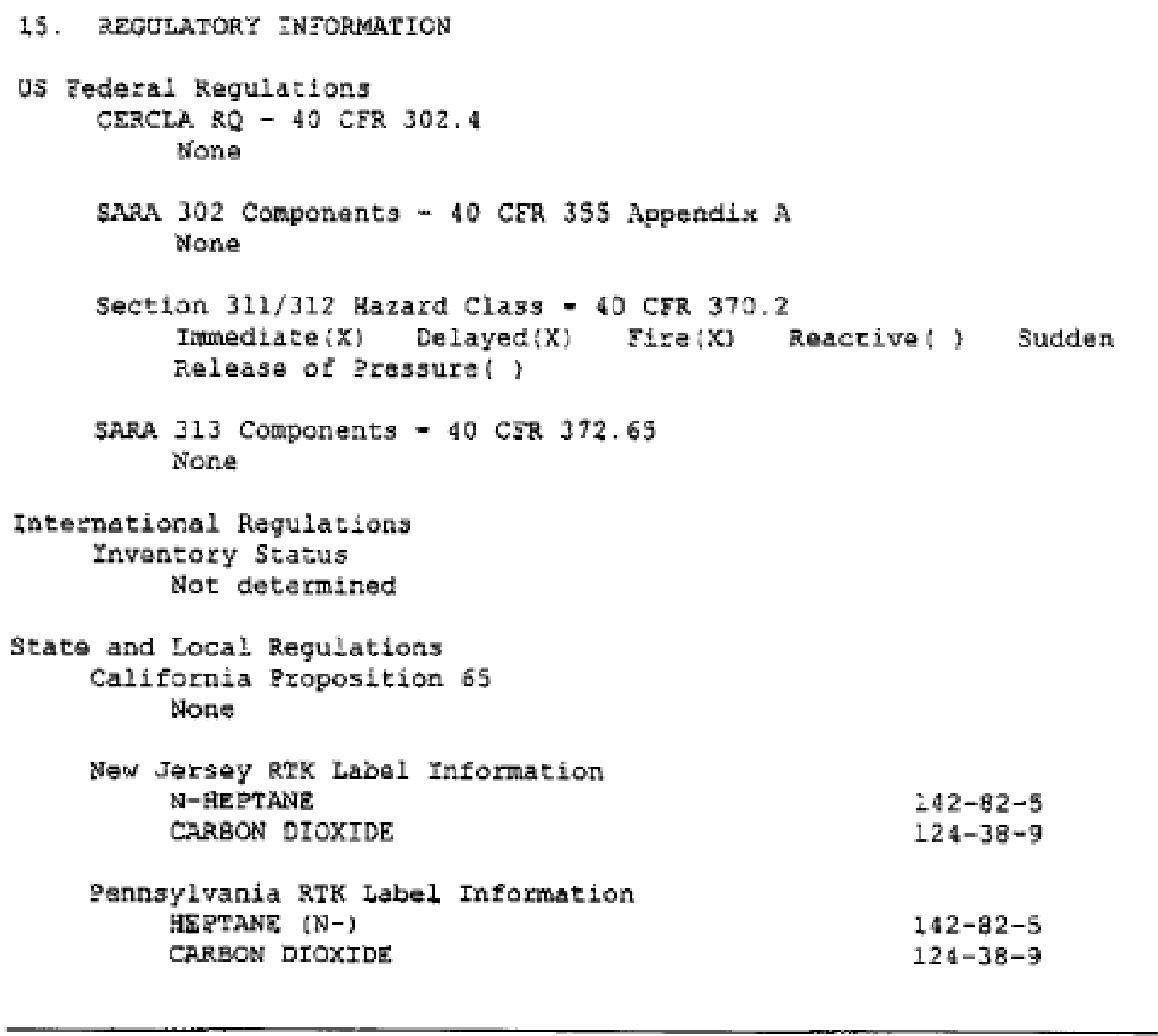

16. OTHER INEORMATION

The information accumulaced herain is believed to be accurate but is not warrantad to be whether originating with the company or not. Recipients are advised to confirm in advance of need that the information is current, applicable, and suitable to cheir circumstances.

Last page 


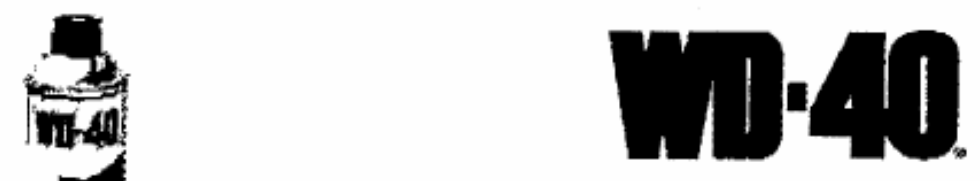

\section{MATERIAL SAFETY DATA SHEET}

1. PRODUCTIDENTIFICATION

\begin{tabular}{|c|c|c|c|}
\hline $\begin{array}{l}\text { Manufacturer: } \\
\text { Address: }\end{array}$ & $\begin{array}{l}\text { WD-40 Company } \\
1061 \text { Cudahy Place }(92110) \\
\text { P.O. Box } 80607 \\
\text { San Diego, California } \\
92138-0607\end{array}$ & $\begin{array}{l}\text { Telephone: } \\
\text { Emergency only: } \\
\text { Information: } \\
\text { Chemical Name: } \\
\text { Trade Name: }\end{array}$ & $\begin{array}{l}\text { 1-(800) 424-9300 (CHEMTREC) } \\
\text { (619) } 275-1400 \\
\text { Organic Mixture } \\
\text { WD-40 Aerosol }\end{array}$ \\
\hline
\end{tabular}

\section{HAZARDOUS INGREDIENTS}

\begin{tabular}{|lccc|}
\hline \multicolumn{1}{|c}{ Chemical Name } & CAS Number & $\%$ & Exposure Limit \\
Aliphatic Petroleum Distillates & $8052-41-3$ & $45-50$ & $100 \mathrm{ppm}$ PEL \\
Petroleum Base Oif & $64742-65-0$ & $15-25$ & $5 \mathrm{mg} / \mathrm{M}^{3} \mathrm{TWA}$ (mist) \\
LVP Hydrocarbon Fluid & $64742-47-8$ & $12-18$ & $1200 \mathrm{mg}^{3} / \mathrm{M}^{3} \mathrm{TWA}$ \\
Carbon Dioxide & $124-38-9$ & $2-3$ & $5000 \mathrm{ppm}$ PEL \\
Non-hazardous ingredients & & $<10$ & \\
\hline
\end{tabular}

\section{PHYSICAL DATA}

\begin{tabular}{|llll|}
\hline Boiling Point: & $323^{\circ} \mathrm{F}$ (minimum) & Evaporation Rate: & Not determined \\
Vapor Density (air=1): & Greater than 1 & Vapor Pressure: & $110 \pm 5$ PSI @ $70^{\circ} \mathrm{F}$ \\
Jlubility in Water: & insoluble & Appearance: & Light amber \\
peciflc Gravity $\left(\mathrm{H}_{2} \mathrm{O}=1\right):$ & $0.817 @ 72^{\circ} \mathrm{F}$ & Odor: & Characteristic odor \\
rercent Volatile (volume): & $74 \%$ & VOC: & 412 grams/liter $(49.5 \%)$ \\
\hline
\end{tabular}

\section{FIRE AND EXPLOSION}

Flash Point:

Flammable Limits:

Extinguishing Media:

Special Fire Fighting Procedures:

Unusual Fire and Explosion Hazards: $131^{\circ} \mathrm{F}$ Tag Closed Cup

(Solvent Portion) [Lel] 1.0\% [Uel] 6.0\%

$\mathrm{CO}_{2}$, Dry Chernical, Foam.

Contents Under Pressure

FLAMMABLE - U.F.C. leve! 3 AEROSOL.

V. HEALTH HAZARD / ROUTE(S) OF ENTRY

Threshold Limit Value
Symptoms of Overexposure
Inhalation (Breathing):
Skin contact:
Eye contact:
ingestion (Swallowed):

First Aid Emergency Procedures Ingestion (Swallowed):

Eye Contact:

Skin Contact:

Inhalation (Breathing):

\section{DANGER!}

Aspiration Hazard:
Aliphatic Petroleum Distillates (Stoddard Solvent) lowest TLV (ACGIH 100 ppm.)

May cause anesthesia, headache, dizziness, nausea and upper respiratory irritation.

May cause drying of skin and/or irritation.

May cause irritation, tearing and redness.

May caused irritation, nausea, vomiting and dianthea.

Do not induce vomiting, seek medical attention.

immediately flush eyes with large amounts of water for 15 minutes.

Wash with soap and water.

Remove to fresh air. Give artificial respiration if necessary.

If breathing is difficult, give oxygen.

Pre-existing medical conditions such as eye, skin and respiratory disorders may be aggravated by exposure.

If swallowed, can enter lungs and may cause chemical pneumonitis.

Do not induce vorniting. Call Physician immediately.

The romnnnante in this mivtu ure have hoon found th he noncarninnnenic hv NTP. 
VI. REACTIVITY DATA

\begin{tabular}{|lll|}
\hline Stability: & Stable $\mathrm{X}$ & Unstable \\
Conditions to avoid: & NA & Strong oxidizing agents \\
:ompatibility: & Thermal decomposition may yield carbon monoxide and/or carbon dioxide. \\
azardous decomposition products: & May occur & Will not occur_X \\
\hline
\end{tabular}

\section{SPILL OR LEAK PROCEDURES}

Spill Response Procedures

Spili unlikely from aerosol cans. Leaking cans should be placed in plastic bag or open pail until pressure has dissipated.

Waste Disposal Method

Empty aerosol cans should not be punctured or incinerated; bury in land fill. Liquid should be incinerated or buried in land fill. Dispose of in accordance with local, state and federal regulations.

\section{VIII, SPECIAL HANDLING INFORMATION}

Ventilation:

Respiratory Protection:

Protective Gloves:

Eyo Protection:

Other Protective Equipment:
Sufficient to keep solvent vapor less than TLV.

Advised when concentrations exceed TLV.

Advised to prevent possible skin irritation.

Approved eye protections to safeguard against potential eye contact, irritation or injury. None required.

\section{SPECIAL PRECAUTIONS}

Keep trom sources of ignition. Avoid excessive inhalation of spray particles, do not take internally. Do not puncture, incinerate or store container above $120^{\circ} \mathrm{F}$. Exposure to heat may cause bursting. Keep can away from electrical current $o r$ battery terminals.

Flectrical arcing can cause bum-through (puncture) which may result in flash fire, causing serious injury. Keep from children.

\section{X.TRANSPORTATION DATA (49 CFR 172.101)}

Domestic Surface

Description: Consumer Commodity

Hazard Class: ORM-D

ID No: None

Label Required: $\quad$ Consumer commodity (ORM-D)

\section{REGULATORY INFORMATION}

All ingredients for this product are listed on the TSCA inventory.

SARA Title III chemicals: None

California Prop 65 chemicals: None

CERCLA reportable quantity: None

RCRA hazardous waste no: $\quad$ D001 (Ignitable)

SIGNATURE: Peter Fougner REVISION DATE:

December. 2004

SUPERSEDES: _. Nevember. 2003

AA: Not applicable NDA: No data available

$\langle=$ Less than $\rangle=$ More than

We believe the satements, technical information and rushumendations contained herain are reliablo. However, the đata is provided without warranty, oxpressad or impled. It is the usar's responsibility both to determine safe conditons for use of this product and assume loss, damage or axpense, diroct or conseguenciai. arieing from its use. Before using product, read tabel. 


\section{arking Paint - Aerosol}

\section{Material Safety Data Sheet}

forms is behicved to be correcl and sufficien tomeet the requirements of OSHA Hazant Conmunication cerning worker's right to know.

sheet covers tie hazardous ingredienls associated with more than one collor aerosol product. As per Whenever the hazards associated with simzilar mixtures sre the same, Gen one isSCS may be prepared

y Markining Paint

\begin{tabular}{|c|c|c|c|c|}
\hline Fluorescent Colors & 16 ox & 2. LAC. & High Delivery & Meialtic \\
\hline $\begin{array}{l}220 \text { Red } \\
222 \text { Orange }\end{array}$ & $\begin{array}{l}\text { 261S Red } \\
\text { 262S Yellont }\end{array}$ & $\begin{array}{l}\text { 270S Flusrescent Red } \\
272 S \text { Fludrescent Orange }\end{array}$ & $\begin{array}{l}281 \text { Red } \\
282 \text { Yellowr }\end{array}$ & 210 Siver \\
\hline 224 Green & 263S Bive & 274 Fluorescent Green & 289 Fluorescenl Otange & \\
\hline 226 Yellow & 265 S Orange & 2rjs Filuorescost RediOrange & & \\
\hline 227 alitur & 267S whine & 279S Fluorescent Pink & & \\
\hline $\begin{array}{l}229 \text { Fudk } \\
230 \text { Redorerme }\end{array}$ & & & & \\
\hline
\end{tabular}

230 Redroreme

\section{SECTION I - MANUFACTURER IDENTIFICATION}

$$
\begin{array}{ll}
\text { ADD Industies, inc. } & \text { ADESS: 1198 Mark Circle, Gardnenile, NV } 89410 \\
.0100 & \text { EMERGENCY PHONE: 1-800-424-9300 } \\
& \text { REASON REVISED: Updated }
\end{array}
$$

\begin{tabular}{|c|c|c|c|c|c|c|c|c|}
\hline$x$ ane & xylene & \begin{tabular}{|l|} 
Aliphádic \\
Petroleum \\
Disstllates \\
\end{tabular} & $\begin{array}{l}\text { Ethyi } \\
\text { Acestas }\end{array}$ & Acebne & $\begin{array}{l}\text { Gycol Ether } \\
\text { EB Acetate }\end{array}$ & $\begin{array}{l}\text { MDaf } \\
\text { Naphtha }\end{array}$ & $\begin{array}{c}\text { Niphalc } \\
\text { Hydrocarbon }\end{array}$ & $\begin{array}{l}\text { n-Buly } \\
\text { Acetale }\end{array}$ \\
\hline j-54-3 & $1330.20-7$ & 64742.83 .7 & 144-786 & $67.64 \cdot 1$ & $112-07-2$ & 64742-89-8 & $64742-47.8$ & $123-86-4$ \\
\hline jppan & $100 \mathrm{ppm}$ & $100 \mathrm{ppon}$ & 400 ppan & $1000 \mathrm{ppm}$ & NIAV & $300 \mathrm{ppm}$ & $N I A V$ & $150 \mathrm{ppm}$ \\
\hline ipom & $103 \mathrm{ppm}$ & $100 \mathrm{ppm}$ & $403 \mathrm{pran}$ & $500 \mathrm{ppm}$ & NIAV & $400 \mathrm{ppm}$ & $N / A V$ & 150 ppm \\
\hline 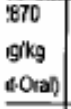 & $\begin{array}{c}4300 \text { molkg } \\
\text { (Rat-aial) }\end{array}$ & N/AV & $\mathrm{N} / \mathrm{AV}$ & $\begin{array}{c}5953 \text { mgikg } \\
\text { (Ral Oal) }\end{array}$ & $N / A V$ & NIAV & Ne/ AV & NIAV \\
\hline IAV & $\begin{array}{l}6702 \text { ppon } \\
4 \mathrm{hr} \text { (Rats }\end{array}$ & NIAV & N/AV & \begin{tabular}{|c|}
$21,030 \mathrm{ppm}$ \\
$8 \mathrm{~K}$ (RRas \\
\end{tabular} & NIAV & WIAV & $N I A V$ & $N / A N$ \\
\hline yes & yes & no & no & no & yes & $n$ & no & no \\
\hline nt $\%$ & wh $\%$ & $w \%$ & $m \%$ & wet \% & wt \% & $m x$ & wt \% & $w \%$ \\
\hline \multicolumn{9}{|l|}{ Eotors } \\
\hline - 13 & $5 \cdot 10$ & 1.5 & 1.5 & $3 \cdot 7$ & 1.5 & & & \\
\hline \multicolumn{9}{|l|}{ s } \\
\hline-13 & & 1.5 & & & & $7 \cdot 13$ & & \\
\hline \\
\hline & $7 \cdot 13$ & & & $30-60$ & & $5 \cdot 10$ & 5.10 & 1.5 \\
\hline
\end{tabular}

\section{EDIENTS / SARA UII INFORMATION / OCCUPATIONAL EXPOSURE LIMIT}

BOLLNG PONT: N IAP

SECTHON III - PHYSTCAL / CHEMICAL CHARAC STICS

SPECIFIC GRAVIY, 41$): 0.8$ to 09

EYAPORAIIOA RATE: Faster than n-Butyi Acetate APPEARANCE. AND ODOR: Oplaque liquid n

FLASH POINT: $\angle 0^{\circ} \mathrm{F}: 19^{\circ} \mathrm{C}$ SECTIOH IV - FIRE AHID EXPLOSION HAZARD DATA

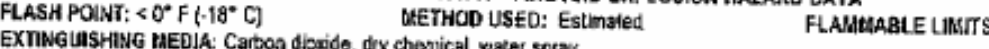

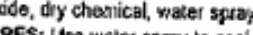

LAMALABLE LIMITS

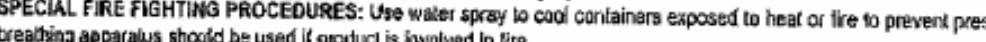
UNUSUAL FIRE AN

pressure buld up from extreme temperature. SENSIINITY TO Aamnatte aerosal under consilitions of sparits, flant, of hol surfaces.

SENSIIVITY TO STAYIC DISCHARGE: Primaray

STABILITY: Stable $\quad$ SECTION V - REACTIVITY OATA

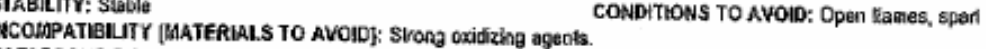
cashy agents.

HAZAROOUS POOM

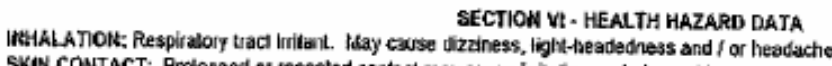

SKAN CONTACT: Prdanged or IepDLted contaci may cause irriletion and Jencattis.

EYE COATACT: PDIDY

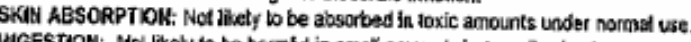

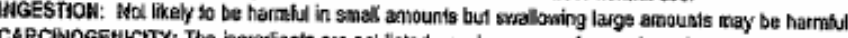

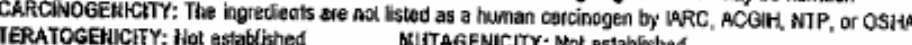

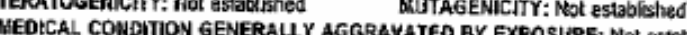

EMERGEHCY AND FIRST AID PROCEOURES: IIHALATION - RenE Mol estedishied

SKII - Wosh slfocted area with 5000 sad water.

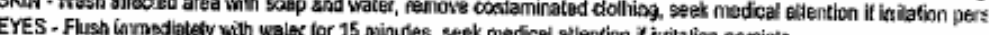

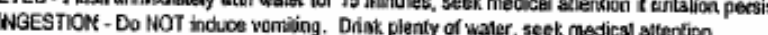

\section{SECTION VII - PRECAUTIONS FOR SAFE HANDLING AND USE}

STEPS TO BE TAKEN IN CASE IAATEREAL IS RELEASED OR SPILLE: Rernove af́ sourcess of ignilition. Ventilato walerocuure. Use an inert absorbent nisterial and asonsparting type bools.

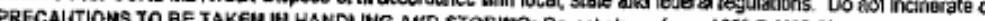

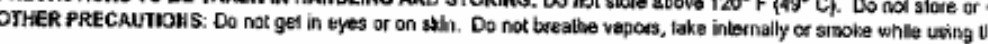

\section{BECTION VUl - CONTROL MEASURES}

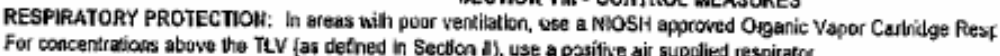

VENTILATION: General ventiation io maintain axposure firiz's belous TLV'S as defined in Section

PROTECTNE GLOVES: Chential resislani gloves such as Neaprene or Nitrie nubber.

EYE PROTECTION: Sately gasses or goggles.

OIHER PROTECTIVE CLOTHENG OR EQULPMENT: Nel ESlablished.

WORK / HYGIENIC PRACTICES: Avoid prolonged or repeated contacl. De not breathe vapors, Wash contaminated HEREIN CONSTTUTES A SPECIFICATION NOR IS IT INTENDED YO WARRANT SUITABILUTY FOR TH 


\section{srking Paint (Regular Colors) - Bulk IMaterial Safety Data Sheet}

ety data sheet as per your teques:

forms is believed to be correct and sufficient to meet the requirements of OSHA Hazard Communicalion weerning worker's cight to know. Ih order for the intormation cantained in the USDS to be most helpfit ie made aveilable to at those who hande or may othenvise be exposed to the product.

sheet covers the hazardous ingredients associated with more thax one color bulk produd. As per 29 venever the hazards associated with similas arixlures are ghe same, then one MSOS may be prepared

encue-Pactic buk products.

$$
\text { MARKIWG PAINT - REGULAR COLORS }
$$

$\begin{array}{lll}204 \text { GREEN } & \text { 207 WHITE } & 210 \text { SILVER } \\ 205 \text { ORANGE } & \text { 208 HIVISBIUTYYYLLOW } & 212 \text { PURPLE } \\ \text { 206 BLACK } & 209 \text { LIGHT BLUE } & 213 \text { BROWN }\end{array}$

\section{WNT. HEL REGULARCOLORS PROOUCT CODE: $17 \mathrm{~B}$ HUIS CODES: $\begin{array}{rrrr}H & F & \mathbf{R} & \mathrm{P} \\ 2 & 3 & 0 & \mathrm{X}\end{array}$}

SECTION I- MANUFACTURER IDENTIFICATEON

$\begin{array}{ll}\text { voe-Pacilic Company, inc. } & \text { ADDRESS: } 1198 \text { Mark Circte, Gardnerville, NV } 89410 \\ \text { ?.0100 } & \text { ELERGENCY PHONE: } 1-800-424-9300 \\ & \text { REASON REVISED: Updaled }\end{array}$

ICTION II - HAZARDOUS INGREDIENTS I SARA III INFORHAATION OCCUPATIONAL EXPOSURE LIMITS

\begin{tabular}{|c|c|c|c|c|}
\hline OSHA PEA & ACGIH TLV & OLHER & $\begin{array}{c}\text { LDSO SPECIES } \\
\text { \& ROUTE }\end{array}$ & $\begin{array}{c}\text { LCSO SPECIES } \\
\text { S ROUTE } \\
\end{array}$ \\
\hline $100 \mathrm{PPM}$ & 100 PPIS & & $430 \mathrm{~m} \mathrm{mg} / \mathrm{kg}$ RAT (ORAE) & 6700 PPW, \& hr RAT (DNHA) \\
\hline 500 PPIA & $100 \mathrm{PPIS}$ & N(A & $3500 \mathrm{mg} / \mathrm{kg}$ RAT (ORAL) & N/A \\
\hline $100 \mathrm{PPM}$ & $100 \mathrm{PPIM}$ & & W/A & NAG \\
\hline KWA & N/A & N/A & N/A & $\mathrm{W} / \mathrm{R}$ \\
\hline $\begin{array}{l}\text { LOWNG: } \\
750 \text { PPAt }\end{array}$ & 750 PPIA & & $9750 \mathrm{cgg} / \mathrm{kg}$ RAT (CRAL) & 6700 PPA S 4 hr RAT (DNE-1K) \\
\hline
\end{tabular}

a the reparing requirements of section 313 of Tilve al and of 40 CFR 372 . Ir not applicabice
PRODUCT COOE: 178

SECTION IQ - PHYSICAL / CHEAICAL CHARAC ITICS

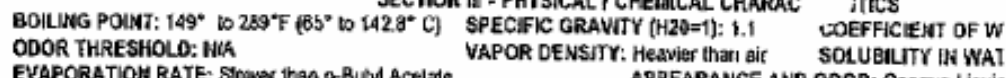

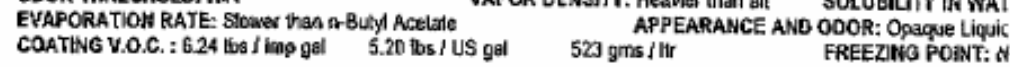

FLASH POET: $59^{\circ}$ iO $74^{\circ} \mathrm{F}\left(15^{\circ} 10233^{\circ}\right.$ SECTION $N$. FRE AND EXPLOSION HAZARD DATA

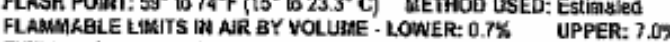

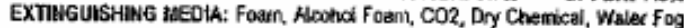

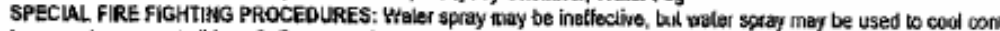

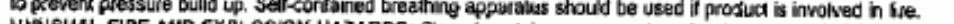
ULAU Yes - Flamenede liquid under condiliens of spu

FEtSITYITY TO MPACT: Do nd pundire SENSITVIIY TO STATIC DISCHARGE: Primanily.
.

STABILITY: Stathe SECTION V - REACTRITY OATA HAZARDOUS DECOMPOSITION OR GY-PRODUCTS: Carbon Hocowide and Carton Dioxide HAZARDOUS POLYHERRIZATION: WIII EOI OCULA

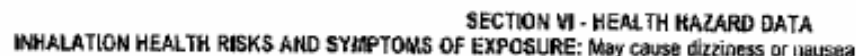

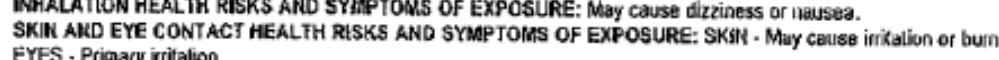
EYES. Primary iritation

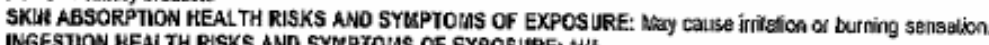
AND SYQRPTOUS OF EXPOSURE: NUA

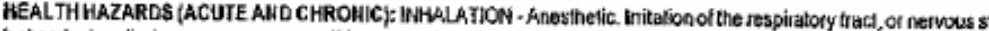

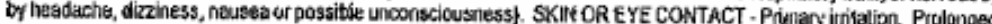
CaRc detinalls - extercise due cere. TERATOE MARC HOLOGRAPHS? No TERATOGENICITY: NUA TAUTAGENACITY: HIIA TOXICOLOGICALYY SVNERGISTIC PROC EMERGENCY AND FIRST AID PROCEDURES: VAPOPS R ROSURE: None kNOW

SPLASH - SKIDNIV

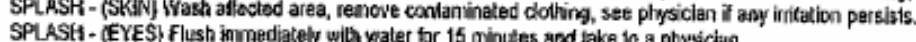

SECTION VII- PRECAUTIONS FOR SAFE HANDLIHE AND USE

STEPS IO BE TAKEN IN CASE WATERLAL IS RELEASED OR SPLLLED: Rernove al sources of ignition - Flanes, electrical. Ventilate artea, avoid run off into sewer by diring. and soak, up with inert absarbent using nom-sparking type VASTE DISPOSAL METHOO: Dispose of in accorduasce wilh local, state and lederal regulations. Do not incinerale PRECAUTIONS TO BE TAKEN WN HAILOLING RNO STORIIUG: Do nol stote above $120^{\circ} 8 / 49^{\circ} \mathrm{C}$. Do not slere or OTHER PRECALTIONS: Do nor get in eyes. Da nof breathe veppors. Avaid skin contact. Do not take inleusally. Sn

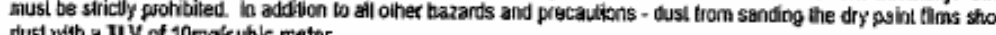

SECTION VIU - CONTROL MEASURES

RESPIRATORY PROTECTLON: Outdoors - Recomnend an approved mechanical particulate Fler to rernove any airts

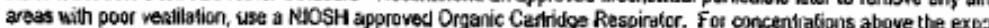
supplied respirator.

VENTILATION: All applcation areas should be adsquately ventilated in onder to beep fie teins in SECTION a befor

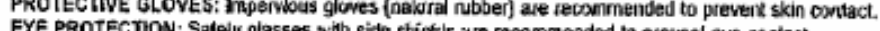

OTHER PROTECTIVE CLOTHIKG OR EQUTPUENT: trapervious apron (natwal rubber) is Iecominended to prevents SORK I HYGEM: PR SECTION IX - DISCLABUER

THE INFORAEAIION CONTANEED HEREN IS BELLEYED TO BE ACCURATE BUT IS NOT WARRANTEO TO BE HEREIN COHSTITUTES A SPECIFICAIICN NOR IS IT NTENOED TO WARRANT SUITABUITY FOR IH 


\section{urking Paint (Fluorescent Colors) - Bulk I Mattial Safety Data Sheet}

aty data sheet as per your request.

formis is believed to be correct and stfficienl to meet the requirements of OSHAH Hazard Communication cerning worker's right to know. th order for the infomafon contained in the MSOS to be most helpful cening orito

sheet oovers the hazardous ingredients associated with more than one color bulk product. As per 29 enever the hazards associated wilh simlar mixtures ate the same, then one MSDS may be prepared

Grve-Padic bulk producls.

MLARKING PAINT - FLUORESCEHT COLORS

$\begin{array}{lll}\text { NGE } & 224 \text { GREEN } & 229 \text { PINK } \\ & 226 \text { YELLOW } & 230 \text { REO FORANGE }\end{array}$

INY-ALL FLUORESCENT COLORS PRODUCT COOE: 1782 HMIS CODES: H F R P

SECTION I- MANUFACTURER IDENTIFICATION

be-Pacilic Company, Inc. ADDRESS: 1198 Mark Circle, Gardnerville, RV 89410 .0100

EMERGENCY PHONE: 1 -800-424-9300

REASON REVISED: Updated

CTIOA II - HAZARDOUS INGREDIENTS I SARA II IMFORMATIOW OCCUPATIOHAL EXPOSURE LIMITS

\begin{tabular}{|c|c|c|c|c|}
\hline OSTH PEL & RCGH ILY & OTMER & $\begin{array}{c}\text { LDED SPECIES } \\
\text { \& ROUTE } \\
\end{array}$ & $\begin{array}{c}\text { LCSO SPECIES } \\
\text { \& ROUTE }\end{array}$ \\
\hline $1006+6 / \mathrm{m}$ & $100 \mathrm{PPH}$ & & 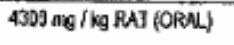 & 6700 PPtS: 4 hr RAT (INHGSG) \\
\hline $300 \mathrm{PPM}$ & to0 PPIS & N/A & $3500 \mathrm{lng} / \mathrm{kg}$ RAT (ORAL) & IW/A \\
\hline $400 \mathrm{PPW}$ & $\triangle 00 \mathrm{PPN}$ & & $\begin{array}{l}>7.10 \mathrm{~g} / \mathrm{kg} \text { RAT (ORAL) } \\
>2.86 \mathrm{~g} / \mathrm{kg} \text { RAT (DERIG) }\end{array}$ & 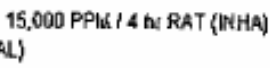 \\
\hline
\end{tabular}

The reporting requienerds of seclioe 313 of Tifte III and of 40 CFR 372 .

not appicicate
PROOUCT CODE: $17 B 2$

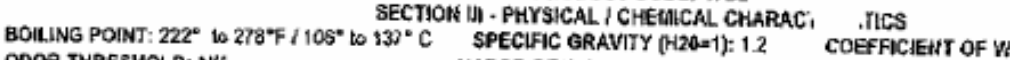
ODOR THRESHOLO: N/A VAPOR DENSTTY: Heavier than air SOLUBIUTY IS WA

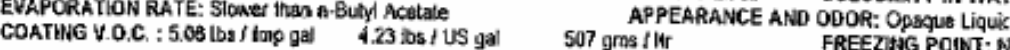

SECTION IV - FIRE AND EXPLOSION HAZARO DATA

FLASH POIKT: $20^{\circ} \mathrm{F} / 7^{\circ} \mathrm{C}$ IMETHOD USED: ExtingLed FLAMIAAABLE LIRITS IN AIR BY VOLUME. LOW

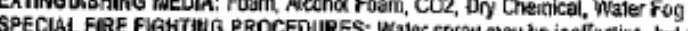

列 ItIUSUAL FIRE AND EXPLOS:ON HAZARDS: Closed conlins should be used il product is involved in fire.

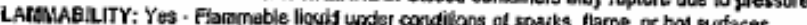

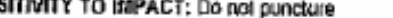

SENSITIVIYY TO STATIC DISCHARGE: Prikardy :

STABILTY: S1BDIC

SECTION V - REACTMITY DATA

CONOHIONS TO AVOID: High vemperature

HAZARDOUS DECOHPPOSITIOU OR BY.PRODUCTS: Carban Honoxide and Carbon Olayide

SECTION VI - MEALTH HAZARD DATA

INHALATION MEALTH RISKS AND SYIMPTONSS OF EXPOSURE: MaY CaUse dizziness or nausea.

SKME ANLO EYE CONTACT HEALTH RISKS AND SYLAPTOWS OF EXPOSURE: SKEN - May causa irritition or bum EYES - Prisusey iritation.

SKIN ABSORPTION HEALTH RISKSS AND SYMPTOMS OF EXPOSURE: IGEy cause irilatios or buning sensalion WEESTON HEALTN RISKS ANO SYMLPYOAS OF EXPOSURE: NIT

by

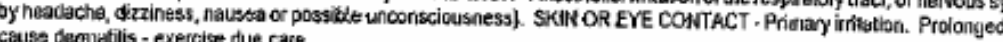
CARCIKOGENICITY: None known

NTP? No NARC BONOGRAPHS? NO

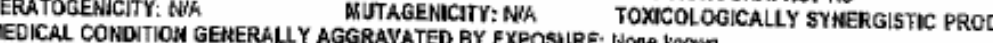

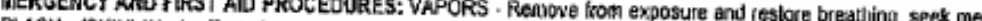

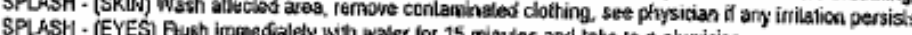

\section{SECTION VII - PRECAUTIONS FOR SAFE HARDLING AND USE}

STEPS TO BE TAKEN IN CASE MATERIAL IS RELEASED OR SPILLED: Renowe all sourcess of ignition- Flarnes,

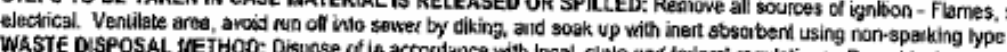

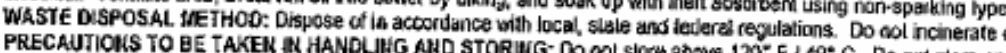

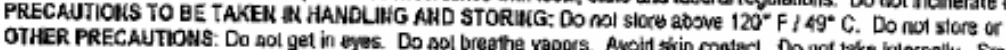

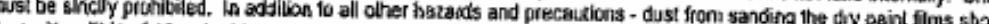
dust with a IL' of toregicuble meter.

\section{SECTHON VJI - CONTROL MEASURES}

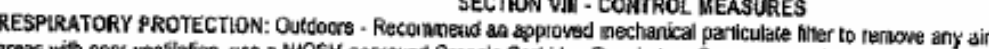
areas with poor verslation, ese a NOSH approved Organic Carthidge Respirator. For cuncentrations above the expos SLppiled respirator.

PROTECTIVE GLOVPSS EYE PROTECTLN: Safely glasses with side shields subet) are recommended to prevert skith contact.

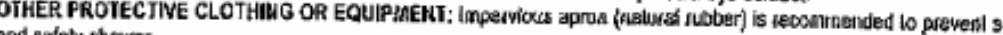

WORK TIGENIC PRACTICES: Avoid prolonged or repe日tes contacl. Do nut breathe vapors. Wash bontaminates

THE INFORIMATION CONTAINED HEREIN IS BELIEVED IO BE ACCURATE BUT IS NOT WARRANTED YO BE HEREIN CONSTITUTES A SPECIFICATION NCR IS IT ATTENDED TO WARRANT SUTIABMITY FOR N 
MATERIAL SAFETY DATA SBERT
The Valvoline Company
Page 001
Dato Propared: $01 / 14 / 02$
Date Printed: $05 / 17 / 04$
MSDS No: 503.0354181-001.004

WINDSEIBLD WASHBR PLUID, 8 METHANOL

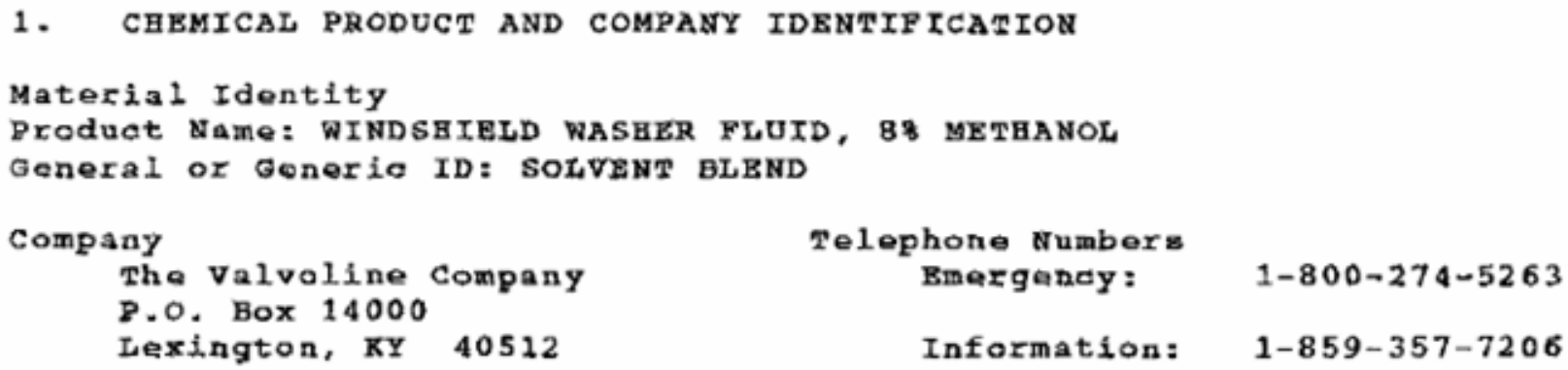

2. COMPOSITION/IEFORMATION ON INGREDIENTS

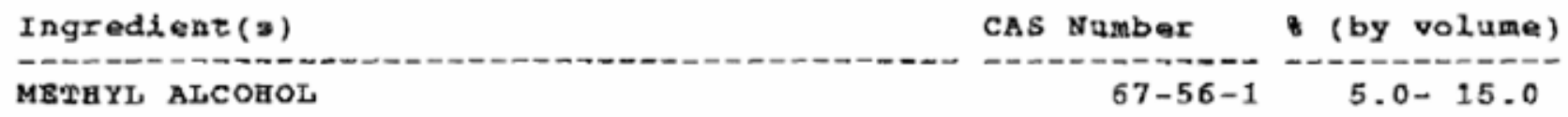

\section{HAZARDS IDERTIFICATION}

Potontial Bealth Befoots

Eye

Can cause eye irritation.

skin

May cause mild skin irritation. Prolonged or repeatod dontact may dry and craok the ekin. Passage through the akin may add to toric effoots from breathing or swallowing.

Swallowing Swallowing this material may bo harmful.

Inhalation

Breathing of vapor or mist is possible. Bresthing small amounts of this material during normal handling is not likely to aauso harmful offocts. Breathing large amounts may be harmful. symptoms usuzlly ooour at alr oonoentrarions higher than the recommended exposure limits (Soo seation 8 ). 
The Valvoline Company

WINDSHIBLD WASEER FLUYD, B\% METHANOL
Paga 002

Dato Prepared: $03 / 14 / 02$

Date Printed: $05 / 17 / 04$

MSDS NO: 503.0354181-001.004

symptoms of Bxposure

signs and symptoms of exposure to this matorial through breathing, swallowing, and/ox passage of the material through the skin may include: stomaoh or intestinal upset (nausea, vomiting, diarrhea) irritation (nose, throat, alrways), central nervous system depression (dizziness, drowsiness, weakness, fatigue, nausea, hoadache, unconsciousness), leg eramps, pain in the abdomen and lower back, blurred visfon, shortness of breath, cyanosis (causes blue coloring of the skin and nails from lack of oxygen), visual impairment (including blindness), coma, and death.

Target Organ $\mathbb{E} f$ fects

Exposure to lethal oonoontrations of methanol has been shown to cause damage to organs including liver, kidneys, panoreas, heart, lungs and brait. Although thig rarely odcura, survivors of severe intoxication may suffer from permanant neurologioal damage. overexposure to this material (or 1ts components) has been suggested as a cause of the following effects in laboratory animals, and may aggravate preexisting disorders of these organs in humans: cardiac abnormalities, liver abnormalities, spleen damage, nervous system damage, eye damage, kidney danage, liver damage, lung damage, central norvous system damage, brain damage, overexposure to this material (or its components) has been ouggested as a cause of the following effocts in humans, and may aggravate preexisting disorders of these organs: eye damage, visual impairment.

Developmental Information Methanol has aaused birth defects in laboratory animalg, but only when iahaled at extremely high vapor concentrations. The rolovance of this finding to humans is uncortain.

Cancer Information

Based on the available information, this material cannot be olassified with regard to oarcinogenicity. This material is not iisted as a darainogen by the International agenay for Resarah on Canaar, the National Toxioology Program, or the Oadupational sifety and Health Administration. 


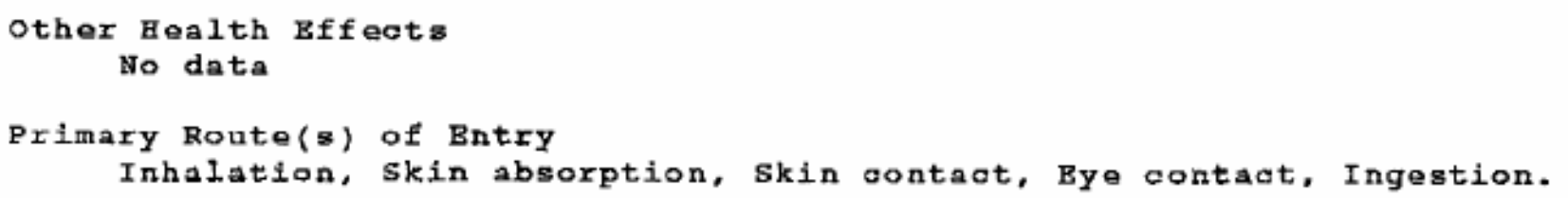

\section{FIRST AID MEASURES}

Eyัด

If symptoms develop, immodiately move individual away from exposure and into fresh air. Flush eyes gently with wator for at least 15 minutes while holding eyelids apart; seok immodiate medical attention.

skin

Remove antaminated clothing. Wash exposed area with soap and water. If symptoms persist, seek medical attention. launder clothing before reuse.

Swallowing

Seek mediaal attention. If individual is drowsy or unconsaious, do not give anything by mouth; place individual on the left side with tho haad down. Contact a physician, modical facility, or poison control conter for advioo about whothex to induce vomiting. If possible, do not leave individual unattended.

Inhalation

If symptoms devolop, immediately move individual away from exposure and into fresh aix. Seek immediate modical attention; keep person warm and quiet. If person $i$ s not breathing, begin artificial respiration. If breathing is difficult, administor oxygen.

Noto to Physicians This product dontains methanol which oan cause intoxication and central norvous system depression. Methanol is metabolized to formio aoid and formaldehyde. These metabolites can cause metabolid adidosis, visual disturbances and blindness. sinoe motabolism is required for thase toxic symptoms, their onsat may be delayed from 6 to 30 hours following ingostion. Ethanol competes for the same motabolic pathway and has been used to provont methanol metabolism. Ethanol administration is indicated in symptomatio patieats or at blood methanol conoentrations above $20 \mathrm{ug} / \mathrm{dl}$. Mothanol is offectively removed by hemodialysis. Continued on next page 
MATERIAI SAFTTY DATA SHBET

The valvolino Company

WINDSBIBLD WASHER FLUID, $8 \%$ METHANOL
Page 004

Date Prepared: 01/14/02

Date Printed: $05 / 17 / 04$

MSDS No: 503.0354181-001.004

Preexisting disorders of the following organs ( or organ systems) may be aggravated by exposure to this material: skin, lung (for oxample, asthma-like conditions), liver, kidneys, a brtral nervous system, panoreas, heart, Exposure to this material may aggravate any pre-axisting condition sensitive to a decrasso in availablo oxygen, such as ohronic lung disease, coronary artery diseaso or anemias.

5. FIRE FIGHTING MEASURES

Flash point

144.5 F $(62.5 \quad$ c) $\mathrm{TCC}$

Explosive Limit

(for component) Lower 6.0 vpper 36.0 8

Autoignition Tomperature

No data

Hazardous Producta of Combustion

May form: carbon dioxide and aarbon monoxide, various hydrocarbons.

Fire and Exploston Bazards

Vapors are heavier than air and may travel along the ground or be moved by ventilation and ignited by heat, pilot lights, other flamos and ignition sources at locationg distant from material handling point. Novor use welding or cutting torch on or near drum (even ompty) because product (even fust residue) oan ignjte explosivoly.

Extinguishing Media regular foam, aloohol foam, water fog, carbon dioxide, dry ohemicai.

Fire Fighting Instructions

Water may be used to keop fire-exposod containers cool until fire is out. Wear a solf-contained breathing apparatus with a full facopiece oporated in the positive pressure demand mode with appropriate turn-out gear and ohemioal resistant personal protective equipment. Refer to tho personal protective equipment soation of this MSDS.

Continued on noxt page 
MATERIAI SAPBTY DATA SEBET

The Valvoline Company

Page 005

Date Prepared: $01 / 14 / 02$

Date Printed: $05 / 17 / 04$

WINDSHIBLD WASEBR FLUID, 8\% MBTHANOL

MSDS No: $503.0354181-001.004$.

\section{ACCIDENTAL RELEASE MBASURBS}

\section{Sma11 spill.}

Bliminate all gouroes of ignition such as flares, flames (inoluding pilot lights), and eloctrioal sparks. Absorb liquid on vermiaulite, floor absorbent or other absorbent material. porsons not wearing proper porsonal protective equipment should bo excluded from area of spill.

Laxge spill

Prevent run-off to sewers, streams or other bodies of water. If run-off ocours, notify proper authorities as required, that a spill has ocaured. Persons not wearing proteotive equipment should be excluded from area of mill until clean-up has been completed. Bliminate all ignition souroes (flaras, flames, inoluding pilot lights, electriaal sparks).

\section{HANDLING AND STORAGE}

Handling

Containers of this material may be hazardous when emptied. Sinco emptied containers rotain product residues (vapor, liquid, and/or solid), all hazard procautiona givon in tho data ghoot must be obsorved. Avoid prolonged or repeated contact.

\section{Storage}

Do not store near extreme heat, open flame, or sources of ignition.

\section{BXPOSURE CONTROLS/PBRSONAL PROTBCTION \\ Byo proteotion \\ Chemical splash goggles in complianos with osHA rogulations are advised; howevor, osHA regulations also permit other type safety glasses. Consult your safety representativo.}


MATERIAL SAFBTY DATA SHEET

$\begin{array}{ll}\text { The Valvoline Company } & \text { Page 006 } \\ & \text { Date Prepared: } 01 / 14 / 02 \\ & \text { Dato Printed: } 05 / 17 / 04 \\ \text { WINDSBIELD WASHER FLUID, B MBTHANOL } & \text { MSDS No: } 503.0354181-001.004\end{array}$

Skin Protection

Wear resistant gloves (consult your safety equipment supplier). To prevent repeated or prolonged skin contact, wear impervious clothing and boots.

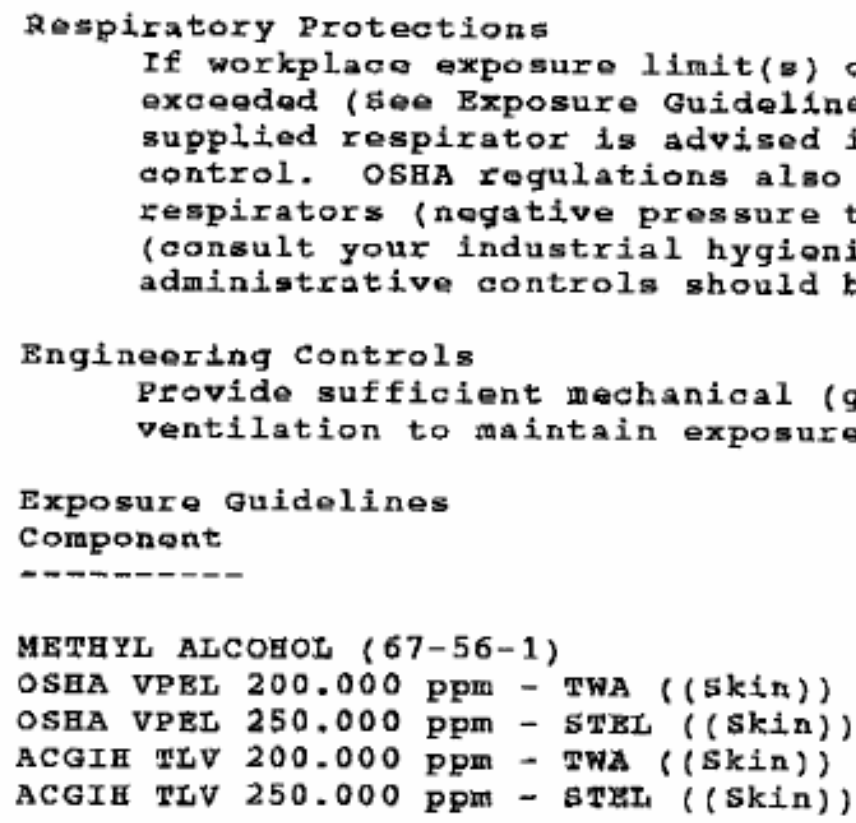

9. PBYSICAL AND CHEMICAL PROPERTIES

Botling Point

(for component) $147.0 \mathrm{~F}(63.8 \mathrm{C})$ e $760 \mathrm{~mm} H$

vapor pressure

(for component) $97.680 \mathrm{ming} 68.00 \mathrm{~F}$

Specifia vapor Density

No data

Continued on next page 


\title{
MATERIAL SAFETY DATA SHEET
}

The Valvolino Company

Page 008

Date Prepared: 01/14/02

Date Printed: $05 / 17 / 04$

WINDSEIBID WASGER FLUID, 8\% METEANOL

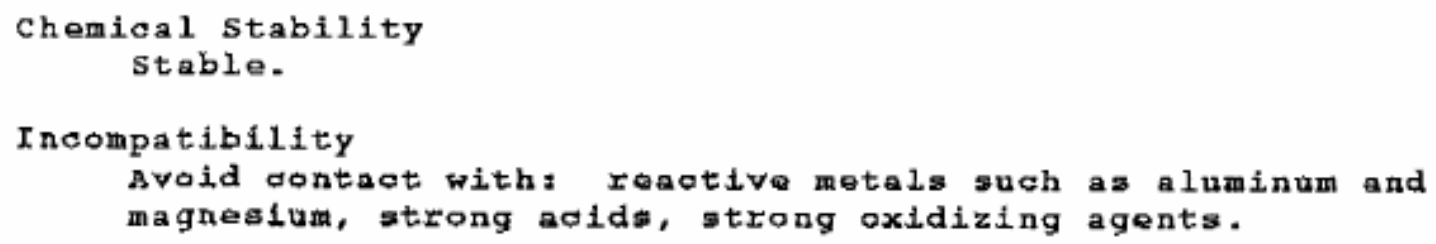

11. TOXICOLOGICAL INFORMATION

No data

\section{ECOLOGICAL INFORMATION \\ No data}

\begin{abstract}
13. DISPOSAL CONSIDERATION
Waste Management Information

Dispose of in accordanoe with all applicable local, state and fodoral regulations.
\end{abstract}

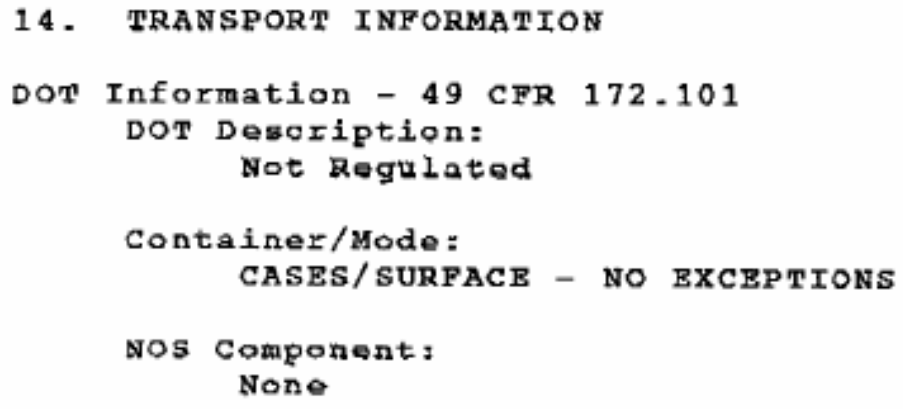




\section{MATERIAL SAFBTY DATA SHBET}

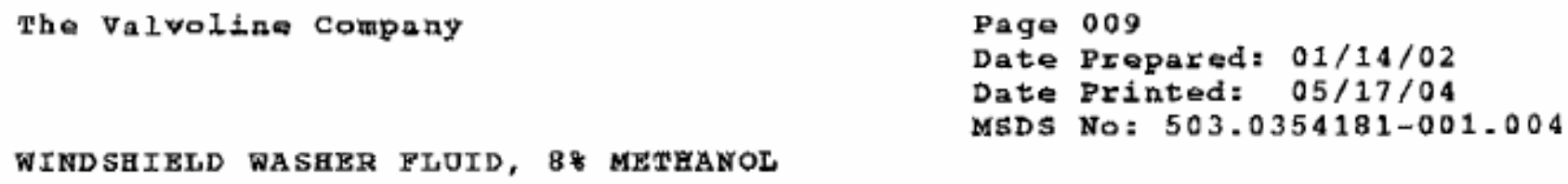

\section{REGULATORY INFORMATION}

us Fedoral Regulations

rSCA (Toxic Substances Control Act) status TSCA (UNITED STATES) Tho intontional ingredients of this product are listed.

CERCLA RQ - 40 CER 302.4 Component component

METHYL ALCOHOL 5000

SARA 302 Components - 40 CFR 355 Appendix A None

Soction 311/312 Hazard Class - 40 CFR 370.2 Immediate(X) Delayed(X) Fire(X) Raactive() Sudden Releaso of Pressure( )

SARA 313 components - 40 CFR 372.65 Seation 313 Component $(\Rightarrow)$ CAs Number METHANOL $67-56-1$

International Regulations

Inventory statug Not determined

State and Local Rogulations

California Ptoposition 65 None

New Jersey RTK Label Information METHYL ALCOEOL

$67-56-1$ 
MATERIAL SAFBTY DATA SERET

The Valvoline Company

Page 010

Date Propared: $01 / 14 / 02$

Date Printed: $05 / 17 / 04$

MSDS No: 503.0354181-001.004

WINDSHIBLD WASBBR FLUYD, 8\% METHANOL

Pennsylvania RTK Label Information METEANOL

$67-56-1$

16. OTHBR INFORMATYON

Tho information acourulated herain 1 s belleved to be acourate but is not warranted to be whether originating with the compang or not. Recipients are advised to confirm in advanoe of nood that tho information is ourront, appliasble, and suitable to thoix ciroumstancos.

Last pago

SOURCE : ASHLAND INC WTR, BASYWTR 
0.3622

100

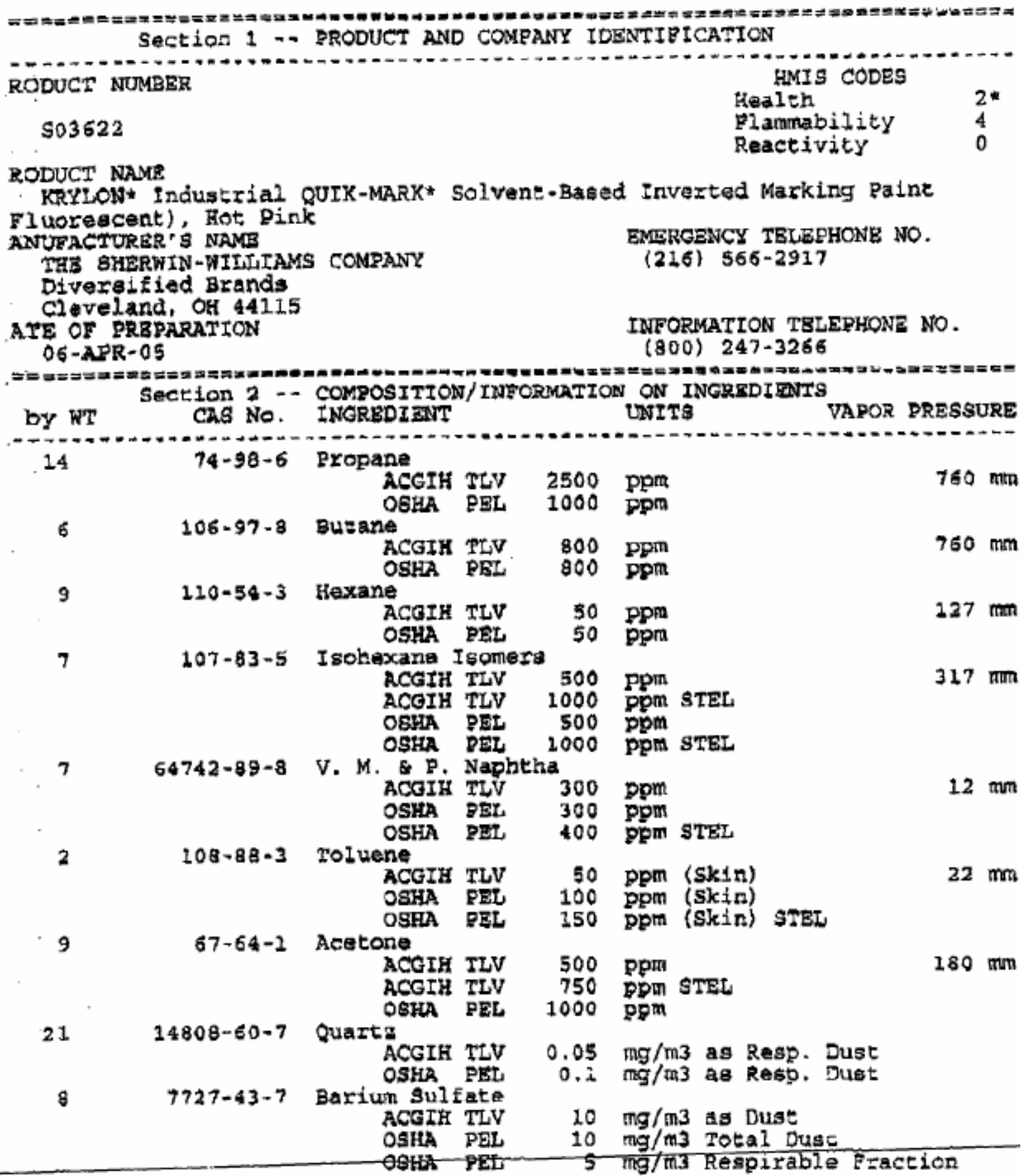

ontinued on page 2 
803622

man Section $3 \ldots$ HAZAKDS IDENTIFICATION

\section{OUTES OF EXPOSURE}

INHALATICN of rapor of spray mist.

BYB or SKIN contact with the product, vapor or spray mist.

FEECTS OF OVEREXPOSURE

BYES: Irritation.

SKIN: Prolonged or repeated exposure may cause irrication.

INAAI.ATION: Irritation of the upper respiratory system.

May cause nervous system depression. Extreme overexposure may result in neonsciousness and possibly death.

IGNS AND SYMPTOMS OF OVEREXPOSURE

Headache, dizziness, nausea, and loss of coordination are indications of xcestaive exposure to vapore or spray mists.

Radness and itching or burning sensation may indicate eye or excessive kin exposure.

BDICAL CONDITIONS AGGRAVATED BY BXPOGURB

None generklly recogni zed.

ANCER INEORMATION

For complet discuasion of toxicology data refer to gection 11.

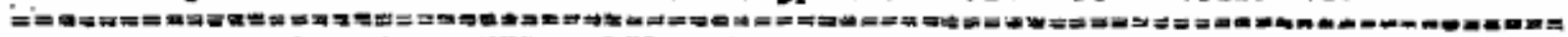
Bection 4 -- FIRST AID MEASURES

EYss: Flush eyes with large amounts of water for 15 minutes. Get medieal attention.

SKIN : Waeh affected area thoroughily with soap and watar. Remove contaminated olothing and launder berors re-use.

INHALATION: If affected, remove from exposure, Restore breathing. Kaep warm and quiet.

INGESTION: Do not induce vomiting. Get medical atcention imnediately.

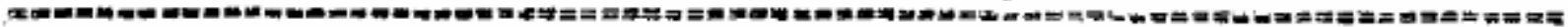
Section 5 - FIRE BIGHTIMG MBRSTRES

LASH POINT

LEL UEL

Prope1.lant < OR $0.9 \quad 12.8$

XTINGUISHING MEDIA

Carbon Dioxide, Dry Chemical, Foam

NUSUAL FIRE AND EXPLOSION HAZARDS

Containerg may explode when exposed to extreme heat.

Application to hot surfaces requires special precautions.

During emergency conditions ovarexposure to decompolition products may ause a health hazard. Symptems may rot be immediacely mpgarent. dbeain edical attention.

PECTAL EIRE FIGHTING PROCBDURES

Full protective equipment including self-contalned breathing apparatus hould be used.

Warex spray may be ineffaceive. If watar is used, fog nozales are referable. Watar may be used to cool closed containers to prevent ressure boildup and possible autoignition or explosion when exposed to xtirentive heat.

ontinued on page 3 
503622

page 3

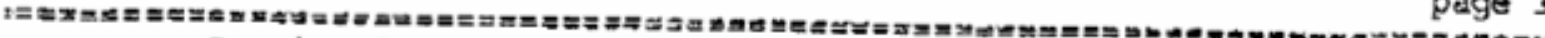
Section 6 - ACCIDBNTAL RELEASB MEASURES

TTEPS TO BE TAKEN IN CASE MATERIAL IS RBLBASED OR SPILIED

Remove ald sources of ignition. Ventilate the area.

Remove with inert absorbent.

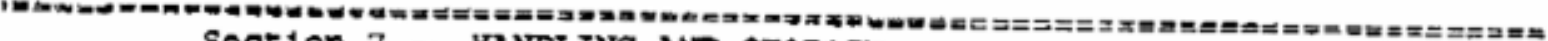
Section 7 -- MANDIING AND STORAGB

ITORAGB CATBGORY

Not Available

'RECAUTIONS TO BE TAKBN IN HANDLING AND STORAGE

Keep away from heat, sparks, and open flame. Vapors will accumulate

eadjly and may ignite explogively.

During use and until all vapors are gone: Keep area ventilated - Do not

moke - Extinguish all flames, pilot 1ights, and heaters - Tuxn off stoves,

loctric tools and appliances, and any other sources of ignition.

Consult NFPA Code. Use approved Bonding and Grounding procedures.

Contents under preseure. Do not guncture, incinerate, or expose to

emperature above 1205. Heat from sunlight, radiators, stoves, hot wate

ad other heat sourees could cause contight, radiators, stoves, hot water,

nternally. Keep out of the reach of childrm.

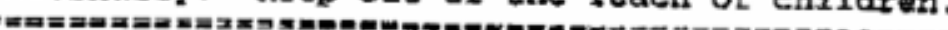

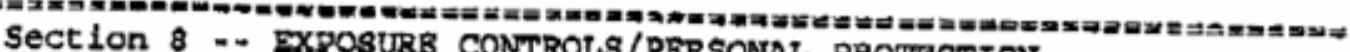
SECLIOn 8 EXPOSURB CONTROLS/PBRSONAL PROTBCTION

RECAUTIONS TO BE TAKEN IN USE

Use only with adequace vontilation.

Avoid contact with akin and eyes. Avoid breathing vapor and spray mist.

This coating may contain materials olassified as nuisance particulateg

listed "as Dust" in section 2) which may be present at hazardous levela re listed in section 2 thading of the dried film. If no specific dust:s IV $10 \mathrm{mg} / \mathrm{m}^{3}$ (total dust) $3 \mathrm{mg} / \mathrm{m3}$ (resle 1 mits for nuisance dusts are ACgIH total dust), $5 \mathrm{mg} / \mathrm{m3}$ (resgirable ENTILATION

Local oxhauct preforable. General exhauat acceptable if the exposure to aterials in Section 2 is maintained below applicable exposure limits. efer to OsHa standards 1910.94, 1910.107, 1910.108.

BSPIRATORY PROTECTION

If personal exposure eannot be controlled below applicable $11 \mathrm{mits}$ by ont1lation, wear a properly fittad organic vapor/particulate respirator pproved by NIOSH/MSHR for protection against materials in Section 2.

When sanding or abrading the dried film, wear a dust/mist raspirator nderlying paint, or cho abrasive. ROTECTIV GLOVES

None required for normal application of aerosol products where minimal

kin contact is expected. For long or repeated contace, wear chemical
esiatant gloves.

YE PROTECTION

Wear safety spectacies with unperforated aideshields. THER PRECAOTIONS Intentional misuse by deliberately concentrating and fnhaling the
ontents can be harmful or fatal.

ontinued on page 4 


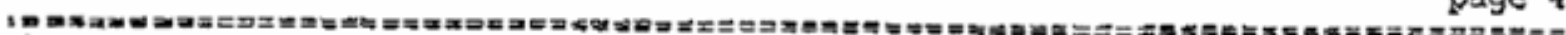
Section 9 - PHYSICAL AND CHBMICAL PROEERTIBg

PRODUCT WEIGHT SPBCIBIC GRAVITY BOILING POINT MELTING POINT VOLATILE VOLUMB SVAPORATION RATE VAPOR DENSITY SOLUBILITY IN WATBR $\mathrm{pH}$

VOLATILE ORGANIC COMPOUNDS

Volatile Weight 47.18 i

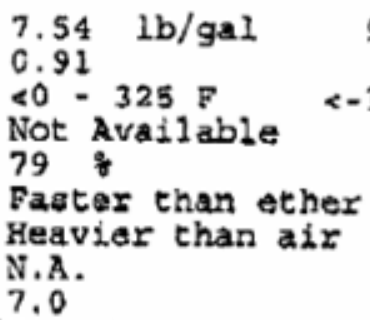

$7.541 \mathrm{~b} / \mathrm{gal}$

0.91

$<0-325 \mathrm{~F}$

Not Available

79 \%

Faster than ether Heavier than air N.A. 7.0 Section 10 - STABILITY AND REACTIUYTY

ITABILITY - stabl.

:ONDITIONS TO AVOID

None known.

NCOMPATIBILITY

None known.

LAZARDOUS DSCOMPOSITION PRODUCTS

By Eire: Carbon Dioxide, Carbon Monoxide

LAZARDOU'S DOLYMERI ZATION

Will not occur

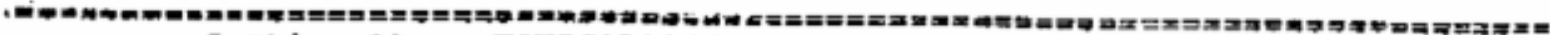
Section $11-2$ TOXICOLOGICAL INFORMATION

THRONIC HEALTH HAZARDS

crystalline Silica (Quartz, Cristobalite) is listed by IARC and NTP. rong texm exposure to high levels of silica dust, which can occur only when anding or abrading the dry film, may cauve lung damage (cilicosis) and ogsibly cancor.

Prolonged and regeated exposure to Hexane may cause damage to nerve 1ssue of the arns and legs (peripheral neuropathy), resulting in miscular 'eakness and loss of sensation. This effect may be increased by the resence of Methyl Ethyl Ketona.

Prolonged overexposure to solvent ingredients in section 2 may cause dverpe effects to the liver, urinary, cardiovascular and reproductive ystems.

Reports have associated repeated and prolonged overexposure to solvents ith permanent brain and nervous sygtem damage. 


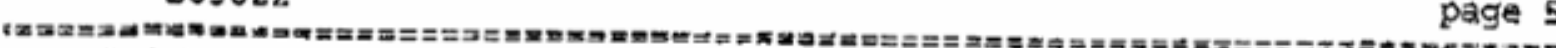
CAS No. Ingredient Name

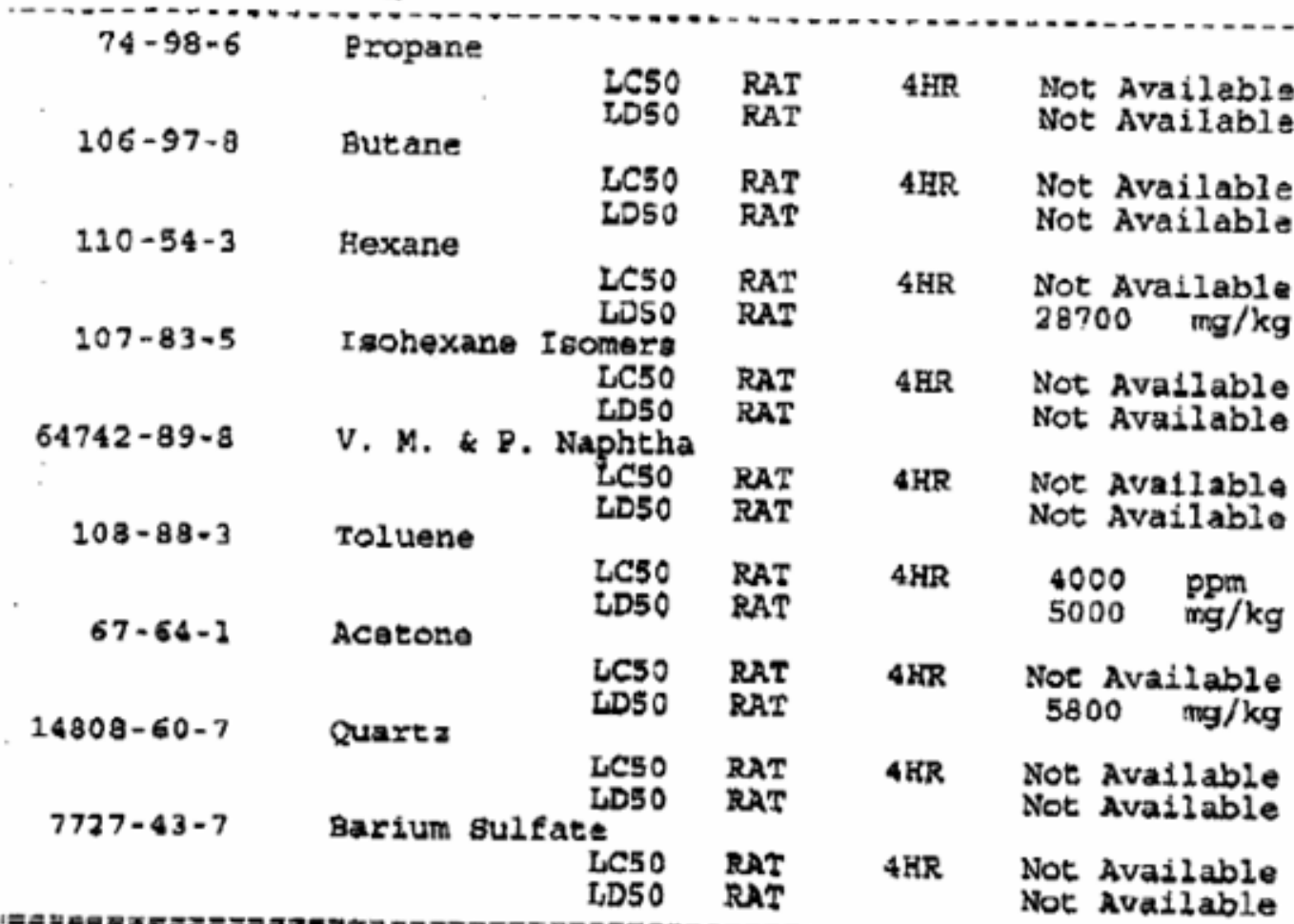

Saction $22 \ldots$ Bcorocreat INEORMATON

COTOXICOLOGICAL INFORMATION

No data available.

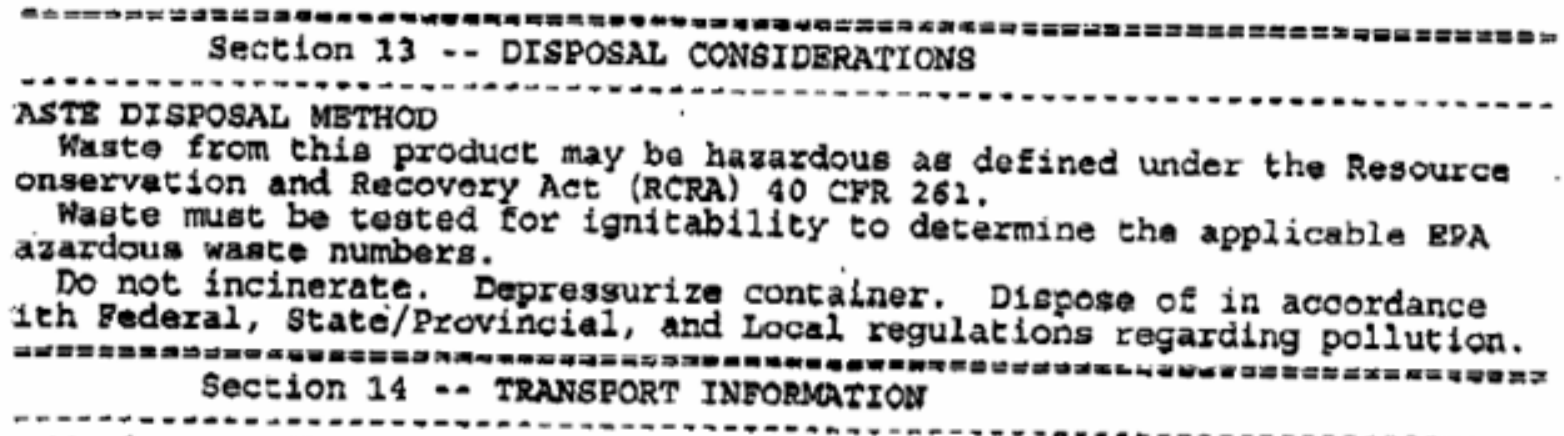

No data avatlable.

ontinued on page 6 


\section{GAIERPILLAR}

Scarch_Again
Print CoverPags
MSDS Number:
5779
Product Name:
Cat DEAC
(Diesel Engine
Antinteze
Coolant)
Concentrate
Manufucturer:
ChevronTexaco
Part Number(s):
1562649 2P9868
8C3684 8C3686
9X6584 9X6585
9X6586

24-hour Emisrgency Contacts:

Rocky Mountain Poison Center

Denver, Colorado

(800) $458-5924$ toll free U.S. \& Canada

(303) $893-1322$

Swiss Toxicological Infomation Centre

Zurich, Switzerland

$+41(0) 12515151$

If you have questions about Caterpillar Material Safety Data Sheets, please contact:

Miranda Katter

Caterpillar Inc.

100 NE Adams Street Peoria, IL USA 61629-3350

(309) 675-5182 phone

(309)675-1376 FAX

e-mail: mskis@eat.com 


\title{
Material Safety Data Sheet
}

\author{
SECTION 1 PRODUCT AND COMPANY IDENTIFICATION \\ Cat? DEACTM (Diesel Engine Antifreeze Coolant) Concentrate \\ Product Use: Antifrseze/Coolant \\ Product Number(s): 07961, CPS227961 \\ Company Identification \\ Chevron Texaco Global Lubricants \\ 6001 Bollinger Canyon Road \\ San Ramon, CA 94683 \\ United States of America \\ Transportation Emergency Response \\ CHEMTREC: (800) 424-0300 or (703) 527-3887 \\ Health Emergency \\ Chevran Texaca Emargency information Center: Located in the USA, International collect cails acteptad. \\ (800) 231.0623 or $(510) 231.0623$ \\ Product informatlon \\ email : lubemsds@chevron.com \\ Product Information: BOO-LUBE-TEK
}

\section{SECTION 2 COMPOSITION/ INFORMATION ON INGREDIENTS}

\begin{tabular}{|l|l|l|}
\hline COMPONENTS & CAS NUMBER & AMOUNT \\
\hline Ethylane Glycol & $107-21-1$ & $80-96 \%$ weight \\
\hline Diethylene glycol & $111.46-6$ & $1-5 \%$ weight \\
\hline Water & $7732-18-5$ & $1-5 \%$ waight \\
\hline
\end{tabular}

SECTIDN 3 GAZARDS DENTIFCATION

\section{EMERGENCY OVERVIEW}

- HARMFUL OR FATAL IF SWALLOWEO

- CAUSES EYE TRRITATION

- POSSIBLE BIRTH DEFECT HAZARD - CONTAINS MATERIAL THAT MAY CAUSE GIRTH DEFECTS

BASED ON ANIMAL DATA

- MAY CAUSE DAMAGE TO:

- KIDNEY

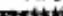

IMMEDIATE HEALTH EFFECTS

Eye: Contact with the eyes causes irritation. Symptoms may inelude pain, taaring, raddening, swelling and impatred vision.

Skin: Contace with the skin is not expectad to causa proianged or signifteant irritation. Not expected to be

Ravision Numbert 2

Ravision Datas 1 troar2002
1 of 7
Gav DEAC (Diesel Engine Antifreaza Coolunt) Comeantrate MSDS : 10891 
harmful to Irttrnal organs if absorbed through the skin. Ingestion: Toxic; may be harmfll or fatal if swallowed. Inhalation: The vapor or fumes from this material may cause respiratory irritation. Symptoms of respiratory irritation may includo coughing end difficulty breathing. Breathing this material at concentrations above the recommended exposure limits may cause central nervous system effects. Central narvous system sffects may include headache, dizziness, nausea, vomiting, weakness. loss of coordination, blumed vision, drowsiness, confusion, or disorientation. At extreme exposures. central nervous system effocts may include respiratory depression, tremors or convulsians, loss of consciousness, coma or death.

DELAYED OR OTHER HEALTH EFFECTS: Reproduction and Birth Defects: Contains material that may be harmful to tha developing fetus based
on animal data.

Target Cirgans: Conteins material that may cause damage to the following organ(s) following repeated ingestion based on animal data: Kidney

See Section 11 for additional information. Risk depends on duration and level of axposure.

SECTION 4 FIRST AID MEASURES

Eye: Flush eyes with water immediately while halding the eyelids open. Remove contact lenses, if worn, after initial flushing, and continus flushing for at least 15 minutes. Set medical attention If irritation
persists. Skin: To remove the material from skin, use soap and water. Discard contaminated clcthing and snoes
or thoroughly clesn before reuse. Ingestion: If swallowed, get immedlate medical attention. Do not induce vomittrng. Never give anything
by mouth to an unconscious person.

Inhalation: Move the exposed person to fresh air. If not bresthing, give artificial respiration. If breathing is difficult, give oxygen. Get medical attention if breathing difficultios continue.

SECTION 5 FIRE FIGHTING MEASURES

FIRE CLASSIFICATION:

OSHA Classification (29 CFR 1910.1200): Not classified by OSHA as flammable or combustible.

NFPA RATINGS: Heath: 2 Flammability: 1 Reactivity: 0

FLAMMABLE PROPERTIES;

Fiashpolint: (Pensky-Martens Closed Cup) 260 PF (127 ${ }^{\circ} \mathrm{C}$ )

Autoignition: NDA

Flammability (Explosive) Llmits ( $\%$ by volume in air): Lower: 3.2 Upper,

EXTINGUISHING MEDIA: Dry Chemical, CO2, AFFF Foam or alcohol resistant foam.

PROTECTION OF FIRE FIGHTERS:

Fire Flghting Instructions; This material will bum aithough it is not aasily ignited. For fres involving this material, do not enter any enclosed or confined fire space without proper protective equipment, including
self-ontained breathing apparatus.

Combustion Produets: Highly dependent on comoustion conditions. A complex mixture of aleberne solids, liquids, and gases including carbon monexide, carbon dioxide, and unidentified organic compounds will be evolved when this material undergoes combustion. Combustion may form oxides of:
Sodium. 
Protectiv Measures: Eliminate all sources of ignition in vcinity of spilled material. Spill Management: Stop the source of the release if you can do it without risk. Contain release to prevent further contamination of scil, surface water or groundwater. Clean up spily as soon as possible, observing preceutions in Exposure Controls/Personal Protection. Uae appropriato techniques such as applying noncombustible absorbent materials or pumping. Where feasible and approprlate, ramove contaminsted soll. Place contaminatod materisis in disposable containers and olispose of in a manner consistent with applicable regulations.

Reporting: Roport spilla to local authorities and/or the U.S. Coast Guard's National Response Center at (800) $424-2802$ as appropriate or required.

SECTION 7 HANDLING AND STORAGE Precautionary Measuras: Do not breathe vapor or fumes. Do not get in eyes. Wash thoroughly after
handing.

Static Hazard: Electrostatic charge may accumulate and create a hazardous condlition when handling this material. To minimize this hazard, bonding and grounding may be necossary but may not, by themselves, be sufficient. Review all operations which have the potential of generating an accumulation of electrostatio charge and/or a flammable atmosphere (including tank and container flling, splash filling. tank cleaning, sampling. gauging, switch loading, filtering. mixing, agitation, and vacuum truek operations) and use appropriate mitigating procedures. For more information, refer to OSMA Standard 29 CFR 1910.108, 'Flammable and Combustlble Liquids', National Fire Protection Association (NFPA 77, 'Recommendad Practice on Static Electricity', and/or ths American Petroleum Institute (API) Recommended Practice 2003, 'Protection Against Ignitions Arising Ourt of Static, Lightring, and Stray
Currents'.

Container Warnings: Container is not dasigned to contain pressure. Do not use pressure to empty container or it may rupture with explosive force. Empty containers retain product residue (salid, liquid, and/or vapor) and cen be dangerous. Do not prossurize, out, weid, braze, solder, drill, grind, or expose soch containers to heat, flame, sparks, static electricity, or other sources of ignition. They may explode retumed to a drum reconditioner or dlsposed af pouid be completely drained, properly closed, and promptsy

SECTION B EXPOSURE CONTROLS/PERSONAL PROTECTION

GENERAL CONSIDERATIONS;

Consider the potential hazards of this materiel (see Section 3). applicasle exposura iimits, job activities, and other substances in the work place when designing enginsering controls and selecting personal protective equipment. If engineering controls or work practices are not adequate to prevent exposure to harmful levels of this material, the personal protectlve squipment liated below is recommended. The user should read and understand all instruetions and limitations supplied with the equipment since protsction is usually provided for a limited time or under cortain eircumstances.

ENGINEERING CONTROLS: Use process enclosures. lowal exhaust ventilation, or cther engineering controls to control airborne levels
below the recommended exposure limits.

PERSQNAL PROTECTIVE EQUIPMENT

Eye/Face Protection: Wear protective equipment to prevent eye contact. Selection of protective equipment may include safety giasaes, chemical goggles, face shieids, ar a combination depanding on the work operations conducted.

Skin Protection: No special protective clothing is normally required. Where splashing is possible, salect protective clothing depending on operations conducted, physical requirements and cther substonces in the workplace. Suggestod materials for protective gloves inciuda: Natural rubber, Neoprene, Nitrile 
Rubber, Polywinyl Chloride (FVC or Vinyl).

Respiratory Frotection: Detemine if airbome concentrations are below the racommended exposure limits. If not, wear an approved respirator that provides adequate protection trom measured concentrations of this material, such as: Air-Purifying Respirator for Organic Vapors, Dusts and Mists. Use a positive pressure air-supplying respirator in circumstances where air-purifying respirators may no: provide adequate protection.

\section{Occupational Exposure Limits:}

\begin{tabular}{|c|c|c|c|c|c|}
\hline Compontent & $\operatorname{Limi}$ & TWA & STEL & Gelling & Notation \\
\hline Extrylene Gilycol & ACGIH_TVV & & & $100 \mathrm{ma} / \mathrm{m3}$ & \\
\hline Ethyiens Giycol & OSHA PEL & & & $125 \mathrm{mg} / \mathrm{m} 3$ & \\
\hline 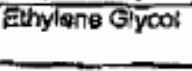 & $\overline{A C G H}$ & & & $\begin{array}{l}39.4 \mathrm{ppm} \\
\text { (wolght) }\end{array}$ & \\
\hline
\end{tabular}

SEGTOON 9 PHYSICAL AND CHEMICAL PROPERTIES

Attention: the data bolow are typical values and do not constitute a specification.

Color: Purple

Fhysical State: Liquid

Ddor: MDA

$\mathrm{pH}: 10 \cdot 31$

Vapor Pressure: $0.12 \mathrm{mmHg}$ (Typical) @ $20^{\circ} \mathrm{C}$

Vapor Density $($ Air $\approx 1): 2.1$

Boilling Point: 228 'F (109 C)

Solubility: Miscibie

Froezing Point: $34^{\circ} \mathrm{F}(37 \mathrm{C})$

Spacific Gravity: 1.13 (Q) $15 . \mathrm{B}^{\circ} \mathrm{C} / 15.6^{\circ} \mathrm{C}$

Viscostly: $8 \mathrm{cst}$ 응 $40^{\circ} \mathrm{C}$

Evaporation Rate: NDA

\section{SECTION 10 STAEILITY AND REACTIVITY}

Chemical Stabillty: This material is considered stable under normal ambient and anticipated storage and handling condivions of termperature and pressure.

Incompatibility With Other Materials: May react with strong oxidizing agants, such as chioratas, nitrates, peroxides, etc.

Hazardous Decomposition Praducts: Aldahydes (Elevated temperatures)

Hazardous Polymerization: hazardous polymerization will not occur.

\section{SECTION 71 TOXICOLOGICALINFORMATION}

IMMEDLATE HEALTH EFFECTS

Eye irritation: The eye iritation hazard is based on evaluation of data for similar cnaterials or product components.

Skin Irritation: The skin irritation hazard is based on evaluation of data for similar materials or product comporients.

Skin Sansitization: No product toxicology data available.

Acute Dermal foxicity: The acute dermal toxicity hazard is based on evaluation of data for similar materials or product components.

Acute Oral Toxicity: The acute oral toxicity hazard is basad on evaluation of data for similar matarials or produck camponents.

Acuta inhafation Toxicity: Tha acute inhalation toxicity hazera is based on evaluation of data for similar

Ravisican Number: 2

Revisiọa Oata: i1/06/2002
4 of 7

CatO DEAC ${ }^{\text {TN }}$ (Dlasal Engine Antifraves Coolant) Concentrato MSDS : 10681 
matarials or product componenis.

ADDITIONAL. TOXICOLOGY INFORMATION:

This product contains athylene glycol (EG). The toxicity of EG via inhalation or skin contact is expected to be slight at room temperature. The estimated oral lethal dose is about $100 \mathrm{cc} \mathrm{(3.3} \mathrm{oz.)} \mathrm{for} \mathrm{an} \mathrm{adult}$ human. Ethylene glycol is oxidized to axalic acid which results in the deposition of calcium oxalate crystals rnainly in the brain and kidneys. Early signs and symptome of EG poisoning may resemble those of alcohol intoxicatlon. Later, the victim may experience nausea, vorniting, weaknest, abdominal and muscle pain, difficulty in breathing and decreased urine output. When EG was heated above the boliling point of water, vapors formed which reportedly caused unconsciousness, increased lymphocyt ceunt, and a rapid, Jerky movement of the eyes in persons chronically exposed. When EG was administered oraily to pregnant rats and mice, there was an increase in fatal deaths and birth defects. Some of these effects occurred at doses that had no toxic effects on the mothers. We are not aware of any reports that EG causes reproductive toxicity in human beings.

This product contains diethylene glycol (DEG). The estimated oral lothal oçse is about 50 ce (1.0̂ oz) for an actult human. DEG has csused the following effects in laboratory animals: liver abnormalities, kidney damage and blood abnormalitias. It has been suggested as a cause of the following effects in humans: liver abnormalities, kidney damage, lung damage and central nervous system damage.

\section{SECTION 12 ECOLOGICAL INFORMATION}

\section{ECOTOXICTY}

The toxicity of this material to aquatic organisms has not been evaluated. Consequently, this material should be kspt out of sewage and drainage systems and all bodies of water.

\section{ENVIRONMENTAL FATE}

This material is expected to be readily bicciegradable.

\section{SECTION 13 DISPOSAL CONSIDERATTONS}

Use material for its intended purpose or recycle if posslble. This material, if it must be discarded, may meet the criteria of a hazardous waste as defined by US EPA under RCRA (4O CFR 2B1) or other State and tocal tegulations. Measurement of certain physical propertios and analysis for regulated components may be necossary to make a correct determination. If this material is classified as a hazardous waste, federal law requires disposal at a icensed nazardous waste disposal facility.

\section{SECTION 14 TRANSPORT INFORMATTON}

The description shown may not appiy to all shipping situations. Consult $\triangle 9 C F R$, or appropriata Dangerous Goods Ragulations, for additional description requirements (ө.g.. technical name) and modespecifle or quantity-specific shipping requirements.

DOT Shipping Name: NOT REGULATED AS A HAZARDOUS MATERIAL FOR TRANSFORTATION UNDER 49 CFR

DOT Hazard Class: NOT APPLICABLE

DOT Identifecation Number: NOT APPLICABLE

DOT Packing Group: NOT APPLICABLE

SECTION 15 REGULATORY INFORMATION

SARA 311/312 GATEGORIES: $\quad$ 1. Immediate (Acute) Health Effects: $\quad$ YES

2. Delaysd (Chronic) Health Effects: YES

3. Fire Hazard:

NO

Revision Number, 2

Ravlaion Datat $11 / 06 / 2002$
5 o! 7

Cato DEAC" (Dieser Engine Antifrosze Coolant Concantrate Mads : 10 est 
$\begin{array}{ll}\text { 4. Sudden Reiease of Pressure Hazard: } & \text { NO } \\ \text { 5. Reactivity Hazard: } & \text { NO }\end{array}$

5. Reactivity Hazard:

REOULATORY LISTS SEARCHED: 4_II=|ARC Group 1

4_|2A=IARC Group 2A

15:SARA Section 313

4_12B=IARC Group 28

16 -CA Proposition 65

O5=NTP Carcinogen

17 =NARTK

08=OSHA Careincgen

$18=N$ N RTK

$09=T_{S C A} 12$ (b)

19 mDOT Marine Pollutant

20=PA RTK

The following components of this material are found on the reguiatory lists indicated.

Ethylene Glycol

$15,17,18,20$

CERCLA REPORTABLE QUANTITIESIRQ)VSARA 302 THRESHOLD PLANNING QUANTITIES(TPQ):

\begin{tabular}{|l|l|l|l|}
\hline Component & Component RQ & Componont TPQ & Product RQ \\
\hline Ethylene Glycal & $5000 \mathrm{lbs}$ & None & $5238 \mathrm{lbs}$ \\
\hline
\end{tabular}

CHEMICAL INVENTORIES:

AUSTRALIA: This material contains components that require notification beforg sale or importation into Australia.

CANADA: All the ecmponents of this material are on the Canadian DSL or have been notifled under the New Substance Notiffeatlon Regulations, but have not yet been published in tha Canada Gazeite.

EUROPEAN UNION: All the components of this material are in compliance with the EU Seventh Amendment Directive 92/32/EEC.

JAPAN: This material contains components that require notification before sale or importation into Japan. KOREA: This materlal contains components that require notification before sale or importation into Korea. PHILIPPINES: All the components of this product are listed on the Philippirie inventory of Chemicais and Chemical Substances (PICCS).

UNITED STATES: All of the components of this material are on the Tcxic Substances Control Act (TSCA) Chemical inventory.

NEW JIERSEY RTK CLASSIFICATION:

Refer to components listed in Section 2.

WHMIS CLASSIFCATION:

Class D, Division 1, Subdivision B: Toxic Materiai -

Acuts Lethallty

Class D, Division 2, Subdivision A: Very Toxic Material -

Chronic Toxic Effects

Teratogenicity and Embryotoxicity

Class D, Dlvision 2, Subdivision B: Toxic Matarial .

Chronic Toxic Effects

Skin or Eye Irritation

\section{SECTION 16 OTHER INFORMATION}

NFPA RATINGS: Health: 2 Flammability; 1 Reactivity: 0

HMIS RATINGS; Hoalth: 2* Flammability: 1 Reactivity: 0

(0-Least, 1-Slight, 2-Maderate, 3-High, 4-Extreme, PPE;- Personal Protection Equipment Index recomenendation." Chronic Effect indicator). These values are obtained using the guidelines or

Revision Number: 2 Rovision Date: $11 \% 0 \mathrm{r} 2002$
6 of 7

Catb DEACTN (Dlesel Engine

Antifrapze Coolant) Concantrate MSDS: 10691 
MAY.13.2004 9:55PM WESTERN STATES PCC

published evaluations prepared by the National Fire Protection Association (NFPA) or the National Paint and Coating Association (for HMIS ratings).

REVISION STATEMENT: This reviston updates the following sections of this Material Satety Data Sheet: 1,16

ABBREVIATIONS THAT MAY HAVE BEEN USED IN THIS DOCUMENT:

TLV - Threshold Limit Value TWA - Nime Weightad Average

STEL - Shart-term Exposure LImit PEL - Permissible Exposure Lmit

NOA - No Oata Avaikable

$\ll \quad$ - Lass Than or Equar To

CAS - Chomical Abstract Service Number

NA - Not Apoliosible

se - Greater Than or Equal To

Prepared according to the OSHA Hazard Communication Standard (29 CFR 1910.1200) and the ANSI MSDS Stardard (Z400.1) by the ChewronTexeco Energy Rssearch \& Technology Company, 100

Chevron Way, RJchmond, Calfomia 94802.

The above information is based on the data of which we are awara and is believed to be corroct as of the date hereof. Since this information may be applied under conditions beyond aur controi and with which wo may be unfamlilar and slneo data made avallable subsequent to the date hervol may suggest modifications of the information, we do not assume any responsibility for the results of its uxe. This information is furntshed upon oondition that tha person recesving it shall make his own determination of the suitability of the materlal for his particular purpoze.

Ravision Numbar: 2

Rovision Data: $11 / 08 / 2002$
7 of 7

Cats DFACr (Diecol Engine Antifraezo Coolant Concentrate Mist : $10 \mathrm{Bs!}$ 


\section{ExxonMobil}

641639-00 RONEX EXTRA DUTY MOLY 2 MATERLAL SAEETY DATA BULLETIN

1. PRODUCT AND COMRANY IDENTIFICATION

PRODUCT NAME: RONEX EXTRA DUTY MOLY 2

SUPPLIER: EXXONMOBIL OIL CORPORATION

3225 GALLOWS RD.

FAIREAX, VA 22037

24 - Hour Health and Safety Emergency (call collect): 609-737-4411

24 - Hour Transportation Emergency:

CHEMTREC: $800-424-9300 \quad 202-433-7616$

LUBES AND FUELS: 281-834-3296

Product and Technical Information:

Lubricants and specialties: B00-662-4525 800-443-9966

Fuels Products: 800-947-9147

MSDS Fax on Demand: 713-613-3661

MSDS Internet Website: http://wWw.exxon.com, http://www.mobil.com

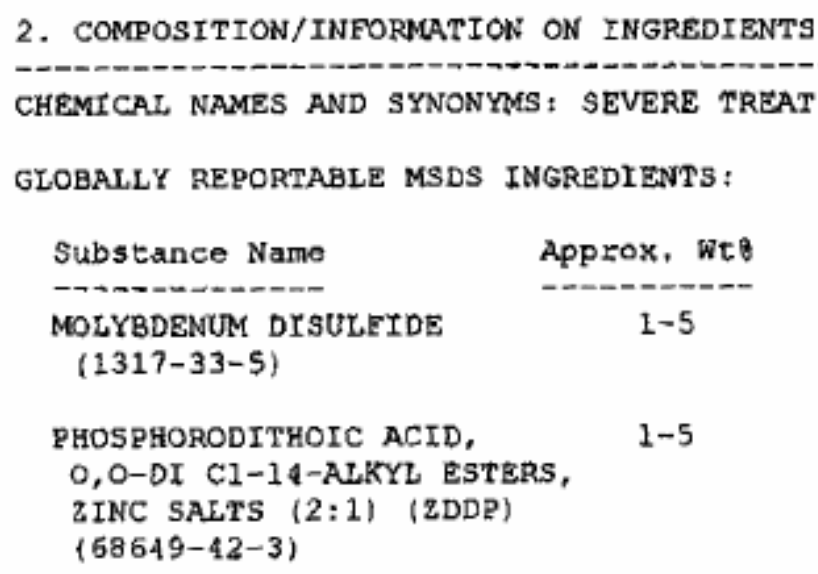


Under normal conditions of use, this product is not considered hazardous according to regulatory guidelines (See section 15).

EMERGENCY OVERVIEW: Dark Gray/Black Grease. DOT ERG No. : NA

POTENTIAL HEALTH EFFECTS: Under normal conditions of intended use, this product does not pose a risk to health. Excessive exposure may result in eye, skin or respiratory irritation.

For further health effects/toxicological data, see Section 11.

4. EIRST AID MEASURES

EYE CONTACT: Elush thoroughly with water. If irritation occurs, call a physician.

SKIN CONTACT: Wesh contact areas with soap and water. Remove and clean oil soaked clothing daily and wash affected area. INJECTION INJURY WARNING: If product is injected into or under the skin, or into any part of the body, regardless of the appearance of the wound or its size, the individual should be evaluated irmediately by a physician as a surgical emergency. Even though initial symptoms from high pressure injection may be minimal or absent, early surgical treatment within the first few hours may significantly reduce the ultimate extent of injury.

INHALATION: Remove from further exposure. If respiratory irritation, dizziness, nausea, or unconsciousness occurs, seek immediate medical assistance. If breaching has stopped, assist ventilation with mechanical device or use mouth-to-mouth resuscitation.

INGESTION: Not expected to be a problem. Seek medical attention if discomfort occurs. Do not induce vomiting.

\section{FIRE-EIGHTING MEASURES}

EXTINGUISHING MEDIA: Carbon dioxide, foam, dry chemical and water fog.

SPECIAL FIRE FIGHTING PROCEDURES: Water or foam may cause frothing. Use water to keep fire exposed containers cool. Water spray may fi used to tish spills away from exposure. Prevent runoff from fire control or dilution from entering streams, sewers, or drinking water supply.

SPECIAL PROTECTIVE EQUIPMENT: FOr fires in enclosed areas, fire fighters must use self-contained breathing apparatus.

UNUSUAL IIRE AND EXPLOSION HAZARDS: NONe.

COMBUSTION PRODUCTS: Fumes, smoke, carbon monoxide, sulfur oxides, aldehydes and ocher decomposition products, in the case of incomplete combustion.

Elash Point C(F): > 200 (392) (ESTIMATED EOR OIL, ASTM D-92 (COC)).

Flammeable Limits (approx. vol, in air) - LEL: NE, UEL: NE

NFPA HAZARD ID: Health: 0, Flammability: 1, Reactivity: 0

\section{ACCIDENTAL RELEASE MEASURES}

NOTIFICATION PROCEDURES: Report spills/releases as required to appropriate authorities. U,S. Coast Guard and EPA regulations require immediate reporting of spills/releases that could reach 
any waterway including intermittent dry creeks. Report spill/release to Coast Guard National Response Center toll free number (800)424-8802. In case of accident or road spldl notify CHEMTREC (800) 424-9300.

PROCEDURES IE MATERIAL IS RELEASED OR SPILLED:

LAND SPILL: Shut off source taking normal safety precautions. Take measures to minimize the effects on ground water. Recover by pumping or contain spilled material with sand or other suitable absorbent and remove mechanically into containers. If necessary, dispose of adsorbed residues as directed in Section 13.

WATER SPILL: Confine the spill immediately with booms. Warn other ships in the vicinity. Notify port and other relevant authorities. Remove from the surface by skimming or with suitable absorbents, If permitted by regulatory authorities the use of suitable dispersants should be consldered where recommended in local oil spill procedures.

ENVIRONMENTAL PRECAUTIONS: Prevent material from entering sewers, water sources or low lying areas; advise the relevant authorities if $1 \mathrm{t}$ has, or if $1 \mathrm{t}$ contaminates soil/vegetation.

PERSONAL PRECAUTIONS: see section 8

\section{HANDLING AND STORAGE}

HANDLING: High pressure injection under the skin may occur due to the rupture of pressurized lines. Always seek medical attention. No special precautions are necessary beyond normal good hygiene practices. See Section 8 for additional personal protection advice when handling this product.

STORAGE: Keep containers closed when not in use. Do not store in open or unlabelled containers. Store away from strong oxidizing agents and combustible materials. Do not store near heat, sparks, flame or strong oxidants. SPECIAL PRECAUTIONS: Prevent small spills and leakages to avoid slip
hazard.

EMPTY CONTAINER WARNING: Empty containers retain residue (1iquid and/or vapor) and can be dangerous. DO NOT PRESSURIzE, CUT, WELD, BRAZE, SOLDER, DRILL, GRIND OR EXPOSE SUCH CONTAINERS TO HEAT, FLAME, SPARKS, STATIC ELECTRICITY, OR OTHER SOURCES OF IGNITION; THEY MAY EXPLODE AND CAUSE INJURY OR DEATH. DO not attempt to refill or clean container since residue is difficult to remove. Empty drums should be completely drained, properly bunged and promptly returned to a drum reconditioner. All containers should be disposed of in an environmentally safe manner and in accordance with governmental regulations.

Substance Name (CAS-No.)

Source

MOLYBDENUM DISULFIDE

$(1317-33-5)$
---TWA--- ----STEL-- NOTE

pprm $\mathrm{mg} / \mathrm{m}^{3}$ ppm $\mathrm{mg} / \mathrm{m}^{3}$ 


$\begin{array}{llr}\text { as Mo Sol Cmpds } & \text { OSHA } & 5 \\ \text { as Mo Insol Cmpds Tot Dust } & \text { OSHA } \\ \text { as Mo Sol Cmpds } & \text { ACGIH } & 5 \\ \text { as Mo Insol Cmpds } & \text { ACGIH } & 10\end{array}$

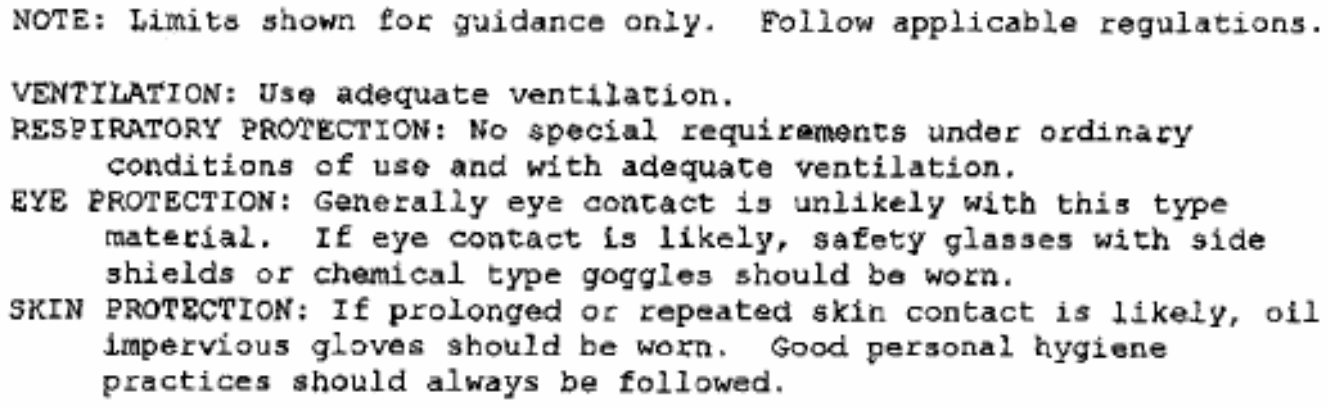


CONOITIONS TO AVOID: Extreme heat and high energy sources of ignition. INCOMPATIBILITY (MATERIALS TO AVOID): SEYong oxidizers,

HAZARDOUS DECOMPOSITION PRODUCTS: Product does not decompose at ambient temperatures.

HAZARDOUS POLYMERIZATION: Will not occur.

11. TOXICOLOGICAL DATA

- -ACUTE TOXICOLOGY---

ORAL TOXICTTY (RATS): Practically non-toxic (LDSO: greater than 2000 $\mathrm{mg} / \mathrm{kg}$ ). --Based on testing of similar products and/or the componenes.

DERMAL TOXICITY (RABBITS): 2ractically non-toxic (LDS0: greater than $2000 \mathrm{mg} / \mathrm{kg}$ ). ---Based on testing of similar products and $/$ or the components.

INHALATION TOXICIPY (RATS): Practically non-toxic (IC50: greater than $5 \mathrm{mg} / 1$ ). ---Based on testing of similar products and/or the components.

EYE IRRITATION (RABBITS): Practically non-irritating. (Draize score: greater than 6 but 15 or less). ---Based on testing of similar products and/or the components.

SKIN IRRITATION (RABBITS); Practically non-izritating. (Primary Irritation Indes: greater than 0.5 but less than 3 ). ---Based on testing of similar products and/or the components.

OTHER ACUTE TOXICITY DATA: Although an acute inhalation study was not performed with this product, a variety of mineral oils and synthetic base oils, such as those in this product have been tested. These samples had virtually no effect other than a nonspecific inflammatory response in the lung to the aerosolized mineral oil. The presence of additives in other tested formulations (in approximately the same amounts as in the present formulation) did not alter the observed effects.

---SUECHRONIC TOXICOLOGY (SUMMARY) w-

No significant adverse effects were found in studies using repeated dermal applications of simliar formulations to the skin of laboratory animals for 13 weeks at doses significantly higher than those expected during nomal industrial exposure. The animals were evaluated extensively for effects of exposure (hematology, serum chemistry, urinalysis, organ weights, microscopic examination of tissues etc.).

---REPRODUCTIVE TOXICOLOGY (SUMMARY) - -

No teratogeric effects would be expected from dermal exposure, based on laboratory developmental toxicity studies of major components in this formulation and/or materials of similar composition.

$\sim$ CHRONIC TOXICOLOGY (SUMARY) - -

Repeated and/or prolonged exposure may cause irricacion to the skin, eyes or respiratory tract. For mineral base olls: Base oils in this product are severely solvent refined and/or severely hydrotreated. Chronic mouse skin painting studies of severely treated oils showed no evidence of carcinogenic effects. These results are confirmed on a continuing basis using various screening methods such as Modified Ames Test, IP-346, and/or other analytical methods. For synthetic base oils: The base 
oils in this product have been tested in the Ames assay and other rests of mutagenicity with negative results. These base oils are not expected to be carcinogenic with chronic demal exposures.

$$
\text { ---SENSITIZATION (SUMMARY)--- }
$$

Not expected to be sensitizing based on tests of this product, components, or similar products.

\section{ECOLOGICAL INPORWATION}

\section{ENVTRONMENTAL FATE AND BFEBCTS:}

This environmental assessment was conducted using information on the individual components as no test data was available for this specific formulation.

ECoTOXICITY: The major components in the formulation show no aquatic toxicity at $1000 \mathrm{mg} / \mathrm{L}$ loading, therefore long-term adverse effects in the aquatic environment are not expected.

MOBILIFY: Not established.

PERSISTENCE AND DEGRADABILITY: This product is expected to be inherently biodegradable, as the principal components have been shown to degrade at slow to moderate rates.

BIOACCUMULATIVE POTENTIAL: Not established.

13. DISPOSAL CONSIDERATIONS

WASTE DISPOSAL: Product is suitablo for burning in an enclosed, controlled burner for fuel value. Such burning may be limited pursuant to the Resource Conservation and Recovery Act. In addition, the product is suitable for processing by an approved recycling facility or can be disposed of at an appropriate government waste disposal facility. Use of these methods is subject to user compliance with applicable laws and regulations and consideration of product characteristics at time of disposal.

RCRA INBOPMATION: The unused product, in our opinion, is not specifically listed by the BPA as a hazardous waste 140 CFR, Part 261D), nor is it formulated to contain materials which are 11sted hazardous wastes. It does not exhibit the hazardous characteristics of ignitability, corrosivity, or reactivity. The unused product is not formulated with substances covered by the Toxicity Characteristic Leaching Procedure (TCLP). However, usod product may be regulated.

14. TRANSPORT INFORYATION

USA DOT: NOC REGULATED BY USA DOT. 
RID/ADR: NOT REGULATED BY RID/ADR.

IMO: NOT REGULATED BY IMO.

IATA: NOT REGULATED BY IATA.

\section{REGULATORY INFORMATION}

US OSHA HAZARD COMMUNICATION STANDARD: When used for its intended purposes, this product is not classified as hazardous in accordance with OSHA 29 CFR 1910.1200.

BU Labeling: Product is not dangerous as defined by the European Union Dangerous Substances/Preparations Directives. EU labeling not required.

Governmental Inventory status: All components comply with TSCA, EINECS/ELINCS and AICS.

V.S. Superfund Amendments and Reauthorization Act (SARA) Title III: This product contains no "EXTREMELY HAZARDOUS SUBSTANCES".

SARA (311/312) REPORTABLE HAZARD CATEGORIES: NONE.

This product contains the following SARA (313) Toxic Release Chemicals:

CHEMICAL NAME

-

PHOSPHORODITHOIC ACID, 0,0 -DI CI-IA-ALKYL ESTERS, ZTNC SALTS

(2:1) (ZDDP)
CAS NUMBER

$$
68649-42-3
$$

CONC.

\begin{tabular}{|c|c|c|}
\hline CHEMTCAL NAME & CAS NUMBER & LIST CITATIONS * \\
\hline 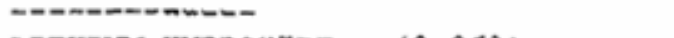 & $--0-0-0--$ & 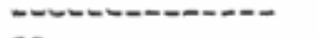 \\
\hline LITHZUM HYOROXIDE $\quad(0.06 \%)$ & $1310-65-2$ & $22-1-2$ \\
\hline MOLYBDENUM (IV) SULFIDE & $1317-33-5$ & $\begin{array}{l}1,10,18,20,2 \\
23\end{array}$ \\
\hline ZINC (ELEMENTAI ANALYSIS) (0.188) & $7440-66-6$ & 22 \\
\hline LITHIUM-SOAP THICKENER $\quad\{7.028\}$ & $7620-77-1$ & 22 \\
\hline $\begin{array}{l}2 \text { TNC DINONYLNAPHTHALENE SULEONATE } \\
(0.408)\end{array}$ & $28016-00-4$ & 22 \\
\hline $\begin{array}{l}\text { PHOSPHORODITHOIC ACID, 0,0-DI } \\
\text { C1-14-ALKYL ESTERS, ZINC SALTS (2: } \\
\text { 1) (ZDDP) (1.298) }\end{array}$ & $68649-42-3$ & $\begin{array}{l}18,20,21,22, \\
25\end{array}$ \\
\hline FATTY ACIDS, C16 22, LITHIUM SALTS & $68783-36-8$ & 22 \\
\hline
\end{tabular}


$(0.878)$

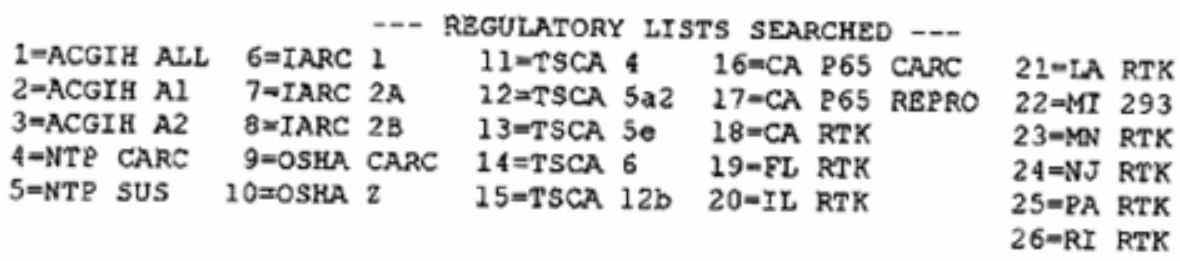

* ERA recently added new chemical substances to its TSCA Section 4 test rules. Please contact the supplier to confirm whother the ingredients in this product curzently appear on a TSCA 4 or TSCA $12 \mathrm{~b}$ list.

Code key:CARC=Carcinogen; SUS=Suspected Carcinogen; REPRO-Reproductive

16. OTHER INFORMATION

USE: GREASE

NOTE: PRODUCTS OE EXXON MOBIL CORPORATION AND ITS AFFILIATED COMPANIES ARE NOT FORMULATED TO CONTAIN PCBS.

Health scudies have shown that many hydrocarbons pose potential human health risks which may vary from person to person. Infozmation provided on this MSDS reflects incended use. This product should not be used for other applications. In any case, the following advice should be considered:

\section{INDUSTRIAL LABEL}

Under normal conditions of intended use, this product does not pose a risk to health. Excessive exposure may result in eye, skin or respiratory irritation. Always observe good hygiene moasures. Eirst Aid: Wash skin with soap and water. Flush eyes with water. If overcome by fumes or vapor, remove to fresh air. If ingested do not induce vomiting. If symptons persist seek medical assistance. Read and understand the MSDS before using this product.

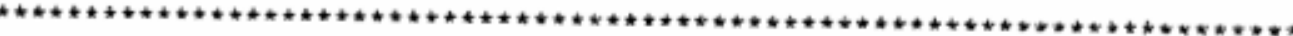

For Internal Use Only: MHC: 1*1*1*1*1*, MPrec: A, TRN: 641639-00, CMCS97: 97P758, REQ: US - MARKETING, SAFE USE: L EHS Approval Date: 13NOV2001 
Information given herein is offered in good faich as accurate, but without guarantee. Conditions of use and suitability of the product for particular uses are beyond our control; all risks of use of the product are therefore assumed by the user and WE EXPRESSLY DISCLAIM ALL MIARRANTIES OE EVERY KIND AND NATURE, INCLUDING WARRANTIES OF MERCHANTABILITY AND FITNESS FOR A EARTICULAR PURPOSE IN RESPECT TO THE USE OR SUITABTLITY OE THE RRODUCT. Nothing is intended as a recommendation for uses which infringe valid patents or as extending license under valid patents. Appropriate warnings and safe handling procedures should be provided to handlers and users. Alteration of this document is stcictly prohibited. Except to the extent required by law, republication or retransmission of this document, in whole or in part, is not permitted. Exxon Mobil Corporation and its affiliated companies assume no responsibility for accuracy of information unless the document is the most current available from an official ExronMobil distribution system. Exxon Mobil Corporation and its affiliated companies neither represent nor warrant that the format, content or product formulas contained in this document comply with the laws of any other country except the United States of America.

Prepared by: ExxonMobil Oil Corporation Enviromental Health and Safety Department, Clinton, USA 


\section{Vendor Data Review System Final Disposition Screen}

This vendor data item has been given the following disposition codes

\begin{tabular}{|c|c|c|c|c|}
\hline Reviewer & $\begin{array}{l}\text { Revision } \\
\text { Level }\end{array}$ & Date & $\begin{array}{l}\text { Disposition } \\
\text { Code }\end{array}$ & Comments \\
\hline VANDEL DOUG S & 0 & $\begin{array}{l}\text { 25- } \\
\text { SEP. } \\
06\end{array}$ & A & \\
\hline TUOTT LEE C & 0 & $\begin{array}{l}25- \\
\text { SEP. } \\
06\end{array}$ & $\mathrm{D}$ & $\begin{array}{l}\text { Chemical info/data has been submitted } \\
\text { and approved by Mikel Driever, Chemical } \\
\text { Coordinator. }\end{array}$ \\
\hline DRIEVER MIKEL K & 0 & $\begin{array}{l}25- \\
\text { SEP. } \\
06\end{array}$ & A & \\
\hline FRITZ KURT D & 0 & $\begin{array}{l}22- \\
\text { SEP. } \\
06\end{array}$ & D & \\
\hline $\begin{array}{l}\text { MCMANAMON } \\
\text { LAWRENCE E }\end{array}$ & 0 & $\begin{array}{l}27- \\
\text { SEP. } \\
06\end{array}$ & A & \\
\hline
\end{tabular}

$\begin{array}{ll}\text { VDR Number: } & \text { VDR-153307 } \\ \text { Revision Level: } & 0 \\ \text { Project Number: } & 23368-152173 \\ \text { Transmittal Number: } & \text { S-507296-01 } \\ \text { Transmittal Status: } & \text { Mandatory Approval } \\ \text { Line Item: } & 1 \\ & \\ \text { Code:: } & \text { Final Comments:: } \\ & \text { No comments }\end{array}$

Disposition Code::

A
No comments 
F-126 


\section{VDS \#3_154111}


F-128 
431.13

$07 / 23 / 2002$

fiev. 04
VENDOR DATA

TRANSMITTAL \& DISPOSITION FORM

ORIGINAL

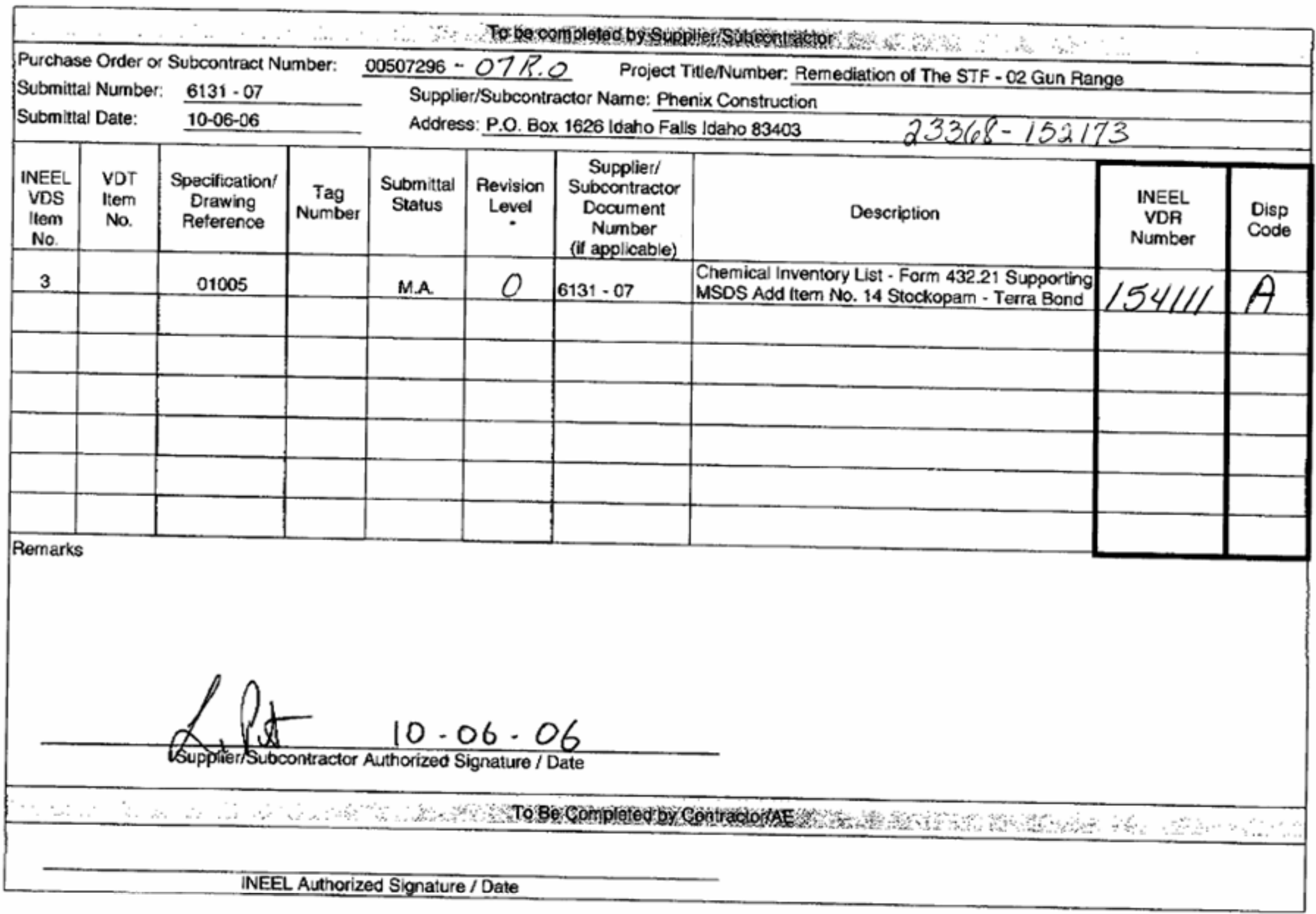


432.21A

04/13/99

Rev. 01

\section{SUBCONTRACTOR REPORTING FORM \\ CHEMICAL INVENTORY LIST \\ (CONTINUATION SHEET)}

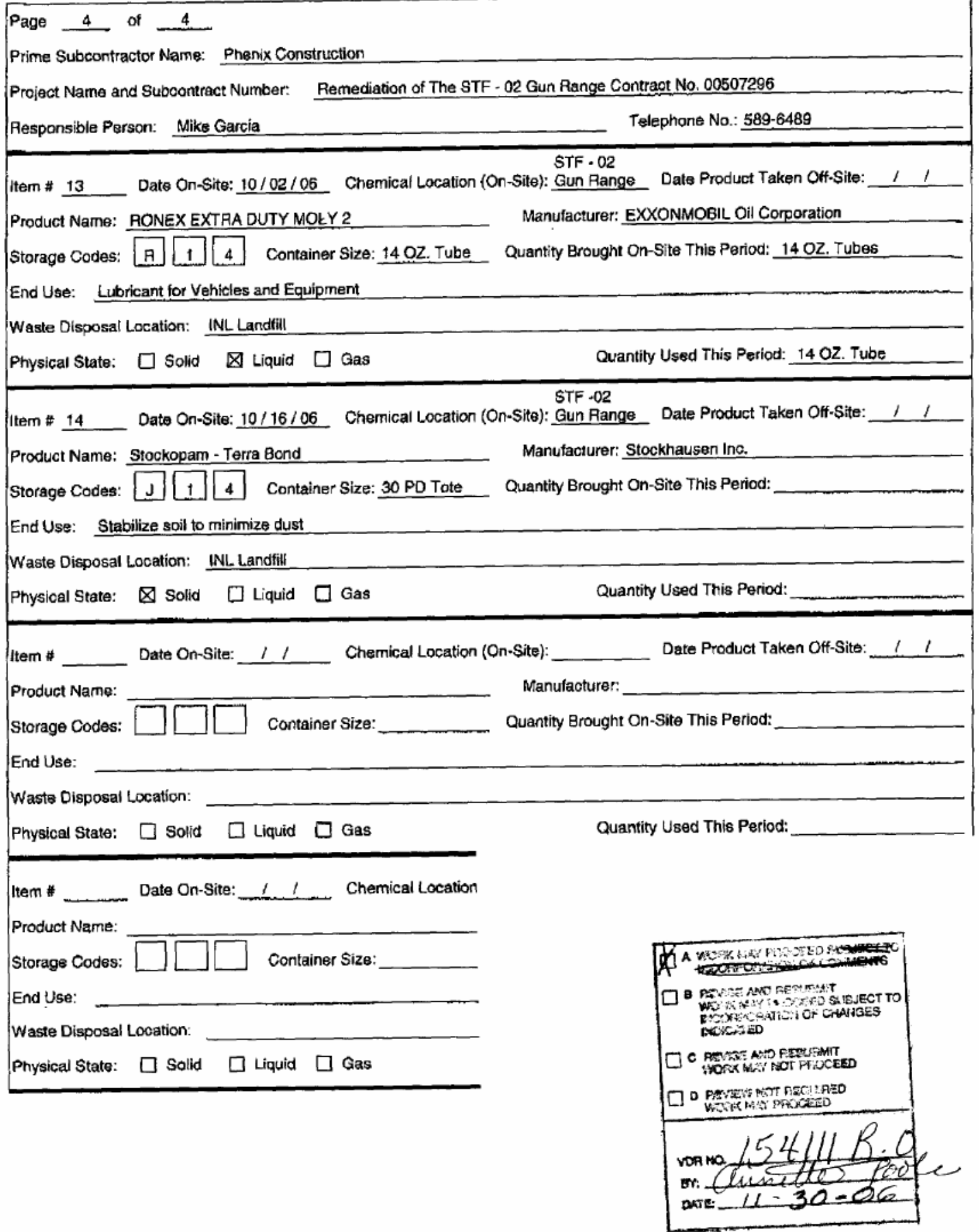




\section{degussa.}

\section{Material Safety Data Sheet STOCKOPAM}

MSDS ID: CR-119

\section{*** Section 1 - Chemical Product and Company Identification}

Chemical Name: Polyacrylamide

Company:

Stockhausen Inc.

2401 Doyle Street

Greensboro, NC 27408

Phone: (800) 242-2271

Emergency : :(800) 424-9300 CHEMTREC

\section{${ }^{* * *}$ Section 2 - Composition / Information on Ingredients}

\begin{tabular}{|l|l|l|}
\hline CAS \# & Component & Pereent \\
\hline $25085-02-3$ & 2-Propenoic acid, sodium salt, polymer with 2-propenamide & \\
\hline
\end{tabular}

Component information/lnformation on Non-Hazardous Components None

** "Section 3 - Hazards Identification

Emergency Overview

Irritant to eyes. Prolonged or repeated skin contact may cause dryness and irritation.

Potential Heaith Effects: Eyes

Irritant

Potential Health Effects: Skin

Irritant

Potential Health Effects: Ingestion

Potential Heaith Effects: Inhalation

HMIS Ratings: Health: 1 Fire: 0 Reactivity: 0 Pers. Prot.: B

Hazard Scale: $0=$ Minimal $1=$ Slight $2=$ Moderate $3=$ Serious $4=$ Severe $*=$ Chronic hazard

*** Section 4 - First Aid Measures

First Aid: Eyes

Immediately flush eyes with water for at least 15 minutes, while holding eyelids open. Seek medical attention.

First Aid: Skin

Wash thoroughly with soap and water.

First Aid: Ingestion

if ingested, get immediate medical attention. Do not induce vomiting unless instructed to do so by medical personnet.

First Aid: Inhalation Move to source of fresh air.

\section{** Section 5 - Fire Fighting Measures $* \star \star$}

\section{General Fire Hazards \\ No particular hazards.}


Upper Flammable Limit (UFL): Not determined Lower Flammable Limit (LFL): Not determined Method Used: DIN 51758

Flash Point: $>200 \mathrm{C}$

Flammability Classification:

Auto Ignition: $>200^{\circ} \mathrm{C}$

Hazardous Combustion Products

On thermal decomposition oxides of carbon and nitrogen.

\section{Extinguishing Media}

Dry chemical, foam, carbon đioxide, water fog.

Fire Fighting Equipment/Instructions

Firefighters should wear full protective clothing including self contained breathing apparatus.

NFPA Ratings: Heaith: 1 Fire: 0 Reactivity: 0

Hazard Scale: $0=$ Minimal $1=$ Slight $2=$ Moderate $3=$ Serious $4=$ Severe

\section{*** Section 6 - Accidental Release Measures ***}

Containment Procedures

Flush residuals to the drain for normal biological treatment. CAUTION- spilled material in contact with water creates very slippery conditions.

Clean-Up Procedures None specified.

Evacuation Procedures

Isolate area. Keep unnecessary personnel away.

Special Procedures

Remove spills promptly as they may make floors slippery. Several washes and/or the use of detergents may be necessary to completely clean any spill.

\section{*** Section 7 - Handling and Storage}

Handling Procedures

Handle as an irritant. Do not get into eyes.

Storage Procedures

None specified.

\section{** Section 8 - Exposure Controls / Personal Protection * * *}

\section{A: Component Exposure Limits}

No information is available.

\section{Engineering Controls}

Provide adequate ventilation to minimize worker exposure.

PERSONAL PROTECTIVE EQUIPMENT

Personal Protective Equipment: Eyes/Face

Safety glasses

Personal Protective Equipment: Skin

Skin contact should be minimized. Impervious gloves (rubber or neoprene) are recommended.

Personal Protective Equipment: Respiratory

Use a nuisance dust mask for dusty conditions.

Personal Protective Equipment: General

CAUTION Extreme slipping hazard when wet. 


\section{* * Section 9 - Physical \& Chemical Properties}

\author{
Appearance: White granular \\ Physical State: Solid \\ Vapor Pressure: N/E \\ Boiling Point: N/E \\ Solubility (H2O): Limited solubility \\ Evaporation Rate: $<1 \quad$ (butyl acetate =1)
}

\author{
Odor: Mild acrylic odor \\ $\mathrm{pH}: \quad 5-7$ at $10 \mathrm{~g} /\left(20^{\circ} \mathrm{C}\right)$ \\ Vapor Density: N/E \\ Melting Point: $N / E$ \\ Specific Gravity: N/E \\ Bulk Density: 44 lbs. $/ \mathrm{ft}^{3}$
}

\section{** " Section 10 - Chemical Stability \& Reactivity Information * *}

Chemical Stability

Stable under usual application conditions.

Chemical Stability: Conditions to Avoid

None.

Incompatibility

None identified.

Hazardous Decomposition

None identified.

Hazardous Polymerization

Hazardous polymerization will not occur.

\section{${ }^{* * *}$ Section 11 - Toxicological Information ${ }^{* *}$ *}

Acute and Chronic Toxicity

A: General Product Information

Eye and skin irritant. May aggravate existing medical conditions such as rashes, allergies or other sensitive areas. Symptoms may include reddening, swelling of affected areas with possible itching, burning or other discomfort. LD50 mouse: $>5000 \mathrm{mg} / \mathrm{kg}$ ( $5 \%$ solution)

B: Acute Toxicity-LD50/LC50

No LD50/LC50's are available for this product's components.

Carcinogenicity

A: General Product Information

NTP: No

IARC: No

OSHA: NO

B: Component Carcinogenicity

No information is available.

Mutagenicity

Results of mutagenicity testing with the Ames Test (Salmonella typhinurium plate test): negative.

\section{$\star * *$ Section 12 - Ecological Information}

\section{Ecotoxicity}

A: General Product Information

Prevent concentrated product from penetrating surface waters without biological waste water treatment.

Dilute solutions in normal use are not to be regarded as hazarcious to aquatic life.

Fish toxicity: LC50 Leuciscus idus $\sim 4000 \mathrm{mgh}, 96$ hour.

B: Component Analysis - Ecotoxicity - Aquatic Toxicity

No information available, 


\section{Environmental Fate}

Biodegradation is negligible, possibly due to the highly polymerized structure of the polyacrylamides. However, the substance is removed from waste water effluent due to flocculation with the bio-siudge.

\section{*** Section 13 - Disposal Considerations}

US EPA Waste Number \& Descriptions

A: General Product Information

Incinerate or dispose of solidified product according to local, state and federal regulations. Unadulterated product is considered non-hazardous.

B: Component Waste Numbers

No EPA Waste Numbers are applicable for this product's components.

Disposal Instructions

Contain and collect using absorbent material if needed. Flush residuals to drain for normal bioiogical treatment. Place collected material into suitable containers for proper disposal.

\section{*** Section 14 - Transportation Information ***}

International Transportation Regulations

This product is not regulated as a hazardous material by the United States (DOT) or Canadian (TDG) transportation regulations.

\section{*** Section $15-$ Regulatory Information $* \star \star *$}

\section{US Federal Regulations}

A: General Product Information

B: Clean Air Act

SARA 313 reportable toxic chemicals - None This product is not Federally regulated as a hazardous material.

No information is available.

C: Component Analysis

No information is available.

D: Food \& Drug Administration

CFR references for the FDA regulated components in this product are listed.

2-Propenoic acid, sodium salt, polymer with 2-propenamide (25085-02-3)

Direct Food 172.710, 173.5, 173.310

Additives:

State Regulations

A: General Product Information

None

B: Component Analysis - State

None of this product's components are listed on the state lists from CA, FL, MA, MN, NJ, or PA.

Component Analysis - WHMIS IDL

No components are listed in the WHMIS IDL.

Component Anaiysis - Inventory

\begin{tabular}{|l|l|l|l|l|}
\hline Component & CAS \# & TSCA & CAN & EEC \\
\hline $\begin{array}{l}\text { 2-Propenoic acid, sodium salt, polymer with 2- } \\
\text { propenamide }\end{array}$ & $25085-02-3$ & YGS & DSL & No \\
\hline
\end{tabular}

\section{${ }^{\star \star \star *}$ Section 16 - Other Information ${ }^{* \star *}$}




\section{Material Safety Data Sheet}

Other Information

Reasonable care has been taken in the preparation of this information, but the manufacturer makes no warranty of merchantability or any other werranty, expressed or implied, with respect to this information. The manufacturer makes no representations and assumes no liability for any direct, incidental or consequential darnages resulting from its use.

MSDS History

This is the U.S. version of the MSDS for STOCKOPAM.

Key/Legend

Contact: Product Compliance Officer

Contact Phone: (800) 242-2271 
degussa.

\section{Technical Data}

\section{STOCKOPAM}

0.5\% Brine Viscosity, mPas

Gel Content, ml/I

Anionic Charge, \%

Residual Acrylamide, ppm
220 Min.

$15 \operatorname{Max}$

$27-33$

$<500$
Slockhausen, inc. 2401 Doyis St.

Greensboro, NC 27406

Tol 1-888-533-7764

Fax 1-336-379-3586

www.stockhausen-inc.com
Stockhausen GmbH

Postfach 570

D.47705, Kresled

Tel 492151381880

Fax 492151381292

unw/slockinausen.com 
dyusso

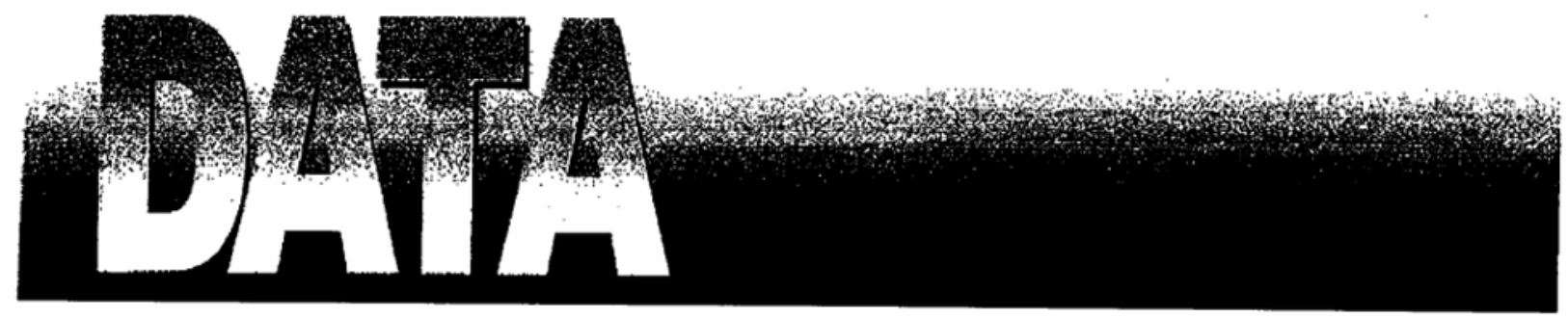

Safety Data of

STOCROPAM

Anionic Polymers

Introduction

STOCKOPAM is an anionic polymer primarily used for hydroseeding and erosion control, e.g., for preventing irrigation-induced erosion. STOCKOPAM is a copolymer of acrylamide and sodium acrylate. STOCKOPAM shows the same physical and chemical properties as other anionic synthetic flocculating agents manufactured by STOCKHAUSEN. Not all of the different variations of the STOCKOPAM types were tested with all the following test systems due to ethical and economical reasons. All studies were performed in the Laboratory for Toxicology and Ecology, Stockhausen, Germany. In the following, STOCKOPAM is referred to as PAM. 


\section{Toxicity Data}

\author{
Acute oral toxicity \\ $L_{50}>1,200 \mathrm{mg} / \mathrm{kg}$ body \\ weight.
}

Up to $1,200 \mathrm{mg} / \mathrm{kg}$ body weight of PAM were applied with a stomach tube to 10 male mice. This was the highest oral dose level applicable due to the high viscosity of PAM in saline solution. Only slight and reversibie toxic symptoms were observed in the highest dosage group; no death was recorded. Body weight development was normal during observation over 14 days after single application.

PAM has to be regarded as essentially non-toxic after oral intake.

\section{HET-CAM-Test}

Hen's egg test (HET) Chorion allantoic membrane (CAM) assay: $200 \mathrm{mg}$ of PAM were applied onto the CAM of hen embryos of 10 days of age. There were moderate effects with respect to vascular injection and hemorrhaging within 2 minutes after application but no effect regarding coagulation. Potential for cell toxicity and adverse effects on membranes seems to be low.

\section{Cytotoxicity in vitro}

PAM was examined regarding its influence on mammalian cells in a cell culture system using a fibroblastic cell line derived from mice. The cells were incubated for 24 hours with a $1 \%(\mathrm{v} / \mathrm{v})$ solution of PAM in cell culture medium. No adverse effects on the morphology or viability of the cells were observed with this concentration.

\section{Ames Test: \\ Salmonella typhimurium reverse mutation assay}

Mutagenicity was tested with the Ames Salmonella plate test (in vitro) with and without metabolic activation by rat liver microsomes. The strains TA 100 and TA 1553 were used as well as TA 98 and TA 1537. An increase in the revertants was not detected in any of the examined cases. Therefore, there was no indication of a mutagenic potential in $\underline{\mathbf{S}}$. typhimurium of PAM

\section{In vivo micronucleus test}

PAM was assayed in an in vivo mouse bone marrow micronucleus test with an oral dose of $600 \mathrm{mg} / \mathrm{kg}$ body weight. Groups of 5 male and 5 female animals were used and were killed at intervals of 24,48 and 72 hours after treatment. At no time point was there a significant increase in micronucleus frequency in the test groups. Therefore it is concluded that PAM is not able to induce micronuclei in polychromatic or normochromatic erythrocytes of bone marrow of mice. 


\section{Ecotoxicity Data}

\begin{abstract}
Chronic bacterial toxicity
Growth behavior and propagation of the microorganism Pseudomonas putida was determined with PAM in concentrations up to $1,600 \mathrm{mg} / \mathrm{l}$. Inhibitory effects on the growth behavior were not observed, i.e. no cytostatic or biocidal effects are to be expected. The EC50-value for haif maximum propagation is higher than the highest concentration tested.
\end{abstract}

Therefore no critical effects to bacteria are expected under relevant use and disposal conditions.

\section{Chronic algae toxicity}

Growth behavior of the single cellular algae Scenedesmus subspicatus was determined with PAM up to a concentration of $500 \mathrm{mg} / \mathrm{l}$. Inhibition of growth was observed in concentrations of $1 \mathrm{mg} / \mathrm{l}$ and higher but the Ecso -value which defines half maximum growth is higher than the highest concentration tested. However, the observed mild toxicity is thought to be of minor practical importance under realistic environmental exposure conditions.

\section{Acute daphnia toxicity}

Acute effects on the swimming ability of the daphnids Daphnia magma were determined with PAM in concentrations up to 640 $\mathrm{mg} / \mathrm{l}$. The ECSO -value for half maximum swimming inhibition is approximately $300 \mathrm{mg} / \mathrm{l}$, the $E C_{0}$-value, the concentration where no effect on the swimming ability occurred, is approximately $40 \mathrm{mg} / \mathrm{l}$. The observed slight toxicity of PAM to daphnids is thought to be of minor relevance when realistic environmental exposure conditions are taken into account.

\section{Acute fish toxicity}

To determine the potential acute lethal effects on fish, the cold water species Leuciscus idus (golden orf) and the warm water species Brachydanio rerio (zebra fish) were exposed to PAM. The $\_$c50 -value which defines the mean lethal concentration is approximately $140 \mathrm{mg} / \mathrm{l}$ and $160 \mathrm{mg} / \mathrm{l}$ for the golden orf and the zebra fish respectively. Therefore, PAM has to be regarded as slightly toxic to fish.

\section{Biodegradability in aquatic systems}

Two different degradation studies on PAM in liquid systems with sewage micro organisms as inoculum have been conducted:

\section{- The Modified Sturm Test (CO2 evolution test)}

- The BODIS Test (closed
bottle test)

In the $\mathrm{CO}_{2}$ evolution test PAM was incubated over $\mathbf{2 8}$ days under aerobic conditions with activated sludge. The amount of carbon dioxide produced from the test substance is measured as a result of biodegradation.

In the BODIS Test degradation is followed by analysis of dissolved oxygen over a period of 28 days. PAM was inoculated with mixed microorganisms and kept in completely full, ciosed bottles. The amount of oxygen taken up by the microbial population during biodegradation is measured.

The results of both laboratory tests indicate that PAM is poorly biodegradable. 


\section{Conclusion}

STOCKOPAM is an anionic polyacrylamides which exhibits a low toxicological profile. There have been no signs of acute oral toxicity and there is no evidence for marked irrita tive properties; no mutagenic potency was found. With respect to the environmental compatibility of STOCKOPAM the data indicate some slight toxic effects on aquatic organisms. However, under realistic environmental exposure conditions no critical deleterious effects are expected.
In standard laboratory test systems biodegradation could not be observed. Nevertheiess, due to results of the latest research there is sufficient and increasing evidence that polyacryfamides do not constitute persistent, non-degradable man-made polymers.

Therefore, STOCKOPAM is regarded as essentially compatible to the environment, especially taking into account the very low dosage levels used for application.
To support this assessment the following facts should be taken into account:

- anionic flocculants have been used successfully in sewage disposal plants for several decades

- for preparation of drinking water similar anionic type polymers are approved by relevant authorities

- the use of polyacrylamides as soil conditioner is well known.

No negative effects on the environment have been reported with respect to these areas of applications. 
Page 1 of 1

\section{Vendor Data Review System Final Disposition Screen}

This vendor data item has been given the following disposition codes

\begin{tabular}{|c|c|c|c|c|}
\hline Reviewer & Revision Level & Date & Disposition Code & Comments \\
\hline JOLLEY WENDELL L & 0 & 09-OCT-06 & $\mathrm{D}$ & \\
\hline VANDEL DOUG S & 0 & 09-OCT-06 & A & \\
\hline DRIEVER MIKEL K & 0 & 09-OCT-06 & A & \\
\hline TUOTT LEE C & 0 & 28-NOV-06 & A & \\
\hline MCMANAMON LAWRENCE E & 0 & 09-OCT-06 & A & \\
\hline LANDIS JOSEPH A & 0 & 09-ОСТ-06 & A & no comments \\
\hline FRITZ KURT D & 0 & 10-OCT-06 & D & \\
\hline VDR Nun & ber: & VDR-154111 & & \\
\hline Revision & evel: & 0 & & \\
\hline Project $\mathrm{N}$ & mber: & $23368-15217$ & & \\
\hline Transmitt & I Number: & S-507296-07 & & \\
\hline Transmitt & I Status: & Mandatory Ap & pproval & \\
\hline Line Item & & 1 & & \\
\hline $\begin{array}{l}\text { Disposition Code:: } \\
\text { A }\end{array}$ & & Final Comm & lents: : & \\
\hline
\end{tabular}


F-142 


\section{VDS \#4_153312}


F-144 


\section{VENDOR DATA
TRANSMITTAL \& DISPOSITION FORM}

To be completed by Supplier/Subcontractor

Purchase Order or Subcontract Number: $00507296-02$ R. 0 Project Title/Number: Remediation of The STF - 02 Gun Range

Submittal Number: 6131.02 Supplier/Subcontractor Narne: Phenix Construction

Submittat Date: $\quad \underline{09-21.06}$ Address: P.O. Box 1626 Idaho Falls Idaho 83403

$23368-152173$

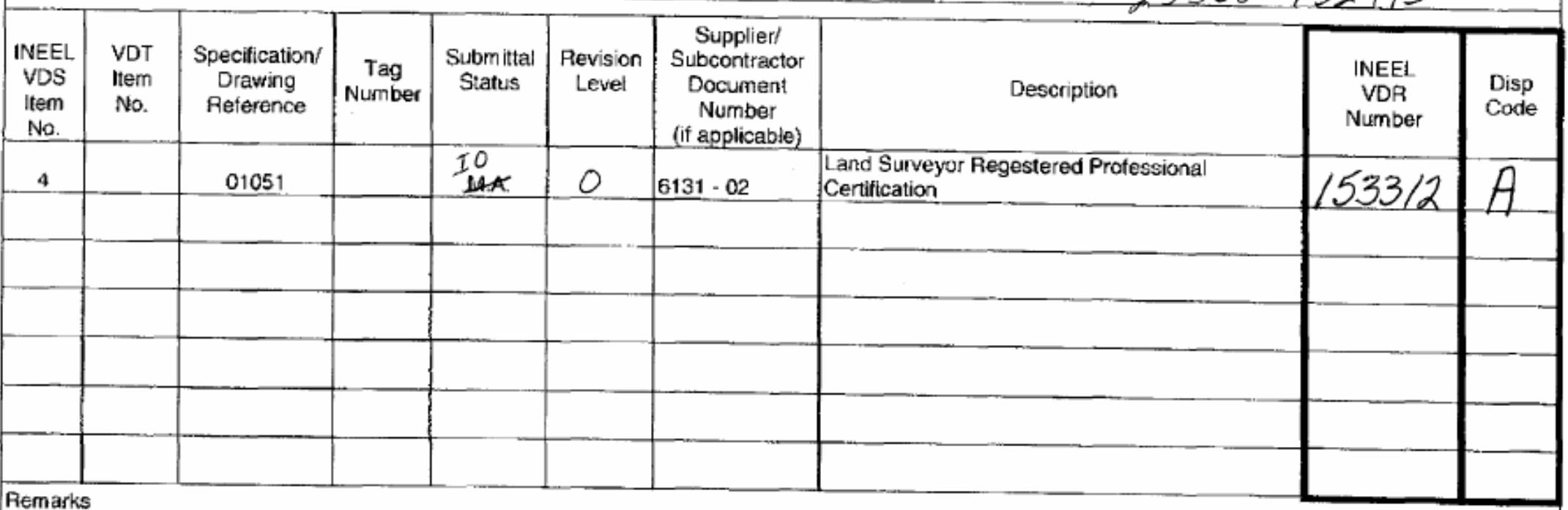

Remarks

$09-21-06$

Suppher/Subcontractor Authorized Signature / Date

To Be Completed by Contractor/AE 


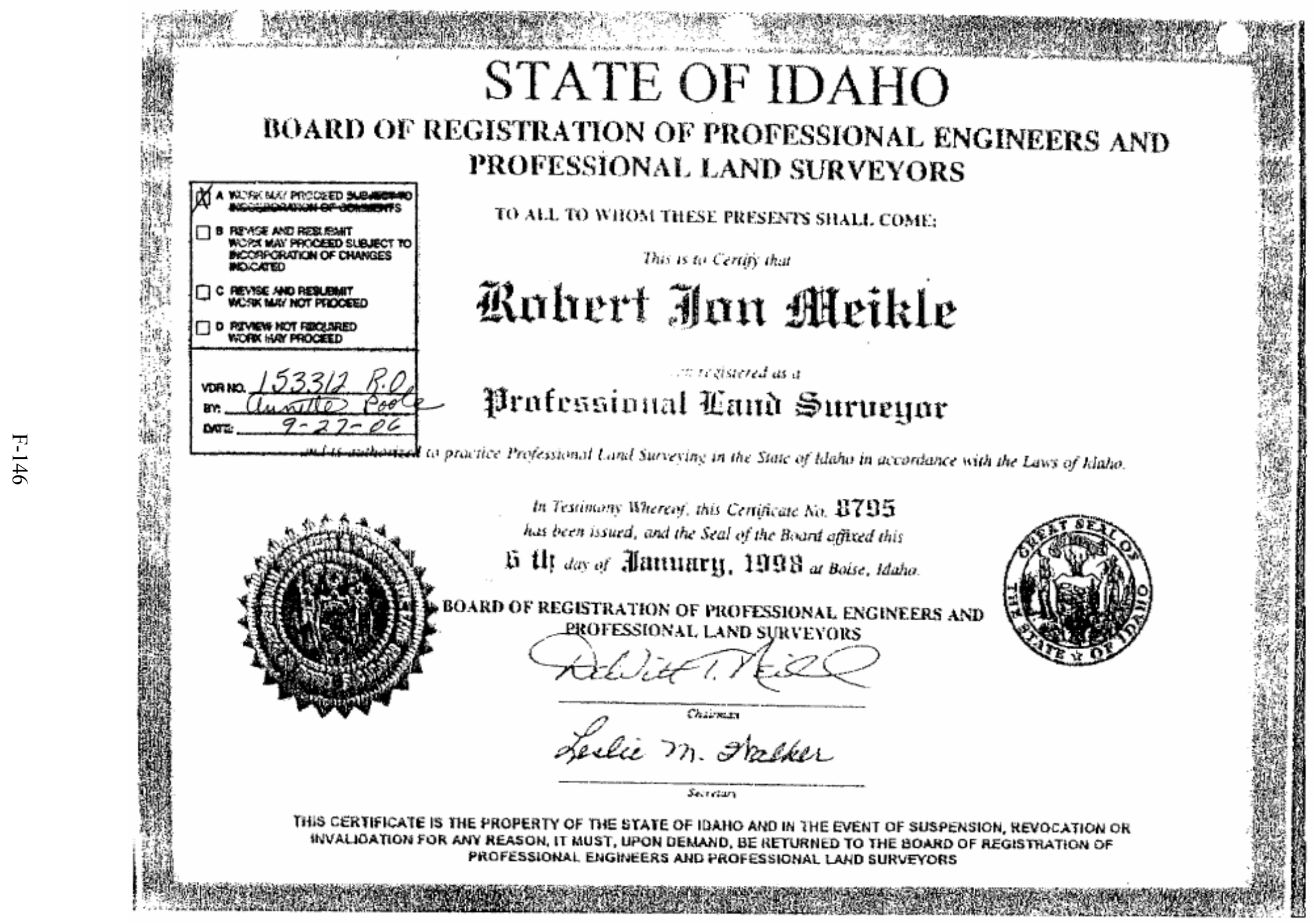




\title{
Vendor Data Review System Final Disposition Screen
}

\author{
This vendor data item has been given the following disposition codes
}

$\begin{array}{ll}\text { Reviewer } & \\ \text { FRITZ KURT D } & 0 \\ \text { VANDEL DOUG S } & 0 \\ \text { DRIEVER MIKEL K } & 0 \\ \text { TUOTT LEE C } & 0 \\ \text { MCMANAMON LAWRENCE E } & 0\end{array}$

VDR Number:

Revision Level:

Project Number:

Transmittal Number:

Transmittal Status:

Line Item:

Disposition Code::

A

$\begin{array}{llll}\text { Revision Level } & \text { Date } & \text { Disposition Code } & \text { Comments } \\ 0 & \text { 22-SEP-06 } & \text { D } \\ 0 & \text { 25-SEP-06 } & \text { A } \\ 0 & \text { 25-SEP-06 } & \text { D } \\ 0 & \text { 25-SEP-06 } & \text { D } \\ 0 & 26-S E P-06 & \text { A }\end{array}$

VDR-153312

0

$23368-152173$

S-507296-02

Information Only

1

Final Comments::

No Comment 
F-148 


\section{VDS \#5_155376}


F-150 


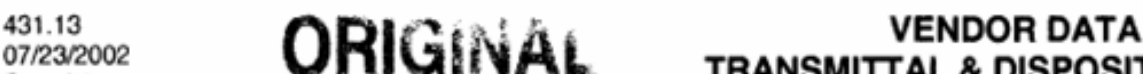

ORANSMITTAL \& DISPOSITION FORM

To be completed by Supplier/Subcontractor

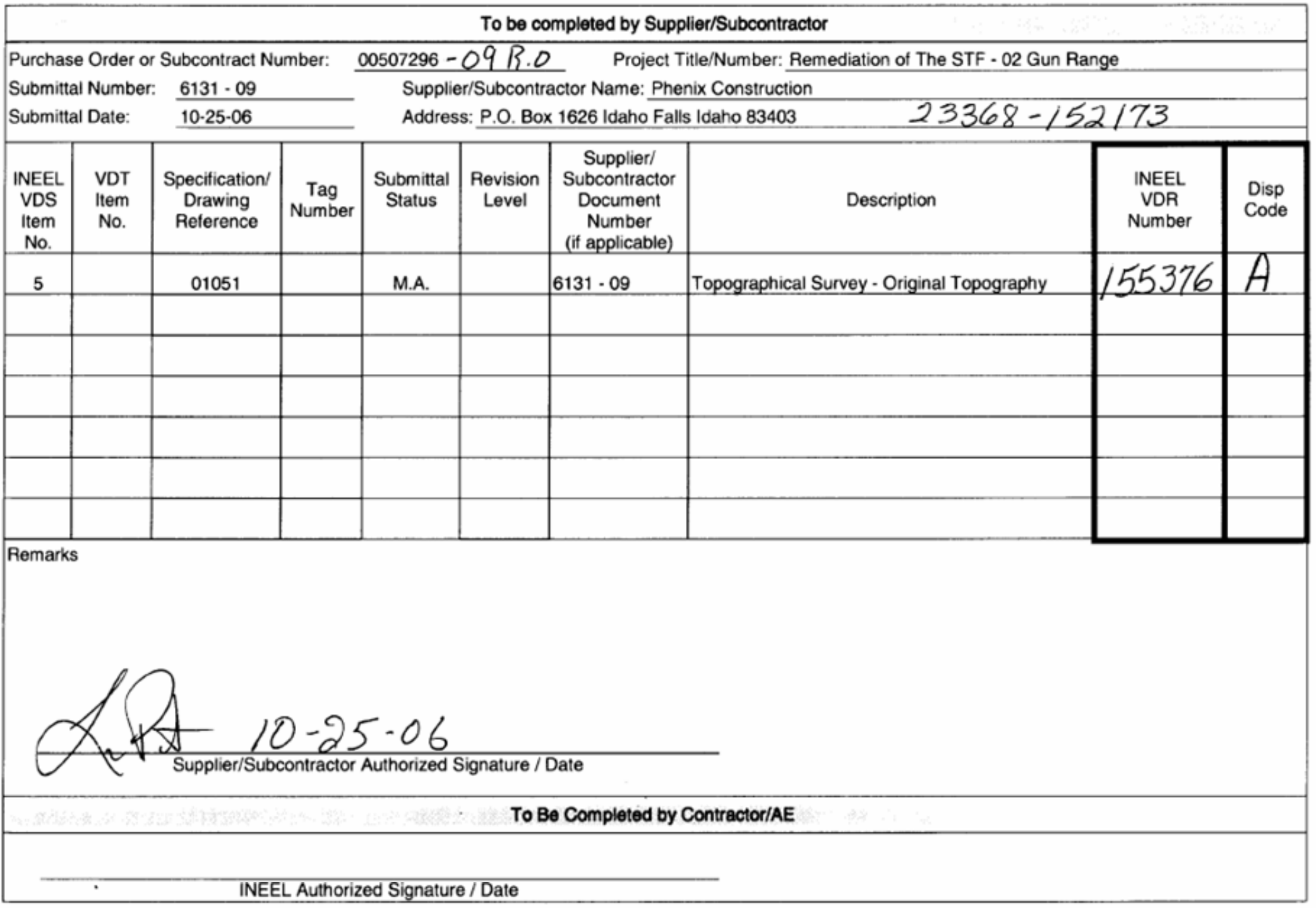




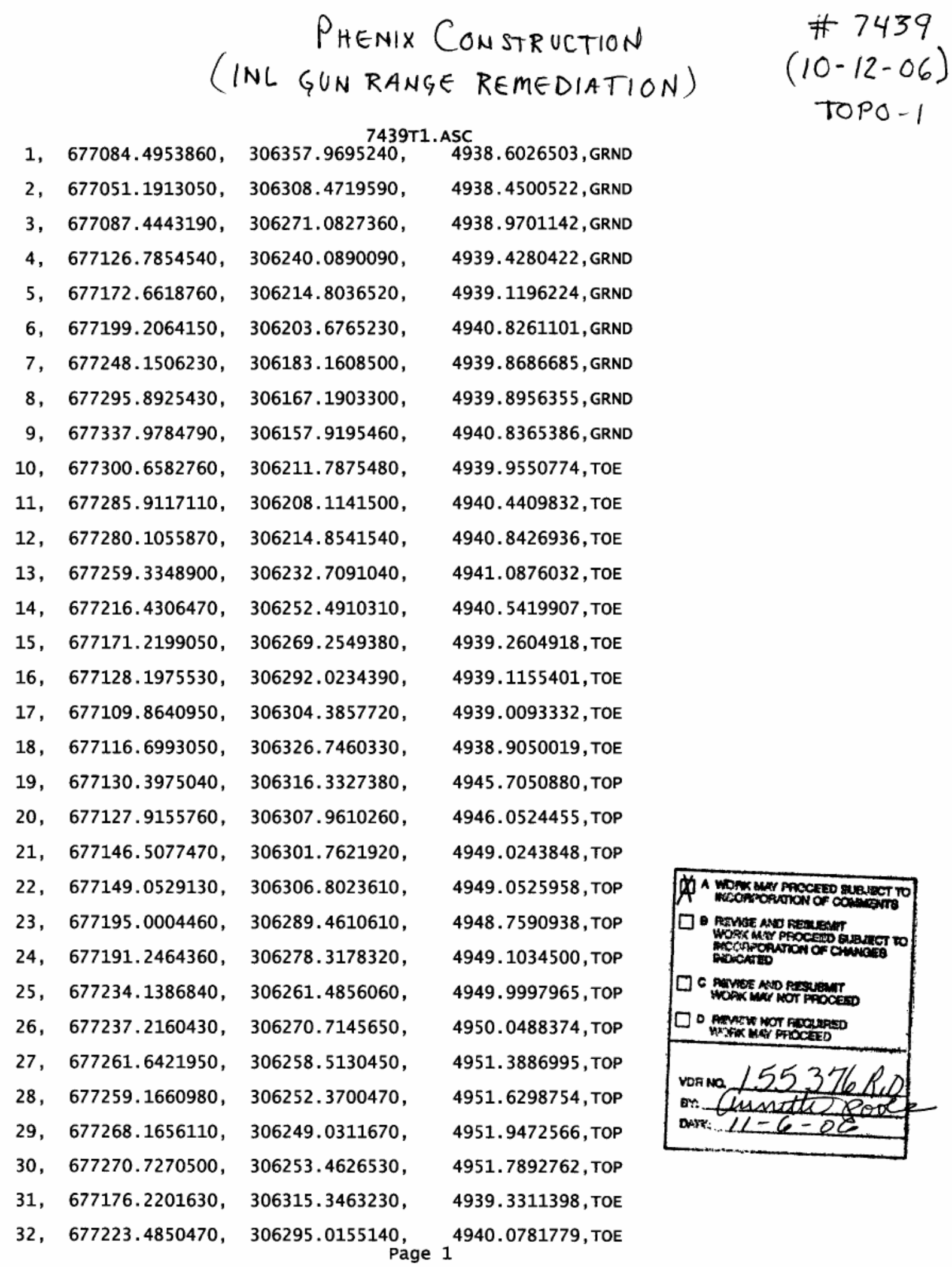


7439T1. ASC

$33,677266.5025370, \quad 306276.1506130, \quad 4940.4998751$, TOE $34,677274.8977990, \quad 306273.3582830, \quad 4941.0033936$, TOE $35,677167.5769680, \quad 306478.4473730, \quad 4939.2655925$, TOE $36,677168.2437820, \quad 306482.7739580, \quad 4940.0472659$,TOP $37,677168.9340240, \quad 306487.1468290, \quad 4939.9163311$, TOP $38,677187.1364110, \quad 306489.2605190, \quad 4941.2321572$, TOP $39,677192.6520880, \quad 306504.8328370, \quad 4941.6992159$,TOP $40,677189.2551440, \quad 306517.1732700, \quad 4939.5744244$,TOP $41,677195.9605990, \quad 306523.9857770, \quad 4939.9986113$,TOP $42,677200.4434680, \quad 306531.2880560, \quad 4939.1558916$, ТОР $43,677193.3495170, \quad 306577.8189170, \quad 4938.6104531$,TOE $44,677188.1434000, \quad 306578.2068250, \quad 4939.5002906$, TOE $45, \quad 677184.3463090, \quad 306576.6917340, \quad 4939.4611317$,TOP $46,677167.5449510,306598.2916000, \quad 4938.5929536$,TOP $47,677168.2780510,306603.7666710, \quad 4938.6464848$, TOP $48,677167.6701040, \quad 306610.0469240, \quad 4937.0956983$, TOE 49, 677127.6401030, 306615.6397610, 4935.7828167, TOE $50,677124.9898890,306609.7150120$, 4937.3830159, TOP $51,677121.6666380,306604.6593060$, 4937.5351267, TOP

$52,677090.6138200,306603.4350570$, 4937.8104427, TOP $53,677086.6578330,306609.8359830$, 4937.6703531, TOP 54, 677082.1748340, 306614.8310590, 4936. 3009123, TOE $55,677079.7334620,306580.1520050$, 4936.5433573, TOE $56,677083.9087880,306577.0969790$, 4937.3327197, TOP $57,677082.4149750,306562.8454680$, $58,677081.0914640,306515.3996510$, 4936.7649786, TOP 59, 677082.1283670, 306497.9095430, 4936.8868309, TOP $60,677076.8830220,306497.0557590$, 4938.6810507, TOP $61,677083.3622780,306477.7044140$, 62 , 677086.3946360, 306484.1219580, $63,677093.7862630,306489.5939750$, 4937.2166475, TOE 4937.9702324, TOE 4939.2470252, TOP 4938.4728596, TOP Page 2 


\begin{tabular}{|c|c|c|c|}
\hline 64, & 677120.9624800 & $\begin{array}{r}74391 \\
306482.8388690\end{array}$ & ISC \\
\hline 65, & 677125.4827350 & 306479.6248970, & 4939.6414140, TOP \\
\hline 66 , & 677124.7981700 & 306474.6058300 & 4938.3748525, TOE \\
\hline 67 , & 677154.5464720 & 306477.0358650 & 4938.7990809, TOE \\
\hline 68, & 677157.7668410 & 306482.1650410 & 4939.8876740, TOP \\
\hline 69 , & 677159.7230310, & 306486.5119660 & 4939.8595641, TOP \\
\hline 70, & 677170.4039150 & 306431.8616640 & 4939.6802088, GRND \\
\hline 71, & 677118.8114700 & 306444.0961500 & 4938.7303555, GRND \\
\hline 72 , & 677070.0184600 & 306467.4632340 & 4937.9576898, GRND \\
\hline 73 , & 677041.9522350, & 306506.5895210 & 4936. 9747211, GRND \\
\hline 74, & 677041.3930150, & 306559.9779130, & 4936. 3928716, GRND \\
\hline 75, & 677045.5898140 & 306608.7052350 & 4936.4952204, GRND \\
\hline 76, & 677055.3661170 & 306639.7593420, & 4936.5739527, GRND \\
\hline 77 , & 677108.6007090, & 306635.2513360 & 4936.0079425, GRND \\
\hline 78, & 677158.3593690 & 306631.3272290 & 4936.7434425, GRND \\
\hline 79, & 677211.4158060 & 306562.1088460 & 4938.9667203, GRND \\
\hline 80, & 677224.8059090 & 306534.3345620 & 4939.4961067, GRND \\
\hline 81, & 677172.1741870 & 306501.3335490 & 4932.4787075, TOE \\
\hline 82 , & 677175.5037070 & 306524.9408180 & 4932.0196105, TOE \\
\hline 83 & 677175.1550240 & 306544.2339380 & 4932.4241491, TOE \\
\hline 84, & 677176.5965670 , & 306564.6424670 & 4932.6879799, TOE \\
\hline 85 & 677168.9863390, & 306569.8321010 & 4931.3828001, TOE \\
\hline 86 , & 677164.7135420 & 306575.0015050 & 4930.8597559, TOE \\
\hline 87 & 677166.9363110 & 306580.9162220 & 4931.6495449, TOE \\
\hline 88, & 677155.0496270 & 306590.8978900 & 4931.4012330, TOE \\
\hline 89, & 677135.8295960 & 306589.9454810 & 4930.3966295, TOE \\
\hline 90, & 677116.2241310 & 306590.7332120 & 4930.1966258, TOE \\
\hline 91, & 677102.8105610 & 306587.7116960 & 4929.8232911, TOE \\
\hline 92 , & 677096.8113990, & 306580.9526520 & 4929.8331566, TOE \\
\hline 93 & 677097.3596100, & 306570.5021460 & 4929.2223630, TOE \\
\hline 94, & 677096.2874380, & 306550.5518800 & 4929.5032709, TOE \\
\hline 95 , & 677096.6088660 & $\begin{array}{r}306536.4835950 \\
\text { Pag }\end{array}$ & 4929.5207609, TOE \\
\hline
\end{tabular}


7439T1.ASC

$96, \quad 677097.3770710, \quad 306529.3437250, \quad 4928.7461540$,TOE $97,677097.1930160, \quad 306510.9581700, \quad 4929.5262989$, TOE $98,677107.4662870, \quad 306501.5297800, \quad 4929.0408688$, TOE $99,677116.9983420, \quad 306501.1075630, \quad 4928.8869737$,TOE $100,677136.4312890, \quad 306501.3548470, \quad 4930.3359966$, TOE $101, \quad 677147.1318320, \quad 306501.0600820, \quad 4931.2979905$, TOE $102,677167.0003200, \quad 306499.5642790, \quad 4931.8457362$, TOE $103,677163.4041940, \quad 306512.0498190, \quad 4931.0092089$, GRND $104,677142.0701860, \quad 306512.8132780, \quad 4931.2380188$, GRND $105,677123.0119990, \quad 306513.7238700, \quad 4928.2030029$, GRND $106,677107.1097980, \quad 306535.4181220, \quad 4928.6281868$, GRND $107,677122.4835280, \quad 306536.7752690, \quad 4930.1762362$, GRND $108,677136.3780510, \quad 306540.0981090, \quad 4928.7811085$, GRND $109,677157.2380480, \quad 306545.9053890, \quad 4929.4870635$, GRND $110,677158.8528610, \quad 306566.4505640, \quad 4930.5223006$, GRND $111,677139.9275130, \quad 306564.6773830, \quad 4930.0130447$, GRND $112, \quad 677129.1265330, \quad 306564.4129990, \quad 4929.3292990$, GRND $113,677119.8866770, \quad 306566.9964900, \quad 4930.3235408$, GRND $114,677112.3145290, \quad 306570.6890720, \quad 4928.8778854$, GRND $115,677117.9748810, \quad 306582.1900110, \quad 4929.2569745$, GRND $116,677139.1497410, \quad 306583.0373800, \quad 4929.8304249$, GRND $117,677159.3800280,306578.6401350, \quad 4930.6273360$, GRND $118,677026.8653260, \quad 306464.6461780, \quad 4937.5195234$,GRND $119,677022.9515740, \quad 306414.2174880, \quad 4937.8646752$, GRND $120,677021.9181290, \quad 306362.4221500, \quad 4937.7546087$, GRND $121,677020.2202880, \quad 306313.2058820, \quad 4938.0858670$, GRND $122,677077.5047910, \quad 306360.4665840, \quad 4938.3901014$, GRND 123, 677094.3763520, 306411.3186260, 4938.8996964, GRND $124,677146.9114450, \quad 306398.3544310, \quad 4939.2431658$, GRND $125,677131.4880420, \quad 306356.1491820, \quad 4939.1169980$, GRND $126,677179.9032670, \quad 306338.7985780, \quad 4939.4193883$, GRND Page 4 


\begin{tabular}{|c|c|c|c|}
\hline 27 & 677199.1923930, & $\begin{array}{r}7439 \\
306379.3469170,\end{array}$ & 4939.7907135, GR \\
\hline 28 , & 677247.0167460 & 306359.5325310, & 4940.0057067, GR \\
\hline 9 & 677232.3044920 & 306316.8134820 & 4939.8823490, GRI \\
\hline 30, & 677273.0785420 & 306309.5742500 & $4940.2356166, \mathrm{GRI}$ \\
\hline 31, & 677286.6085510 & 306341.3909260 & 4940.3022564, GR \\
\hline 32, & 677280.9257360 & 306281.4760840 & 4941.1621790, TOE \\
\hline 33, & 677296.4300790, & 306316.3340240 & 4940.7863278 , TOE \\
\hline 34, & 677310.9557430, & 306352.6064910 & 4940.5831365 , TOE \\
\hline 35 & 677323.4591570 & 306379.0997320 & 4940.9590094, TOE \\
\hline 136, & 677334.8085400 & 306403.4115930 , & 4940.4643567, TOE \\
\hline 37, & 677340.0679730 & 306413.5328720 & 4941.0197934, TOE \\
\hline 38, & 677336.2450900, & 306421.9799840 & 4940.8000660, TOE \\
\hline 39, & 677311.1023250, & 306432.3304200 & 4940.5959236, TOE \\
\hline 140, & 677293.6933670, & 306440.2801660 & 4940.6082241 , TOE \\
\hline 141, & 677278.9963880 & 306447.6337020 & 4940.9853971, TOE \\
\hline 42 , & 677271.0990610, & 306427.1050760 & 4940.5903760, TOE \\
\hline 143, & 677268.4666100 & 306417.7014050 & .6231744, TOE \\
\hline 144 & 677319.3505960, & 306389.5657680 & 4940.5550153, TOE \\
\hline 145, & 677318.4143150 & 306377.6289030 & 4941.0040861, TOE \\
\hline 46 , & 677309.6708790, & 306361.3604660 & 4940.8502950, TOE \\
\hline 147 & 677298.1512630, & 306355.7727670 & 4940.2811020, TOE \\
\hline 148 & 677260.9420240 & 306369.9703640, & 4940.3564740, TOE \\
\hline 149, & 677221.4698700 & 306390.2459950 & 4940.0588973, TOE \\
\hline 150 & 677214.8080020, & 306407.6833220 & 4940.2886023, TOE \\
\hline 151, & 677226.8889380, & 306437.9492620 & 4940.1950365, TOE \\
\hline 152, & 677237.4951970 & 306465.9145360 & 4940.1062775, TOE \\
\hline 153 & 677212.5313640 & 306476.3479340 , & 4940.5696198 , TOE \\
\hline 154, & 677195.4583090 & 306477.1162470 & 4940.7373561, TOE \\
\hline 155 & 677199.2673730, & 306497.5810250 & 4941.6866034, TOE \\
\hline 156, & 677205.8264520 & 306510.2716140 & 4940.5676533, TOE \\
\hline 157 & 677215.0661820 & 306512.8893100, & 4939.9158827, TOE \\
\hline & 677248.1646510, & 306502.8563420 & 4940.41 \\
\hline
\end{tabular}


7439T1. ASC

159, 677289.1840440, 306487.5778830, 4940.9864158, TOE $160,677325.2675350, \quad 306475.7772560, \quad 4939.8548295$, TOE $161,677351.3846070, \quad 306463.3143420, \quad 4940.7333189$, TOE $162,677376.6461380, \quad 306457.6580470, \quad 4941.3940546$, TOE 163, 677396.4904370, 306456.3823410, 4942.1304753, TOE $164,677401.5561830, \quad 306451.2012720, \quad 4940.6913376$, TOE $165,677399.8911600, \quad 306438.8974090, \quad 4940.5477485$, TOE $166,677310.0065350, \quad 306223.5692160, \quad 4940.0569790$, TOE $167,677331.0966030,306272.3013240, \quad 4940.0569560$, TOE $168,677350.7686630, \quad 306316.5799960, \quad 4940.2274121$, TOE $169,677370.0937800,306360.0497470$, $170,677388.2028720,306401.8938870$, $171,677397.4872250,306422.6247560$, $172,677454.6936710,306405.9445660$, $173,677433.9358680,306351.2943570$, $174,677413.1805830,306301.7915310$, $175,677391.3310310,306250.6638300$, $176,677360.9418140,306204.1073800$, $177,677281.0659040,306249.4655960$, $178,677279.8603390,306244.2793320$, 179, 677292.8591910, 306241.2698070, $180,677282.5169530,306243.1120620$, $181,677293.5771980,306252.5828270$, $182,677298.6197280,306250.8536440$, $183,677311.0419090,306286.7648910$, 184, 677316.1322320, 306286.4521690, $185,677330.7202060,306319.3906970$, $186,677325.7239770,306321.6331940$, $187,677343.5775090,306363.3935940$, $188,677348.6553870,306361.9959740$, $189,677362.0711710,306394.0694480$, 4940.0647028 , TOE 4939. 4884481, TOE 4939. 4153319, TOE 4939.1565819, GRND 4938.6432963, GRND 4939. 2883081, GRND 4939.6382240, GRND 4939.9606507, GRND 4952.1107992, TOP 4952. 2576958, TOP 4952.4173525 , TOP 4952. 4009094, TOP 4952.6112121, TOP 4952.8387655, TOP 4952.9414405, TOP 4952.8169182, TOP 4953.4427888, TOP 4953.0233496, TOP 4953. 2767772 , TOP 4953.4018525, TOP 4953.4574901, TOP Page 6 


\begin{tabular}{|c|c|c|c|}
\hline 90 & 677358.2316590, & $\begin{array}{r}7439 \\
306396.6696940\end{array}$ & $\begin{array}{l}\text { ASC } \\
4953.3076209, \text { TOP }\end{array}$ \\
\hline 191, & 677364.5505050 & 306416.3762900 , & 4953.6685163 , TOP \\
\hline 192 & 677369.8378200 & 306417.2130070 & 4953.5617364, TOP \\
\hline 193, & 677374.0374810 & 306432.3564400 & 4953.9363196, TOP \\
\hline 194, & 677362.5308730 & 306425.9637930 & 4953.7588234, TOP \\
\hline 195, & 677354.9805770 & 306434.7901050 & 4953.4163775, TOP \\
\hline 196, & 677357.0942980 & 306440.6326360 & 4953.1624269, TOP \\
\hline 197, & 677318.3653260 & 306450.3373690 & 4951.2654286, TOP \\
\hline 198, & 677321.3271320 & 306457.5598030 , & 4951.2282080 , TOP \\
\hline 199, & 677291.7916760, & 306460.5912730 , & 4950.5851746 , TOP \\
\hline 200 & 677293.8053180, & 306468.9874620 & 4950.5477542 , TOP \\
\hline 201, & 677272.7789120 & 306468.2759860 & 4950.0941296 , TOP \\
\hline 202 , & 677272.9027930 & 306478.1781100 & 4950.0954471 , TOP \\
\hline 203 & 677257.2368500 & 306475.4678010 & 4949.7028583 , TOР \\
\hline 204 & 677258.0585160 & 306483.8463340 & 4949.7198565 , TOP \\
\hline 205, & 677220.0411090 & 306495.3248000 & 4949.0748010, TOР \\
\hline 206, & 677219.9632340, & 306489.1499410, & 4948.9059155 , TOP \\
\hline 207 & 677260.3147450 & 306465.7587600 & 4953.2819831 ,TOP \\
\hline 208 & 677263.9621110 & 306464.3860640 & 4953.3 \\
\hline 209, & 677246.9112340 & 306431.6840540 & 4952.8359799, TOP \\
\hline 210 & 677249.9582230, & 306430.8486280 & 4952.8156777, TOP \\
\hline 211 & 677243.6305430 & 306413.3600290 & 4952.7673200, TOP \\
\hline 212 & 677240.0567320 & 306414.6072350 & 4952.8233331 , TOP \\
\hline 13 & 677237.2220840 & 306406.9676460 & 4952.6301127 , ТОР \\
\hline 214 & 677243.5983780 & 306408.6879900 & 4952.3636004, TOP \\
\hline 215 & 677247.5920230 & 306404.8297980 & 4952.3362364, TOP \\
\hline 16 & 677246.5508430 & 306401.8134540 & 4952.5900784, TOP \\
\hline 217, & 677268.6576270 & 306391.8051340 & 4952.6669954, ТОР \\
\hline 218 & 677271.7458270, & 306392.8369440 & 4952.8056711 , TOP \\
\hline 219, & 677298.8102970 & 306380.7850310 & 4952.5072904, TOP \\
\hline 20 & 677297.7516910, & 306378.8942410 & 4952.5617583, TOP \\
\hline & 677255.8935200 & & 4940.506678 \\
\hline
\end{tabular}




\section{T1. ASC}

$222, \quad 677297.7262740, \quad 306568.9985730, \quad 4939.2164554$, GRND $223,677339.3457320, \quad 306544.4924060, \quad 4939.6646266$, GRND $224,677324.0752460, \quad 306515.9998000, \quad 4939.4670792$, GRND $225,677274.8852200, \quad 306529.5818000, \quad 4939.6422106$, GRND $226,677230.5538290, \quad 306533.5314840, \quad 4939.8831241$, GRND $1000,677106.0800000,306220.1600000,4939.4500000$, $1001,676836.9000000,306489.7000000,4934.7000000$, $1002,677259.9800000,306459.7200000,4953.3000000$, 


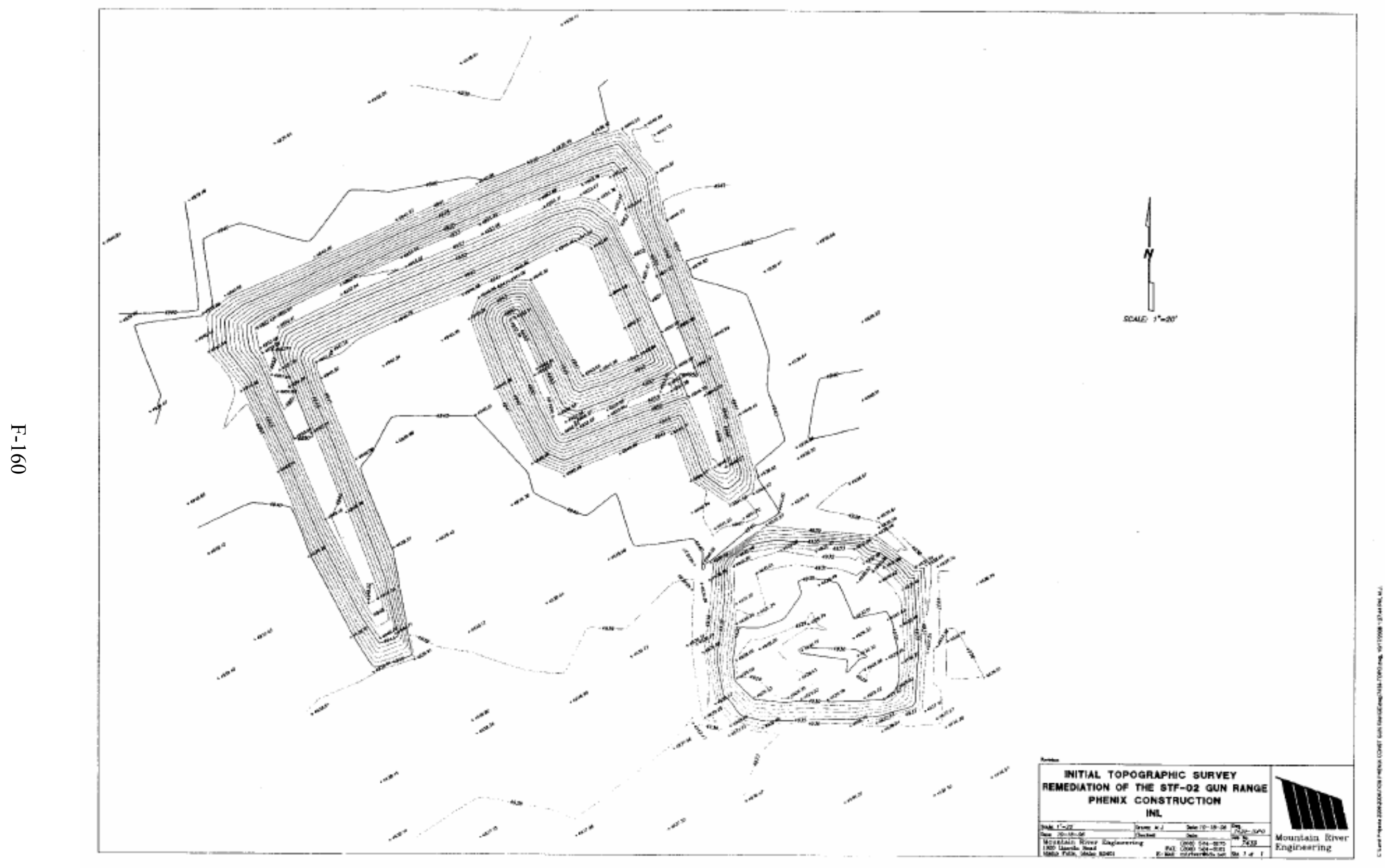




\section{Vendor Data Review System Final Disposition Screen}

This vendor data item has been given the following disposition codes

\begin{tabular}{|c|c|c|c|c|}
\hline Reviewer & $\begin{array}{l}\text { Revision } \\
\text { Level }\end{array}$ & Date & $\begin{array}{l}\text { Disposition } \\
\text { Code }\end{array}$ & Comments \\
\hline TUOTT LEE C & 0 & ${ }_{\text {OCT-06 }}^{31-}$ & A & No comment. \\
\hline $\begin{array}{l}\text { MCMANAMON } \\
\text { LAWRENCE E }\end{array}$ & 0 & $\begin{array}{l}06- \\
\text { NOV- } \\
06\end{array}$ & $\mathrm{D}$ & \\
\hline DRIEVER MIKEL K & 0 & $\begin{array}{l}30- \\
\text { ОСТ-06 }\end{array}$ & & $\begin{array}{l}\text { Is there supposed to be an Initial or } \\
\text { Resubmital CHemical Inventory List with } \\
\text { this? }\end{array}$ \\
\hline LANDIS JOSEPH A & 0 & $\begin{array}{l}30- \\
\text { OCT-06 }\end{array}$ & & no comment \\
\hline FRITZ KURT D & 0 & $\begin{array}{l}02- \\
\text { NOV- } \\
06\end{array}$ & D & $\begin{array}{l}\text { Can I have the electronic data sent to } \\
\text { me? }\end{array}$ \\
\hline $\begin{array}{l}\text { JOLLEY WENDELL } \\
\text { L }\end{array}$ & 0 & $\begin{array}{l}31- \\
\text { OCT-06 }\end{array}$ & & \\
\hline VANDEL DOUG S & 0 & $\begin{array}{l}\text { 31- } \\
\text { OCT-06 }\end{array}$ & A & \\
\hline
\end{tabular}

VDR Number:

VDR-155376

Revision Level:

0

Project Number:

$23368-152173$

Transmittal Number:

S-507296-09

Transmittal Status:

Line Item:

Mandatory Approval

1

Disposition Code::

A

Final Comments: 
F-162 


\section{VDS \#6_155906}


F-164 
431.13

$07 / 23 / 2002$

Rev. 04
1.

To be completed by Supplier/Subcontractor
VENDOR DATA

TRANSMITTAL \& DISPOSITION FORM

Page 2 of 3

ज)

Purchase Order or Subcontract Number: $00507296-0 / 0$ R. O Project Title/ Number: Remediation of The STF . 02 Gun Range

Submittal Number: $6131-10 \quad$ Supplier/Subcontractor Name: Phenix Construction

Submittal Date: $\quad \underline{11-07-06} \quad$ Address: P.O. Box 1626 Idaho Falls Idaho $83403 \quad 23368-152173$

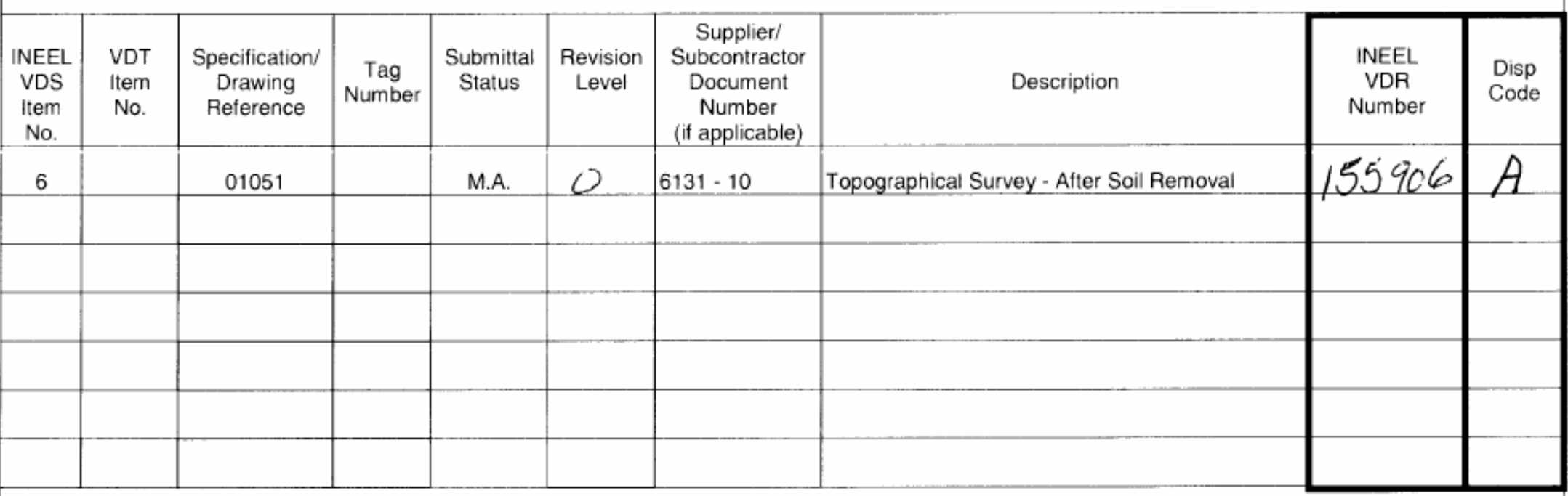

Remarks

Berm Area only.

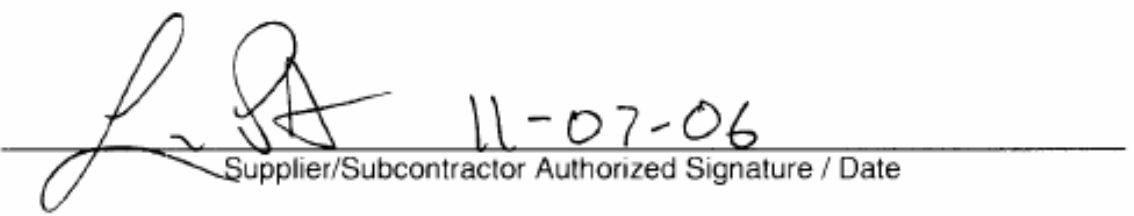

To Be Completed by Contractor/AE

INEEL Authorized Signature / Date 


\begin{tabular}{|c|c|c|}
\hline 229 & 677307.9017 & 306352.2785 \\
\hline 230 & 677346.358 & 306350.9647 \\
\hline 231 & 677366.0459 & 306398.4236 \\
\hline 232 & 677373.7306 & 306419.1645 \\
\hline 233 & 677370.6138 & 306436.1409 \\
\hline 234 & 677354.1811 & 306445.3552 \\
\hline 235 & 677331.6412 & 306455.5945 \\
\hline 236 & 677326.8817 & 306448.6954 \\
\hline 237 & 677309.6811 & 306433.4285 \\
\hline 238 & 677307.2315 & 306428.3342 \\
\hline 239 & 677333.165 & 306409.726 \\
\hline 240 & 677320.5757 & 306393.0222 \\
\hline 241 & 677302.4563 & 306359.7621 \\
\hline 242 & 677283.6206 & 306365.7641 \\
\hline 243 & 677280.8224 & 306357.9465 \\
\hline 244 & 677305.1116 & 306347.263 \\
\hline 245 & 677297.0501 & 306321.3969 \\
\hline 246 & 677276.2213 & 306275.3991 \\
\hline 247 & 677266.9971 & 306270.0733 \\
\hline 248 & 677266.1954 & 306266.395 \\
\hline 249 & 677269.4711 & 306251.9884 \\
\hline 250 & 677268.3642 & 306245.9043 \\
\hline 251 & 677280. & 306243.4828 \\
\hline 252 & 677291.8377 & 306241.6467 \\
\hline 253 & 677305.3493 & 306258.043 \\
\hline 254 & 677326.4495 & 303.6829 \\
\hline 2000 & 677266.7077 & 306266.3973 \\
\hline 2001 & 677267.4386 & 306269.7509 \\
\hline 2002 & 677276.6105 & 306275.0465 \\
\hline 2003 & 677297.5182 & 306321.2187 \\
\hline 2004 & 677305.7218 & 306347.5408 \\
\hline 2005 & 677281.4502 & 306358.2166 \\
\hline 2006 & 677283.9287 & 306365.1412 \\
\hline 2007 & 677302.6979 & 306359.1603 \\
\hline 2008 & 677320.9972 & 306392.7506 \\
\hline 2009 & 677333.8724 & 306409.8338 \\
\hline 2010 & 677307.8639 & 306428.4958 \\
\hline 2011 & 677310.0881 & 306433.1213 \\
\hline 2012 & 677312.8042 & 306430.9414 \\
\hline 2013 & 677316.4348 & 306434.5857 \\
\hline 2014 & 677339.9192 & 306412.7207 \\
\hline 2015 & 677335.8219 & 306410.3169 \\
\hline 2016 & 677330.3642 & 306391.723 \\
\hline 2017 & 677320.9284 & 306390.1843 \\
\hline 2018 & 677307.8707 & 306362.4918 \\
\hline 2019 & 677314.7474 & 306357.7336 \\
\hline 2020 & 677313.2729 & 306350.0733 \\
\hline 2021 & 677284.0469 & 306362.0726 \\
\hline 2022 & 677304.0195 & 306337.465 \\
\hline 2023 & 677292.2276 & 306303.9072 \\
\hline 2024 & 677295.3004 & 306300.0705 \\
\hline 2025 & 677283.7073 & 306273.3064 \\
\hline
\end{tabular}

4938.62 TOE 4951.9517 TOE 4951.9796 TOE 4951.7797 TOE 4952.7737 TOE 4950.9763 TOE 4950.0171 TOE 4951.6713 TOE 4940.8217 TOE 4940.5963 TOE 4940.5464 TOE 4940.5475 TOE 4941.4968 TOE 4942.688 TOE 4940.3157 TOE 4940.5489 TOE 4940.7336 TOE 4941.0331 TOE 4943.9629 TOE 4946.2056 TOE 4951.8344 TOE 4950.3269 TOE 4951.9998 TOE 4952.419 TOE 4950.975 TOE 4951.484 TOE 4941.0927 TOP 4940.7248 TOP 4940.7599 TOP 4939.9562 TOP 4939.7617 TOP 4940.0254 TOP 4939.8238 TOP 4940.3246 TOP 4939.338 TOP 4939.7631 TOP 4939.5949 TOP 4939.6388 TOP 4938.3051 TOE 4938.5605 TOE 4939.3097 TOE 4939.3793 TOE 4938.4098 TOE 4938.6012 TOE 4939.2791 TOE 4938.4355 TOE 4938.5345 TOE 4939.0005 TOE 4938.8177 TOE 4938.9357 TOE 4939.1449 TOE 4939.2533 TOE

\author{
Phenix Const. \\ EXCAVATION \\ FROM THE \\ STF-O2 GUN RANGE
}

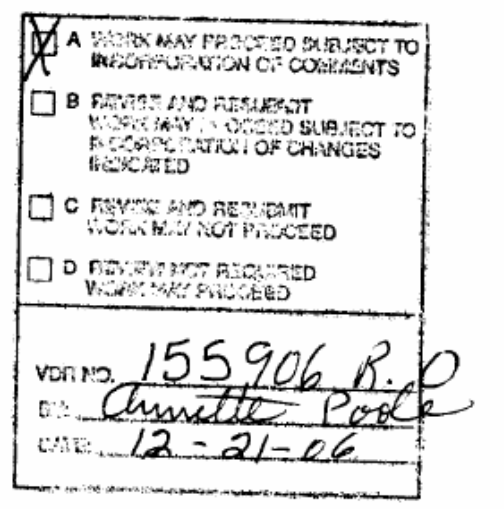




$\begin{array}{rrrr}2026 & 677279.1367 & 306274.6334 & 4939.1658 \text { TOE } \\ 2027 & 677272.8023 & 306269.6203 & 4939.6839 \text { TOE } \\ 2028 & 677268.9436 & 306246.2952 & 4950.2688 \text { TOP } \\ 2029 & 677269.9813 & 306251.9995 & 4949.6045 \text { TOP } \\ 2030 & 677277.2546 & 306248.8621 & 4950.4285 \text { TOP } \\ 2031 & 677280.183 & 306243.9749 & 4950.4802 \text { TOP } \\ 2032 & 677291.6332 & 306242.1848 & 4951.2649 \text { TOP } \\ 2033 & 677286.804 & 306251.3297 & 4950.2801 \text { TOP } \\ 2034 & 677299.306 & 306259.4324 & 4951.121 \text { TOP } \\ 2035 & 677304.9225 & 306258.3112 & 4951.6534 \text { TOP } \\ 2036 & 677325.992 & 306303.8849 & 4951.922 \text { TOP } \\ 2037 & 677320.6314 & 306307.455 & 4951.3647 \text { TOP } \\ 2038 & 677339.3097 & 306354.6798 & 4951.4093 \text { TOP } \\ 2039 & 677345.9229 & 306351.2196 & 4951.6308 \text { TOP } \\ 2040 & 677365.5803 & 306398.6063 & 4951.7422 \text { TOP } \\ 2041 & 677361.0691 & 306404.672 & 4951.4643 \text { TOP } \\ 2042 & 677363.1821 & 306416.4573 & 4951.9763 \text { TOP } \\ 2043 & 677373.214 & 306419.2094 & 4951.9753 \text { TOP } \\ 2044 & 677370.1645 & 306435.8197 & 4952.969 \text { TOP } \\ 2045 & 677359.0908 & 306427.1285 & 4951.6422 \text { TOP } \\ 2046 & 677351.9959 & 306434.823 & 4950.6175 \text { TOP } \\ 2047 & 677353.955 & 306444.9088 & 4951.0006 \text { TOP } \\ 2048 & 677331.8152 & 306454.9663 & 4950.1995 \text { TOP } \\ 2049 & 677327.2589 & 306448.3616 & 4949.6667 \text { TOP }\end{array}$




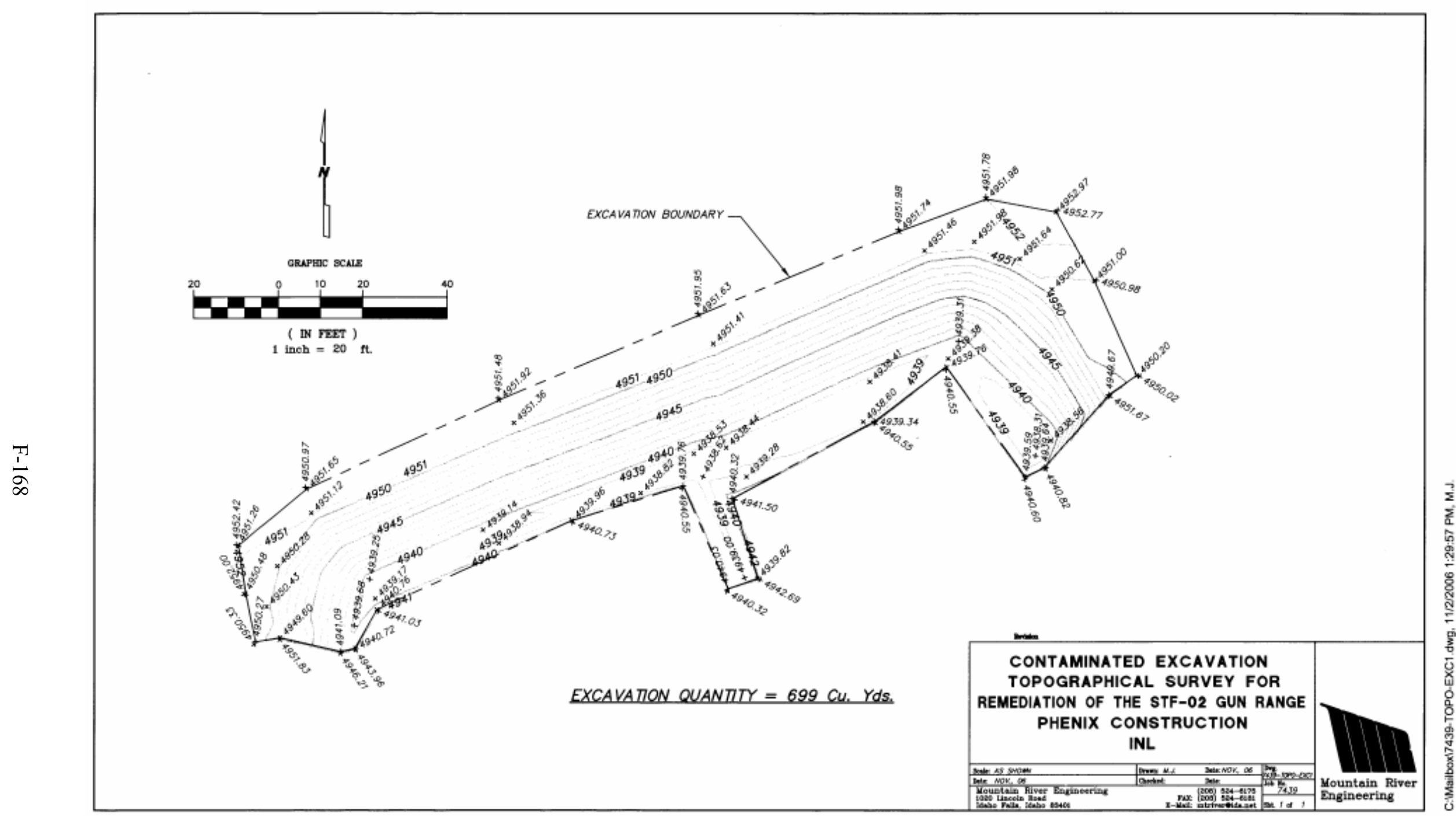




\section{Vendor Data Review System Final Disposition Screen}

This vendor data item has been given the following disposition codes

\begin{tabular}{|c|c|c|c|c|}
\hline Reviewer & $\begin{array}{l}\text { Revision } \\
\text { Level }\end{array}$ & Date & $\begin{array}{l}\text { Disposition } \\
\text { Code }\end{array}$ & Comments \\
\hline TUOTT LEE C & 0 & $\begin{array}{l}08- \\
\text { NOV- } \\
06\end{array}$ & B & $\begin{array}{l}\text { A survey is also needed of the areas where the } \\
\text { contaminated soils were stored. This should occur } \\
\text { after the contaminated soils and the } 6 " \text { of soil } \\
\text { beneath the piles, have been removed. This } \\
\text { survey will be used to document the performance } \\
\text { of the removal of the additional } 6 \text { " of soil beneath } \\
\text { the remediation soil piles. }\end{array}$ \\
\hline $\begin{array}{l}\text { MCMANAMON } \\
\text { LAWRENCE E }\end{array}$ & 0 & $\begin{array}{l}11- \\
\text { NOV- } \\
06\end{array}$ & $D$ & \\
\hline $\begin{array}{l}\text { VANDEL DOUG } \\
\mathrm{S}\end{array}$ & 0 & $\begin{array}{l}\text { 08- } \\
\text { NOV- } \\
06\end{array}$ & A & \\
\hline $\begin{array}{l}\text { DRIEVER } \\
\text { MIKEL K }\end{array}$ & 0 & $\begin{array}{l}\text { 09- } \\
\text { NOV- } \\
06\end{array}$ & B & $\begin{array}{l}\text { Should there be a Chemical Inventory List } \\
\text { associated with this submital? Form } 432.21\end{array}$ \\
\hline FRITZ KURT D & 0 & $\begin{array}{l}28- \\
\text { NOV- } \\
06\end{array}$ & B & Please submit electronic data to me. \\
\hline $\begin{array}{l}\text { LANDIS } \\
\text { JOSEPH A }\end{array}$ & 0 & $\begin{array}{l}27- \\
\text { NOV- } \\
06\end{array}$ & A & no comment \\
\hline
\end{tabular}

The following reviewers have NOT yet reviewed this vendor data item

JOLLEY WENDELL L

$\begin{array}{ll}\text { VDR Number: } & \text { VDR-155906 } \\ \text { Revision Level: } & 0 \\ \text { Project Number: } & 23368-152173 \\ \text { Transmittal Number: } & \text { S-507296-010 } \\ \text { Transmittal Status: } & \text { Mandatory Approval } \\ \text { Line Item: } & 1 \\ \text { Code:: } & \text { Final Comments:: }\end{array}$

Disposition Code::

A 
F-170 


\section{VDS \#7_158614}


F-172 
VENDOR DATA

To be completed by Supplier/Subcontractor

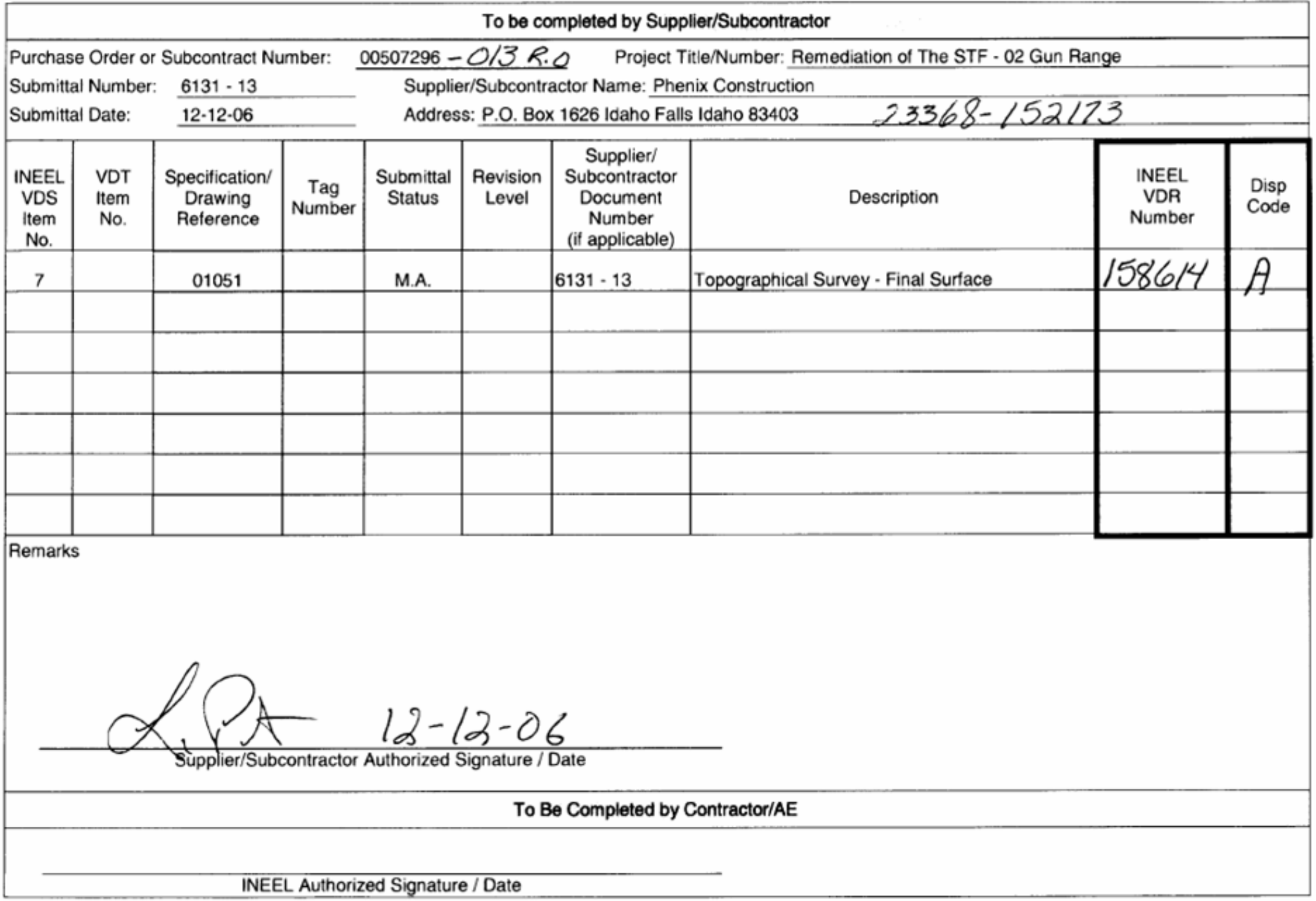


7439-ASBLT.ASC

Final AsBuILT TOPOg.

\begin{tabular}{|c|c|}
\hline 1 & 5000 \\
\hline 1005 & 676990.7353 \\
\hline 1007 & 677106.08 \\
\hline 1008 & 676836.9 \\
\hline 3000 & 677033.9316 \\
\hline 3001 & 677022.3284 \\
\hline 3002 & 677024.0269 \\
\hline 3003 & 677025.7535 \\
\hline 3004 & 677027.6431 \\
\hline 3005 & 677027.3319 \\
\hline 3006 & 677026.2525 \\
\hline 3007 & 677082.7787 \\
\hline 3008 & 677082.2436 \\
\hline 3009 & 677085.3319 \\
\hline 3010 & 677086.1354 \\
\hline 3011 & 677084.5605 \\
\hline 3012 & 677085.1141 \\
\hline 3013 & 677060.6832 \\
\hline 3014 & 677112.7785 \\
\hline 3015 & 677127.9273 \\
\hline 3016 & 677133.078 \\
\hline 3017 & 677140.134 \\
\hline 3018 & 677146.5648 \\
\hline 3019 & 677157.3825 \\
\hline 3020 & 677172.70 \\
\hline 3021 & 677205.0015 \\
\hline 3022 & 677199.1338 \\
\hline 3023 & 677190.3699 \\
\hline 3024 & 677183.5535 \\
\hline 3025 & 677173.080 \\
\hline 3026 & 677158.601 \\
\hline 3027 & 677141.044 \\
\hline 3028 & 677189.439 \\
\hline 3029 & 677213.817 \\
\hline 3030 & 677238.112 \\
\hline 3031 & 677256.152 \\
\hline 3032 & 677276.363 \\
\hline 3033 & 677291.252 \\
\hline 3034 & 677302.434 \\
\hline 3035 & 677346.712 \\
\hline 3036 & 677337.772 \\
\hline 3037 & 677326.77 \\
\hline 3038 & 677305.439 \\
\hline 3039 & 677281.989 \\
\hline 3040 & 677261.880 \\
\hline 3041 & 677241.658 \\
\hline 3042 & 677224.457 \\
\hline 3043 & 677266.133 \\
\hline 3044 & 677280.755 \\
\hline 3045 & 677307.437 \\
\hline 3046 & 677329.762 \\
\hline 3047 & 677358.22 \\
\hline
\end{tabular}

5000
306402.593
306220.1
306489

306304.0949

306350.3104

306406.0695

306457.5801

306509.3631

306561.9043

306611.4765

306636.1836

306584.9011

306532.4927

306479.8127

306427.2505

306374.7542

306302.0475

306282.5237

306331.6588

306384.2244

306439.7645

306494.7684

306548.5195

306612.4984

306561.1923

306505.9228

306450.8791

306400.4688

306352.7895

306311.0385

306272.0584

306254.3361

306301.9687

306351.687

306403.2847

306442.5244

306470.6261

306491.4816

306466.1865

306440.432

306414.7689

306367.8548

306319.9666

306276.809

306246.3773

306219.219

306182.963

306211.2537

306265.6267

306316.9641

306363.3962

$$
\begin{gathered}
0 \\
4937.803407 \mathrm{CP} \\
4939.45 \\
4934.7
\end{gathered}
$$

4938.252294 GRND

4938.239475 GRND

4937.897457 GRND

4937.519121 GRND

4936.802297 GRND

4936.577526 GRND

4936.329608 GRND

4936.303928 GRND

4937.772612 GRND

4938.46574 GRND

4939.103785 GRND

4939.450218 GRND

4939.454074 GRND

4938.68047 GRND

4939.057022 GRND

4939.666801 GRND

4939.808398 GRND

4939.891999 GRND

4939.951592 GRND

4939.924577 GRND

4938.257758 GRND

4939.962415 GRND

4940.964821 GRND

4940.419923 GRND

4940.390253 GRND

4939.808367 GRND

4939.449626 GRND

4939.896253 GRND

4940.774694 GRND

4940.84335 GRND

4940.395323 GRND

4940.773167 GRND

4942.167252 GRND

4940.371304 GRND

4942.504235 GRND

4943.448778 GRND

4941.67901 GRND

4941.837168 GRND

4941.398093 GRND

4941.778803 GRND

4940.554069 GRND

4940.686556 GRND

4939.74652 GRND

4940.262894 GRND

4941.879792 GRND

4941.872918 GRND

4941.622373 GRND
4940.185159 GRND

\author{
PHENIX CONST. \\ STF-O2 GUN RANGE
}




$\begin{array}{lrrr}3048 & 677386.0835 & 306402.7012 & 4940.434553 \text { GRND } \\ 3049 & 677403.7889 & 306434.4713 & 4943.475669 \text { GRND } \\ 3050 & 677409.0726 & 306447.7259 & 4942.502926 \text { GRND } \\ 3051 & 677451.194 & 306439.455 & 4940.906913 \text { GRND } \\ 3052 & 677443.3469 & 306411.4052 & 4939.291262 \text { GRND } \\ 3053 & 677418.0461 & 306363.9896 & 4939.254398 \text { GRND } \\ 3054 & 677387.6303 & 306308.9039 & 4939.91735 \text { GRND } \\ 3055 & 677372.6339 & 306256.4872 & 4939.788445 \text { GRND } \\ 3056 & 677353.2123 & 306208.6922 & 4939.955021 \text { GRND } \\ 3057 & 677321.5915 & 306167.7128 & 4939.96133 \text { GRND }\end{array}$





\section{Vendor Data Review System Final Disposition Screen}

This vendor data item has been given the following disposition codes

\begin{tabular}{|c|c|c|c|c|}
\hline Reviewer & $\begin{array}{l}\text { Revision } \\
\text { Level }\end{array}$ & Date & $\begin{array}{l}\text { Disposition } \\
\text { Code }\end{array}$ & Comments \\
\hline FRITZ KURT D & 0 & $\begin{array}{l}\text { 14-DEC- } \\
06\end{array}$ & $\mathrm{D}$ & $\begin{array}{l}\text { I can't do anything without the } \\
\text { electronic data. }\end{array}$ \\
\hline VANDEL DOUG S & 0 & $\begin{array}{l}\text { 13-DEC- } \\
06\end{array}$ & A & \\
\hline $\begin{array}{l}\text { MCMANAMON } \\
\text { LAWRENCE E }\end{array}$ & 0 & $\begin{array}{l}\text { 14-DEC- } \\
06\end{array}$ & $D$ & \\
\hline TUOTT LEE C & 0 & $\begin{array}{l}\text { 18-DEC- } \\
06\end{array}$ & A & \\
\hline DRIEVER MIKEL K & 0 & $\begin{array}{l}\text { 13-DEC- } \\
06\end{array}$ & $\mathrm{D}$ & \\
\hline LANDIS JOSEPH A & 0 & $\begin{array}{l}\text { 13-DEC- } \\
06\end{array}$ & $A$ & no comment \\
\hline
\end{tabular}

The following reviewers have NOT yet reviewed this vendor data item

JOLLEY WENDELL L

VDR Number:

Revision Level:

Project Number:

Transmittal Number:

Transmittal Status:

Line Item:
VDR-158614

0

$23368-152173$

S-507296-013

Mandatory Approval

1

Final Comments:

A 
F-178 


\section{VDS \#8_153746}


F-180 
VENDOR DATA TRANSMITTAL \& DISPOSITION FORM
ORIGINAL

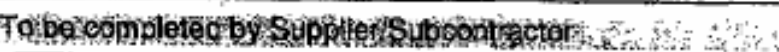

Purchase Order or Subcontract Number: $00507296-04 \mathrm{R} .1 \quad$ Project Title/Number: Remediation of The STF - 02 Gun Range

Submittal Number: 613 - 05 R-01

Supplier/Subcontractor Narne: Phenix Construction

Submittal Date: $\quad 10-06-06 \quad$ Address: P.O. Box 1626 idaho Falls Idaho 83403

$23368-152173$

\begin{tabular}{|c|c|c|c|c|c|c|c|c|c|}
\hline $\begin{array}{l}\text { INEEL } \\
\text { VDS } \\
\text { Item } \\
\text { No. }\end{array}$ & $\begin{array}{l}\text { VDT } \\
\text { item } \\
\text { No. }\end{array}$ & $\begin{array}{c}\text { Specification/ } \\
\text { Drawing } \\
\text { Reference }\end{array}$ & $\begin{array}{l}\text { Tag } \\
\text { Number }\end{array}$ & $\begin{array}{l}\text { Submittal } \\
\text { Status }\end{array}$ & $\begin{array}{c}\text { Revision } \\
\text { Level }\end{array}$ & $\begin{array}{c}\text { Supplier/ } \\
\text { Subcontractor } \\
\text { Document } \\
\text { Number } \\
\text { (if applicable) }\end{array}$ & Description & $\begin{array}{l}\text { INEEL } \\
\text { VOR } \\
\text { Number }\end{array}$ & $\begin{array}{l}\text { Disp } \\
\text { Code }\end{array}$ \\
\hline 8 & & 02200 & & M.A. & R. 01 & $6131-05 R-01$ & Emissions and Dust Control Plan & 153746 & A \\
\hline & & & & & & & & & \\
\hline & & & & & & & & & \\
\hline & & & & & & & & & \\
\hline & & & & & & & & & \\
\hline & & & & & & & & & \\
\hline & & & & & & & & & \\
\hline
\end{tabular}

Remarks

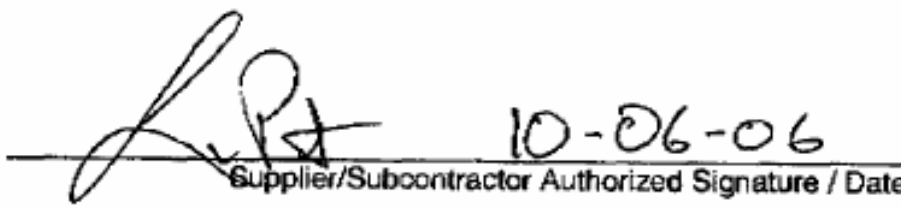

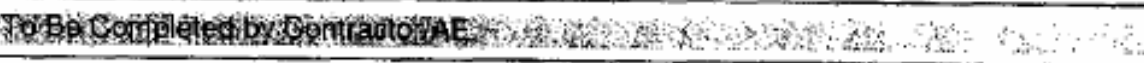




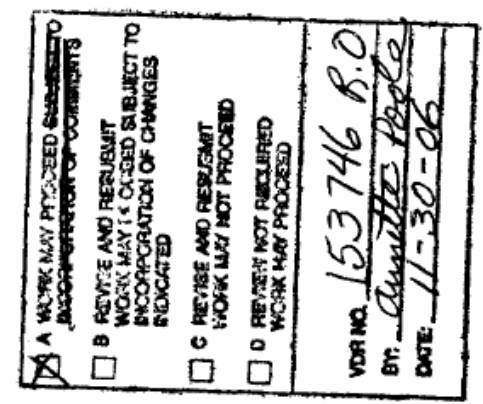

\title{
REMEDIATION OF THE STF-02 GUN RANGE EMISSIONS AND DUST CONTROL PLAN
}

\author{
DEMOLITION OF STF-02 SHOOT HOUSE
}

During demolition activities materials will be sprayed lightly with water supplied by $4000 \mathrm{gal}$ water truck with hose to minimize dust.

\section{EARTHWORK}

During excavation of the 1.5' of lead contaminated soil from the berm a 4000 gal water truck with hose will be used to spray soils in front of excavation activities and while excavation is taking place. This material will be moved and stockpiled on $40 \mathrm{Mil}$ liner or another suitable bartier. Soil will be sprayed with water truck while being stockpiled. Stockpiles will be covered with $10 \mathrm{Mil}$ liner at end of each day. A written Exposure Monitoring Plan will be submitted to CWX and approved by CWI prior to the start of any lead contaminated soil excavation and any screening activities.

During excavation and grading of the remaining berms to the Leach Pond a 4000 gal water truck will be used to minimize dust.

\section{SOIL SCREENING}

During screening operations stockpiles will be sprayed with water supplied by $4000 \mathrm{gal}$ water truck with hose and screening operation lightly sprayed with water to minimize dust. A written Exposure - Monitoring Plan will be submitted to CWI and approved by CWI prior to the start of any lead contaminated soil excavation and any screening activities.

Note:

If water does not adequately minimize dust during excavation and screening of lead contaminated soils water amended with Sockopam (Terra Bond) may also be applied with use of a Hydro Seeder Unit. Water amended with Stockopam (Terra Bond) may also be used to cover stockpiled soils at end of each day in lieu of 10 Mil liner. 


\title{
Vendor Data Review System Final Disposition Screen
}

\author{
This vendor data item has been given the following disposition codes
}

\begin{tabular}{|c|c|c|c|c|}
\hline Reviewer & $\begin{array}{l}\text { Revision } \\
\text { Level }\end{array}$ & Date & $\begin{array}{l}\text { Disposition } \\
\text { Code }\end{array}$ & Comments \\
\hline TUOTT LEE C & 1 & $\begin{array}{l}28- \\
\text { NOV- } \\
06\end{array}$ & A & \\
\hline $\begin{array}{l}\text { DRIEVER MIKEL } \\
\mathrm{K}\end{array}$ & 1 & $\begin{array}{l}\text { O9- } \\
\text { OCT } \\
06\end{array}$ & A & \\
\hline $\begin{array}{l}\text { DRIEVER MIKEL } \\
K\end{array}$ & 0 & $\begin{array}{l}\text { 02- } \\
\text { OCT. } \\
06\end{array}$ & $\mathrm{D}$ & \\
\hline FRITZ KURT D & 0 & $\begin{array}{l}\text { O4- } \\
\text { OCT. } \\
06\end{array}$ & A & \\
\hline VANDEL DOUG S & 0 & $\begin{array}{l}02- \\
\text { OCT. } \\
06\end{array}$ & A & \\
\hline $\begin{array}{l}\text { MCMANAMON } \\
\text { LAWRENCEE }\end{array}$ & 1 & $\begin{array}{l}\text { 09- } \\
\text { OCT. } \\
06\end{array}$ & A & \\
\hline $\begin{array}{l}\text { MCMANAMON } \\
\text { LAWRENCEE }\end{array}$ & 0 & $\begin{array}{l}\text { O5- } \\
\text { OCT. } \\
06\end{array}$ & A & \\
\hline $\begin{array}{l}\text { JOLLEY } \\
\text { WENDELL L }\end{array}$ & 1 & $\begin{array}{l}\text { O9- } \\
\text { OCT- } \\
06\end{array}$ & $D$ & \\
\hline $\begin{array}{l}\text { LANDIS JOSEPH } \\
\text { A }\end{array}$ & 1 & $\begin{array}{l}\text { 09- } \\
\text { OCT. } \\
06\end{array}$ & $A$ & NO COMMENT \\
\hline TUOTT LEE C & 0 & $\begin{array}{l}\text { 02- } \\
\text { OCT- } \\
06\end{array}$ & B & $\begin{array}{l}\text { Please revise/clarify the produced used for the } \\
\text { "amended water." There needs to be a } 1: 1 \text { tie } \\
\text { with this produce/chemical and the approved } \\
\text { list of chemicals. }\end{array}$ \\
\hline $\begin{array}{l}\text { JOLLEY } \\
\text { WENDELL L }\end{array}$ & 0 & $\begin{array}{l}\text { O3. } \\
\text { OCT. } \\
\text { OE }\end{array}$ & $D$ & \\
\hline VANDEL DOUG S & & $\begin{array}{l}\text { O9. } \\
\text { OCT. } \\
06\end{array}$ & A & \\
\hline FRITZ KURT D & 1 & $\begin{array}{l}10- \\
\text { OCT- } \\
06\end{array}$ & A & \\
\hline $\begin{array}{l}\text { LANDIS JOSEPH } \\
\text { A }\end{array}$ & 0 & $\begin{array}{l}05- \\
\text { OCT. } \\
06\end{array}$ & A & no comments \\
\hline
\end{tabular}


Page 2 of 2

$\begin{array}{ll}\text { VDR Number: } & \text { VDR-153746 } \\ \text { Revision Level: } & 1 \\ \text { Project Number: } & 23368-152173 \\ \text { Transmittal Number: } & \text { S-507296-04R.1 } \\ \text { Transmittal Status: } & \text { Mandatory Approval } \\ \text { Line Item: } & 1\end{array}$

Disposition Code::

A

Final Comments:: 


\section{VDS \#9_155291}


F-186 
VENDOR DATA

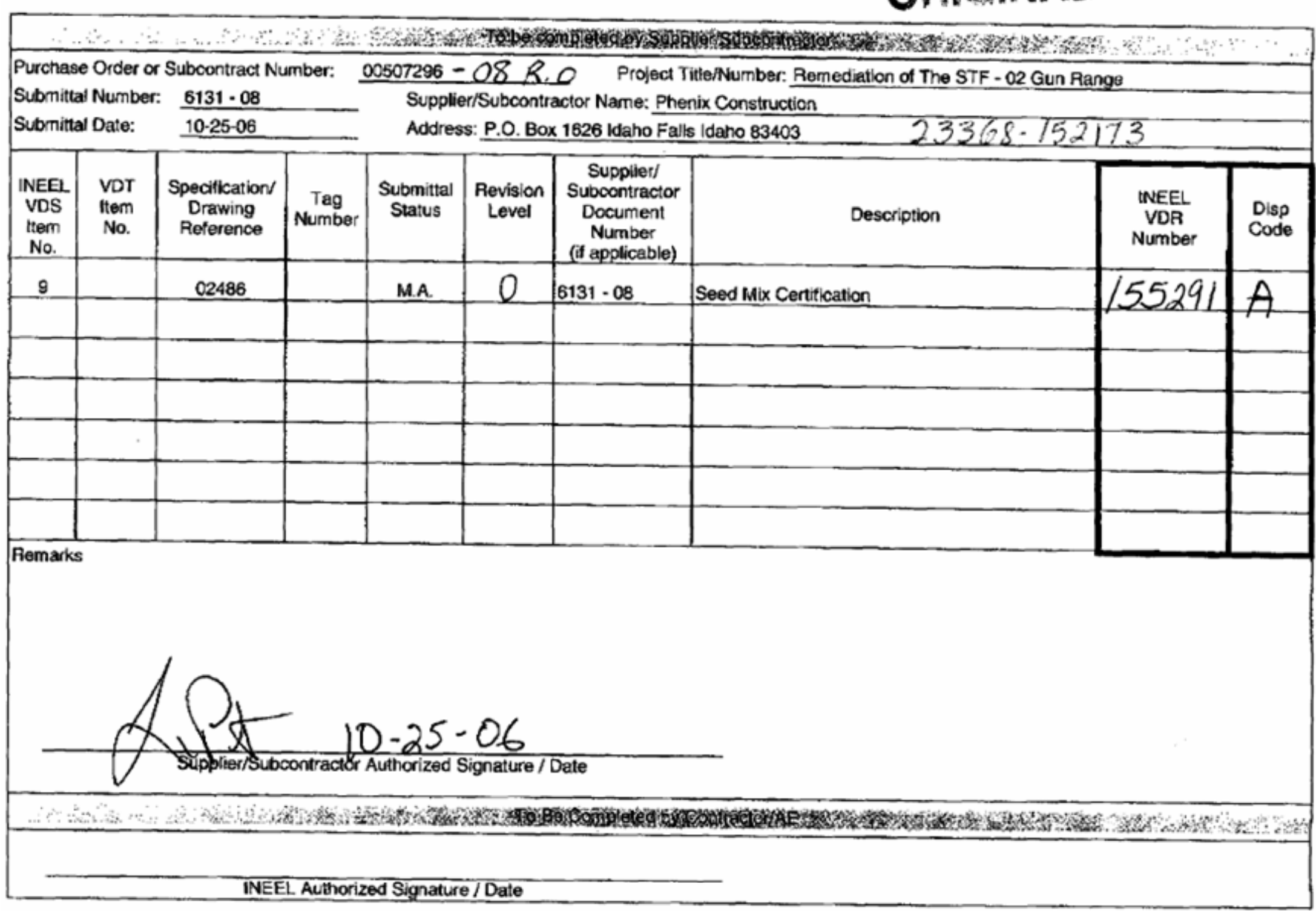


DATB SHIPPED: 10/23/2006

SOLD TO:

PHENIX OF IDAHO, INC.

P.O. BOX 1626

IDAHO PALLS, ID 83403-1626

PROJECT NAMB: STE-02 GUN RANGE

SEED MIX NUMBER; 57515

Letter of Certification

This memo is written to certify that this seed mix is true to label and has been duly tested by a fully accredited seed testing laboratory using rules sanctioned by the Association of official seed Analysts. The specifications of the seed uged in the mix are as follows:

\begin{tabular}{|c|c|c|c|c|c|c|c|}
\hline Common Name & variety & origin & & $a t \#$ & Puricy & $\begin{array}{l}\text { Germ + } \\
\text { Dorm. }\end{array}$ & 8PLS \\
\hline LOW RABBITBRUSH & WNS & UT & CHVI & -30899 & 32.18 & 67 & 20.89 \\
\hline INDIAN RICEGRASS & RIMROCK & $M T$ & ORHY & -36207 & 99.70 & 97 & 96.71 \\
\hline SQUIRRELTAIL & vNS & WA & SIFY & -36358 & 96.49 & 89 & 85.88 \\
\hline WESTRRN WHBATGRASS & ROSANA & WA & AGSM & -36286 & 96.64 & 95 & 91.81 \\
\hline BLUEBUNCH WHEATGRASS & GOLDAR & WA & AGSP & -35608 & 91.95 & 93 & 85.51 \\
\hline BOREAL SWEETVETCH & TIMP & WY & HEBO & -36071 & 98.78 & 86 & 84.95 \\
\hline MUNRO GLOBEMALLOW & vNS & $\mathrm{Az}$ & SPMU & -32558 & 98.05 & 90 & 88.25 \\
\hline THICKSPIKE WHEATGRAS & BANNOCK & WA & AGDA & $-363 \times 7$ & 95.64 & 94 & 89.90 \\
\hline
\end{tabular}

Furthermore, we certify that said seed was packed as follows:

Number of Bags Bulk Lbs. Per Bag

\begin{tabular}{ll}
\hline 1 & 50.00 \\
1 & 12.47 \\
\hline
\end{tabular}

The number of pounds and percent of bulk for this mix are as follows:

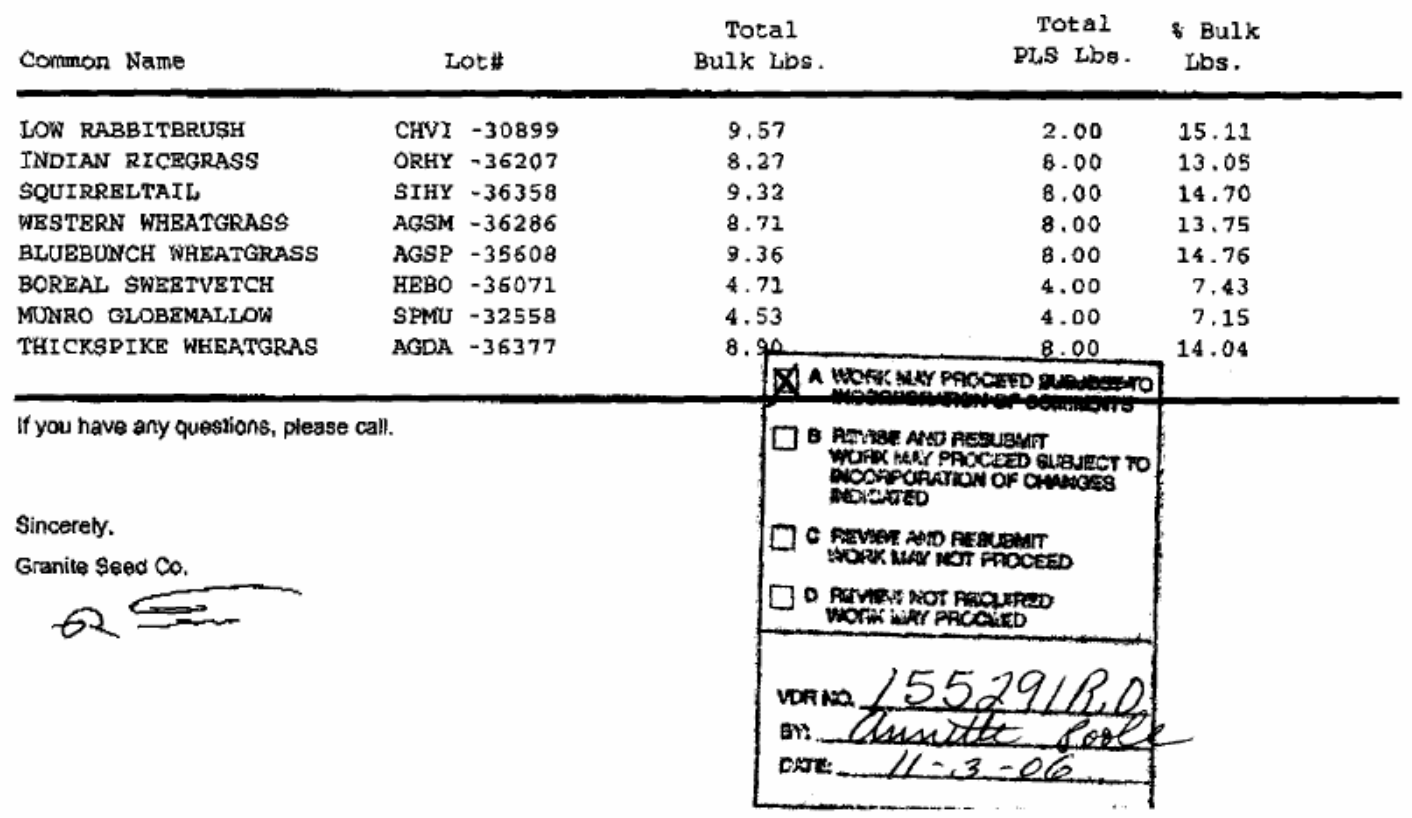


Lot Number: CHVI $\quad-30899$

Kind and Variety: VNS - LOW RABBITBRUSH

Scientific Name: CHRYSOTHAMNUS VISCIDIFLOR

Pure Seed \%: 31.18 Total Viable \%: 67.00

Crop \%: 0.07 Germination \%: 67.00

Inert \%: 68.33 Hard Seed \%: 0.00

Weed \%: 0.42 Date of Test: 11/30/2005

Pure Live Seed \%: 20.89 Origin: UT

Name of $L a b ; I D=P \quad$ UT $=T Z$

Noxious or Restricted Weeds (all 50 States):

DOWNY BROME 674/\# COMMON YARROW 225/\#

NEEDEDLEGRASS $225 / \#$

Lot Number: ORHY -36207

Kind and Variety: RIMROCK - INDIAN RICEGRASS

Scientific Name: ACHNATHERUM HYMENOIDES

Pure Seed \%: 99.70 Total Viable \%: 97.00

Crop \%: 0.00 Germination \%: 97.00

Inert \%: 0.27 Hard Seed \%: 0.00

Weed \%: 0.03 Date of Test: 12/29/2005

Pure Live Seed \%: 96.71 Origin: MT

Name of Lab: MT=P\&TZ

Noxious or Restricted Weeds (all 50 States):

NONE

Granite Seed Company certifies that this analysis accurately represents the original copy of seed analysis and, where applicable, all testing has been conducted by a certified Seed Technologist using the sanctioned rules established by the Association of Official Seed Analysts.

Signature: ar 
Lot Number: SiHY -36358

Kind and Variety: VNS - SQUIRRELTAIL

Scientific Name: ELYMUS ELYMOIDES

Pure Seed \%: 96.49 Total Viable \%: 89.00

Crop \%: 0.04 Germination \%: 89.00

Inert \%: 3.47 Hard Seed \%: 0.00

Weed \%: 0.00 Date of Test: 10/10/2006

Pure Live Seed \%: 85.88 Origin: WA

Name of Lab: WY=TZ $\quad A G R I-Q U A L=T Z$

Noxious or Restricted Weeds (all 50 States):

NONE

Lot Number: AGSM -36286

Kind and Variety: ROSANA - WESTERN WHEATGRASS

Scientific Name: PASCOPYRUM SMITHII

Pure Seed \%: 96.64 Total Viable \%: 95.00

Crop \%: 0.38 Germination \%: 95.00

Inert \%: 2.89 Hard Seed \%: 0.00

Weed \%: 0.09 Date of Test: 09/22/2006

Pure Live Seed \%: 91.81 Origin: WA

Name of Lab: $M T=P \& T Z$

Noxious or Restricted Weeds (all 50 States):

Saisola kall $5 / 1.00 \mathrm{lb}$

Granite Seed Company certifies that this analysis accurately represents the original copy of seed analysis and, where applicable, all testing has been conducted by a certified Seed Technoiogist using the sanctioned rules established by the Association of Official Seed Analysts.

Signature: कर $=2$ Date: $10-23-06$ 
Lot Number: AGSP $\quad-35608$

Kind and Variety: GOLDAR - BLUEBUNCH WHEATGRASS

Scientific Name: PSEUDOROEGNERIA SPICATA SSP. SPICATA

Pure Seed \%: 91.95 Total Viable \%: 93.00

Crop \%: 2.04 Germination \%: 93.00

Inert \%: 6.01 Hard Seed \%: 0.00

Weed \%: 0.00 Date of Test: 08/24/2006

Pure Live Seed \%: 85.51 Origin: WA

Name of $L a b: W A=G \quad W A=G$

Noxious or Restricted Weeds (all 60 States):

NONE

Lot Number: HEBO - 36071

Kind and Variety: TIMP - BOREAL SWEETVETCH

Scientific Name: HEDYSARUM BOREALE

Pure Seed \%: 98.78 Total Viable \%: 86.00

Crop \%: 0.00 Germination \%: 54.00

Inert \%: 1.22 Hard Seed \%: 32.00

Weed \%: 0.00 Date of Test: 08/28/2006

Pure Live Seed \%: 84.95 Origin: WY

Name of Lab: $W Y=P \& G$

Noxious or Restricted Weeds (all 50 States):

NONE

Granite Seed Company certifies that this analysis accurately represents the original copy of seed analysis and, where applicable, all testing has been conducted by a certifled Seed Technologist using the sanctioned rules established by the Association of Offlciel Seed Analysts.

Signature: $\rightarrow 25$

Date: $10-23-06$ 
Granite Seed Company

Lehi, Utah 84043

CERTIFIED COPY OF SEED ANALYSIS

Lot Number: SPMU 32558

Kind and Variety: VNS - MUNRO GLOBEMALLOW

Scientific Name: SPHAERALCEA MUNROANA

Pure Seed \%: 98.05 Total Viable \%: 90.00

Crop \%: 0.00 Germination \%: 90.00

Inert \%: 1.67 Hard Seed \%: 0.00

Weed \%: 0.28 Date of Test: 05/10/2006

Pure Live Seed \%: 88.25 Origin: AZ

Name of Lab: ID $=P \& T Z \quad A Z=T Z$

Noxious or Restricted Weeds (all 50 States):

GRAMA 1002/\#

UNKNO WN 200/\#

Lot Number: AGDA $\quad-36377$

Kind and Variety: BANNOCK - THICKSPIKE WHEATGRASS

Scientific Name: ELYMUS LANCEOLATUS SSP. LANCEOLATUS

Pure Seed \%: 95.64 Total Viable \%: 94.00

Crop \%: $0.00 \quad$ Germination \%: 94.00

Inert \%: 4.07 Hard Seed \%: 0.00

Weed \%: 0.29 Date of Test: 09/27/2006

Pure Live Seed \%: 89.90 Origin: WA

Name of Lab: WA=P,G,\&T

Noxious or Restricted Weeds (all 50 States):

NONE

Granite Seed Company certifles that this analysis accurately represents the original copy of seed analysis and, where applicable, all testing has been conducted by a certified Seed Technologist using the sanctioned rules established by the Association of Official Seed Analysts.

Sianature:

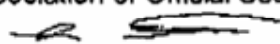

nate. $10-2.3-106$ 


\section{Vendor Data Review System Final Disposition Screen}

This vendor data item has been given the following disposition codes

\begin{tabular}{|c|c|c|c|c|}
\hline Reviewer & $\begin{array}{l}\text { Revision } \\
\text { Level }\end{array}$ & Date & $\begin{array}{l}\text { Disposition } \\
\text { Code }\end{array}$ & Comments \\
\hline LANDIS JOSEPH A & 0 & $\begin{array}{l}26- \\
\text { OCT- } \\
06\end{array}$ & A & no comment \\
\hline DRIEVER MIKEL K & 0 & $\begin{array}{l}30- \\
\text { OCT- } \\
06\end{array}$ & A & \\
\hline FRITZ KURT D & 0 & $\begin{array}{l}\text { 02- } \\
\text { NOV- } \\
06\end{array}$ & $\mathrm{D}$ & \\
\hline $\begin{array}{l}\text { JOLLEY WENDELL } \\
\mathrm{L}\end{array}$ & 0 & $\begin{array}{l}26- \\
\text { OCT } \\
06\end{array}$ & $\mathrm{D}$ & \\
\hline $\begin{array}{l}\text { JOLLEY WENDELL } \\
\text { L }\end{array}$ & 0 & $\begin{array}{l}26- \\
\text { OCT- } \\
06\end{array}$ & $\mathrm{D}$ & \\
\hline VANDEL DOUG S & 0 & $\begin{array}{l}\text { 26- } \\
\text { OCT- } \\
06\end{array}$ & A & \\
\hline $\begin{array}{l}\text { JOLLEY WENDELL } \\
L\end{array}$ & 0 & $\begin{array}{l}26- \\
\text { OCT. } \\
06\end{array}$ & A & \\
\hline $\begin{array}{l}\text { MCMANAMON } \\
\text { LAWRENCE E }\end{array}$ & 0 & $\begin{array}{l}26- \\
\text { OCT. } \\
06\end{array}$ & $\mathrm{D}$ & \\
\hline TUOTT LEE C & 0 & $\begin{array}{l}02- \\
\text { NOV- } \\
06\end{array}$ & A & $\begin{array}{l}\text { Approve - have less than } 4 \text { ac. to seed } \\
\text { so the amounts will be sufficient per the } \\
\text { spec. }\end{array}$ \\
\hline
\end{tabular}

$\begin{array}{ll}\text { VDR Number: } & \text { VDR-155291 } \\ \text { Revision Level: } & 0 \\ \text { Project Number: } & 23368-152173 \\ \text { Transmittal Number: } & \text { S-507296-08 } \\ \text { Transmittal Status: } & \text { Mandatory Approval } \\ \text { Line Item: } & 1\end{array}$

Disposition Code::

Final Comments::

A 
F-194 
VDS \#10_153317

F-195 
F-196 
431.13

07/23/2002

Rev. 04
VENDOR DATA TRANSMITTAL \& DISPOSITION FORM

\section{ORIGINAL}

To be completed by Supplier/Subcontractor

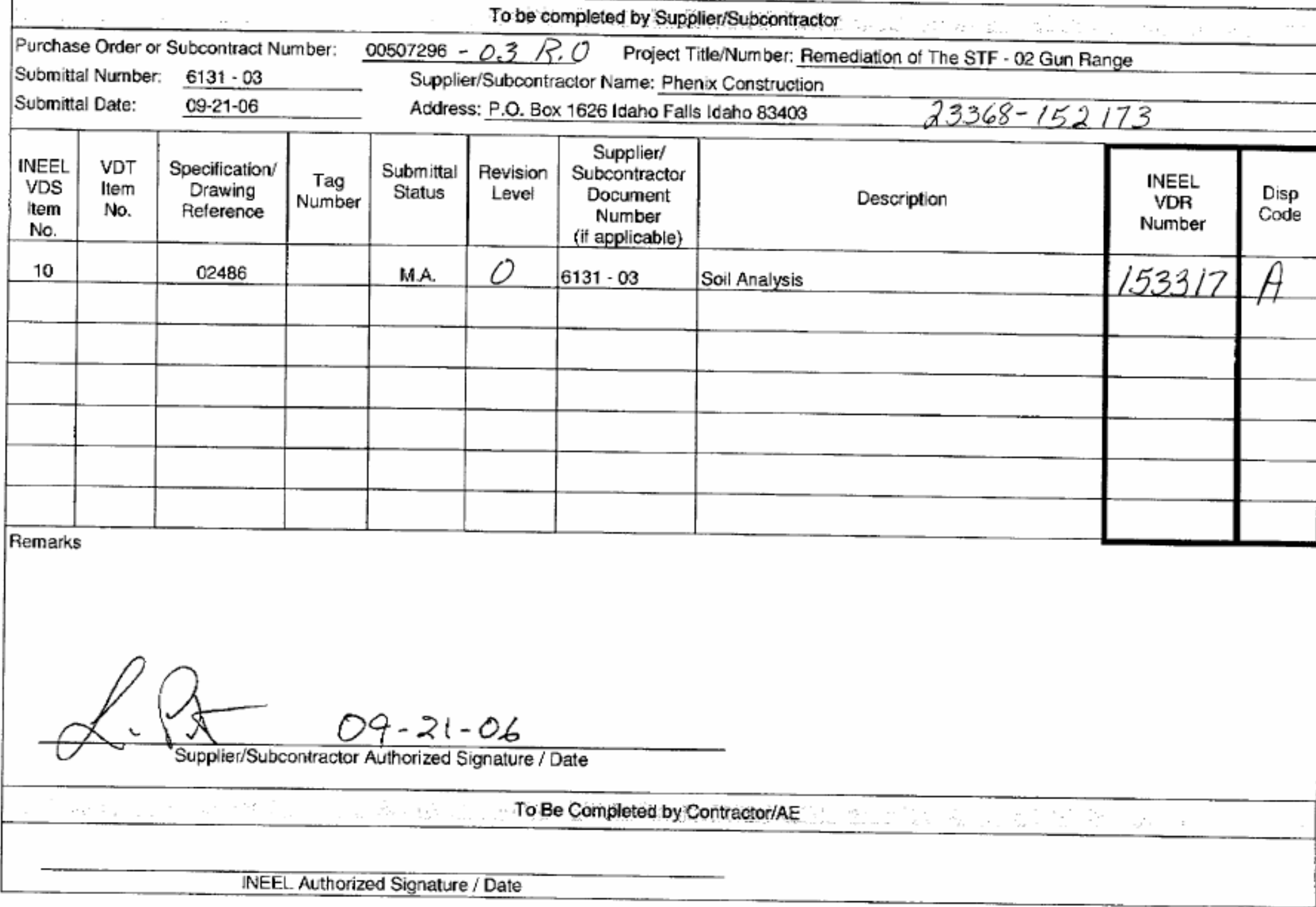




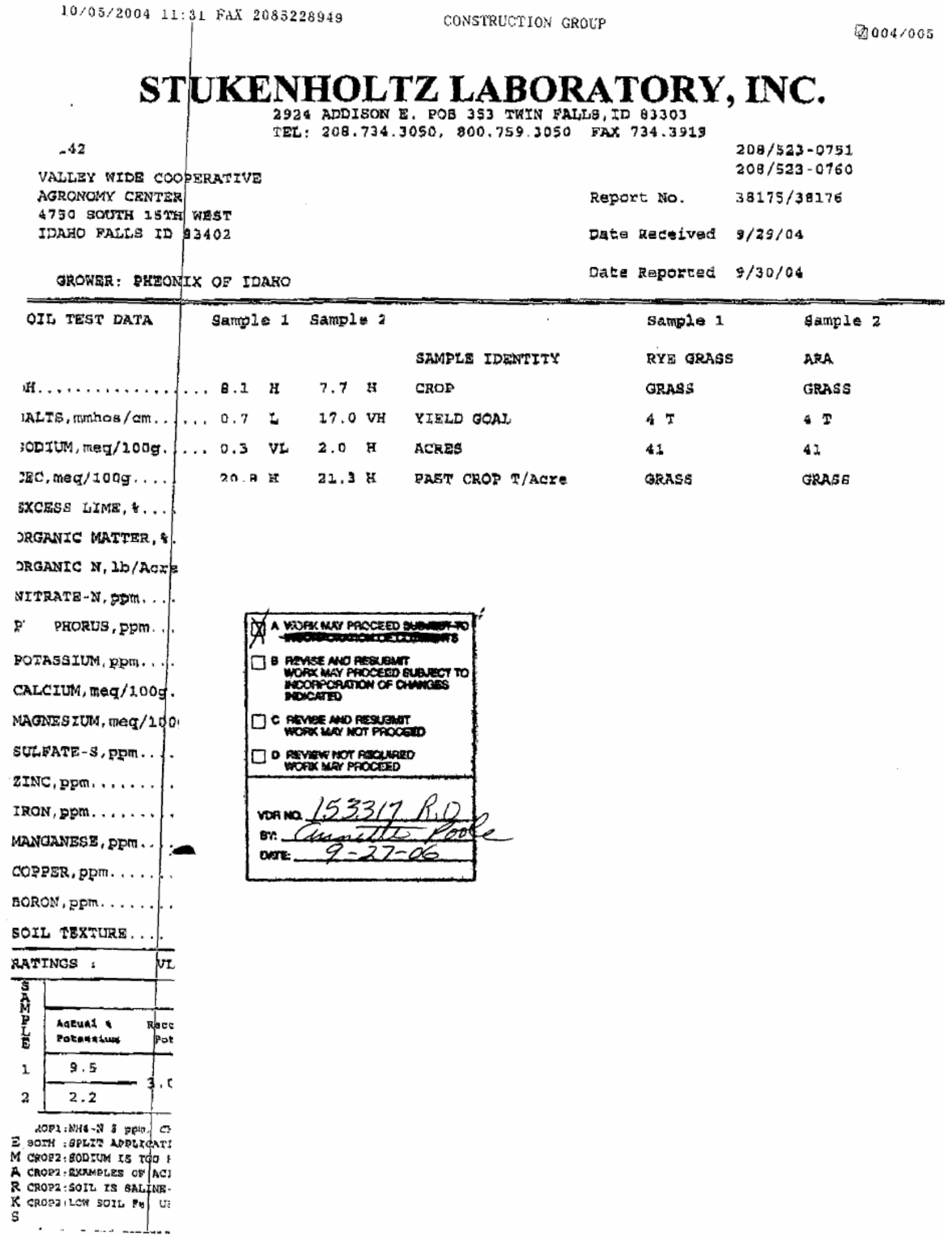


$-42$

VALLEY WIDE COOPERATIVE

AGRONOMY CENTER

4750 SCUTH $152 \mathrm{YH}$ WEST

IDAHO FALIS ID 93402

OROWER: PHEONIX OF IDAHO

OIL TEST DATA Samgle 1 sample

OIL TEST DATA Sample 1 Sample 2

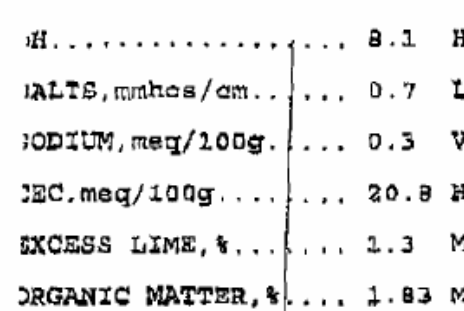$$
\text { IRGANIC N, Ib/ACXF. . } 70
$$$$
\text { NITRATE-N, DDM...... } 13 \text { M }
$$$$
\text { g' PRORUS, ggm. . ... } 7 \text { r }
$$$$
\text { BotassIUM, ppm.......590 VH } 140 \text { I }
$$$$
\text { CALCILM, meg/100g.... } 13.9 \mathrm{H} \quad 13.0 \mathrm{M}
$$$$
\text { MAGNESIUM, meg/100g, 4.6 VH } 5.8 \text { VH }
$$$$
\text { sutbate-s,ppm... ...4 VL } 49 \text { VH }
$$$$
\text { zINC, ppm. ..... } \ldots . \ldots 0.6 \text { I } 0.4 \text { vL }
$$$$
\text { IRON, gpm.......... } 4.8 \text { M. } 3.0 \text { L }
$$$$
\text { MANGANESE, DPM,..... } 2.0 \text { z }
$$$$
\text { COpgER,ppm........ } 1.6 \text { H }
$$$$
\text { BORON, RPM........... 0.90 M }
$$$$
5.4 \mathrm{H}
$$$$
0,6 \mathrm{~L}
$$$$
2,45 \mathrm{H}
$$

SAMELE IDANTIT

Report No.

Date Rectived

Date Reported $9 / 30 / 04$

208/523-0751

208/523-0760

$38175 / 38176$

CROP

YIELD GOAI

Ackas

PAST CROP T/ACY

Sample 1

sample 2

RYI GRASS ARA

GRASS GRASS

$4 \mathrm{~T}+\mathrm{T}$

4142

GRASS GRASE

MRNURE T/ACre 0 o

EREV, AQRLIED NUTRIENTS 0

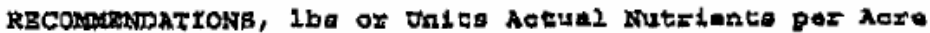

NITROogN. . . . . . . 110

$\mathrm{P}_{2} \mathrm{O}_{5}-$ pHOSPHATE.....70 110

$\mathrm{K}_{3} \mathrm{O}$ - gOTASH......... O So

CAICIUM. ............ a

MAGNEGIUM.......... 0 0

SULEATE-SULRUR. ...., 40

ZINC............8 80

Iron ............. o

MANGAANBSE........... 3

COERER............ o

BORON............. 0

ELEMENTAL SUTEUR. .....

$200 * \pi *$

\begin{tabular}{l} 
SOII TEXTURE... \\
\hline RATINGS :
\end{tabular}

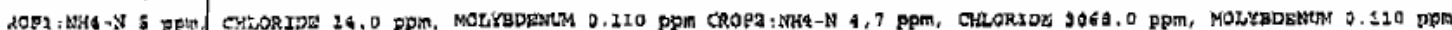

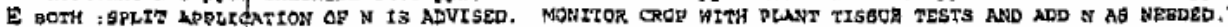

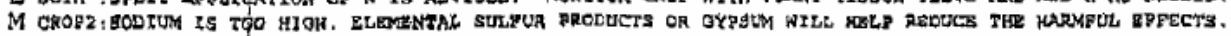

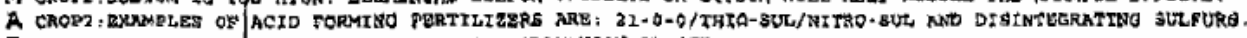

$R$ CROP2:SOIZ IS GALFNE-ALKALI AND NEEDS RECTAMATION PRACOTCES.

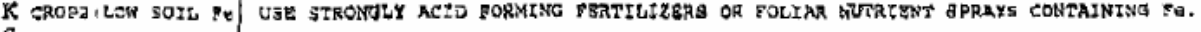

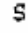




\section{Vendor Data Review System Final Disposition Screen}

This vendor data item has been given the following disposition codes

\begin{tabular}{|c|c|c|c|c|}
\hline Reviewer & Revision Level & Date & Disposition Code & Comments \\
\hline DRIEVER MIKEL K & 0 & 25-SEP-06 & $\mathrm{D}$ & \\
\hline VANDEL DOUG S & 0 & 25-SEP-06 & A & \\
\hline TUOTT LEE C & 0 & 25-SEP-06 & $\mathrm{D}$ & No comments. \\
\hline FRITZ KURT D & 0 & 22-SEP-06 & A & \\
\hline MCMANAMON LAWRENCE E & 0 & 26-SEP-06 & A & None \\
\hline
\end{tabular}

VDR Number: $\quad$ VDR-153317

Revision Level: $\quad 0$

Project Number: $\quad 23368-152173$

Transmittal Number: S-507296-03

Transmittal Status: Mandatory Approval

Line Item:

1

Disposition Code::

Final Comments::

A

No comment 
VDS \#11_153751 
F-202 


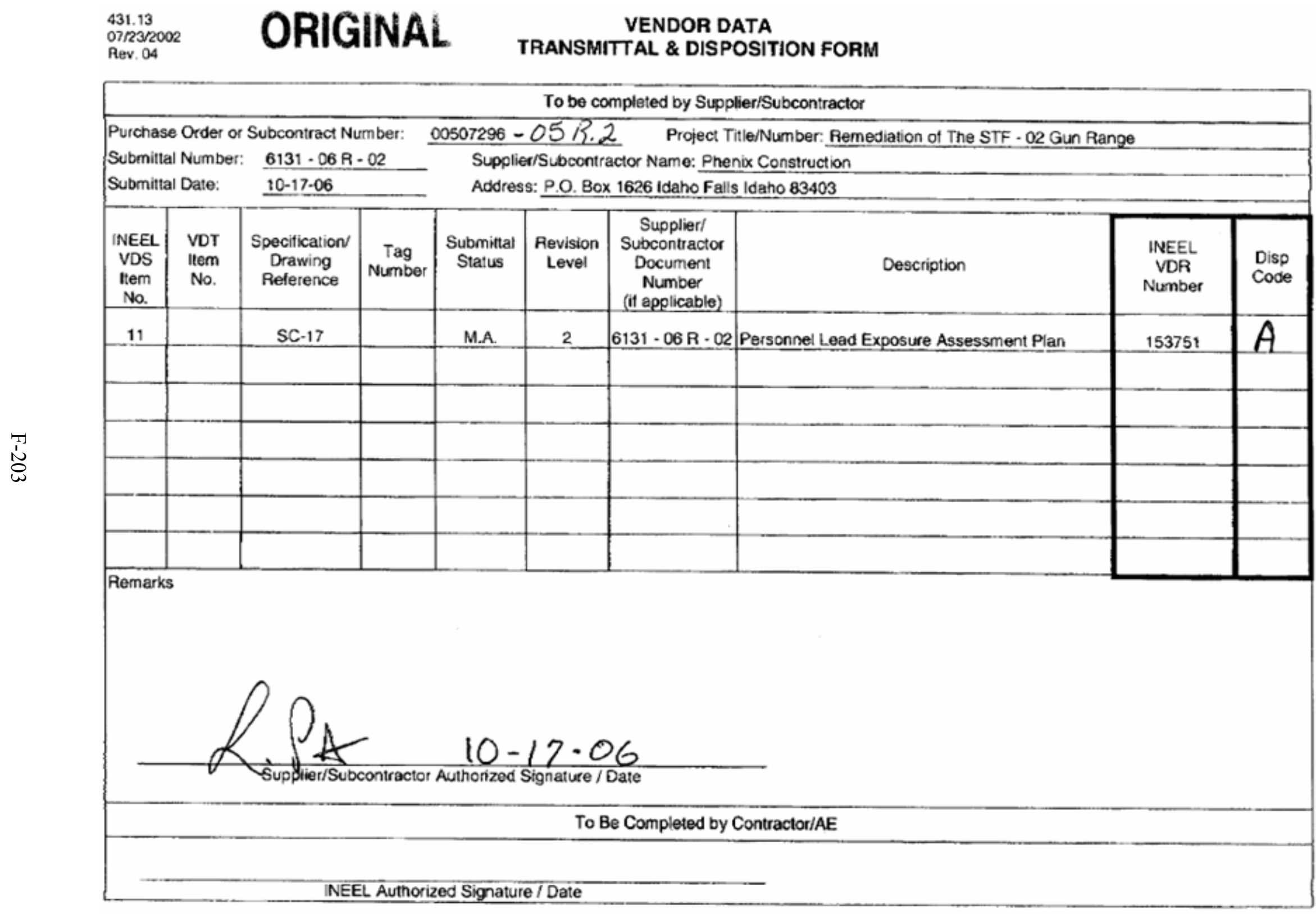




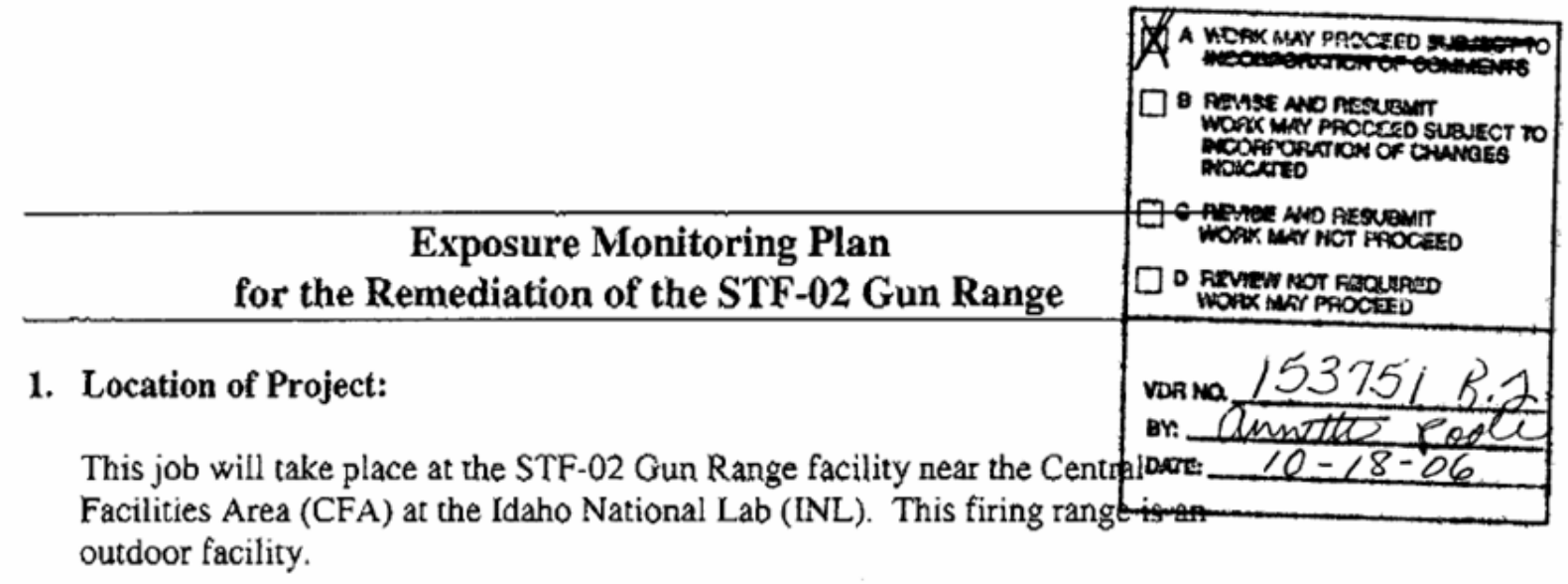

\section{Brief Description of Job:}

Phenix Construction will perform remediation of the STF-02 Gun Range site at the INL. Remediation requires the removal of lead and treatment and disposal of lead contaminated soil found within the STF-02 Gun Range. Spent ammunition was the source of lead and lead contamination.

Phenix shall furnish labor, material, equipment and supplies (other than government-furnished materials and/or equipment) and perform work and operations necessary to remediate the lead contaminated soil site to remediation action goals, in accordance with the construction drawings and these specifications.

3. Personnel and companies involved in the project are:

$\begin{array}{lll}\text { Organization } & \text { Individual } & \underline{\text { Phone }} \\ \text { CWI Project Oversight } & \text { Joseph Landis } & (208) 526-6311 \\ \text { CWI Safety POC } & \text { Larry McManamon } & (208) 526-3658 \\ \text { Phenix Project Manager } & \text { Lance Peterson } & (208) 524-6488 \\ \text { Health and Safety Lead } & \text { Mike Garcia } & (208) 524-6488 \\ \text { HSO Level II and } & \text { Adam Hult } & \text { (208) } 351-2325 \\ \text { Lead Competent Person } & & \end{array}$

\section{Schedule}

Prior to arriving at the site, Phenix personnel will submit this exposure monitoring plan to the CWI - Safety Contact (Larry McManamon) and the CWI STR (Jody Landis) for review and comments. Work will begin when the CWI STR and Safety Contact provide approval. Phenix is anticipating beginning work in midOctober 2006 with duration of one to two months.

\section{Sequence of work}

Table 1, located at the end of this exposure monitoring plan provides a description of potential and anticipated project exposures associated with the following remediation tasks. All tasks are designated to be performed in Level D PPE, but Level C (use of half-faced respirators) will be wom for tasks specified in Table 1 
until lead exposure levels are known (see Section 12). Tasks associated with the remediation and cleanup at the STF-02 Gun Range are defined as:

\section{- Mobilization}

\section{- Constructing Staking and Surveying}

Construction surveys will be conducted throughout and following the execution of the project, from baseline topography to post-remediation of specific areas receiving clean fill and revegetation.

\section{- Demolition and Removal}

During and after the clean soil excavation, site demolition will occur. Care will be exercised in excavating, loading, hauling, screening, and dumping of contaminated waste to minimize dust generation. Dust minimization practices will be implemented to mitigate any release of airborme contaminants. Suspect contaminated materials will be sprayed down by the water truck to minimize dust from demolition activities. Some materials removed will be loaded and hauled on site to designated disposal locations or landfills (i.e., bulky waste). Contaminated sand will be carefully excavated and screened. Appropriate Personal Protective Equipment, as required, will be worn by all operators and laborers performing this work. Demolished materials will be properly segregated. Baseline exposure monitoring will occur during demolition and removal of suspect materials.

\section{- Excavation, Stockpiling, and Screening of Lead-Contaminated Soils}

Care will be taken to minimize contact with and dust generation from moving and handling materials. Baseline exposure monitoring will occur during these activities.

\section{- Vehicle Loading}

Sieved soil will be loaded onto vehicles for shipment to an off-site treatment, storage, or disposal facility. Care will be taken to minimize dust generation. Baseline exposure monitoring will occur during vehicle loading of sieved soils.

\section{- Grading and Revegetation of Disturbed Areas}

Clean soil and seeding will be added to area as part of the remediation. Care will be taken to minimize dust generation.

\section{- Demobilization}

After the remedial action has been completed and all equipment has been decontaminated, all materials, supplies, tools, equipment, and project-derived wastes will be removed from the site. 
The task requiring Level C PPE during the initial exposure assessment are Demolition and Removal, Excavation. Stockpiling, and Screening of LeadContaminated Soils, and Vehicle Loading. Once monitoring results are known, PPE levels can be downgraded if work practices and controls are proven to be effective.

\section{Equipment and Materials}

Excavators, front end loaders, dozers, shovels, rakes, protective clothing, cotton and leather gloves, hoses and sprayers, power and other hand tools as needed.

\section{Crew}

The initial setup of the work areas, contamination areas, waste segregation, and soil screening will be established by the Phenix crew members and the health and safety person. Key crew assignments include:

Phenix Project Lead:

Phenix Health and Safety Lead:

Lance Peterson

Lead Competent Person: Mike Garcia

Adam Hult

\section{Competent Person}

A designated Competent Person for occupational health and safety issues will be on site at all times. This person will conduct daily inspections of the work areas to ensure that control measures, work practices, personnel protective equipment, and hygiene facilities are used as prescribed in this document. Additional Lead Competent Person's for specific tasks such as excavation will be identified during the project. Other personnel may be trained to act as a backup competent person on this project. This person is also responsible for all exposure monitoring activities and ensuring that samples are sent to a lab accredited by the American Industrial Hygiene Association (AIHA) for analysis.

\section{Control Measures}

During all phases of the scope of work, controls (primarily dust suppression with water) will be in implemented to minimize the lead dust exposure. Lead containing dust has a high density and does not easily disperse like many other materials (e.g. asbestos, etc.). Physical containment and proper work practices will be sufficient to prevent untoward spread of dusts outside the immediate working zone. All due caution will be exercised by field staff to avoid actions that would tend to transport project derived dusts from leaving the work zone. Personnel working in potential or known lead contaminated areas must complete Lead Awareness and Lead Worker training. 
Baseline sampling (personal and area sampling) will be performed to assess the effectiveness of controls in places. As a precaution, personnel with potential lead exposure will wear respiratory protection during the initial monitoring period until exposure levels are known.

\section{Medical Surveillance}

Lead workers assigned to work full-time to the project ( 40 hours/week) who enter controlled areas during excavation or lead contaminated soil handling will have an initial blood- $\mathrm{Pb}$ sample taken prior to beginning work at the site. At the direction of the Health and Safety Lead or the Competent Person, additional blood-Pb levels will be taken if monitoring data concludes the action level can be or was exceeded. Worker blood lead increases of $10 \mathrm{mg} / \mathrm{dl}$ or more will trigger an investigation of protective equipment and work practices. Workers will be removed from high risk exposure activities if blood- $\mathrm{Pb}$ levels are greater then 50 $\mu \mathrm{g} / \mathrm{dl}$. All workers on this project are informed of their blood lead levels as soon as results are received.

\section{Technology Considered in Meeting Permissible Exposure Limit (PEL)}

The only specialized mechanism that will be utilized for this project is the use of water and/or amended water for dust suppression and natural ventilation as work is performed in an outdoor environment.

A designated Lead Competent Person for occupational health and safety issues will be on site at all times. This person will conduct daily inspections of the work areas to ensure that control measures, work practices, personnel protective equipment. and hygiene facilities are used as prescribed in this document. Additional personnel may be trained to act as a backup competent person on this project.

\section{Exposure Monitoring}

During all phases of the scope of work controls will be in operation to minimize the lead dust exposure, primarily with administrative controls and dust suppression with water. Sampling will be performed as outlined in Table 1. Lead containing dust has a high density and does not easily disperse like many other materials (e.g. asbestos, etc.). All due caution will be exercised by field staff to avoid actions that would tend to transport project derived dusts from leaving the work zone. Administrative controls, such as job rotation and stay times, will also be used to maintain a workers 8 -hr time-weight average (TWA) below the action level (AL) or permissible exposure level (PEL). The AL for lead is $30 \mu \mathrm{g} / \mathrm{m}^{3}$ and the PEL is $50 \mu \mathrm{g} / \mathrm{m}^{3}$ for 8 -hr time weighted averages. All personnel working in potential or known lead contaminated areas must complete Lead Awareness and Lead Worker training. 
NOTE 1: Engineering and administrative controls are key to keeping employees below the PEL. As stated in PRD-2105, Lead, when a work environment $>$ or equal to the PEL additional requirements must be met.

Baseline sampling (personal and area sampling) of personnel and tasks will occur as part of performing an initial exposure assess as outlined in Table 1 . IF the initial exposure assessment shows the possibility of an employee exposure at or above the action level, THEN representative monitoring shall be conducted for each employee in the workplace who is exposed to lead, according to the following schedule:

- IF exposure > PEL, THEN monitoring shall be conducted quarterly until at least 2 consecutive measurements, taken at least 7 days apart, are at or below the PEL, after which monitoring shall be performed as in (B) below.

- IF the exposure is < PEL and >AL, THEN monitoring shall be conducted every six months until at least 2 consecutive measurements, taken at least 7 days apart, are below the action level, after which monitoring may be discontinued.

NOTE 2: If sampling results indicate that an unprotected worker' sample results $>P E L$ then the worker must be immediately notified of the sample results and the Miscellaneous Sites Completion Point of Contact must also be notified within two hours of knowledge of that exposure (per PRD-2111).

NOTE 3: If unprotected workers have sample results $>A L$ but $<P E L$ then the Miscellaneous Sites Completion Point of Contact must be notified of the exposures.

When administrative controls are used as a means of reducing employee exposure to lead, job rotation or a stay time schedule shall be established and implemented using the following:

1. Name or identification number of each affected employee

2. Duration and exposure levels at each job or work station where each affected employee is located, and

3. Any other applicable information that may be useful in assessing the reliability of the administrative controls.

When airborne lead concentrations exceed the PEL during a work activity, written compliance documentation shall be provided, using any of the following:

1. Work order 
2. Approved procedure

3. Task sheet

4. Exposure assessment

5. Any other hazard evaluation (see PRD-1501, Work Control).

Lead workers assigned to work full-time to the project ( 40 hours/week) will have an initial blood-Pb sample taken prior to beginning work at the site. Blood- $\mathrm{Pb}$ levels will be taken if monitoring indicates the AL can be or was exceeded. Worker blood lead increases of $10 \mathrm{mg} / \mathrm{dl}$ or more will trigger an investigation of protective equipment and work practices. Workers with blood-Pb levels $>40$ $\mu \mathrm{g} / \mathrm{dl}$ must be informed in writing within five working days of result receipt. Workers will be removed from high risk exposure activities if blood-Pb levels are greater then $50 \mu \mathrm{g} / \mathrm{dl}$. All workers on this project will be informed of their blood lead levels as soon as results are received.

\section{Technology Considered in Meeting Permissible Exposure Limit}

The only specialized mechanism that will be utilized for this project is the use of water and/or amended water for dust suppression and natural ventilation as work is performed in an outdoor environment. If sample results indicate workers are exposed above the AL or PEL-TWA then additional controls may need to be instituted and personal will be required to wear tespiratory protection

\section{Respirators}

As all workers are deemed to be performing incidental work. but respirators will be worn during baseline sampling activities as indicated in Table 1, until sample results are obtained. Once known, PPE levels can be downgraded if work practices and controls are proven to be effective. Respirators will be selected in accordance with PRD-2109, Respiratory Protection and Appendix B of PRD2105, Lead.

Phenix shall have and follow a written Respiratory Protection Program and be responsible to confirm to the requirement of 29 CFR 1926.103 and 29 CFR 1910.134, and American National Standards Institute (ANSI) Z88.2 standard. Medical exam and fit test records will be submitted as part of the training documentation to the CWI Safety POC.

If initial and routine sampling indicate the $\mathrm{AL}\left(30 \mu \mathrm{g} / \mathrm{m}^{3}\right)$, but $<\mathrm{PEL}$ $\left(50 \mu \mathrm{g} / \mathrm{m}^{3}\right)$.may be or has been exceeded then a respirator can be prescribed either at the direction of the Health and Safery Lead or at a worker's request.

\section{Protective Clothing}

Level D PPE will be prescribed for this project, but Level C (use of half-faced respirators) will be wom for tasks specified in Table 1 until lead exposure levels 
are known. Used disposable or designated work clothing (coveralls) will be sealed in poly bags or drums provided by Phenix. Work clothing will be donned and doffed in designated areas and shall not be the same clothing worn home by workers. Phenix will be responsible for the proper laundering and disposal of all PPE, paper and misc. debris generated during the work phase of this project.

\section{Hygiene Facilities}

Proper hygiene facilities will help eliminate or reduce cross-contamination and ingestion of lead-contaminated dust. Hand washing facilities will be used to decontaminate workers. A waterless wash area will be set up inside the range itself. Portable toilets and washroom facilities at the job-site must be provided and maintained by the Phenix. Water, soap and towels will be provided. Hands and face will be washed before all breaks and at the end of the day. If employees are working in an environment > or equal to the PEL then they will be provided a clean change area, PPE, and a shower facility. It is recommended to have disposable PPE to doff at a designated area. Follow proper guidelines for labeling and disposal of lead contaminated PPE. Employees are not permitted to enter eating facilities or leave work area wearing lead-contaminated PPE or equipment. Contact CWI Safety Contact about shower facility locations at the INL.

\section{Air Monitoring Data}

As stated previously, air sampling will be performed on this job. Based on expected results, the greatest potential for lead exposure will occur during the demolition and removal, excavation, stockpiling, screening of lead contaminated soil, and vehicle loading. No previous exposure monitoring is available so baseline sampling will be performed. It is expected that 8-hour time weighted average exposures on the job will be lower then the action level. See Table 1 for prescribed sampling methods.

\section{Workers Right to Know}

Sample results must be disseminated to each employee within 24 hours of laboratory analysis receipt. Affected employees have the right to be informed about sampling methods, the monitoring process, and may request copies laboratory results. Identified exposures in excess of the established limits must be reported to the contractor within one hour of receipt. If results are greater than the AL or PEL then the prescribed guidelines of PRD-2105 shall be implemented. All workers on this project are informed of their blood lead levels as soon as results are received.

\section{Training}


All workers entering the controlled work areas will have Lead Awareness and Lead Worker training to be informed as the hazards associated with lead exposure ways to eliminate and reduce exposure to lead.

\section{Work Practice Program}

Safety is first and foremost on every project undertaken by Phenix. Each employee is expected to review and adhere to the safety practices and procedures outlined in the site specific Health and Safety Plan. Daily discussions are held as needed to review any potentially hazardous tasks or procedures to be undertaken that day or any events from the previous day that could have had a potential for causing an accident. 
T'able 1. Activities and associated potential or anticipated exposure hazard.

\begin{tabular}{|c|c|c|c|c|c|}
\hline Activity & $\begin{array}{l}\text { Potential or } \\
\text { Anticipated } \\
\text { Exposures }\end{array}$ & $\begin{array}{l}\text { Anticipated } \\
\text { Exposure } \\
\text { Level }\end{array}$ & $\begin{array}{l}\text { Actions Used to Eliminate or Mitigate } \\
\text { Exposures }\end{array}$ & $\begin{array}{l}\text { Sample } \\
\text { Method }\end{array}$ & Sample Frequency \\
\hline Mobilization $^{\top}$ & Lead' - airboxise & $<A L$ & Lead Awareness and Lead Worker training. & $\mathrm{NA}$ & No Sumpling. \\
\hline $\begin{array}{l}\text { Cunstruction Staking and } \\
\text { Surveying' }\end{array}$ & Leud ${ }^{3}-$ airborne & <Al. & Lead Awareness and Lead Worker truning. & NA & No Sampling. \\
\hline Derrotition and Rerwoval ${ }^{?}$ & Lead'- airborve & $\angle A L$ & \multirow{3}{*}{$\begin{array}{l}\text { - Lead Awarentess and Lead Worker } \\
\text { training. } \\
\text { Level D PPE } \\
\text { - Dust suppression with water. } \\
\text { - Persomel will work with closed cabs. } \\
\text { - Personnel will work upwind from } \\
\text { visible dust. }\end{array}$} & \multirow{2}{*}{$\begin{array}{l}\text { NIOSH } \\
7300 \\
\text { (air } \\
\text { samples) }\end{array}$} & \multirow{3}{*}{ 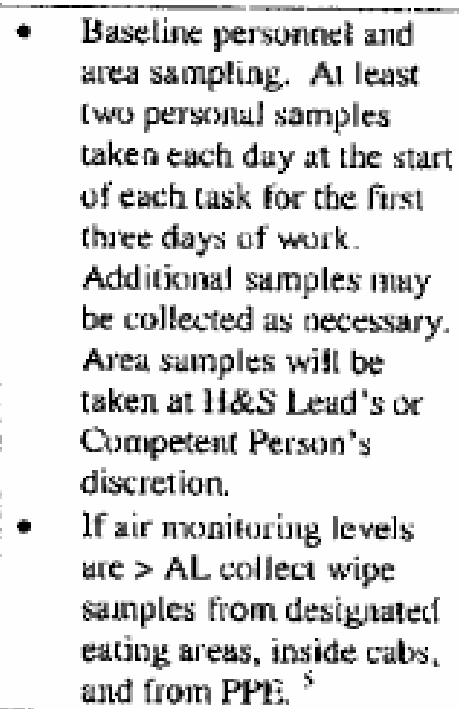 } \\
\hline $\begin{array}{l}\text { Excuvation, Stock piling, } \\
\text { und Scretening of Lead- } \\
\text { Contaninated Soil' }\end{array}$ & $\begin{array}{l}\text { Leud } \\
\text { Lead - airberne } \\
\text { contamination }\end{array}$ & $<\mathrm{Al}$ & & & \\
\hline Vehick hading? & Lead? - ainborne & $<\overline{A L}$ & & $\begin{array}{l}\text { NIOSH } \\
91(x) \\
\text { (wipe } \\
\text { samples) }\end{array}$ & \\
\hline $\begin{array}{l}\text { Final Grading and } \\
\text { Revegetation of Disturbed } \\
\text { Areus }\end{array}$ & Lead ${ }^{3}$ - airborne & $<A L$ & $\begin{array}{l}\text { - Lead Awareness and Lead Worker } \\
\text { training. } \\
\text { - If deerned neccessary, dust suppression } \\
\text { with water. }\end{array}$ & NA & No sampling. \\
\hline Denobjitization ${ }^{1}$ & Lead $^{3}$ - airborne & $<\mathrm{AL}$ & Lead A wareness and Lead Worker training. & NA & No sampline. \\
\hline 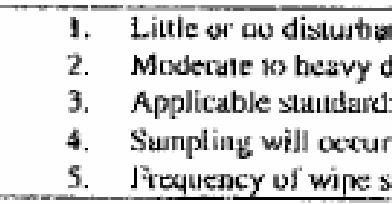 & $\begin{array}{l}\text { unikipated. } \\
\text { urbatce auricipated. } \\
\text { JCFR } 1926.62 \text {, Letad } \\
\text { results frum other at } \\
\text { ples will uccur at the }\end{array}$ & $\begin{array}{l}\text { ies }>\text { AL. (AL }=\text { ? } \\
\text { eelion of the } H\end{array}$ & $\begin{array}{l}\left.\text { g/ } / \mathrm{m}^{3}\right) \text {. } \\
\text { th and Safety Lead or Comperent Person. }\end{array}$ & & \\
\hline
\end{tabular}




\section{Vendor Data Review System Final Disposition Screen}

This vendor data item has been given the following disposition codes

\begin{tabular}{|c|c|c|c|c|}
\hline Reviewer & $\begin{array}{l}\text { Revision } \\
\text { Level }\end{array}$ & Date & $\begin{array}{l}\text { Disposition } \\
\text { Code }\end{array}$ & Comments \\
\hline TUOTT LEE C & 0 & $\begin{array}{l}02- \\
\text { OCT- } \\
06\end{array}$ & D & \\
\hline TUOTT LEE C & 0 & $\begin{array}{l}02- \\
\mathrm{OCT} \text { - } \\
06\end{array}$ & & \\
\hline FRITZ KURT D & 0 & $\begin{array}{l}04- \\
\text { OCT- } \\
06\end{array}$ & D & \\
\hline $\begin{array}{l}\text { LANDIS } \\
\text { JOSEPH A }\end{array}$ & 0 & $\begin{array}{l}05- \\
\text { OCT- } \\
06\end{array}$ & A & no comments \\
\hline
\end{tabular}

1. Reference to the construction Lead standard is identified as 29CFR 1910.62 on footnote 3 of Table 1. Suggest change to 29CFR 1926.62, "Lead". 2. Section 5 suggest delete incidental lead work. 3 . Section 12 , Note 2 and Note 3, delete "INL construction Managemeent and INL Construction Management IH" and change to "Miscellaneous Sites Completion Project Point of contact" 4. Section 12, Number 5 , change to PRD-1501, "Work Control". 5. Section 15, Level D PPE is prescribed for this project. Personnel working in close proximity to lead contaminated

$\begin{array}{ll}\text { MCMANAMON } 17- & 17- \\ & \text { OCT- B }\end{array}$ LAWRENCE E $\quad 06$

$\begin{array}{lll}\begin{array}{l}\text { MCMANAMON } \\ \text { LAWRENCE E }\end{array} & \begin{array}{l}18- \\ \text { OCT-A } \\ \end{array} & 06 \\ \text { VANDEL } & 0 & 02- \\ \text { DOUG S } & & \text { OCT-A } \\ & & 06 \\ & & \\ \text { DRIEVER } & & 02-\end{array}$
materials may need to wear some type of coveralls to ensure we don't allow contaminated clothing to leav controlled areas. Suggest this is not the same clothing they wear home every evening. 6 . editorial only: Section 13, last sentense, insert "wear" or "continue with" between the words "to" and respirator" and change respirator to "respiratory" .7 . Since we are to use respiratory protection, PRD-2109, Respiratory Protection" applies and section 3.1.1 requires each subcontractor to have a written Respiratory Protection Program that complies with all requirements of $29 \mathrm{CFR}$ 1926.103, 29 CFR 1910.134, and American National Standards Institute (ANSI) Z88.2 standard. Both medical exams and fit test records will need to be submitted as part of the training documentation. 


$\begin{array}{lll}\text { MIKELK } & 0 & \text { OCT- D } \\ & & 06 \\ & & 03- \\ \text { JOLLEY } & & \text { OCT. D } \\ \text { WENDELL L } & 0 & \end{array}$

$\begin{array}{ll}\text { MCMANAMON } & 11- \\ \text { LAWRENCE E } & \text { OCT- C } \\ & 06\end{array}$

1. The assumption in this plan is there will be no potential for lead exposure at or above the action level or exposure limits and therefore no respiratory protection is anticipated unless dusty conditions are generated. It is recommended personel with potential lead exposure that are to be monitored for lead exposure begin wearing respiratory protection until exposure levels are known. Once known, PPE could be downgraded if exposure levels are controlled by work practices, ect. If levels are found to be in excess of teh $\mathrm{AL}$ or $\mathrm{PEL}$, then additional controls may be instituted and the personnel were protected by the respiratory protection. We don't want to start without respiratory protection; over expose workers to lead: and prove it by monitoring. We need to be proactive, not reactive. 2. General I would make sure that they include a statement that if the subcontractor has an unprotected exposure over the AL or PEL they are required to inform you with in the required time frame as listed in PRD-2111. 3. Section 8 I would add that this person is responsible for all exposure monitoring activities and ensuring that samples are sent to an AlHA accredited lab. 4. Section 12। would rename EXPOSURE MONITORING OR SAMPLING and DATA COLLECTION. I would then state that all sampling will be performed as outlined in TABLE 1. Here is where an expanded decision logic could be incorporated if sample results are $>A L$ but PEL then ............. In section 12 it also talks about written

compliance documentation shall be provided using any of the following: They need to be sure that if they do exceed the PEL and have to create a compliance plan that they are able to cover all of the code driven requirements of the plan in those documents listed. I doubt that can be done with a SWP. 5. TABLE 1 The information in the column Sample Frequency should indicate number of and how often samples will be taken. Is 1 sample enough or should 5 samples be taken? They also talk about medical surveillance, which could be removed since it really isn't sampling. They also have listed in the footnotes that 29 CFR 1910.62 is the applicable standard. 6. Section 14 They say all of the work is incidental work (I am assuming that this means they believe all exposures will be below the $\mathrm{AL}$, but the mention that respirators will be used in dusty environments. I would better define expected dusty environments maybe even to the task and worker level because they don't want an unprotected over exposure. They could expand section 5 to include a little more detail and expected exposures and to whom for each of the task listed in this section. 


$\begin{array}{ll}\text { VDR Number: } & \text { VDR-153751 } \\ \text { Revision Level: } & 2 \\ \text { Project Number: } & 23368-152173 \\ \text { Transmittal Number: } & \text { S-507296-05R.2 } \\ \text { Transmittal Status: } & \text { Mandatory Approval } \\ \text { Line Item: } & 1\end{array}$

Disposition Code:

A

Final Comments: 
F-216 
VDS \#12_153756 
F-218 


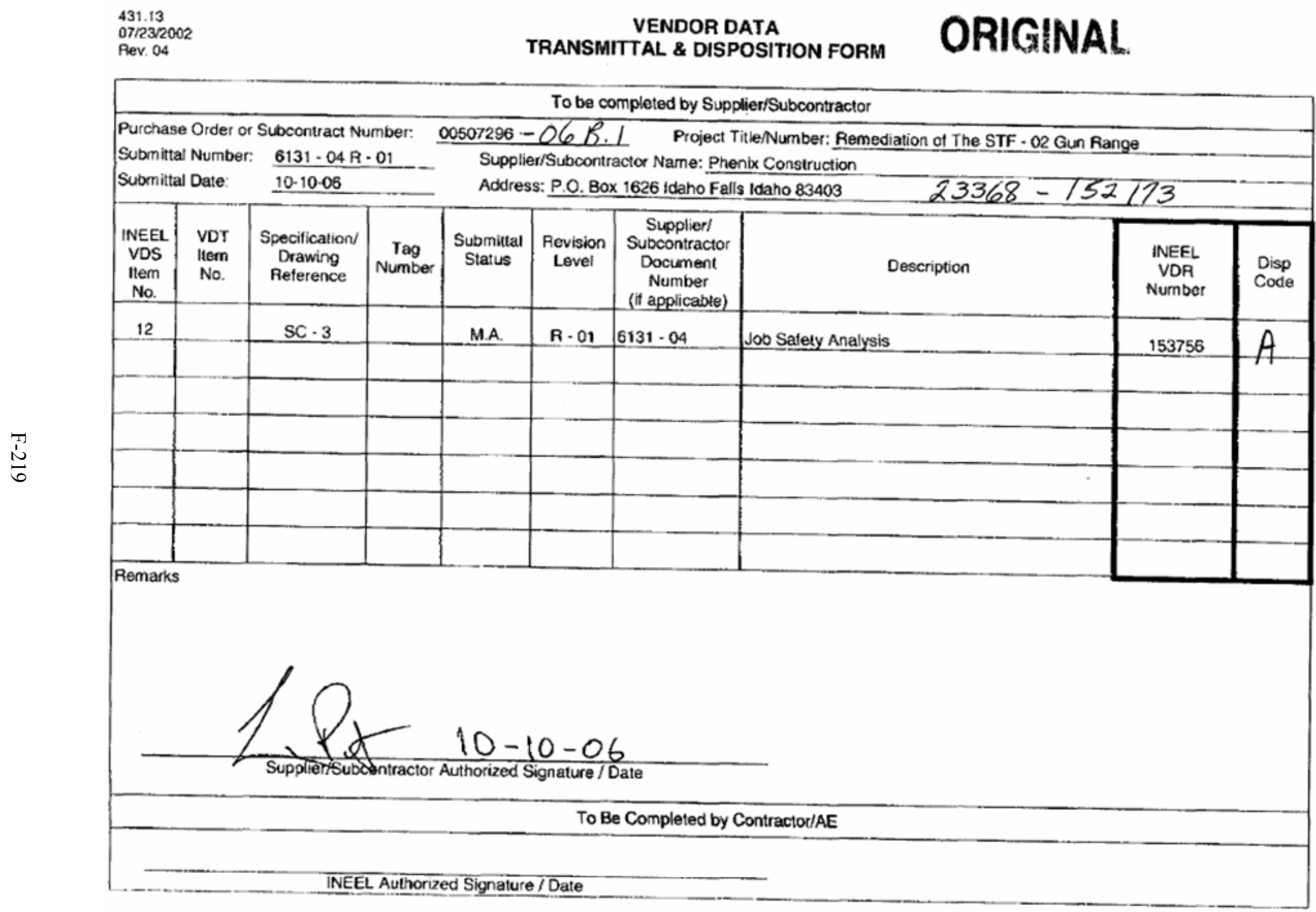


432.58

$6 / 13 / 05$

Pev. 05

LISTING OF PERSONNEL WHO DEVELOPED JSA

\begin{tabular}{|c|l|l|l|}
\hline S-Number & Print Name & Signature & ite \\
\hline 51896 & Lance Peterson & & All \\
\hline 56864 & Kelly Eborn & & All \\
\hline B5054 & Whe Garcia & & AL \\
\hline & & & \\
\hline & & & \\
\hline
\end{tabular}

$\stackrel{T}{N}$

SIGNATURE VERIFIES I HAVE READ AND UNDERSTAND THE CONTENTS OF THE JSA

Ñ
CONSTRUCTION MANAGEMENT

JOB SAFETY ANALYSIS

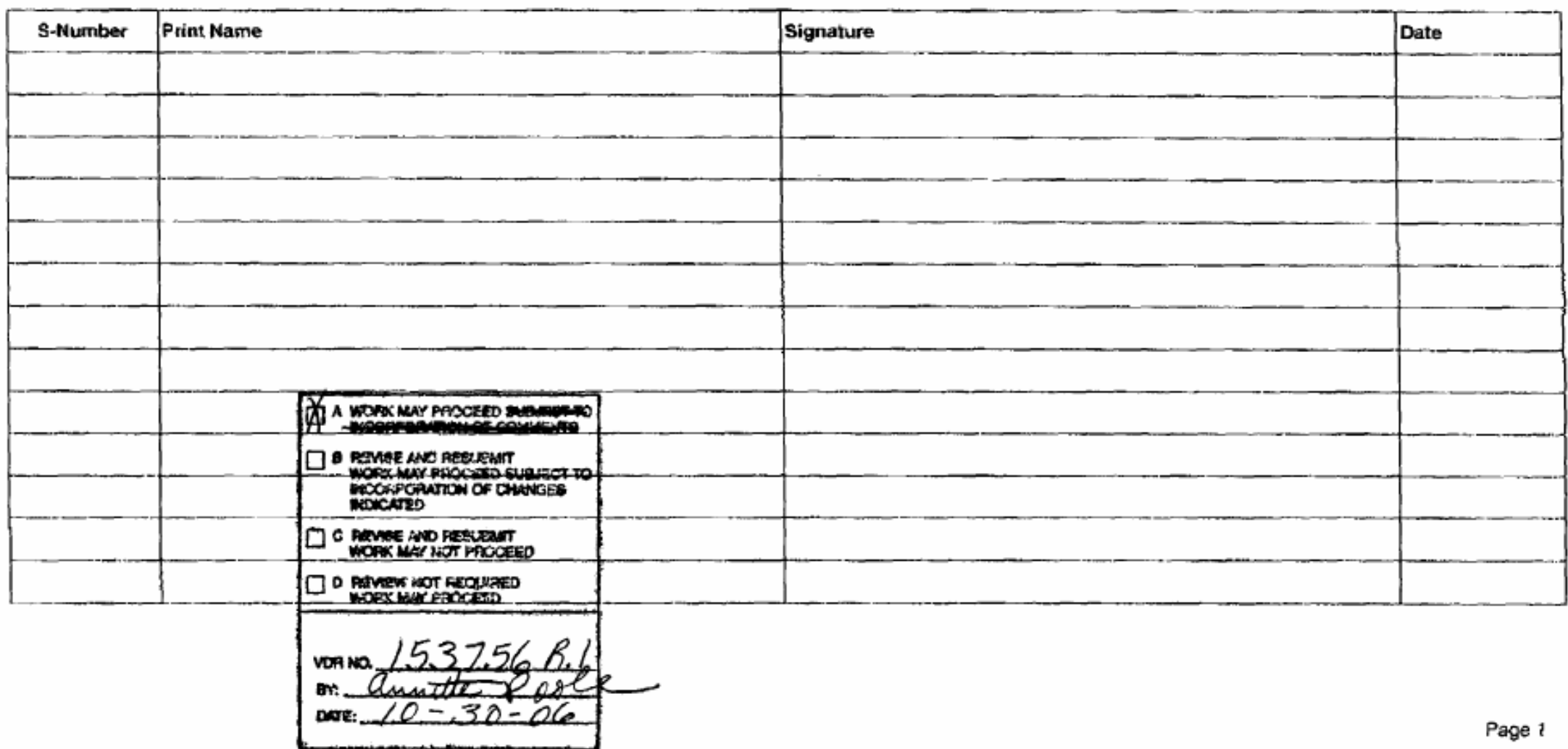

\begin{tabular}{|l|l|l|}
\hline items developed & Date \\
\hline All & Rev: & \\
\hline ALL & & \\
\hline
\end{tabular}




\begin{tabular}{|c|c|c|c|c|}
\hline \multicolumn{2}{|c|}{$\begin{array}{l}\text { Job } \\
\text { femediation of The STF - O2 Gun Range Contract No. } 00507296\end{array}$} & \multicolumn{3}{|c|}{$\begin{array}{l}\text { JSA By } \\
\text { Lance Peterson }\end{array}$} \\
\hline \multirow[t]{3}{*}{$\begin{array}{l}\text { FacikityfProject \& Location } \\
\text { INL STF - 02 Gun Range }\end{array}$} & \begin{tabular}{|l|} 
Date Ot Analysis \\
$09-23-06$
\end{tabular} & \multirow{2}{*}{\multicolumn{2}{|c|}{$\begin{array}{l}\text { Reviewed By } \\
\text { CM Point of Contact }\end{array}$}} & \multirow{3}{*}{$\begin{array}{l}\text { Approved By } \\
\text { SubcontractoriFA/Supt. } \\
\text { S\&H Fepresentative / Subcontractor Safety }\end{array}$} \\
\hline & Revision Review & & & \\
\hline & & \multicolumn{2}{|c|}{ S\&H Representative / CM Safety } & \\
\hline \multicolumn{4}{|c|}{$\begin{array}{l}\text { Briefly Describe The Job and Expected hesult } \\
\text { Miobization, surveying. excavation and greding berm soil to leach pond, remove electrical circuits and poles, asphalt removat, } \\
\text { remove creosote railroad ties, remove burn barrel and test stand, excavation of lead contaminated soil, screening lead } \\
\text { contaminated soil, remove shoot house, de con pad construction, remove perimeter fence, reclaim borrow area and re vegetation. }\end{array}$} & $\begin{array}{l}\text { SME REVIEW REQ'D* DNO TJ YES } \\
\text { Evaluated by: } \frac{}{\text { Signed CM POC }} \text { Date }\end{array}$ \\
\hline \multicolumn{4}{|c|}{ 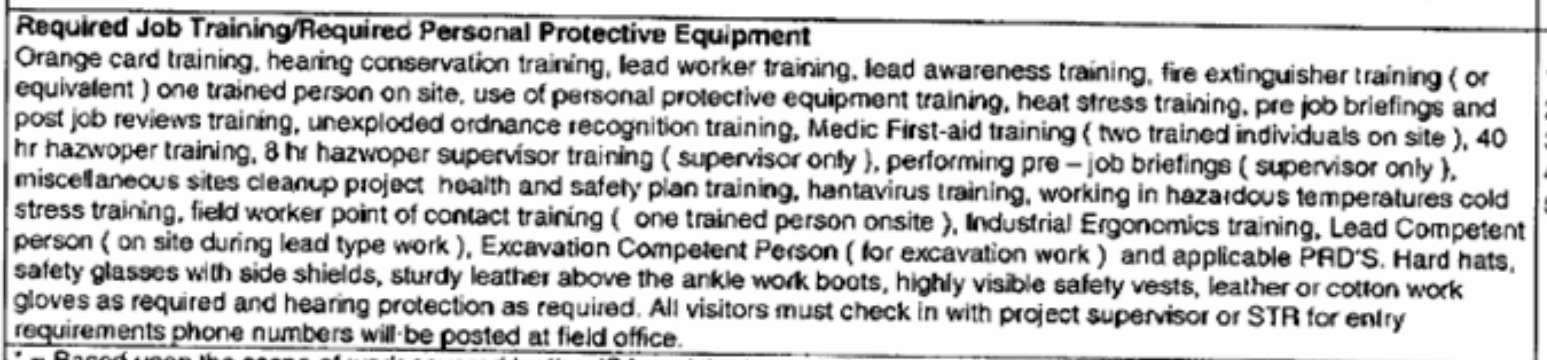 } & $a_{3}=$ Discipline \\
\hline \multicolumn{5}{|c|}{ JSA $: 607024$} \\
\hline Sequence Of Basic Job Steps & \multicolumn{2}{|c|}{\begin{tabular}{|c|} 
Potential Mazards \\
\end{tabular}} & \multicolumn{2}{|c|}{ Hazard ControlvPPE } \\
\hline 1. Mobiization & \multicolumn{2}{|c|}{ 1.A Heavy machinery i.e.; struck by, noise } & \multicolumn{2}{|c|}{$\begin{array}{l}\text { 1. Al Stay out of pinch points, swing and working areas of equipment. Workers to } \\
\text { wear high visibility vests } \\
\text { 1. A2 Ensure back up alaims are cperational or use spotter. }\end{array}$} \\
\hline & \multicolumn{2}{|c|}{ 1. B Back strain and Pinch point } & \multicolumn{2}{|c|}{$\begin{array}{l}\text { 1. B1 Use proper litting procedures do not itt more than } 50 \mathrm{bs} \text { or } 1 / 3 \text { body weight } \\
\text { for unloading and loading equipment and materials. }\end{array}$} \\
\hline 2. Serting up work areas & \multicolumn{2}{|c|}{$\begin{array}{l}\text { 2. A Open trench and heavy machinery } \\
\text { working. }\end{array}$} & \multicolumn{2}{|c|}{$\begin{array}{l}\text { 2. A1 Construction area will be roped oft (as required) and properly posted. Ensure } \\
\text { that excavalion checklist has been filled out by competent person. }\end{array}$} \\
\hline & \multicolumn{2}{|c|}{ 2.B CERCLANAZWOPER hazards } & \multicolumn{2}{|c|}{ 2.B1 Post area with proper CERCLAMHAZWOPER postings on perimeter. } \\
\hline
\end{tabular}




\section{CONSTRUCTION MANAGEMENT} JOB SAFETY ANALYSIS

\begin{tabular}{|c|c|c|}
\hline \multirow{4}{*}{ 3. Survey work } & Potential Hazards & Hazard ControlPPE \\
\hline & $\begin{array}{l}\text { 3.A Heary machinery working i.e.: struck } \\
\text { by noise }\end{array}$ & $\begin{array}{l}\text { 3. A1 Ensure back-up alarms are operational or use spotter. } \\
\text { 3. A2 Be aware of equipment movement at al times, grourd people will wear highly } \\
\text { visible safery vests. } \\
\text { 3. A3 Employees wear proper P.P.E. hard hats, sately glasses with side shields, } \\
\text { sturdy leather above the ankle work boots, leather gloves and hearing protection } \\
\text { using previous data. }\end{array}$ \\
\hline & 3.8 Possible eye and hand injury & $\begin{array}{l}\text { 3. Bi Use proper eye protection safety glasses with side shields and leather } \\
\text { gloves. }\end{array}$ \\
\hline & $\begin{array}{l}\text { 3.CPotential slips, trips lalls whise working } \\
\text { on stopes }\end{array}$ & 3.C1 Ensure good footing while wotking on slopes no ruming up and down stopes. \\
\hline \multirow[t]{5}{*}{$\begin{array}{l}\text { 4. Giading and excavation of berm soil to leach } \\
\text { pond. }\end{array}$} & $\begin{array}{l}\text { 4.A. Existing utifties possible electrical } \\
\text { shock, contamination }\end{array}$ & $\begin{array}{l}\text { 4.A1 Layout excavation area and notify CWI lor S.I.T. to locate and identify } \\
\text { existing utilities. Review utifity plan as needed. } \\
\text { 4.A2 Use } 2^{\prime}, 5^{\circ} \text { method in locating existing lines in excavation area. } \\
\text { 4.A3 If unidentified utilities or unknowns are encountered during excavation } \\
\text { activities, stop work and notily CWI POC. } \\
\text { 4.A4 Obtain clearance from power management follow direction from power } \\
\text { management while working under overhead electrical. }\end{array}$ \\
\hline & 4.B Heavy machinery i.e.: struck by, noise & $\begin{array}{l}\text { 4.B1 Stay out of way of pinch points, swing and working areas of equipment. } \\
\text { Workers to wear high visibility vests. } \\
\text { 4.B2 Ensure backup alarms are operational or use spotter. } \\
\text { 4.83 Be aware of equipment movement at all times. Operators maintain eye } \\
\text { contact with ground people. Ground people will wear highly visible safety vests. } \\
\text { 4.B4 Ensure equipment is in safe condition - backup alarms working. no broken } \\
\text { windows, fire extinguishers in place etc. ( Equipment inspection tag by CWI prior to } \\
\text { equipment use.) } \\
\text { 4.85 Employees wear proper P.P.E. equipment such as: hard hats, salety glasses } \\
\text { with side shields, sturdy leather above the ankle work boots, and hearing protection } \\
\text { using previous data. }\end{array}$ \\
\hline & 4.C Flammable Bums. & $\begin{array}{l}\text { 4.C1 No open flames or burning, turn off equipment while refuefing equipment( } \\
\text { avoid any spills ) }\end{array}$ \\
\hline & $\begin{array}{l}\text { 4.D Heavy machinery accident, roll over ( } \\
\text { bodily injury) }\end{array}$ & $\begin{array}{l}\text { 4.D1 Ensure equipment is equipped with proper rol over protection per } 1926 \\
\text { Subpart } " W \text { - Wear seat bets. Follow operators manual } \\
\text { 4. }\end{array}$ \\
\hline & 4.E Cave - inn's & $\begin{array}{l}\text { 4.E1 Implement OSHA procedures for class C soil Subpart "P" } \\
\text { 4.E2 Excavation checklist completed daily by competent person prior to } \\
\text { excavation. } \\
\text { 4.E3 If trench exceeds } 5 \text { ' in depth, } 1.5 \text { to } 1 \text { sioping required. } \\
\text { 4.E4 Place axcavated material minimum 2' away from edge of trench. } \\
\text { 4.ES Slope trench over } 4 \text { ' deep } 1.5 \text { to } 1 \text { for access and egress. }\end{array}$ \\
\hline
\end{tabular}




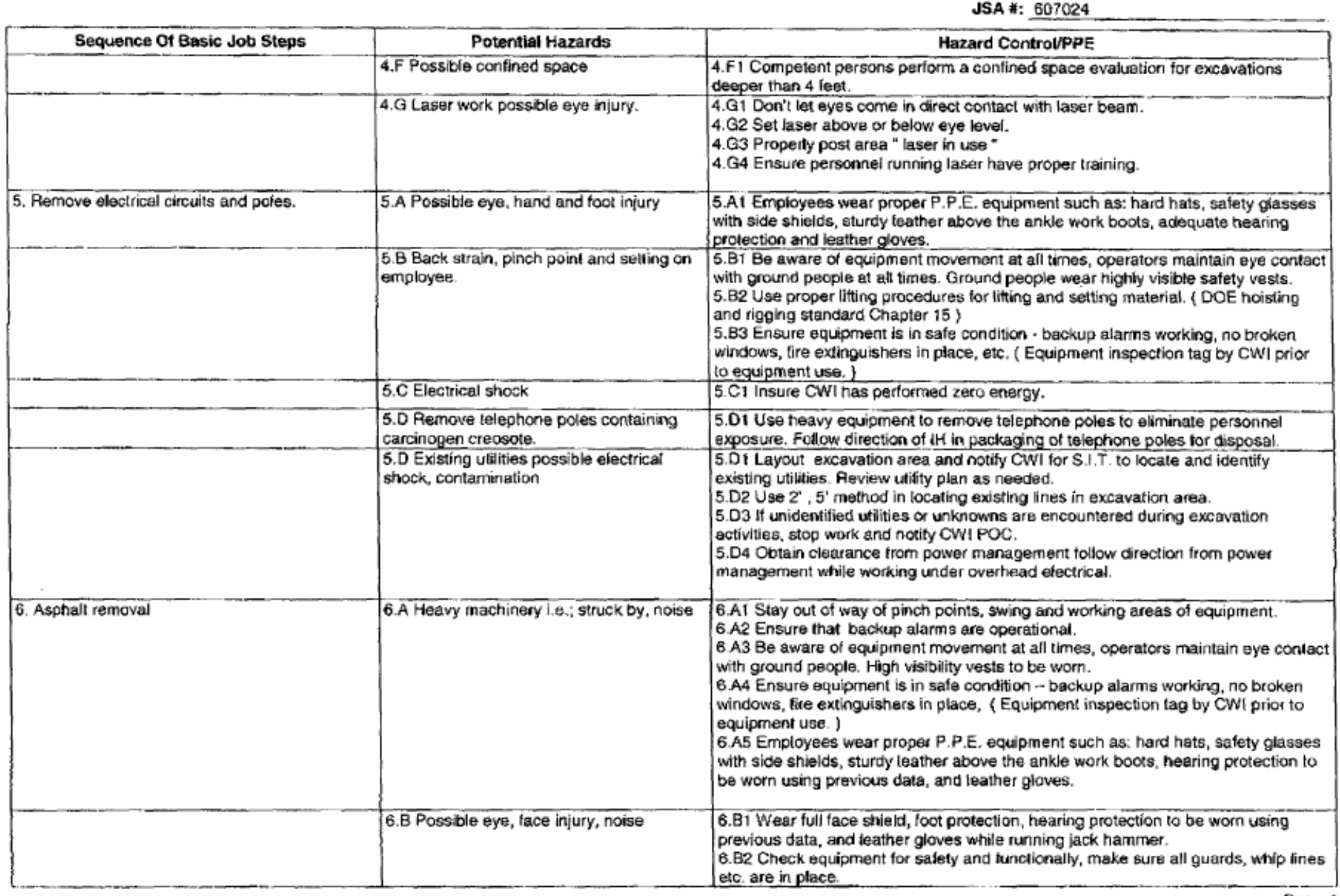




\section{CONSTRUCTION MANAGEMENT JOB SAFETY ANALYSIS}

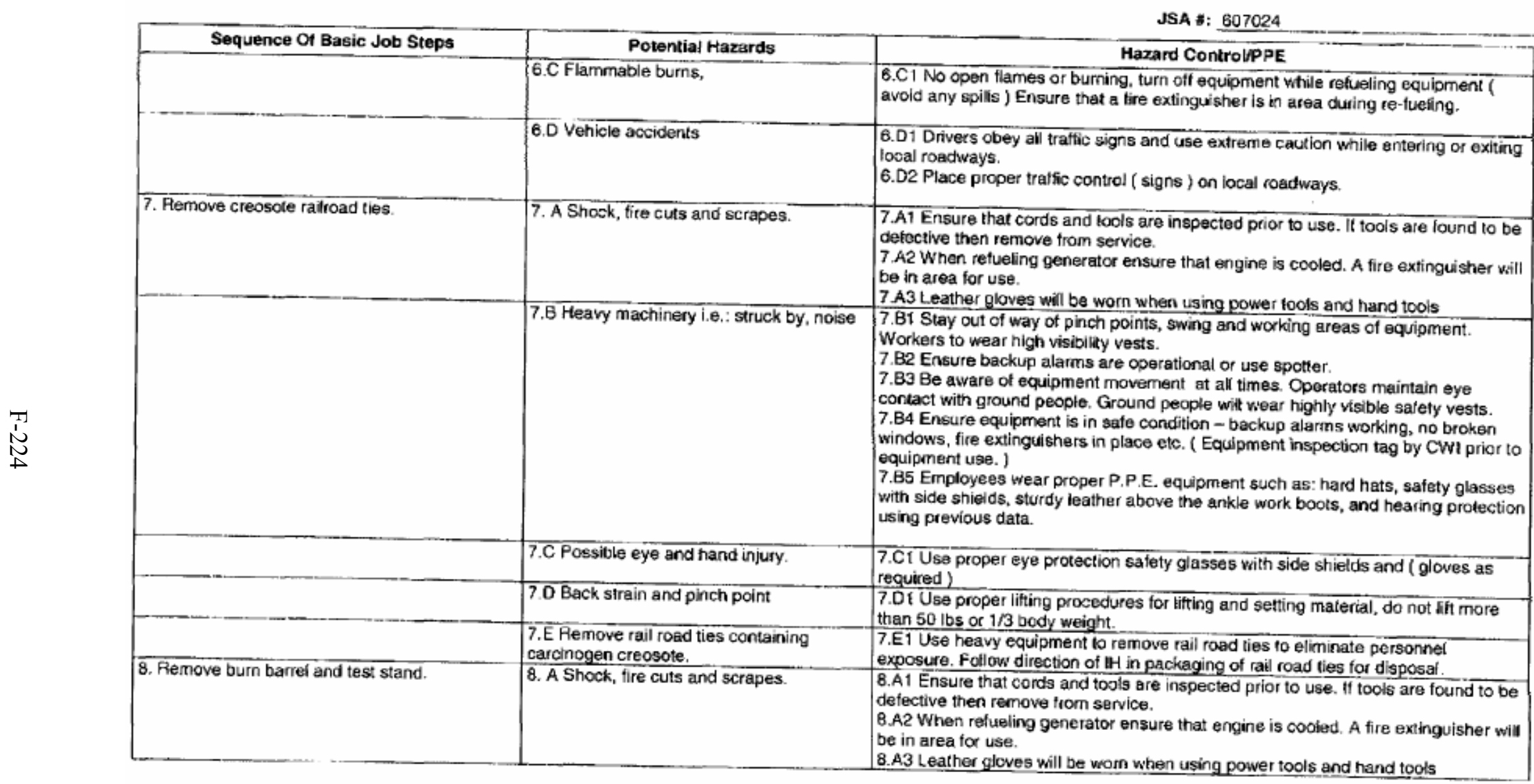




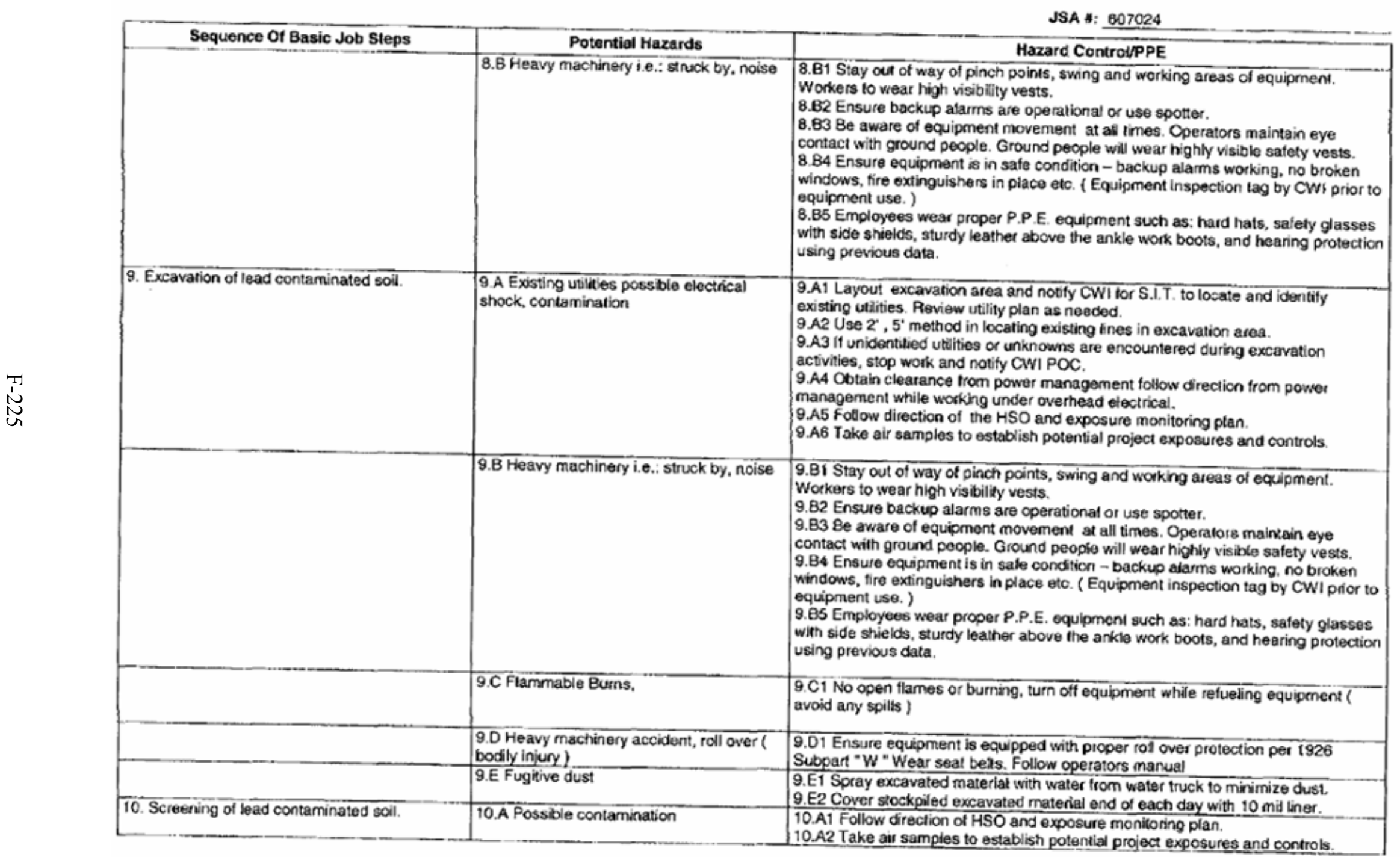




\section{JOB SAFETY ANALYSIS}

JSA : 607024

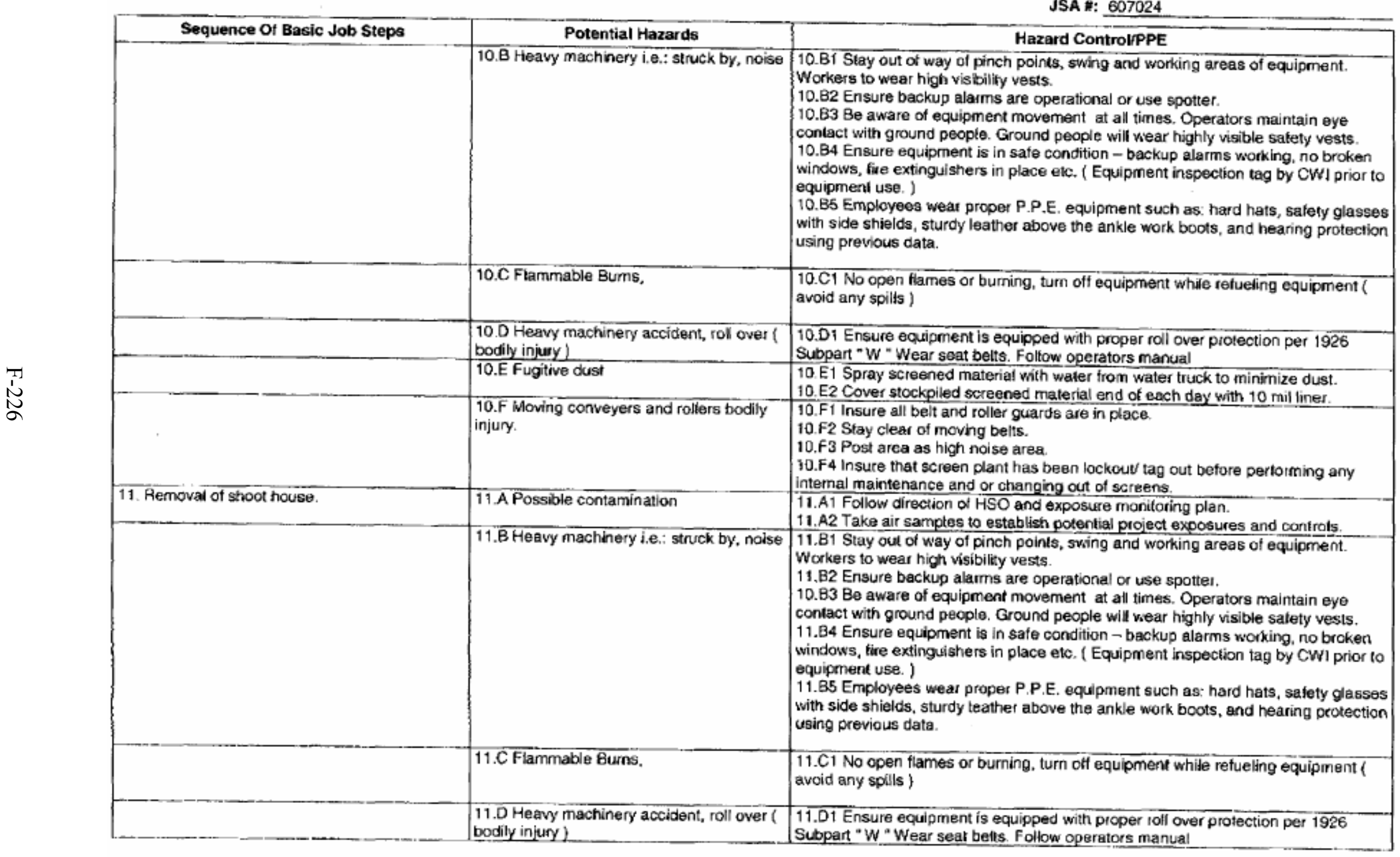




\section{CONSTRUCTION MANAGEMENT JOB SAFETY ANALYSIS}

\begin{tabular}{|c|c|c|}
\hline & & JSA : 607024 \\
\hline Sequence Of Basic Job Steps & Potential Hazards & Hazard Control/PPE \\
\hline & 11.E Fugitive dust & 11.E1 Spray demolished material with water from water truck to minimize dust. \\
\hline & 11.F Pigeon/Bird feces in shooting shack. & $\begin{array}{l}\text { 11.F1 Use heavy equipment to demolish shooting shack to eliminate personnel } \\
\text { exposure. Such as excavator with thumb. }\end{array}$ \\
\hline & $\begin{array}{l}\text { 11.G Over head hazards during removal of } \\
\text { shoot house. }\end{array}$ & $\begin{array}{l}\text { 11.G1 Use heavy equipment to demolish shooting shack to eliminate personnel } \\
\text { exposure. Such as excavator with thumb. }\end{array}$ \\
\hline & $\begin{array}{l}\text { 11. H Remave rail road ties containing } \\
\text { carcinogen creosote. }\end{array}$ & $\begin{array}{l}\text { 11.H1 Use heavy equipment to remove rail road ties to eliminate personnel } \\
\text { exposure. Follow direction of iH in packaging of rail road ties for disposal. }\end{array}$ \\
\hline & 11.F Vehicle accidents & $\begin{array}{l}\text { 11. F1 Drivers obey all traffic signs and use extreme caution while entering or } \\
\text { exiting local roadways. } \\
\text { 11.F2 Place proper tuaffic control ( signs f on local roadways. }\end{array}$ \\
\hline 12. De con pad construction. & 12. A Heavy machinery i.e.: struck by, noise & $\begin{array}{l}\text { 12.A1 Stay out of way of pinch points, swing and working areas of equipment. } \\
\text { Workers to wear high visibility vests. } \\
\text { 12.A2 Ensure backlp atarms are operational or use spotter. } \\
\text { 12.A3 Be aware of equipment movement at all times. Operators maintain eye } \\
\text { contact with ground people. Ground people will wear highly visible salefy vests. } \\
12 . \text { A4 Ensure equipment is in safe condition - backup alarms working, no broken } \\
\text { windows, fite extinguishers in place etc. ( Equipment inspection tag by CWH prior to } \\
\text { equipment use.) } \\
\text { 12.A5 Employees wear proper P.P.E. equipment such as: hard hats, safety glasses } \\
\text { with side shieids, sturdy leather above the ankle work boots, and hearing protection } \\
\text { using previous dala. }\end{array}$ \\
\hline & 12.8 Flammable Bums, & $\begin{array}{l}\text { 12.B1 No open flarnes or burring, turn off equipmert while refueling equipment ( } \\
\text { avoid any spills ) }\end{array}$ \\
\hline & $\begin{array}{l}\text { 12.C Heavy machinery accident, roll over ( } \\
\text { bodily injury) }\end{array}$ & $\begin{array}{l}\text { 12. Ci Ensure equipment is equipped with proper roll over protection per } 1926 \\
\text { Subpart " } W \text { " Wear geat betts. Follow operators manual }\end{array}$ \\
\hline & 12.D Possible eye and hand injury. & $\begin{array}{l}12.01 \text { Use proper eye protection safely glasses with sióe shields and (gloves as } \\
\text { required) }\end{array}$ \\
\hline & 12.E Back strain and pinch poin! & $\begin{array}{l}\text { 12. E1 Use proper lifting procedures for lifting and setting material, do not fitt more } \\
\text { than } 50 \text { bs or } 1 / 3 \text { body weight. }\end{array}$ \\
\hline
\end{tabular}




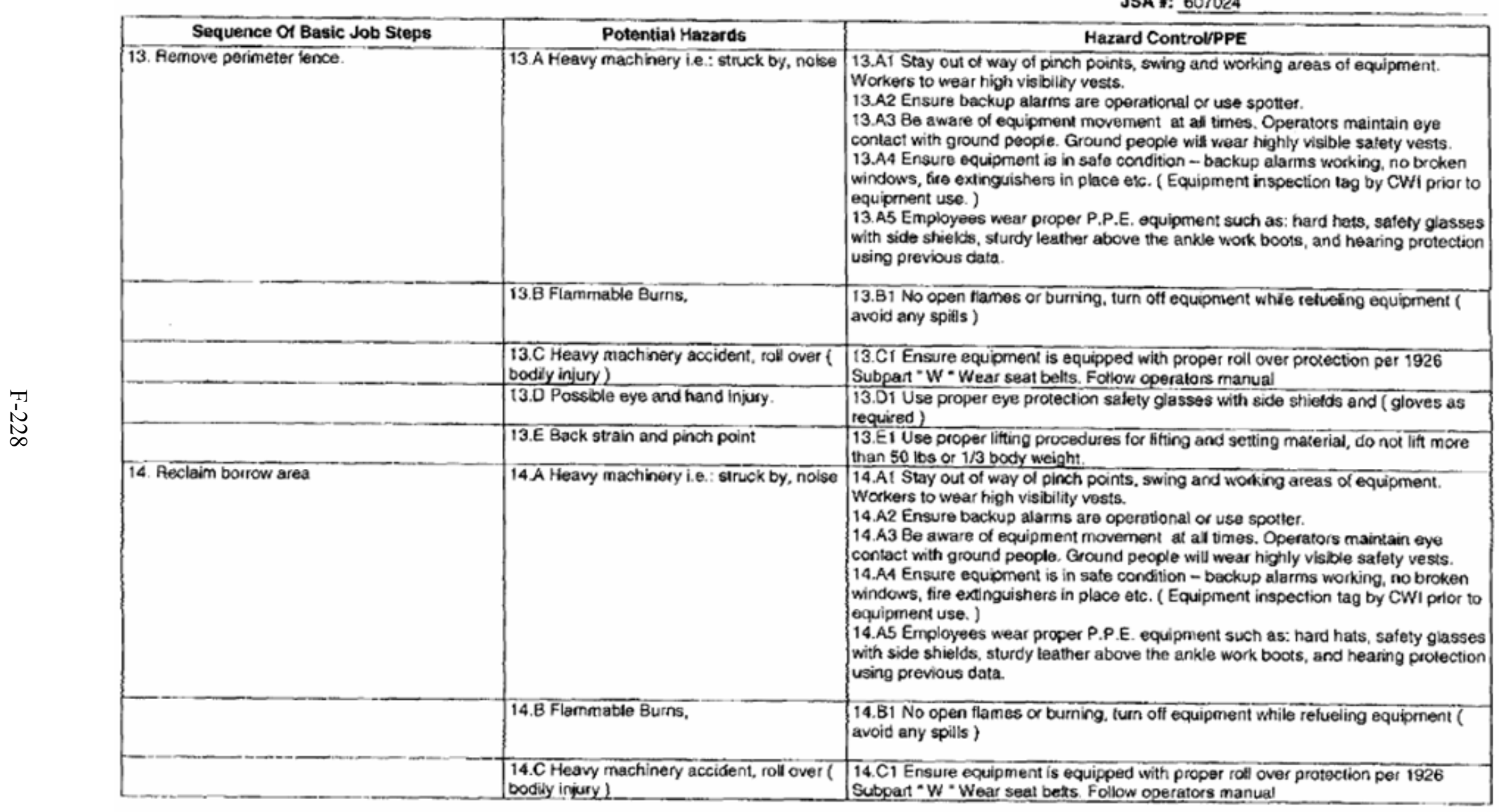


JSA \#: 607024

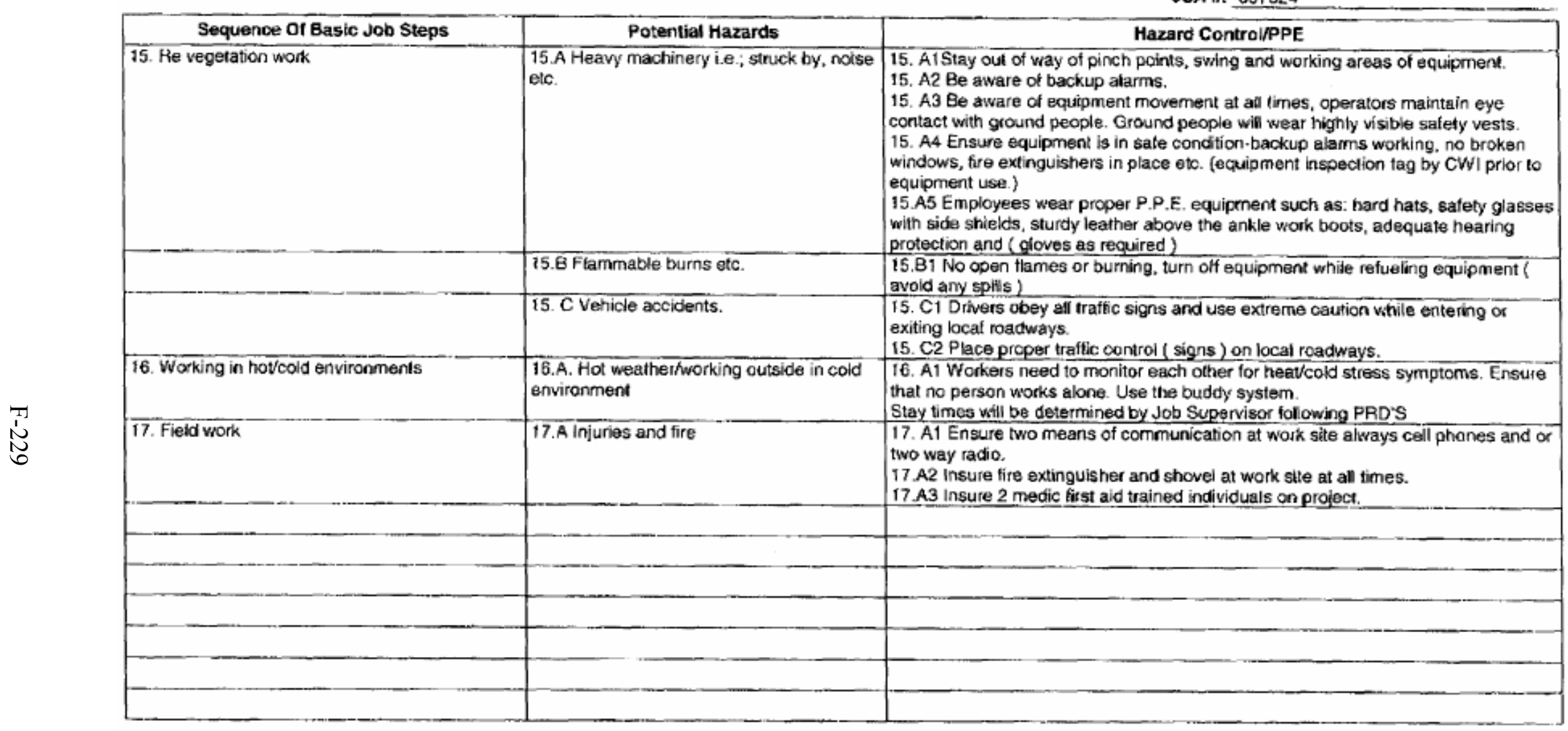


Page 1 of 2

\section{Vendor Data Review System Final Disposition Screen}

This vendor data item has been given the following disposition codes

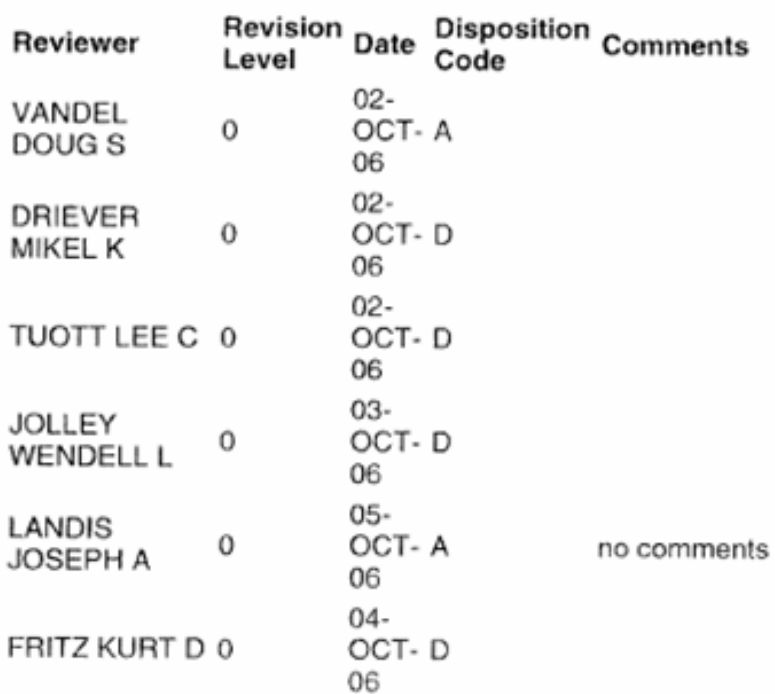

\footnotetext{
Missing hazards need ing to be addressed include the following: 1. Performing work on steep slopes with gravel creating a potential slip, trip, and fall hazard. Uneven walking/working surfaces. (suggest 3c) 2. CERCLA/HAZWOPER hazards and postings on perimeter. 3. Lack of connumication, proper emergency supplies for field work as this is field work. 4. Conveyor belts and machinery specialized for this operation- hazards including rollers, inrunning nip points, noise, vibration, moving with other equipment including setup and adjustments. 5. Striking unidentified lines- subsurface utilities (Suggest 5d) 6.

Independant verification that lines have had zero energy verification per PRD-2011, Appendix A 7. Pigeon/bird feces in shooting shack (suggest 7E) 8. Chemical exposure to rail-road ties and telephone poles containing a Carcinogen Creosote wood preservative- have $\mathrm{IH}$ provide proper controls (suggest 7F) 9. Overhead hazards during removal of shoot house in addition to Creosote wood preservative Training section : Add 2-medic first-aid minimal; Industrial Ergonomics; Lead Competent person: Excavation competent person; UXO; and must diffrenciate training between zones to identify what a visitor will need to enter zone who will not be conducting work. Assign JSA number 607024 as this is the work order number.
}

MCMANAMON 09LAWRENCE E

OCT. B 06 


\begin{tabular}{|c|c|c|c|}
\hline TUOTT LEE C & 1 & $\begin{array}{l}\text { 12- } \\
\text { OCT- D } \\
06\end{array}$ & no comments \\
\hline $\begin{array}{l}\text { LANDIS } \\
\text { JOSEPH A }\end{array}$ & 1 & $\begin{array}{l}26 \cdot \\
\text { OCT-A } \\
06\end{array}$ & no comment \\
\hline FRITZ KURT D & 1 & $\begin{array}{l}26- \\
\text { OCT- A } \\
06\end{array}$ & \\
\hline $\begin{array}{l}\text { DRIEVER } \\
\text { MIKEL K }\end{array}$ & 1 & $\begin{array}{l}11- \\
\text { OCT- A } \\
06\end{array}$ & \\
\hline $\begin{array}{l}\text { VANDEL } \\
\text { DOUG S }\end{array}$ & 1 & $\begin{array}{l}11 \cdot \\
\text { OCT. A } \\
06\end{array}$ & \\
\hline $\begin{array}{l}\text { MCMANAMON } \\
\text { LAWRENCE E }\end{array}$ & 1 & $\begin{array}{l}17 \cdot \\
\text { OCT-A } \\
06\end{array}$ & \\
\hline $\begin{array}{l}\text { JOLLEY } \\
\text { WENDELL L }\end{array}$ & 1 & $\begin{array}{l}26- \\
\text { OCT- D } \\
06\end{array}$ & \\
\hline
\end{tabular}

$\begin{array}{ll}\text { VDR Number: } & \text { VDR-153756 } \\ \text { Revision Level: } & 1 \\ \text { Project Number: } & 23368-152173 \\ \text { Transmittal Number: } & \text { S-507296-06R.1 } \\ \text { Transmittal Status: } & \text { Mandatory Approval } \\ \text { Line Item: } & 1\end{array}$

Disposition Code::

Final Comments::

A 
F-232 